
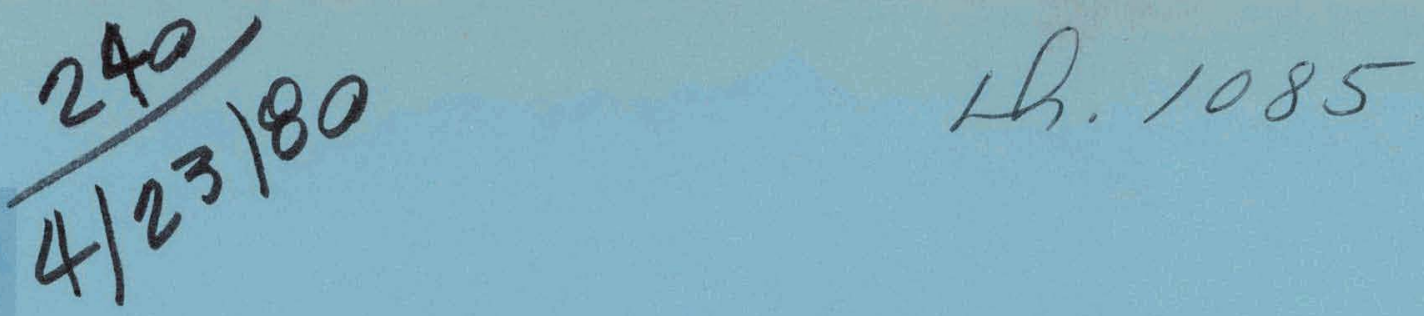

\title{
DATA SUMMARY FOR EVALUATION OF THE TRANSPORT AND DIFFUSION CLIMATOLOGY OF THE UNITED STATES EAST AND GULF COASTS
}

Gilbert S. Raynor and Janet V. Hayes

July 1979

MASTER

\section{ATMOSPHERIC SCIENCES DIVISION DEPARTMENT OF ENERGY AND ENVIRONMENT}

BROOKHAVEN NATIONAL LABORATORY ASSOCIATED UNIVERSITIES, INC.

UNDER CONTRACT NO. EY-76-C-02-0016 WITH THE

UNITED STATES DEPARTMENT OF ENERGY 


\section{DISCLAIMER}

This report was prepared as an account of work sponsored by an agency of the United States Government. Neither the United States Government nor any agency Thereof, nor any of their employees, makes any warranty, express or implied, or assumes any legal liability or responsibility for the accuracy, completeness, or usefulness of any information, apparatus, product, or process disclosed, or represents that its use would not infringe privately owned rights. Reference herein to any specific commercial product, process, or service by trade name, trademark, manufacturer, or otherwise does not necessarily constitute or imply its endorsement, recommendation, or favoring by the United States Government or any agency thereof. The views and opinions of authors expressed herein do not necessarily state or reflect those of the United States Government or any agency thereof. 


\section{DISCLAIMER}

Portions of this document may be illegible in electronic image products. Images are produced from the best available original document. 
BNL 51098

UC-11

(Environmental Control Technology and

Earth Sciences - TID-4500)

\section{DATA SUMMARY FOR EVALUATION OF THE TRANSPORT AND DIFFUSION CLIMATOLOGY OF THE UNITED STATES EAST AND GULF COASTS}

Gilbert S. Raynor and Janet V. Hayes

July 1979

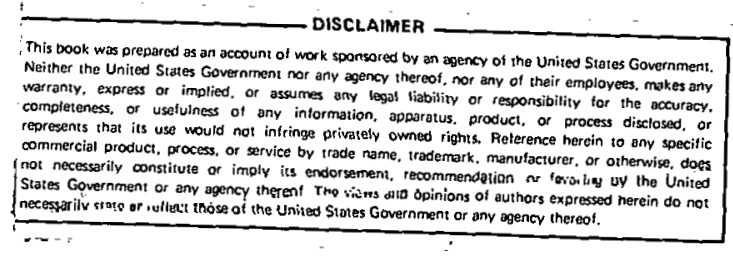

ATMOSPHERIC SCIENCES DIVISION DEPARTMENT OF ENERGY AND ENVIRONMENT

BROOKHAVEN NATIONAL LABORATORY ASSOCIATED UNIVERSITIES, INC. UPTON, NEW YORK 11973 


\section{NOTICE}

This rcport was preparcd as alt acrount of wark sponsored by the United States Government. Neither the United States nor the United States Departunent of Eneigy (DOE), nnr any of their employees, nor any of their contractors, subcontractors, or their employees, makes any warranty, express or implior, nr assumes any legal liability or respuisibility fur ilic accuracy, rompleteness or usefulness of any information, apparatus, product or process disclosed, or reprèsents llät its ube wishlil not infringe. privately owned rights.

Printed in the United States of America

$$
\text { Available from }
$$

National Teclinical Information Service

$$
\begin{aligned}
& \text { U.S. Department of Commerce } \\
& 5285 \text { Port Royal Road }
\end{aligned}
$$$$
\text { Springfield, VA } 22161
$$

Price: Printed Copy $\$ 1450$; Microfiche $\$$

$$
A 20
$$




\section{ABSTRACT}

A study of the atmospheric transport and diffusion climatology of the United States east and Gulf coasts was conducted as part of a larger coastal meteorology and diffusion program to aid in planning and site selection. Synoptic data were obtained from thirty coastal stations from Maine to Texas and analyzed in terms of conditions important to emission transport and diffusion. The thirty stations included four pairs with one of each pair at a greater distance from the coast than the other but near the same latitude.

For each station, wind directions were classified into eight groups with reference to orientation of the local coastline. For some studies, these were combined into three groups--onshore, alongshore and offshore. Wind speeds were divided into four classes. A diffusion class for each observation was computed by a modified Pasquill method. This gave eight classes which were combined into three--unstable, neutral and stable--for some studies. A diffusion rating was derived from combinations of wind speed and diffusion class ranging from very good to very poor. Finally, the joint frequency distributions of wind direction and diffusion rating were calculated for each station. Data were then classified by season, time of day, wind direction, wind speed, diffusion class and combinations of these variables and the percent of hours in each subgroup determined. Results are presented in a series of tablco. 
Table 1. Description of stations. . . . . . . . . . . . . . . . . 9

Table 2. Diffusion rating by wind speed and stability class......... . 20

Tables 3.1 to 3.30. Percent of hours in each combined wind direction class by season and time of day at 30 stations. . . . . . . . . . . . . 11

Tables 4.1 to 4.30 . Percent of hours in each of the combined stability classes by combined wind direction classes and wind speed at 30 stations. . . . 41

Tables 5.1 to 5.30. Percent of hours in each of the elght stability classes by the combined wind dirertion classes, season and time of day at 30 stations. 71

Tables 6.1 to 6.30 . Percent of hours in each wind speed class by combined wind direction classes, season and time of day at 30 stations. . . . . . . 191

Tables 7.1 to 7.30. Percent of hours in each diffusion class by combined wind direction classes, season and time of day at 30 stations. . . . . . . 311

Tables 8.1 to 8.30. Percent of hours in each combination of wind direction class and diffusion rating class at 30 stations. . . . . . . . . . . 431

\section{LIST OF FIGURES}

Figure 1. Map of the study area showing Brookhaven National Laboratory (BNL) and the stations from which data were used. Station names are given

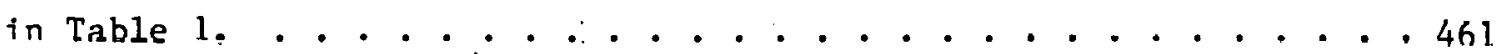

Figure 2. Number and designation of wind direction sectors relative to the coastline. 
INTRODUCTION

Coastal locations are used for a variety of installations and activities which are actual or potential sources of atmospheric pollutants. Additional facilities and activities are continually being proposed. Among the current and proposed installations are both floating and shore-based nuclear power plants, fossil-fueled power plants, industries and refineries. Activities include oildrilling, shipment of fuel and transport of hazardous material. Such facilities and activities are commonly concentrated in coastal locations because of proximity to population centers, access to transportation and availability of cooling water.

In the past, sites for such facilities and activities have generally been selected on the basis of economic or political considerations with little effort to select locations which would minimize the effects of airborne emissions. In recent years, environmental impact studies are often required after a site has been selected, but usually these merely document, more or less adequately, existing conditions. In a few cases, particularly for nuclear installations, impact studies may affect the design or operating requirements of the plant, but seldom the location. Thus, studies of diffusion are rarely made as part of the site selection process.

This study is part of a larger study of coaslal meteorology and diffusion ${ }^{1-3}$ and was planned to assist in site selection by describing the transport and diffusion climatology of the United States east and Gulf coasts in as much detail as can be extracted from readily available meteorological data. S1te specific studies may still be necessary for particular locations especially if the site is not representative of the coastline in the region, but such: studies could be based on and guided by the information presented here. In 
other cases, the information below may be adequate to guide a choice between potential sites with respect to air pollution transport and diffusion. No consideration was given in this study to other environmental, economic or political conditions that may also be important in site selection although a planned extension of this study will rate each section of coastline for severe weather potential. The effects on atmospheric diffusion of meteorological processes in coastal zones were discussed earlier. ${ }^{4}$ A prior report ${ }^{5}$ briefly described this study and presented some preliminary results.

AREA

The area covered in this study is the United States easL and Gulf coasts. from Maine to Texas. The inland extent of the coastal zunt is conoidered rn lut that distance to which sea breezes normally penetrate, about $30 \mathrm{~km}$ in the north and $60 \mathrm{~km}$ in the south. However, boundaries are somewhat indefinite due to the irregular configuration of the coast and the presence of partially or completely enclosed bodies of water such as bays, sounds, harbors and estuaries. The seaward extent of the coastal zone is even lüre indefinite, but conditions measured on land should probably not be extrapulated.morc than $10 \mathrm{~km}$ offshore without appropriate adjustments, particularly in wind speed and stability.

The region studied is all within the coastal plain and is generally characterized by flat beaches and very gentle slopes inland exrept in New England where the coast is more rugged and the terrain hilly cluse to the sid. Local geography should be considered in applying or extrapolating results presenced below.

\section{VARIABLES}

Meteorological variables of primary concern in this study are those which govern or influence transport and diffusion of airborne gases and particles. 
The most important are wind direction and speed and some measure of diffusive capacity such as turbulence, gustiness or lapse rate. Other pertinent parameters include amount, type and height of clouds, solar radiation, air, land and water temperatures, humidity and precipitation. Other important variables are time of year, time of day, latitude, local geography, topography and surface roughness. Not all of these variables were included in the data available and some that were available were used indirectly, to determine a stability category, for instance.

\section{DATA}

Data for the portion of the study reported here were obtained from the National Climatic Center and consist of hourly or three-hourly synoptic observa-. tions usually for consecutive two-year periods from 30 stations. It was not possible to obtain the same two-year period for all stations since all were not operative at the same time and not all data taken at the stations were available on tape. The years 1970-1971 were obtained for most stations.

The stations selected are shown in Figure 1 and listed in Table 1 with their designation, latitude, longitude, elevation, angle of nearest coastline and period of data. Since these weather stations are at varying distances from the coast, the distance to the nearest large body of water (usually a bay) and the distance to the open occan or Gulf are also tabulated.

Stations were selected to give a reasonably continuous and evenly spaced. distribution along the coast while avoiding non-representative locations as much as possible. Moreover, selection was limited to those stations for which data on tape was available.

Eight stations were chosen to give four pairs for comparison between. a coastal station and another somewhat further inland. The pairs are Boston and 
Bedford, Massachusetts; Belmar and Lakehurst, New Jersey; Cape Kennedy and orlando, Florida; and Galveston and Houston, Texas. The same years of data were obtained for both stations in each pair.

The synoptic data normally include station designation, date and time, type, height and amount of clouds in as many as four layers, total sky cover, ceiling height, visibility, weather and/or obstructions to vision, sea level and station barometric pressure, dry bulb and wet bulb air temperature, dew point, relative humidity, wind diriection and wind speed. Most data were three-hourly observac1ons. Table liootnotes those years with 24-liuurly data or 16 to 18hourly data. In the latter case observations were not taken during nighttime hours.

ANALYTICAL METHODS

Synoptic data were converted to forms most useful for the purposes of this study. Wind directions were divided into efght numbered classes relative to the coastline nearest to the station (Figure 2): For some analyses, these eight classes were combined into three: onshore, alongshore and uffsliure. In oome cases (e.8., IMH, ACK, ORF, ILAT, BVE and MSY) cnastal waters were present in two directions from the station due to a location on an island, on a penninsula or near a bend in the coast. In these cases, the nearest coast representative of the open ocean or Gulf was chosen. The coastal oriencation chosen is lieted in Tahle 1.

Wind speeds were changed from knots to $\mathrm{m} \mathrm{sec}^{-1}$ and grouped into five classes, calm, $0.5-2.4,2.5-4.9,5.0-9.9$ and $\geq 10.0 \mathrm{~m} \sec ^{-1}$

A stability class was calculated for each observation using a modification of the Pasquill-Gifford method described by Turner ${ }^{6}$ by modifying the STAR computer program obtained from the National Weather Service. The original program 
uses date, time of day, geographic location, total sky cover, ceiling height and wind speed to compute a stability-class. Modifications used here were described earlier. 7 The eight stability classes computed are designated extremely unstable, unstable, slightly unstable, neutral day, neutral night, slightly stable, stable and extremely stable. For some analyses, the eight classes were combined into three: unstable, neutral and stable.

For classifying the data by season, four classes were formed: spring, March-May; summer; June-August ; fa11, September-November; winter, DecemberFebruary.

For classifying data byctimerófdday, two classes were formed, day and night. In order to obtaint the ibest =agreement between these classes and atmospheric stability, day'isc considered the period from one hour after sunrise to one hour before.sunset as used in the STAR program.

A diffusion rating classification was derived from a combination of the wind speed and stability classes as shown in Table 2. Diffuston rating classes were determined from ranges of dilution factors $\left(\bar{u}, \sigma_{y}, \sigma_{z}\right)$ at $1 \mathrm{~km}$ as shown at the bottom of the table. These divisions agreed well with subjective determinations of several meteorologists experienced in diffusion studies. Values of $\sigma_{y}$ and $\sigma_{z}$ at $1 \mathrm{~km}$ were taken from Turner ${ }^{6}$. Some combinations of wind speed and stability may rarely or never occur, but all are filled in for completeness. The diffusion rating was used as another variable.

Finally, the joint frequency of the wind direction classes and the diffusion rating classes was computed for each station.

For each station, percentage frequency distributions were computed for selected variables or combinations of variables. Results are presented in a series of tables described below. 


\section{RESULTS}

Results: presented here are derived from the two years of data averaged. Results are presented in six groups of 30 similar tables, one for each station. Within each group, stations are arranged in order along the coast from Maine to Texas.

The percent of hours in each of the three combined wind direction classes and the percent with calm winds are presented in tables 3.1 to 3.30 by season and time of day.

The percent of hours in each of the three combined stability classes by the combined wind direction classes and wind speed is tabulated in tables 4.1 to 4.30 for all hours with non-calm wind speeds.

The percent of hours in each of the eight stability classes by the comblned wind direction classes, season and time of day is shown in tables 5.1 to 5.30 for all non-calm hours.

The percent of hours in each wind speed class by the combined wind direction classes, season and time of day is listed in tables 6.1 to 6.30 for all HuH"'calm livurs.

The percent of hours in each diffusion rating class. by combined wind direction class, season and time of day is given in tables 7.1 to 7.30 .

The joint percentage frequency of hours in each combination of 'wind direćtion class and diffusion rating class is given in tables 8.1 to 8.30 .

More detailed results were computed and are available for inspection, but are not included here. A11 computations were made for the two years separately as well as for the two years combined. All computations were made for the eight wind direction sectors as well as for the three combined sectors. All computations were made for the eight stability classes as well as for the three 
combined classes. Numerical frequencies as well as percentage frequencies were computed for all cases.

\section{APPLICATION}

The results presented here are arranged for easy use with diffusion models in which the primary meteorological inputs are wind speed and measures of lateral and vertical diffusion. The latter-can readily be derived from the stability classes by established relationships. Presentation of data by season, time of day and wind direction classes permits ready selection of the other meteorological parameters for any desired combination of conditions. Interpolation can be employed for locations between stations. Use of the information in tables.7.1 to 7.30 and 8.1 to 8.30 may permit preliminary site evaluation without diffusion modeling.

\section{FUTURE WORK}

Additional studies are planned as part of this investigation. An attempt will be made to determine and classify diffusion conditions over the water which may be quite different from those over land. Data from lightships and other sources will be used. The frequency of several types of severe weather will be determined as a function of location along the coast. Data from BNL and several outlying stations will be analyzed to fill in the gap between New England and New Jersey and to compare results from an east-west coastline with those from nearly north-south coastlines. Certain data included in this report will be the subjects of one or more analytical papers. 
REFERENCESS

1. Raynor, G.S. Michael, P., Brown, R.M. andí SèthuRaman-S.e, Studies of atmospheric diffusion from a nearshore oceanic siteis. J.? Appl. Meteor. 14: 1080-1094. 1975.

2. Raynor, G.S., Brown, R.M. and SethuRaman, S., A comparison of diffusionfrom a small island and an undisturbed ocean site. J. App1. Meteor. 17: 129-139. 1978.

3. Raynor, G.S., SethuRaman, S. and Brown, R.M., Formation and characteristics of coastal internal boundary layers during onshore flows. Boundary Layer Meteor., in press. Report BNL 25115, Brookhaven National Laboratory, Upton, NY. 1979.

4. Raynor, G.S., Effects on atmospheric diffusion of meteorological processes in coastal zones. pp. 199-212 in: Air quality meteorology and atmospheric ozone. A.L. Morris and R.C. Barras (eds.) ASTM Special Tech. Pub. 653, Amer. Soc. for Testing and Materials, Phil., PA. 1978.

5. Raynor, G.S. and Hayes, J.V., A study of the transport and diffusion climatology of the U.S. east coast. p. 110-111 in preprint vol. Amer. Metcor. Soc. Conf. on Coastal Meteor. Sept. 21-23, 1976, Virginia Beach, VA, Amer. Meteor. Soc., Boston, MA, 1976.

6. Turner, D.B., $\Lambda$ diffusion model for an urban area. J. Appl. Meteor. $3: 83-91 . \quad 1964$.

7. Raynor, G. $S_{0}$ and Hayes, $J_{0} V_{0}$, A comparative study of diffusion classifleation by lapse rate, gustiness and a modified Pasquill method. Proc. NATO/CCMS 9th Internat. Tech. Meeting on Air Pollution Modelling and its Application, Aug. 28-31, 1978, Toronto, Can. 1978. 
Table 1

Description of Stations

\begin{tabular}{|c|c|c|c|c|c|c|c|c|c|}
\hline Station & $\begin{array}{l}\text { Desig- } \\
\text { nation }\end{array}$ & Lat. & Long. & Elev. (m) & $\begin{array}{l}\text { Distance } \\
\text { Nearest } \\
\text { Water }\end{array}$ & $\begin{array}{l}(k m) \text { to } \\
\text { Open } \\
\text { Coast }\end{array}$ & $\begin{array}{l}\text { Direction of } \\
\text { Coast (degrees) }\end{array}$ & Period & of Data \\
\hline Portland, ME & PWM & $43^{\circ} 39^{\prime}$ & $70^{\circ} 19^{\prime}$ & 19.0 & 1.3 & 8.8 & $028-208$ & 1970 & 1971 \\
\hline Boston, MA & BOS & $42^{\circ} 22^{\prime}$ & $71^{\circ} 02^{\prime}$ & 4.6 & 3.8 & 10.2 & $354-174$ & 1969 & 1970 \\
\hline Bedford, MA & BED & $42^{\circ} 28^{\prime}$ & $71^{\circ} 17^{\prime}$ & 43.6 & 25.4 & 31.8 & $354-174$ & $1969 *$ & $1970 *$ \\
\hline Falmouth, MA & FMH & $41^{\circ} 39^{\prime}$ & $70^{\circ} 31^{\prime}$ & 41.8 & 8.3 & 11.4 & $076-256$ & $1968 *$ & $1969 *$ \\
\hline Nantucket, MA & ACK & $41^{\circ} 15^{\prime}$ & $70^{\circ} 04^{\prime}$ & 3.7 & 0.4 & 0.4 & $271-091$ & 1968 & 1969 \\
\hline Belmar, NJ & BLM & $40^{\circ} 11^{\prime}$ & $74^{\circ} 04^{\prime}$ & 25.6 & 1.3 & 5.1 & $012-192$ & $1960 *$ & 1970 \\
\hline Lakehurst, NJ & NEL & $40^{\circ} 02^{\prime}$ & $74^{\circ} 20^{\prime}$ & 36.9 & 16.6 & 24.1 & $010-190$ & $1960 *$ & 1970 \\
\hline Atlantic City, NJ & ACY & $39^{\circ} 27^{\prime}$ & $74^{\circ} 35^{\prime}$ & 20.4 & 8.8 & 16.6 & $040-220$ & 1970 & 1971 \\
\hline Wilmington, DE & ILG & $39^{\circ} 40^{\prime}$ & $75^{\circ} 36^{\prime}$ & 24.1 & 2.6 & 96.5 & $037-217$ & 1970 & 1971 \\
\hline Wallops Island, Va & WAL & $37^{\circ} 51^{\prime}$ & $75^{\circ} 29^{\prime}$ & 2.1 & 0.2 & 0.2 & $032-212$ & 1970 & 1971 \\
\hline Norfolk, VA & ORF & $36^{\circ} 53^{\prime}$ & $76^{\circ} 12^{\prime}$ & 9.1 & 6.4 & 17.8 & $000-180$ & 1970 & 1971 \\
\hline Cape Hatteras, NC & HAT & $35^{\circ} 16^{\prime}$ & $75^{\circ} 33^{\prime}$ & 7.6 & 0.0 & 2.6 & $046-226$ & 1970 & 1971 \\
\hline Wilmington, NC & ILM & $34^{\circ} 14^{\prime}$ & $77057^{\prime}$ & 14.0 & 11.4 & 13.4 & $033-2.13$ & 1970 & 1971 \\
\hline Charleston, SC & CHS & $32^{\circ} 54^{\prime}$ & $80^{\circ} 02^{\prime}$ & 14.0 & 17.8 & 25.4 & $056-236$ & 1970 & 1971 \\
\hline Brunswick, GA & SSI & $310^{\circ} 5^{\prime}$ & $81^{\circ} 28^{\prime}$ & 9.5 & 6.3 & 8.7 & $016-196$ & 1970 & 1971 \\
\hline Cape Kennedy, FL & KEN & $28^{\circ} 29^{\prime}$ & $80^{\circ} 33^{\prime}$ & 4.9 & 1.0 & 1.0 & $340-160$ & 1970 & 1971 \\
\hline Orlando, FL & ORL & $28033^{\prime}$ & $81^{\circ} 20^{\prime}$ & 33.5 & 50.8 & 63.6 & $340-160$ & 1970 & 1971 \\
\hline Miam1, FL & MIA & $25^{\circ} 49^{\prime}$ & $80^{\circ} 17^{\prime}$ & 7.3 & 2.4 & 15.8 & $027-207$ & 1970 & 1971 \\
\hline Ft. Myers, FL & FMY & $26^{\circ} 34^{\prime}$ & $81^{\circ} 52^{\prime}$ & 6.1 & 12.7 & 16.6 & $329-149$ & 1970 & 1971 \\
\hline Tampa, FL & TPA & $27^{\circ} 58^{\prime}$ & $82^{\circ} 32^{\prime}$ & 11.0 & 1.3 & 29.3 & $350=170$ & 1970 & 1971 \\
\hline Tallahassee, FL & TLH & $30^{\circ} 26^{\prime}$ & $84^{\circ} 20^{\prime}$ & 20.7 & 42.0 & 42.0 & $084-264$ & 1970 & 1971 \\
\hline Pensacola, FL & PNS & $30^{\circ} 28^{\prime}$ & $87^{\circ} 12^{\prime}$ & 35.4 & 2.6 & 16.6 & $080-260$ & 1970 & 1971 \\
\hline Mob1le, AL & MOB & $300^{\prime} 1^{\prime}$ & $88^{\circ} 14^{\prime}$ & 66.1 & 17.9 & 48.3 & $092-272$ & 1970 & 1971 \\
\hline B1lox1, MS & BIX & $30^{\circ} 24^{\prime}$ & $88^{\circ} 55^{\prime}$ & 7.9 & 0.6 & 19.1 & $083-263$ & $1969 * \star$ & $1970 * \star$ \\
\hline New Orleans, LA & MSY & $29059^{\prime}$ & $90^{\circ} 15^{\prime}$ & 2.1 & 6.4 & 76.3 & $084-264$ & 1972 & 1973 \\
\hline Boothville, LA & BVE & $290^{\prime} 0^{\prime}$ & $89^{\circ} 24^{\prime}$ & 0.9 & 2.5 & 15.3 & $095-275$ & 1972 & 1973 \\
\hline Lake Charles, LA & $\mathrm{LCH}$ & $30^{\circ} 07^{\prime}$ & $93^{\circ} 13^{\prime}$ & 4.3 & 10.1 & 36.8 & $090-270$ & 1970 & 1971 \\
\hline Galveston, TX & GLS & $29^{\circ} 16^{\prime}$ & $94^{\circ} 51^{\prime}$ & 2.7 & 1.3 & 1.3 & $051-231$ & $1961 *$ & $1962 *$ \\
\hline Houston, $\mathrm{TX}$ & , HOU & $29^{\circ} 39^{\prime}$ & $95^{\circ} 17^{\prime}$ & 18.9 & 25.4 & .59 .7 & $051-231$ & 1961 * & $1962 *$ \\
\hline Brownsville, TX & BRO & $25^{\circ} 55^{\prime}$ & $97^{\circ} 28^{\prime}$ & 6.1 & 21.6 & 31.9 & $000-180$ & 1970 & 1971 \\
\hline
\end{tabular}

*24 observations per day.

* 16 to 18 observations per day. 
Table 2

Diffusion Rating by Wind Speed and Stability Classes

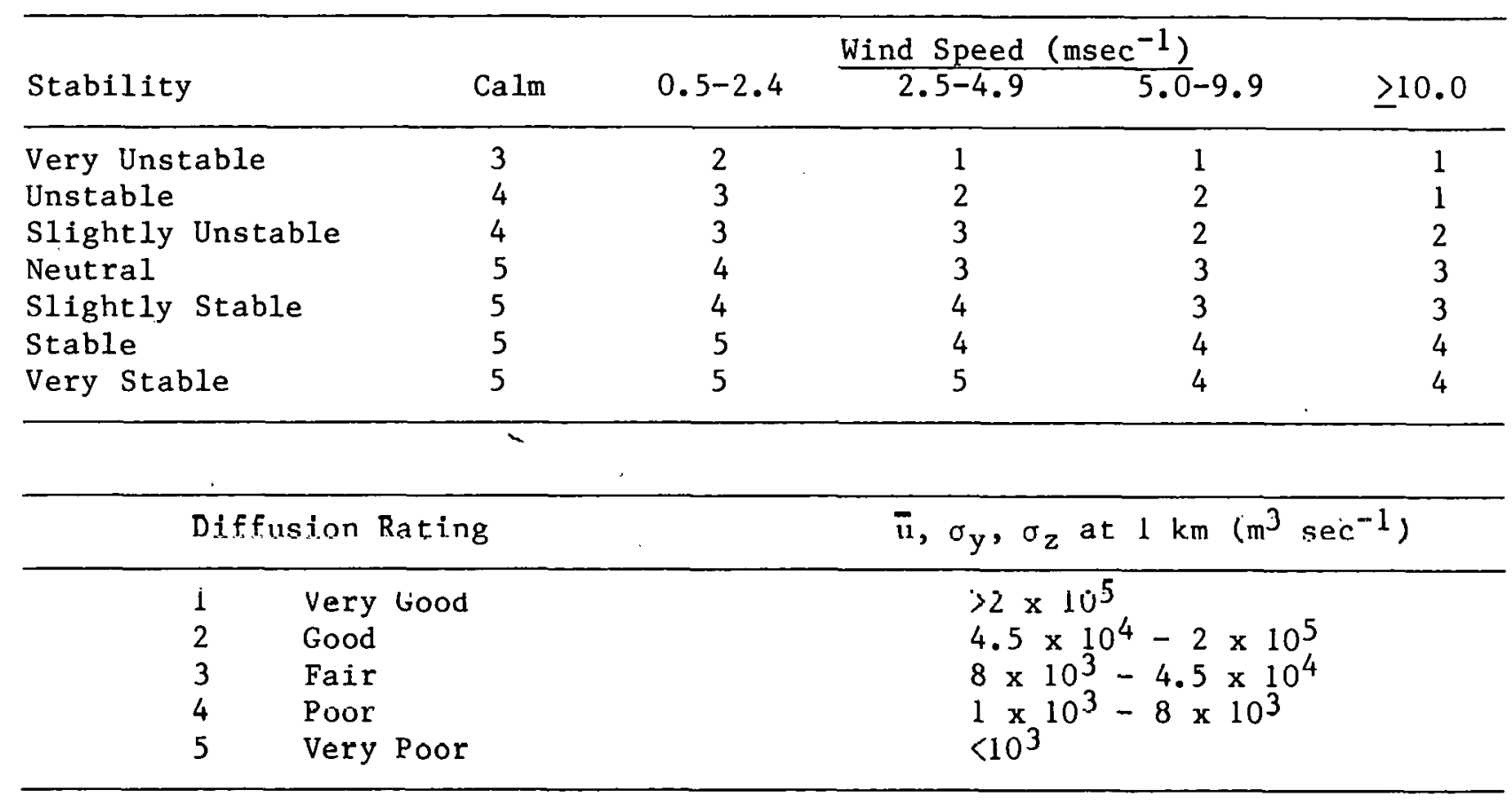




\begin{tabular}{|c|c|c|c|}
\hline Percer & $\begin{array}{r}\text { Tort } \\
\text { of Hours in } \\
\text { by Season }\end{array}$ & $\begin{array}{l}\text { ection } \\
\text { ay }\end{array}$ & \\
\hline Direction & $\begin{array}{c}\text { All } \\
\text { Hours }\end{array}$ & Day & Night \\
\hline \multicolumn{4}{|c|}{ Annual } \\
\hline Onshore & 21.7 & 31.8 & 14.6 \\
\hline Alongshore & 25.2 & 22.9 & 26.8 \\
\hline Offshore & 50.9 & 44.3 & 55.5 \\
\hline Calm & 2.1 & 0.9 & 3.0 \\
\hline \multicolumn{4}{|c|}{ Spring } \\
\hline Onshore & 25.5 & 34.6 & 17.1 \\
\hline Alongshore & 22.2 & 21.8 & 22.6 \\
\hline of $f$ shore & 49.5 & 42.4 & 56.1 \\
\hline Calm & 2.8 & 1.3 & .4 .2 \\
\hline \multicolumn{3}{|c|}{ Summer } & \\
\hline Onshore & 28.1 & 38.2 & 17.9 \\
\hline Alongshore & 27.5 & 22.8 & 32.2 \\
\hline offshore & 43.0 & 38.9 & 47.1 \\
\hline Calm & 1.4 & 0.1 & 2.7 \\
\hline \multicolumn{4}{|c|}{ Fall } \\
\hline Onshore & 22.7 & 31.9 & 17.1 \\
\hline Alongshore & 28.1 & 27.0 & 28.8 \\
\hline offshore & 47.2 & 40.6 & 51.2 \\
\hline Calm & 2.0 & 0.5 & 2.9 \\
\hline \multicolumn{4}{|c|}{ Winter } \\
\hline Onshore & 10.3 & 15.4 & 8.3 \\
\hline Alongshore & 23.1 & 19.4 & 24.5 \\
\hline offshore & 64.3 & 62.9 & 64.8 \\
\hline $\mathrm{Ca} 1 \mathrm{~m}$ & 2.4 & 2.2 & 2.4 \\
\hline
\end{tabular}




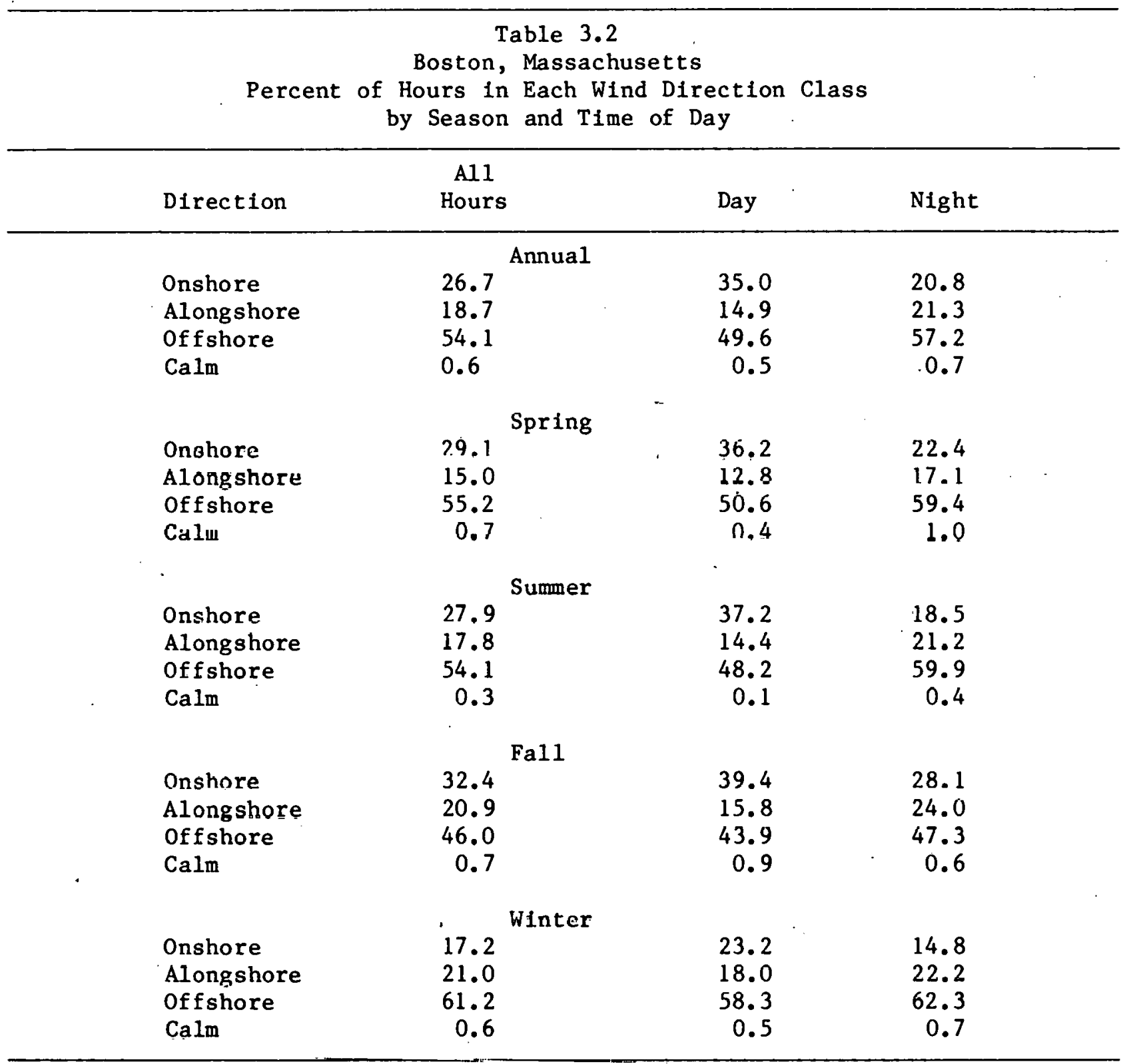




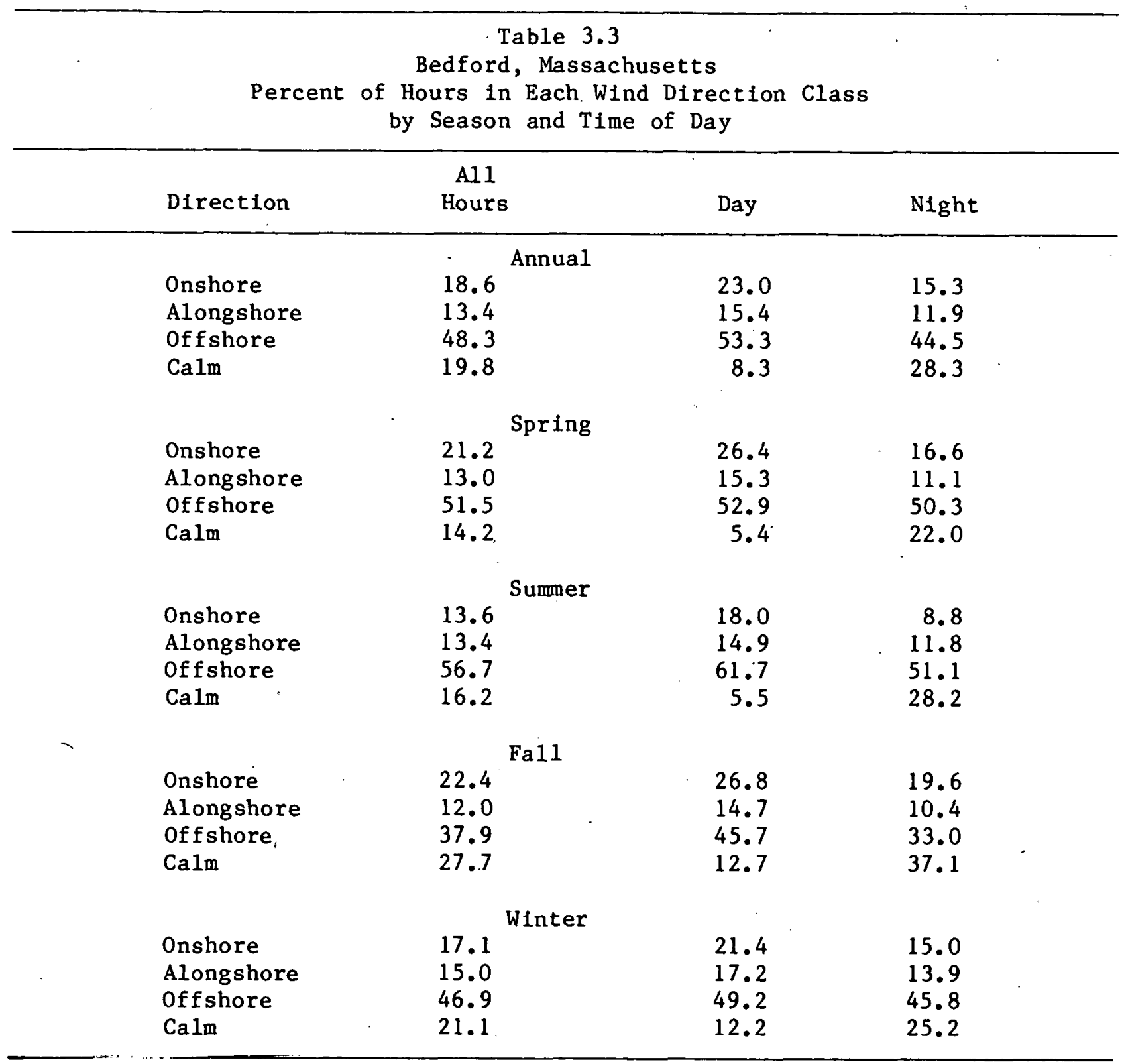




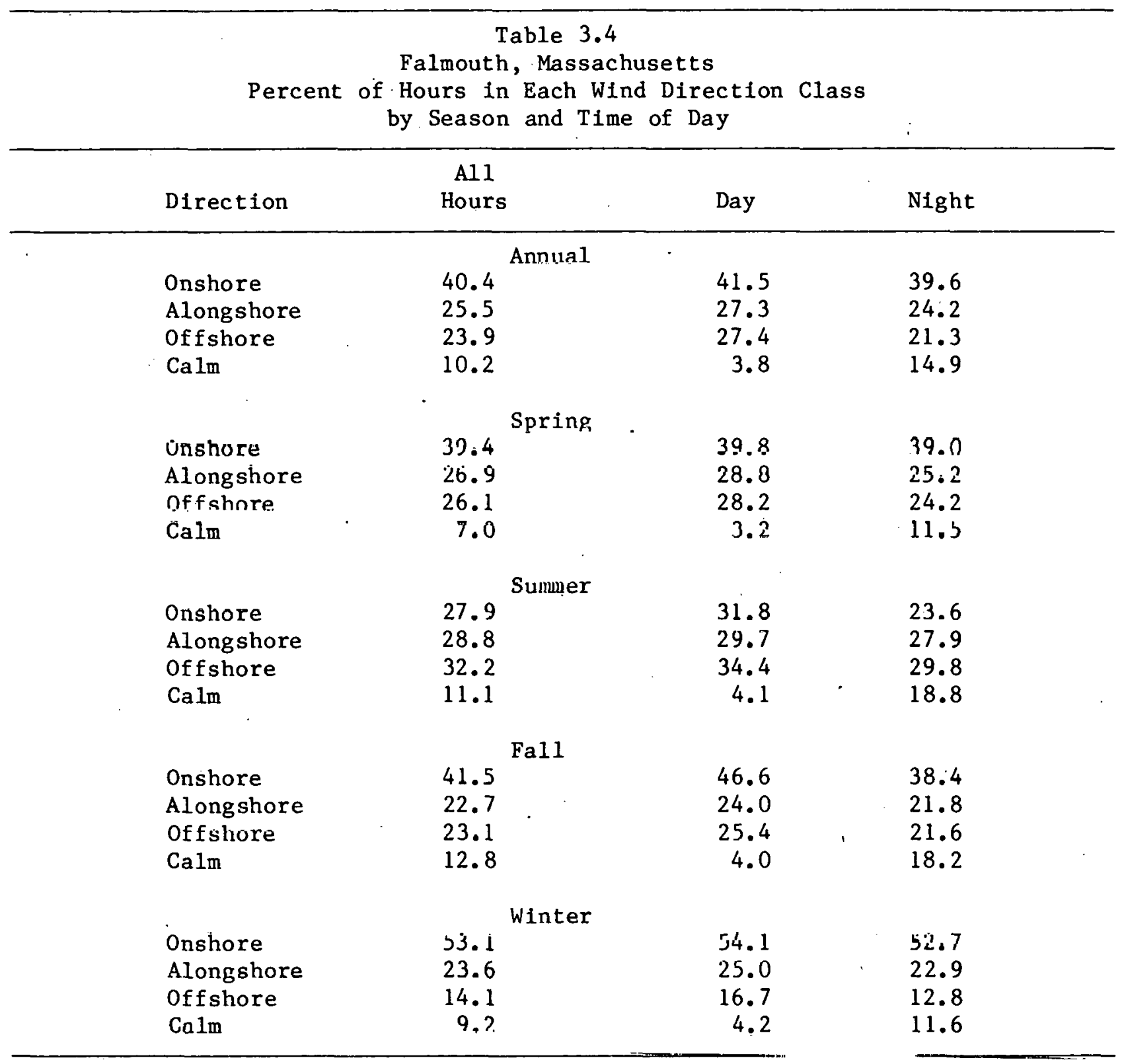


Table 3.5

Nantucket, Massachusetts

Percent of Hours in Each Wind Direction Class

by Season and Time of Day

\begin{tabular}{|c|c|c|c|}
\hline Direction & $\begin{array}{c}\text { Al1 } \\
\text { Hours }\end{array}$ & Day & Night \\
\hline \multicolumn{4}{|c|}{ Annual } \\
\hline Onshore & 40.4 & 38.2 & 42.1 \\
\hline Alongshore & 24.7 & 23.8 & 25.3 \\
\hline Offshore & 34.3 & 37.9 & 31.7 \\
\hline Calm & 0.6 & 0.1 & 1.0 \\
\hline \multicolumn{4}{|c|}{ Spring } \\
\hline Onshore & 37.6 & 36.5 & 38.8 \\
\hline Alongshore & 23.6 & 23.3 & 23.8 \\
\hline Offshore & 38.5 & 40.2 & 36.8 \\
\hline $\mathrm{Calm}$ & 0.3 & 0.0 & 0.7 \\
\hline \multicolumn{4}{|c|}{ Summer } \\
\hline Onshore & 25.8 & 27.9 & 23.8 \\
\hline Alongshore & 20.4 & 19.0 & 21.7 \\
\hline Offishore & 52.8 & 53.0 & 52.5 \\
\hline Calm & 1.0 & 0.1 & 1.9 \\
\hline \multicolumn{4}{|c|}{ Fal1 } \\
\hline Onshore & 42.3 & 43.4 & 41.6 \\
\hline Alongshore & 29.7 & 27.6 & 31.3 \\
\hline Offshore & 27.4 & 28.8 & 26.2 \\
\hline Calm & 0.6 & 0.2 & 0.9 \\
\hline \multicolumn{4}{|c|}{ Winter } \\
\hline Onshore & 56.2 & 52.5 & 57.7 \\
\hline Alongshore & 25.7 & 28.2 & 24.7 \\
\hline Offshore & 17.6 & 19.0 & 17.0 \\
\hline Calm & 0.5 & 0.2 & 0.6 \\
\hline
\end{tabular}




\begin{tabular}{|c|c|c|c|}
\hline \multicolumn{4}{|c|}{$\begin{array}{c}\text { Percent of Hours in Each Wind Direction Class } \\
\text { by Season and Time of Day }\end{array}$} \\
\hline Direction & $\begin{array}{c}\text { All } \\
\text { Hours }\end{array}$ & Day & Night \\
\hline \multicolumn{4}{|c|}{ Annual } \\
\hline $\begin{array}{l}\text { Onshore } \\
\text { Alongshore } \\
\text { Offshore } \\
\text { Calm }\end{array}$ & $\begin{array}{l}21.0 \\
21.3 \\
46.8 \\
10.9\end{array}$ & $\begin{array}{r}26.3 \\
23.8 \\
43.6 \\
6.4\end{array}$ & $\begin{array}{l}17.3 \\
.19 .4 \\
49.1 \\
14.2\end{array}$ \\
\hline \multicolumn{4}{|c|}{ Spring } \\
\hline $\begin{array}{l}\text { Onshore. } \\
\text { Alongshore } \\
\text { Offohore } \\
\text { Calm }\end{array}$ & $\begin{array}{r}26.9 \\
18.0 \\
45.5 \\
9.6\end{array}$ & $\begin{array}{r}33.4 \\
19.5 \\
43.4 \\
3.7\end{array}$ & $\begin{array}{l}21.1 \\
16.8 \\
47.3 \\
14.8\end{array}$ \\
\hline \multicolumn{4}{|c|}{ Summer } \\
\hline $\begin{array}{l}\text { Onshore } \\
\text { Alongshore } \\
\text { Offshore } \\
\text { Calm }\end{array}$ & $\begin{array}{l}15.9 \\
30.5 \\
39.7 \\
13.9\end{array}$ & $\begin{array}{r}22.7 \\
30.8 \\
37.5 \\
9.0\end{array}$ & $\begin{array}{r}8.8 \\
30.1 \\
41.9 \\
19.1\end{array}$ \\
\hline \multicolumn{4}{|c|}{$\mathrm{Fa} 11$} \\
\hline $\begin{array}{l}\text { Onshore } \\
\text { Alongshore } \\
\text { Offshore } \\
\text { Calm }\end{array}$ & $\begin{array}{l}28.3 \\
21.5 \\
39.4 \\
10.8\end{array}$ & $\begin{array}{r}30.0 \\
21.7 \\
38.1 \\
7.2\end{array}$ & $\begin{array}{l}27.2 \\
19.5 \\
40.2 \\
13.0\end{array}$ \\
\hline \multicolumn{4}{|c|}{ Winter } \\
\hline $\begin{array}{l}\text { Onshore } \\
\text { Alongshore } \\
\text { Offshore } \\
\text { Calm }\end{array}$ & $\begin{array}{r}13.0 \\
15.0 \\
62.8 \\
9.2\end{array}$ & $\begin{array}{r}16.5 \\
17.6 \\
61.0 \\
4.9\end{array}$ & $\begin{array}{l}11.4 \\
13.7 \\
63.7 \\
11.2\end{array}$ \\
\hline
\end{tabular}




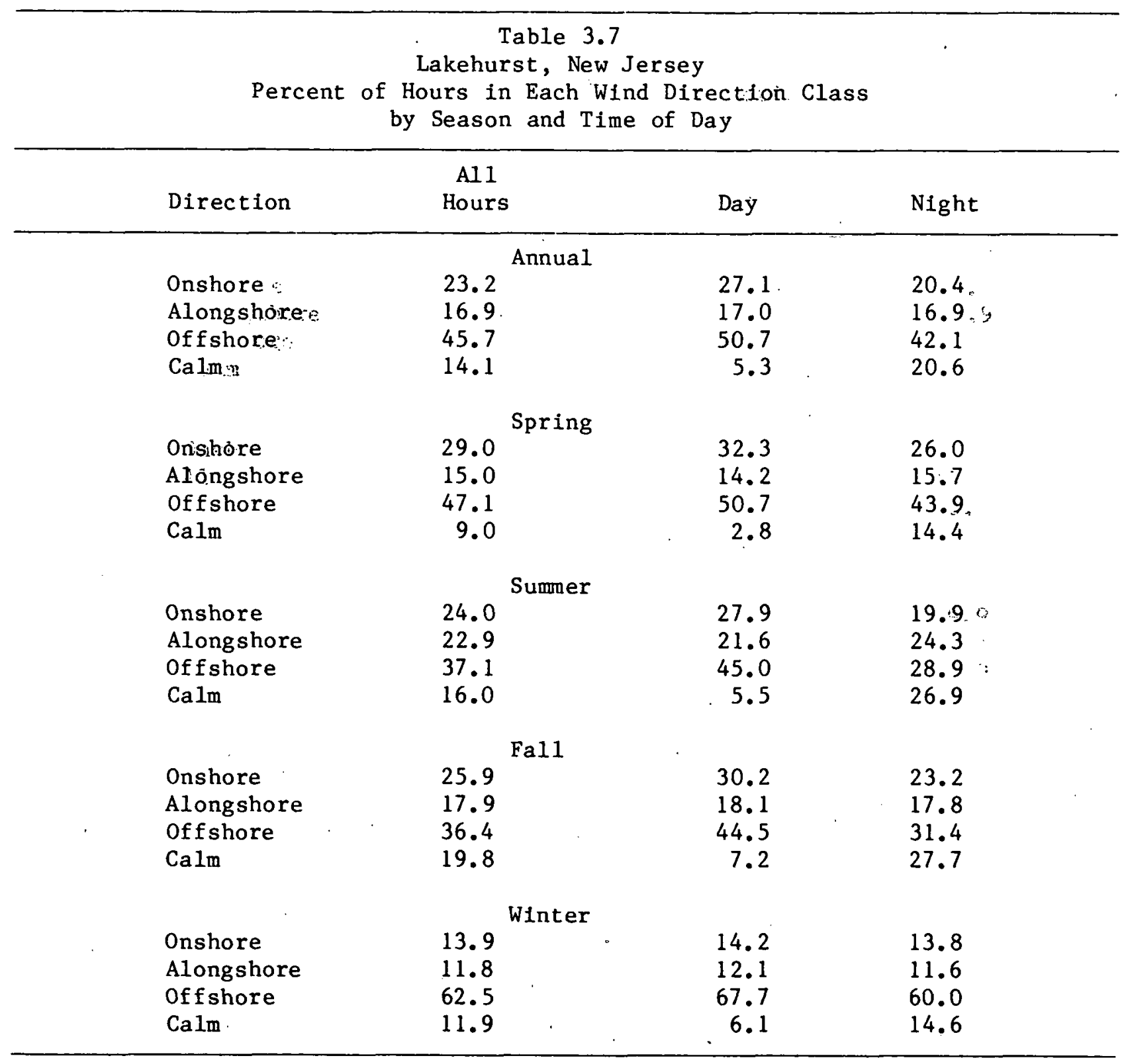


Table 3.8

Atlantic City, New Jersey

Percent of Hours in Each Wind Direction Class

by Season and Time of Day

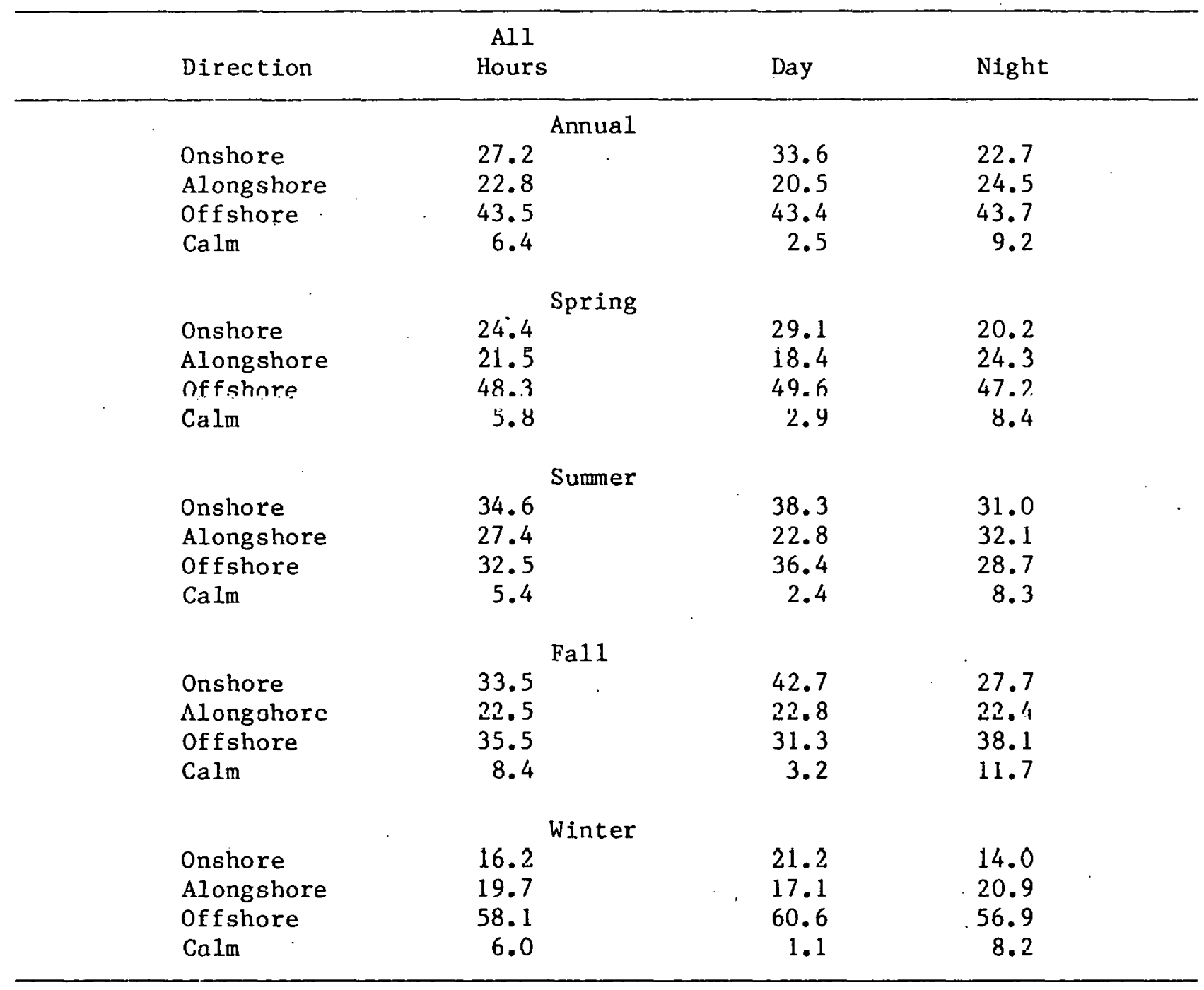




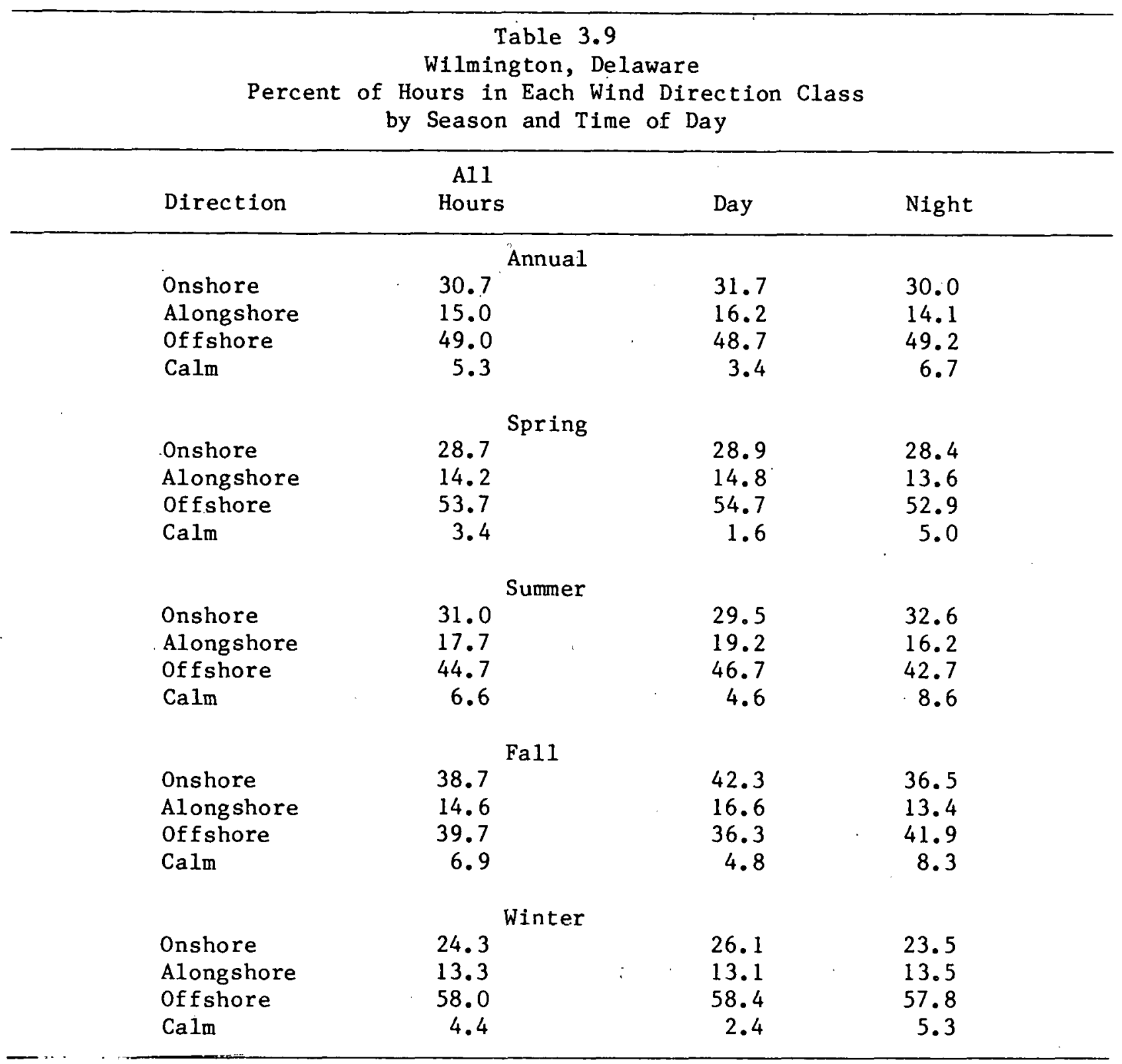




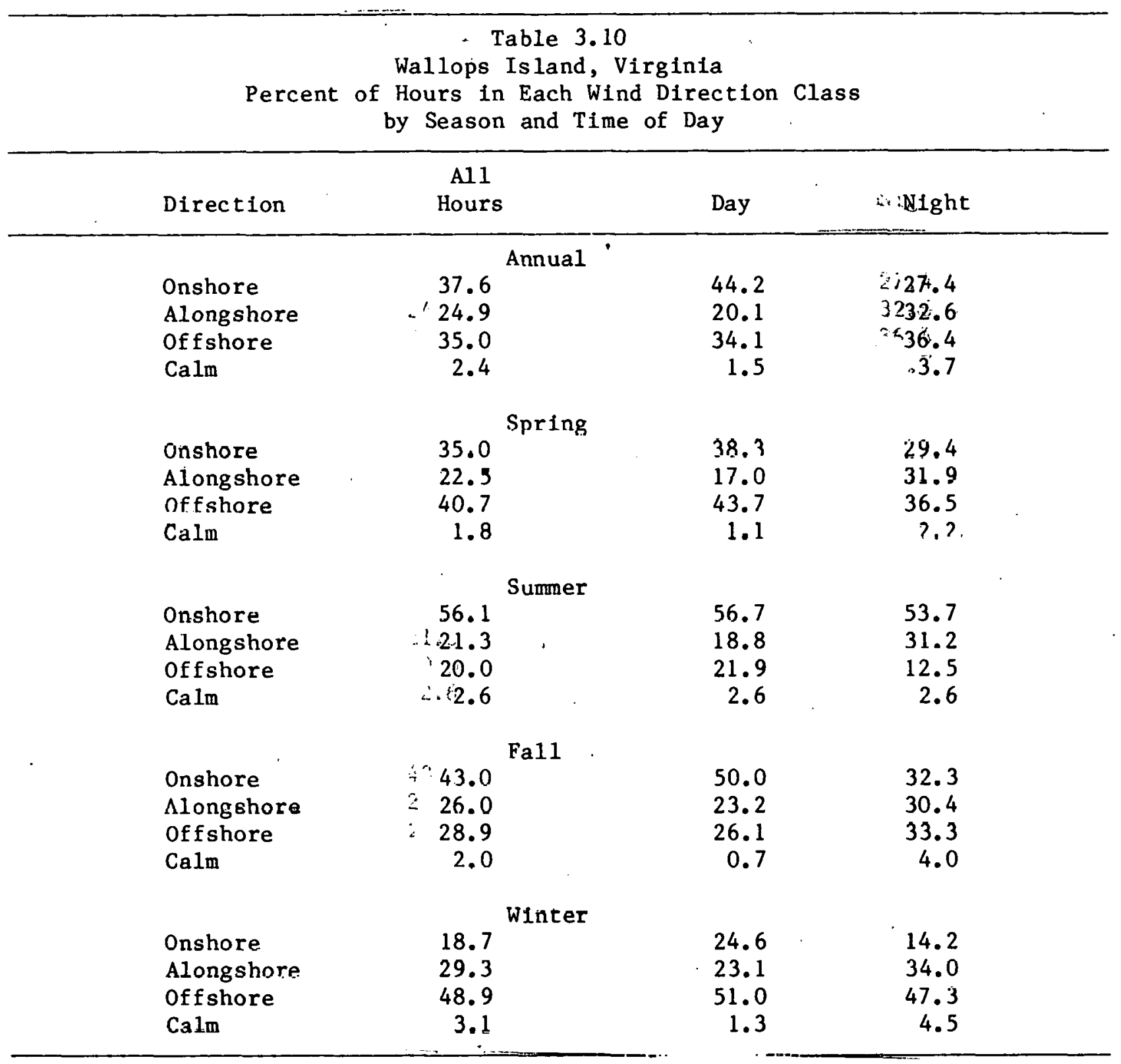




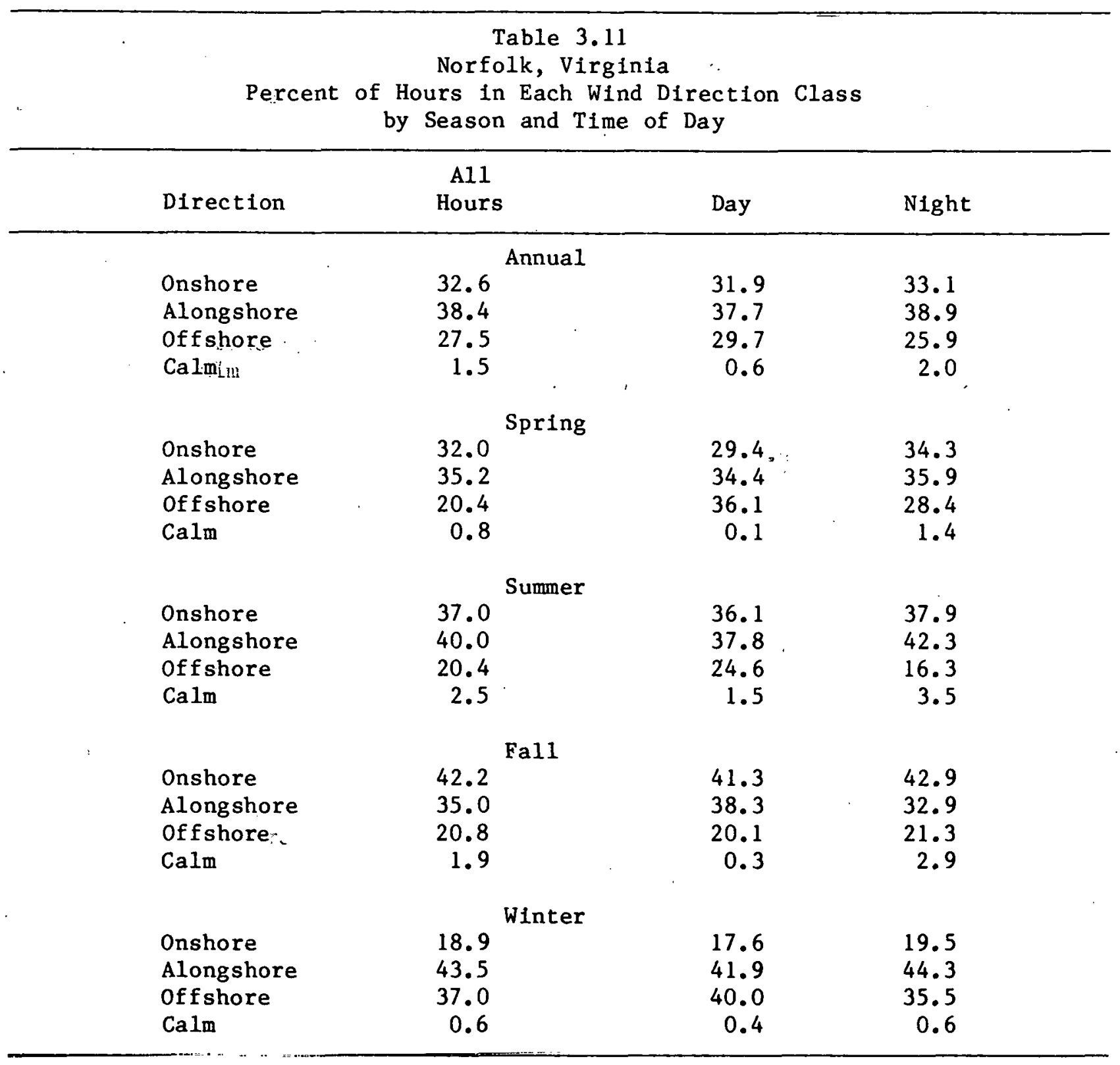




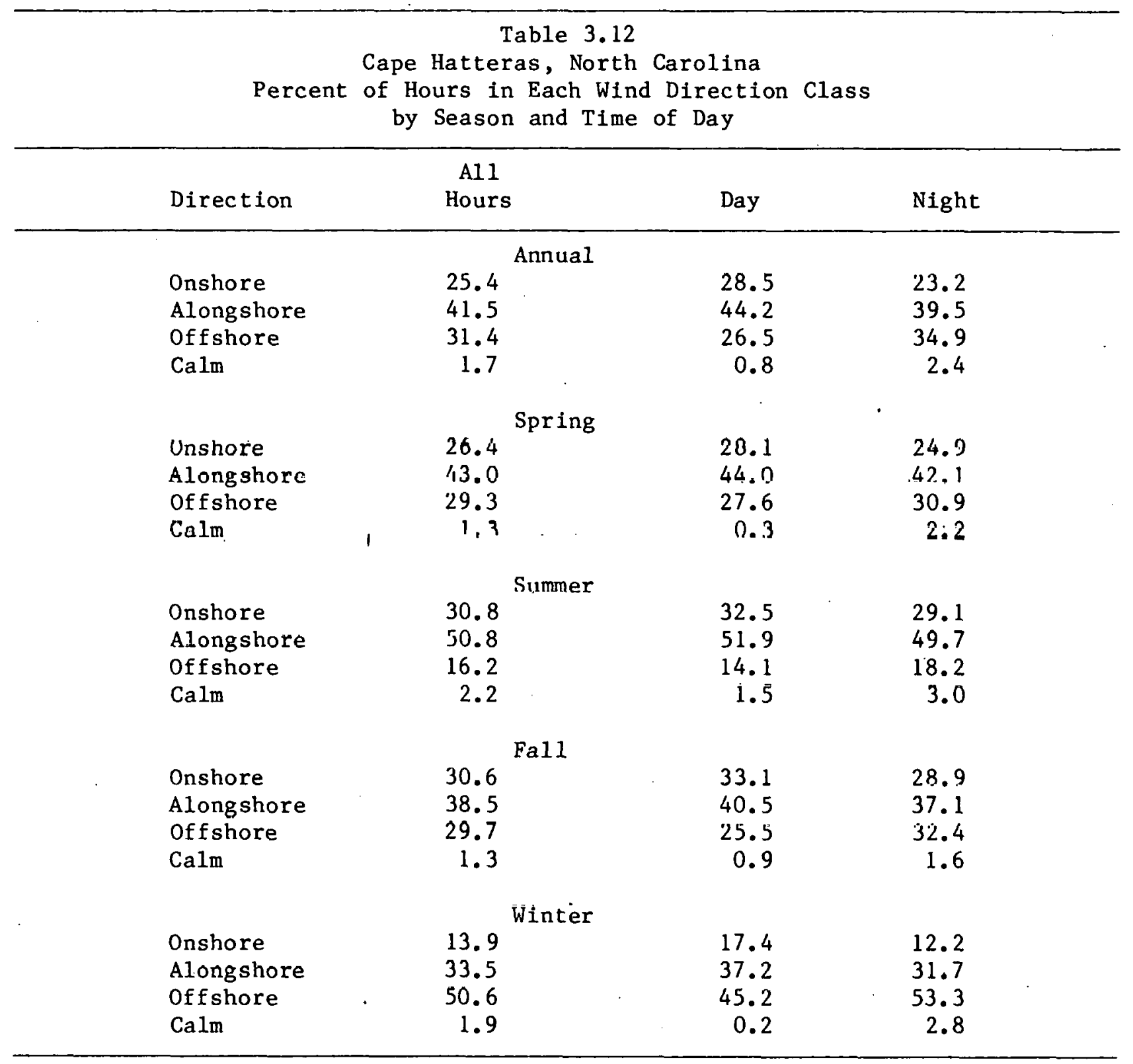




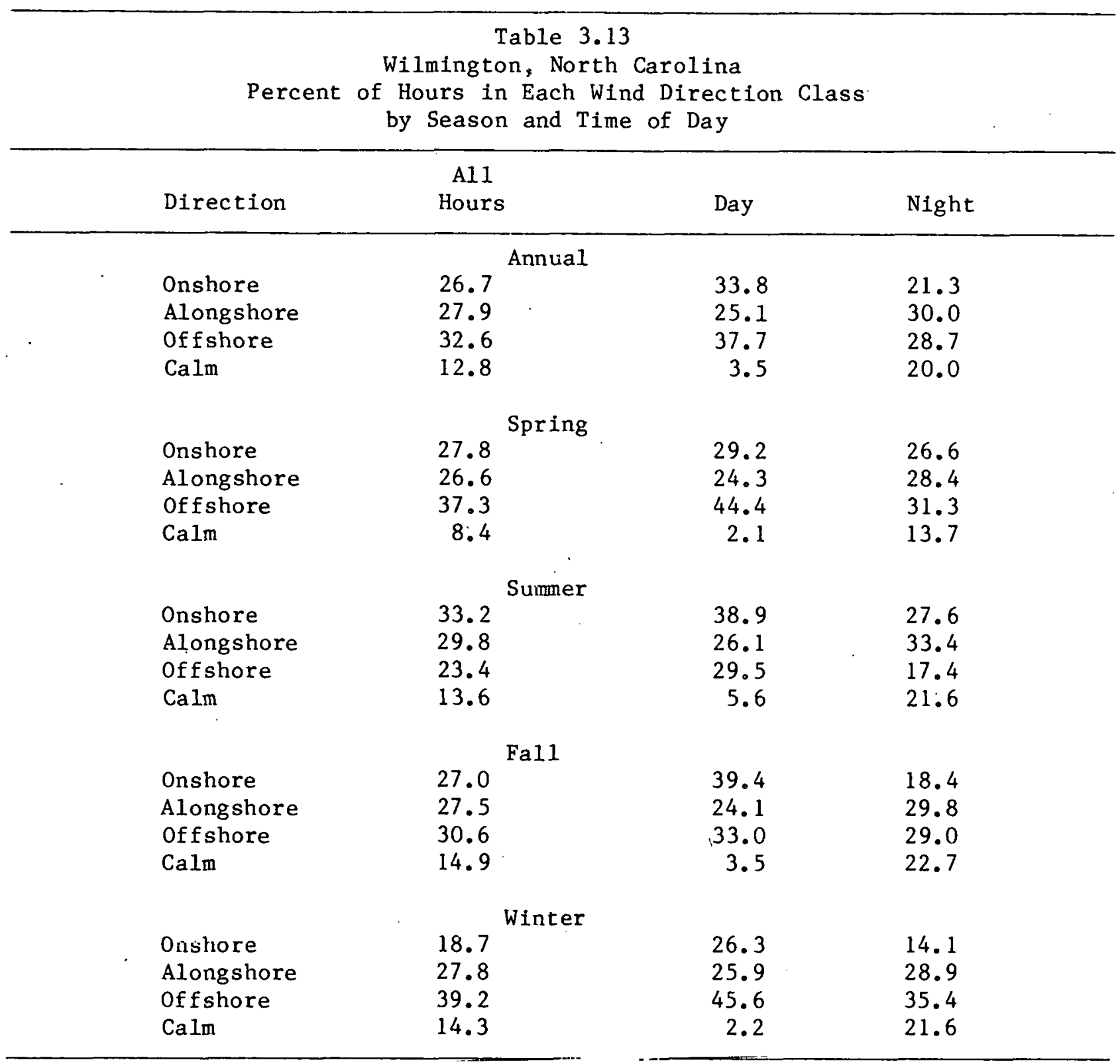




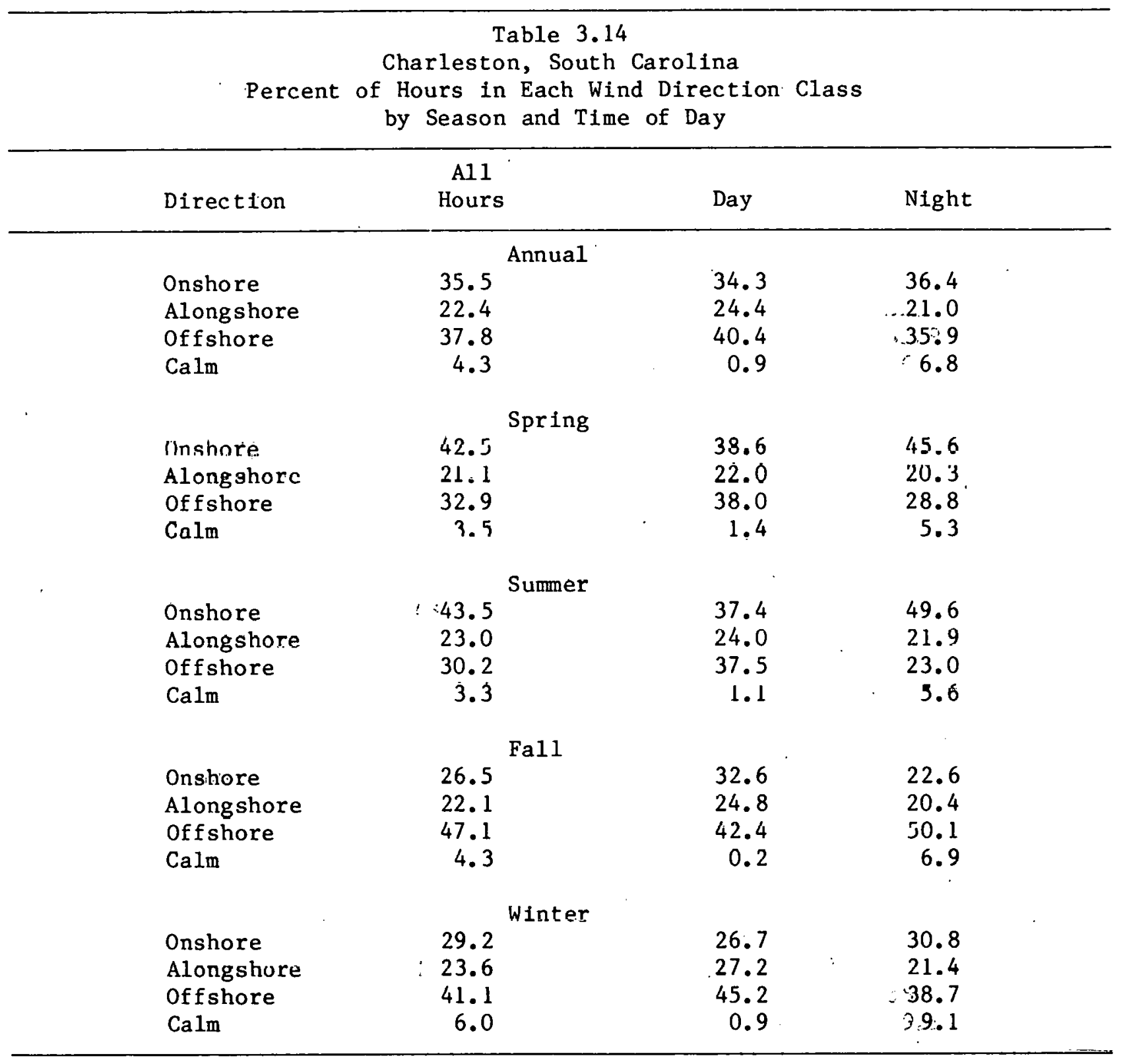




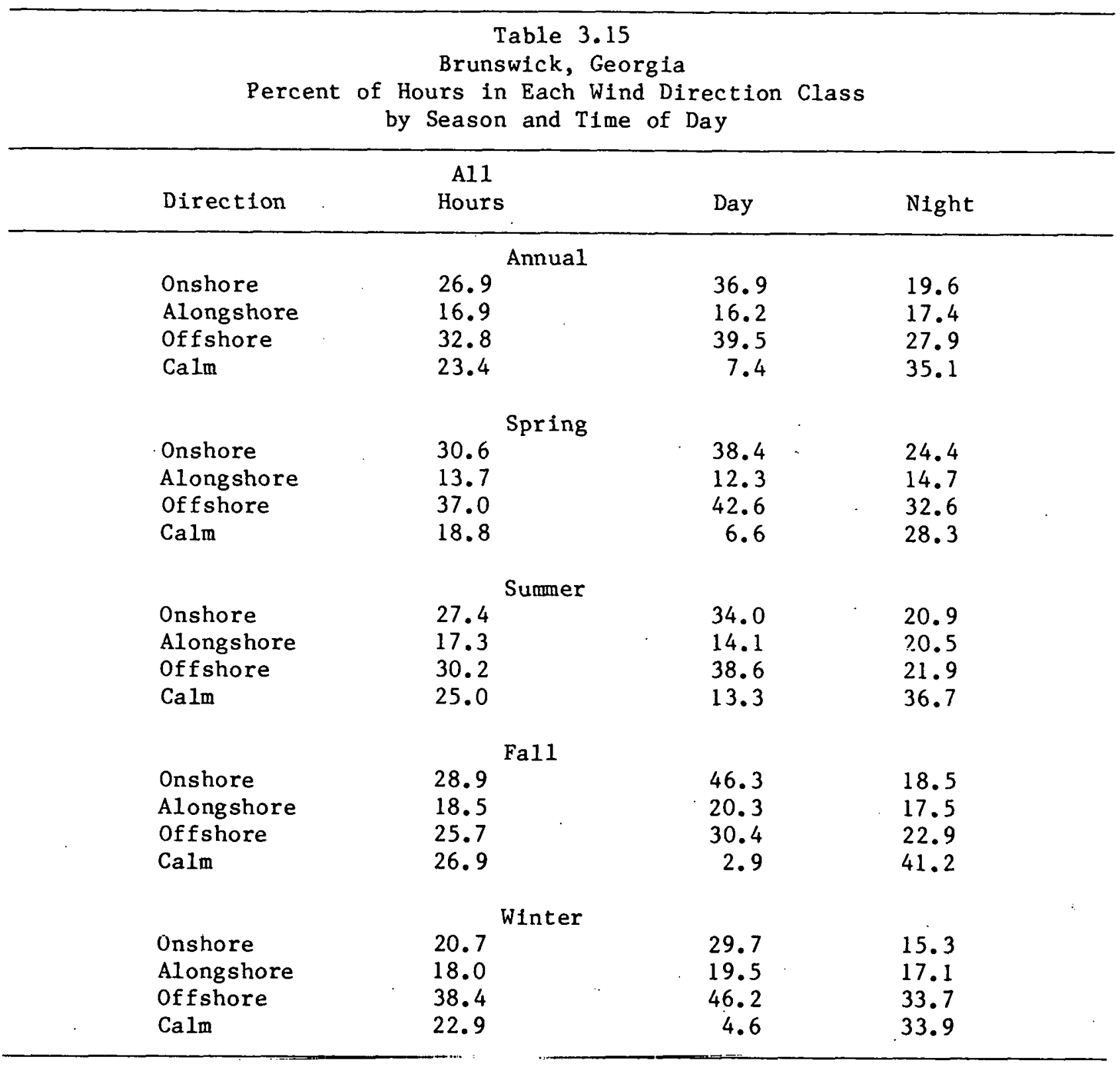




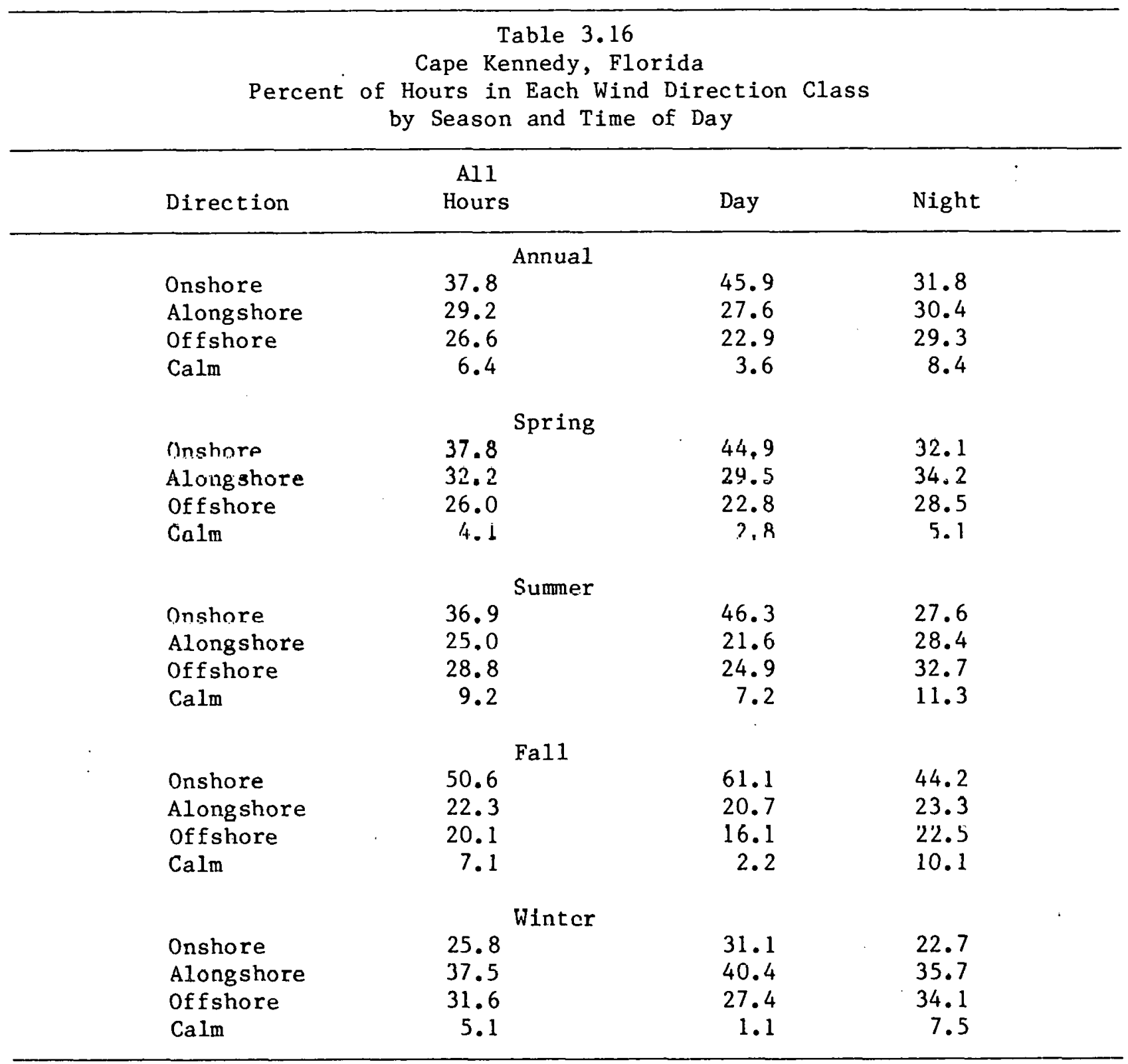




\begin{tabular}{|c|c|c|c|c|}
\hline \multicolumn{5}{|c|}{$\begin{array}{c}\text { Percent of Hours in Each Wind Direction Class } \\
\text { by Season and Time of Day }\end{array}$} \\
\hline & Direction & $\begin{array}{l}\text { Al1 } \\
\text { Hours }\end{array}$ & Day & Night \\
\hline \multicolumn{5}{|c|}{ Annual } \\
\hline 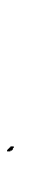 & $\begin{array}{l}\text { Onshore } \\
\text { Alongshore } \\
\text { Offshore } \\
\text { Calm }\end{array}$ & $\begin{array}{r}38.0 \\
22.6 \\
29.9 \\
9.5\end{array}$ & $\begin{array}{r}39.6 \\
21.0 \\
36.3 \\
3.1\end{array}$ & $\begin{array}{l}36.9 \\
23.7 \\
25.3 \\
14.1\end{array}$ \\
\hline \multicolumn{5}{|c|}{ Spring } \\
\hline & $\begin{array}{l}\text { Onshore } \\
\text { Alongshore } \\
\text { Offshore } \\
\text { Calm }\end{array}$ & $\begin{array}{r}33.6 \\
22.0 \\
36.1 \\
8.4\end{array}$ & $\begin{array}{r}35.0 \\
21.4 \\
40.4 \\
3.3\end{array}$ & $\begin{array}{l}32.4 \\
22.5 \\
32.7 \\
12.3\end{array}$ \\
\hline \multicolumn{5}{|c|}{ Summer } \\
\hline & $\begin{array}{l}\text { Onshore } \\
\text { Alongshore } \\
\text { Offshore } \\
\text { Calm }\end{array}$ & $\begin{array}{r}33.9 \\
22.1 \\
34.2 \\
9.7\end{array}$ & $\begin{array}{r}33.7 \\
21.6 \\
39.0 \\
5.6\end{array}$ & $\begin{array}{l}34.1 \\
22.6 \\
29.5 \\
13.7\end{array}$ \\
\hline \multicolumn{5}{|c|}{ Fall } \\
\hline & $\begin{array}{l}\text { Onshore } \\
\text { Alongshore } \\
\text { Offshore } \\
\text { Calm }\end{array}$ & $\begin{array}{r}50.8 \\
20.9 \\
18.5 \\
9.8\end{array}$ & $\begin{array}{r}55.9 \\
19.0 \\
24.0 \\
1.1\end{array}$ & $\begin{array}{l}47.7 \\
22.1 \\
15.3 \\
14.9\end{array}$ \\
\hline \multicolumn{5}{|c|}{ Winter } \\
\hline & $\begin{array}{l}\text { Onshore } \\
\text { Alongshore } \\
\text { Offshore } \\
\text { Calm }\end{array}$ & $\begin{array}{l}34.0 \\
25.1 \\
30.8 \\
10.1\end{array}$ & $\begin{array}{r}36.9 \\
21.7 \\
40.0 \\
1.5\end{array}$ & $\begin{array}{l}32.2 \\
27.2 \\
25.3 \\
15.2\end{array}$ \\
\hline
\end{tabular}




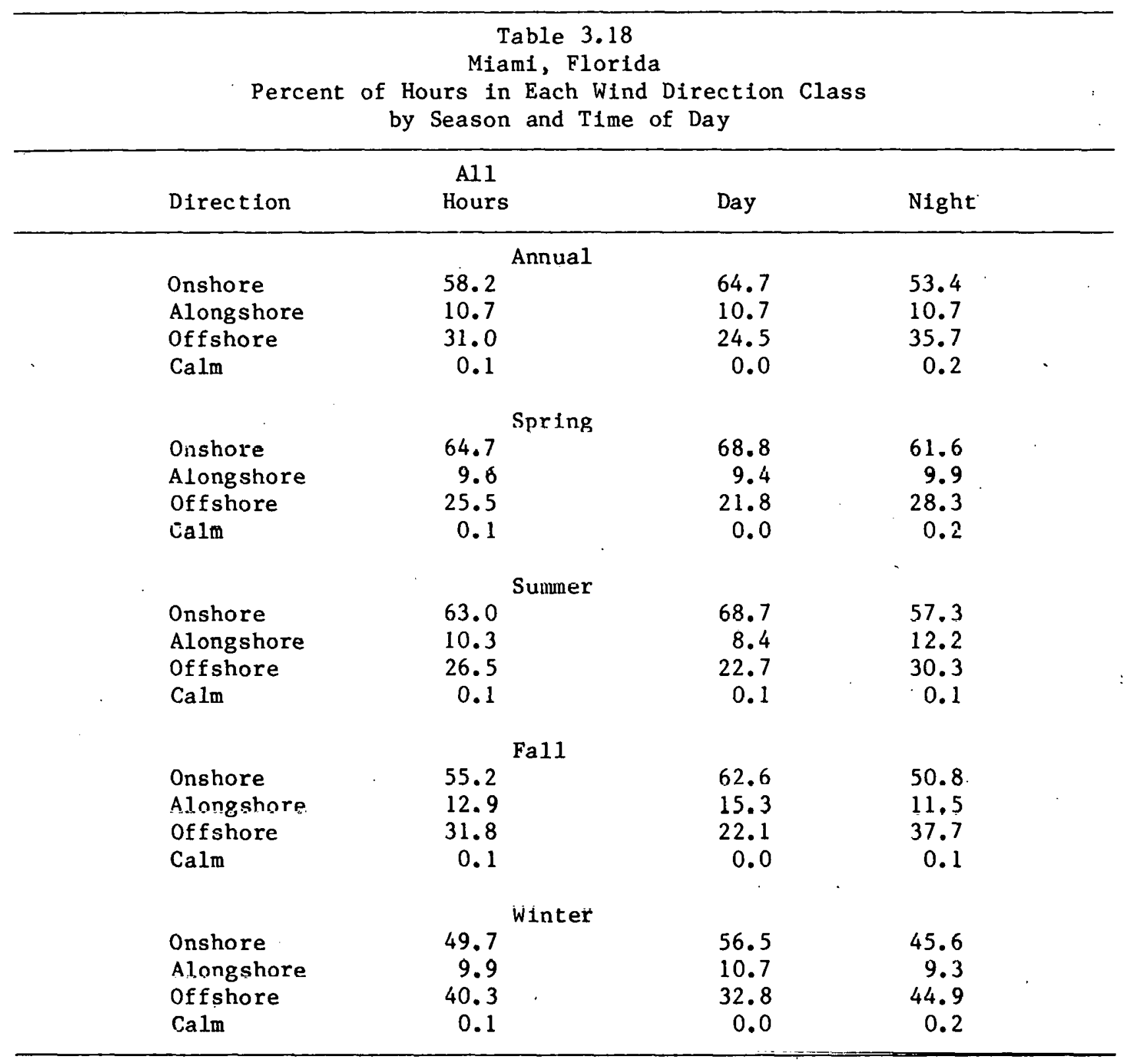


Table 3.19

Fort Myers, Florida

Percent of Hours in Each Wind Direction Class

by Season and Time of Day

\begin{tabular}{|c|c|c|c|}
\hline Direction & $\begin{array}{c}\text { All } \\
\text { Hours }\end{array}$ & Day & Night \\
\hline \multicolumn{4}{|c|}{ Annual } \\
\hline $\begin{array}{l}\text { Onshore } \\
\text { Alongshore } \\
\text { Offshore } \\
\text { Calm }\end{array}$ & $\begin{array}{l}22.9 \\
16.5 \\
50.1 \\
10.6\end{array}$ & $\begin{array}{r}33.3 \\
17.0 \\
47.3 \\
2.3\end{array}$ & $\begin{array}{l}15.4 \\
16.0 \\
52.0 \\
16.5\end{array}$ \\
\hline \multicolumn{4}{|c|}{ Spring } \\
\hline $\begin{array}{l}\text { Onshore } \\
\text { Alongshore } \\
\text { Offshore } \\
\text { Calm }\end{array}$ & $\begin{array}{r}33.2 \\
18.7 \\
38.9 \\
9.3\end{array}$ & $\begin{array}{r}42.8 \\
16.1 \\
38.6 \\
2.5\end{array}$ & $\begin{array}{l}25.8 \\
20.6 \\
39.1 \\
14.5\end{array}$ \\
\hline \multicolumn{4}{|c|}{ Summer } \\
\hline $\begin{array}{l}\text { Onshore } \\
\text { Alongshore } \\
\text { offshore } \\
\text { Calm }\end{array}$ & $\begin{array}{l}24.7 \\
17.4 \\
45.9 \\
12.0\end{array}$ & $\begin{array}{r}35.1 \\
17.6 \\
42.8 \\
4.5\end{array}$ & $\begin{array}{l}15.0 \\
17.2 \\
48.7 \\
19.1\end{array}$ \\
\hline \multicolumn{4}{|c|}{$\mathrm{Fa} 11$} \\
\hline $\begin{array}{l}\text { Onshore } \\
\text { Alongshore } \\
\text { Offshore } \\
\text { Calm }\end{array}$ & $\begin{array}{l}12.7 \\
10.5 \\
65.2 \\
11.6\end{array}$ & $\begin{array}{r}22.7 \\
13.6 \\
63.0 \\
0.7\end{array}$ & $\begin{array}{r}6.7 \\
8.7 \\
66.5 \\
18.1\end{array}$ \\
\hline \multicolumn{4}{|c|}{ Winter } \\
\hline $\begin{array}{l}\text { Onshore } \\
\text { Alongshore } \\
\text { Offshore } \\
\text { Calm }\end{array}$ & $\begin{array}{r}20.8 \\
19.2 \\
50.5 \\
9.4\end{array}$ & $\begin{array}{r}30.6 \\
20.9 \\
47.6 \\
0.9\end{array}$ & $\begin{array}{l}15.0 \\
18.2 \\
52.2 \\
14.6\end{array}$ \\
\hline
\end{tabular}




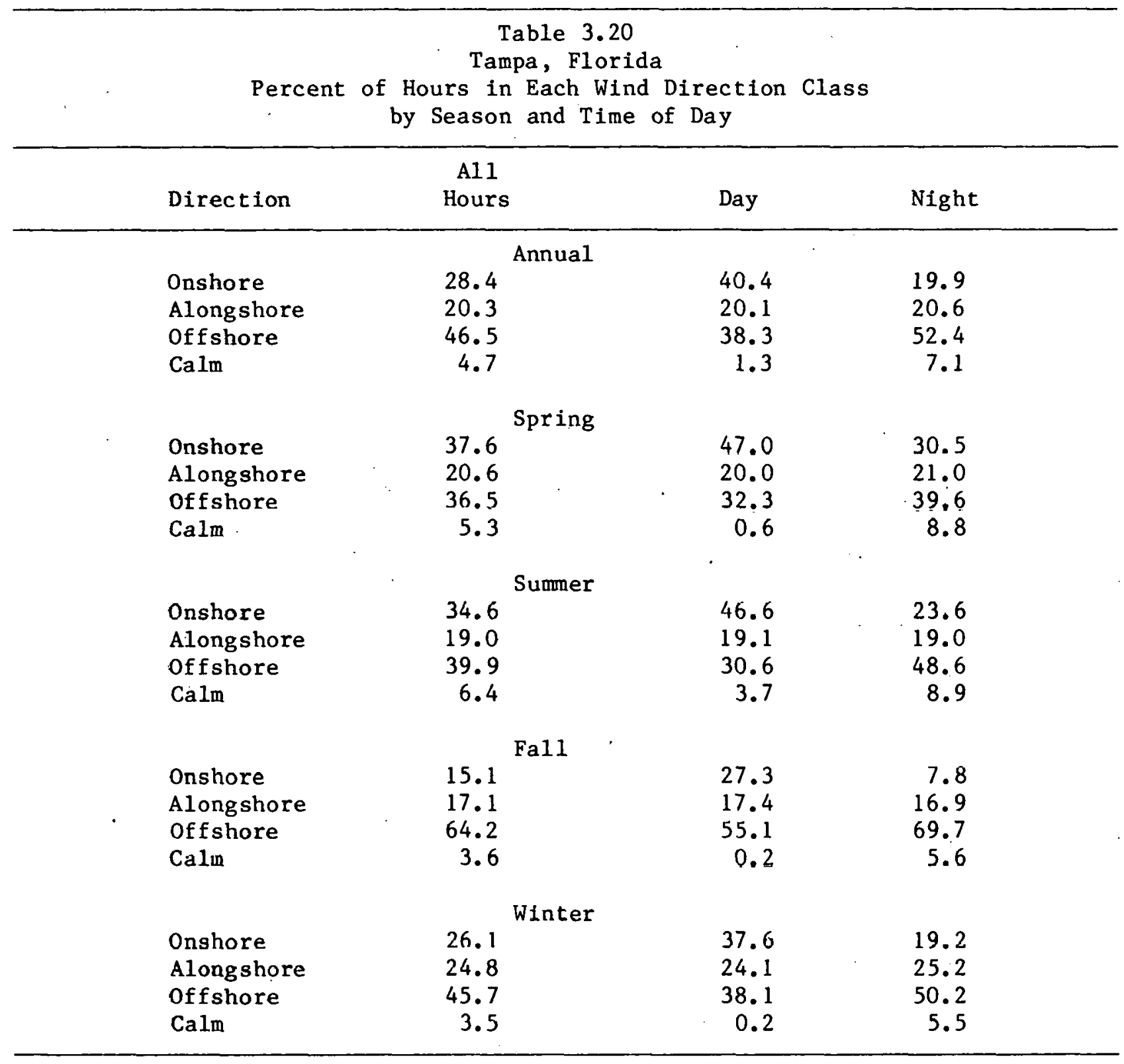


Table 3.21

Tallahassee, Florida

Percent of Hours in Each Wind Direction Class by Season and Time of Day

\begin{tabular}{|c|c|c|c|}
\hline Direction & $\begin{array}{c}\text { Al1 } \\
\text { Hours }\end{array}$ & Day & Night \\
\hline \multicolumn{4}{|c|}{ Annual } \\
\hline Onshore & 30.9 & 36.9 & 26.6 \\
\hline Alongshore & 15.6 & 16.0 & 15.3 \\
\hline Offshore & 36.6 & 41.7 & 33.0 \\
\hline Calm & 16.9 & 5.4 & 25.0 \\
\hline \multicolumn{4}{|c|}{ Spring } \\
\hline Onshore & 37.8 & 43.9 & 33.2 \\
\hline Alongshore & 12.7 & 12.3 & 13.0 \\
\hline offshore & 33.5 & 38.7 & 29.7 \\
\hline $\mathrm{Calm}$ & 16.0 & 5.1 & 24.1 \\
\hline \multicolumn{4}{|c|}{ Summer } \\
\hline Onshore & 35.9 & 37.9 & 34.2 \\
\hline Alongshore & 18.5 & 16.7 & 20.1 \\
\hline offshore & 27.6 & 35.4 & 20.7 \\
\hline Calm & 17.9 & 9.9 & 25.1 \\
\hline \multicolumn{4}{|c|}{ Fall } \\
\hline Onshore & 18.5 & 26.7 & 13.6 \\
\hline Alongshore & 20.2 & 21.2 & 19.6 \\
\hline Offshore & 45.0 & 50.4 & 41.8 \\
\hline Calm & 16.3 & 1.6 & 25.1 \\
\hline \multicolumn{4}{|c|}{ Winter } \\
\hline Onshore & 31.1 & 37.8 & 27.1 \\
\hline Alongshore & 11.0 & 14.1 & 9.2 \\
\hline Offshore & 40.5 & 44.6 & 38.0 \\
\hline $\mathrm{Calm}$ & 17.4 & 3.5 & 25.7 \\
\hline
\end{tabular}




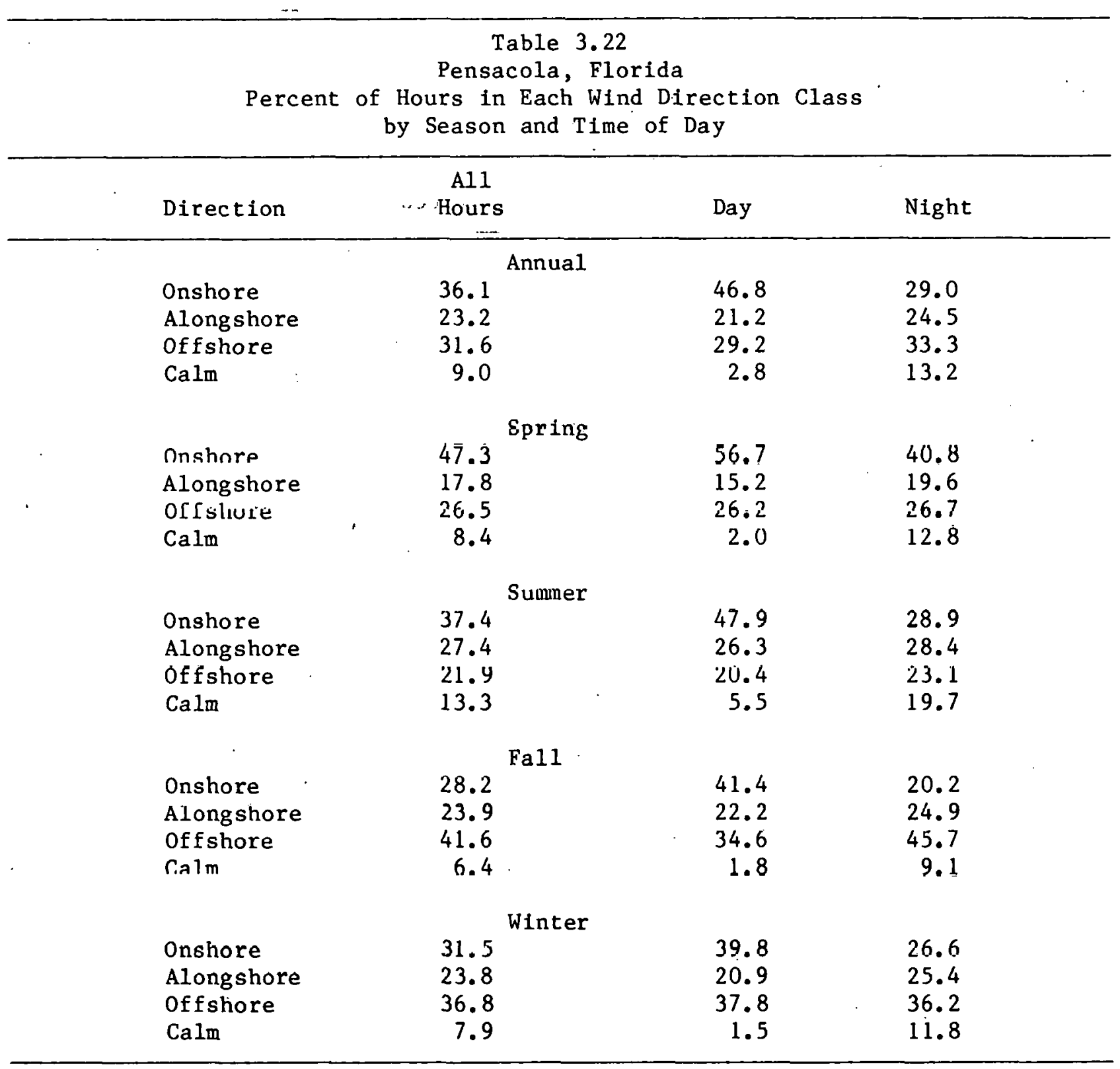




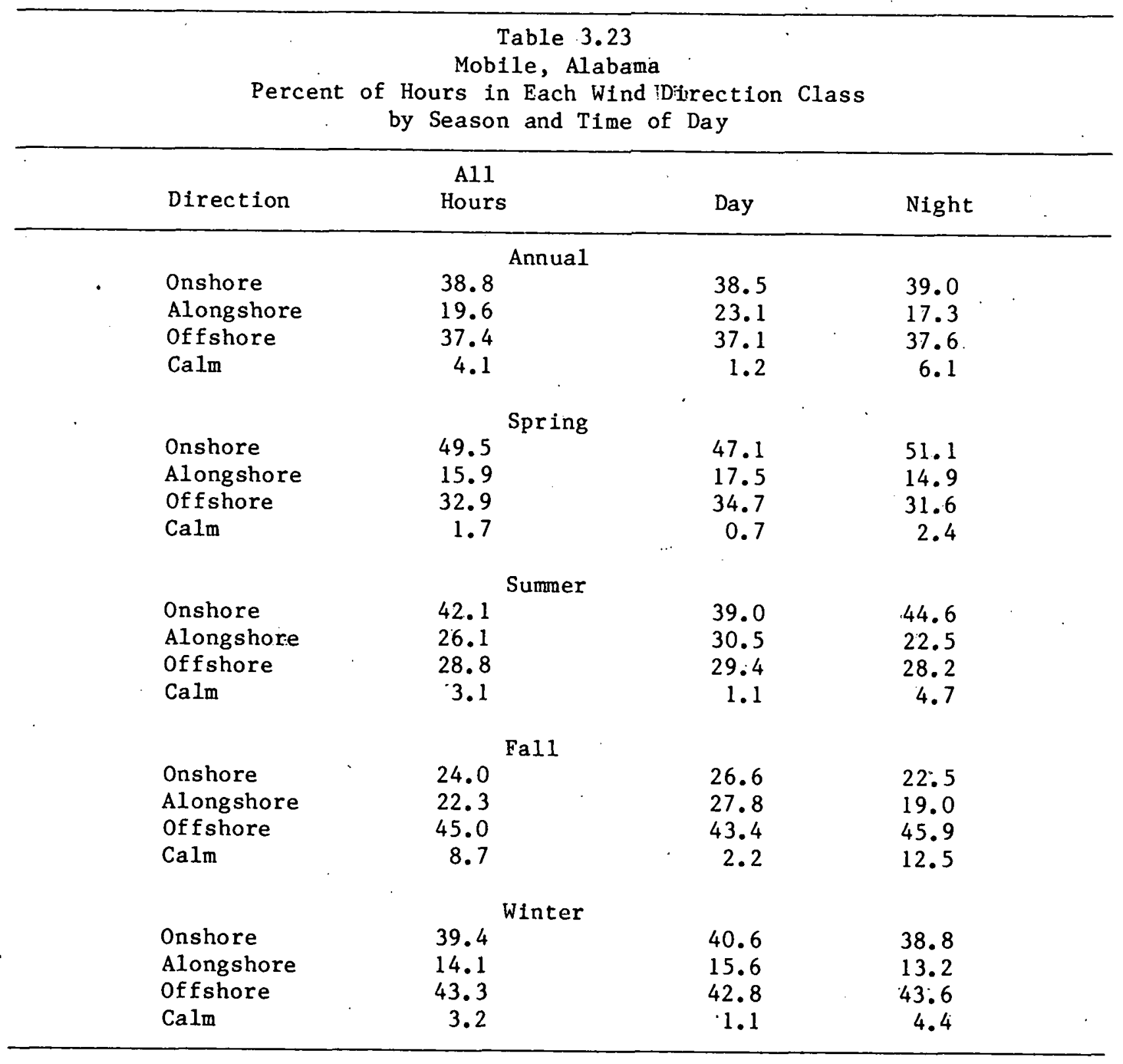




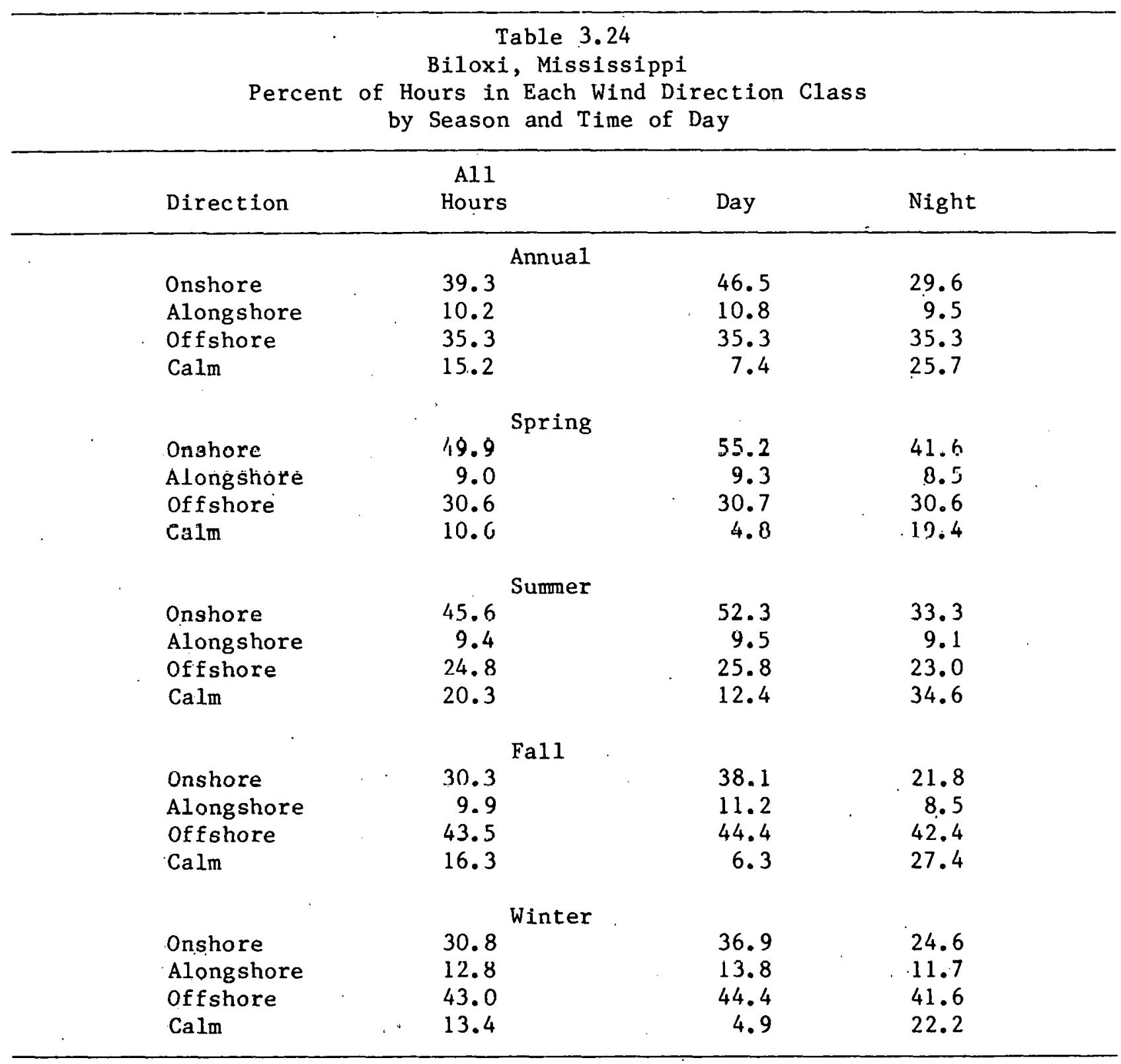




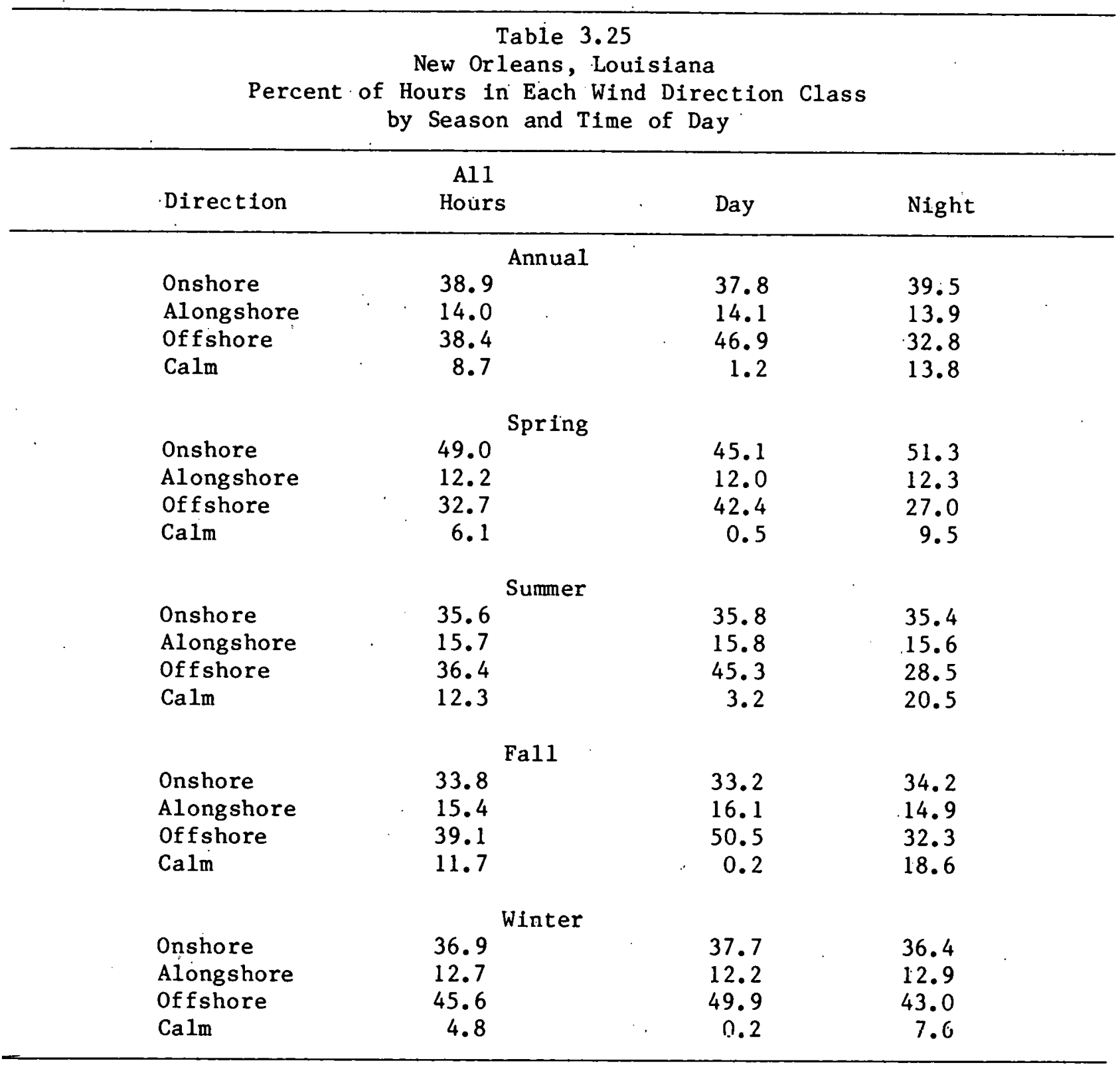


Table 3.26

Boothville, Louisiana

Percent of Hours in. Each. Wind Direction Class

by Season and Time of Day

\begin{tabular}{|c|c|c|c|}
\hline Direction & $\begin{array}{l}\text { All } \\
\text { Hours }\end{array}$ & Day & Night \\
\hline \multicolumn{4}{|c|}{ Annual } \\
\hline Onshore & .36 .7 & 36.1 & 37.1 \\
\hline Alongshore & 15.4 & 14.0 & 16.3 \\
\hline offshore & 36.6 & 46.5 & 30.1 \\
\hline $\mathrm{Calm}$ & 11.3 & 3.4 & 16.6 \\
\hline \multicolumn{4}{|c|}{ Spring } \\
\hline Onshore & 48.0 & 43.8 & 50.6 \\
\hline Alongshore & 18.6 & 15.4 & 20.6 \\
\hline Gifeshure & 29.1 & 39.2 & 22.9 \\
\hline Calm & 4.3 & 1.6 & 5,9 \\
\hline \multicolumn{4}{|c|}{ Summer } \\
\hline Onshore & 39.5 & 38.2 & 40.6 \\
\hline Alongshore & 14.6 & 14.2 & 14.9 \\
\hline Offshore & 28.1 & 41.7 & 17.0 \\
\hline Calm & 17.9 & 6.0 & 27.5 \\
\hline \multicolumn{4}{|c|}{ Fa11 } \\
\hline Onshore & 27.0 & 29.5 & 25.5 \\
\hline Alongshore & 16.8 & 16.7 & 16.8 \\
\hline of fshore & 39.8 & 50.5 & 33.3 \\
\hline Calm & 16.5 & 3.3 & 24.4 \\
\hline \multicolumn{4}{|c|}{ Winter } \\
\hline Onshore & 32.1 & 32.3 & 31.9 \\
\hline Alongshore & $11: 3$ & 9.4 & 12.5 \\
\hline Offshore & 503.0 & 56.0 & 46.3 \\
\hline $\mathrm{Calm}$ & 6.6 & 2.2 & 9.4 \\
\hline
\end{tabular}




\begin{tabular}{|c|c|c|c|c|}
\hline \multicolumn{3}{|c|}{$\begin{array}{c}\text { Table } 3.27 \\
\text { Lake Charles, Louisiana } \\
\text { Percent of Hours In Each Wind Direction } \\
\text { by Season and Time of Day }\end{array}$} & Class & \multirow[b]{2}{*}{ Night } \\
\hline Direction & $\begin{array}{c}\text { A11 } \\
\text { Hours }\end{array}$ & Day & & \\
\hline \multicolumn{5}{|c|}{ Annual } \\
\hline $\begin{array}{l}\text { Onshore } \\
\text { Alongshore } \\
\text { Offshore } \\
\text { Calm }\end{array}$ & $\begin{array}{r}43.2 \\
19.3 \\
30.8 \\
6.6\end{array}$ & $\begin{array}{r}49.1 \\
19.7 \\
29.0 \\
2.2\end{array}$ & . & $\begin{array}{r}39.2 \\
19.1 \\
32.1 \\
9.7\end{array}$ \\
\hline \multicolumn{5}{|c|}{ sppring } \\
\hline $\begin{array}{l}\text { Onshore } \\
\text { Alongshore } \\
\text { offshore } \\
\text { Calm }\end{array}$ & $\begin{array}{r}52.9 \\
1577 \\
228.5 \\
2.9\end{array}$ & $\begin{array}{r}56.7 \\
16.5 \\
25.6 \\
1.2\end{array}$ & & $\begin{array}{r}50.3 \\
15.2 \\
30.4 \\
4.0\end{array}$ \\
\hline \multicolumn{5}{|c|}{ Summer } \\
\hline $\begin{array}{l}\text { Onshore } \\
\text { Alongshore } \\
\text { Off shore } \\
\text { Calm }\end{array}$ & $\begin{array}{l}46.1 \\
21.0 \\
21.9 \\
11.1\end{array}$ & $\begin{array}{r}54.5 \\
21.3 \\
21.4 \\
2.9\end{array}$ & & $\begin{array}{l}38.5 \\
20.7 \\
22.3 \\
18.4\end{array}$ \\
\hline \multicolumn{5}{|c|}{$\mathrm{Fal1}$} \\
\hline $\begin{array}{l}\text { Onshore } \\
\text { Alongshore } \\
\text { Offshore } \\
\text { Calm }\end{array}$ & $\begin{array}{r}32.2 \\
20.6 \\
39.6 \\
7.6\end{array}$ & $\begin{array}{r}39.2 \\
18.9 \\
38.6 \\
3.3\end{array}$ & & $\begin{array}{l}28.0 \\
21.6 \\
40.1 \\
10.2\end{array}$ \\
\hline \multicolumn{5}{|c|}{ Winter } \\
\hline $\begin{array}{l}\text { Onshore } \\
\text { Alongshore } \\
\text { offshore } \\
\text { Calm }\end{array}$ & $\begin{array}{l}41.5 \\
20.1 \\
33.4 \\
4.9\end{array}$ & $\begin{array}{r}44.0 \\
22.2 \\
32.5 \\
1.3\end{array}$ & & $\begin{array}{r}40.0 \\
18.9 \\
34.0 \\
7.1\end{array}$ \\
\hline
\end{tabular}




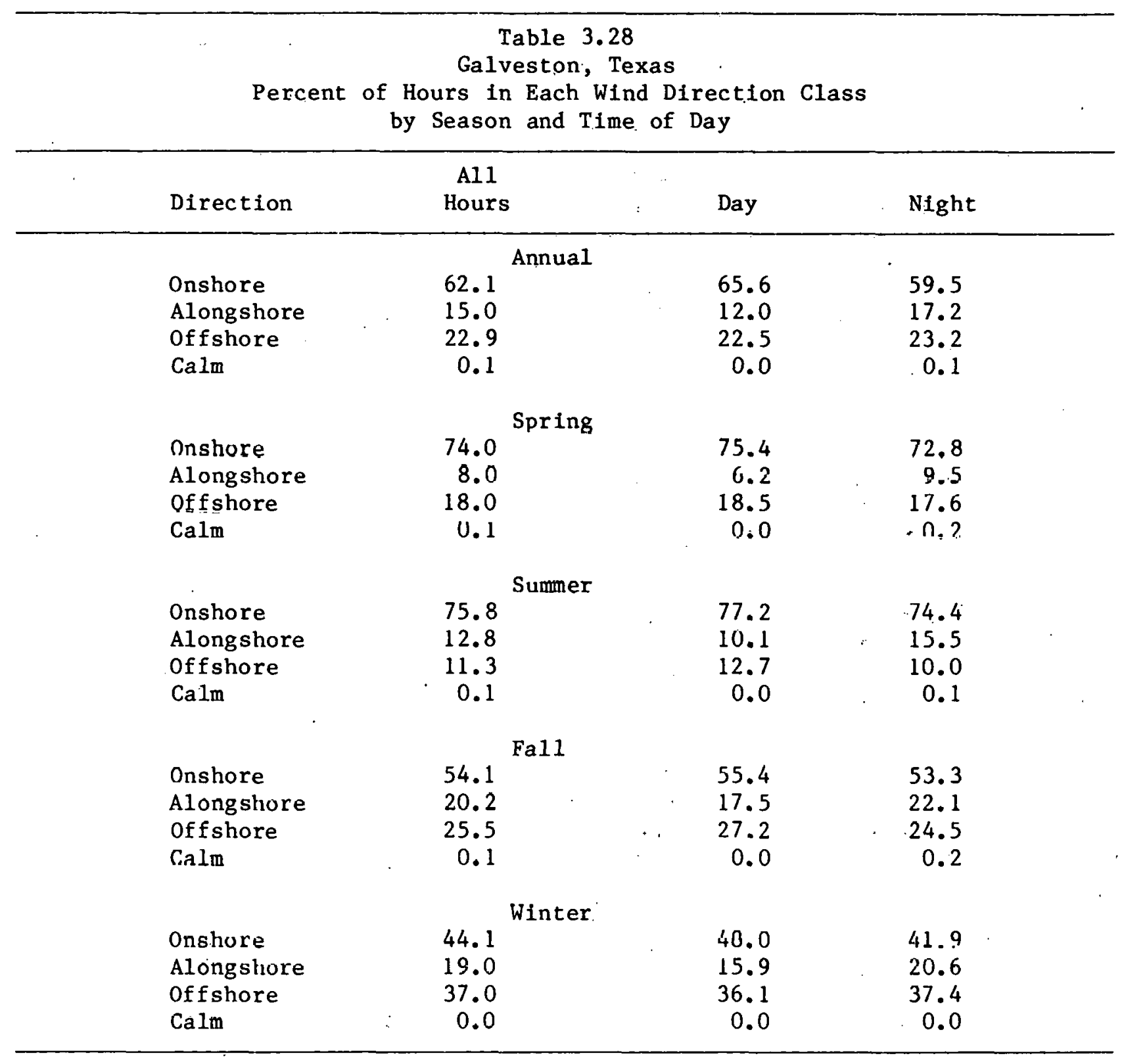


Table 3.29

Houston, Texas

Percent of Hours in Each Wind Direction Class

by Season and Time of Day

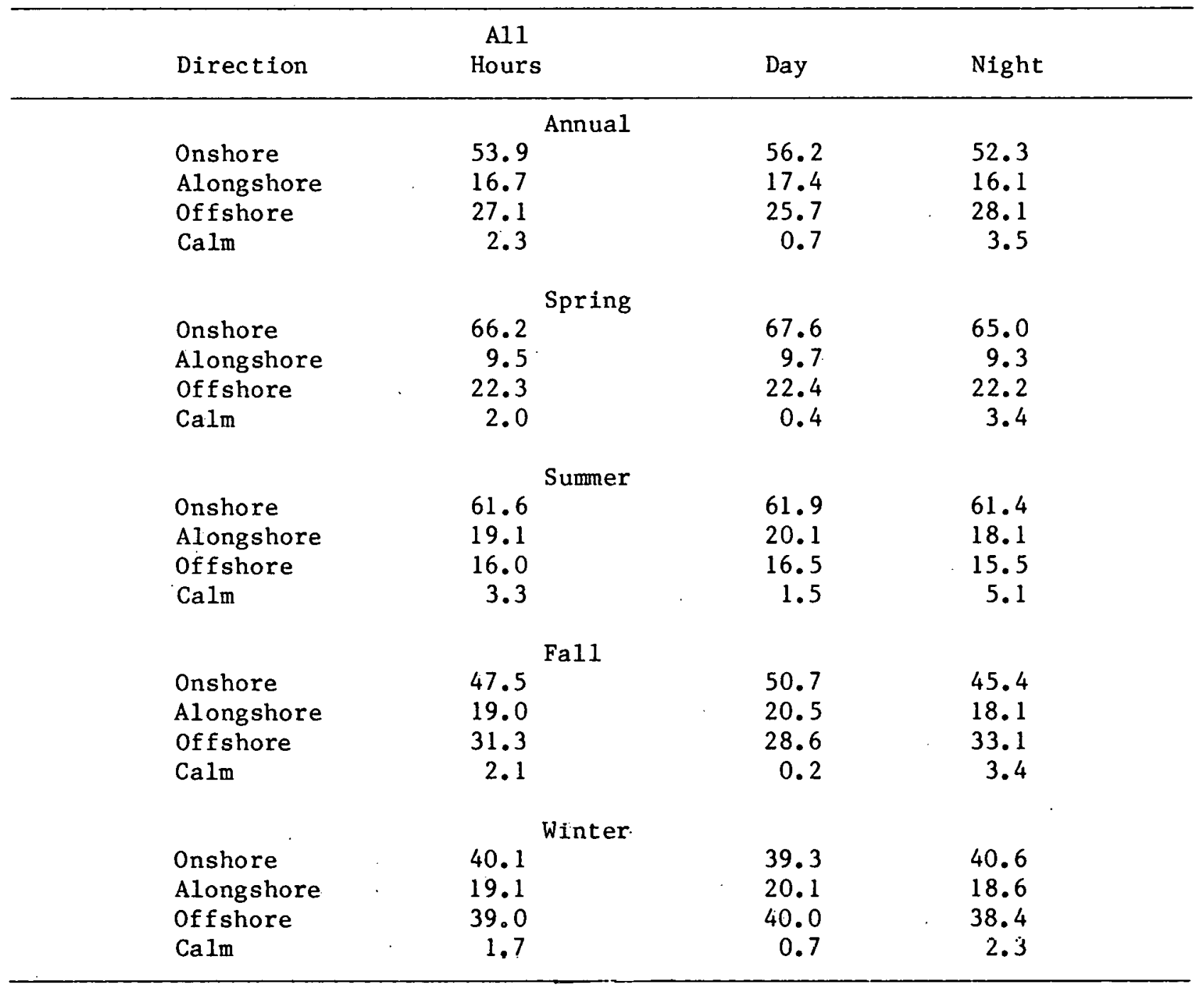


Table: 3.830

Browns.ville;. Texas

Percent of Hours in Each: Wind Direction Class

by Seasoni and 'Time of Day.

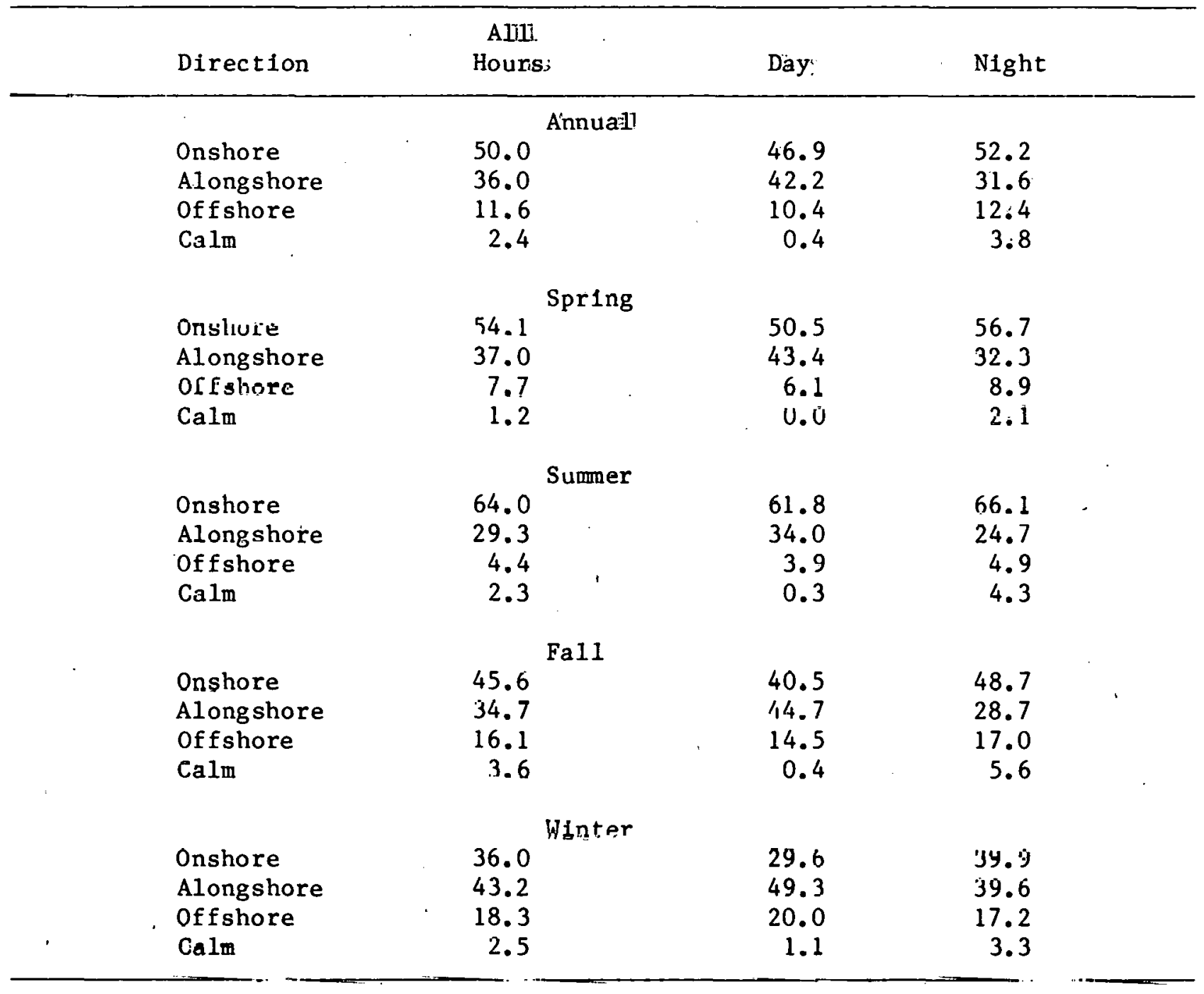




\begin{tabular}{|c|c|c|c|c|}
\hline & $\begin{array}{r}\text { Tab } \\
\text { Portlar } \\
\text { Hours it } \\
\text { Ind Dire }\end{array}$ & $\begin{array}{l}\text { e } 4.1 \\
\text { id, Maine } \\
\text { Each Stabi } \\
\text { tion and Spe }\end{array}$ & Class & \\
\hline \multirow[b]{2}{*}{ Stability } & \multirow[b]{2}{*}{$0.5-2.4$} & \multicolumn{2}{|c|}{ Wind Speed $\left(\mathrm{msec}^{-1}\right)$} & \multirow[b]{2}{*}{$\geq 10.0$} \\
\hline & & $2.5-4.9$ & $5.0-9.9$ & \\
\hline $\begin{array}{l}\text { Unstable } \\
\text { Neutral } \\
\text { Stable }\end{array}$ & $\begin{array}{l}\quad \text { A11 } \\
15.0 \\
18.6 \\
66.4\end{array}$ & $\begin{array}{c}\text { Directions } \\
18.5 \\
41.6 \\
39.9\end{array}$ & $\begin{array}{r}9.0 \\
85.2 \\
5.8\end{array}$ & $\begin{array}{r}3.8 \\
96.2 \\
0.0\end{array}$ \\
\hline $\begin{array}{l}\text { Unstable } \\
\text { Neutral } \\
\text { Stable }\end{array}$ & $\begin{array}{l}\quad \text { Onst } \\
18.7 \\
30.0 \\
51.3\end{array}$ & lore $\begin{array}{l}31.3 \\
54.6 \\
14.1\end{array}$ & $\begin{array}{r}17.8 \\
81.0 \\
1.3\end{array}$ & $\begin{array}{r}0.0 \\
100.0 \\
0.0\end{array}$ \\
\hline $\begin{array}{l}\text { Unstable } \\
\text { Neutral } \\
\text { Stable }\end{array}$ & $\begin{array}{l}\quad \text { Alor } \\
19.5 \\
21.6 \\
58.9\end{array}$ & $\begin{array}{r}\text { Igshore } \\
9.1 \\
52.5 \\
38.4\end{array}$ & $\begin{array}{r}5.2 \\
87.8 \\
7.0\end{array}$ & $\begin{array}{r}18.8 \\
81.3 \\
0.0\end{array}$ \\
\hline $\begin{array}{l}\text { Unstable } \\
\text { Neutral } \\
\text { Stable }\end{array}$ & $\begin{array}{l}\quad \text { offs } \\
12.5 \\
14.1 \\
73.4\end{array}$ & $\begin{array}{l}\text { hore } \\
\qquad \begin{array}{r}17.3 \\
29.3 \\
53.4\end{array}\end{array}$ & $\begin{array}{r}7.5 \\
85.5 \\
7.0\end{array}$ & $\begin{array}{r}0.0 \\
100.0 \\
0.0\end{array}$ \\
\hline
\end{tabular}




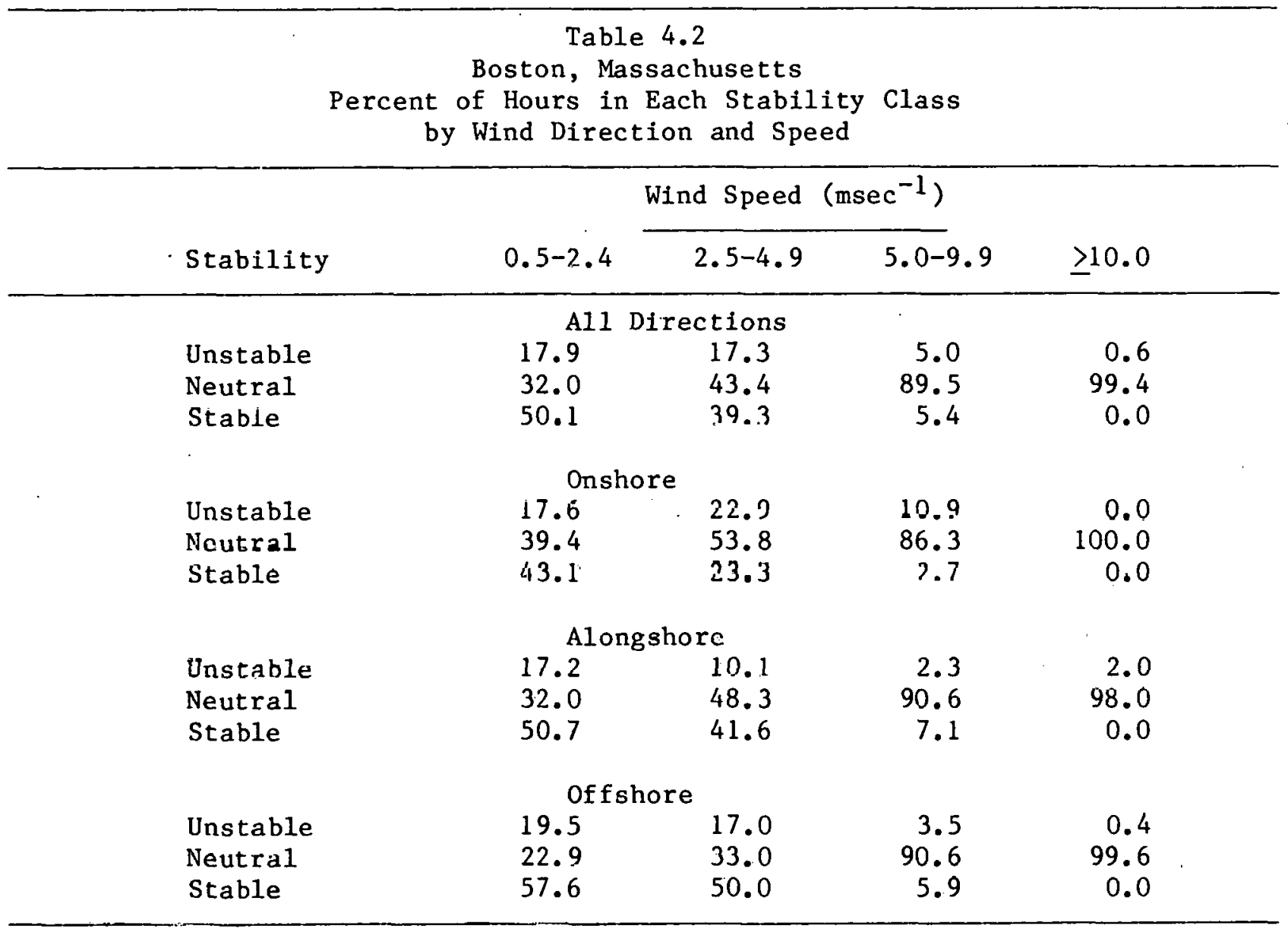




\begin{tabular}{|c|c|c|c|c|}
\hline \multirow[b]{3}{*}{ Stability } & $\begin{array}{r}\text { Tabl } \\
\text { edford, M } \\
\text { Hours in } \\
\text { ind Direc }\end{array}$ & $\begin{array}{l}\text { e } 4.3 \\
\text { lassachusett } \\
\text { Each Stabi } \\
\text { tion and Spe }\end{array}$ & Class & \\
\hline & \multirow[b]{2}{*}{$0.5-2.4$} & \multicolumn{2}{|c|}{ Wind Speed $\left(\mathrm{msec}^{-1}\right)$} & \multirow[b]{2}{*}{$\geq 10.0$} \\
\hline & & $2.5-4.9$ & $5.0-9.9$ & \\
\hline $\begin{array}{l}\text { Unstable } \\
\text { Neutral } \\
\text { Stable }\end{array}$ & $\begin{array}{l}\quad \text { A11 } \\
23.6 \\
23.5 \\
52.8\end{array}$ & $\begin{array}{c}\text { Directions } \\
23.0 \\
45.7 \\
31.2\end{array}$ & $\begin{array}{r}8.6 \\
85.1 \\
6.4\end{array}$ & $\begin{array}{r}0.0 \\
100.0 \\
0.0\end{array}$ \\
\hline $\begin{array}{l}\text { Unstable } \\
\text { Neutral } \\
\text { Stable }\end{array}$ & $\begin{array}{l}\quad \text { Onsh } \\
23.6 \\
39.6 \\
36.8\end{array}$ & lore $\begin{array}{r}20.8 \\
68.5 \\
10.7\end{array}$ & $\begin{array}{r}6.7 \\
92.5 \\
0.8\end{array}$ & $\begin{array}{r}0.0 \\
100.0 \\
0.0\end{array}$ \\
\hline $\begin{array}{l}\text { Unstable } \\
\text { Neutral } \\
\text { Stable }\end{array}$ & $\begin{array}{l}\quad \text { Alon } \\
24.7 \\
29.7 \\
45.5\end{array}$ & $\begin{array}{l}\text { gshore } \\
23.4 \\
55.5 \\
21.0\end{array}$ & $\begin{array}{r}10.4 \\
84.3 \\
5.3\end{array}$ & $\begin{array}{r}0.0 \\
100.0 \\
0.0\end{array}$ \\
\hline $\begin{array}{l}\text { Unstable } \\
\text { Neutral } \\
\text { Stable }\end{array}$ & $\begin{array}{l}\quad \text { Offs } \\
23.4 \\
14.7 \\
61.9\end{array}$ & $\begin{array}{r}\text { hore } \\
\begin{array}{r}23.9 \\
32.9 \\
43.2\end{array}\end{array}$ & $\begin{array}{r}8.8 \\
82.9 \\
8.3\end{array}$ & $\begin{array}{r}0.0 \\
100.0 \\
0.0\end{array}$ \\
\hline
\end{tabular}


Table 4.4

Falmouth, Massachusetts

Percent of Hours in Each Stability Class

by Wind Direction and Speed

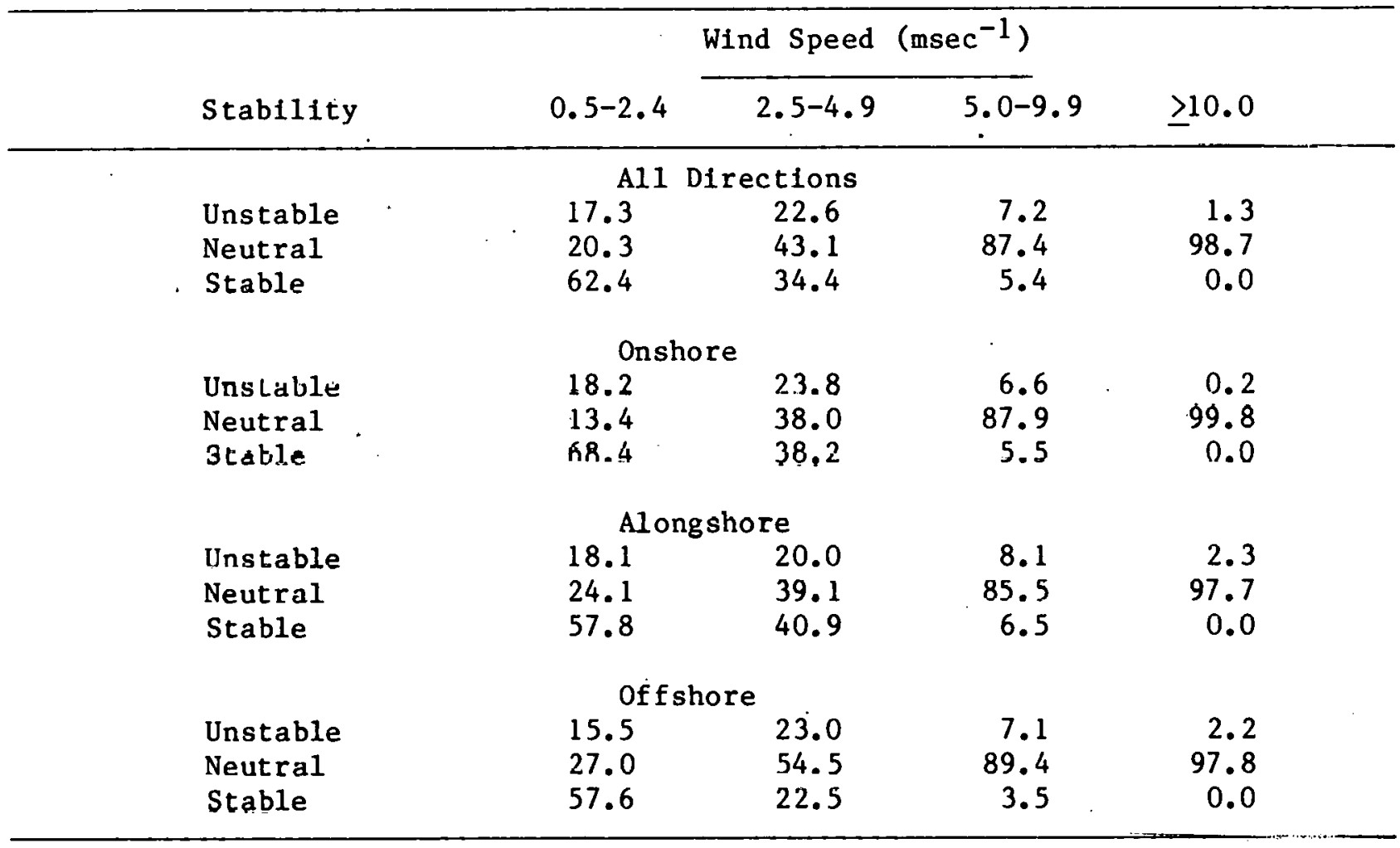




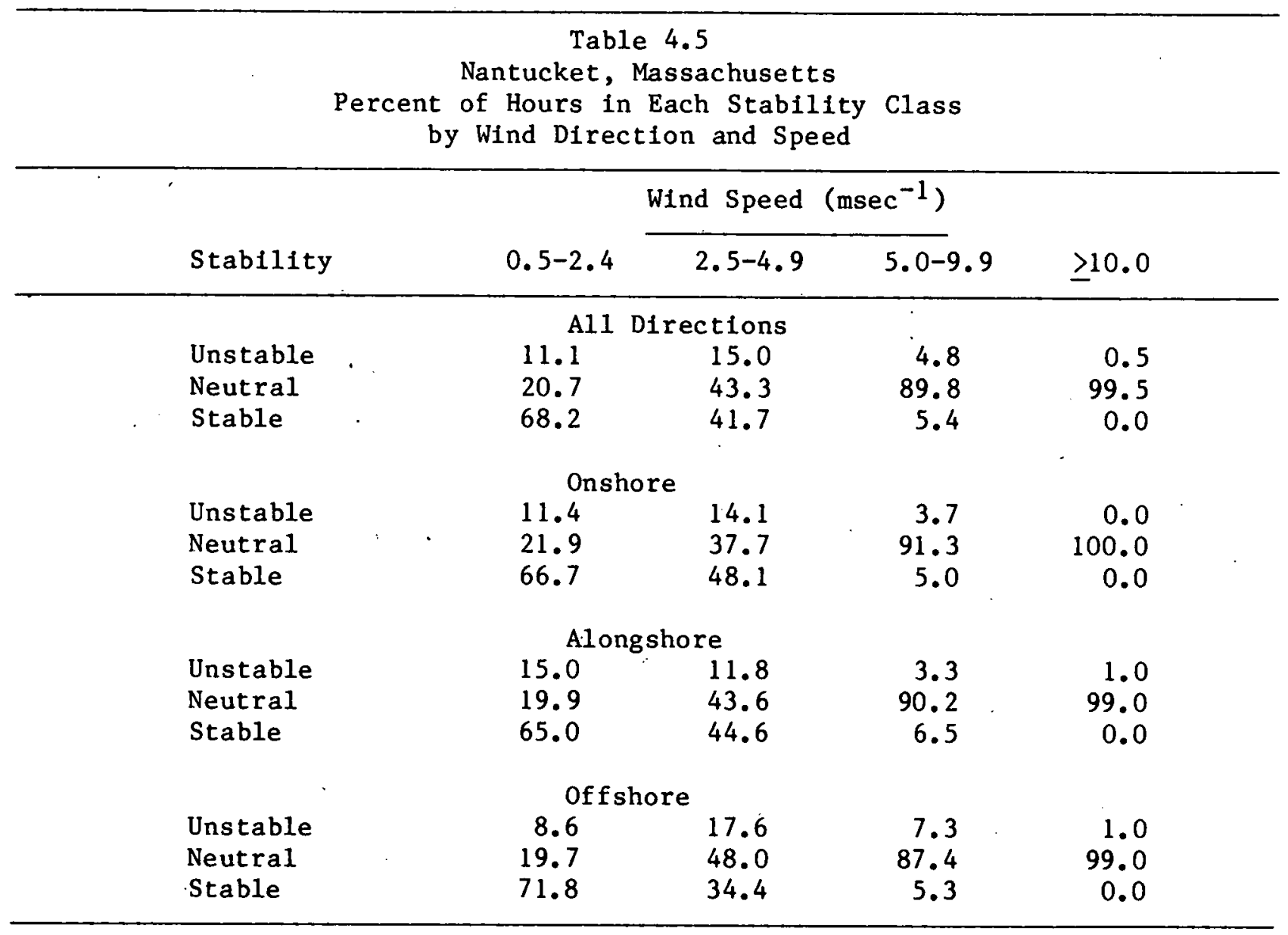




\begin{tabular}{|c|c|c|c|c|}
\hline \multicolumn{5}{|c|}{$\begin{array}{c}\text { Percent of Hours in Each Stability Class } \\
\text { by Wind Direction and Speed }\end{array}$} \\
\hline \multirow[b]{2}{*}{ Stability } & \multirow[b]{2}{*}{$0.5-2.4$} & \multicolumn{2}{|c|}{ Wind Speed $\left(\mathrm{msec}^{-1}\right)$} & \multirow[b]{2}{*}{$\geq 10.0$} \\
\hline & & $2.5-4.9$ & $5.0-9.9$ & \\
\hline $\begin{array}{l}\text { Unstable } \\
\text { Neutral } \\
\text { Stable }\end{array}$ & $\begin{array}{l}\quad \text { A11 } \\
28.2 \\
13.8 \\
57.9\end{array}$ & $\begin{array}{c}\text { ections } \\
28.6 \\
37.5 \\
33.9\end{array}$ & $\begin{array}{r}6.8 \\
84.9 \\
8.3\end{array}$ & $\begin{array}{r}0.0 \\
100.0 \\
0.0\end{array}$ \\
\hline $\begin{array}{l}\text { Unistable } \\
\text { Neutral } \\
\text { Stable }\end{array}$ & $\begin{array}{l}\quad \text { Onst } \\
34.6 \\
23.8 \\
36.5\end{array}$ & $\begin{array}{l}28.4 \\
54.6 \\
17.0\end{array}$ & $\begin{array}{r}1.8 \\
96.1 \\
2.1\end{array}$ & $\begin{array}{r}0.0 \\
100.0 \\
0.0\end{array}$ \\
\hline $\begin{array}{l}\text { Unstable } \\
\text { Neutral } \\
\text { Stable }\end{array}$ & $\begin{array}{l}\quad \text { Alor } \\
28.4 \\
16.6 \\
55.0\end{array}$ & $\begin{array}{l}\text { re } \\
33.3 \\
39.4 \\
27.3\end{array}$ & $\begin{array}{r}15.1 \\
80.0 \\
5.0\end{array}$ & $\begin{array}{r}0.0 \\
100.0 \\
0.0\end{array}$ \\
\hline $\begin{array}{l}\text { Unstable } \\
\text { Neutral } \\
\text { Stable }\end{array}$ & $\begin{array}{l}\text { Off } \\
23.1 \\
8.1 \\
68.7\end{array}$ & $\begin{array}{l}26.3 \\
27.3 \\
46.4\end{array}$ & $\begin{array}{r}6.3 \\
82.9 \\
10.8\end{array}$ & $\begin{array}{r}0.0 \\
100.0 \\
0.0\end{array}$ \\
\hline
\end{tabular}


Table 4.7

Lakehurst, New Jersey

Percent of Hours in Each Stability Class

by Wind Direction and Speed

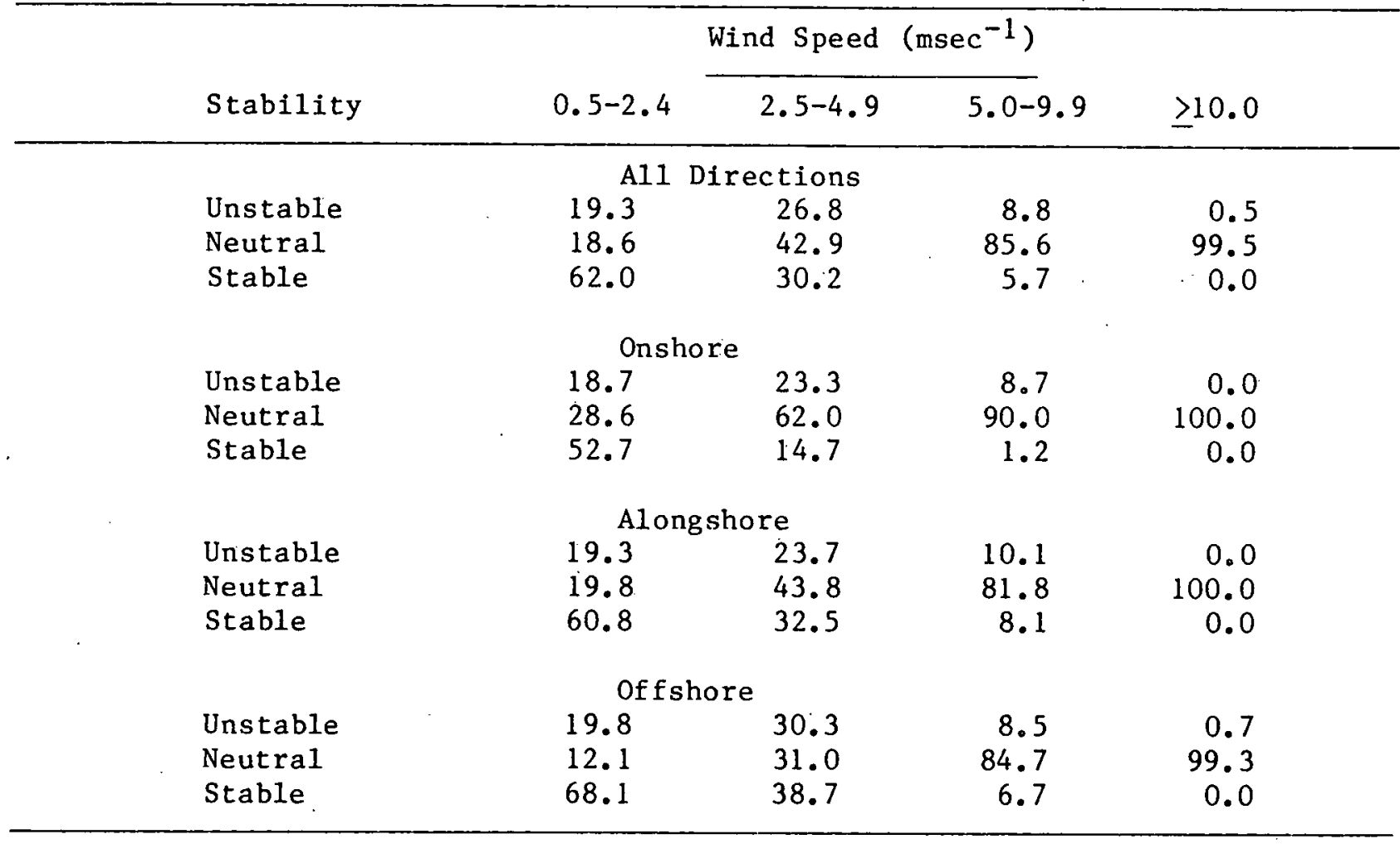




\begin{tabular}{|c|c|c|c|c|}
\hline \multirow[b]{3}{*}{ Stability } & $\begin{array}{l}\text { Tabl } \\
\text { antic Cit } \\
\text { Hours in } \\
\text { ind Direc }\end{array}$ & $\begin{array}{l}4.8 \\
\text {, New Jerse } \\
\text { Each Stabil } \\
\text { ion and Spe }\end{array}$ & $\begin{array}{l}\text { y } \\
\text { ity Class } \\
\text { ed }\end{array}$ & \\
\hline & \multirow[b]{2}{*}{$0.5-2.4$} & \multicolumn{2}{|c|}{ Wind Speed $\left(\mathrm{msec}^{-1}\right)$} & \multirow[b]{2}{*}{$\geq 10.0$} \\
\hline & & $2.5-4.9$ & $5.0-9.9$ & \\
\hline $\begin{array}{l}\text { Unstable } \\
\text { Neutra1. } \\
\text { SLalle: }\end{array}$ & $\begin{array}{l}\quad \text { Al1 } \\
16.5 \\
24.7 \\
58.8\end{array}$ & $\begin{array}{c}\text { irections } \\
17.2 \\
38.0 \\
44.7\end{array}$ & $\begin{array}{r}9.1 \\
84.9 \\
6.0\end{array}$ & $\begin{array}{r}0.4 \\
99.6 \\
0.0\end{array}$ \\
\hline $\begin{array}{l}\text { Unstable } \\
\text { Nentral } \\
\text { Stable }\end{array}$ & $\begin{array}{l}\text { Onsh } \\
17.1 \\
34.4 \\
18.1\end{array}$ & $\begin{array}{r}20.9 \\
48.5 \\
30.6\end{array}$ & $\begin{array}{r}13.6 \\
82.7 \\
3.6\end{array}$ & $\begin{array}{r}0.0 \\
100.0 \\
0.0\end{array}$ \\
\hline $\begin{array}{l}\text { Unstable } \\
\text { Neutral } \\
\text { Stable }\end{array}$ & $\begin{array}{l}\quad \text { Alon } \\
19.9 \\
25.0 \\
55.1\end{array}$ & $\begin{array}{l}\text { shore } \\
10.4 \\
41.4 \\
48.2\end{array}$ & $\begin{array}{r}6.0 \\
87.4 \\
6.6\end{array}$ & $\begin{array}{r}0.0 \\
100.0 \\
0.0\end{array}$ \\
\hline $\begin{array}{l}\text { Unstable } \\
\text { Neutral } \\
\text { Stable }\end{array}$ & $\begin{array}{l}\quad \text { Offs } \\
14.2 \\
19.7 \\
66.1\end{array}$ & $\begin{array}{r}18.8 \\
28.7 \\
52.5\end{array}$ & $\begin{array}{r}7.6 \\
85.1 \\
7.3\end{array}$ & $\begin{array}{r}0.7 \\
99.3 \\
0.0\end{array}$ \\
\hline
\end{tabular}




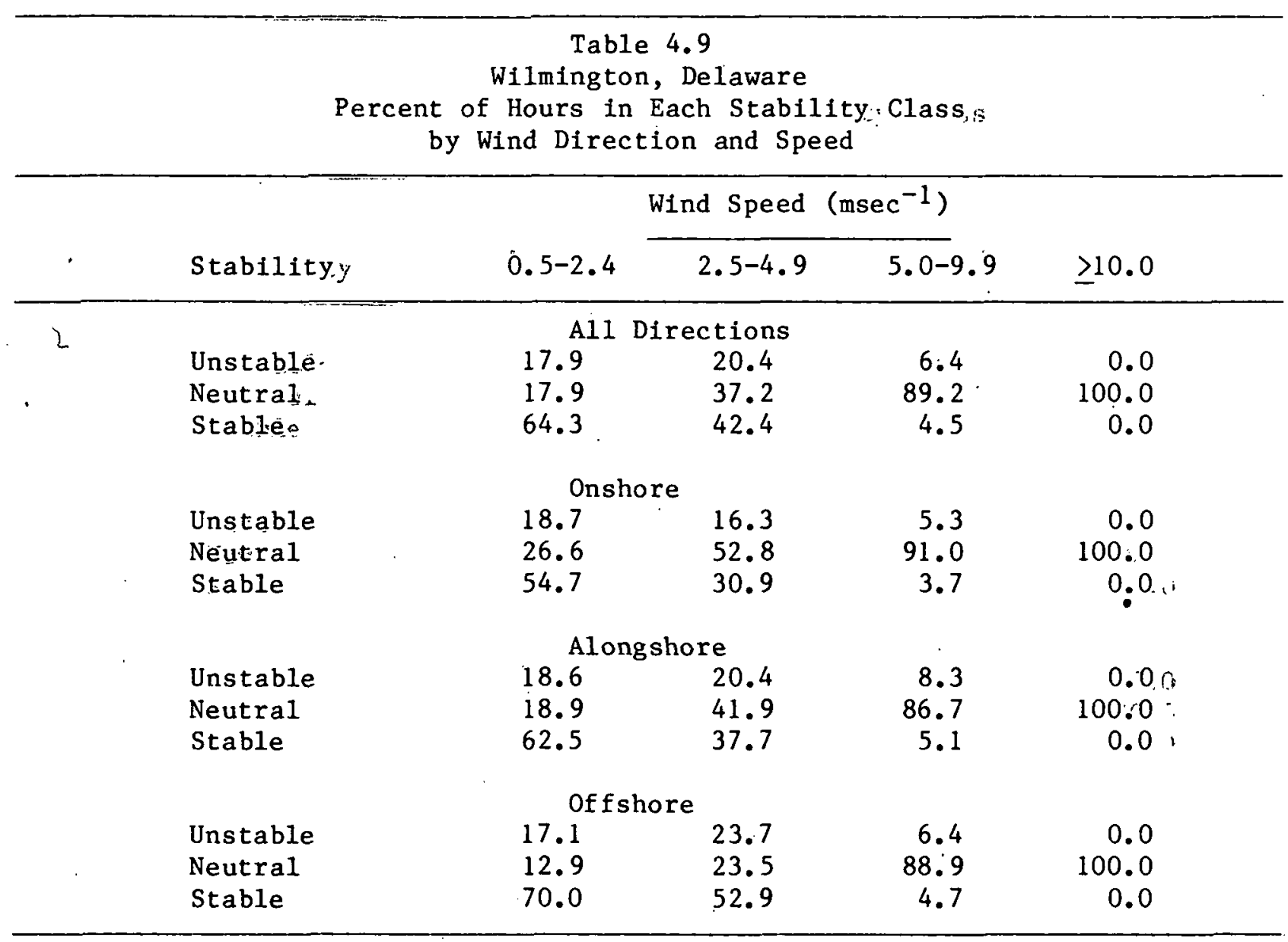




\begin{tabular}{|c|c|c|c|c|}
\hline & $\begin{array}{r}\text { Tab1 } \\
\text { 1lops Is } 1 \\
\text { Hours in } \\
\text { ind Direc }\end{array}$ & $\begin{array}{l}4.10 \\
\text { and, Virgin } \\
\text { Each Stabi } \\
\text { ion and Spe }\end{array}$ & Class & \\
\hline \multirow[b]{2}{*}{ Stability } & \multirow[b]{2}{*}{$0.5-2.4$} & \multicolumn{2}{|c|}{ Wind Speed $\left(\mathrm{msec}^{-1}\right)$} & \multirow[b]{2}{*}{$\geq 10.0$} \\
\hline & & $2.5-4.9$ & $5.0-9.9$ & \\
\hline $\begin{array}{l}\text { Unstable } \\
\text { Neutral } \\
\text { Stable }\end{array}$ & $\begin{array}{l}\quad \text { A11 } \\
37.7 \\
19.6 \\
12.7 \\
\end{array}$ & $\begin{array}{c}\text { Directions } \\
38.6 \\
29.6 \\
31.8\end{array}$ & $\begin{array}{r}11.4 \\
83.2 \\
5.4\end{array}$ & $\begin{array}{r}2.4 \\
97.6 \\
0.0\end{array}$ \\
\hline $\begin{array}{l}\text { Unstable } \\
\text { Neutral } \\
\text { Stable }\end{array}$ & $\begin{array}{l}\quad \text { Oneh } \\
44.5 \\
23.5 \\
32.0\end{array}$ & $\begin{array}{l}53.3 \\
26.6 \\
20.1\end{array}$ & $\begin{array}{r}19.8 \\
76.1 \\
4.1\end{array}$ & $\begin{array}{r}3.8 \\
96.2 \\
0.0\end{array}$ \\
\hline $\begin{array}{l}\text { Unstable } \\
\text { Neutral } \\
\text { Stable }\end{array}$ & $\begin{array}{l}\quad \text { Alon } \\
30.9 \\
18.3 \\
50.8\end{array}$ & $\begin{array}{l}\text { gshore } \\
\qquad \begin{array}{r}18.2 \\
39.1 \\
42.8\end{array}\end{array}$ & $\begin{array}{r}4.1 \\
89.6 \\
6.3\end{array}$ & $\begin{array}{r}5.9 \\
94.1 \\
0.0\end{array}$ \\
\hline $\begin{array}{l}\text { Unstable } \\
\text { Neutral } \\
\text { SLable }\end{array}$ & $\begin{array}{l}\quad \text { Offs } \\
34.5 \\
16.6 \\
48.9\end{array}$ & 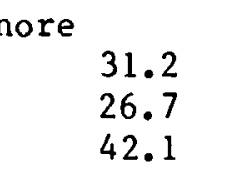 & $\begin{array}{r}9.6 \\
84.7 \\
5.7\end{array}$ & $\begin{array}{r}0.9 \\
99.1 \\
0.0\end{array}$ \\
\hline
\end{tabular}




\begin{tabular}{|c|c|c|c|c|}
\hline & $\begin{array}{r}\text { Tabl } \\
\text { Norfolk } \\
\text { Hours in } \\
\text { ind Direo }\end{array}$ & $\begin{array}{l}4.11 \\
\text { Virginia } \\
\text { Each Stabil } \\
\text { tion and Spe }\end{array}$ & Class & \\
\hline \multirow[b]{2}{*}{ Stability } & \multirow[b]{2}{*}{$0.5-2.4$} & \multicolumn{2}{|c|}{ Wind Speed $\left(\mathrm{msec}^{-1}\right)$} & \multirow[b]{2}{*}{$\geq 10.0$} \\
\hline & & $2.5-4.9$ & $5.0-9.9$ & \\
\hline $\begin{array}{l}\text { Unstable } \\
\text { Neutral } \\
\text { Stable }\end{array}$ & $\begin{array}{l}\quad A 11 \\
15.0 \\
13.2 \\
71.8\end{array}$ & $\begin{array}{c}\text { Directions } \\
22.6 \\
32.6 \\
44.7\end{array}$ & $\begin{array}{r}8.5 \\
84.7 \\
6.8\end{array}$ & $\begin{array}{r}0.0 \\
100.0 \\
0.0\end{array}$ \\
\hline $\begin{array}{l}\text { Unstable } \\
\text { Neutral } \\
\text { Stable }\end{array}$ & $\begin{array}{l}\quad \text { Onsh } \\
12.9 \\
13.4 \\
73.7\end{array}$ & $\begin{array}{l}21.7 \\
34.4 \\
43.9\end{array}$ & $\begin{array}{r}11.4 \\
83.5 \\
5.1\end{array}$ & $\begin{array}{r}0.0 \\
100.0 \\
0.0\end{array}$ \\
\hline $\begin{array}{l}\text { Unstable } \\
\text { Neutral } \\
\text { Stable }\end{array}$ & $\begin{array}{l}\text { Alon } \\
16.7 \\
13.8 \\
69.5\end{array}$ & $\begin{array}{l}\text { shore } \\
\begin{array}{r}22.1 \\
30.3 \\
47.6\end{array}\end{array}$ & $\begin{array}{r}7.6 \\
85.4 \\
7.0\end{array}$ & $\begin{array}{r}8.0 \\
100.0 \\
0.0\end{array}$ \\
\hline $\begin{array}{l}\text { Unstable } \\
\text { Neutral } \\
\text { Stable }\end{array}$ & $\begin{array}{l}\quad \text { Offs } \\
18.3 \\
12.2 \\
69.5\end{array}$ & $\begin{array}{l}25.1 \\
33.0 \\
41.9\end{array}$ & $\begin{array}{r}7.4 \\
84.9 \\
7.6\end{array}$ & $\begin{array}{r}0.0 \\
100.0 \\
0.0\end{array}$ \\
\hline
\end{tabular}




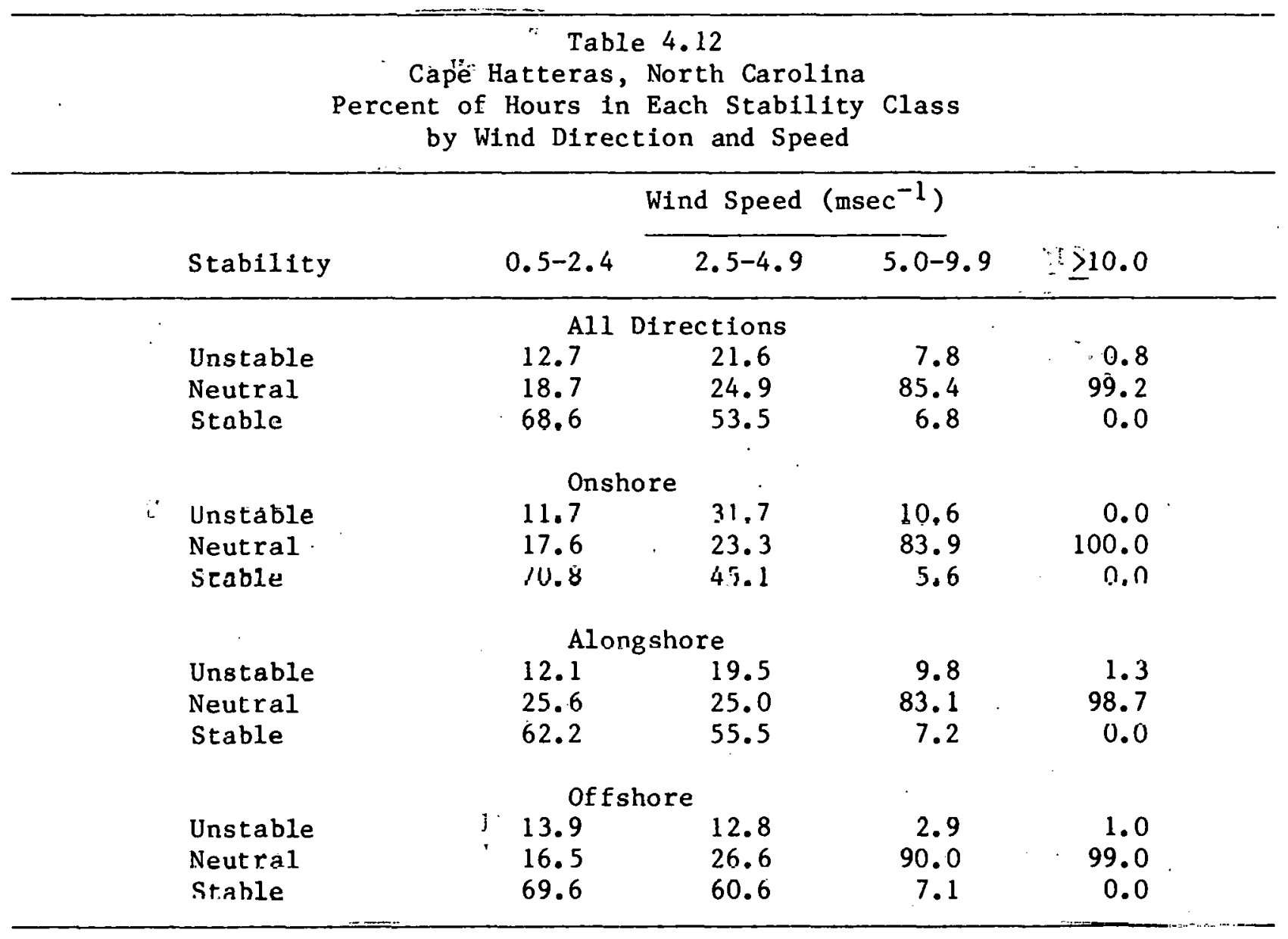




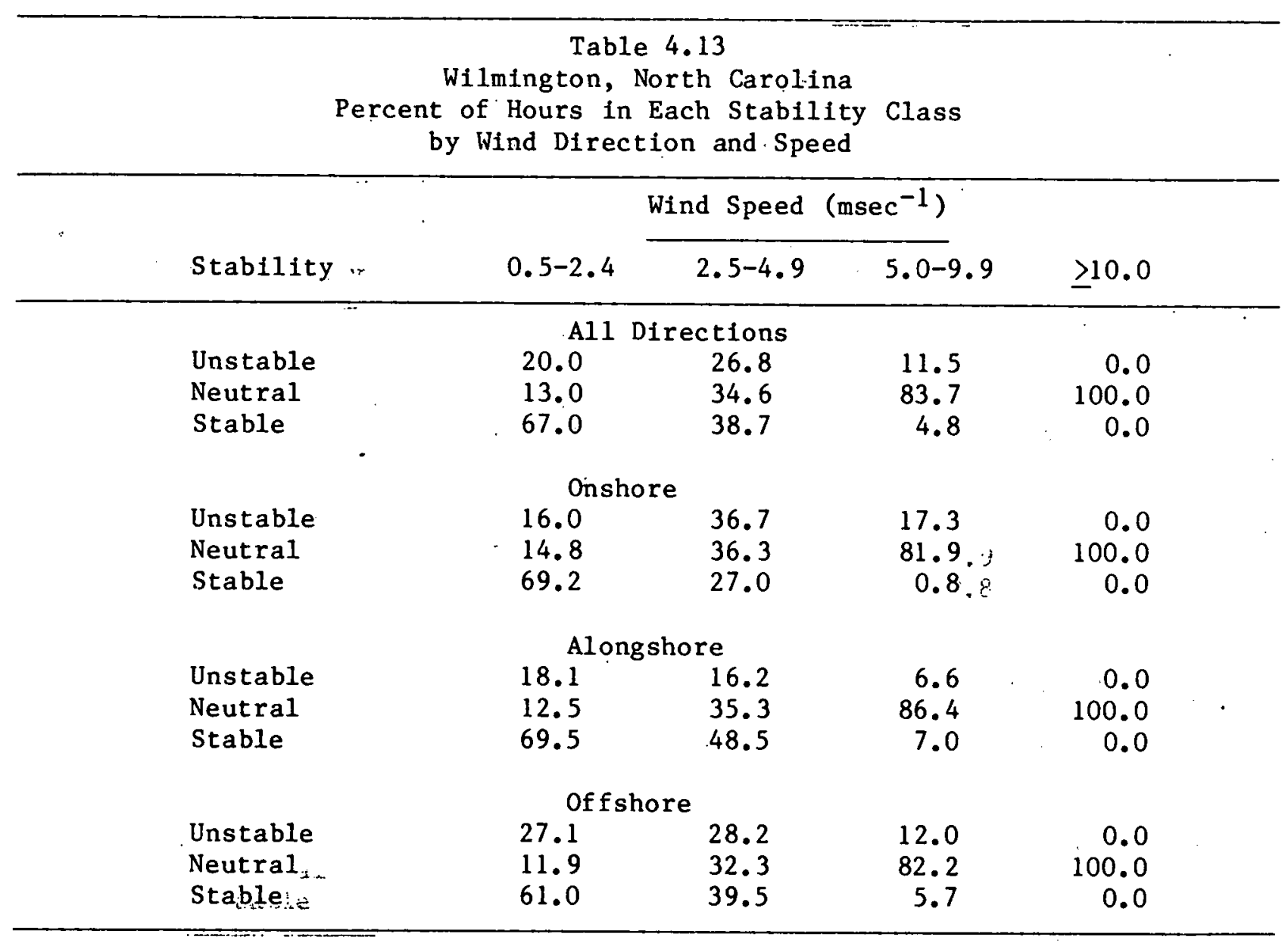


Table 4.14

Charleston, South Carolina

Percent of Hours in Each Stability Class

by Wind Direction and Speed

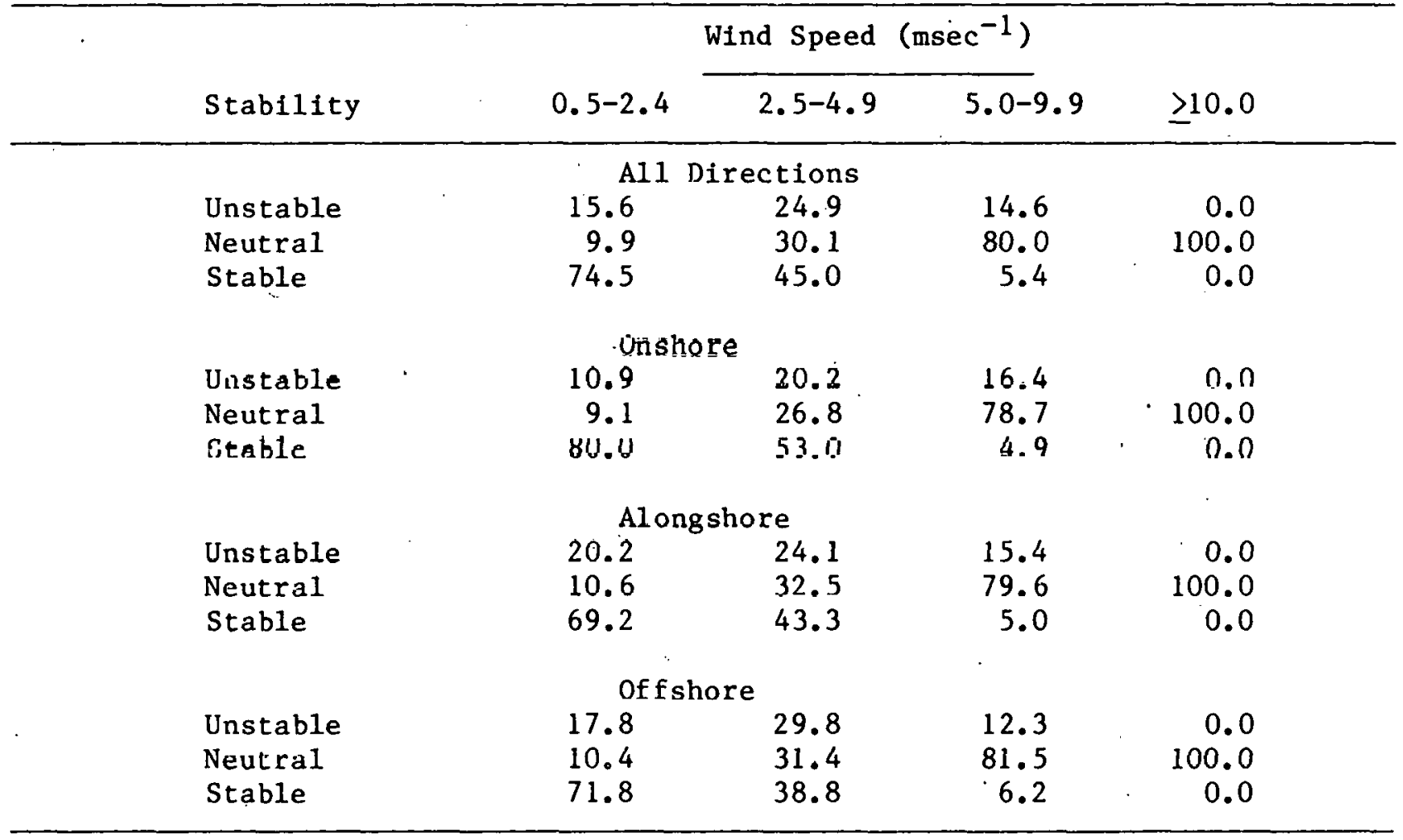


Table 4.15

Brunswick, Georgia

Percent of Hours in Each Stability Class

by Wind Direction and Speed

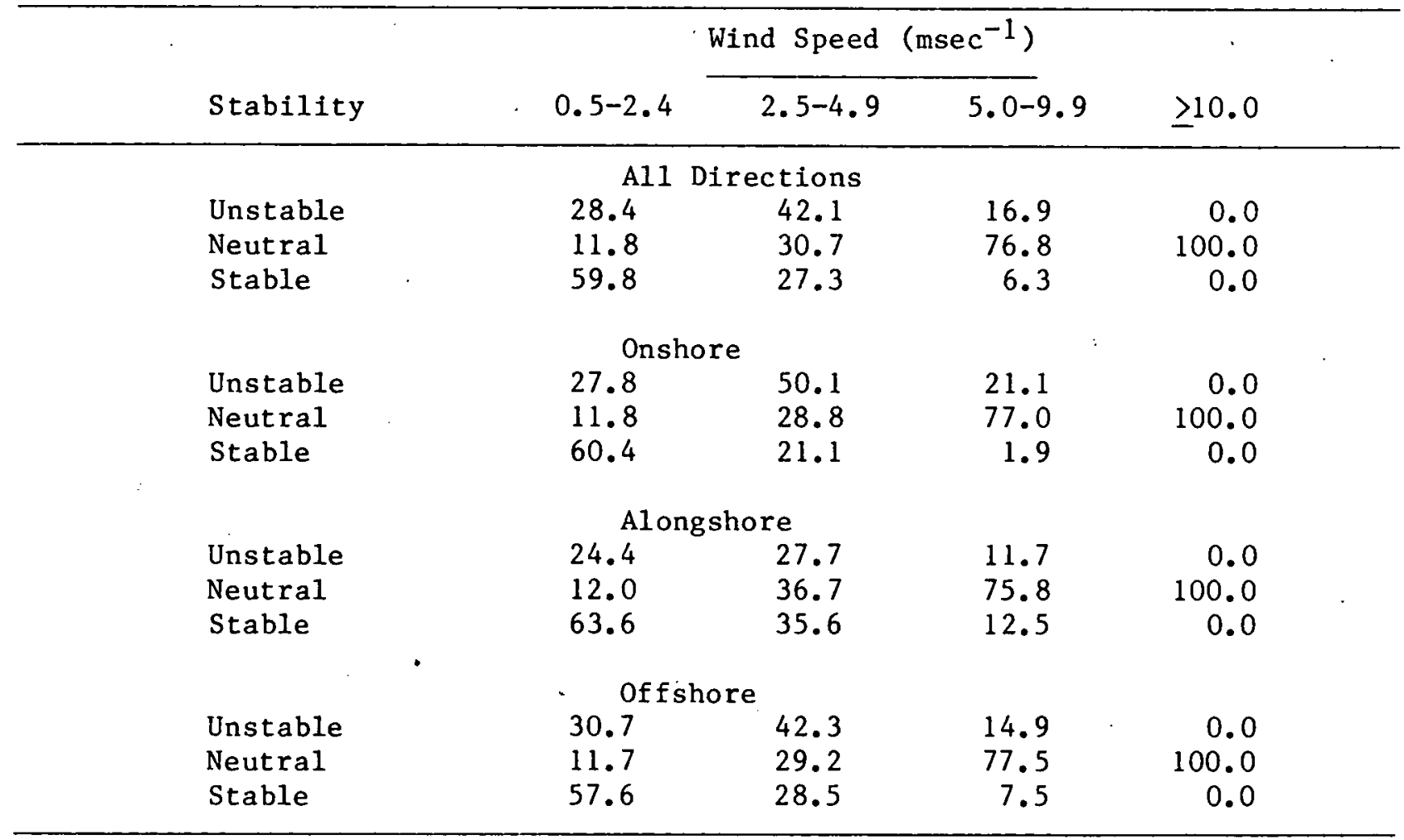




\begin{tabular}{|c|c|c|c|c|}
\hline & $\begin{array}{r}\text { Tab1 } \\
\text { ape Kenne } \\
\text { Hours in } \\
\text { ind Direc }\end{array}$ & $\begin{array}{l}\text { e } 4.16 \\
\text { dy, Florida } \\
\text { Each Stabil } \\
\text { tion and Spe }\end{array}$ & Class & \\
\hline \multirow[b]{2}{*}{ Stability } & \multirow[b]{2}{*}{$0.5-2.4$} & \multicolumn{2}{|c|}{ Wind Speed $\left(\mathrm{msec}^{-1}\right)$} & \multirow[b]{2}{*}{$\Rightarrow \geq 10.0$} \\
\hline & & $2.5-4.9$ & $5.0-9.9$ & \\
\hline $\begin{array}{l}\text { Unstable } \\
\text { Neutral } \\
\text { Stable }\end{array}$ & $\begin{array}{c}\text { A11 } \\
16.2 \\
7.0 \\
76.9\end{array}$ & $\begin{array}{c}\text { Directions } \\
43.4 \\
16.8 \\
39.8\end{array}$ & $\begin{array}{r}21.9 \\
69.8 \\
8.3\end{array}$ & $\begin{array}{r}0.0 \\
100.0 \\
0.0\end{array}$ \\
\hline $\begin{array}{l}\text { Unstable } \\
\text { Neutral } \\
\text { Stable }\end{array}$ & $\begin{array}{l}\text { Onsh } \\
17.8 \\
6.8 \\
75.3\end{array}$ & $\begin{array}{l}\text { ore } \\
\begin{array}{r}55.5 \\
13.9 \\
30.7\end{array}\end{array}$ & $\begin{array}{r}31.8 \\
60.5 \\
7.8\end{array}$ & $\begin{array}{r}0.0 \\
100.0 \\
0.0\end{array}$ \\
\hline $\begin{array}{l}\text { Unstable } \\
\text { Neutral } \\
\text { Stable }\end{array}$ & $\begin{array}{c}\text { A.1nn } \\
11.2^{8} \\
8.1 \\
80.6\end{array}$ & $\begin{array}{r}\text { gshore } \\
35.0 \\
17.9 \\
47.1\end{array}$ & $\begin{array}{r}16.3 \\
76.6 \\
7.1\end{array}$ & $\begin{array}{r}0.0 \\
100.0 \\
0.0\end{array}$ \\
\hline $\begin{array}{l}\text { Unstable } \\
\text { Neutral } \\
\text { Stable }\end{array}$ & $\begin{array}{l}\text { Offs } \\
18.5 \\
6.2 \\
75.2\end{array}$ & $\begin{array}{l}\text { hore } \\
\begin{array}{r}31.1 \\
20.9 \\
48.0\end{array}\end{array}$ & $\begin{array}{l}16.1 \\
72.5 \\
11.5\end{array}$ & $\begin{array}{r}0.0 \\
100.0 \\
0.0\end{array}$ \\
\hline
\end{tabular}




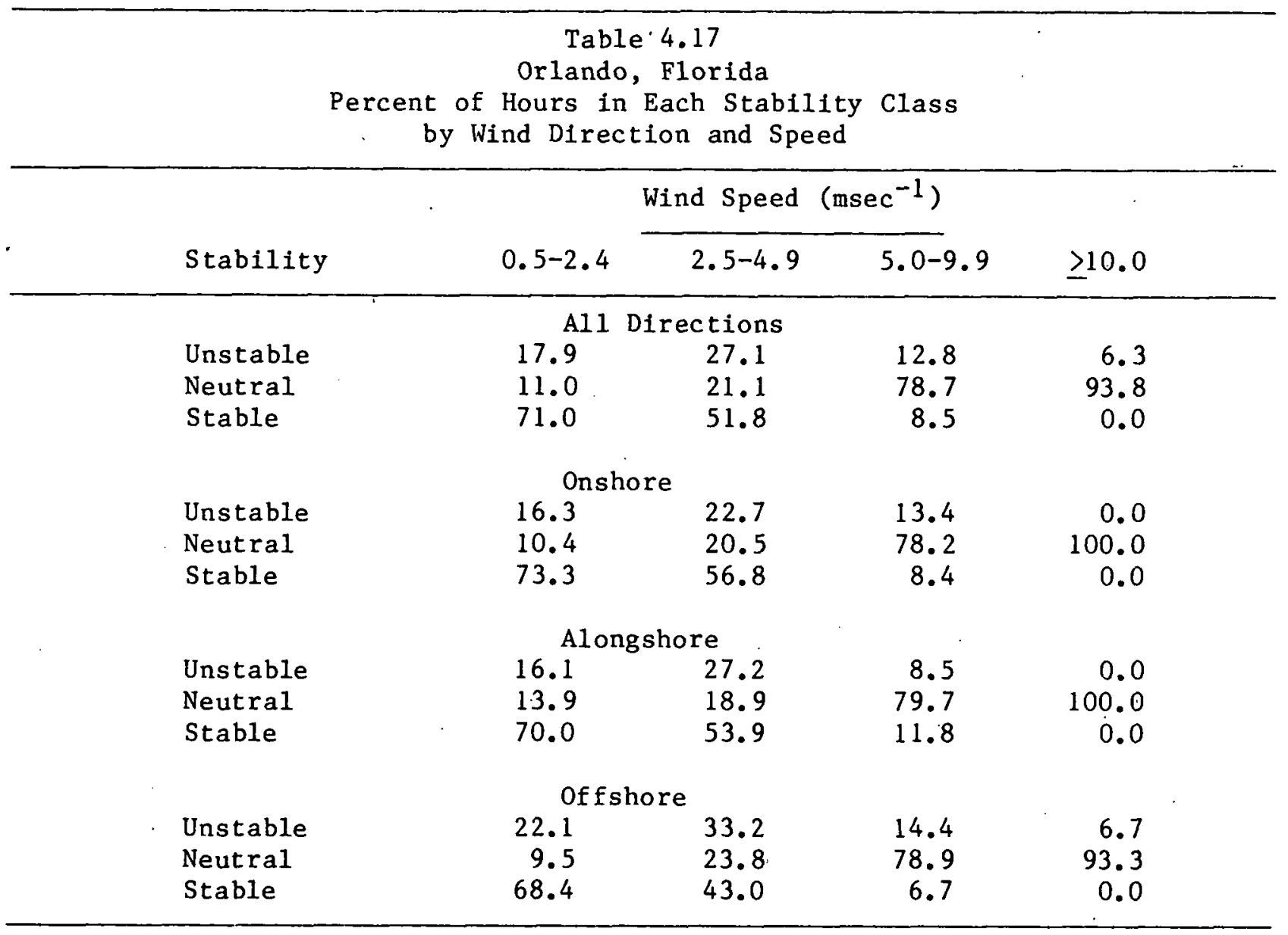




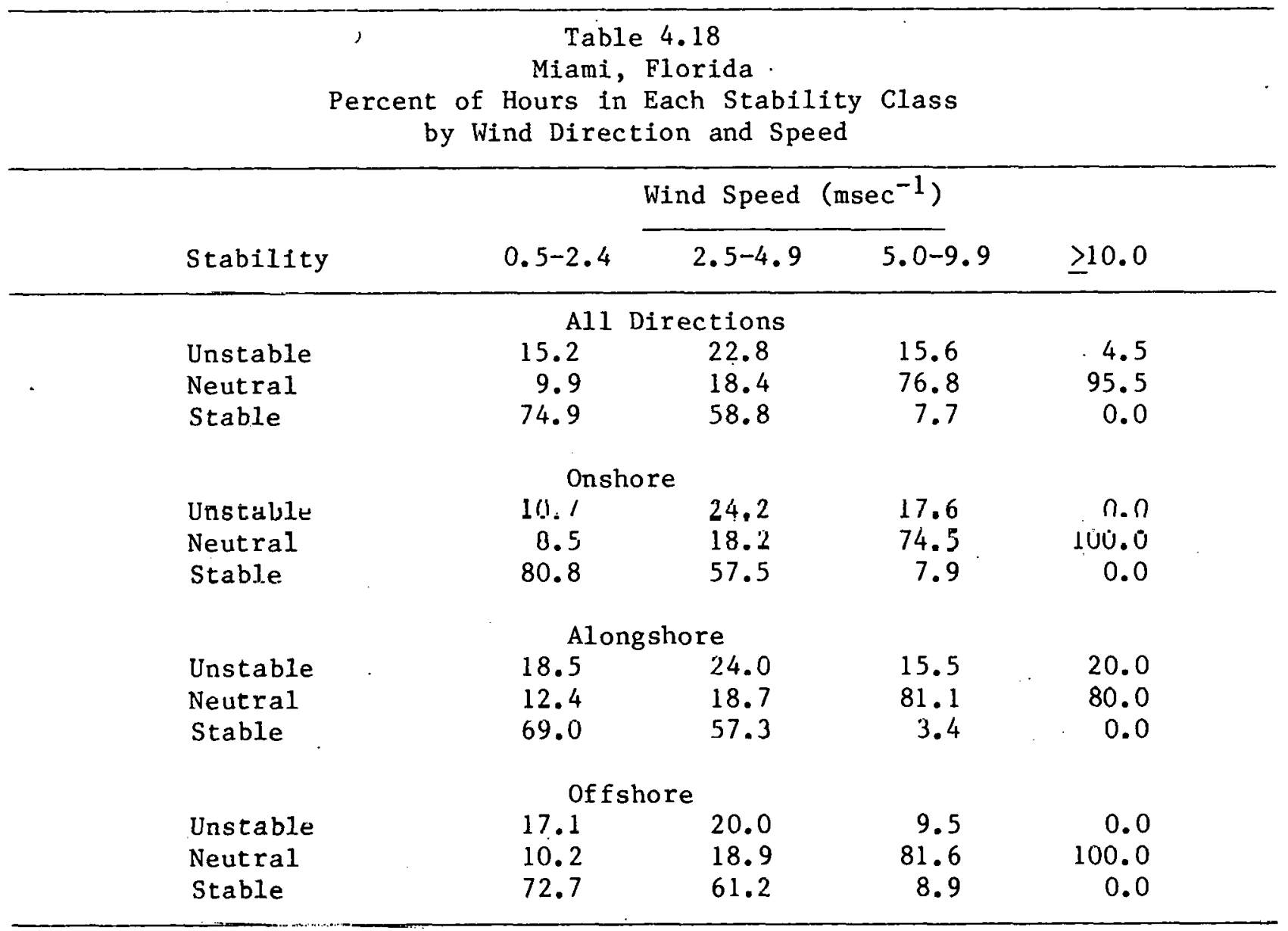




\begin{tabular}{|c|c|c|c|c|}
\hline \multicolumn{4}{|c|}{$\begin{array}{c}\text { Table } 4.19 \\
\text { Fort Myers, Florida } \\
\text { Percent of Hours in Each Stability Class } \\
\text { by Wind Direction and Speed }\end{array}$} & \multirow[b]{3}{*}{$\geq 10.0$} \\
\hline & \multirow[b]{2}{*}{$0.5-2.4$} & \multicolumn{2}{|c|}{ Wind Speed $\left(\mathrm{msec}^{-1}\right)$} & \\
\hline Stability & & $2.5-4.9$ & $5.0-9.9$ & \\
\hline $\begin{array}{l}\text { Unstable } \\
\text { Neutral } \\
\text { Stable }\end{array}$ & $\begin{array}{l}\text { A11 } \\
12.5 \\
1.0 .1 \\
77.4\end{array}$ & $\begin{array}{c}\text { Directions } \\
29.0 \\
16.4 \\
54.5\end{array}$ & $\begin{array}{r}20.6 \\
70.9 \\
8.5\end{array}$ & $\begin{array}{r}0.0 \\
100.0 \\
0.0\end{array}$ \\
\hline $\begin{array}{l}\text { Unstable } \\
\text { Neutral } \\
\text { Stable }\end{array}$ & $\begin{array}{l}\text { Onsh } \\
18.3 \\
18.5 \\
63.2\end{array}$ & $\begin{array}{l}39.0 \\
15.2 \\
45.8\end{array}$ & $\begin{array}{r}24.9 \\
69.7 \\
5.4\end{array}$ & $\begin{array}{r}0.0 \\
100.0 \\
0.0\end{array}$ \\
\hline $\begin{array}{l}\text { Unstable } \\
\text { Neutral } \\
\text { Stable }\end{array}$ & $\begin{array}{l}\quad \text { Alon } \\
11.9 \\
13.0 \\
75.2\end{array}$ & $\begin{array}{l}\text { Igshore } \\
\qquad \begin{array}{r}28.7 \\
21.3 \\
50.0\end{array}\end{array}$ & $\begin{array}{r}14.4 \\
75.9 \\
9.7\end{array}$ & $\begin{array}{r}0.0 \\
100.0 \\
0.0\end{array}$ \\
\hline $\begin{array}{l}\text { Unstable } \\
\text { Neutral } \\
\text { Stable }\end{array}$ & $\begin{array}{r}\text { Offs } \\
11.6 \\
7.8 \\
80.6\end{array}$ & $\begin{array}{l}\text { hore } \\
\qquad \begin{array}{r}25.0 \\
15.4 \\
59.6\end{array}\end{array}$ & $\begin{array}{l}19.4 \\
70.5 \\
10.1\end{array}$ & $\begin{array}{r}0.0 \\
100.0 \\
0.0\end{array}$ \\
\hline
\end{tabular}




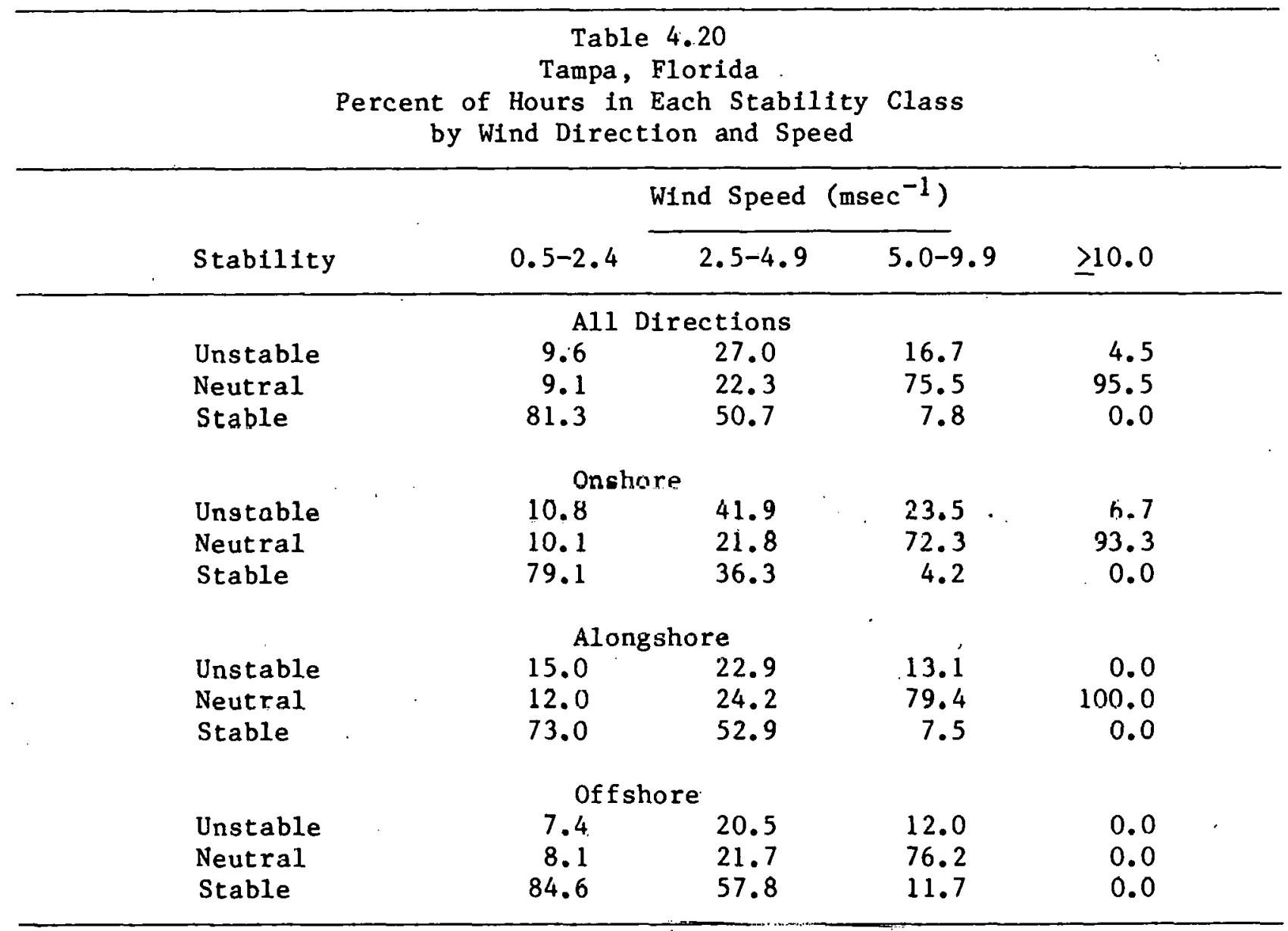


Table 4.21

Tallahassee, Florida

Percent of Hours in Each Stability Class

by Wind Direction and Speed

\begin{tabular}{|c|c|c|c|c|c|}
\hline & \multirow[b]{2}{*}{ Stability } & \multirow[b]{2}{*}{$0.5-2.4$} & \multicolumn{3}{|c|}{ Wind Speed $\left(\mathrm{msec}^{-1}\right)$} \\
\hline & & & $2.5-4.9$ & $5.0-9.9$ & $\geq 10.0$ \\
\hline & $\begin{array}{l}\text { Unstable } \\
\text { Neutral } \\
\text { Stable }\end{array}$ & $\begin{array}{l}\quad \text { A11 } \\
18.6 \\
11.6 \\
69.9\end{array}$ & $\begin{array}{c}\text { Directions } \\
34.6 \\
30.9 \\
34.5\end{array}$ & $\begin{array}{r}17.5 \\
78.8 \\
3.7\end{array}$ & $\begin{array}{r}0.0 \\
100.0 \\
0.0\end{array}$ \\
\hline & $\begin{array}{l}\text { Unstable } \\
\text { Neutral } \\
\text { Stable }\end{array}$ & $\begin{array}{l}\quad \text { Onsh } \\
20.7 \\
14.1 \\
65.1\end{array}$ & lore $\begin{array}{l}31.4 \\
39.2 \\
29.4\end{array}$ & $\begin{array}{r}16.6 \\
80.6 \\
2.8\end{array}$ & $\begin{array}{r}0.0 \\
100.0 \\
0.0\end{array}$ \\
\hline - & $\begin{array}{l}\text { Unstable } \\
\text { Neutral } \\
\text { Stable }\end{array}$ & $\begin{array}{l}\quad \text { Alor } \\
15.6 \\
10.2 \\
74.2\end{array}$ & $\begin{array}{l}\text { Igshore } \\
32.5 \\
29.7 \\
37.8\end{array}$ & $\begin{array}{r}22.9 \\
69.1 \\
8.0\end{array}$ & $\begin{array}{r}0.0 \\
100.0 \\
0.0\end{array}$ \\
\hline & $\begin{array}{l}\text { Unstable } \\
\text { Neutra1 } \\
\text { Stable }\end{array}$ & $\begin{array}{l}\quad \text { Offs } \\
18.1 \\
10.0 \\
71.9\end{array}$ & $\begin{array}{l}\text { hore } \\
\begin{aligned} 38.1 \\
24.9 \\
37.0\end{aligned}\end{array}$ & $\begin{array}{r}16.6 \\
80.0 \\
3.4\end{array}$ & $\begin{array}{l}0.0 \\
0.0 \\
0.0\end{array}$ \\
\hline
\end{tabular}




\begin{tabular}{|c|c|c|c|c|}
\hline \multirow[b]{3}{*}{ Stability } & $\begin{array}{r}\text { Tabl } \\
\text { Pensacol } \\
\text { Hours in } \\
\text { ind Direc }\end{array}$ & $\begin{array}{l}\text { e } 4.22 \\
\text {, Florida } \\
\text { Each Stabil } \\
\text { tion and Spe }\end{array}$ & Class & \\
\hline & \multirow[b]{2}{*}{$0.5-2.4$} & \multicolumn{2}{|c|}{ Wind Speed $\left(\mathrm{msec}^{-1}\right)$} & \multirow[b]{2}{*}{$\geq 10.0$} \\
\hline & & $2.5-4.9$ & $5.0-9.9$ & \\
\hline $\begin{array}{l}\text { Unstable } \\
\text { Neutral } \\
\text { Stable }\end{array}$ & $\begin{array}{l}\quad \text { A11 } \\
14.1 \\
17.8 \\
68.1\end{array}$ & $\begin{array}{c}\text { Directions } \\
24.5 \\
28.5 \\
47.0\end{array}$ & $\begin{array}{r}15.2 \\
77.4 \\
7.4\end{array}$ & $\begin{array}{r}0.0 \\
100.0 \\
0.0\end{array}$ \\
\hline $\begin{array}{l}\text { Unotable } \\
\text { Neutral } \\
\text { Stable }\end{array}$ & $\begin{array}{l}\quad \text { Onsh } \\
16.4 \\
17.6 \\
65.9\end{array}$ & ore $\begin{array}{r} \\
33.0 \\
29.2 \\
37.8\end{array}$ & $\begin{array}{r}19.1 \\
75.7 \\
5.2\end{array}$ & $\begin{array}{r}0.0 \\
100.0 \\
0.0\end{array}$ \\
\hline $\begin{array}{l}\text { Unstable } \\
\text { Neutral } \\
\text { Stable }\end{array}$ & $\begin{array}{l}\quad \text { Alon } \\
14.5 \\
22.0 \\
63.5\end{array}$ & $\begin{array}{l}\text { gshore } \\
\qquad \begin{array}{r}16.3 \\
35.4 \\
48.3\end{array}\end{array}$ & $\begin{array}{l}11.6 \\
76.1 \\
12.3\end{array}$ & $\begin{array}{l}0.0 \\
0.0 \\
0.0\end{array}$ \\
\hline $\begin{array}{l}\text { Unstable } \\
\text { Neutra1 } \\
\text { Stable }\end{array}$ & $\begin{array}{l}\quad \text { Offs } \\
12.8 \\
14.7 \\
72.5\end{array}$ & $\begin{array}{l}\text { hore } \\
\qquad \begin{array}{r}21.8 \\
22.4 \\
55.8\end{array}\end{array}$ & $\begin{array}{r}10.6 \\
80.8 \\
8.6\end{array}$ & $\begin{array}{r}0.0 \\
100.0 \\
0.0\end{array}$ \\
\hline
\end{tabular}




\begin{tabular}{|c|c|c|c|c|}
\hline \multirow[b]{3}{*}{ Stability } & $\begin{array}{r}\text { Tab. } \\
\text { Mobile } \\
\text { Hours ir } \\
\text { ind Dire }\end{array}$ & $\begin{array}{l}4.23 \\
\text { Alabama } \\
\text { Each Stabi } \\
\text { tion and Spe }\end{array}$ & Class & \\
\hline & \multirow[b]{2}{*}{$0.5-2.4$} & \multicolumn{2}{|c|}{ Wind Speed $\left(\mathrm{msec}^{-1}\right)$} & \multirow[b]{2}{*}{$\geq 10.0$} \\
\hline & & $2.5-4.9$ & $5.0-9.9$ & \\
\hline $\begin{array}{l}\text { Unstable } \\
\text { Neutral } \\
\text { Stable }\end{array}$ & $\begin{array}{l}\quad \text { A11 } \\
14.6 \\
15.9 \\
69.5\end{array}$ & $\begin{array}{c}\text { Directions } \\
20.7 \\
30.7 \\
48.6\end{array}$ & $\begin{array}{r}9.5 \\
84.8 \\
5.7\end{array}$ & $\begin{array}{r}0.0 \\
100.0 \\
0.0\end{array}$ \\
\hline $\begin{array}{l}\text { Unstable } \\
\text { Neutral } \\
\text { Stable }\end{array}$ & $\begin{array}{l}\quad \text { Onst } \\
12.7^{12} \\
16.8 \\
70.4\end{array}$ & 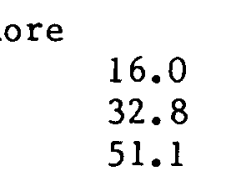 & $\begin{array}{r}9.1 \\
85.9 \\
4.9\end{array}$ & $\begin{array}{r}0.0 \\
100.0 \\
0.0\end{array}$ \\
\hline $\begin{array}{l}\text { Unstable } \\
\text { Neutral } \\
\text { Stable }\end{array}$ & $\begin{array}{l}\quad \text { Alor } \\
20.4 \\
15.1 \\
64.4\end{array}$ & $\begin{array}{l}\text { gshore } \\
27.5 \\
31.8 \\
40.7\end{array}$ & $\begin{array}{r}13.2 \\
81.6 \\
5.2\end{array}$ & $\begin{array}{r}0.0 \\
100.0 \\
0.0\end{array}$ \\
\hline $\begin{array}{l}\text { Unstable } \\
\text { Neutral } \\
\text { Stable }\end{array}$ & $\begin{array}{l}\quad \text { Offs } \\
12.2^{2} \\
16.4 \\
71.4\end{array}$ & $\begin{array}{l}\text { hore } \\
\qquad \begin{array}{r}21.5 \\
28.4 \\
50.0\end{array}\end{array}$ & $\begin{array}{r}8.6 \\
84.8 \\
6.6\end{array}$ & $\begin{array}{r}0.0 \\
100.0 \\
0.0\end{array}$ \\
\hline
\end{tabular}




\begin{tabular}{|c|c|c|c|c|}
\hline \multirow[b]{3}{*}{$=\cdots$ stability } & $\begin{array}{r}\text { Tab1 } \\
\text { Biloxi, M } \\
\text { Hours in } \\
\text { ind Direc } \\
\end{array}$ & $\begin{array}{l}\text { e } 4.24 \\
\text { ississippi } \\
\text { Each Stabil } \\
\text { tion and Spe }\end{array}$ & Class & \\
\hline & \multirow[b]{2}{*}{$0.5-2.4$} & \multicolumn{2}{|c|}{ Wind Speed $\left(\mathrm{msec}^{-1}\right)$} & \multirow[b]{2}{*}{$\geq 10.0$} \\
\hline & & $2.5-4.9$ & $5.0-9.9$ & \\
\hline $\begin{array}{l}\text { Unstable } \\
\text { Neutral } \\
\text { Stable }\end{array}$ & $\begin{array}{l}\quad \text { A11 } \\
37.6 \\
14.8 \\
47.6\end{array}$ & $\begin{array}{c}\text { Directions } \\
45.0 \\
29.9 \\
25.1\end{array}$ & $\begin{array}{r}20.9 \\
72.9 \\
6.2\end{array}$ & $\begin{array}{r}5.5 \\
94.5 \\
0.0\end{array}$ \\
\hline $\begin{array}{l}\text { IInstable } \\
\text { Neutral } \\
\text { Stable }\end{array}$ & $\begin{array}{l}\quad \text { Onsh } \\
36.7 \\
13.0 \\
50.4\end{array}$ & ore $\begin{array}{r}52.1 \\
25.4 \\
22.5\end{array}$ & $\begin{array}{r}31 . .5 \\
63.2 \\
5.3\end{array}$ & $\begin{array}{r}10.4 \\
89.6 \\
0.0\end{array}$ \\
\hline $\begin{array}{l}\text { Unstable } \\
\text { Neutral } \\
\text { Stable }\end{array}$ & $\begin{array}{l}\quad \text { Alon } \\
37.2 \\
17.3 \\
45.5\end{array}$ & $\begin{array}{r}\text { gshore } \\
39.1 \\
37.3 \\
23.6\end{array}$ & $\begin{array}{r}16.4 \\
78.9 \\
4.6\end{array}$ & $\begin{array}{r}0.0 \\
100.0 \\
0.0\end{array}$ \\
\hline $\begin{array}{l}\text { Unstable } \\
\text { Neutral } \\
\text { Stable }\end{array}$ & $\begin{array}{l}\quad \text { offs } \\
38.2 \\
15.6 \\
46.2\end{array}$ & 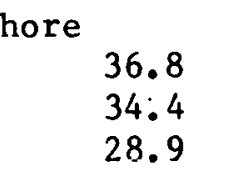 & $\begin{array}{r}10.0 \\
82.0 \\
8.0\end{array}$ & $\begin{array}{r}0.0 \\
100.0 \\
0.0\end{array}$ \\
\hline
\end{tabular}




\begin{tabular}{|c|c|c|c|c|}
\hline \multirow[b]{3}{*}{ Stability } & \multirow{2}{*}{\multicolumn{3}{|c|}{$\begin{array}{c}\text { Table } 4.25 \\
\text { New Orleans, Louisiana } \\
\begin{array}{c}\text { Percent of Hours in Each Stability Class } \\
\text { by Wind Direction and Speed }\end{array} \\
\text { Wind Speed }\left(\mathrm{msec}^{-1}\right)\end{array}$}} & \multirow[b]{3}{*}{$\geq 10.0$} \\
\hline & & & & \\
\hline & $0.5-2.4$ & $2.5-4.9$ & $5.0-9.9$ & \\
\hline $\begin{array}{l}\text { Unstable } \\
\text { Neutral } \\
\text { Stable }\end{array}$ & $\begin{aligned} & \mathrm{Al1} \\
& 15.3^{-} \\
& 8.5 \\
& 76.2\end{aligned}$ & $\begin{array}{c}\text { Directions } \\
: 27.8 \\
26.6 \\
45.6\end{array}$ & $\begin{array}{r}14.6 \\
80.0 \\
5.44\end{array}$ & $\begin{array}{r}0.0 \\
100.0 \\
0.0\end{array}$ \\
\hline $\begin{array}{l}\text { Unstable } \\
\text { Neutral } \\
\text { Stable }\end{array}$ & $\begin{array}{r}\text { Onsh } \\
14.0 \\
9.2 \\
76.8\end{array}$ & ore $\begin{array}{l}17.3 \\
29.4 \\
53.3\end{array}$ & $\begin{array}{r}11.6 \\
82.3 \\
6.1\end{array}$ & $\begin{array}{r}0.0 \\
100.0 \\
0.0\end{array}$ \\
\hline $\begin{array}{l}\text { Unstable } \\
\text { Neutral } \\
\text { Stable }\end{array}$ & $\begin{array}{l}\text { Alor } \\
10.7 \\
8.9 \\
80.4\end{array}$ & $\begin{array}{l}\text { gshore } \\
23.3 \\
27.0 \\
49.7\end{array}$ & $\begin{array}{r}15.3 \\
81.1 \\
3.6\end{array}$ & $\begin{array}{l}0.0 \\
0.0 \\
0.0\end{array}$ \\
\hline $\begin{array}{l}\text { Unstable } \\
\text { Neutra1 } \\
\text { Stable }\end{array}$ & $\begin{array}{c}\text { Offs } \\
19.2^{2} \\
7.9 \\
72.9\end{array}$ & $\begin{array}{l}\text { hore } \\
\begin{array}{r}40.6 \\
23.4 \\
35.9\end{array}\end{array}$ & $\begin{array}{r}17.5 \\
77.2 \\
5.3\end{array}$ & $\begin{array}{r}0.0 \\
100.0 \\
0.0\end{array}$ \\
\hline
\end{tabular}




\begin{tabular}{|c|c|c|c|c|}
\hline & $\begin{array}{c}\text { Bab] } \\
\text { Boothvill } \\
\text { Percent of Hours ir } \\
\text { by Wind Dired }\end{array}$ & $\begin{array}{l}4.26 \\
\text { Louisiana } \\
\text { Each Stabil } \\
\text { tion and Spe }\end{array}$ & ity Class & \\
\hline \multirow[b]{2}{*}{ Stability } & \multirow[b]{2}{*}{$0.5-2.4$} & \multicolumn{2}{|c|}{ Wind Speed $\left(\mathrm{msec}^{-1}\right)$} & \multirow[b]{2}{*}{$\geq 10.0$} \\
\hline & & $2.5-4.9$ & $5.0-9.9$ & \\
\hline $\begin{array}{l}\text { Unstable } \\
\text { Neutral } \\
\text { Stable }\end{array}$ & $\begin{array}{l}\text { A11 } \\
15.0 \\
9.3 \\
75.7\end{array}$ & $\begin{array}{c}\text { Directions } \\
30.4 \\
24.3 \\
45.3\end{array}$ & $\begin{array}{r}12.6 \\
79.2 \\
8.2\end{array}$ & $\begin{array}{r}5.5 \\
94.5 \\
0.0\end{array}$ \\
\hline $\begin{array}{l}\text { Unstable } \\
\text { Neutral } \\
\text { Stable }\end{array}$ & $\begin{array}{l}\text { Onsl } \\
15.1 \\
8.0 \\
76.9\end{array}$ & $\begin{array}{l}22.1 \\
25.0 \\
52.9\end{array}$ & $\begin{array}{r}11.4 \\
18.8 \\
9.8\end{array}$ & $\begin{array}{r}28.8 \\
71.2 \\
0.0\end{array}$ \\
\hline $\begin{array}{l}\text { Unstable } \\
\text { Neutral } \\
\text { Stable }\end{array}$ & $\begin{array}{l}\text { Alor } \\
10.4 \\
8.6 \\
81.1\end{array}$ & $\begin{array}{l}\text { Igshore } \\
20.4 \\
24.1 \\
55.5\end{array}$ & $\begin{array}{l}19.2 \\
70.1 \\
10.7\end{array}$ & $\begin{array}{l}0.0 \\
0.0 \\
0.0\end{array}$ \\
\hline $\begin{array}{l}\text { Unstable } \\
\text { Neutral } \\
\text { Stable }\end{array}$ & $\begin{array}{l}\quad \text { off } \\
18.7 \\
11.4 \\
69.9\end{array}$ & $\begin{array}{l}\text { shore } \\
\begin{array}{r}44.1 \\
23.8 \\
32.0\end{array}\end{array}$ & $\begin{array}{r}12.0 \\
81.8 \\
6.1\end{array}$ & $\begin{array}{r}0.0 \\
100.0 \\
0.0\end{array}$ \\
\hline
\end{tabular}




\begin{tabular}{|c|c|c|c|c|c|}
\hline & \multicolumn{5}{|c|}{$\begin{array}{l}\text { Lake Charles, Louisiana } \\
\text { Percent of Hours in Each Stability Class } \\
\text { by Wind Direction and Speed }\end{array}$} \\
\hline & \multirow[b]{2}{*}{ Stability } & \multirow[b]{2}{*}{$0.5-2.4$} & \multicolumn{2}{|c|}{ Wind Speed $\left(\mathrm{msec}^{-1}\right)$} & \multirow[b]{2}{*}{$\geq 10.0$} \\
\hline & & & $2.5-4.9$ & $5.0-9.9$ & \\
\hline & $\begin{array}{l}\text { Unstable } \\
\text { Neutral } \\
\text { Stable }\end{array}$ & $\begin{array}{l}\quad \text { A11 } \\
13.9 \\
11.1 \\
74.9\end{array}$ & $\begin{array}{c}\text { Directions } \\
23.4 \\
30.4 \\
46.2\end{array}$ & $\begin{array}{r}10.2 \\
86.8 \\
3.0\end{array}$ & $\begin{array}{r}3.5 \\
96.5 \\
0.0\end{array}$ \\
\hline . & $\begin{array}{l}\text { Unstable } \\
\text { Neutral } \\
\text { Stable }\end{array}$ & $\begin{array}{l}\quad \text { Onsh } \\
13.1 \\
10.6 \\
76.2\end{array}$ & 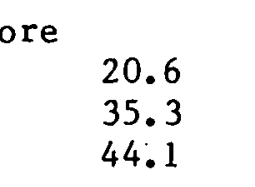 & $\begin{array}{r}11.9 \\
85.7 \\
2.5\end{array}$ & $\begin{array}{r}4.5 \\
95.5 \\
0.0\end{array}$ \\
\hline & $\begin{array}{l}\text { Unstable } \\
\text { Neutral } \\
\text { Stable }\end{array}$ & $\begin{array}{l}\quad \text { Alon } \\
13.2 \\
11.9 \\
74.9\end{array}$ & $\begin{array}{l}\text { gshore } \\
\qquad \begin{array}{r}27.4 \\
28.1 \\
44.5\end{array}\end{array}$ & $\begin{array}{r}13.8 \\
83.5 \\
2.6\end{array}$ & $\begin{array}{r}0.0 \\
100.0 \\
0.0\end{array}$ \\
\hline & $\begin{array}{l}\text { Unstable } \\
\text { Neutral } \\
\text { Stable }\end{array}$ & $\begin{array}{l}\quad \text { Offs } \\
15.4^{\prime} \\
11.1 \\
73.5\end{array}$ & 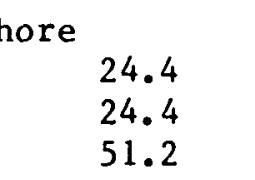 & $\begin{array}{r}7.0 \\
89.2 \\
3.8\end{array}$ & $\begin{array}{r}2.8 \\
97.2 \\
0.0\end{array}$ \\
\hline
\end{tabular}




\begin{tabular}{|c|c|c|c|c|c|}
\hline \multicolumn{6}{|c|}{$\begin{array}{l}\text { Table } 4.28 \\
\text { Galveston, Texas } \\
\text { Hours in Each Stability Class } \\
\text { Lnd Direction and Speed }\end{array}$} \\
\hline & \multirow[b]{2}{*}{ Stability } & \multirow[b]{2}{*}{$0.5-2.4$} & \multicolumn{2}{|c|}{ Wind Speed $\left(\mathrm{msec}^{-1}\right)$} & \multirow[b]{2}{*}{$\geq 10.0$} \\
\hline & & & $2.5-4.9$ & $5.0-9.9$ & \\
\hline . & $\begin{array}{l}\text { Unstable } \\
\text { Neutral } \\
\text { Stable }\end{array}$ & $\begin{array}{l}\text { A.1 } \\
27.6 \\
15.0 \\
57.4\end{array}$ & $\begin{array}{c}\text { Directions } \\
39.4 \\
27.4 \\
33.2\end{array}$ & $\begin{array}{r}15.3 \\
80.0 \\
4.6\end{array}$ & $\begin{array}{r}1.0 \\
99.0 \\
0.0\end{array}$ \\
\hline & $\begin{array}{l}\text { Unstable } \\
\text { Neutral } \\
\text { Stable }\end{array}$ & $\begin{array}{l}\quad \text { nnsh } \\
27.4 \\
16.0 \\
56.7\end{array}$ & $\begin{array}{r}41.3 \\
25.2 \\
33.5\end{array}$ & $\begin{array}{r}19.4 \\
75.3 \\
5.3\end{array}$ & $\begin{array}{r}2.8 \\
97.2 \\
0.0\end{array}$ \\
\hline & $\begin{array}{l}\text { Ungtable } \\
\text { Neutral } \\
\text { Stable }\end{array}$ & $\begin{array}{l}\text { Alor } \\
23.9 \\
11.3 \\
64.8\end{array}$ & $\begin{array}{r}\text { gshore } \\
32.7 \\
35.5 \\
31.8\end{array}$ & $\begin{array}{r}9.5 \\
87.2 \\
3.4\end{array}$ & $\begin{array}{r}0.0 \\
100.0 \\
0.0\end{array}$ \\
\hline & $\begin{array}{l}\text { Unstable } \\
\text { Neutral } \\
\text { Stable }\end{array}$ & $\begin{array}{l}\quad \text { offs } \\
31.2 \\
16.7 \\
52.1\end{array}$ & $\begin{array}{l}\text { hore } \\
\begin{aligned} 37.1 \\
29.8 \\
33.1\end{aligned}\end{array}$ & $\begin{array}{r}7.0 \\
89.7 \\
3.3\end{array}$ & $\begin{array}{r}1.1 \\
98.9 \\
0.0\end{array}$ \\
\hline
\end{tabular}




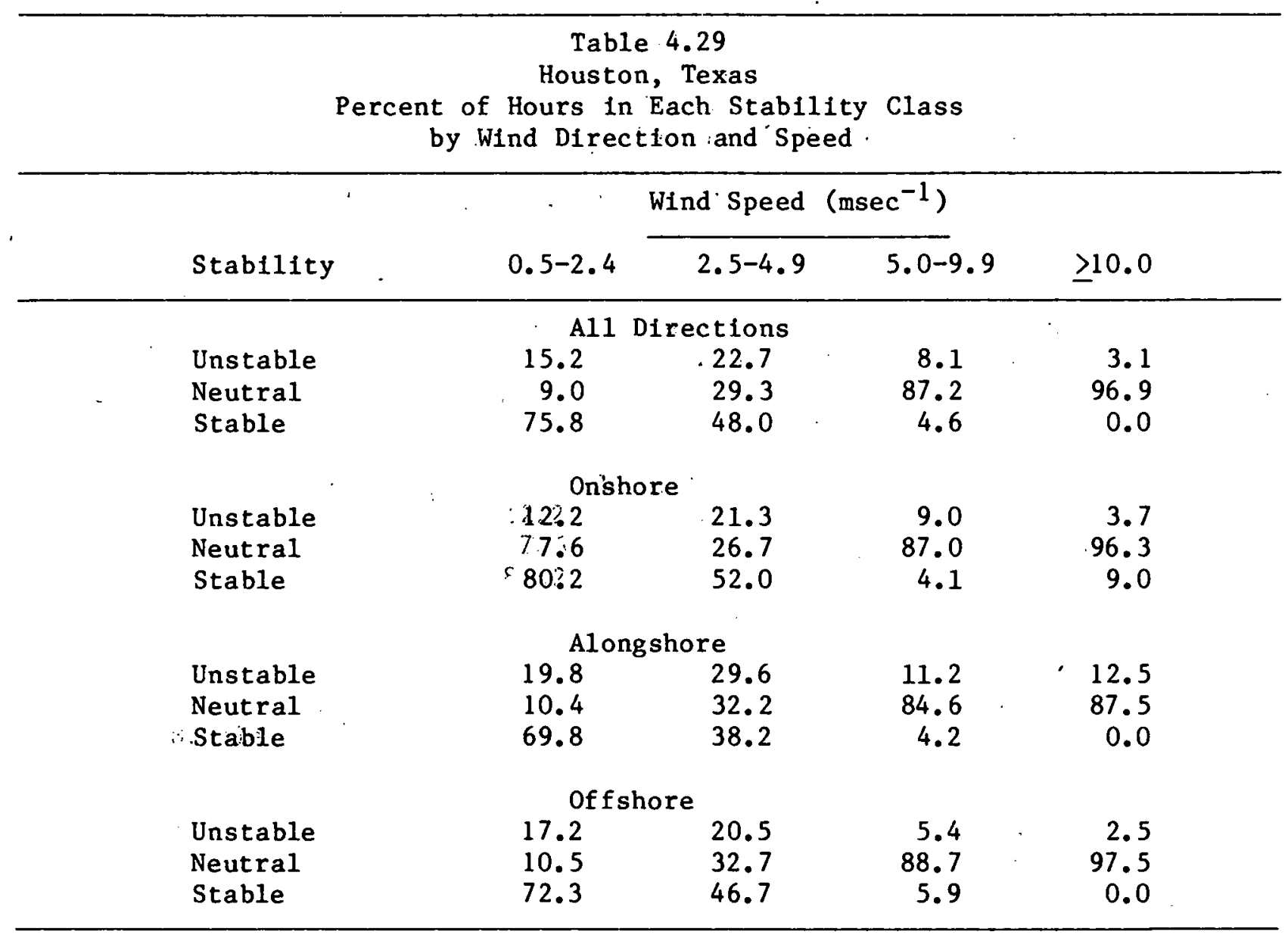




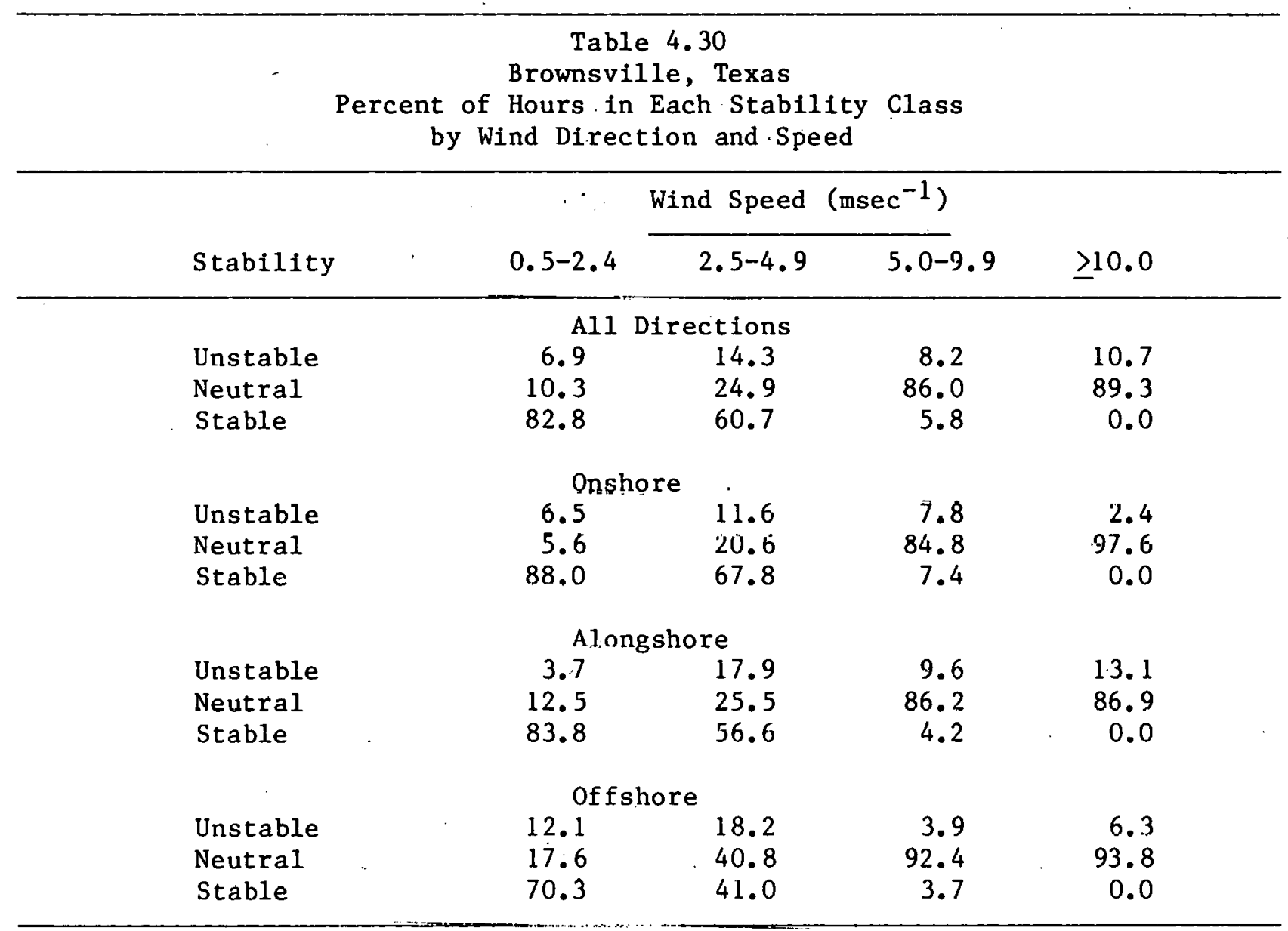




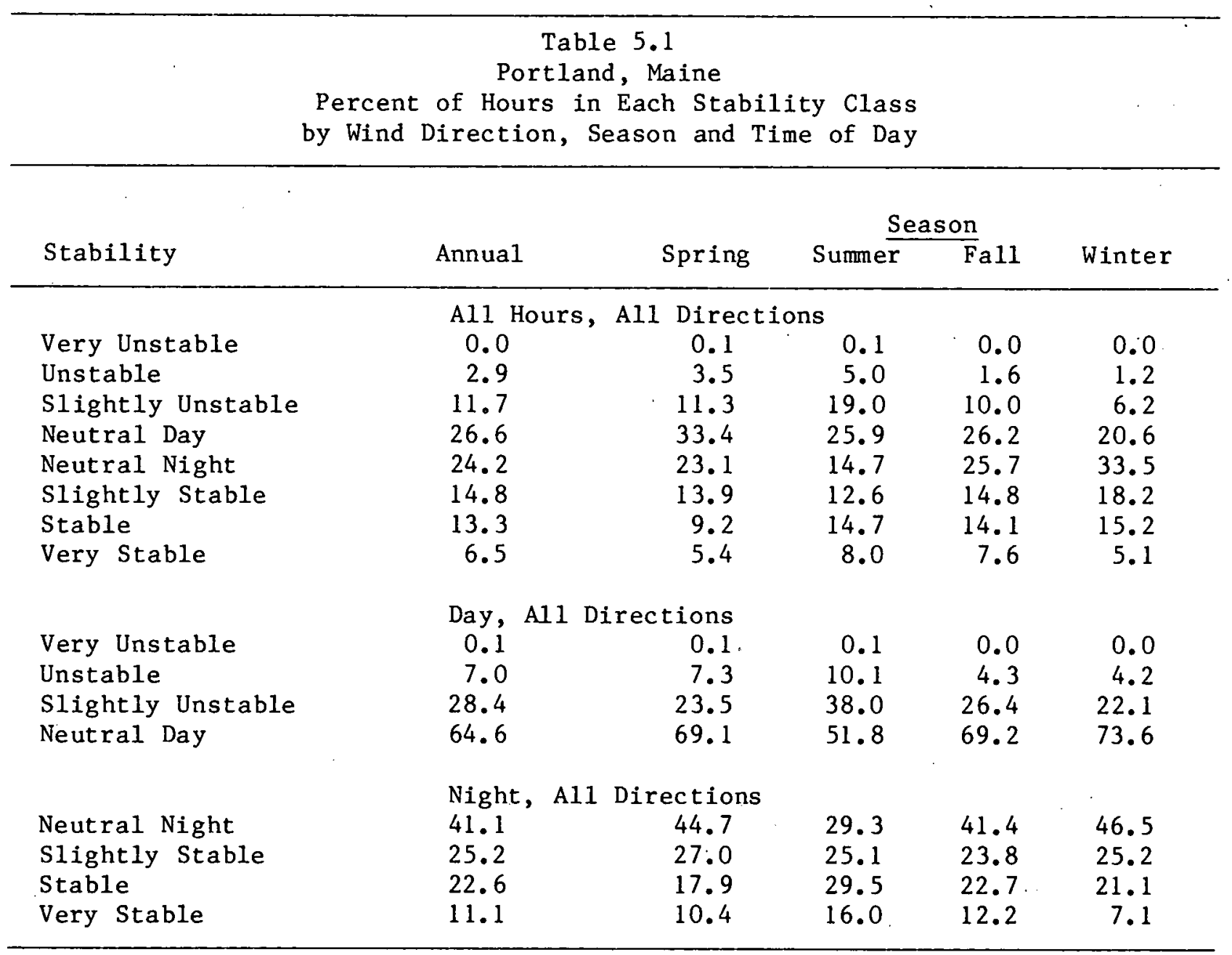


Täbile: 5i.ll (continued)

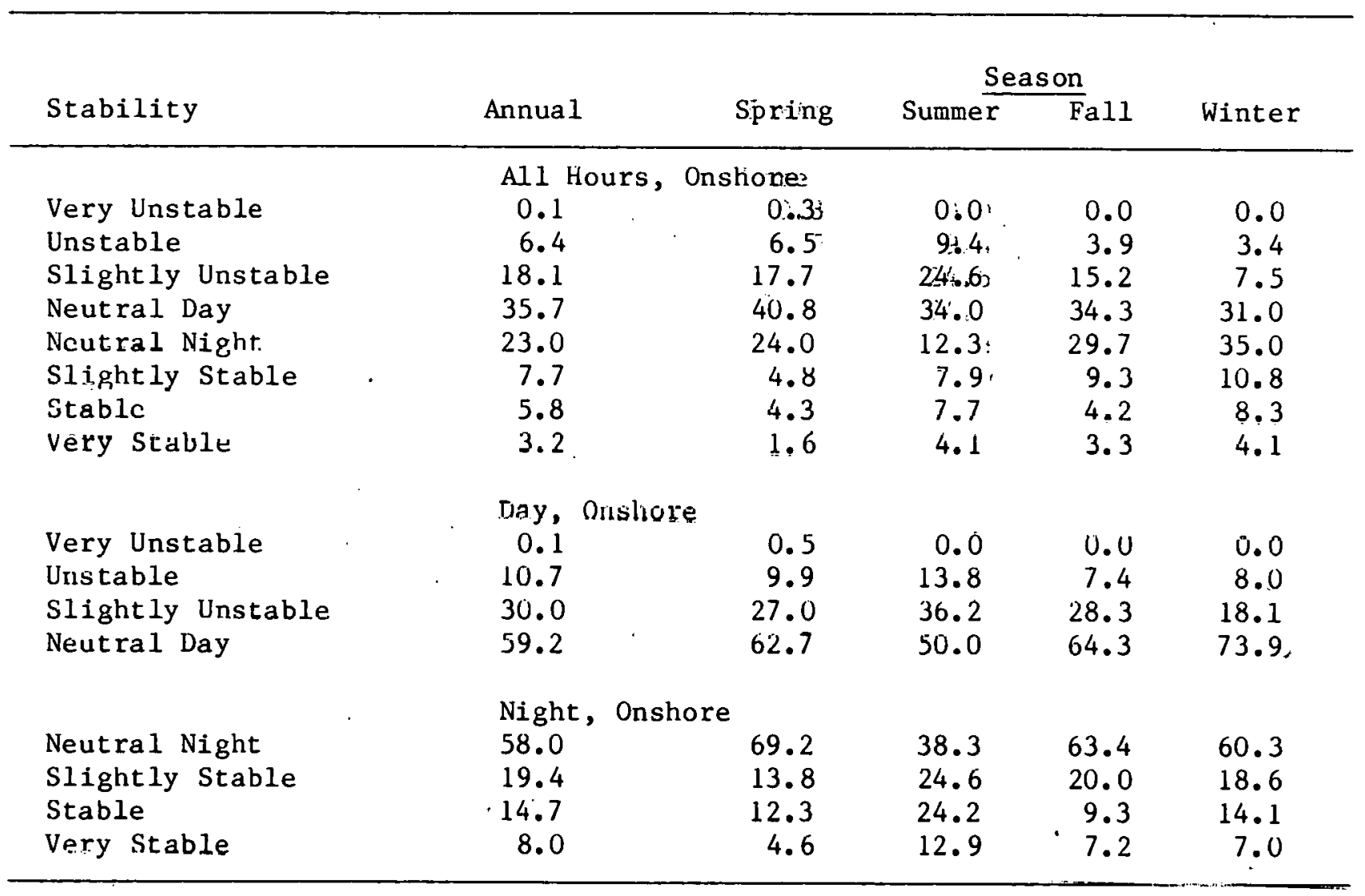


Table 5.1 (continued)

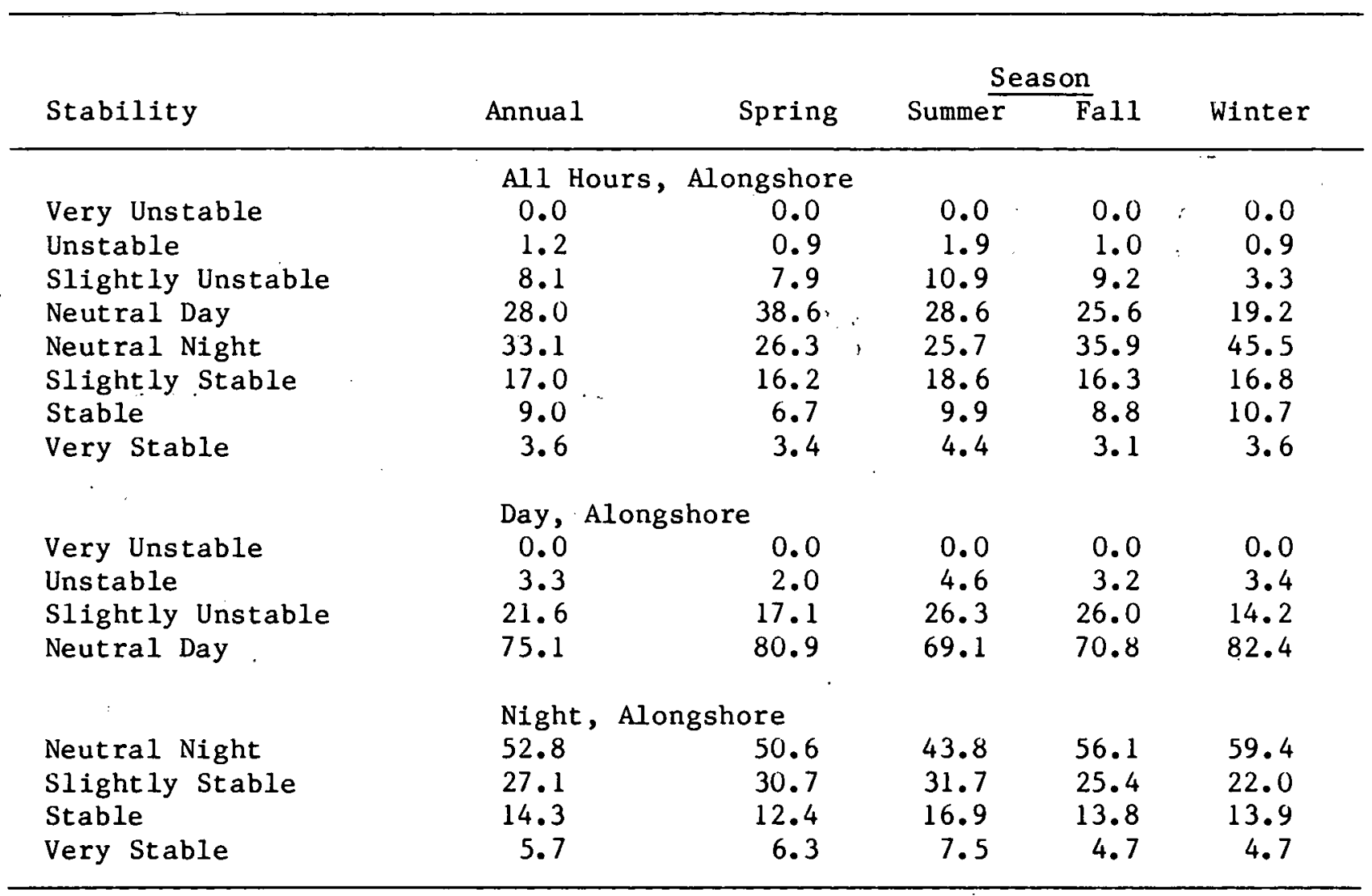


Table 5.1 (continued)

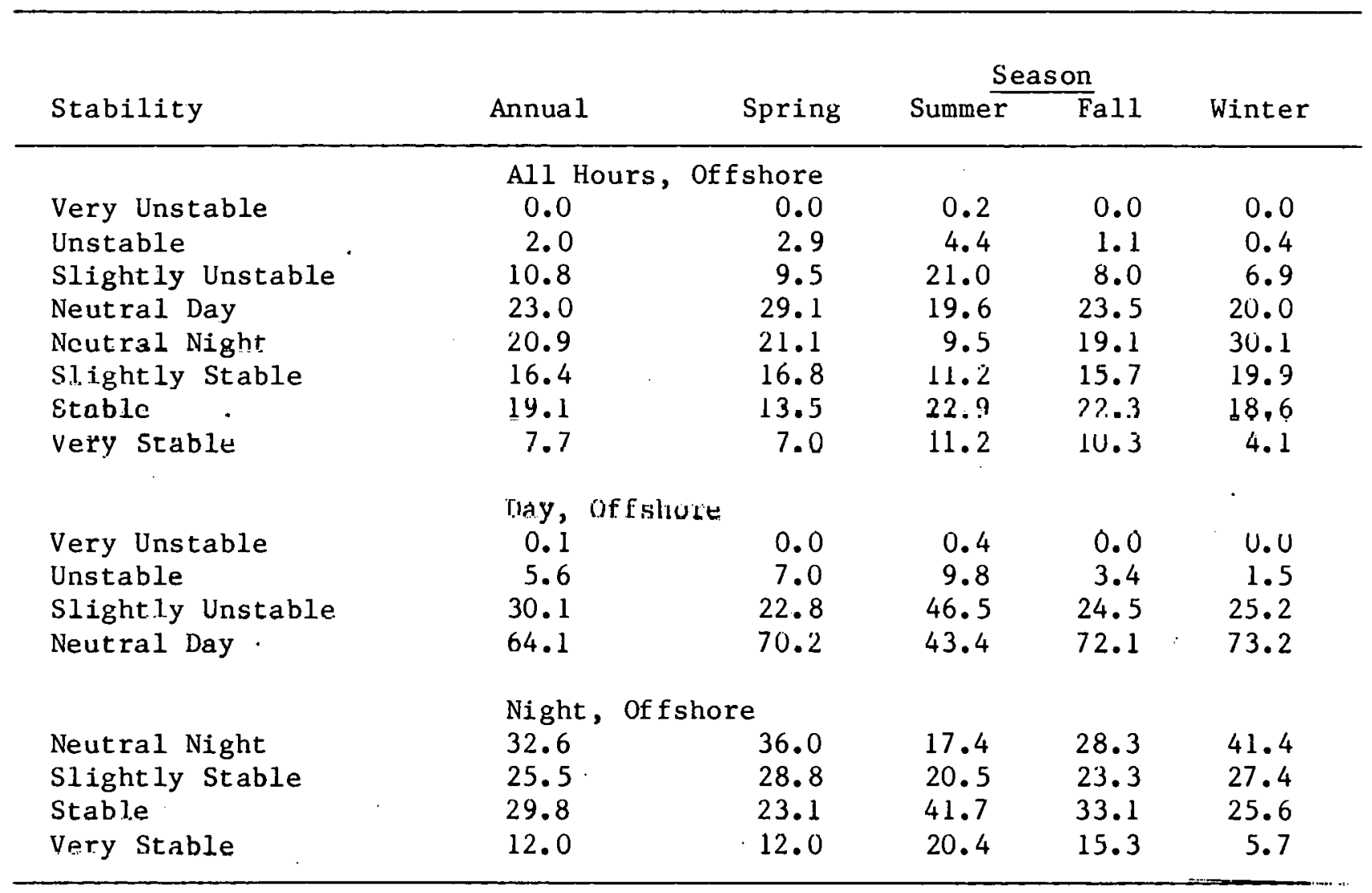




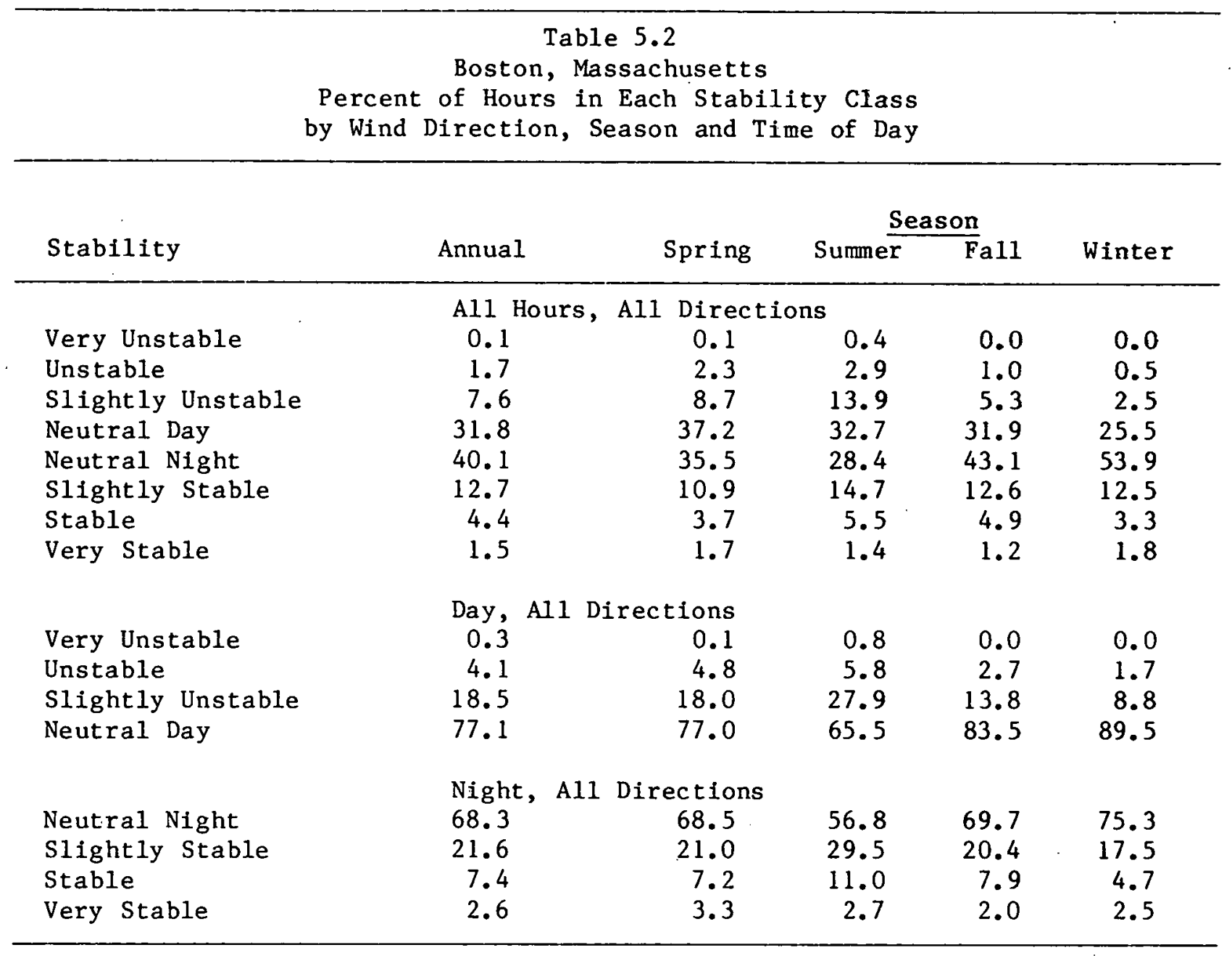


Table 5.2 (continued)

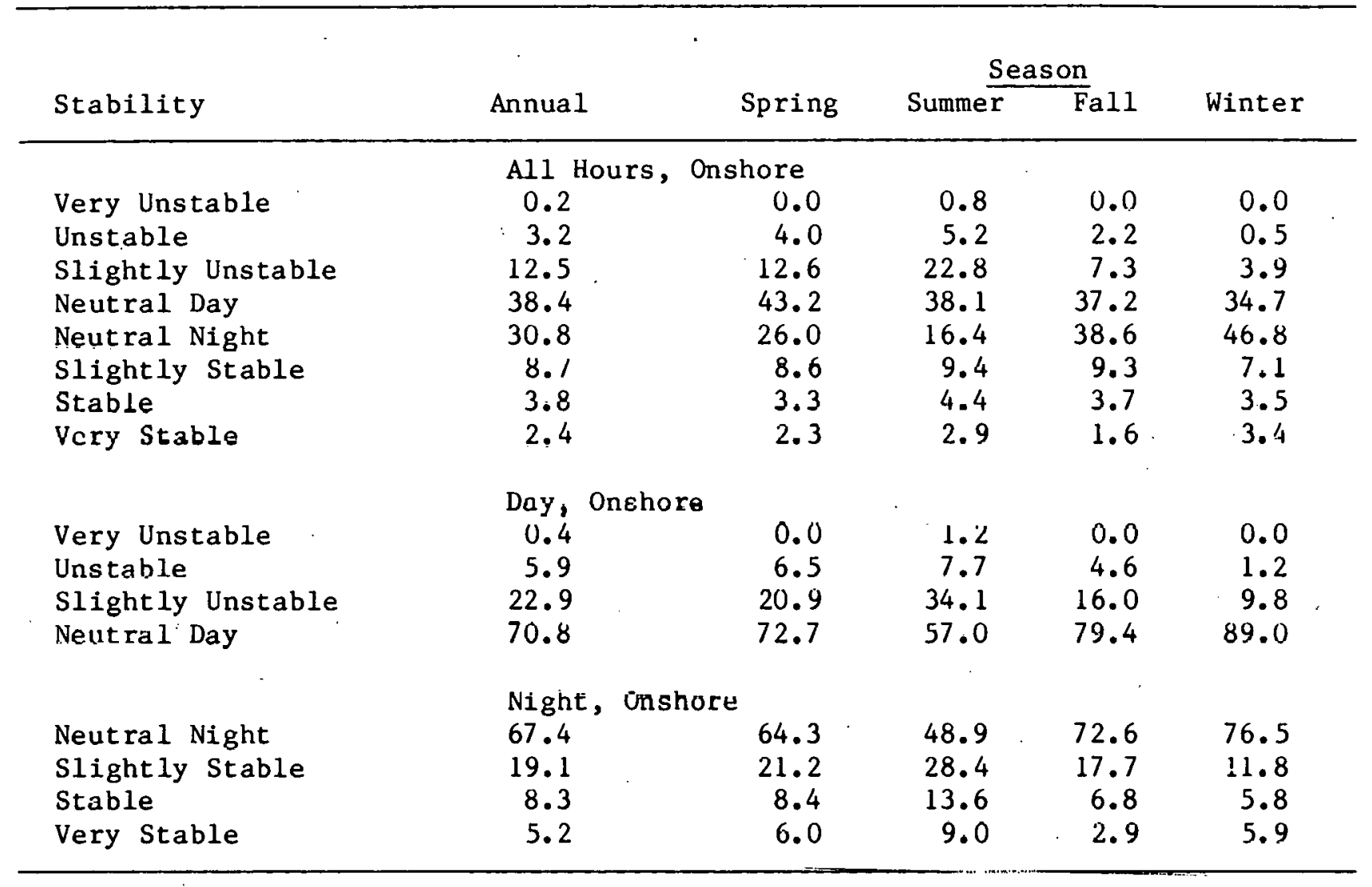


Table 5.2 (continued)

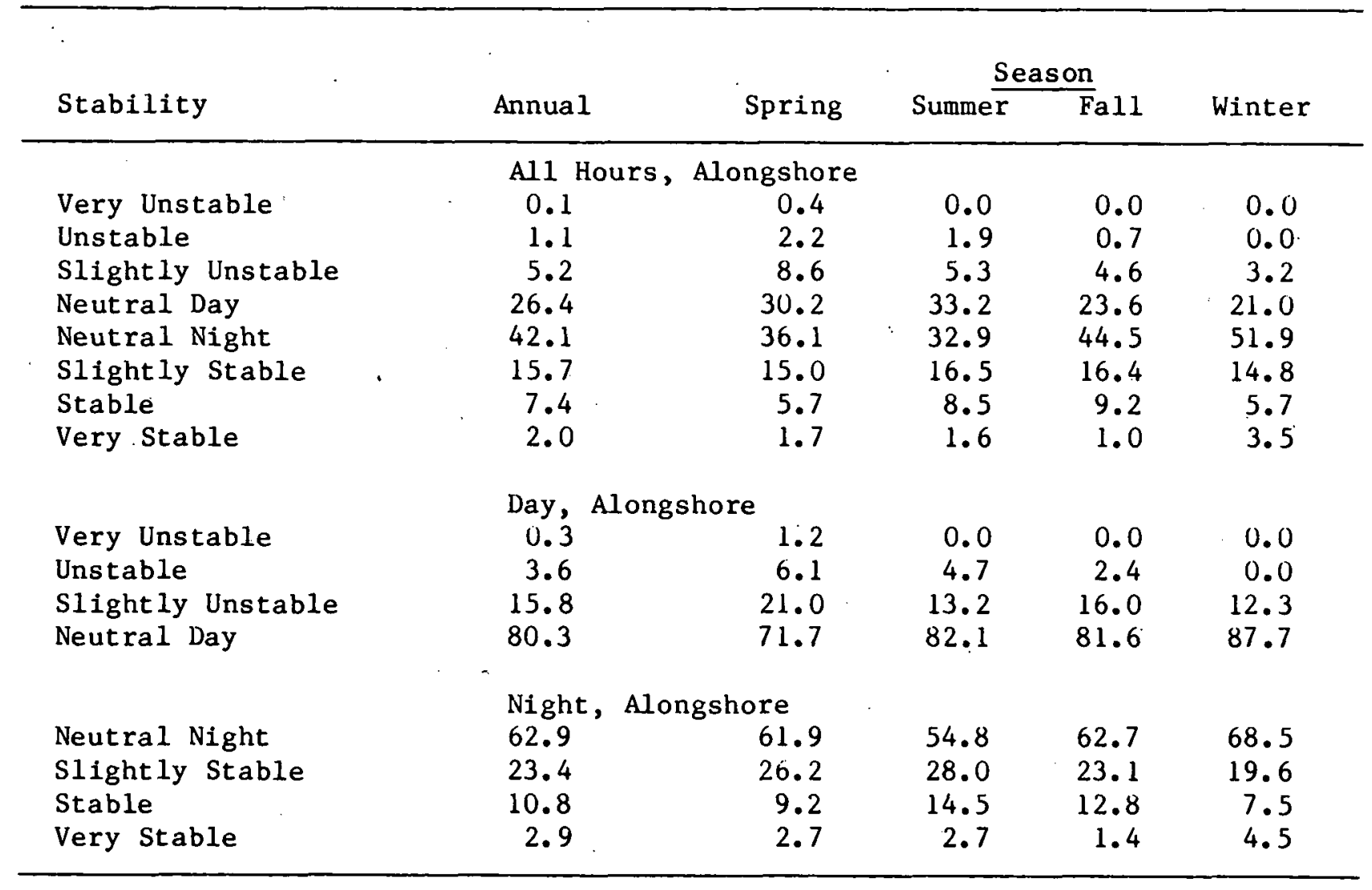


Table 5.2 (continued)

\begin{tabular}{|c|c|c|c|c|c|}
\hline \multirow[b]{2}{*}{ Stability } & \multirow[b]{2}{*}{ Annual } & \multirow[b]{2}{*}{ Spring } & \multicolumn{2}{|c|}{ Season } & \multirow[b]{2}{*}{ Winter } \\
\hline & & & Summer & Fall & \\
\hline \multicolumn{6}{|c|}{ All Hours, of fshore } \\
\hline Very Unstable & 0.1 & 0.0 & 0.2 & 0.0 & 0.0 \\
\hline Unstable & 1.0 & 1.2 & 2.2 & 0.3 & 0.4 \\
\hline Slight1y Unstable & 6.1 & 6.5 & 12.1 & 3.8 & 1.9 \\
\hline Neutral Day & 30.7 & 36.5 & 30.0 & 32.5 & 24.7 \\
\hline Neulial Niglit & 44.3 & 40.6 & 33.1 & 45.5 & 56.6 \\
\hline Slightly Stable & 13.5 & 11.0 & 16.7. & 13.4 & 13.1 \\
\hline Scable & 3.7 & 3.5 & 5.1 & 4.1 & $2: 5$ \\
\hline Very S̄table & 0.6 & U.I & U.b & 0.5 & 0.7 \\
\hline \multicolumn{6}{|c|}{ Nay, Utishore } \\
\hline Very Unstable & 0.2 & 0.0 & 0.5 & 0.0 & 0.0 \\
\hline Unstable & 2.7 & 2.7 & 5.0 & 0.8 & 1.5 \\
\hline Slightly Unstable & 16.0 & 14.8 & 27.1 & 10.2 & 7.2 \\
\hline \multirow[t]{2}{*}{ Neutral Day } & 81.1 & 82.5 & 67.3 & 89.0 & 91.3 \\
\hline & Night & Offshore & & & \\
\hline Neutral N1ght & 71.3 & 72.9 & 59.7 & 71.3 & 77.6 \\
\hline Slightly Stable & 21.7 & 19.6 & 30.2 & 21.4 & 17.9 \\
\hline Stable & 6.0 & 6.3 & 9.2 & 6.6 & 3.4 \\
\hline Very Stable & 1.0 & 1.2 & 0.9 & 0.8 & 1.0 \\
\hline
\end{tabular}


Table 5.3

Bedford, Massachusetts

Percent of Hours in Each Stability Class

by Wind Direction, Season and Time of Day

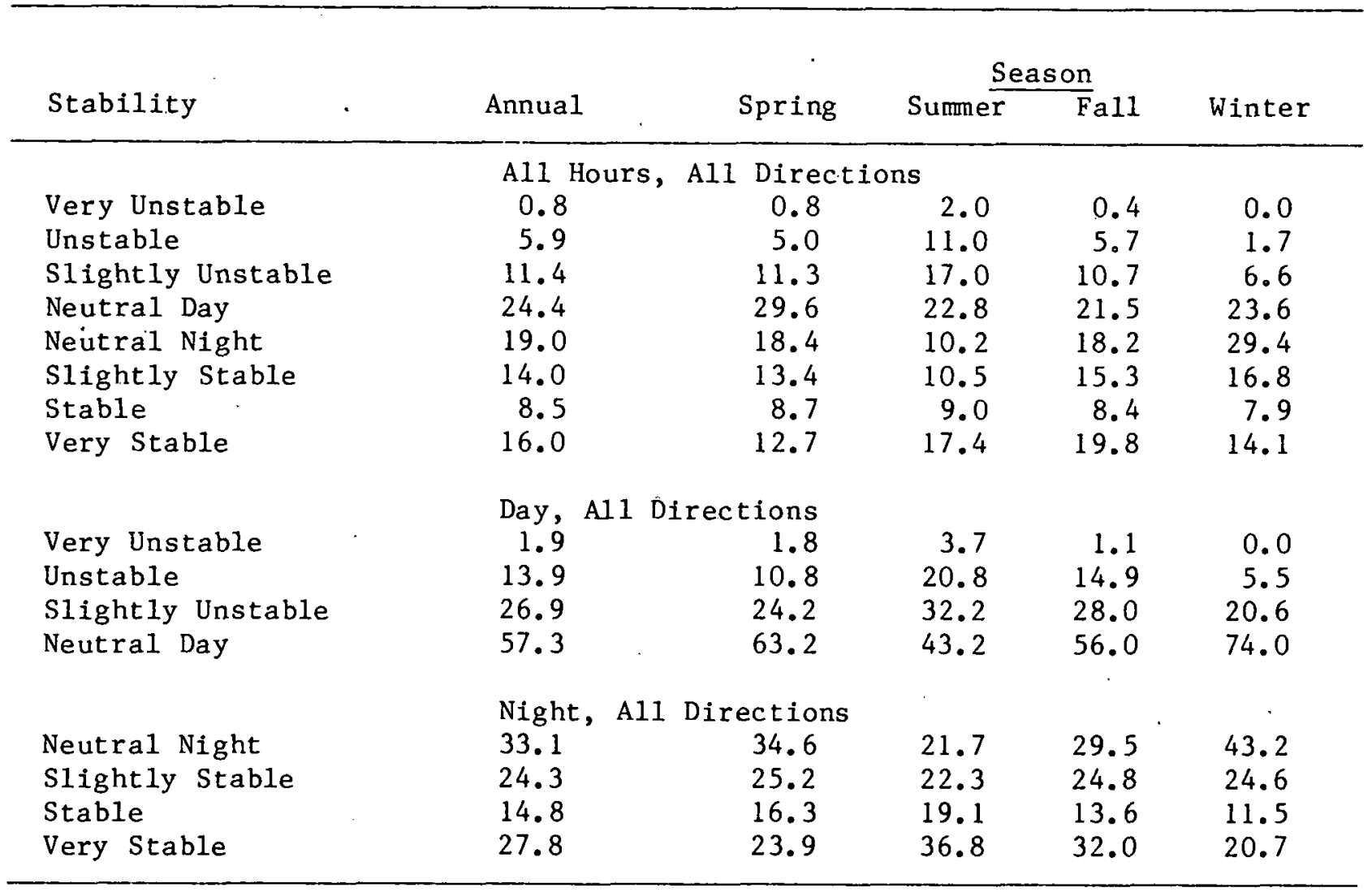


Table 5.3 (continued)

\begin{tabular}{|c|c|c|c|c|c|}
\hline \multirow[b]{2}{*}{ Stability } & \multirow[b]{2}{*}{ Annual } & \multirow[b]{2}{*}{ Spring } & \multicolumn{2}{|c|}{ Season } & \multirow[b]{2}{*}{ Winter } \\
\hline & & & Summer & $\bar{F} a 11$ & \\
\hline & All Hours, & Onshore & & & \\
\hline Very Unstable. & 0.6 & 0.7 & 2.0 & 0.0 & 0.0 \\
\hline Unstable & 6.6 & 7.7 & 13.2 & 5.8 & 1.6 \\
\hline Slightly Unstable & 10.4 & 12.8 & 17.4 & 8.6 & 4.8 \\
\hline Neutrál Day & 34.9 & 37.0 & 37.0 & 31.8 & 33.4 \\
\hline Neutral NIglit & 32.0 & 26.6 & 13.9 & 35.5 & 48.8 \\
\hline Elight y. Stable & 8.5 & 6.6 & 9.6 & 10.5 & 7.1 \\
\hline Stable & 4.1 & 5.4 & 4.2 & 4.4 & 2.4 \\
\hline \multirow[t]{2}{*}{ Very Stable } & 2.8 & 3.2 & 2.8 & 3.3 & 2.0 \\
\hline & Day, Onshor & & & & \\
\hline Very Unstable & 1.1 & 1.3 & 2.9 & 0.0 & 0.0 \\
\hline Unstable & 12.5 & 13.3 & 18.9 & 12.2 & 4.0 \\
\hline Slight:ly: Unstable & 19.9 & 21.9 & 24.9 & 18.5 & 12.0 \\
\hline Neutral Day. & 66.5 & 63.5 & 53.2 & 69.3 & 84.0 \\
\hline & Night, Onsh & hore & & & \\
\hline Neutral Night & 67.4 & 63.6 & 45.4 & 66.0 & 81.0 \\
\hline Slightly Stable & 17.9 & 15.9 & 31.5 & 19.5 & 11.8 \\
\hline Stable & 8.7 & 12.9 & 13.8 & 8.3 & 4.0 \\
\hline Very Stable & 6.0 & 7.6 & 9.2 & 6.2 & 3.3 \\
\hline
\end{tabular}


Table 5.3 (continued)

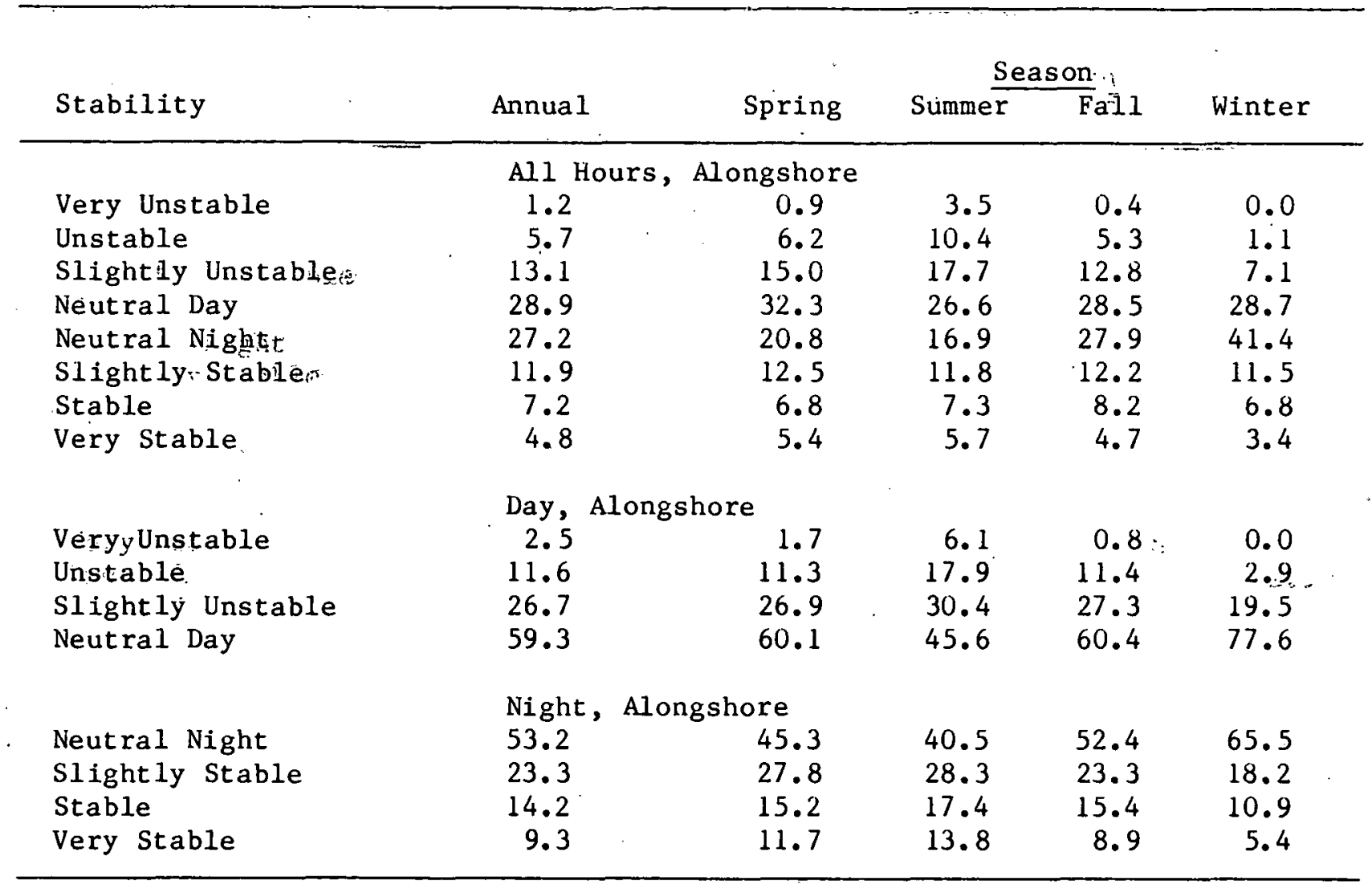


Table 5.3 (continued)

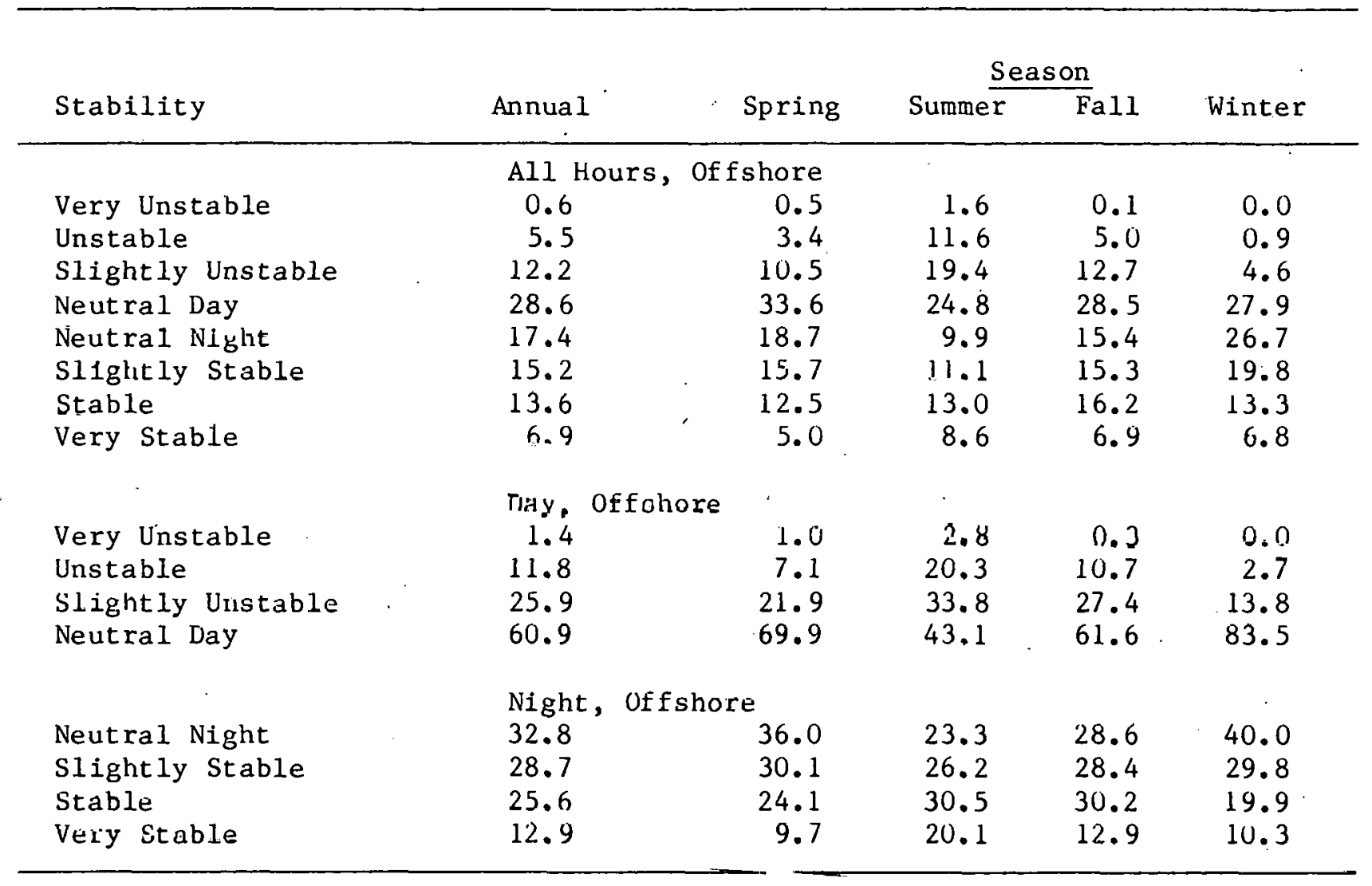


Table 5.4

Falmouth, Massachusetts

Percent of Hours in Each Stability Class

by Wind Direction, Season and Time of Day

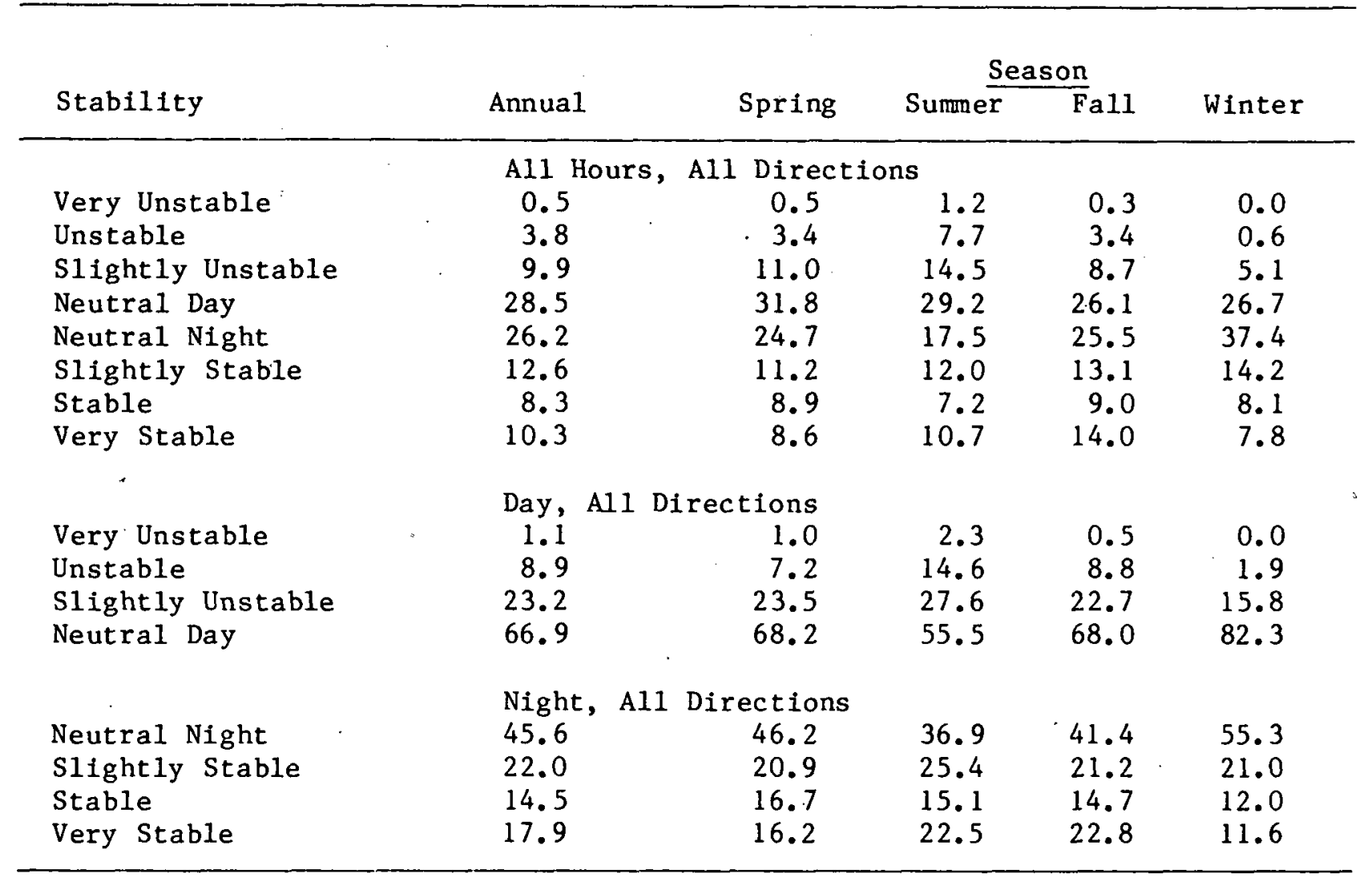


Table 5.4 (continued)

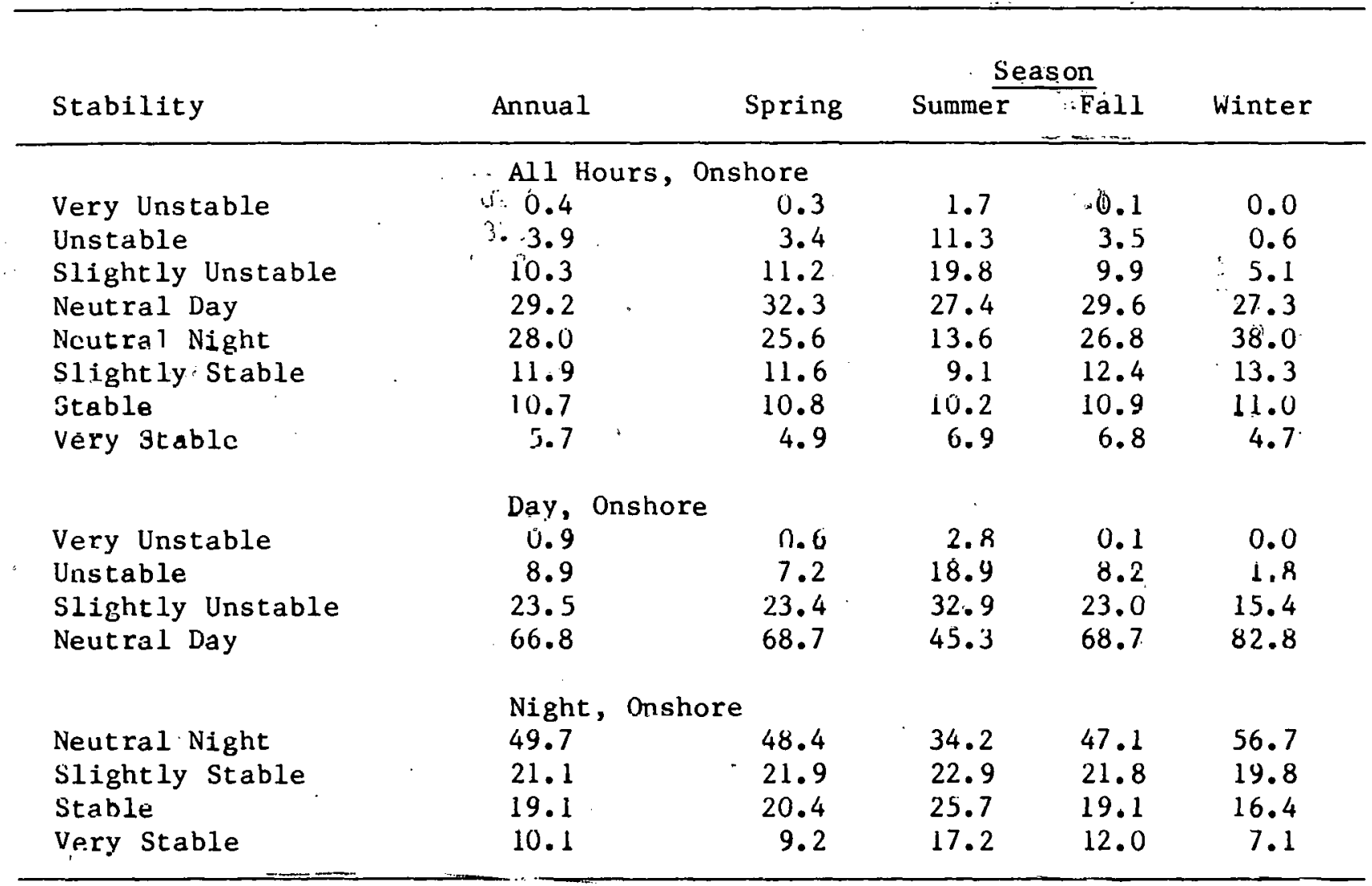


Table 5.4 (continued)

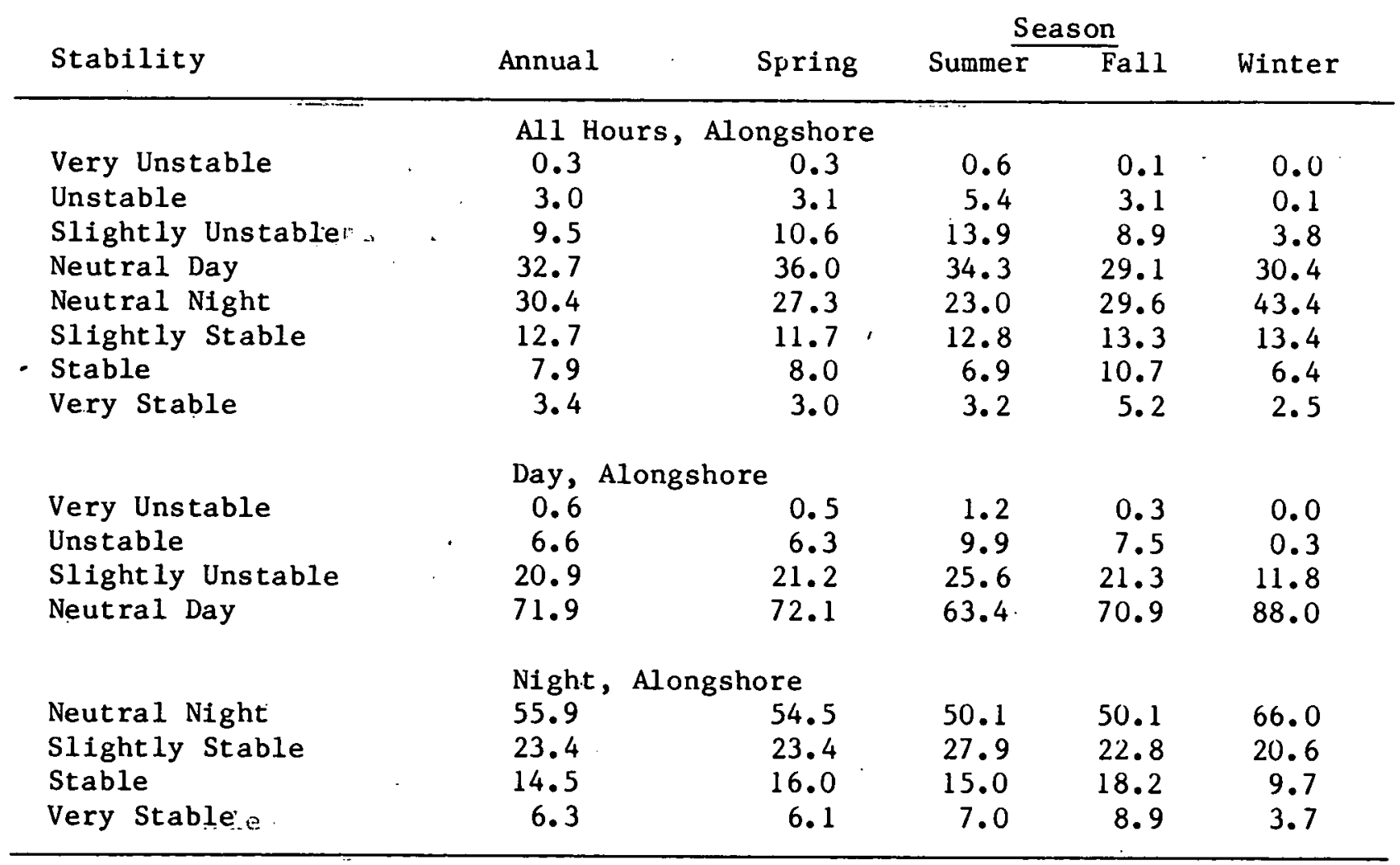


Table 5.4 (continued)

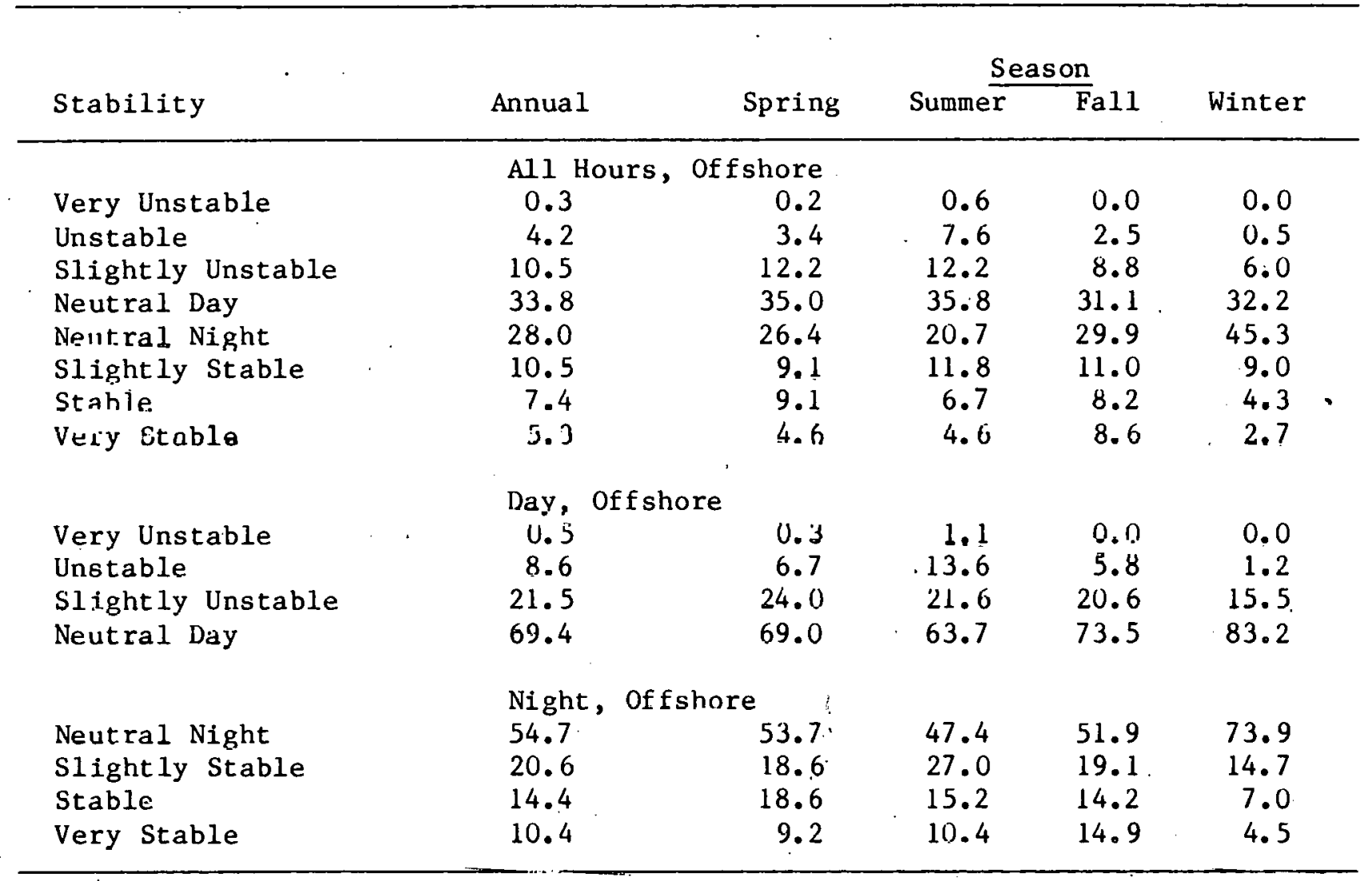


Table 5.5

Nantucket, Massachusetts

Percent of Hours in Each Stability Class

by Wind Direction, Season and Time of Day

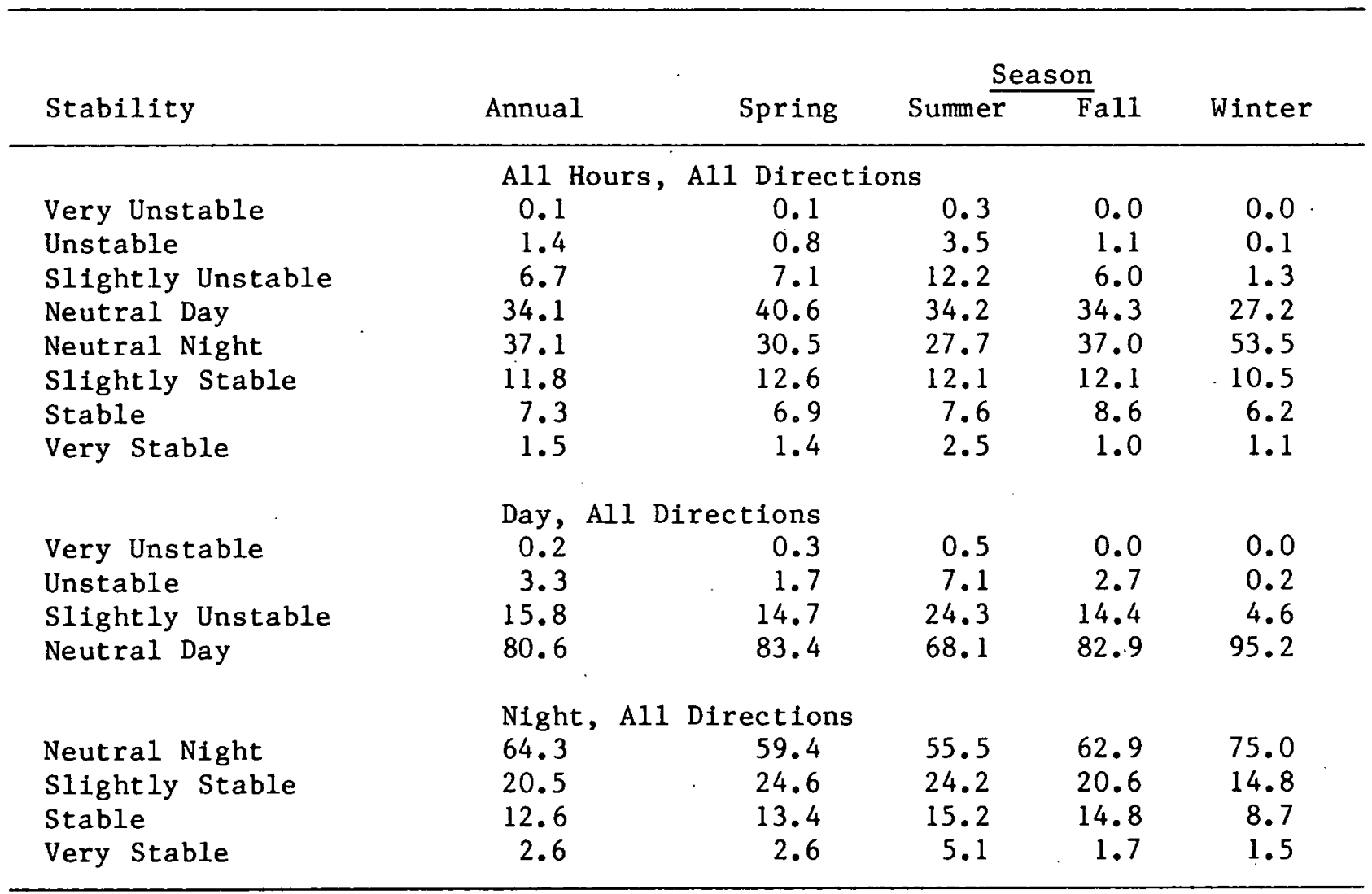


Table 5.5 (continued)

\begin{tabular}{|c|c|c|c|c|c|}
\hline \multirow[b]{2}{*}{ Stability } & \multirow{2}{*}{ Annual } & \multirow[b]{2}{*}{ Spring } & \multicolumn{2}{|c|}{ Season } & \multirow[b]{2}{*}{ Winter } \\
\hline & & & Summer & Fall & \\
\hline & \multicolumn{2}{|c|}{ All Hours, Onshore } & & & \\
\hline Very Unstable & 0.0 & 0.0 & 0.0 & 0.0 & 0.0 \\
\hline Unstable & 1.1 & 0.9 & 3.7 & 1.0 & 0.1 \\
\hline Slightly Unstabile & 5.6 & 5.1 & 14.5 & 6.8 & 1.0 \\
\hline Neutral Day & 33.2 & 41.2 & 35.9 & 34.5 & 25.5 \\
\hline Neutral Night & 40.1 & 32.9 & 23.7 & 37.7 & 53.9 \\
\hline slightly stable & 11.2 & 11.2 & 11.9 & 10.9 & 11.2 \\
\hline SLable & 7.7 & 7.6 & 7.9 & 8.7 & 7.1 \\
\hline Very Stable & 1.1 & 1.3 & 2.4 & 0.3 & 1.0 \\
\hline \multicolumn{3}{|c|}{ Day, Onshore } & & & \\
\hline Very Unstable & 0.0 & 0.0 & 0.0 & 0.0 .0 & 0.0 \\
\hline Unstable & 2.8 & 1.9 & 6.7 & 2.4 & 0.5 \\
\hline Slightly Unstable & 14.0 & 10.6 & 26.7 & 16.0 & 3.9 \\
\hline \multirow[t]{2}{*}{ Neutral Day } & 83.2 & 87.5 & 66.5 & 81.6 & 95.6 \\
\hline & Night & , Onshore & & & \\
\hline Neutral Night & 66.6 & 62.1 & 51.7 & 65.4 & 73.6 \\
\hline Slightly Stable & 18.7 & 21.1 & 26.3 & 18.8 & 15.3 \\
\hline Stable & 12.8 & 14.4 & 17.0 & 15.1 & 9.7 \\
\hline Very Stable & 1.9 & 2.4 & 5.1 & 0.6 & 1.4 \\
\hline
\end{tabular}


Table 5.5 (continued)

\begin{tabular}{|c|c|c|c|c|c|}
\hline \multirow[b]{2}{*}{ Stability } & \multirow[b]{2}{*}{ Annual } & \multicolumn{4}{|c|}{ Season } \\
\hline & & Spring & Summer & Fall & Winter \\
\hline \multicolumn{6}{|c|}{ All Hours, Alongshore } \\
\hline Very Unstable & 0.3 & 0.6 & 0.7 & 0.0 & 0.0 \\
\hline Unstable. & 1.2 & 0.9 & 2.8 & 1.2 & 0.0 \\
\hline Slightly Unstable & 5.1 & 7.5 & 9.4 & 4.0 & 0.5 \\
\hline Neutral Day & 34.2 & 38.8 & 34.1 & 33.2 & 30.8 \\
\hline Neutral Night & 38.2 & 28.4 & 28.4 & 40.1 & 53.4 \\
\hline Slightly Stable & 12.9 & 16.4 & 13.5 & 11.6 & 10.8 \\
\hline Stable & 7.4 & 6.8 & 9.8 & 8.9 & 4.0 \\
\hline Very Stable & 0.8 & 0.7 & 1.3 & 1.0 & 0.5 \\
\hline \multicolumn{6}{|c|}{ Day, Alongshore } \\
\hline Very Unstable & 0.7 & 1.3 & 1.4 & 0.0 & 0.0 \\
\hline Unstable & 2.9 & 2.0 & 5.8 & 3.2 & 0.0 \\
\hline Slightly Unstable & 12.4 & 15.8 & 20.0 & 10.3 & 1.7 \\
\hline Neutral Day & 84.0 & 80.9 & 72.8 & 86.5 & 98.3 \\
\hline \multicolumn{6}{|c|}{ Night, Alongshore } \\
\hline Neutra1 Night & 64.4 & 54.6 & 53.3 & 65.0 & 77.7 \\
\hline Slightly Stable & 21.8 & 31.2 & 25.5 & 18.9 & 15.8 \\
\hline Stable & 12.4 & 12.9 & 18.7 & 14.6 & 5.8 \\
\hline Very Stable & 1.4 & 1.3 & 2.4 & 1.6 & 0.7 \\
\hline
\end{tabular}


Table 5.5 (continued)

\begin{tabular}{|c|c|c|c|c|c|}
\hline \multirow[b]{2}{*}{ Stability } & \multirow[b]{2}{*}{ Annual } & \multirow[b]{2}{*}{ Spring } & \multicolumn{2}{|c|}{ Season } & \multirow[b]{2}{*}{ Winter } \\
\hline & & & Summer & Fa 11 & \\
\hline \multicolumn{6}{|c|}{ Al1 Hours, Of fshore } \\
\hline Very Unstable & 0.1 & 0.0 & 0.3 & 0.0 & 0.0 \\
\hline Unstable & 1.9 & 0.8 & 3.8 & 1.0 & 0.0 \\
\hline Slightly Unstable & 9.3 & 9.3 & 12.4 & 6.7 & 3.7 \\
\hline Neutral Day & 35.4 & 40.9 & 33.9 & 35.9 & 27.1 \\
\hline Neutral Night & 33.3 & 29.0 & 29.7 & 33.2 & 53.8 \\
\hline Slightly stable & 11.8 & 12.4 & 11.5 & 14.3 & 7.6 \\
\hline Etablo & 6.8 & 6.4 & 6.5 & 8.0 & 6.8 \\
\hline Very Stable & 1.4 & 1.3 & 1.9 & 0.8 & 1.0 \\
\hline \multicolumn{6}{|c|}{ Day, offshore } \\
\hline Very Unstable & 0.2 & 0.0 & 0.5 & 0.0 & 0.0 \\
\hline Unstable & 4.1 & 1.5 & 7.5 & 2.5 & 0.0 \\
\hline Slightly Unstable & 19.9 & 18.2 & 24.7 & 15.4 & 12.2 \\
\hline Neutral Day & 75.9 & 80.3 & 67.3 & 82.1 & 87.8 \\
\hline \multicolumn{6}{|c|}{ Night, offshore } \\
\hline Neutral Night. & 62.6 & 59.1 & 59.9 & 58.9 & 77.8 \\
\hline Slightly Stable & 21.9 & 25.4 & 23.2 & 25.4 & 11.0 \\
\hline Stable & 12.8 & 13.2 & 13.0 & 14.4 & 9.8 \\
\hline Very Stable & 2.7 & 2.3 & 3.9 & 1.4 & 1.5 \\
\hline
\end{tabular}




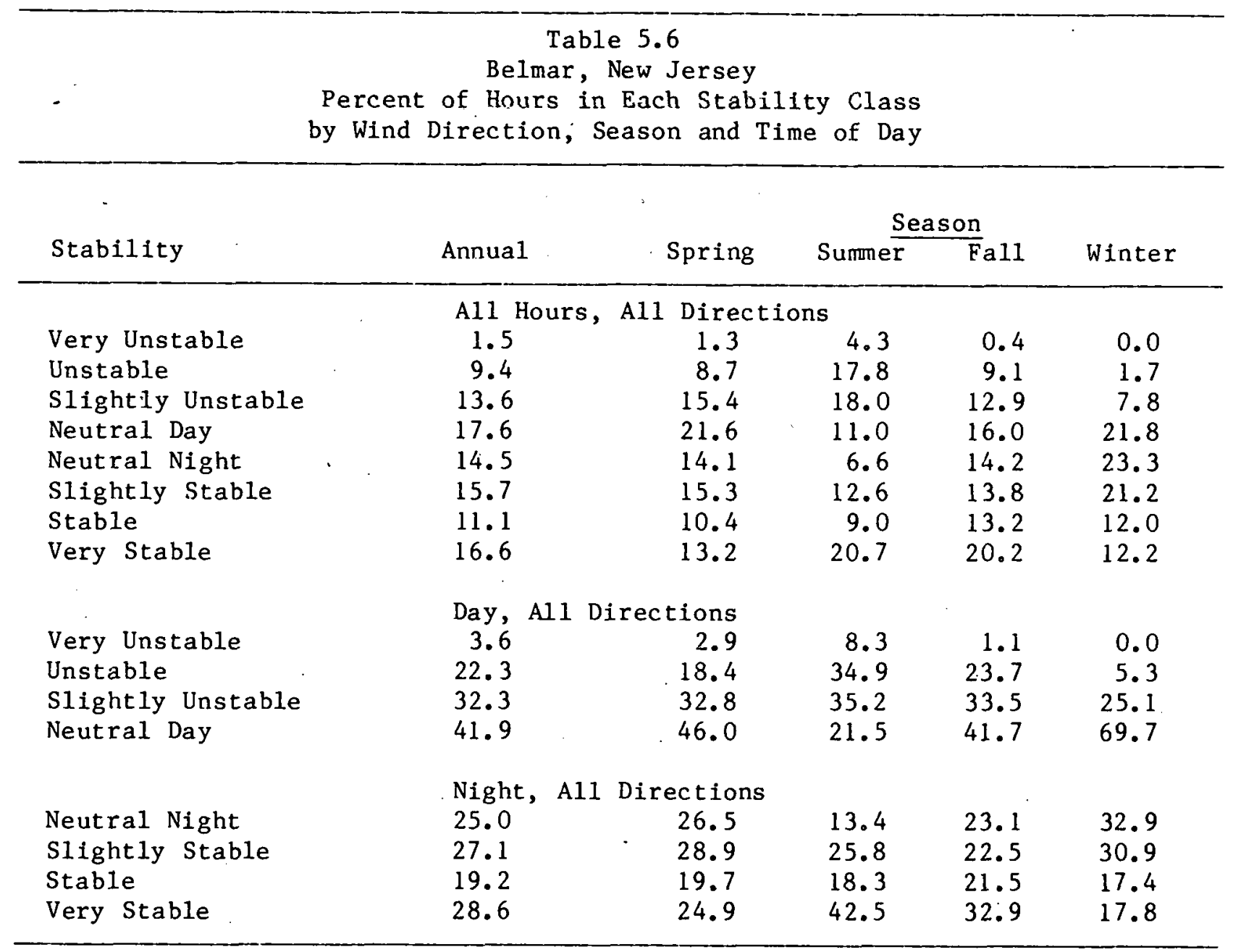


Table 5.6 (continued)

\begin{tabular}{|c|c|c|c|c|c|}
\hline \multirow[b]{2}{*}{ Stability } & \multirow[b]{2}{*}{ Annual } & \multirow[b]{2}{*}{ Spring } & \multicolumn{2}{|c|}{ Season } & \multirow[b]{2}{*}{ Winter } \\
\hline & & & Summer & Fall & \\
\hline \multicolumn{6}{|c|}{ All Hours, Onshore } \\
\hline Very Linstable & 2.3 & 2.4 & 7.8 & 0.0 & 0.0 \\
\hline Unstable & 13.6 & 12.3 & 30.5 & 10.4 & 2.7 \\
\hline Slightly Unstable & 13.7 & 17.3 & 19.8 & 9.7 & 7.5 \\
\hline Neutral Day & 22.8 & 26.2 & 15.4 & 20.7 & 29.2 \\
\hline Neutral Night & 24.1 & 23.7 & 8.3 & 25.4 & . 41.8 \\
\hline Slightly stable & 12.4 & 10.4 & 8.7 & 17.5 & 10.4 \\
\hline Stable & 5.8 & 4.2 & 3.8 & 9.3 & 4.1 \\
\hline Veiy Stable & 5.3 & $3: 5$ & 5.6 & 7.0 & 4.4 \\
\hline \multicolumn{6}{|c|}{ Day, Onshore } \\
\hline Very Unstable & 4.4 & 4.3 & 10.8 & 0.0 & 10.0 \\
\hline Unstable & 26.0 & 21.2 & 41.4 & 25.4 & 6.7 \\
\hline Slightly Unstable & $26.2^{\circ}$ & 29.8 & 27.0 & 23.8 & 19.1 \\
\hline Neutral Day & 43.4 & 44.7 & 20.8 & 50.8 & 74.2 \\
\hline \multicolumn{6}{|c|}{ Night, Onshore } \\
\hline Neutral Night & 51.0 & 57.2 & 32.0 & 42.9 & 69.6 \\
\hline Slightly Stable & 26.0 & 24.7 & 33.7 & 29.5 & 16.9 \\
\hline Stable & 12.2 & 10.0 & 14.9 & 15.7 & 6.7 \\
\hline Very Stable & 10.8 & 8.1 & 19.3. & 11.9 & 6.8 \\
\hline
\end{tabular}


Table 5.6 (continued)

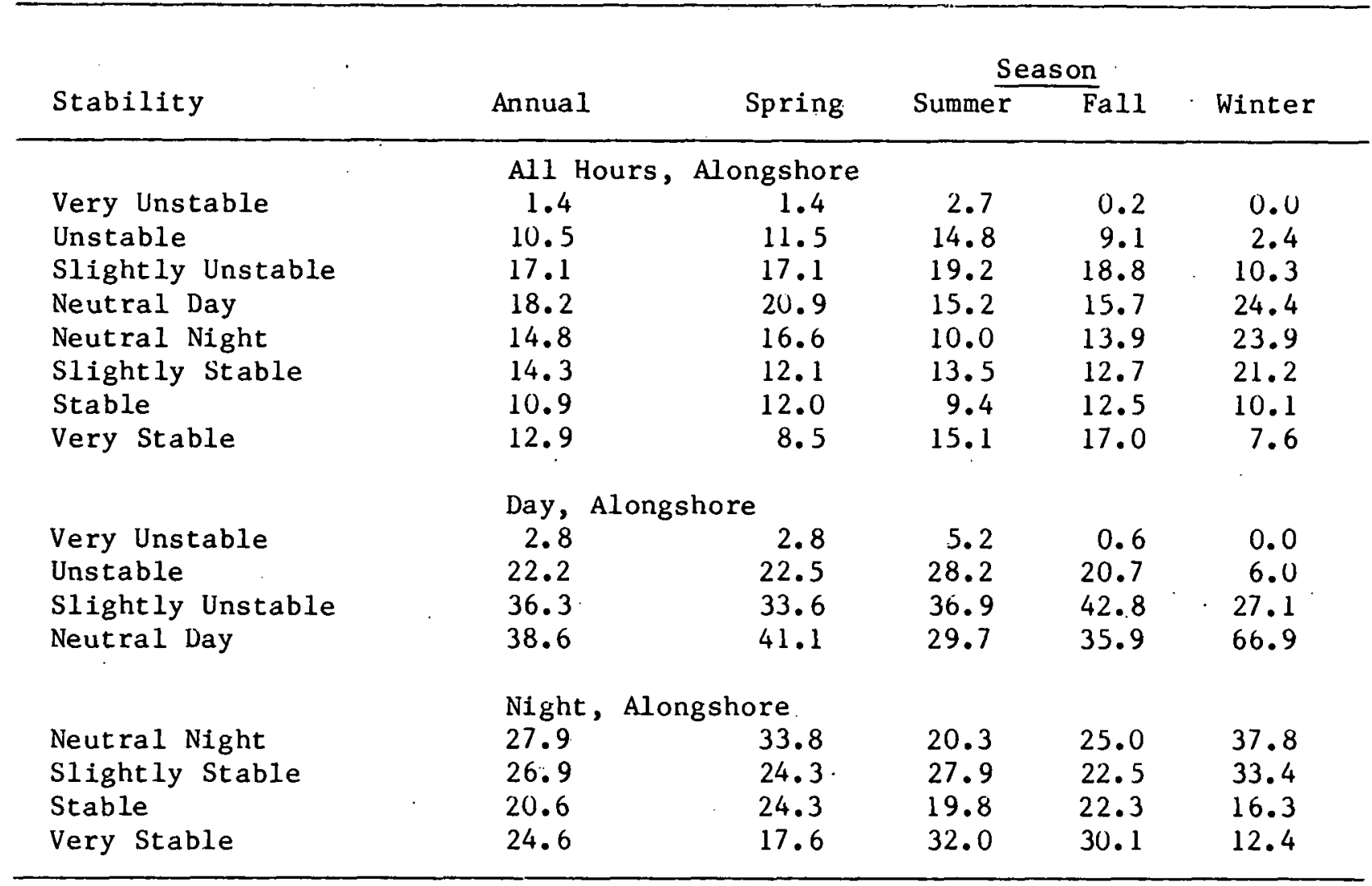


Table 5.6 (continued)

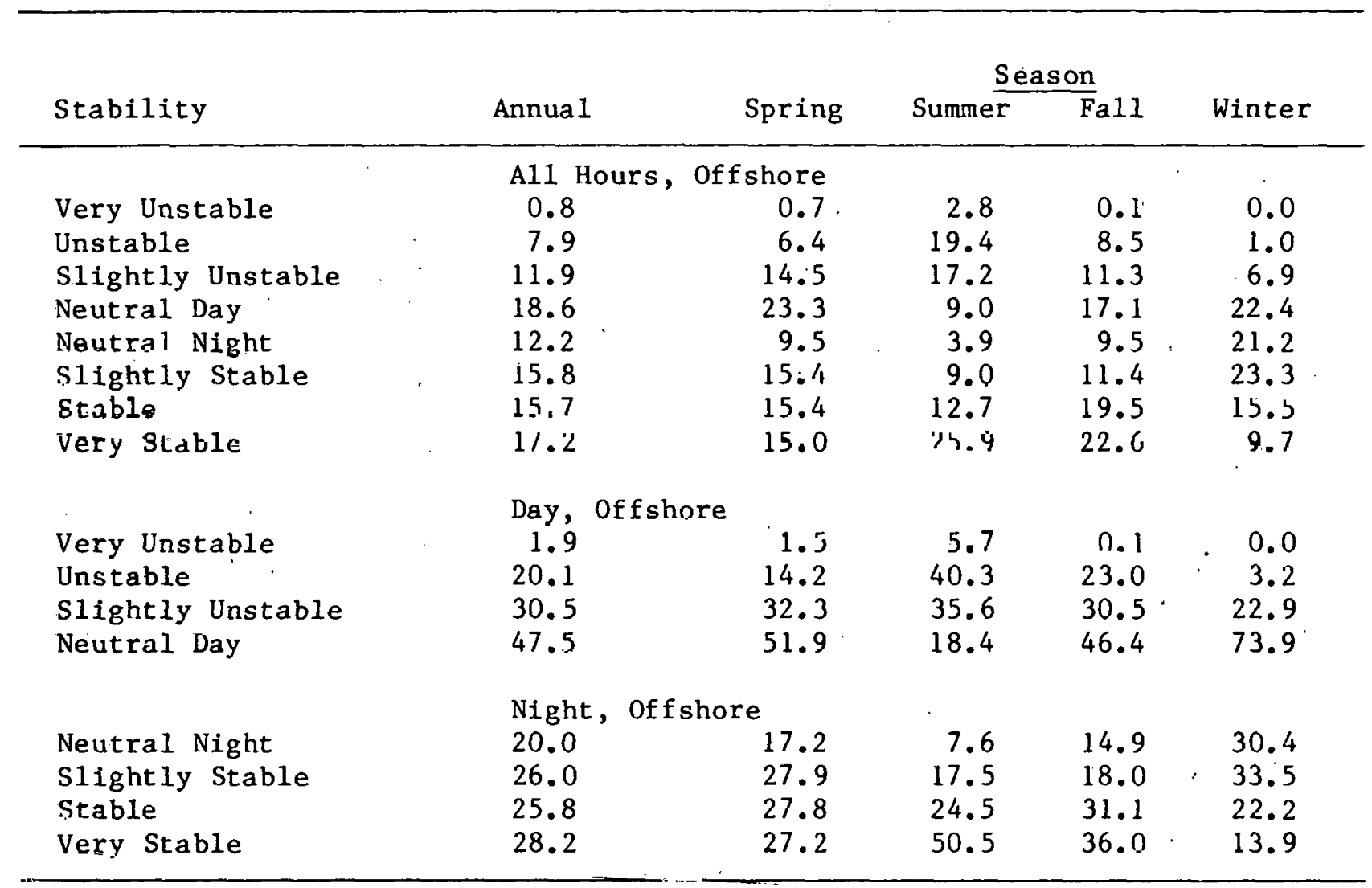




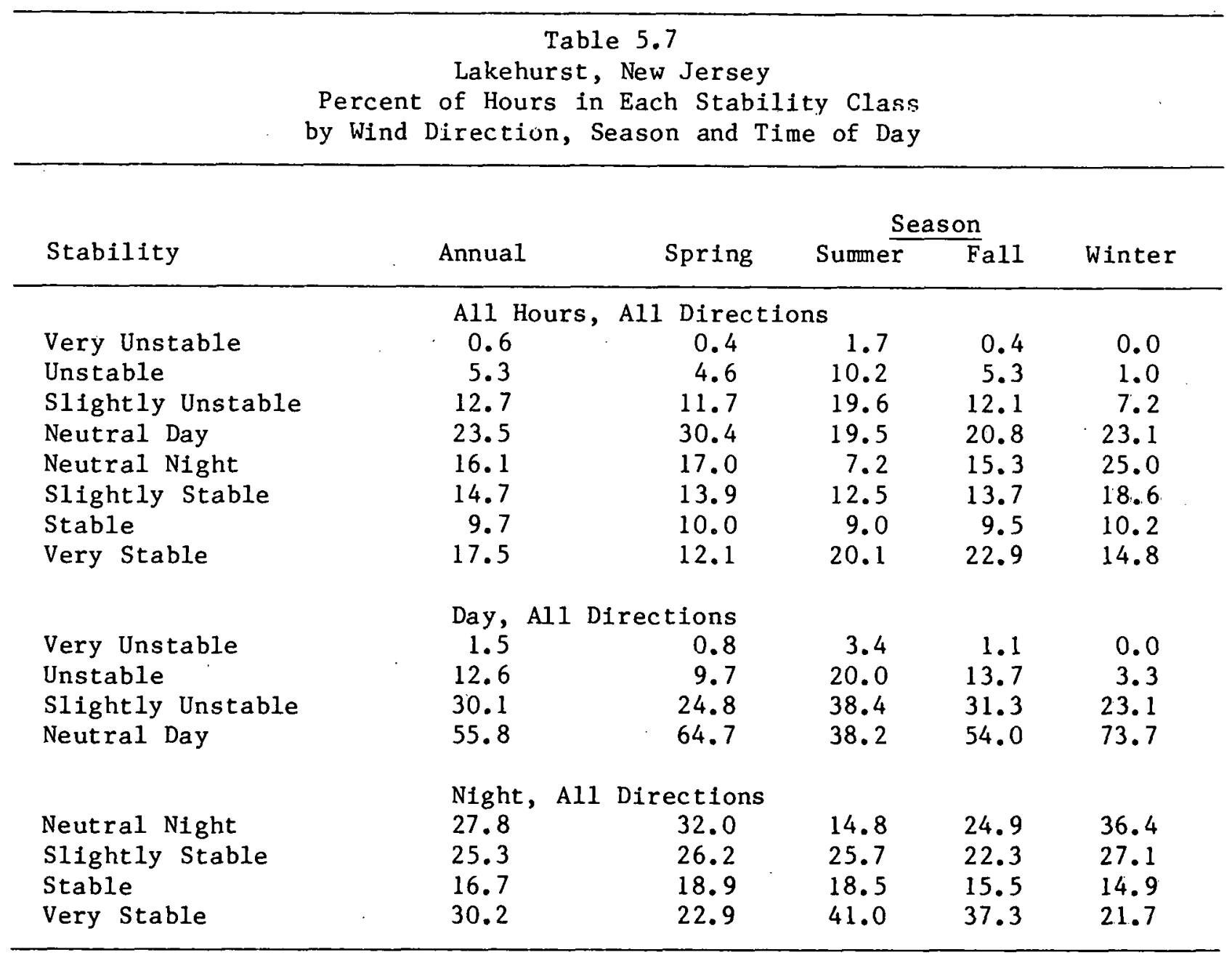


Table 5.7 (continued)

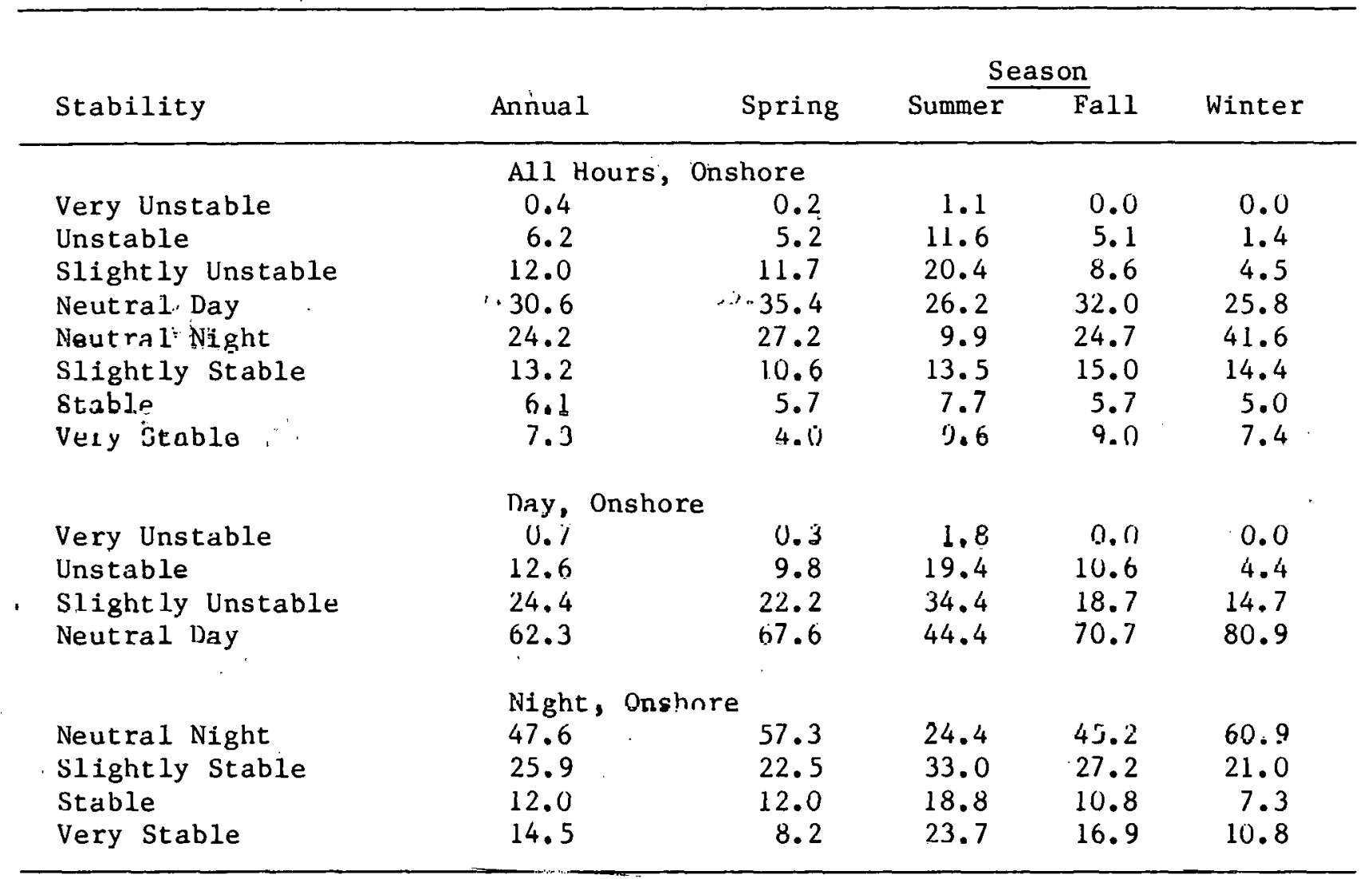


Table 5.7 (continued)

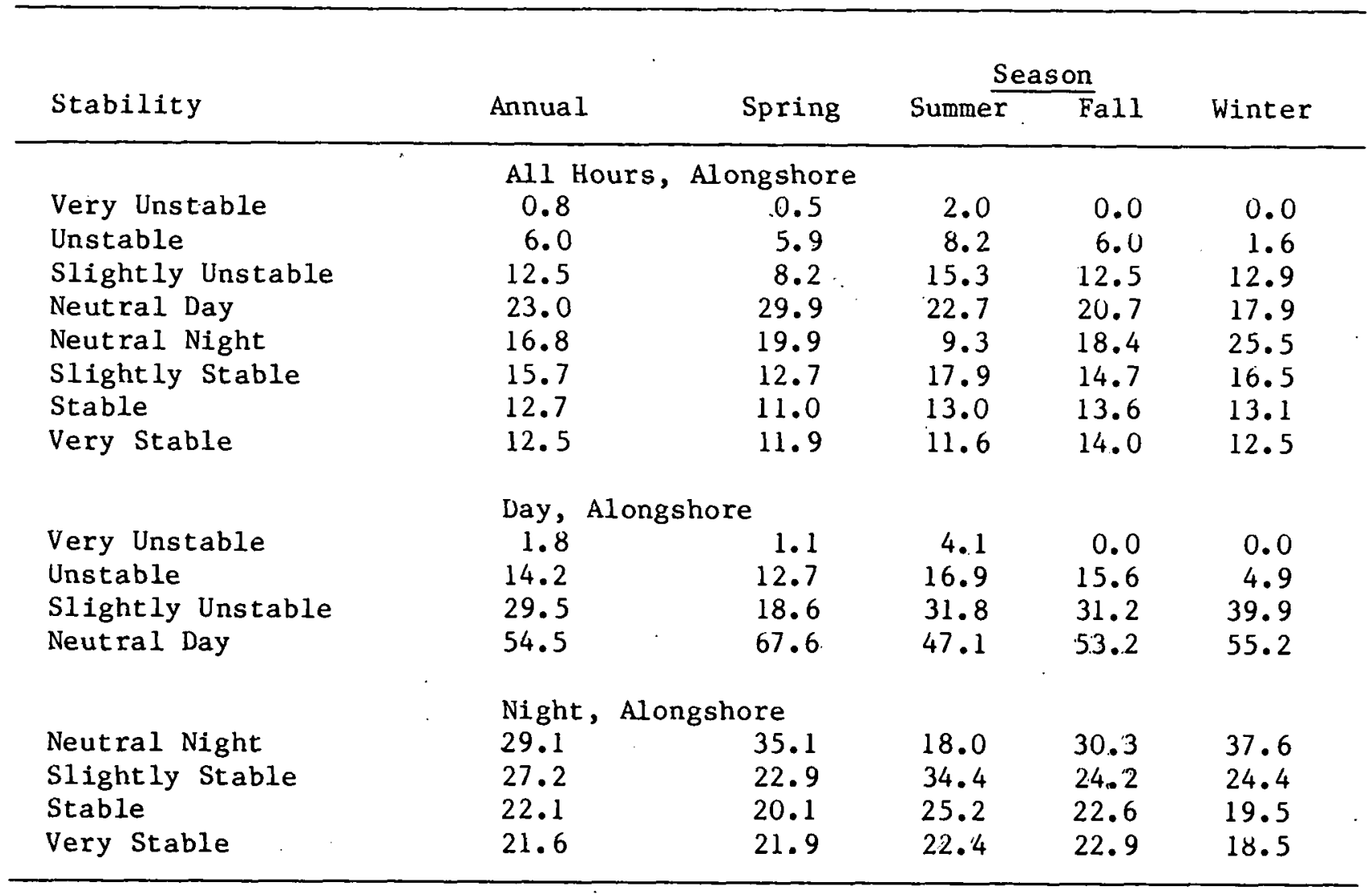


Table 5:7 (continued)

\begin{tabular}{|c|c|c|c|c|c|}
\hline \multirow[b]{2}{*}{ Stability } & \multirow[b]{2}{*}{ Annual } & \multirow[b]{2}{*}{ Spring } & \multicolumn{2}{|c|}{ Season } & \multirow[b]{2}{*}{ Winter } \\
\hline & & & Summer & Fall & \\
\hline \multicolumn{6}{|c|}{ All Hours, of fshore } \\
\hline Very Unstable & 0.5 & 0.4 & 1.8 & 0.1 & 0.0 \\
\hline Unstable & 4.9 & 3.7 & 12.3 & 5.9 & 0.6 \\
\hline Slightly Unstable & 14.3 & 13.3 & 26.1 & $17: 1$ & 6.4 \\
\hline Neutral Day & 26.9 & 33.2 & 21.6 & 24.2 & 27.1 \\
\hline Neutral Night & 15.5 & 12.5 & 5.5 & 13.7 & 24.9 \\
\hline Slight ly Stable & 13.4 & 13.9 & 6.4 & 10.0 & 19.1 \\
\hline Stable & 12.9 & 14.1 & 10.7 & 14.7 & 12.1 \\
\hline Very Stable & 11.7 & 8.9 & 15.6 & 14.3 & 9.8 \\
\hline \multicolumn{6}{|c|}{ Day, Offshore } \\
\hline Very Unstable & 1.0 & 0.8 & 3.0. & 0.1 & 0.0 \\
\hline Unstable & 10.4 & 7.5 & 19.9 & 12.5 & 1.7 \\
\hline Slightly Unstable & 30.8 & 26.3 & 42.3 & 36.0 & 18.8 \\
\hline Neutral Day & 57.7 & 65.5 & 34.9 & 51.3 & 79.6 \\
\hline \multicolumn{6}{|c|}{ Night, of fshore } \\
\hline Neutral Night & 29.0 & $\cdot 25.7$ & 14.3 & 26.1 & 37.7 \\
\hline Slight ly Stable & 25.1 & 28.3 & 16.7 & 18.9 & 29.1 \\
\hline Stable & 24.1 & 28.3 & 28.0 & 27.9 & 18.3 \\
\hline Very Stable & 21.8 & 17.8 & 41.0 & 27.1 & 14.9 \\
\hline
\end{tabular}


Table 5.8

Atlantic City, New Jersey

Percent of Hours in Each Stability Class

by Wind Direction, Season and Time of Day

\begin{tabular}{lccrrr}
\hline & \multicolumn{5}{c}{ Season } \\
Stability & Annual & Spring & Sumer & Fall & Winter \\
\hline & Al1 Hours, All Directions & & \\
Very Unstable & 0.3 & 0.3 & 0.6 & 0.1 & 0.0 \\
Unstable & 3.0 & 2.6 & 7.5 & 1.6 & 0.1 \\
Slightly Unstable & 10.1 & 9.6 & 18.1 & 9.1 & 3.3 \\
Neutral Day & 28.4 & 34.6 & 23.7 & 27.7 & 27.4 \\
Neutral Night & 26.3 & 24.8 & 16.0 & 27.3 & 37.2 \\
Slightly Stable & 15.6 & 14.5 & 14.9 & 15.2 & 17.8 \\
Stable & 11.5 & 9.2 & 14.5 & 12.8 & 9.2 \\
Very Stable & 5.0 & 4.3 & 4.6 & 6.0 & 4.9 \\
& & & & & \\
Very Unstable & Day, Al1 Directions & & & \\
Unstable & 0.6 & 0.6 & 1.2 & 0.4 & 0.0 \\
Slightly Unstable & 7.2 & 5.5 & 15.1 & 4.3 & 0.5 \\
Neutral Day & 24.2 & 20.5 & 36.3 & 23.7 & 10.8 \\
& 68.0 & 73.5 & 47.4 & 71.7 & 88.7 \\
Neutral Night & Night, All Directions & & & \\
Slightly Stable & 45.0 & 46.9 & 31.9 & 44.4 & 53.8 \\
Stable & 26.8 & 27.5 & 29.8 & 24.8 & 25.7 \\
Very Stable & 19.7 & 17.4 & 29.1 & 20.9 & 13.4 \\
\hline
\end{tabular}


Table 5.8 (continued)

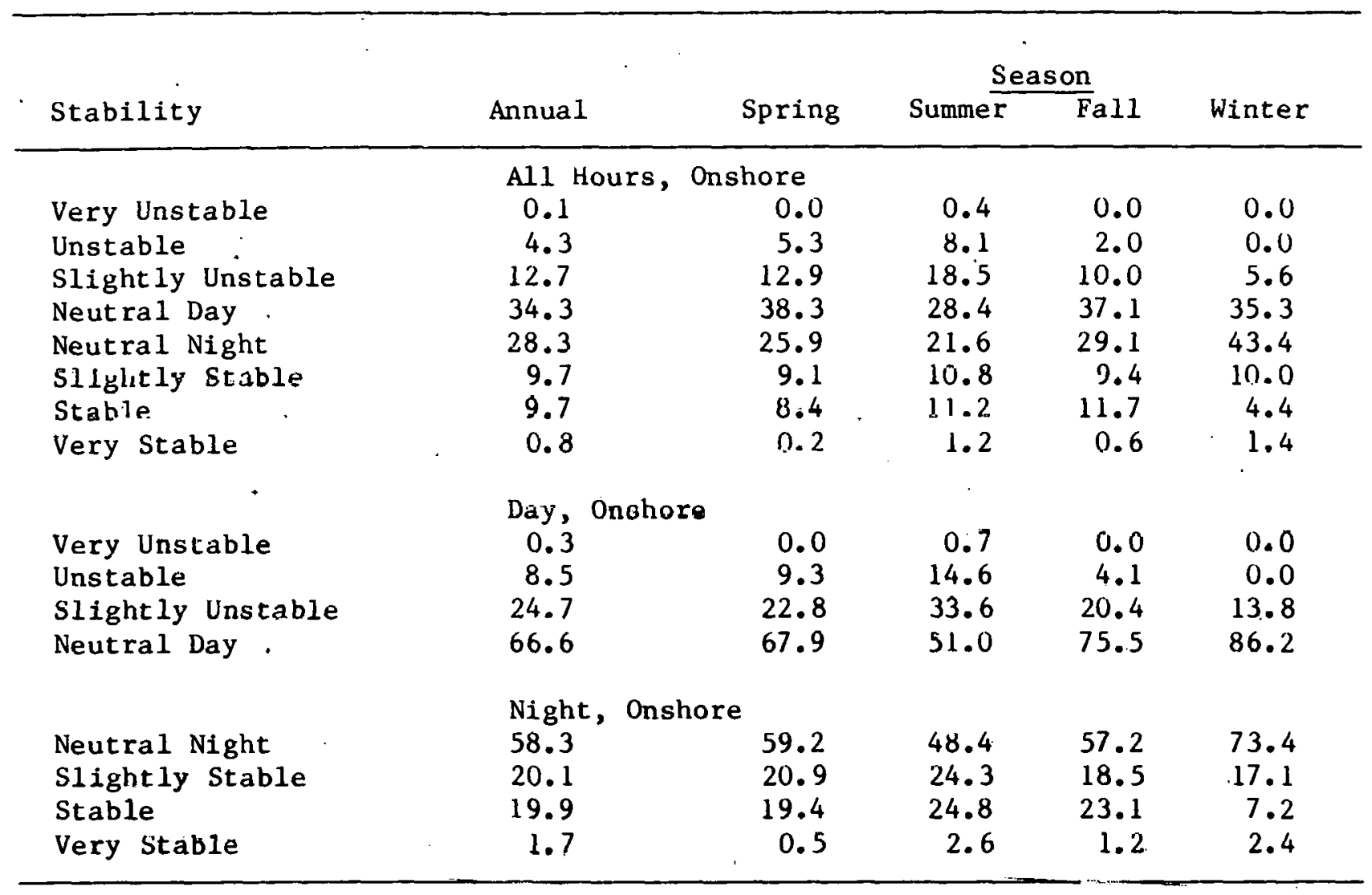


Table 5.8 (continued)

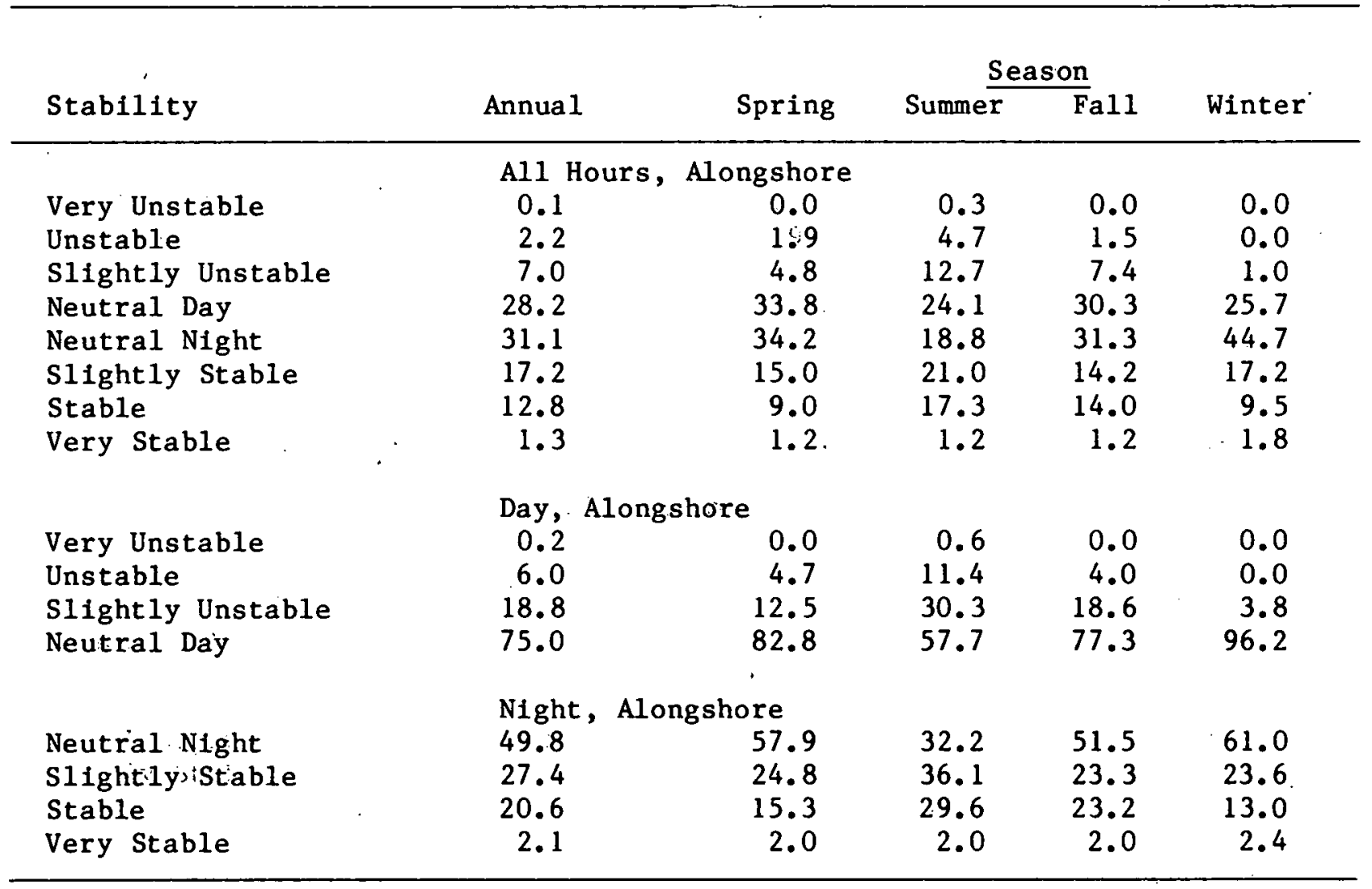


Table 5.8 (continued)

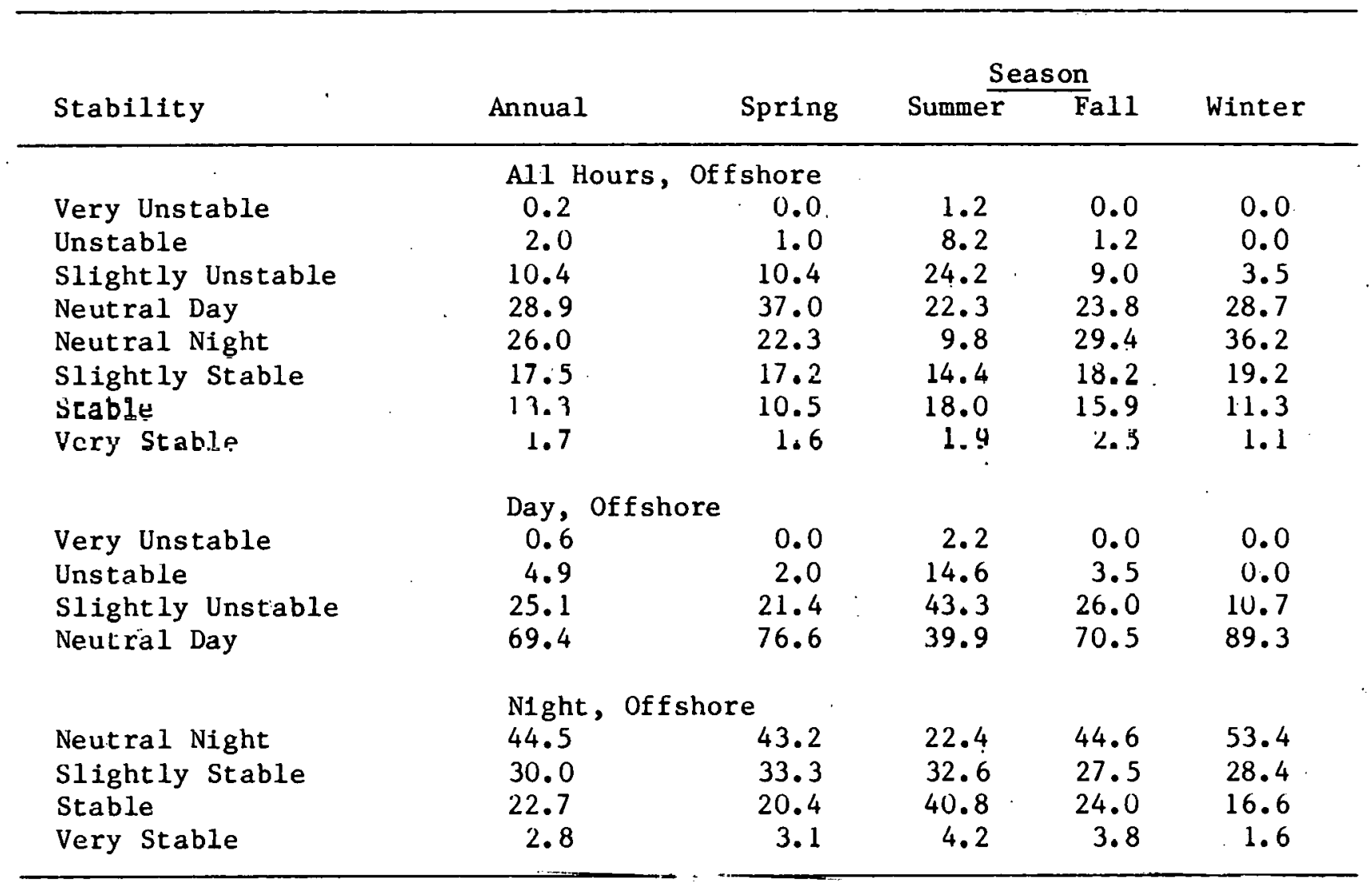


Table 5.9

Wilmington, Delaware

Percent of Hours in Each Stability Class

by Wind Direction, Season and Time of Day

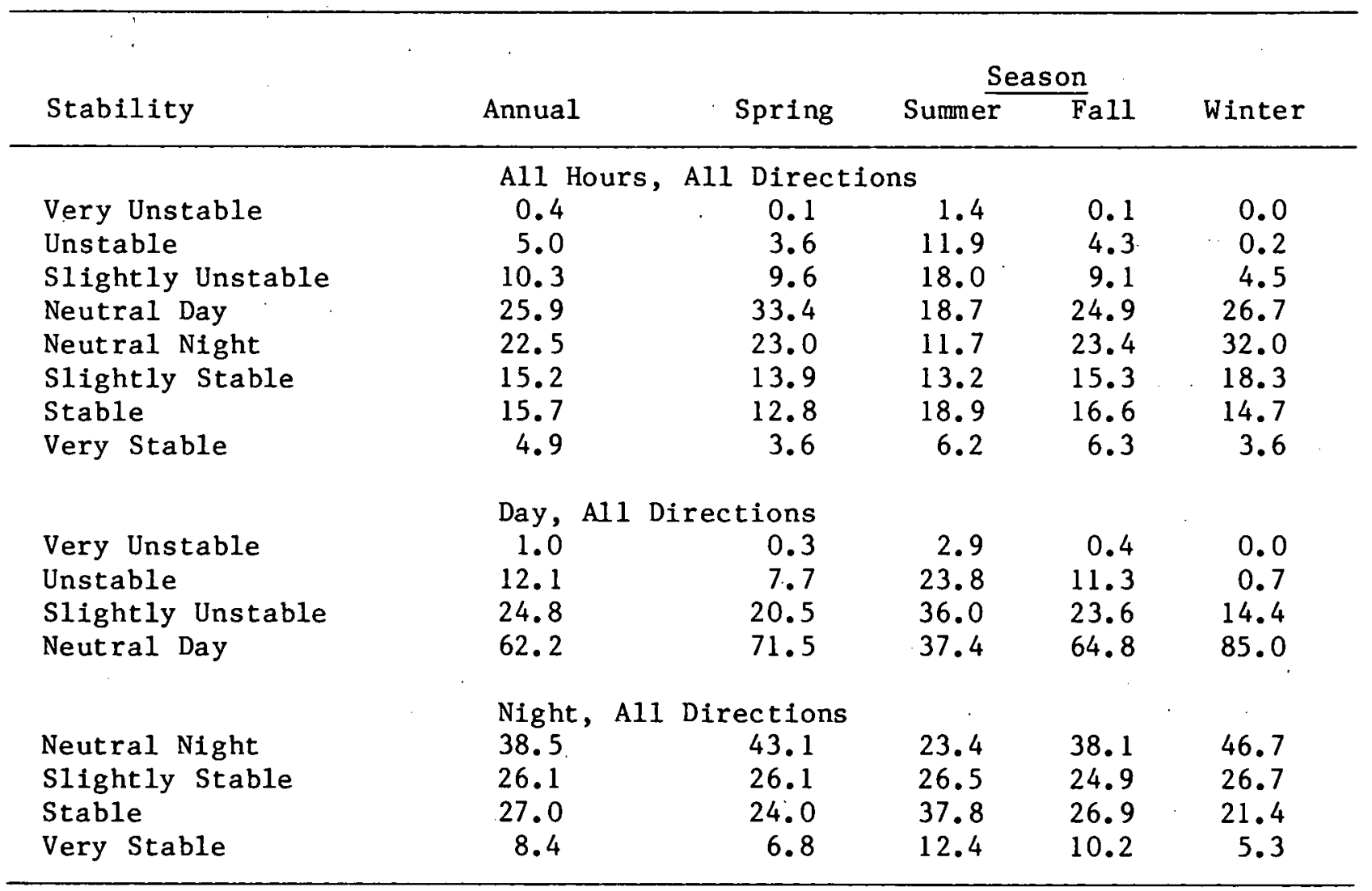


Table 5..9. (continued)

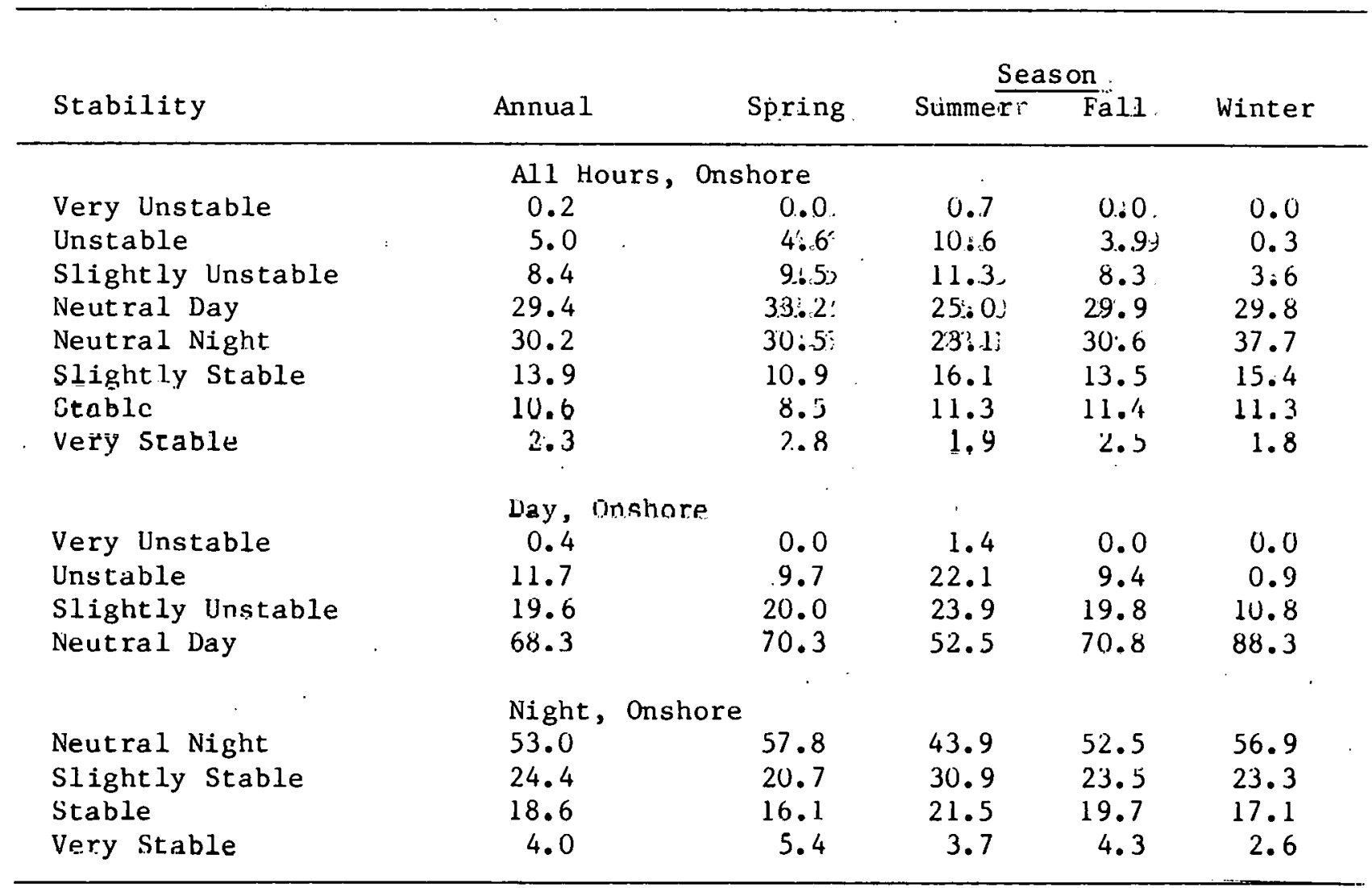


Table 5.9 (continued)

\begin{tabular}{|c|c|c|c|c|c|}
\hline \multirow[b]{2}{*}{ Stability } & \multirow[b]{2}{*}{ Annua 1} & \multirow[b]{2}{*}{ Spring } & \multicolumn{2}{|c|}{ Season } & \multirow[b]{2}{*}{ Winter } \\
\hline & & & Summe $\bar{r}$ & $\overline{F a l l}$ & \\
\hline \multicolumn{6}{|c|}{ All Hours, Alongshore } \\
\hline Very Unstable & 0.7 & 0.0 & 2.5 & 0.0 & 0.0 \\
\hline Unstable & 5.1 & 4.1 & 9.7 & 4.7 & 0.0 \\
\hline Slightly Unstable & 10.4 & 9.4 & 16.2 & 8.4 & 5.1 \\
\hline Neutral Day & 29.1 & 35.3 & 26.2 & 30.8 & 25.5 \\
\hline Neutra1 Night & 21.9 & 26.3 & 11.2 & 21.8 & 31.2 \\
\hline Slightly Stable & 13.4 & 8.9 & 11.8 & 16.3 & 16.7 \\
\hline Stable & 16.3 & 14.2 & 17.6 & 14.1 & 20.0 \\
\hline Very Stable & 3.1 & 1.9 & 4.9 & 3.8 & 1.5 \\
\hline \multicolumn{6}{|c|}{ Day, Alongshore } \\
\hline Very Unstable & 1.5 & 0.0 & 4.5 & 0.0 & 0.0 \\
\hline Unstable & 11.1 & 8.4 & 17.8 & 10.8 & 0.0 \\
\hline \multirow{3}{*}{$\begin{array}{l}\text { Siight ly Unstable } \\
\text { Neutral Day }\end{array}$} & 23.0 & 19.3 & 29.8 & 19.4 & 15.8 \\
\hline & 64.3 & 72.2 & 47.9 & 69.8 & 84.2 \\
\hline & Night & , Alongshore & & & \\
\hline Neutral Night & 39.8 & 51.4 & 24.1 & 38.4 & 45.1 \\
\hline Slightly Stable & 24.5 & 17.3 & 26.0 & 29.1 & 24.1 \\
\hline Stable & 29.9 & 27.7 & 39.1 & 25.5 & 28.5 \\
\hline Very Stable & 5.8 & 3.6 & 10.9 & 7.0 & 2.3 \\
\hline
\end{tabular}


Table 5.9 (continued)

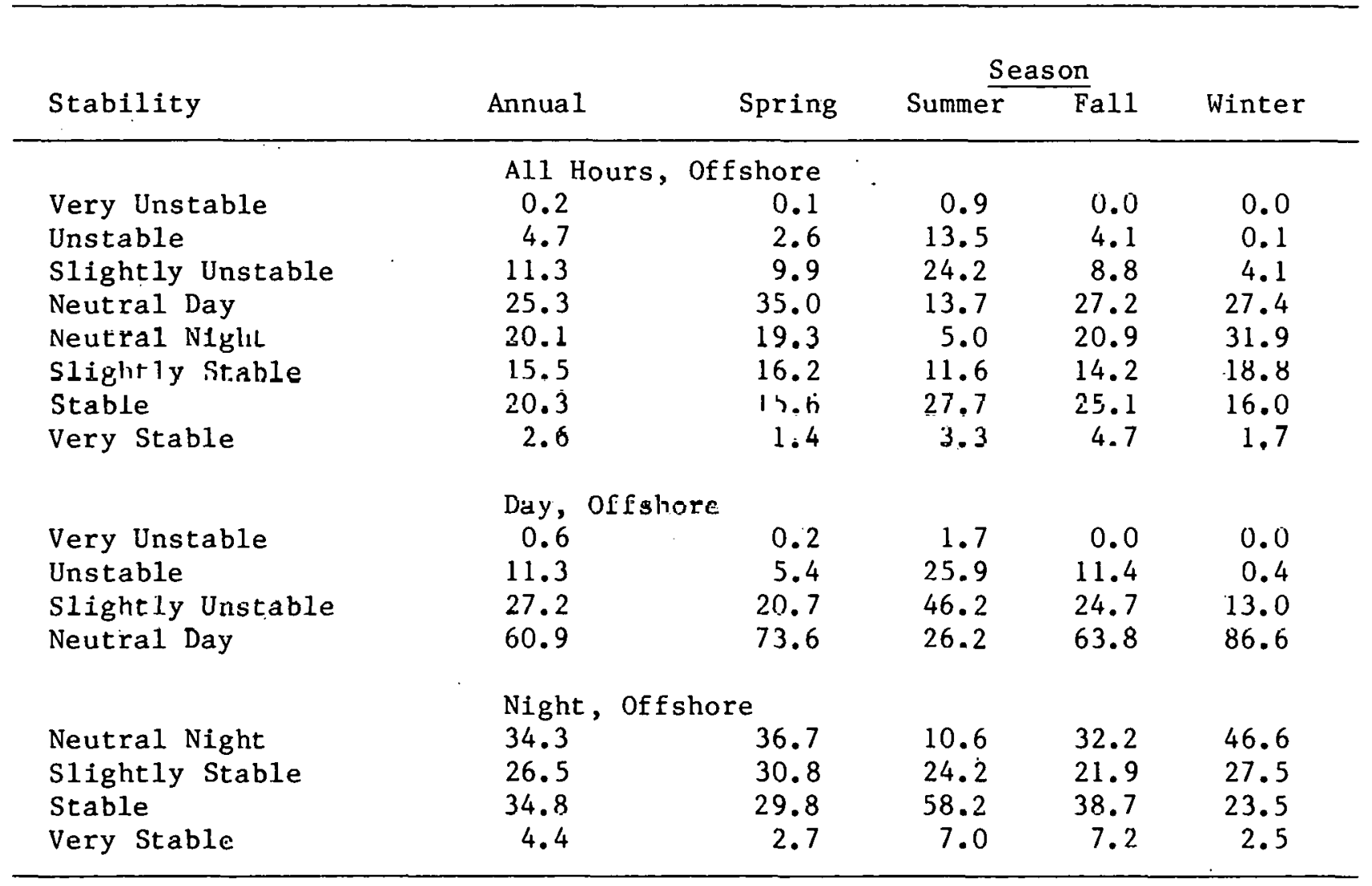




\begin{tabular}{|c|c|c|c|c|c|}
\hline \multirow[b]{3}{*}{ Stability } & \multicolumn{3}{|c|}{$\begin{array}{l}\text { Table } 5.10 \\
\text { os Island, Virginia } \\
\text { ars in Each Stability Class } \\
\text { on, Season and Time of Day }\end{array}$} & ' & \multirow[b]{3}{*}{ Winter } \\
\hline & \multirow[b]{2}{*}{ Annual } & \multirow[b]{2}{*}{ Spring } & \multicolumn{2}{|c|}{ Season } & \\
\hline & & & Summer & $\bar{F} a 11$ & \\
\hline $\begin{array}{l}\text { Very Unstable } \\
\text { Unstable } \\
\text { Slightly Unstable } \\
\text { Neutral Day } \\
\text { Neutral Night } \\
\text { Slightly Stable } \\
\text { Stable } \\
\text { Very Stable }\end{array}$ & $\begin{array}{l}\text { A11 H } \\
0.5 \\
7.9 \\
17.2 \\
35.3 \\
17.5 \\
10.9 \\
7.5 \\
3.3\end{array}$ & $\begin{array}{c}\text { ours, All Direct } \\
0.0 \\
6.3 \\
15.5 \\
40.2 \\
17.9 \\
10.6 \\
7.0 \\
2.6\end{array}$ & $\begin{array}{r}2.1 \\
18.7 \\
28.3 \\
30.1 \\
6.1 \\
6.7 \\
6.3 \\
1.7\end{array}$ & $\begin{array}{r}0.1 \\
5.8 \\
18.7 \\
35.8 \\
18.0 \\
9.3 \\
7.8 \\
4.5\end{array}$ & $\begin{array}{r}0.0 \\
1.5 \\
7.7 \\
35.2 \\
26.5 \\
16.3 \\
8.5 \\
4.2\end{array}$ \\
\hline $\begin{array}{l}\text { Very Unstable } \\
\text { Unstable } \\
\text { Slightly Unstable } \\
\text { Neutral Day }\end{array}$ & $\begin{array}{r}\text { Day, } \\
0.8 \\
12.9 \\
28.3 \\
57.9\end{array}$ & $\begin{array}{r}\text { All Directions } \\
0.0 \\
10.3 \\
24.6 \\
65.1\end{array}$ & $\begin{array}{r}2.6 \\
23.6 \\
35.8 \\
38.1\end{array}$ & $\begin{array}{r}0.2 \\
9.6 \\
31.0 \\
59.2\end{array}$ & $\begin{array}{r}0.0 \\
3.5 \\
17.6 \\
79.0\end{array}$ \\
\hline $\begin{array}{l}\text { Neutral Night } \\
\text { Slightly Stable } \\
\text { Stable } \\
\text { Very Stable }\end{array}$ & $\begin{array}{l}\text { Night, } \\
44.5 \\
27.8 \\
19.3 \\
8.4\end{array}$ & $\begin{array}{c}\text { A11 Directions } \\
47.5 \\
27.4 \\
19.0 \\
6.1\end{array}$ & $\begin{array}{r}29.2 \\
32.3 \\
30.2 \\
8.3\end{array}$ & $\begin{array}{l}45.4 \\
23.5 \\
19.7 \\
11.4\end{array}$ & $\begin{array}{r}47.5 \\
29.6 \\
15.3 \\
7.6\end{array}$ \\
\hline
\end{tabular}


Table $5 \cdot 10 \cdot($ continued)

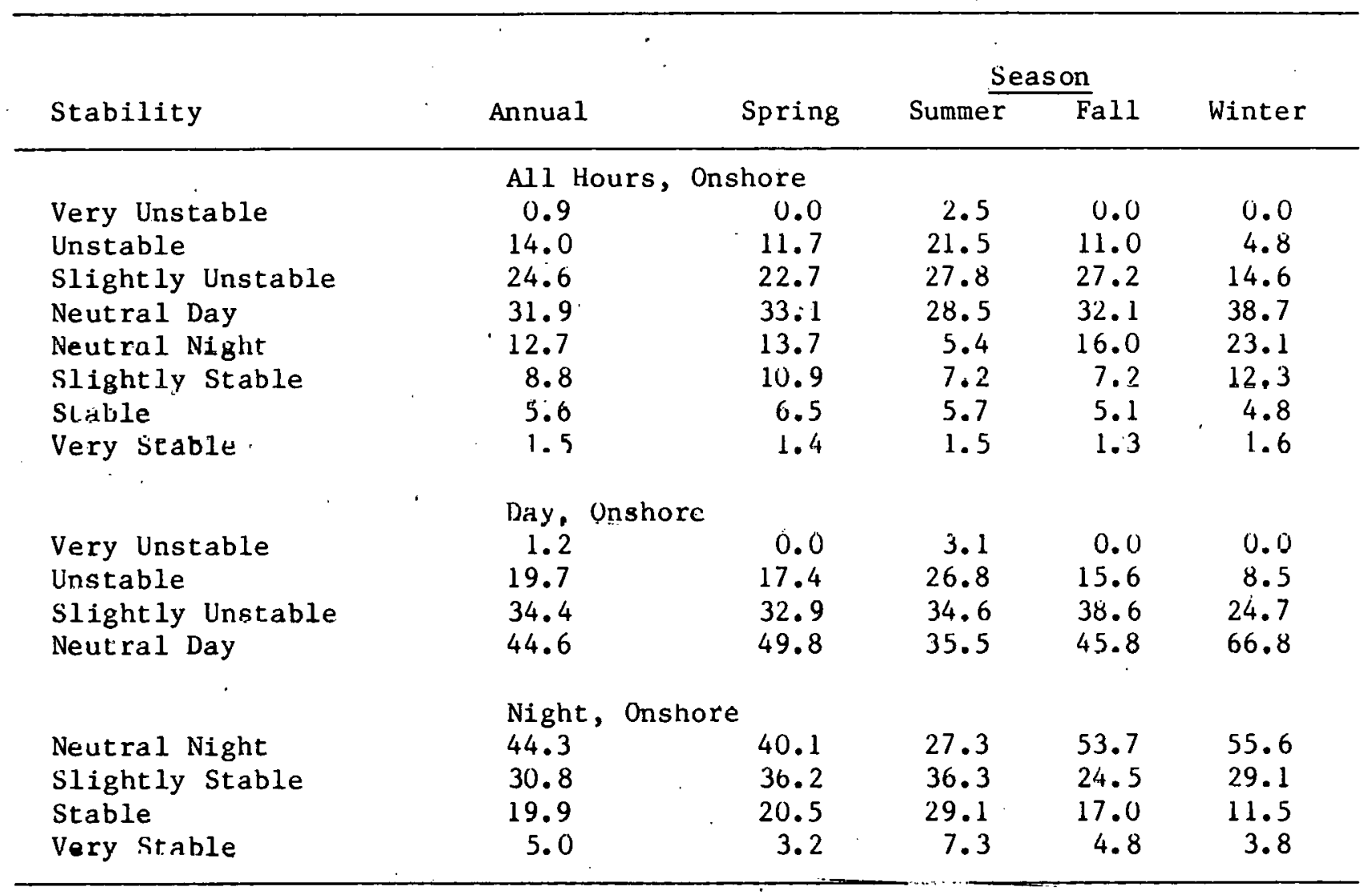


Table 5.10 (continued)

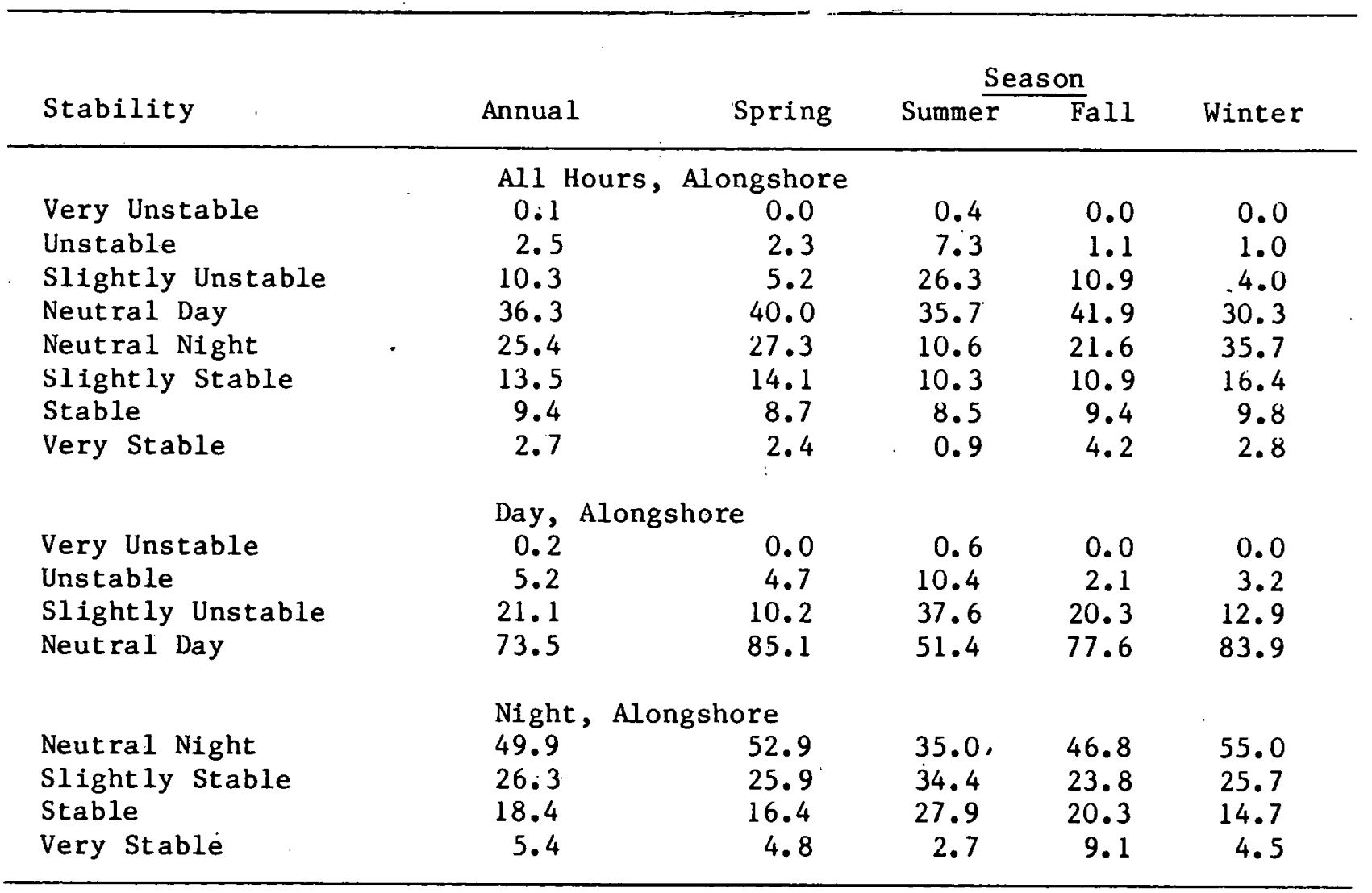


Table 5.10. (continued)

\begin{tabular}{|c|c|c|c|c|c|}
\hline \multirow[b]{2}{*}{ Stability } & \multirow[b]{2}{*}{ Annual } & \multirow{2}{*}{ Spring } & \multicolumn{2}{|c|}{ Season } & \multirow[b]{2}{*}{ Winter } \\
\hline & & & Summer & Fal1 & \\
\hline \multicolumn{6}{|c|}{ All Hours, Offshore } \\
\hline Very Unstable & 0.3 & 0.0 & 2.1 & 0.0 & 0.0 \\
\hline Unstable & 4.6 & 3.8 & 20.6 & 2.2 & 0.4 \\
\hline Slightly Unstable & 14.1 & 14.5 & 33.7 & 13.6 & 6.7 \\
\hline Neutral Day & 40.4 & 47.8 & 30.6 & 38.6 & 39.1 \\
\hline Ncutral Night & 17.9 & 16.9 & 3.8 & 18.2 & 23.6 \\
\hline Slightly Stable & 10.6 & 7.5 & 1.6 & 10.4 & 16.9 \\
\hline Stable & 8.6 & 6.8 & 6.5 & $10 . \overline{7}$ & 9.8 \\
\hline Very Stable & 3.5 & $2: 8$ & 1.1 & 6.3 & 3.5 \\
\hline \multicolumn{6}{|c|}{ Day, offshore } \\
\hline Very Unstable & 0.5 & 0.0 & 2.5 & 0.0 & 0.0 \\
\hline Unstable & 7.7 & 6.1 & 23.7 & 4.0 & $0 . \dot{9}$ \\
\hline Slight ly Unstable & 23.9 & $22.0^{\circ}$ & 38.7 & 24.6 & 14.7 \\
\hline Neutra1 Day & 67.9 & 71.9 & 35.1 & 71.4 & 84.4 \\
\hline \multicolumn{6}{|c|}{ Night, offshore } \\
\hline Neutral Night & 43.8 & 50.6 & 29.0 & 39.8 & 43.4 \\
\hline Slightly Stable & 26.1 & 20.8 & 12.9 & 22.7 & 31.7 \\
\hline Stable & 21.6 & 20.9 & 49.0 & 23.6 & 18.6 \\
\hline Very Stable & 8.5 & 7.8 & 9.1 & 13.8 & 6.3 \\
\hline
\end{tabular}




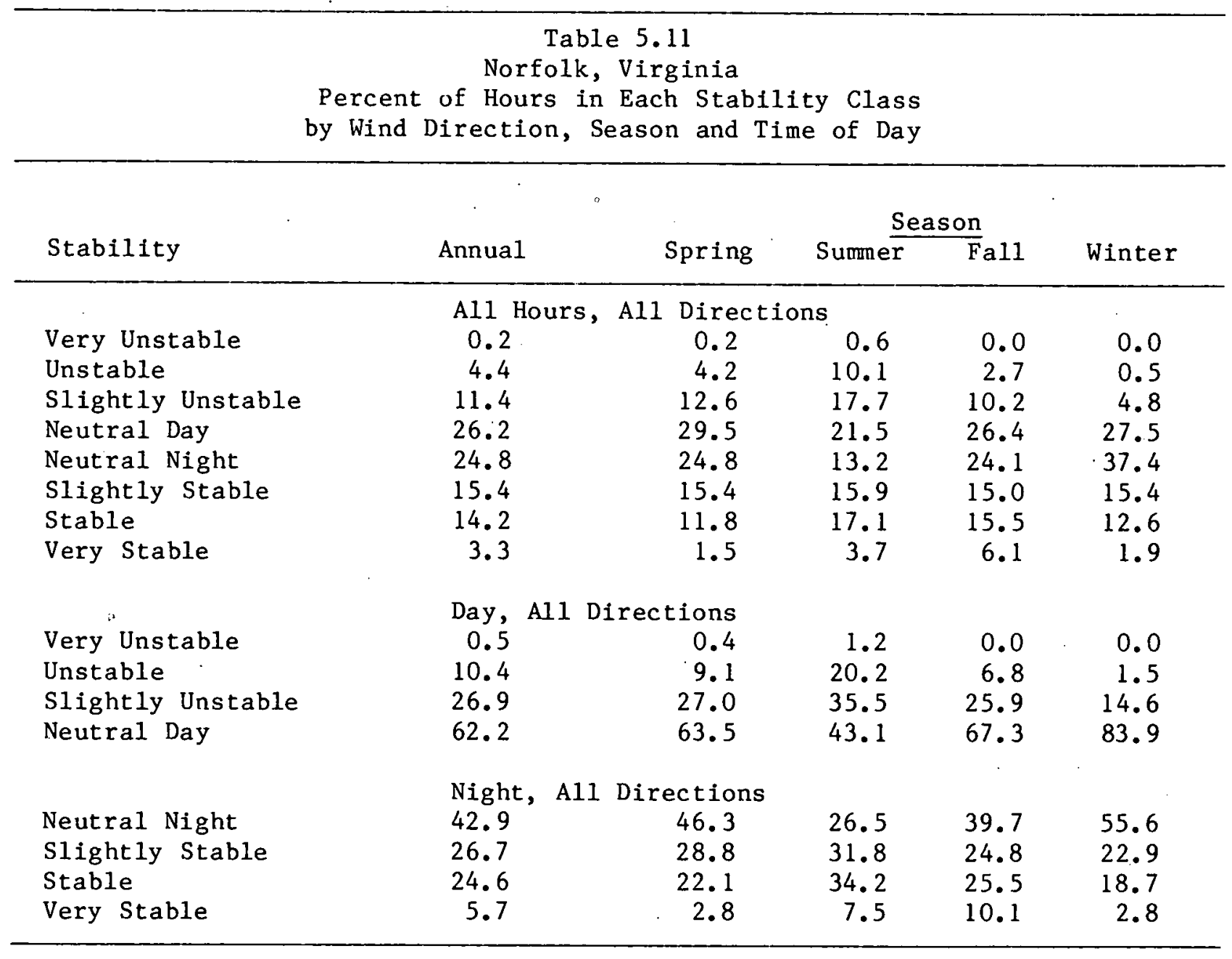


Table 5.11 (continued)

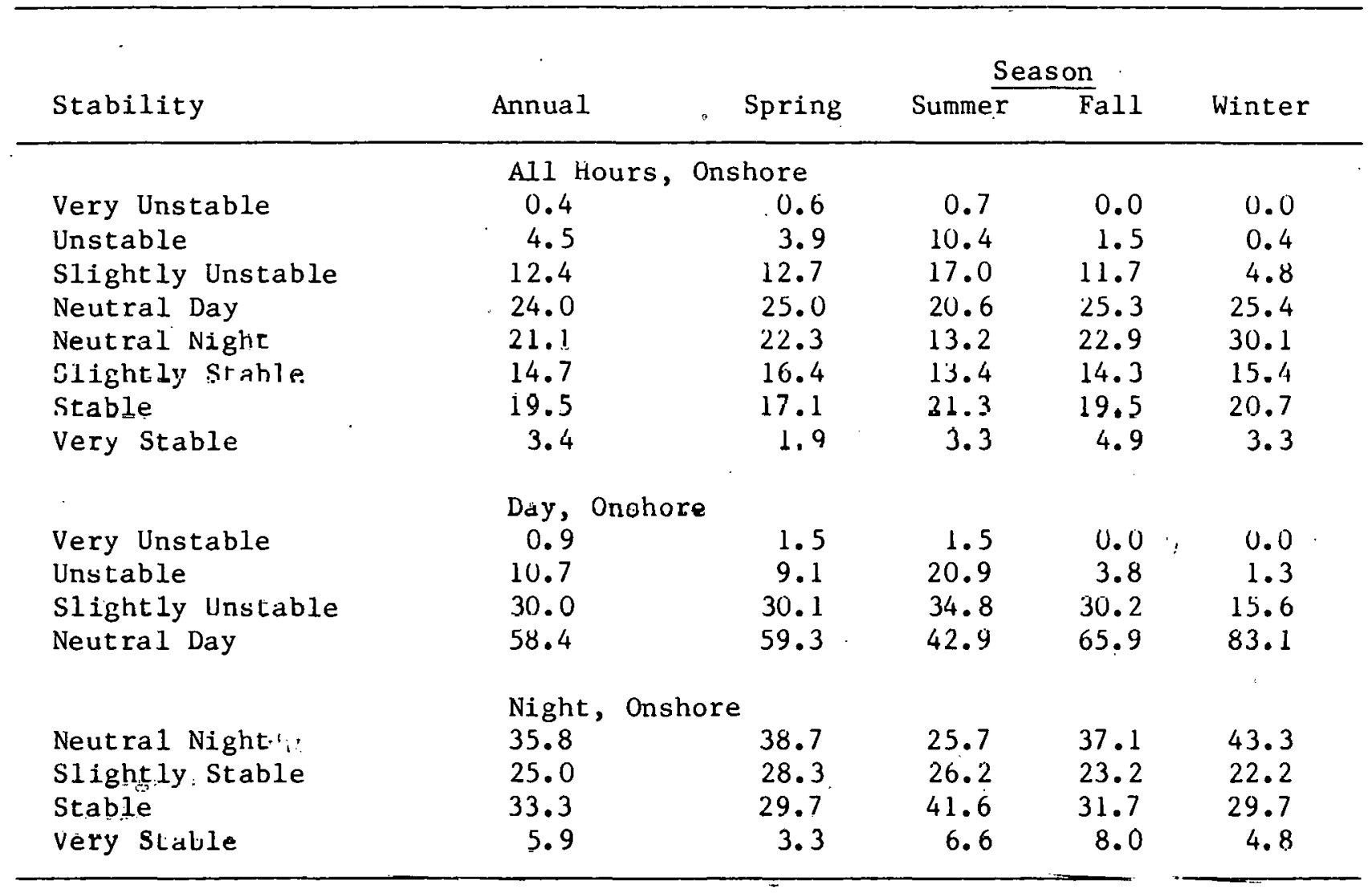


Table 5.11 (continued)

\begin{tabular}{|c|c|c|c|c|c|}
\hline \multirow[b]{2}{*}{ Stability } & \multirow[b]{2}{*}{ Annua 1} & \multicolumn{4}{|c|}{ Seàson } \\
\hline & & Spring & Summer & Fal1! & Winter \\
\hline & \multicolumn{2}{|c|}{ All Hours, Alongshore } & \multicolumn{2}{|c|}{$\checkmark$} & \\
\hline Very Unstable: & 0.2 & 0.0 & 0.9 & 0.0 & 0.0 \\
\hline Unstable & 4.4 & 5.0 & 8.5 & 4.3 & 0.3 \\
\hline Slightly Unstabliee & 10.3 & 10.9 & 16.5 & 9.5 & 4.7 \\
\hline Neutral Day & 26.4 & 29.3 & 21.4 & 29.5 & 26.6 \\
\hline Neutral Night: & 26.8 & 27.7 & 14.3 & 22.0 & 42.0 \\
\hline Slight ly Stablé & 16.1 & 15.8 & 20.2 & 15.9 & 12.6 \\
\hline Stable & 13.3 & 10.6 & 16.0 & 14.6 & 12.0 \\
\hline \multirow[t]{2}{*}{ Very Stable } & 2.2 & 0.6 & 2.4 & 4.2 & 1.8 \\
\hline & \multicolumn{2}{|c|}{ Day, Alongshore } & & & \\
\hline Vëry Unstable & 0.6 & 0.0 & 1.8 & $0.0 !$ & 0.0. \\
\hline Unistabledes & 10.8 & 11.2 & 18.1 & 10.1 & 1.06 \\
\hline Slight ly Unstable & 25.0 & 24.1 & 34.8 & 22.5 & 15.0 \\
\hline \multirow[t]{2}{*}{ Neutra 11 Day } & 63.6 & 64.7 & 45.3 & 67.5 & 84.1 \\
\hline & \multicolumn{2}{|c|}{ Night, Alongshore } & & & \\
\hline Neutral Night & 46.0 & 50.9 & 27.0 & 39.7 & 61.5 \\
\hline Slightly Stable & 27.5 & 28.8 & 38.2 & 27.6 & 18.4 \\
\hline Stable & 22.7 & 19.2 & 30.2 & 25.3 & 17.5 \\
\hline Very Stable & 3.8 & 1.0 & 4.5 & 7.3 & 2.6 \\
\hline
\end{tabular}


Table 5.11 (continued)

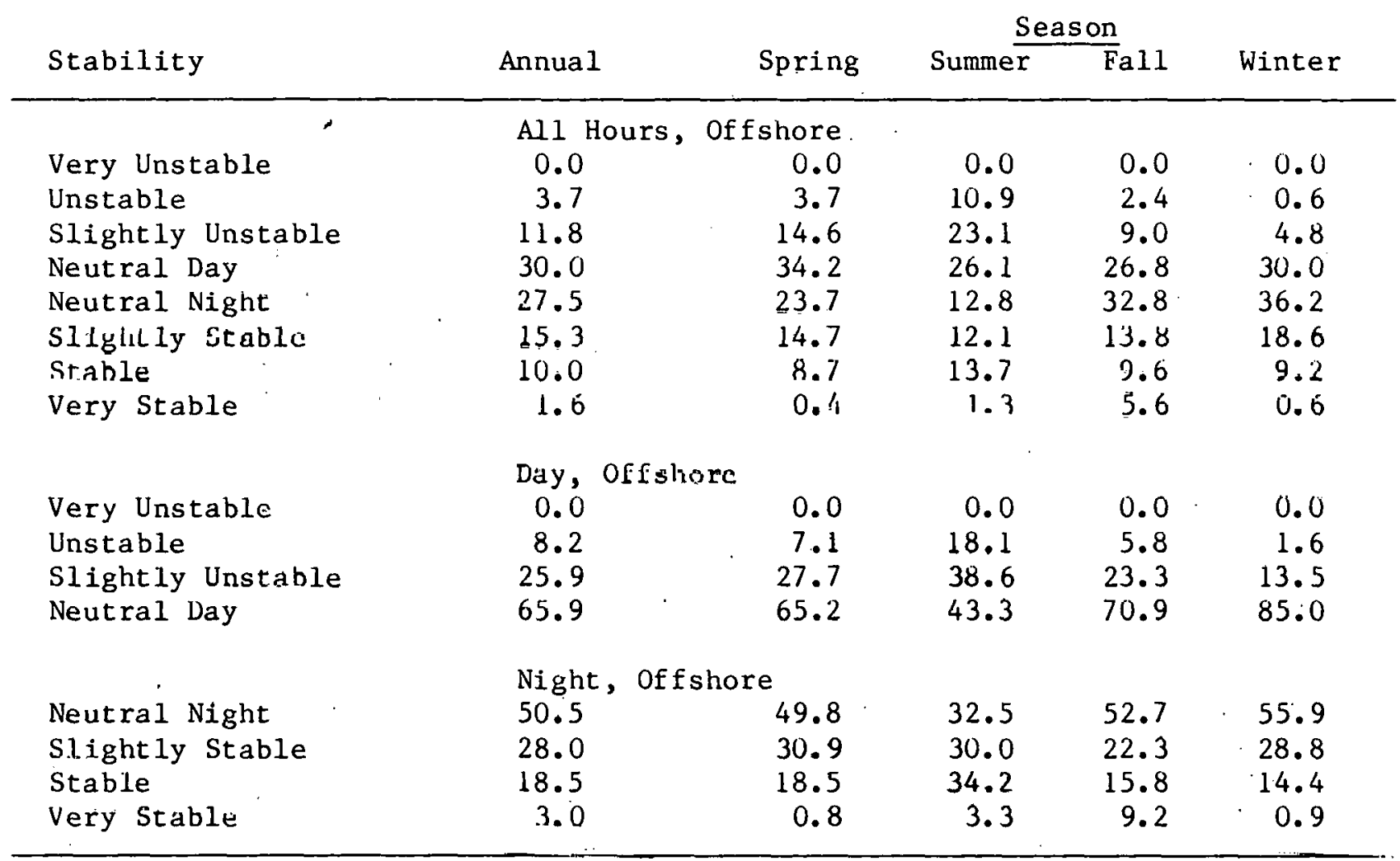


Table 5.12

Cape Hatteras, North Carolina

Percent of Hours in Each Stability Class

by Wind Direction, Season and Time of Day

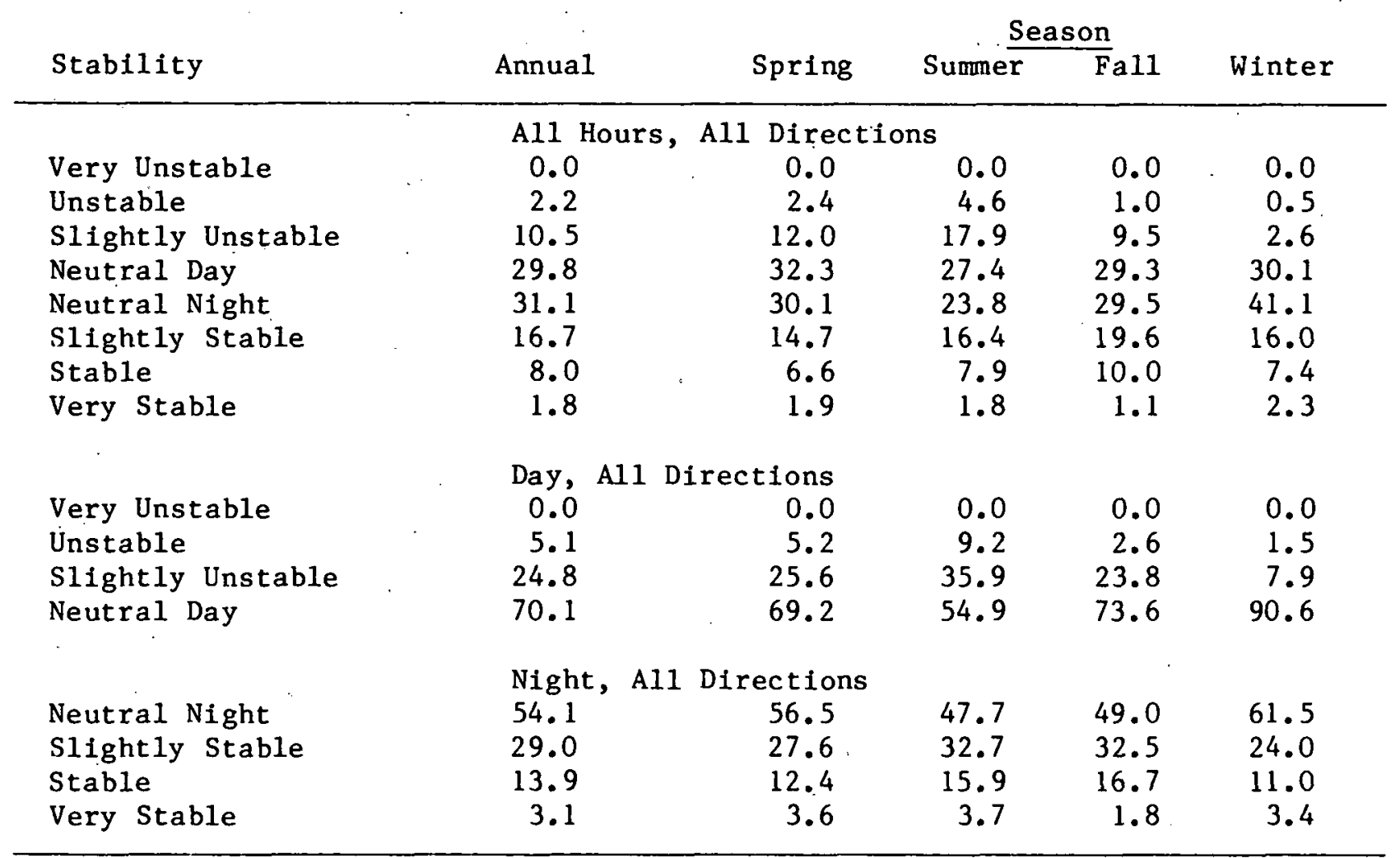


Tatable 5.12 (continued)

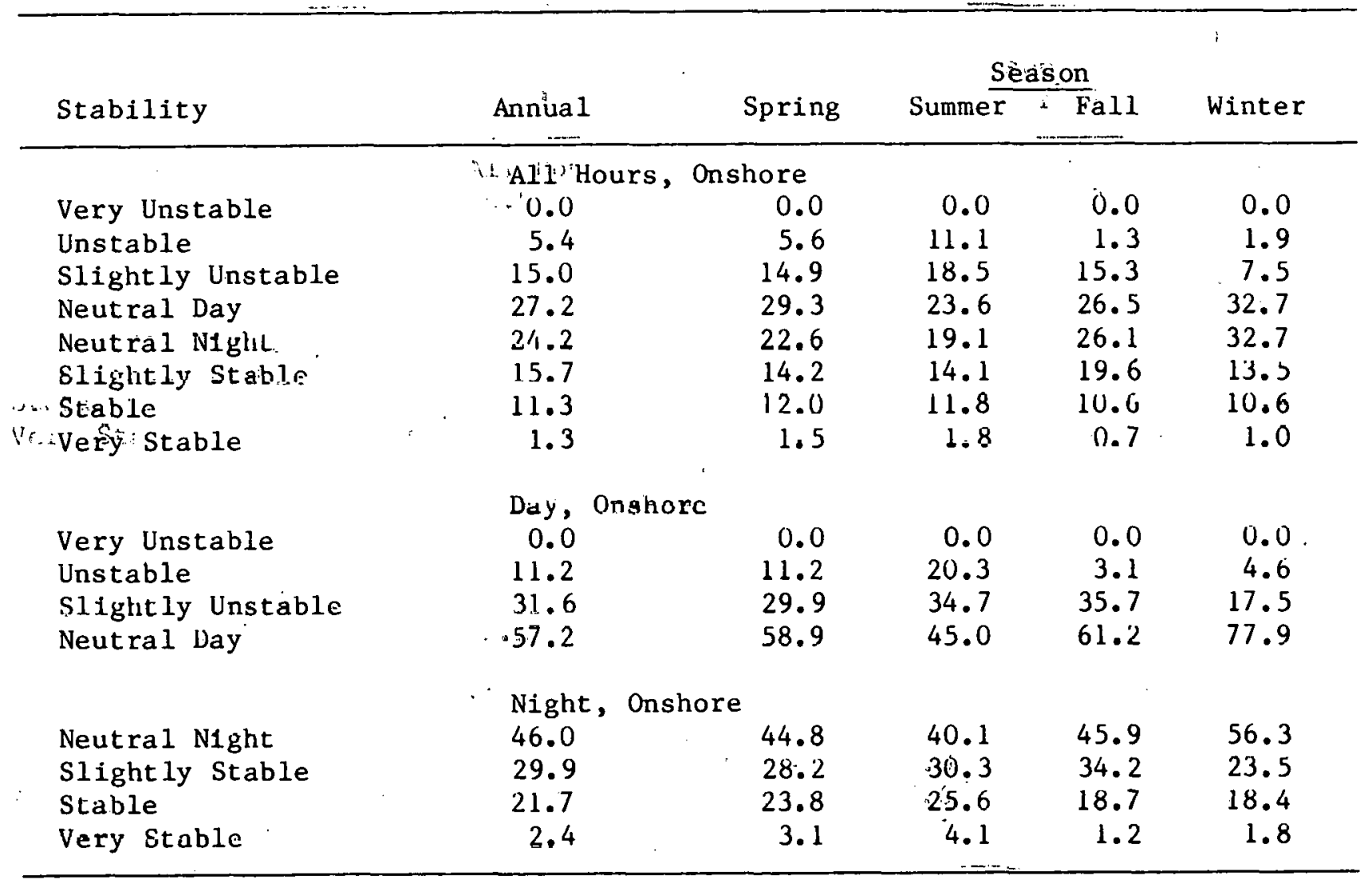


Table 5.12 (continued)

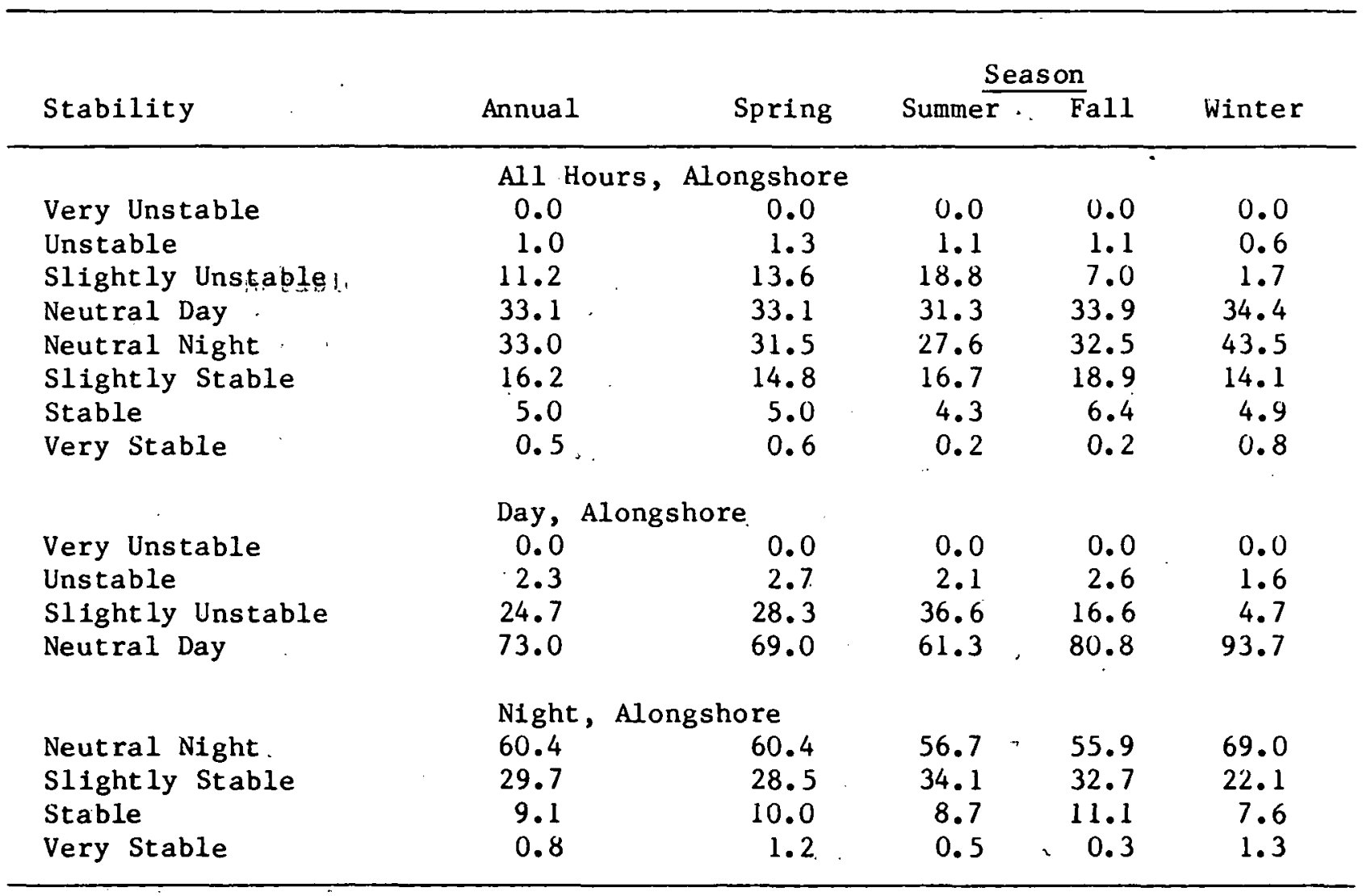


Table 5.12 (continued)

\begin{tabular}{|c|c|c|c|c|c|}
\hline \multirow[b]{2}{*}{ Stability } & \multirow[b]{2}{*}{ Annual } & \multirow[b]{2}{*}{ Spring } & \multicolumn{2}{|c|}{ Season } & \multirow[b]{2}{*}{ Winter } \\
\hline & & & Summer & $\bar{F} a 11$ & \\
\hline \multicolumn{6}{|c|}{ All Hours, of fshore } \\
\hline Very Unstable & 0.0 & 0.0 & 0.0 & 0.0 & 0.0 \\
\hline Unstable . & 1.0 & 1.0 & 4.6 & 0.7 & 0.0 \\
\hline Slightly Unstable & 5.8 & 7.2 & 13.8 & 6.0 & 2.1 \\
\hline Neutral Day & 29.1 & 35.9 & 25.1 & 27.6 & 27.4 \\
\hline Neutral Night & 35.5 & 35.4 & 22.7 & 30.1 & 43.0 \\
\hline Slight ly Stable & 18.5 & 15.0 & 19.7 & 21.0 & 18.6 \\
\hline Stable & 9.6 & 4.4 & 13.1 & 14.4 & 0.5 \\
\hline Very Stable & 0.5 & 0.6 & 0.8 & 0.2 & 0.4 \\
\hline \multicolumn{6}{|c|}{ Day, offshore } \\
\hline Very Unstable & 0.0 & 0.0 & 0.0 & 0.0 & 0.0 \\
\hline Unstable & 2.9 & 2.4 & 10.8 & 2.1 & 0.0 \\
\hline Slightly Unstable & 16.1 & 16.5 & 31.7 & $17.2:$ & 7.3 \\
\hline Neutral Day & 80.9 & 81.1 & 57.5 & 80.8 & 92.7 \\
\hline \multicolumn{6}{|c|}{ Night, of fshore } \\
\hline Neutral Night & 55.4 & 63.3 & 40.3 & 45.7. & 61.1 \\
\hline Slightly Stable & 28.9 & 26.9 & 35.1 & 32.1 & 26.4 \\
\hline Stable & 14.9 & 8.7 & 23.1 & 21.8 & 12.0 \\
\hline Very Stable & 0.8 & 1.1 & 1.5 & 0.3 & 0.5 \\
\hline
\end{tabular}




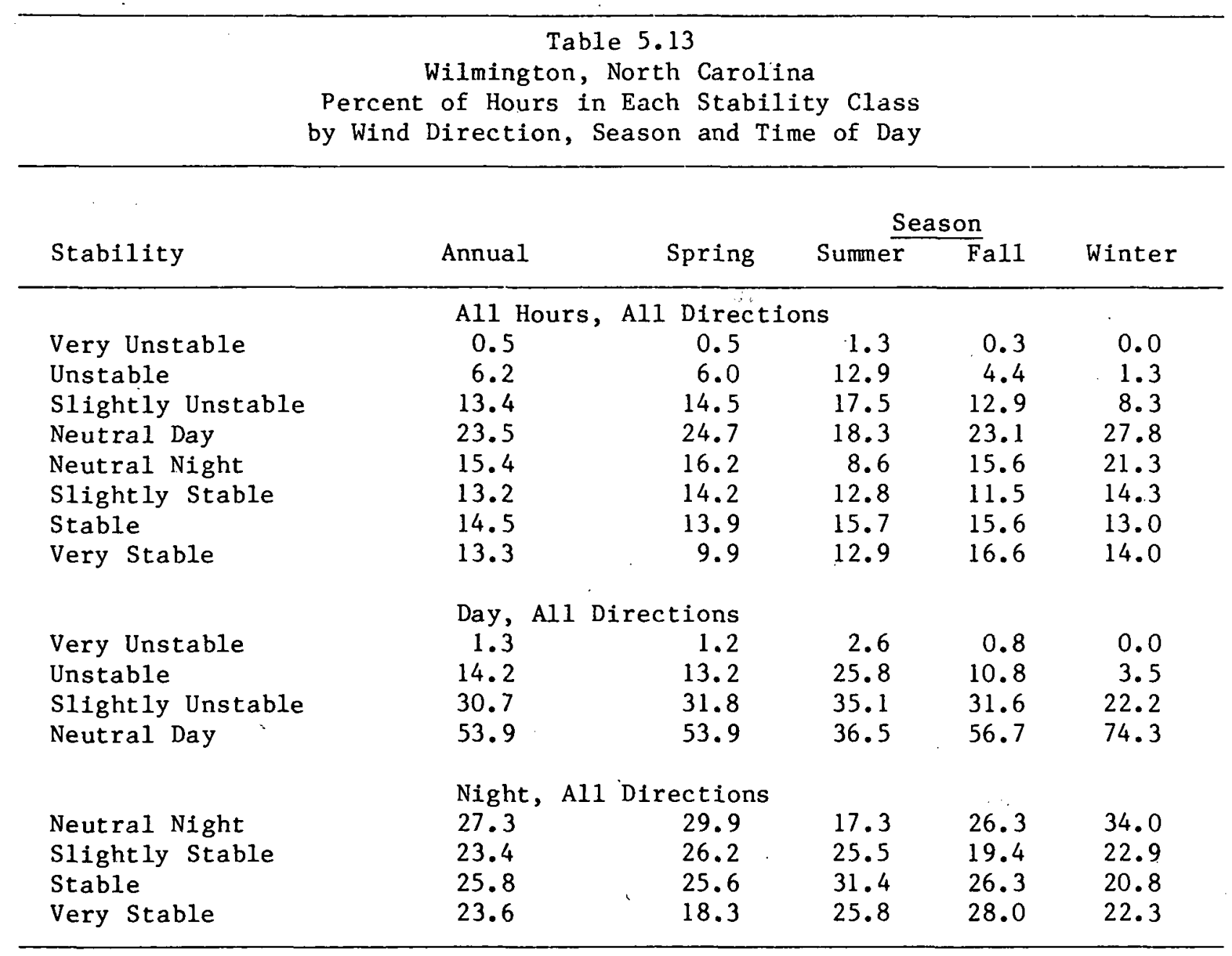


Table 5.13 (continued)

\begin{tabular}{|c|c|c|c|c|c|}
\hline \multirow[b]{2}{*}{ Stability } & \multirow[b]{2}{*}{ Annual } & \multirow[b]{2}{*}{ Spring } & \multicolumn{2}{|c|}{ Season } & \multirow[b]{2}{*}{ Winter } \\
\hline & & & Summer & Fall & \\
\hline \multicolumn{6}{|c|}{ All Hours, Onshore } \\
\hline Very Unstab:le & 0.6 & 1.1 & 1.0 & 0.0 & 0.0 \\
\hline Unstable & 7.9 & 7.0 & 12.5 & 7.7 .0 & 2.1 \\
\hline Slightly Unstable & 19.0 & 17.3 & 21.2 & 22.2 .6 & 12.9 \\
\hline Neutral Day & 27.8 & 23.4 & 24.0 & 30.1 & 37.9 \\
\hline Neutral Night & 13.9 & 18.2 & 8.2 & 111.8 & 19.1 \\
\hline Slightly Stable & 8.6 & 8.2 & 11.0 & 5.1 & 9.6 \\
\hline Stahlè & 15.9 & 18.1 & lih.? & $: 16.8$ & 11.9 \\
\hline Very Stablo & 6.3 & 6.7 & 6.0 & 6.6 & 6.6 \\
\hline \multicolumn{6}{|c|}{ Day, Onshore } \\
\hline Very Unstable & 1.1 & 2.1 & 1.8 & 0.0 & 0.0 \\
\hline Unstable & 14.2 & 14.3 & 21.3 & 11.8 & 4.0 \\
\hline Slightly Unstable & 34.4 & 35.3 & 36.3 & 37.9 & 24.4 \\
\hline Neutra 1 Day & 50.3 & 48.3 & 40.7 & 50.3 & 71.6 \\
\hline \multicolumn{6}{|c|}{ Night, Onshore } \\
\hline Neutral Night & 31.2 & 35.5 & 19.9 & 29.2 & 40.2 \\
\hline Slightly Stable & 19.2 & 15.8 & 26.4 & 12.7 & 20.4 \\
\hline Stable & 35.7 & 35.4 & 39.4 & 41.9 & $\therefore 2.5 .4$ \\
\hline Very Stable & 14.0 & 13.3 & 14.2 & 16.2 & 14.0 \\
\hline
\end{tabular}


Table 5.13 (continued)

\begin{tabular}{|c|c|c|c|c|c|}
\hline \multirow[b]{2}{*}{ Stability } & \multirow[b]{2}{*}{ Annual } & \multirow[b]{2}{*}{ Spring } & \multicolumn{2}{|c|}{ Season } & \multirow[b]{2}{*}{ Winter } \\
\hline & & & Summer & Fall & \\
\hline \multicolumn{6}{|c|}{ All Hours, Alongshore } \\
\hline Very Unstable & 0.2 & 0.3 & 0.5 & 0.3 & 0.0 \\
\hline Unstable & 4.2 & 4.1 & 8.0 & $\therefore 3.1$ & 1.1 \\
\hline Slight ly Unstable & 9.4 & 9.5 & 14.1 & .7 .7 & 5.9 \\
\hline Neutral Day & 25.5 & 28.1 & 21.4 & 724.6 & 27.8 \\
\hline Neutral Night & 20.4 & 20.7 & 12.5 & 19.0 & 30.0 \\
\hline Slight ly Stable & 19.5 & 20.2 & 20.0 & 18.7 & 18.8 \\
\hline Stable & 17.6 & 13.8 & 20.3 & 21.8 & 15.1 \\
\hline Very Stable & 3.2 & 3.3 & 3.3 & 4.8 & 1.3 \\
\hline \multicolumn{6}{|c|}{ Day, Alongshore } \\
\hline Very Unstable & 0.6 & 0.7 & 1.0 & 0.8 & 0.0 \\
\hline Unstable & 10.6 & 9.8 & 18.2 & 8.8 & 3.3 \\
\hline Slightly Unstable & 23.9 & 22.7 & 31.9 & 21.6 & 17.0 \\
\hline \multirow[t]{2}{*}{ Neutral Day } & 64.9 & 66.8 & 48.9 & 68.8 & 79.8 \\
\hline & Night & Alongshore & & & \\
\hline Neutral Night & 33.5 & 35.4 & 22.4 & 29.5 & 46.1 \\
\hline Slightly Stable & 32.2 & 35.1 & 35.7 & 29.1 & 28.9 \\
\hline Stable & 29.1 & 23.7 & 36.0 & 33.9 & 23.0 \\
\hline Very Stable & 5.3 & 5.7 & 5.9 & 7.5 & 2.0 \\
\hline
\end{tabular}


Table 5.13 (continued)

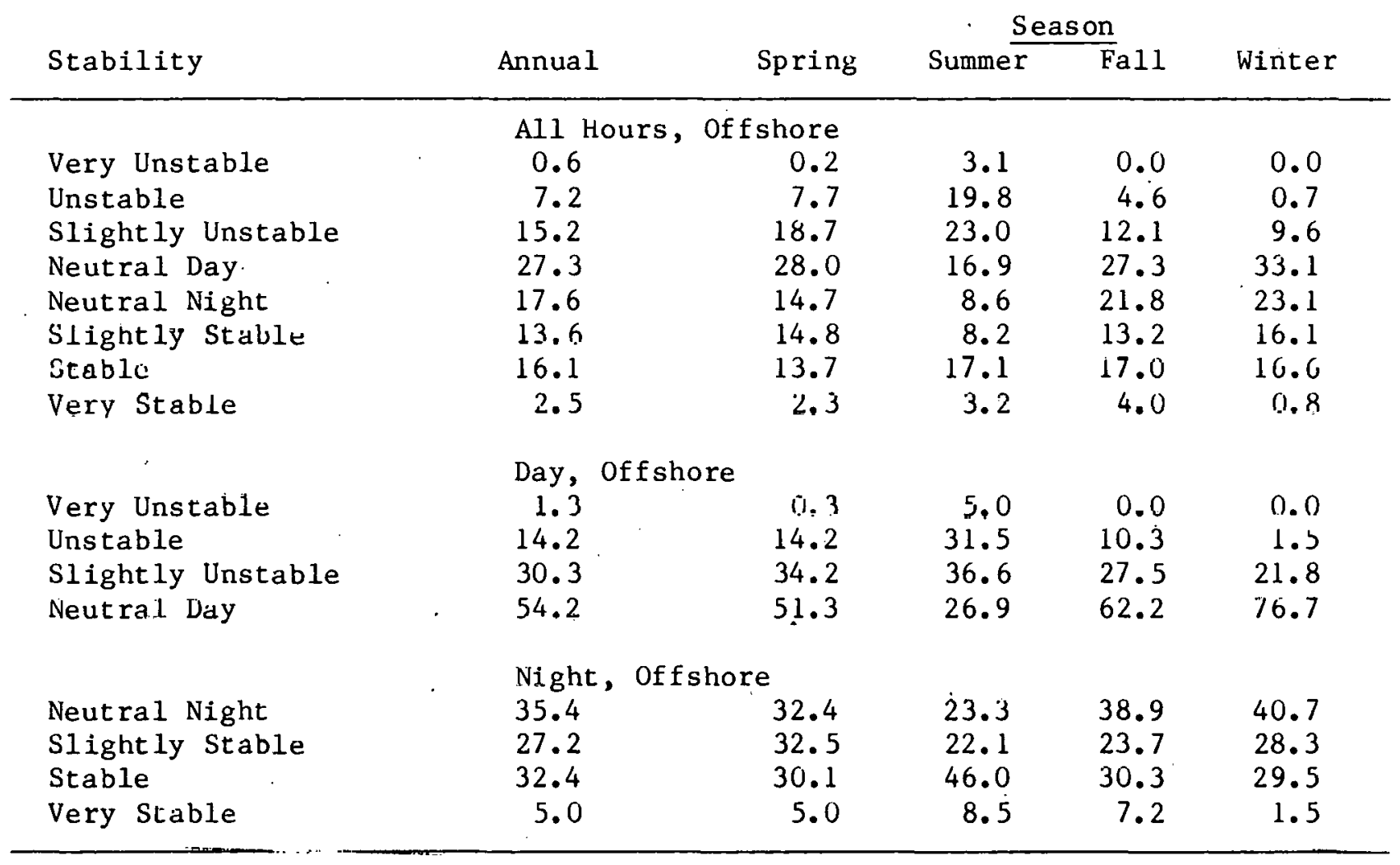




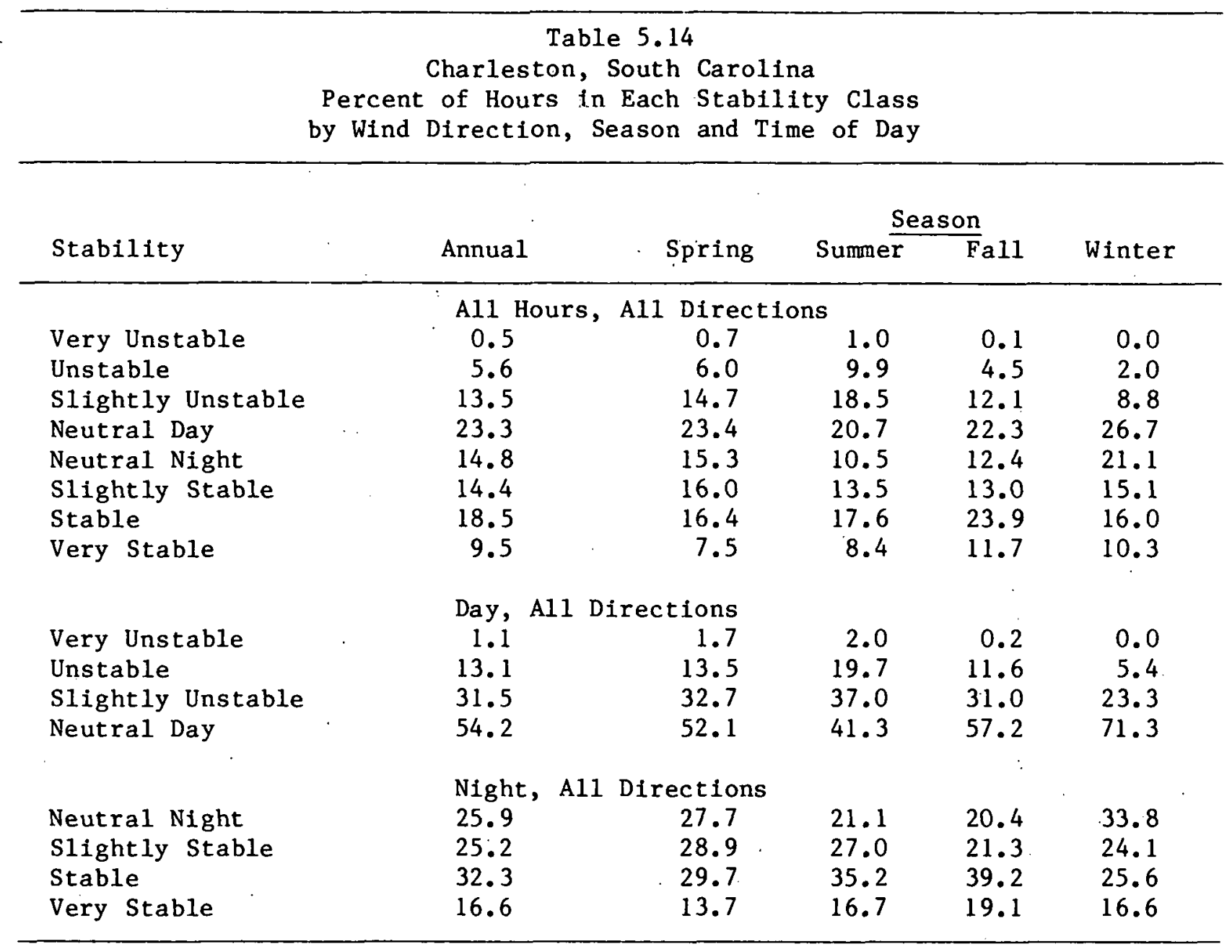


Table 5.14 (continued)

\begin{tabular}{|c|c|c|c|c|c|}
\hline \multirow[b]{2}{*}{ Stability } & \multirow[b]{2}{*}{ Annual } & \multirow[b]{2}{*}{ Spring } & \multicolumn{2}{|c|}{ Seas on } & \multirow[b]{2}{*}{ Winter } \\
\hline & & & Summer & Fall & \\
\hline \multicolumn{6}{|c|}{ All Hours, Onshore } \\
\hline Very Unstable & 0.4 & 0.5 & 0.8 & 0.0 & 0.0 \\
\hline Unstable & 4.2 & 4.6 & 5.7 & 3.8 & 1.7 \\
\hline Slightly Unstable & 12.5 & 12.1 & 15.1 & 15.7 & 6.3 \\
\hline Neutral Day & 24.3 & 23.6 & 21.6 & 27.6 & 26.3 \\
\hline Neutral Night & 13.6 & 15.2 & 11.1 & 6.9 & 20.4 \\
\hline Slightly Stable & 15.9 & 17.7 & 16.6 & 13.4 & 14.1 \\
\hline St.able & 21.7 & 19.0 & 22.2 & 23.3 & 23.9 \\
\hline Vcry Stable & 7.4 & 7.3 & 6.8 & 4.3 & 7.3 \\
\hline \multicolumn{6}{|c|}{ Day, Onshore } \\
\hline Very Unstable & 1.0 & 1.2 & 1.8 & 0.0 & 0.0 \\
\hline Unstable & 10.1 & 11.2 & 13.1 & 7.5 & 4.9 \\
\hline Slightly Unstable & 30.2 & 29.7 & 34.6 & 33.3 & 18.1 \\
\hline Neutral Day. & 58.7 & 57.8 & 50.5 & 59.3 & 77.0 \\
\hline \multicolumn{6}{|c|}{ Night, Onshore } \\
\hline Neutral Night & 23.2 & 25.7 & 19.4 & 13.0 & 31.0 \\
\hline Slightly Stable & 27.1 & 29.9 & 29.0 & 25.1 & 21.3 \\
\hline Stable & 37.1 & 32.1 & 39.4 & 44.2 & 36.5 \\
\hline Very Stable & 12.6 & 12.3 & 12.2 & 17.8 & 11.2 \\
\hline
\end{tabular}


Table 5.14 (continued)

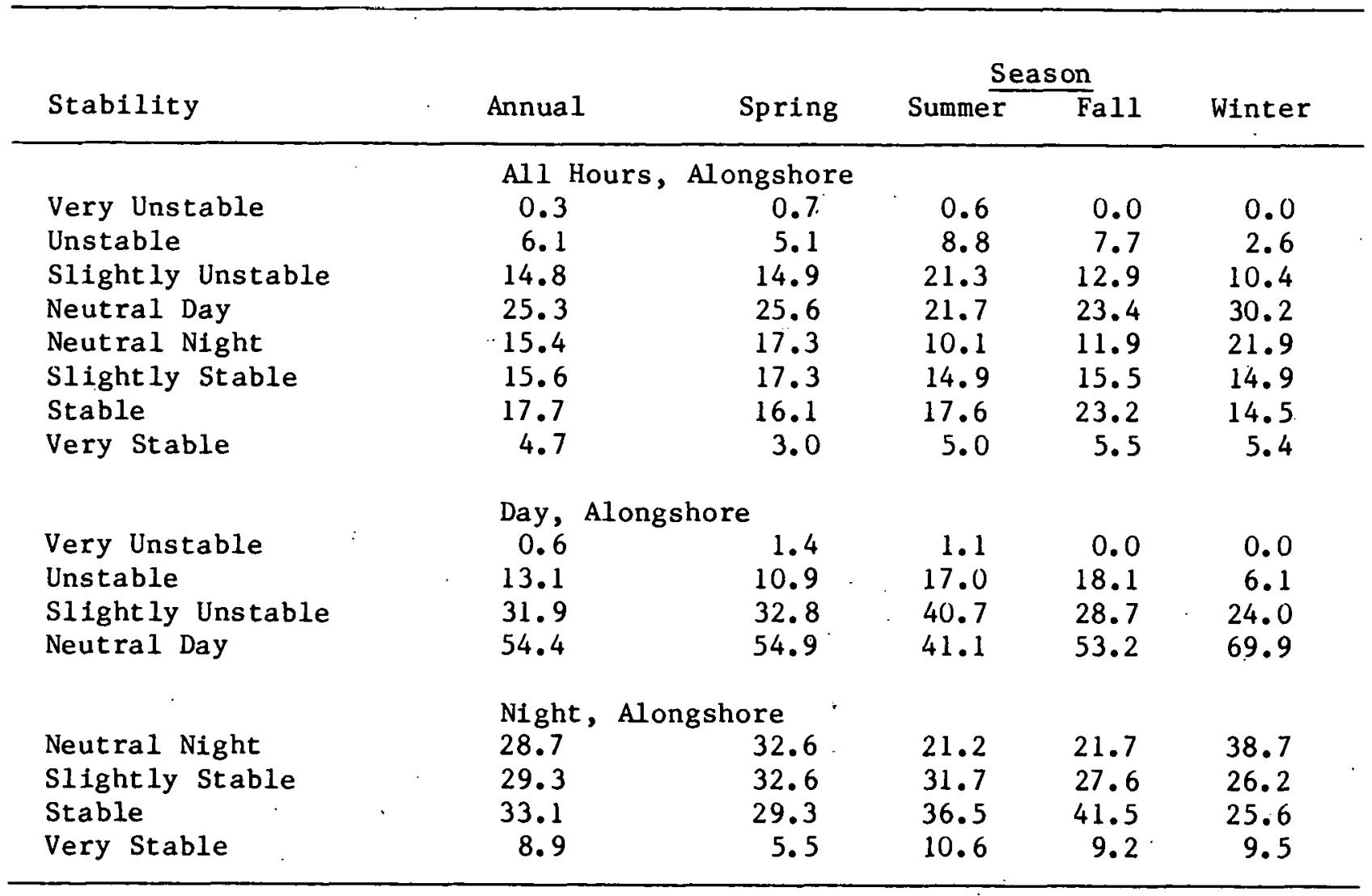


Table 5.14 (continued)

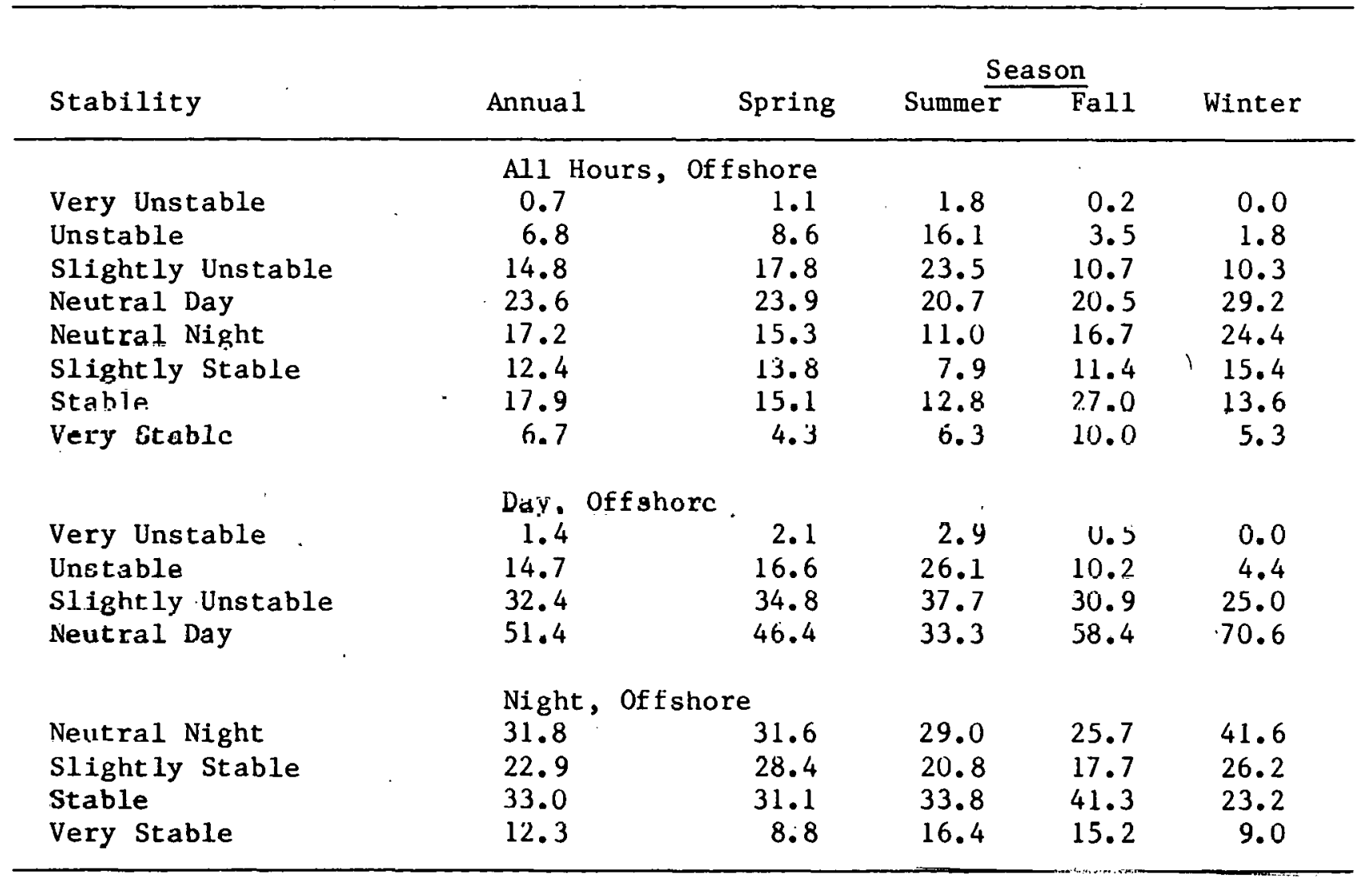


Table 5.15

Brunswick, Georgia

Percent of Hours in Each Stability Class

by Wind Direction, Season and Time of Day

\begin{tabular}{lccrrr} 
& & & \multicolumn{2}{c}{ Season } \\
Stability & Annual & Spring & Sumer & Fall & Winter \\
\hline & All Hours, & All Directions & & \\
Very Unstable & 1.7 & 2.4 & 3.7 & 0.6 & 0.2 \\
Unstable & 11.9 & 13.5 & 19.4 & 8.0 & 6.3 \\
Slightly Unstable & 14.6 & 14.5 & 16.3 & 13.6 & 14.0 \\
Neutral Day & 14.1 & 13.7 & 10.5 & 15.2 & 17.0 \\
Neutral Night & 7.5 & 7.7 & 4.8 & 5.6 & 11.7 \\
Slightly Stable & 10.8 & 10.7 & 9.3 & 10.2 & 13.1 \\
Stable & 10.6 & 12.2 & 9.0 & 12.2 & 9.0 \\
Very Stable & 28.8 & 25.3 & 26.9 & 34.5 & 28.7 \\
& & & & & \\
Very Unstable & Day, All Directions & 5.4 & 7.5 & 1.6 & 0.6 \\
Unstable & 4.1 & 30.7 & 38.9 & 21.4 & 16.7 \\
Slightly Unstable & 28.0 & 32.9 & 32.6 & 36.3 & 37.5 \\
Neutral Day & 34.5 & 31.0 & 21.1 & 40.7 & 45.3 \\
$\quad$ & 33.3 & & & & \\
Neutral Night & Night, All Directions & 13.8 & 9.6 & 9.0 & 18.8 \\
Slightly Stable & 12.9 & 19.2 & 18.6 & 16.4 & 21.0 \\
Stable & 18.8 & 21.7 & 17.9 & 19.5 & 14.3 \\
Very Stable & 18.3 & 45.3 & 53.8 & 55.2 & 45.9 \\
\hline
\end{tabular}


Table 5.15 (continued)

\begin{tabular}{|c|c|c|c|c|c|}
\hline \multirow[b]{2}{*}{ Stability } & \multirow[b]{2}{*}{ Annual } & \multirow[b]{2}{*}{ Spring } & \multicolumn{2}{|c|}{ Season } & \multirow[b]{2}{*}{ Winter } \\
\hline & & & Summer & Fall & \\
\hline \multicolumn{6}{|c|}{ All Hours, Onshore } \\
\hline Very Unstable & 1.7 & 2.8 & 3.2 & 0.3 & 0.0 \\
\hline Unstable & 13.8 & 15.5 & 21.3 & 8.4 & 8.6 \\
\hline Slightly Unstable & 22.3 & 20.7 & 22.3 & 22.7 & 24.0 \\
\hline Neutral Day & 20.2 & 16.2 & 15.1 & 28.7 & 21.9 \\
\hline Neutral Night & 7.8 & 6.5 & 4.7 & 5.0 & 17.9 \\
\hline Slightly Stable & 9.5 & $1 U .1$ & 10.4 & 8.2 & 9.0 \\
\hline stable & 11.3 & 12.9 & 11.9 & 12.5 & h. .3 \\
\hline Very Stable & 13.5 & 15.3 & 11.1 & 14.3 & 12.3 \\
\hline \multicolumn{6}{|c|}{ Day, Onohore } \\
\hline Very Unstable & 3.0 & 5.0 & 5.2 & 0.4 & 0.0 \\
\hline Unstable & 23.8 & 28.1 & 34.1 & 14.1 & 15.2 \\
\hline Slightly Unstable & 38.4 & 37.5 & 36.3 & 37.8 & 44.7 \\
\hline Neutral Day & 34.8 & 29.4 & 24.4 & 47.7 & 40.1 \\
\hline \multicolumn{6}{|c|}{ Night, Unshore } \\
\hline Neutral Night & 18.5 & 14.5 & 12.5 & 12.5 & 40.2 \\
\hline Slightly Stable & 22.7 & 22.6 & 26.8 & 20.6 & 19.3 \\
\hline Stable & 26.8 & 28.7 & 30.9 & .31 .2 & 13.8 \\
\hline Very Stable & 32.0 & 34.1 & 29.9 & 35.7 & 26.7 \\
\hline
\end{tabular}


Table $5.15^{\prime}$ (continued)

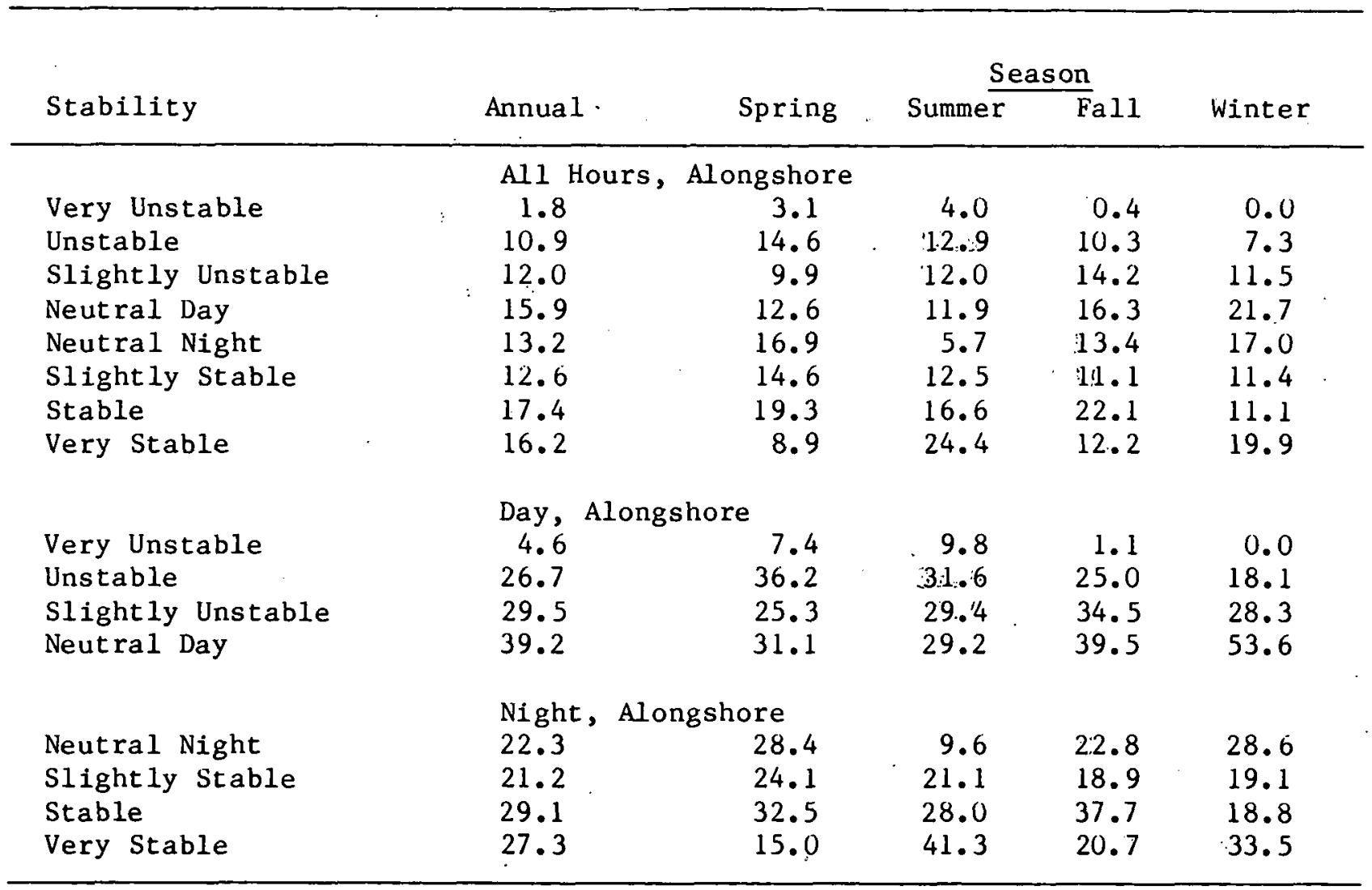


Table 5.15 (continued)

\begin{tabular}{|c|c|c|c|c|c|}
\hline \multirow[b]{2}{*}{ Stability } & \multirow[b]{2}{*}{ Annual } & \multicolumn{4}{|c|}{ Season } \\
\hline & & Spring & Summe $\bar{r}$ & $\overline{F a}$ all & Winter \\
\hline $\begin{array}{l}\text { Very Unstable } \\
\text { Unstable } \\
\text { Slightly Unstable } \\
\text { Neutral Day } \\
\text { Neutral Night } \\
\text { Slightly Stable } \\
\text { Stable } \\
\text { Very SLable }\end{array}$ & $\begin{array}{l}\text { A11 H } \\
1.6 \\
15.2 \\
16.8 \\
17.5 \\
8.0 \\
11.0 \\
13.0 \\
17.1\end{array}$ & $\begin{array}{r}\text { Hours, offshore } \\
1.7 \\
13.8 \\
16.6 \\
19.0 \\
8.3 \\
10.5 \\
14.3 \\
15.9\end{array}$ & $\begin{array}{r}4.3 \\
28.4 \\
18.7 \\
12.4 \\
6.0 \\
6.6 \\
8.7 \\
14.9\end{array}$ & $\begin{array}{r}0.5 \\
14.0 \\
15.6 \\
14.7 \\
5.4 \\
11.1 \\
15.3 \\
23.3\end{array}$ & $\begin{array}{l}0.0 \\
6.8 \\
16.7 \\
21.7 \\
11.5 \\
14.4 \\
12.9 \\
15.9\end{array}$ \\
\hline $\begin{array}{l}\text { Very Unstable } \\
\text { Unstable } \\
\text { Slight ly Unstable } \\
\text { Neutral Day }\end{array}$ & $\begin{array}{r}\text { Day, } \\
3.1 \\
29.7 \\
33.0 \\
34.2\end{array}$ & $\begin{array}{l}\text { off shore } \\
\qquad \begin{array}{r}3.4 \\
27.0 \\
32.3 \\
37.3\end{array}\end{array}$ & $\begin{array}{l}6.8 \\
44.7 \\
29.3 \\
19.1\end{array}$ & $\begin{array}{r}1.2 \\
30.9 \\
35.8 \\
32.2\end{array}$ & $\begin{array}{l}0.11 \\
15.2 \\
36.7 \\
48.1\end{array}$ \\
\hline $\begin{array}{l}\text { Neutral Night } \\
\text { Slightly Stable } \\
\text { Stable } \\
\text { Very Stable }\end{array}$ & $\begin{array}{l}\text { Night } \\
16.3 \\
22.5 \\
26.5 \\
34.7\end{array}$ & $\begin{array}{l}\text {, uff shore } \\
\qquad \begin{array}{r}17.0 \\
21.3 \\
29.3 \\
32.4\end{array}\end{array}$ & $\begin{array}{l}16.8 \\
18.8 \\
24.3 \\
40.1\end{array}$ & $\begin{array}{r}9.8 \\
20.2 \\
27.9 \\
42.1\end{array}$ & $\begin{array}{l}21.2 \\
26.4 \\
23.4 \\
29.0\end{array}$ \\
\hline
\end{tabular}


Table 5.16

Cape Kennedy, Florida

Percent of Hours in Each Stability Class

by Wind Direction, Season and Time of Day

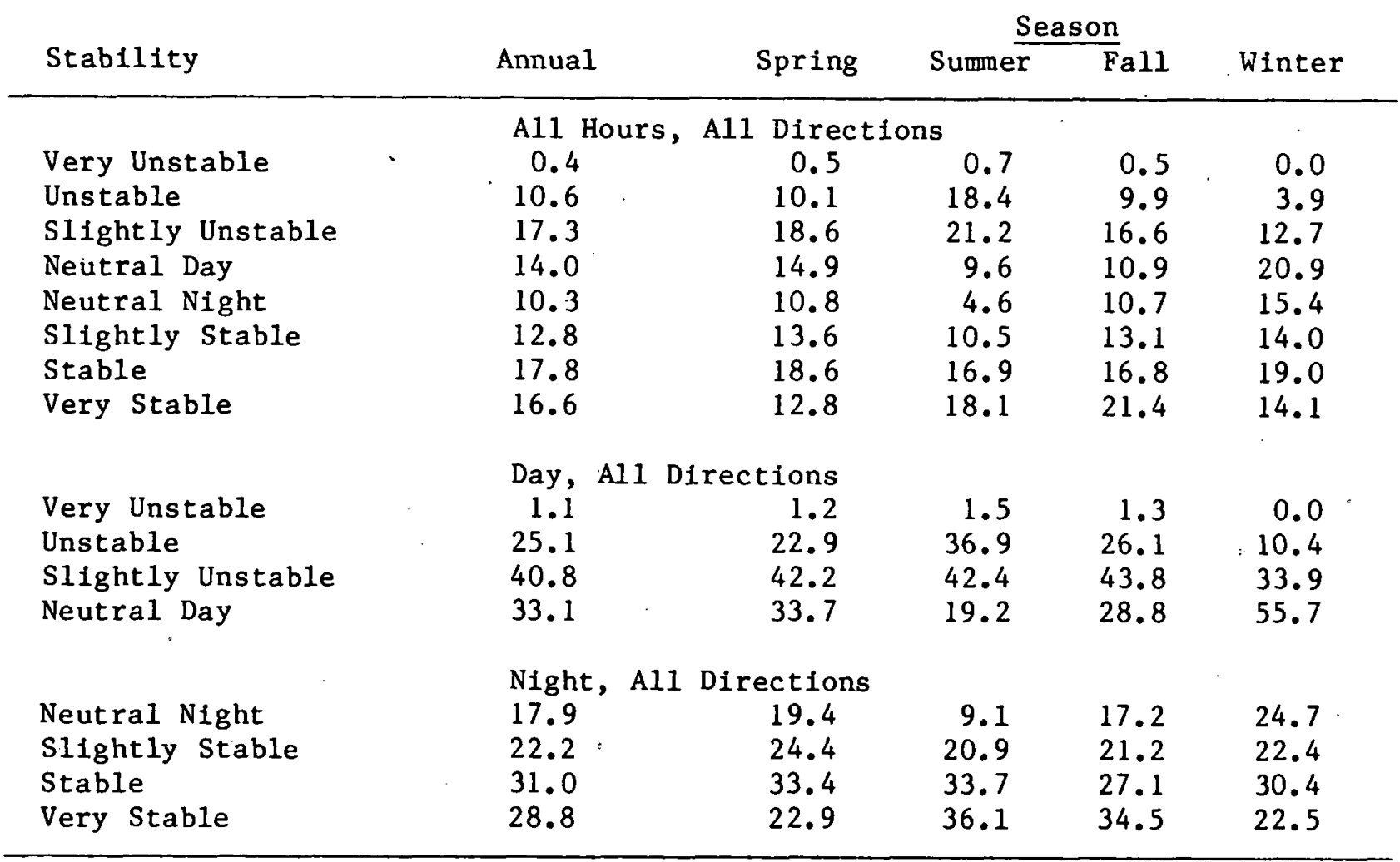


Table 5.16 (continued)

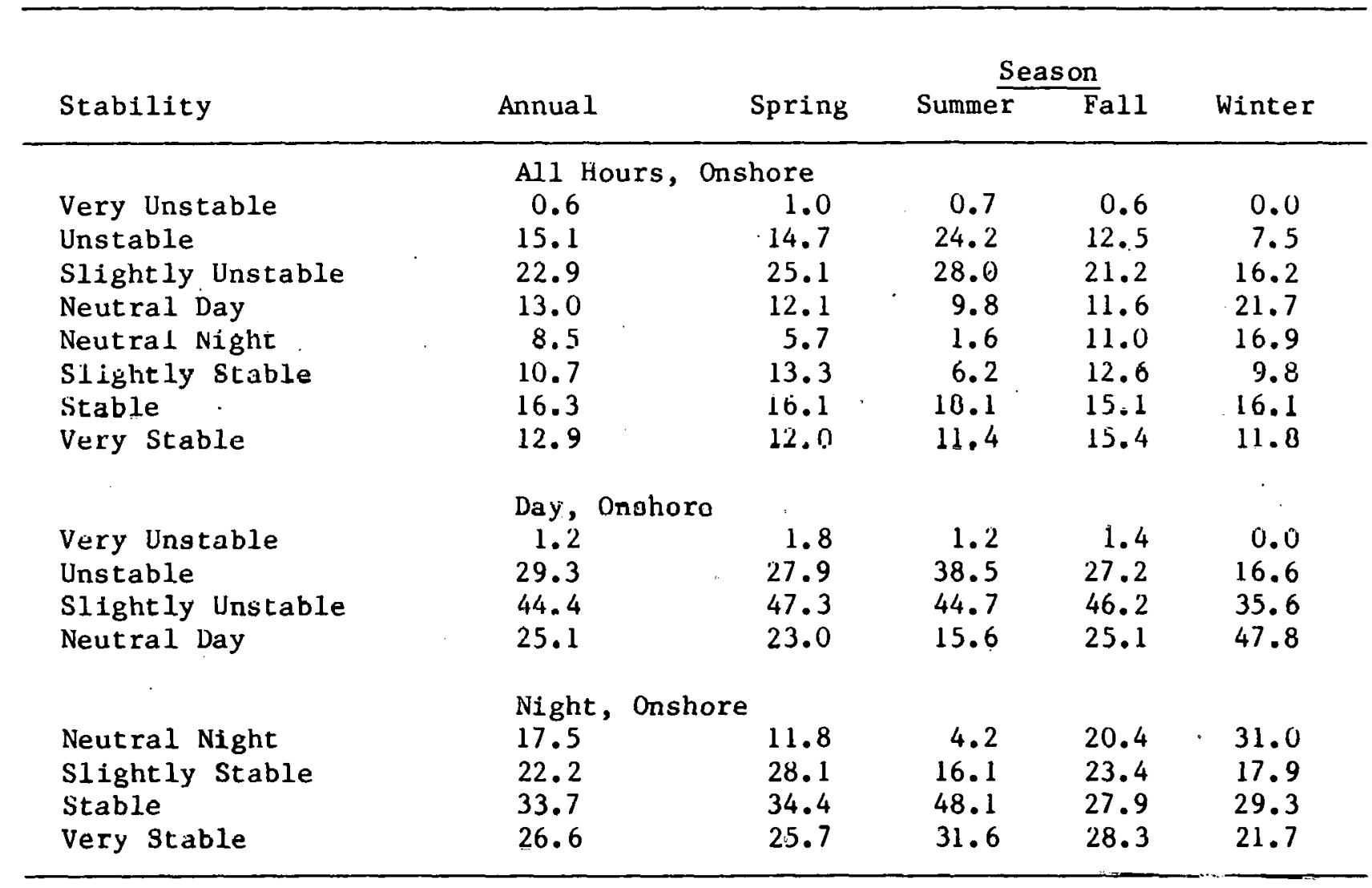


Table 5.16 (continued)

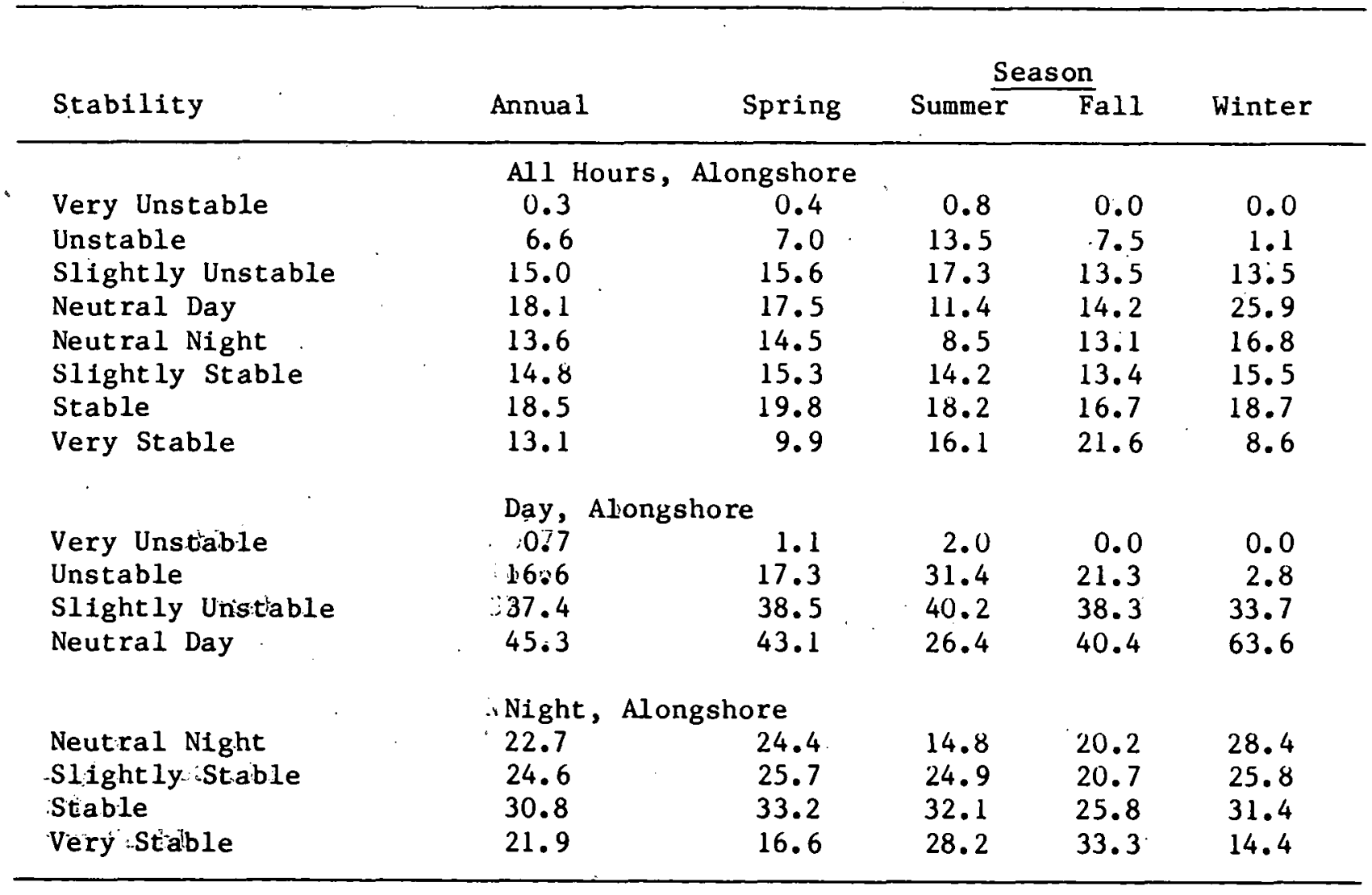


Table 5.16 (continued)

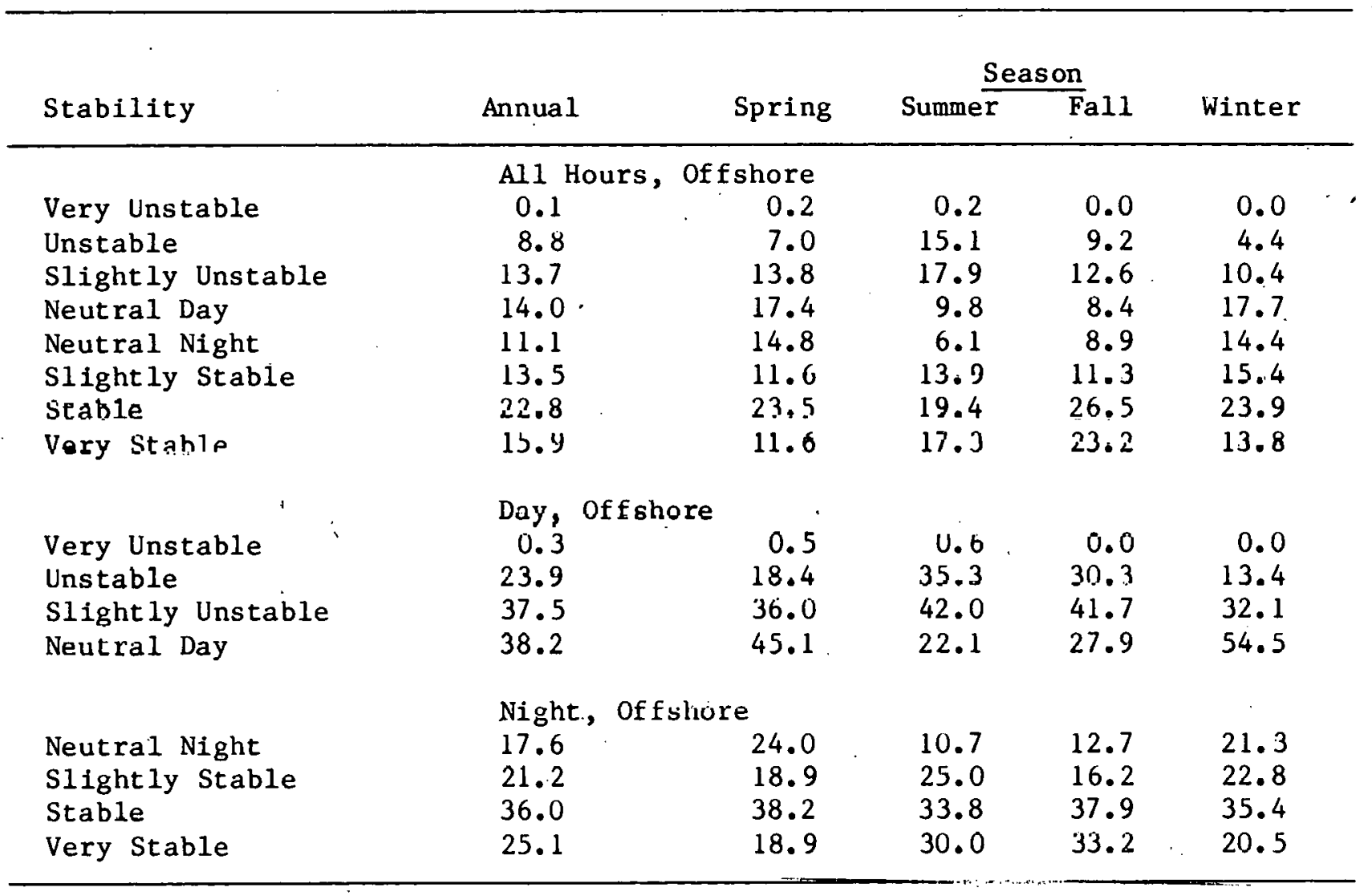




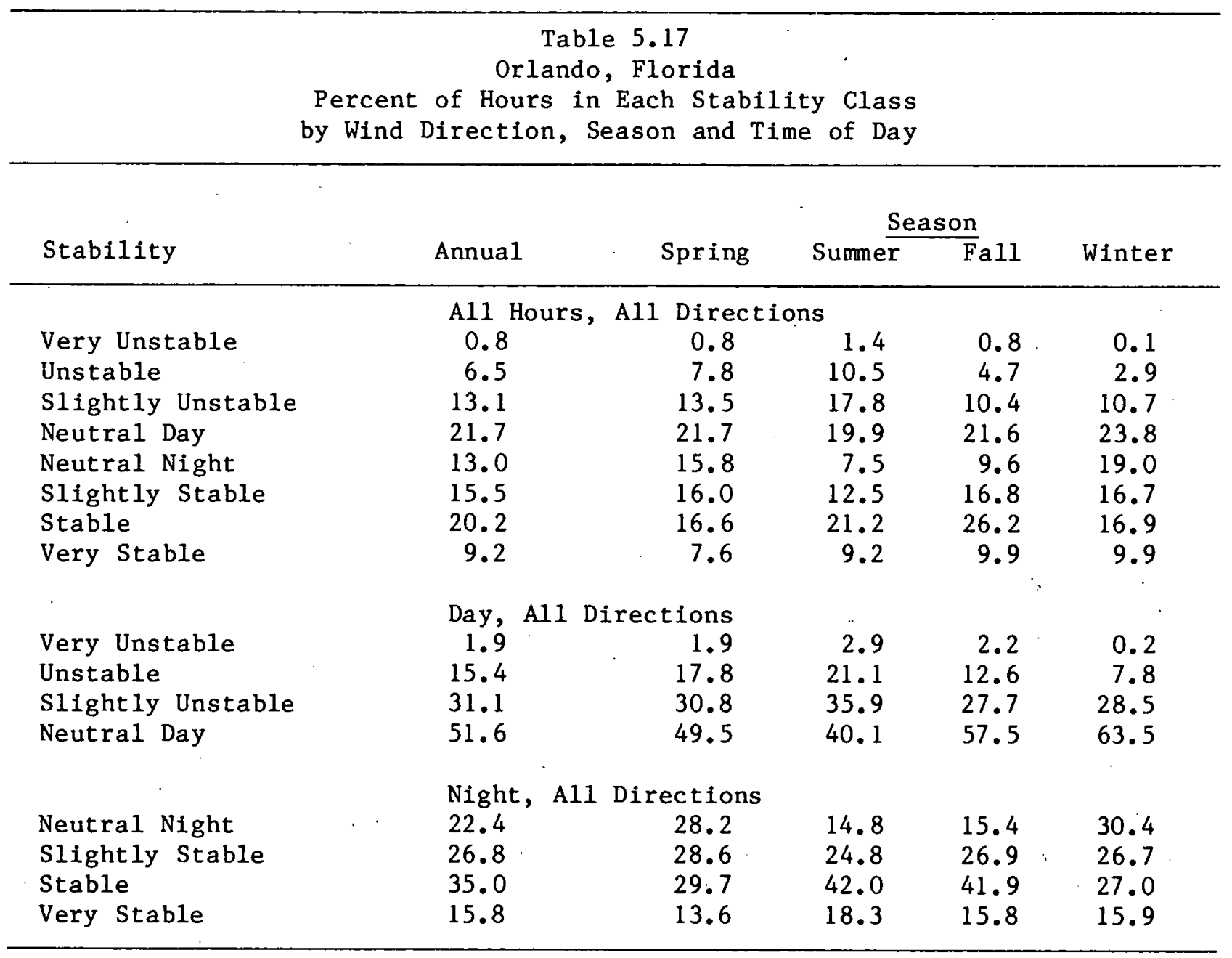


Table 5.17 ((continued))

\begin{tabular}{|c|c|c|c|c|c|c|}
\hline \multirow[b]{2}{*}{ Stability } & & \multirow[b]{2}{*}{ Annual } & \multicolumn{4}{|c|}{ Season: } \\
\hline & & & Spring & Summe $\bar{r}$ & Fäll: & Winter \\
\hline \multicolumn{7}{|c|}{ All Hours, Onshore } \\
\hline Very Unstable & - & 0.7 & 0.7 & 1.2 & 0.7 & 0.0 \\
\hline Unstable & & 5.5 & 8.4 & 7.6 & 3.6 & 3.5 \\
\hline S1ight1y Unstable & & 12.6 & 15.0 & 13.7 & 10.5 & $12: 7$ \\
\hline Neutral Day & & 25.2 & 21.9 & 27.1 & 26.7 & 24.7 \\
\hline Neutral Night & & 12.0 & 15.6 & 8.8 & 7.1 & 18.6 \\
\hline slightly Stable & & 15.8 & 17.7 & 12.3 & 16.3 & 16.1 \\
\hline Stable & & 25.5 & 18.3 & 25.8 & 32.6 & 21.0 \\
\hline Very Stable & & 2.7 & 2.3 & 3.4 & 2.6 & 2.8 \\
\hline \multicolumn{7}{|c|}{ Day, Onohore } \\
\hline Very Unstable & & 1.5 & 1.4 & 2.4 & 1.7 & 0.0 \\
\hline Unstable & & 12.4 & 18.2 & 15.4 & 8.7 & 8.5 \\
\hline Slightly Unstable & & 28.8 & 32.6 & 28.0 & 25.3 & 31.2 \\
\hline Neutral Day & & 57.3 & 47.8 & 54.1 & 64.3 & 60.3 \\
\hline \multicolumn{7}{|c|}{ Night, Onshore } \\
\hline Neutral Night & & 21.4 & 28.8 & 17.6 & 12.1 & 31.5 \\
\hline Slightly. Stable & & 28.3 & 32.8 & 24.5 & 27.8 & 28.3 \\
\hline Stable & & 45.6 & 34.2 & 51.0 & 55.7 & 35.4 \\
\hline Very Stahle & & 4.7 & 4.2 & 6.9 & 4.4 & 4.9 \\
\hline
\end{tabular}


Table 5.17 (continued)

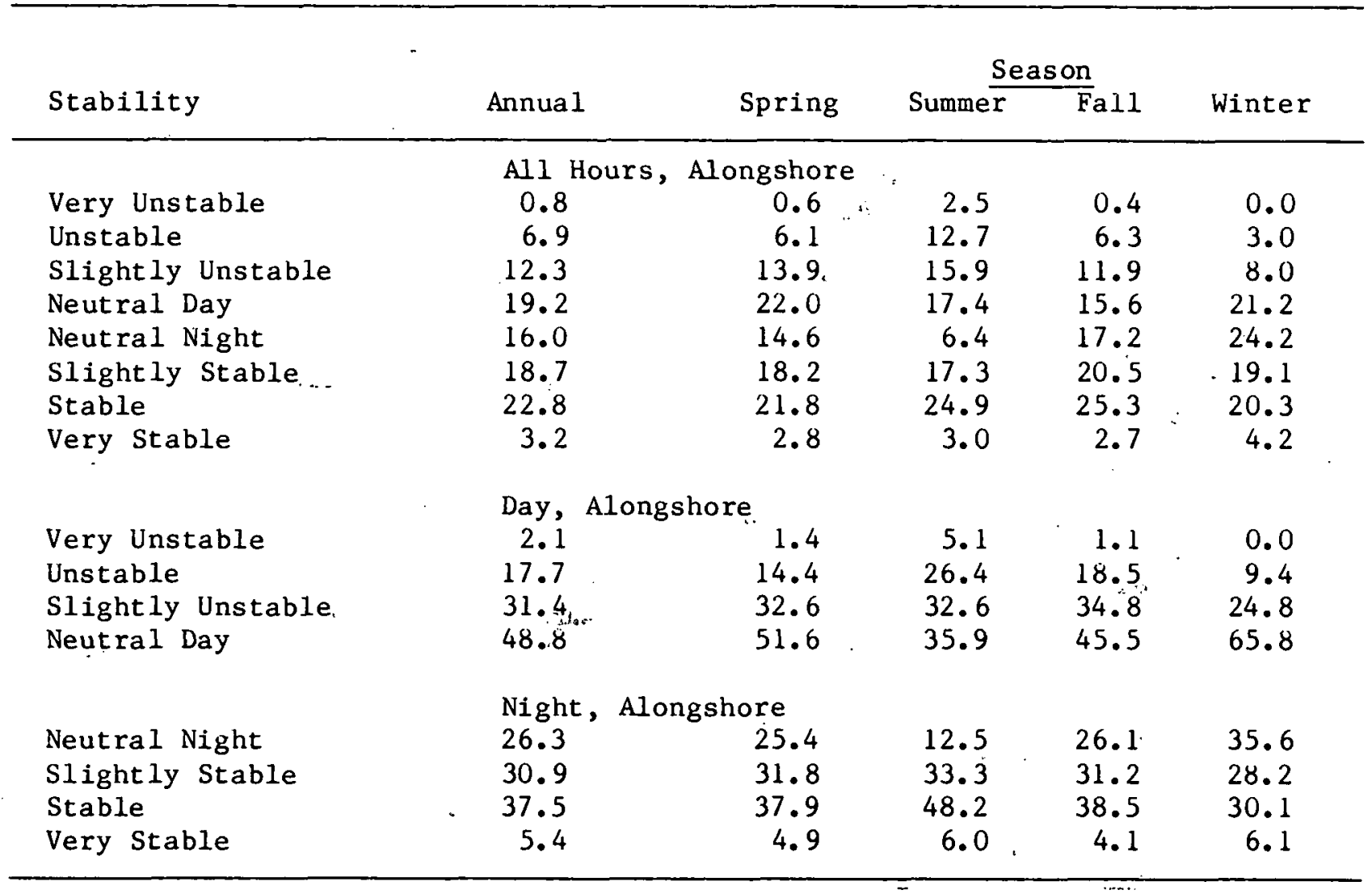


Table 5.17 (continued)

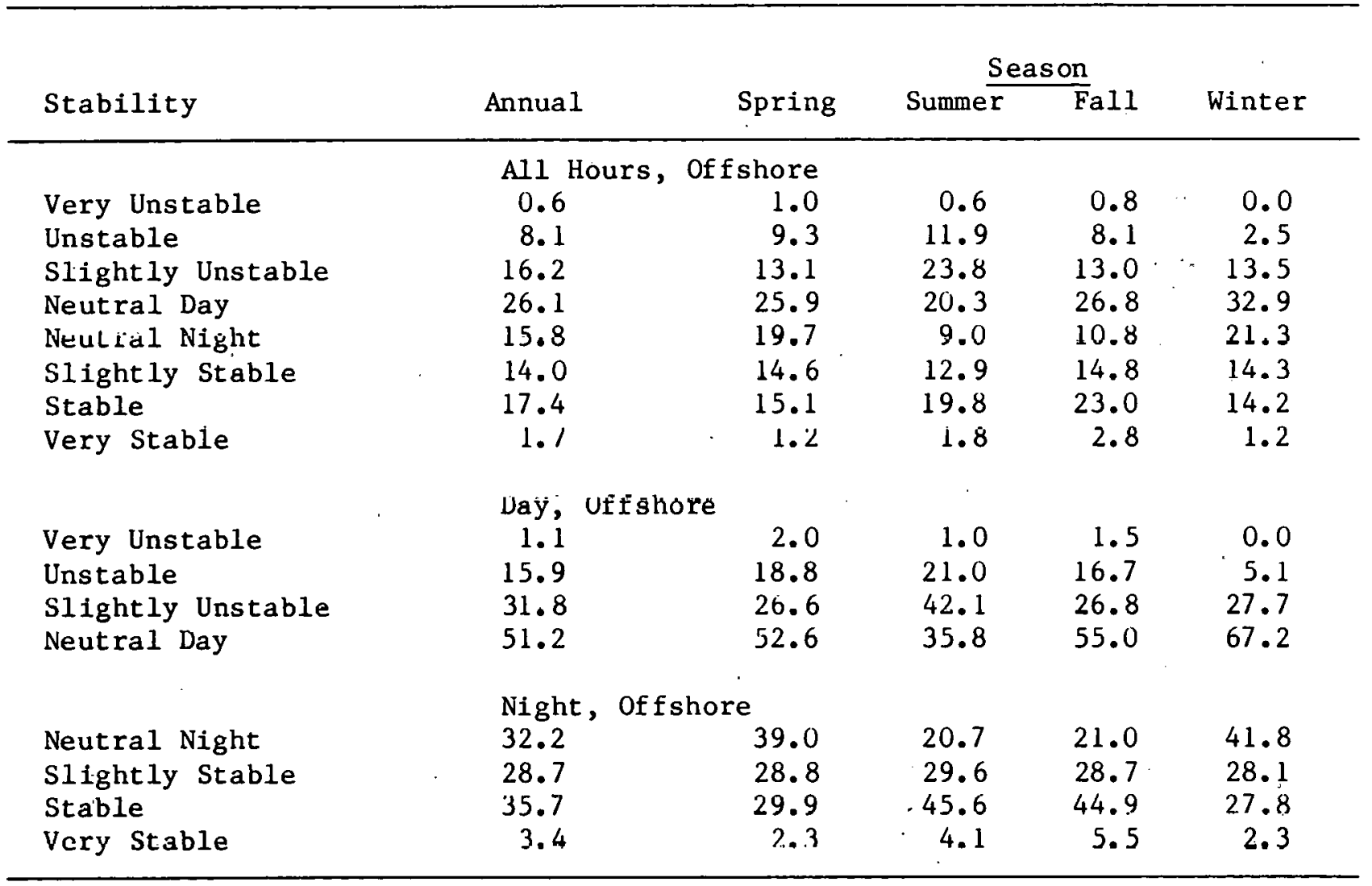




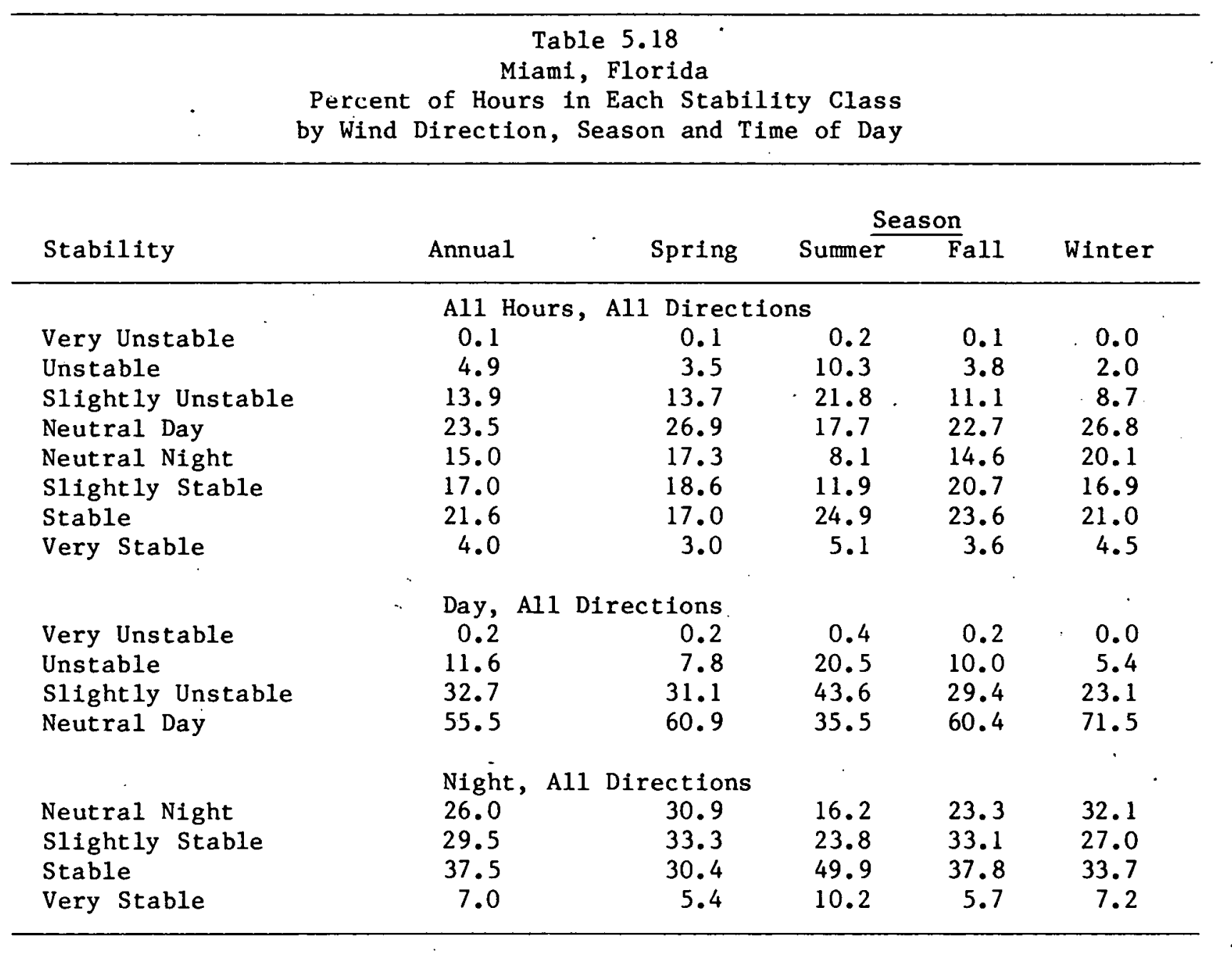


Table 5.18 (continued)

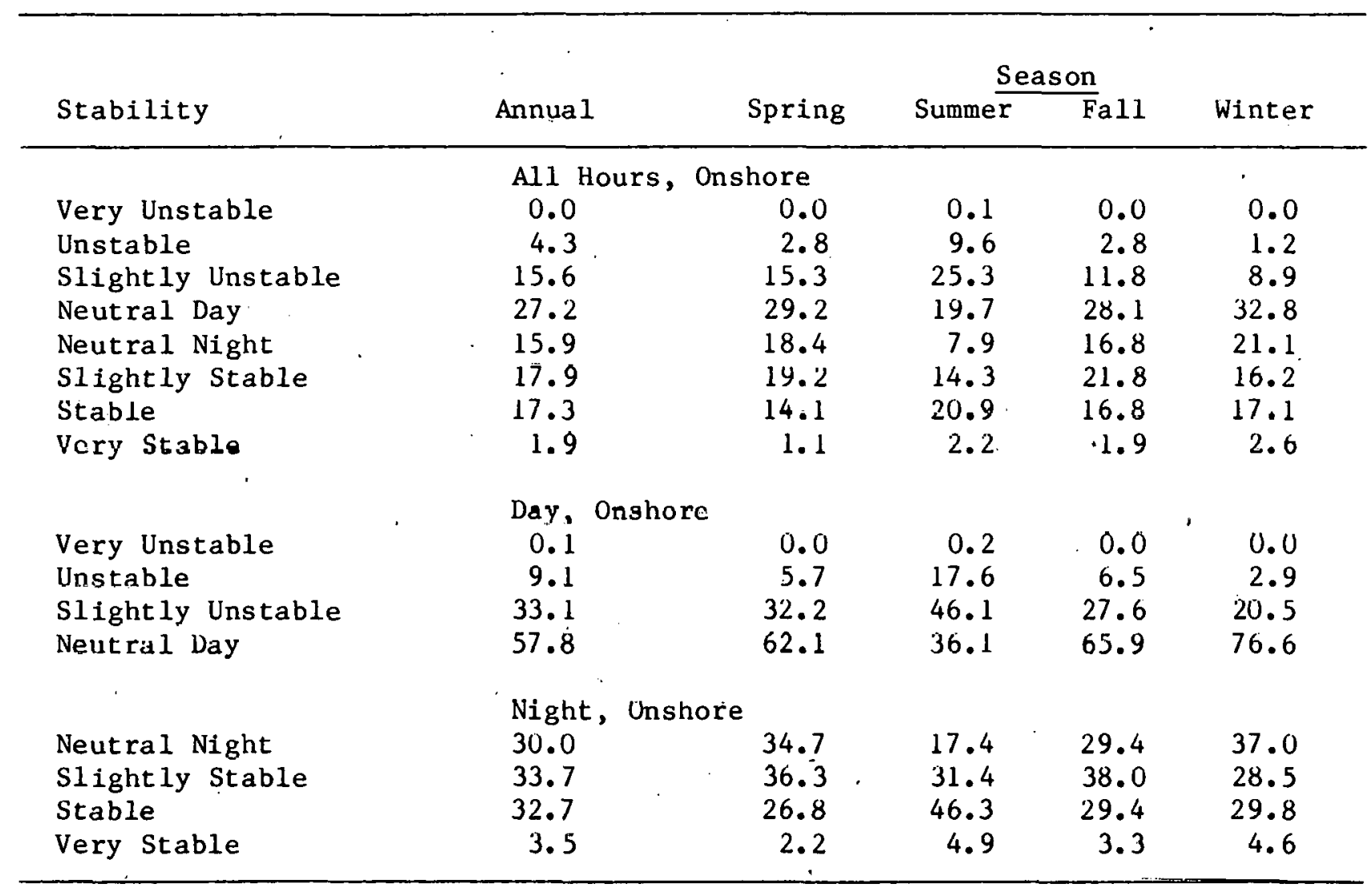


Table 5.18 (continued)

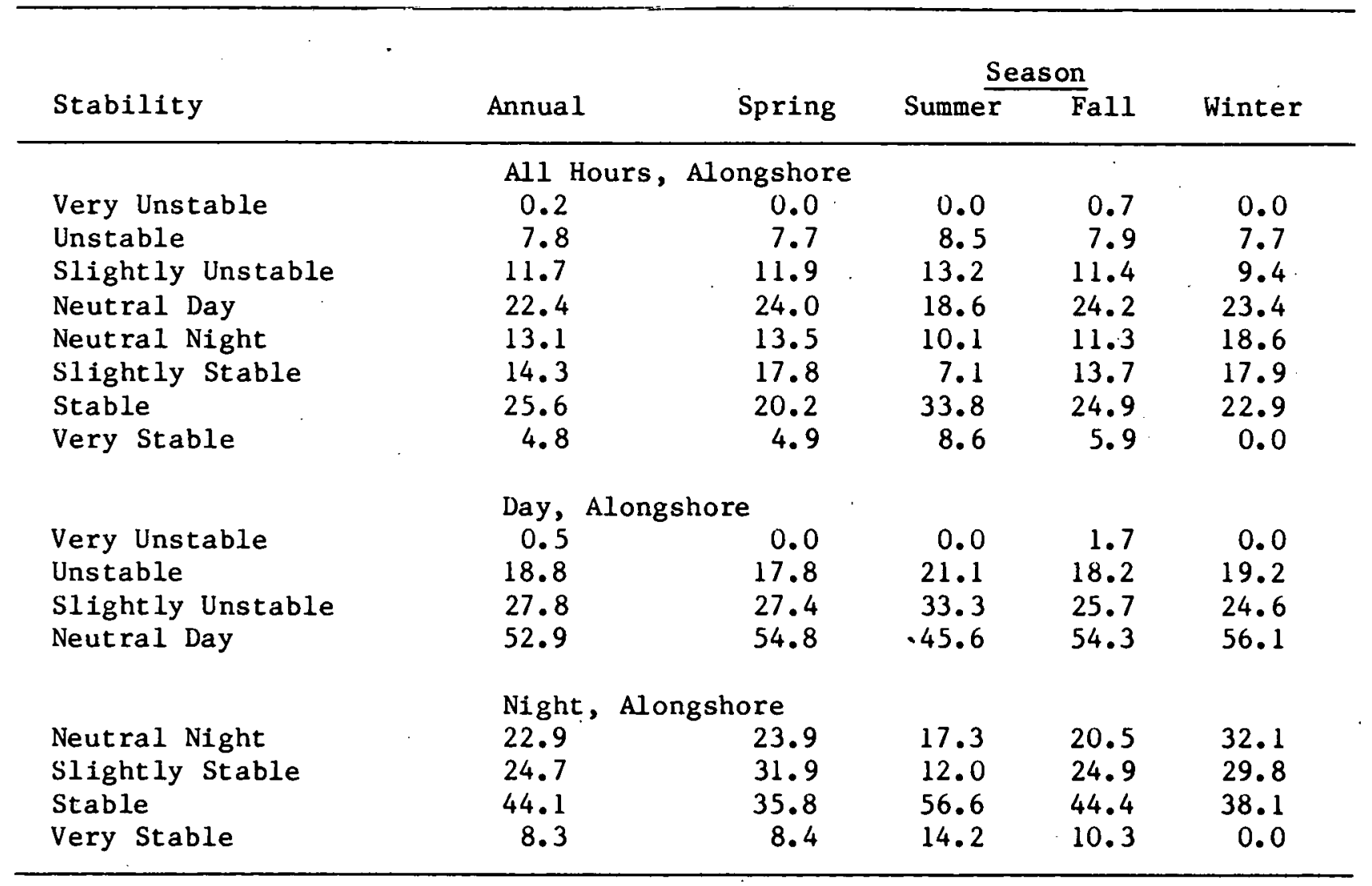


Table 5.18 (continued)

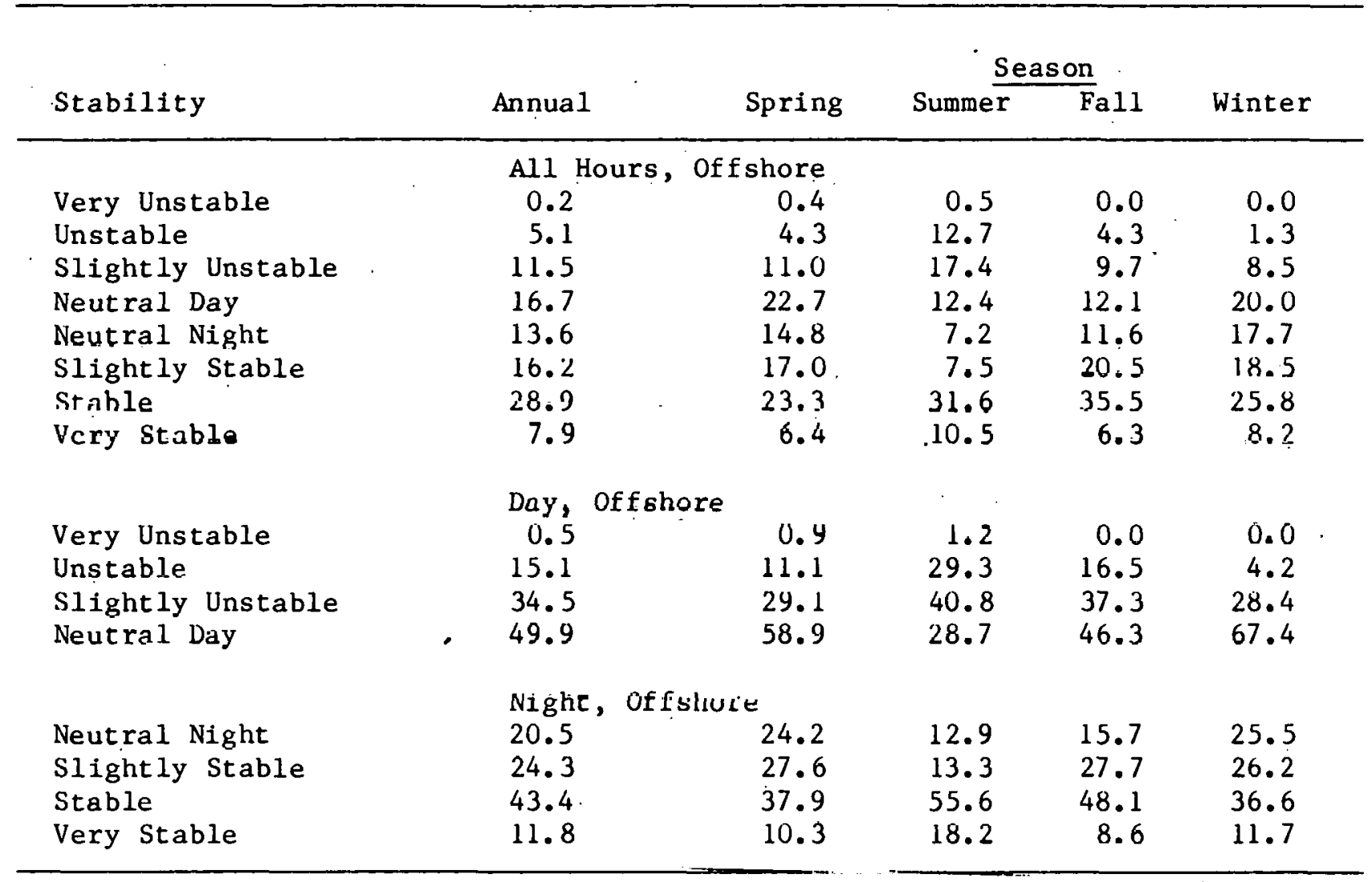


Table 5.19

Fort Myers, Florida

Percent of Hours in Each Stability Class.

by Wind Direction, Season and Time of Day

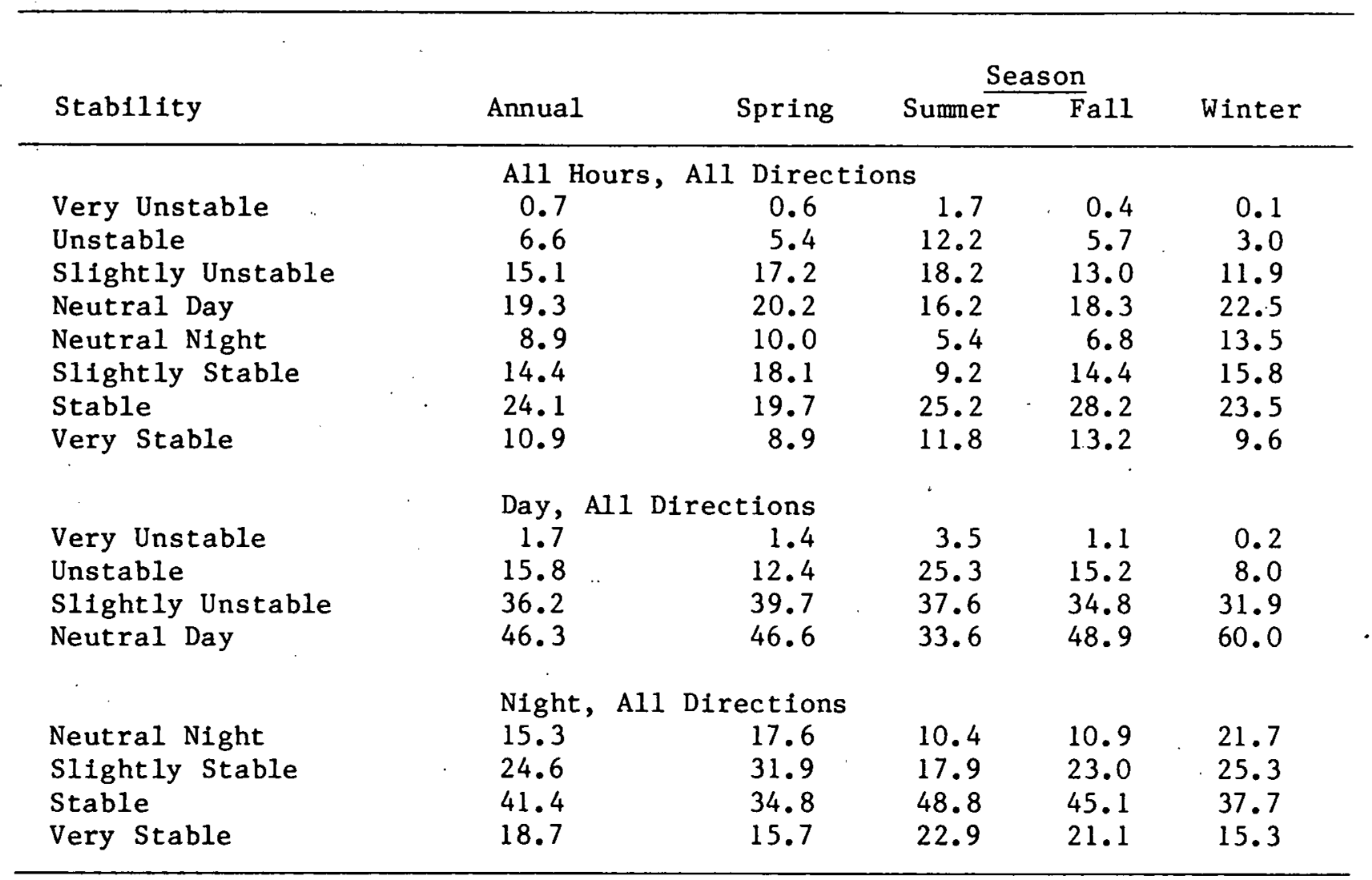


Table 5.19 (continued)

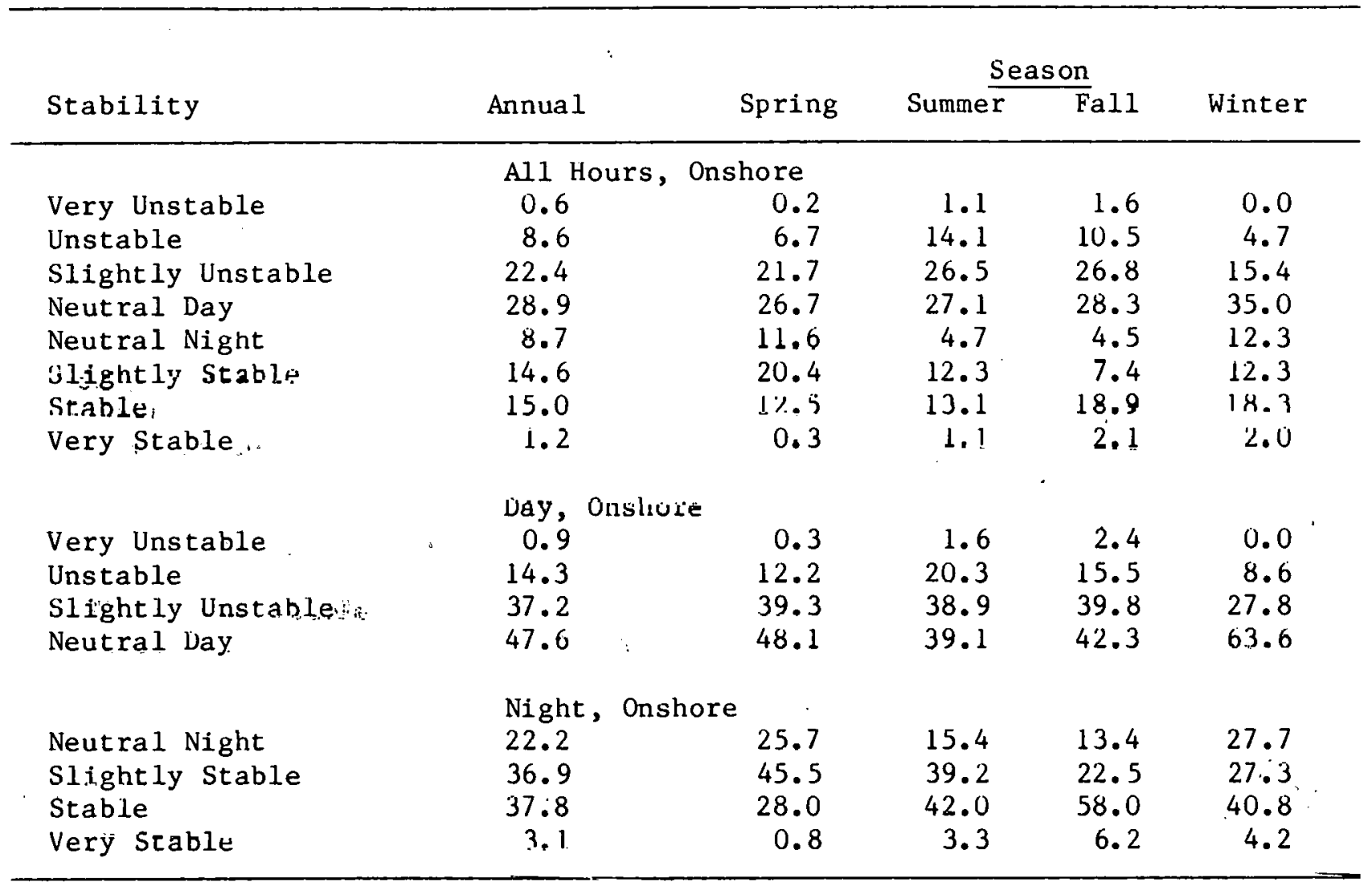


Table 5.19 (continued)

\begin{tabular}{|c|c|c|c|c|c|}
\hline \multirow[b]{2}{*}{ Stability } & \multirow[b]{2}{*}{ Annual } & \multicolumn{4}{|c|}{ Season } \\
\hline & & Spring & Summer & Fall & Winter \\
\hline \multicolumn{6}{|c|}{ All Hours, Alongshore } \\
\hline Very Unstable & 0.9 & 0.8 & 2.8 & 0.0 & 0.0 \\
\hline Unstable & 6.7 & 5.4 & 10.8 & 7.8 & 3.2 \\
\hline Slightly Unstablee & 14.4 & 13.8 & 17.3 & 15.4 & 11.7 \\
\hline Neutral Day & 21.2 & 17.4 & 18.0 & 24.8 & 26.2 \\
\hline Neutral Night.t & 14.2 & 15.5 & 10.0. & 7.2 & 20.2 \\
\hline Slightly stable & 14.9 & 20.4 & 6.6 & 14.4 & 17.3 \\
\hline Stable & 24.8 & 24.5 & 30.2 & 26.4. & 19.6 \\
\hline Very Stable & 2.9 & 2.2 & 4.4 & 4.0 & 1.7 \\
\hline \multicolumn{6}{|c|}{ Day, Alongshore } \\
\hline Very, Uñstablè & 2.2 & 2.1 & 5.7 & 0.0 & 0.0 .0 \\
\hline Unstable & 15.4 & 14.6 & 22.0 & 16.3 & 8.2 \\
\hline Slightly Unstable & 33.2 & 37.1 & 35.5 & 31.0 & 28.2 \\
\hline Neutral Day. & 49.2 & 46.3 & 36.8 & 52.7 & 63.6 \\
\hline \multicolumn{6}{|c|}{ Night, Alongshore } \\
\hline Neutralinight & 25.0 & 24.9 & 19.6 & 14.1 & 33.8 \\
\hline Slightly Stable & 26.2 & 32.6 & 12.9 & 28.0 & 29.7. \\
\hline Stable & 43.7 & 39.1 & 59.0 & 50.5 & 33.6 \\
\hline Very Stable & 5.1 & 3.5 & 8.5 & 7.5 & 2.8 \\
\hline
\end{tabular}


Table 5.19 (continued)

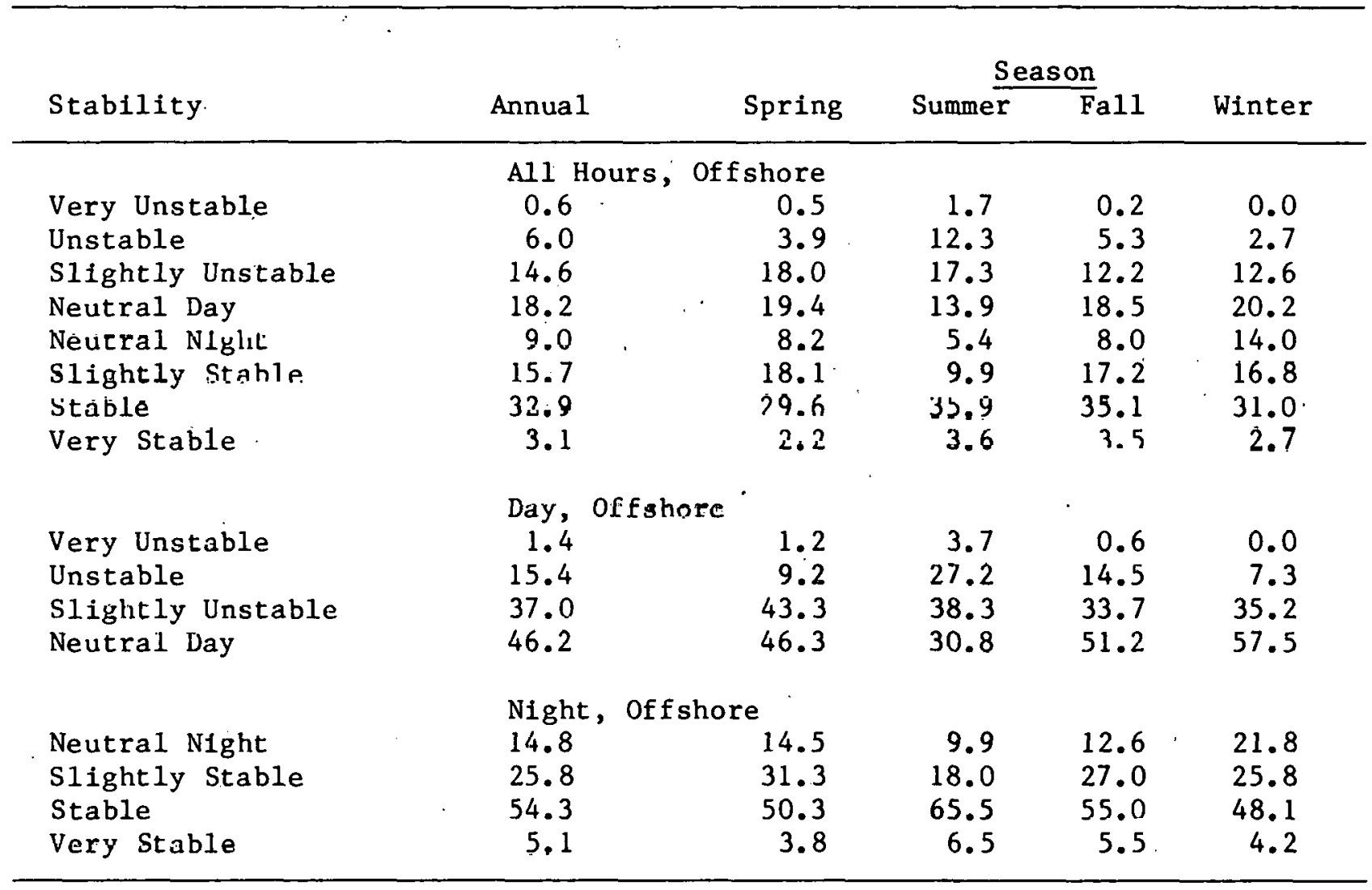




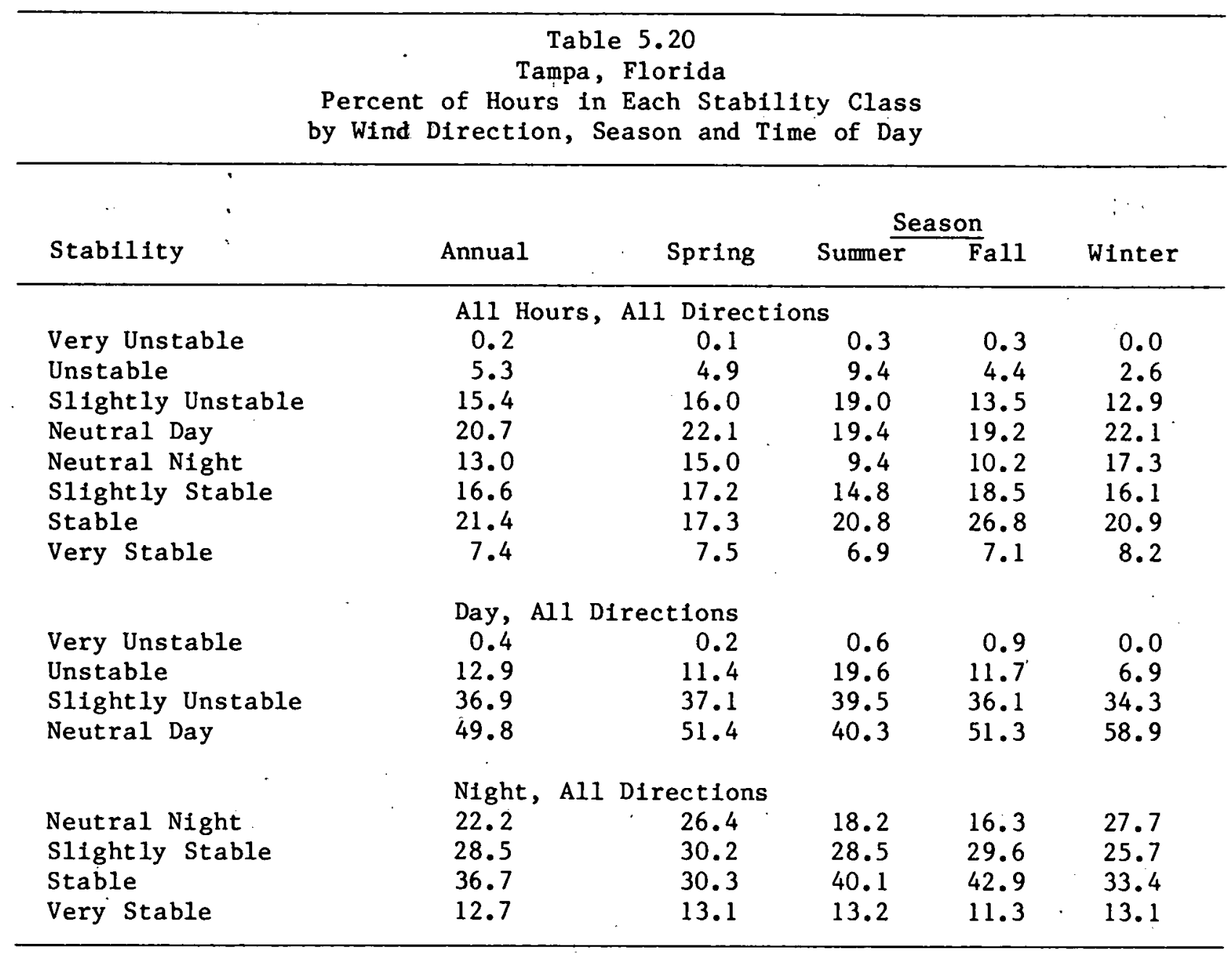


Table, 5.20 (continued)

\begin{tabular}{|c|c|c|c|c|c|}
\hline \multirow[b]{2}{*}{ Stability } & \multirow[b]{2}{*}{$\therefore$ Annual } & \multirow[b]{2}{*}{ Spring } & \multicolumn{2}{|c|}{ Seatso on } & \multirow[b]{2}{*}{ Winter } \\
\hline & & & Summer $\bar{r}$ & in & \\
\hline \multicolumn{6}{|c|}{ Al1 Hours, Onshore } \\
\hline Very Unstable & 0.2 & 0.0 & 0.4 & 0.5 & 0.0 \\
\hline Unstable & 7.9 & 6.3 & 11.6 & 10.2 & 4.6 \\
\hline Slightly Unstable & 23.8 & 22.1 & 26.1 & 27.2 & 21.1 \\
\hline Neutral Day & 27.1 & 25.2 & 26.7 & 30.2 & 28.7 \\
\hline Ncutral Night & 12.0 & 16.0 & 8.1 & 6.8 & 14.5 . \\
\hline Slight ly Stable & 13.2 & 14.7 & 16.1 & 7.3 & 10.1 \\
\hline Stable & 13.1 & 13.7 & 10.2 & 13.1 & 16.1 \\
\hline Very staable & 2.8 & 2.1 & $1 . n$ & 4.6 & 4.8 \\
\hline \multicolumn{6}{|c|}{ Day, Onehore } \\
\hline Very Unstable & 0.3 & 0.0 & 0.6 & 0.7 & 0.0 \\
\hline Unstable & .13 .5 & 11.9 & 17.9 & 15.1 & 8.4 \\
\hline Slightly Unstable & $=40.3$ & 41.3 & 40.3 & 39.8 & 39.1 \\
\hline \multirow[t]{2}{*}{ Neutral Day } & $\therefore \quad 45.9$ & 46.8 & 41.2 & 44.3 & 52.5 \\
\hline & \multicolumn{5}{|l|}{ (2) } \\
\hline Neutral Night & 29.4 & 34.5 & 23.0 &. .21 .3 & 32.0 \\
\hline Slightly Stable & 32.1 & 31.7 & 45.3 & 23.2 & 22.4 \\
\hline Stable & 31.8 & 29.3 & 29.0 & 41.5 & 35.1 \\
\hline Very Stable & 6.7 & 4.6 & 2.8 & 13.9 & 10.6 \\
\hline
\end{tabular}


Table 5.20 (continued)

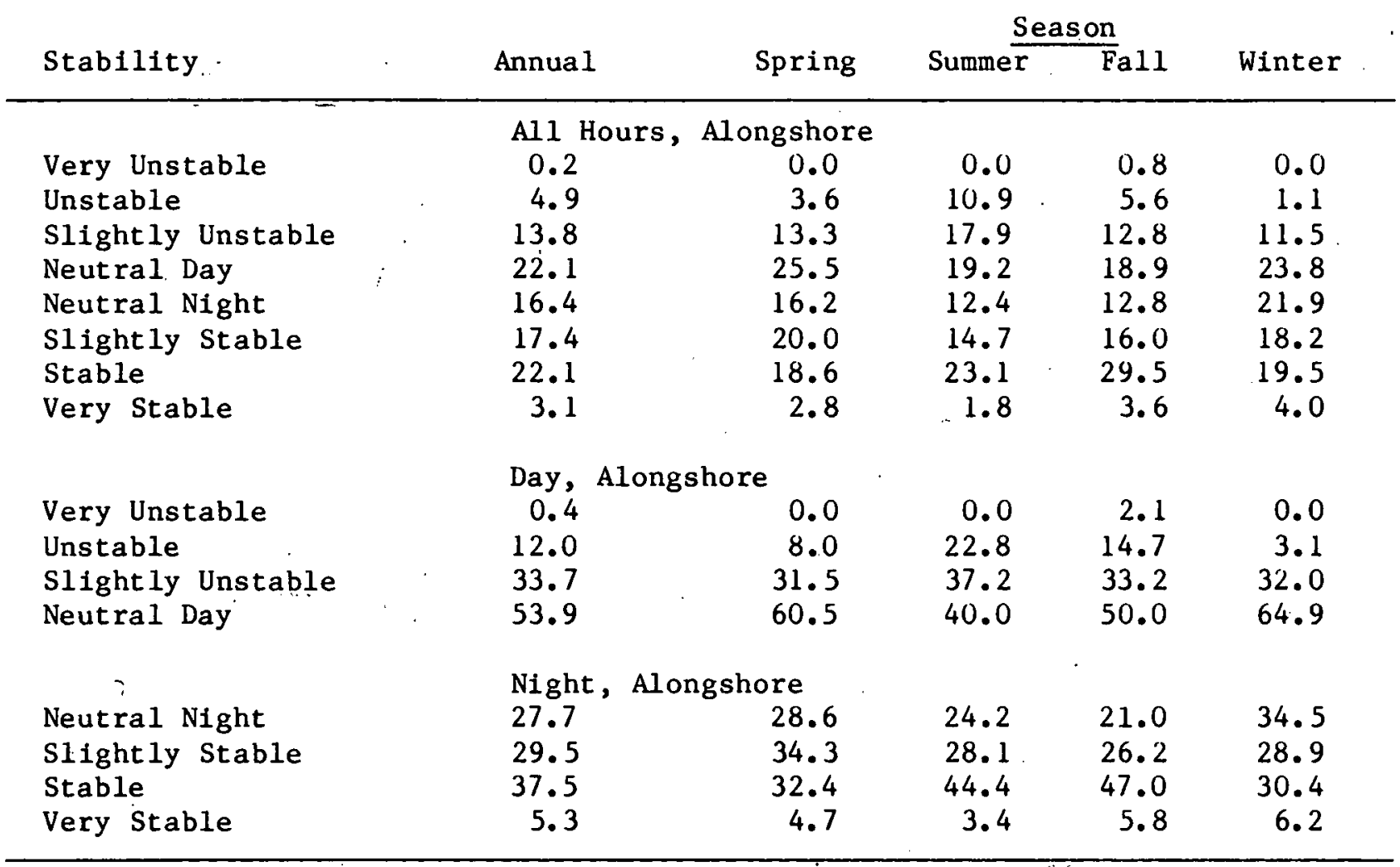


Table 5.20 (continued)

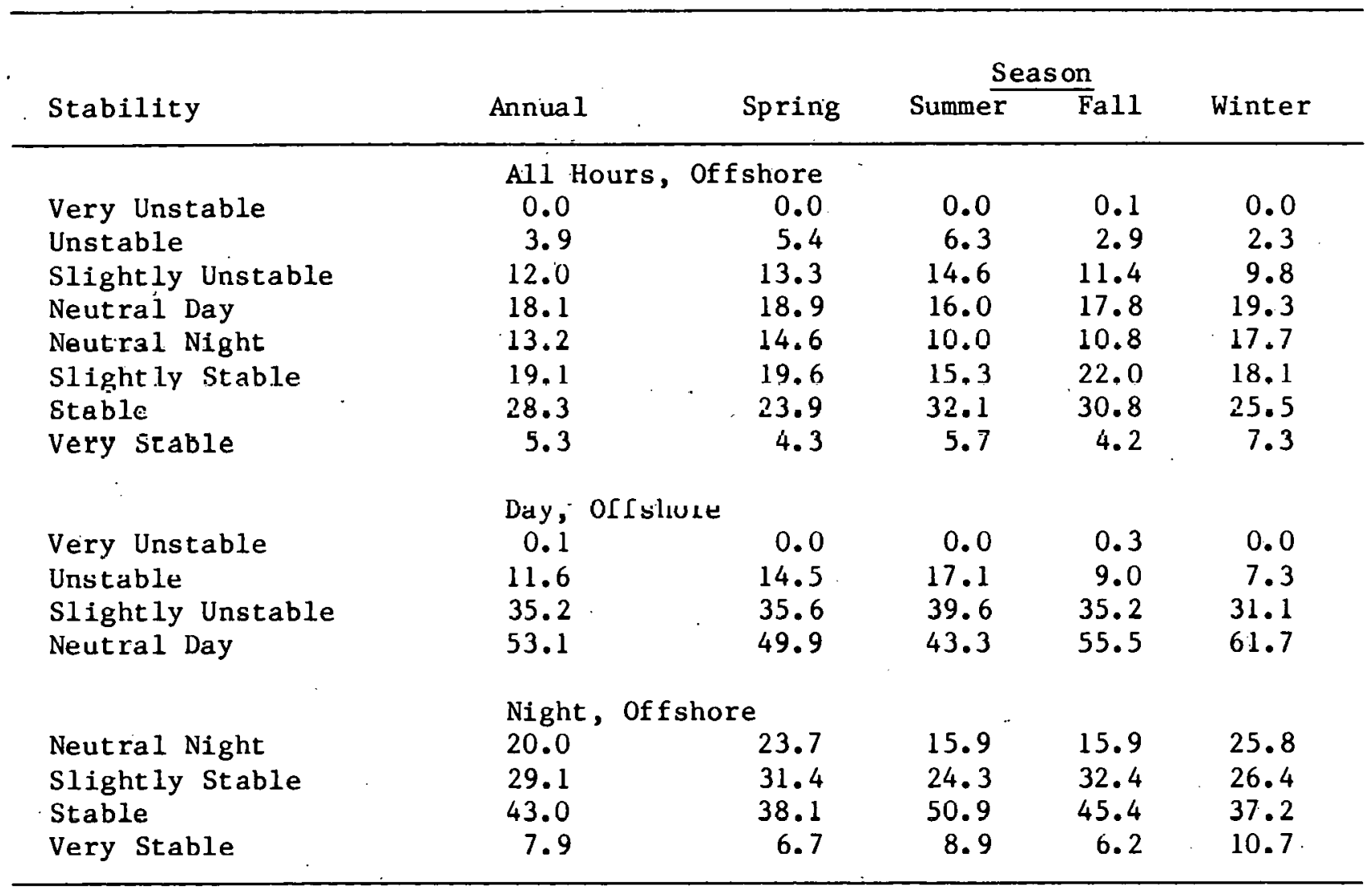


Table 5.21

Tallahassee, Florida

Percent of Hours in Each Stability Class

by Wind Direction, Season and Time of Day

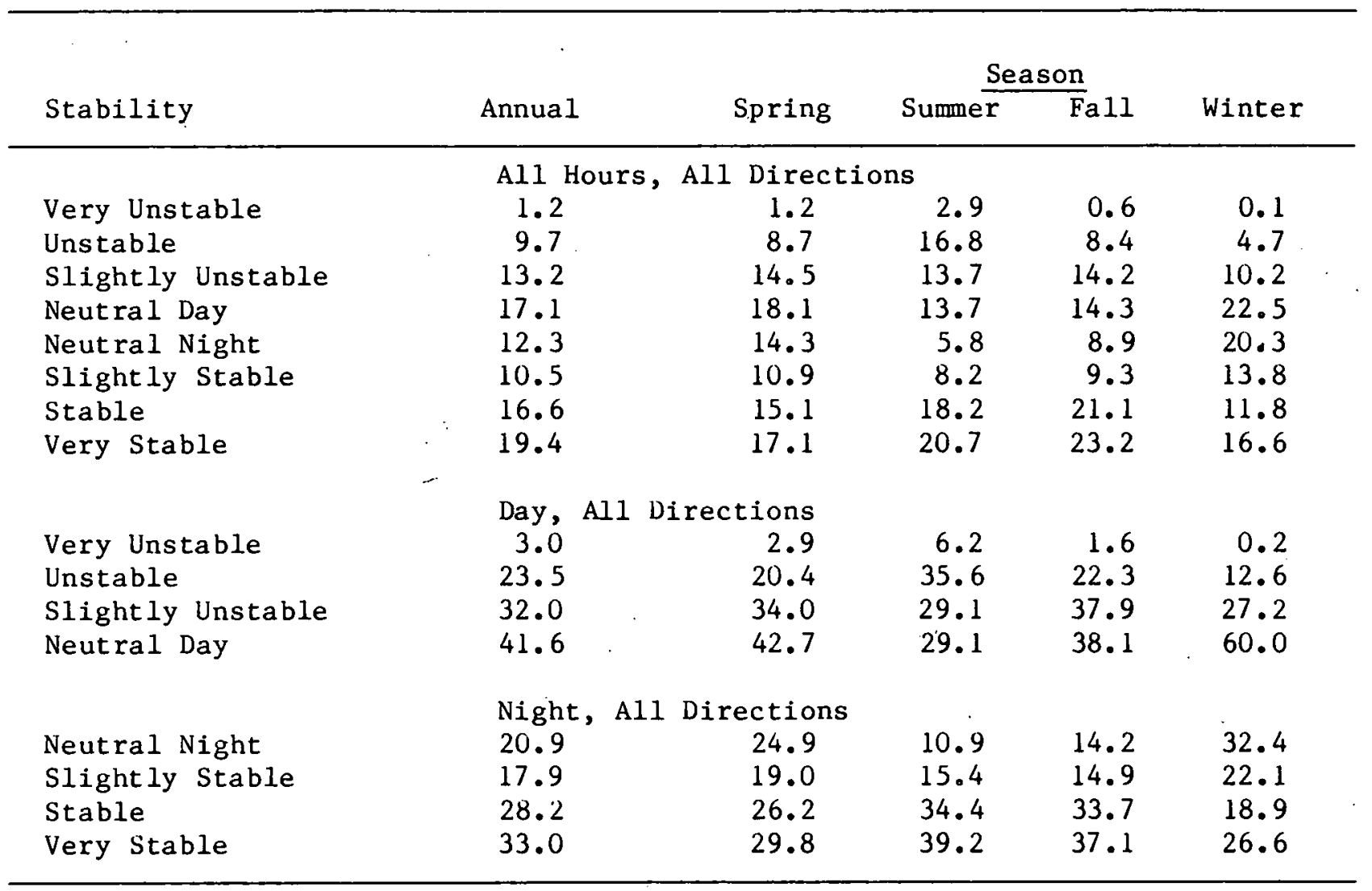


Table 5.21 (continued)

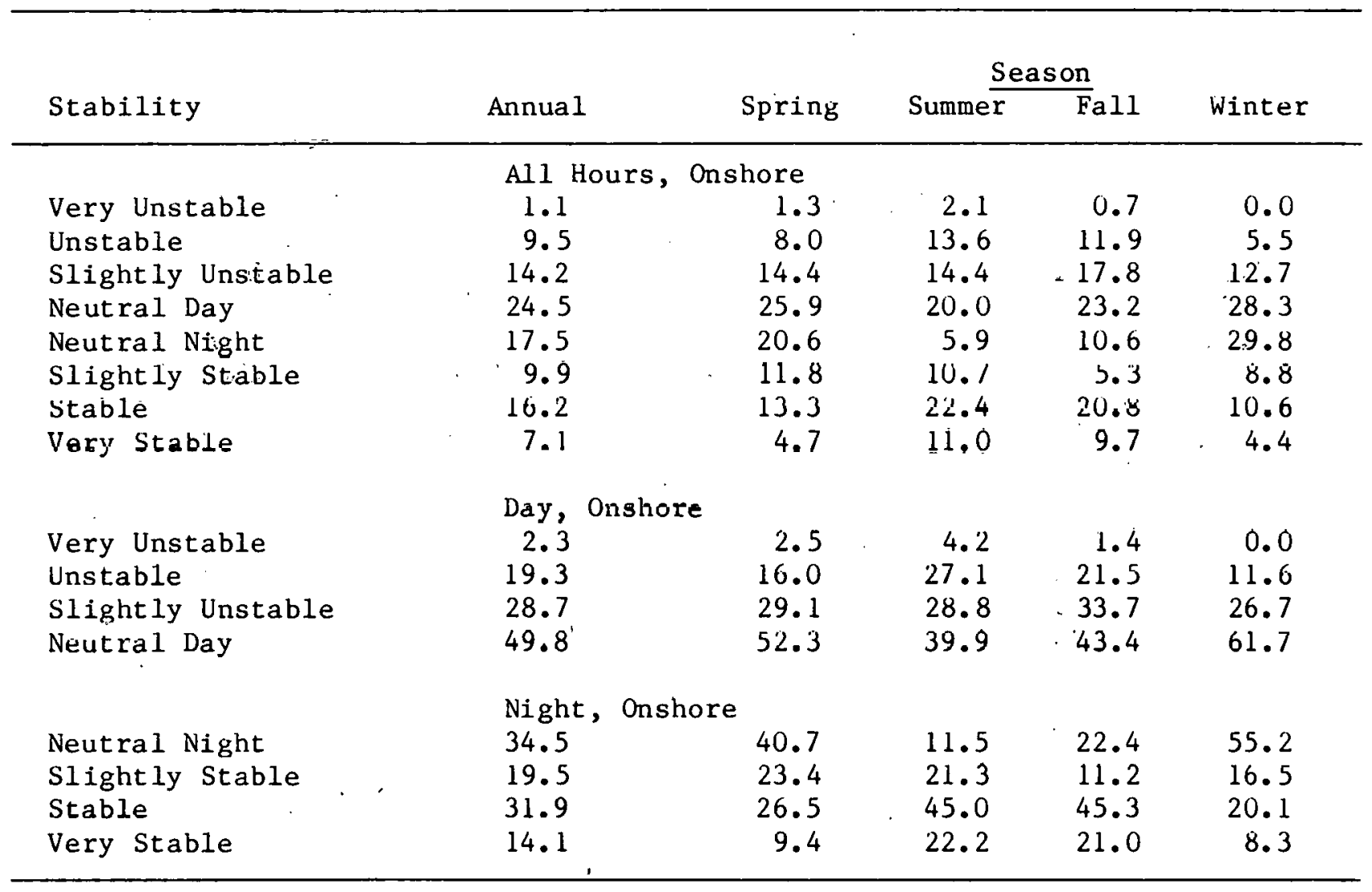


Table 5.21 (continued)

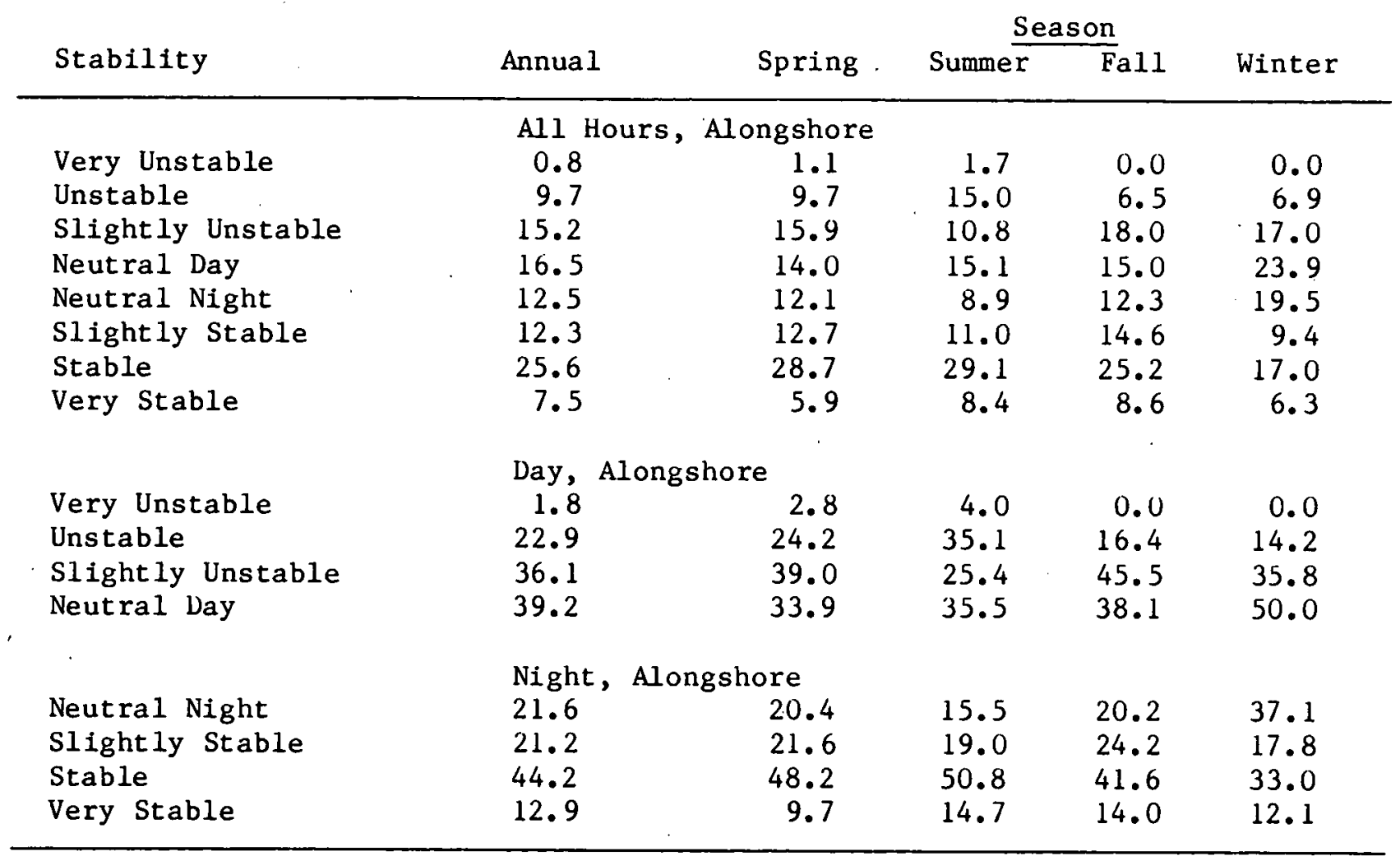


Table 5.21 (continued)

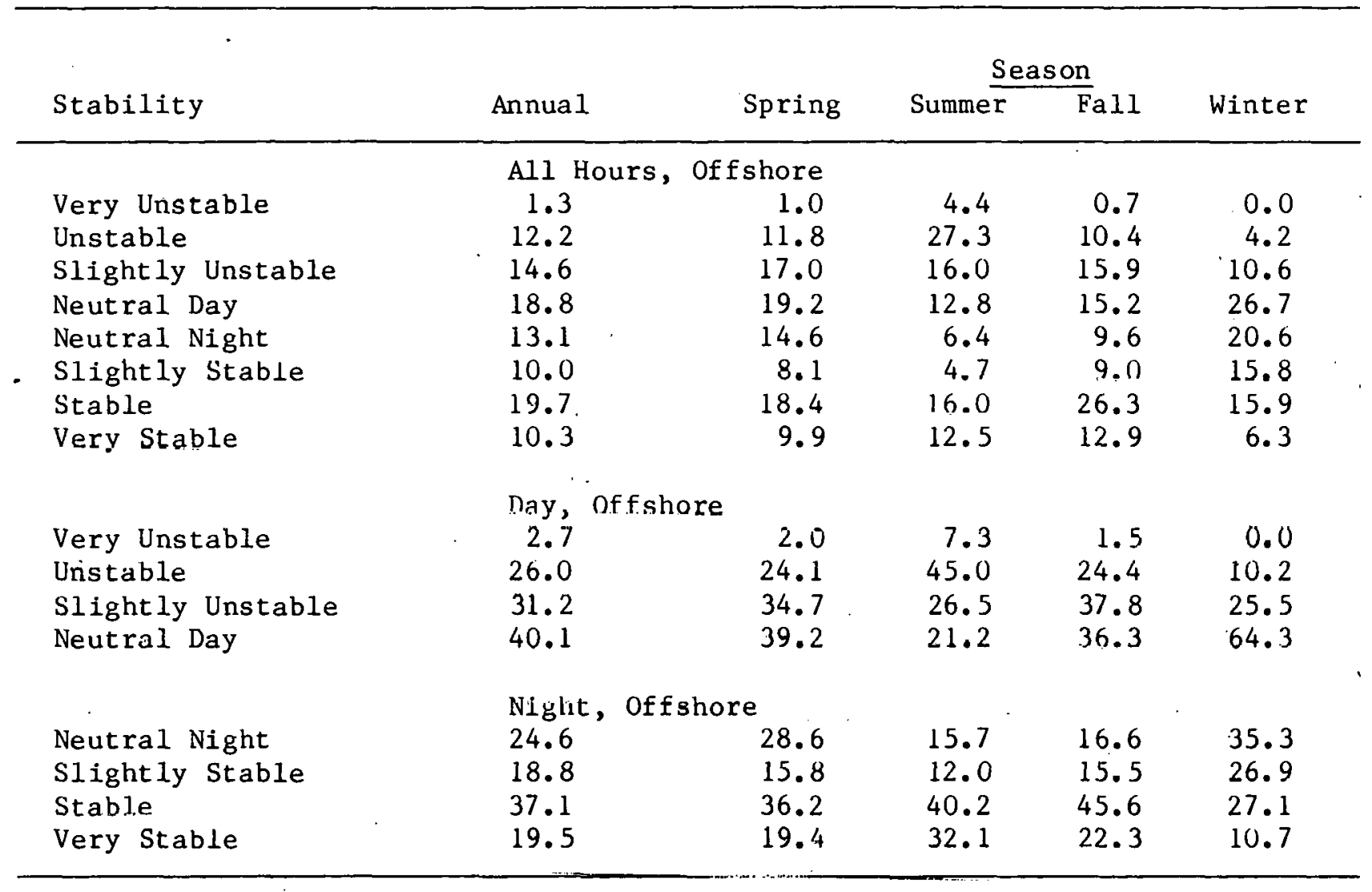




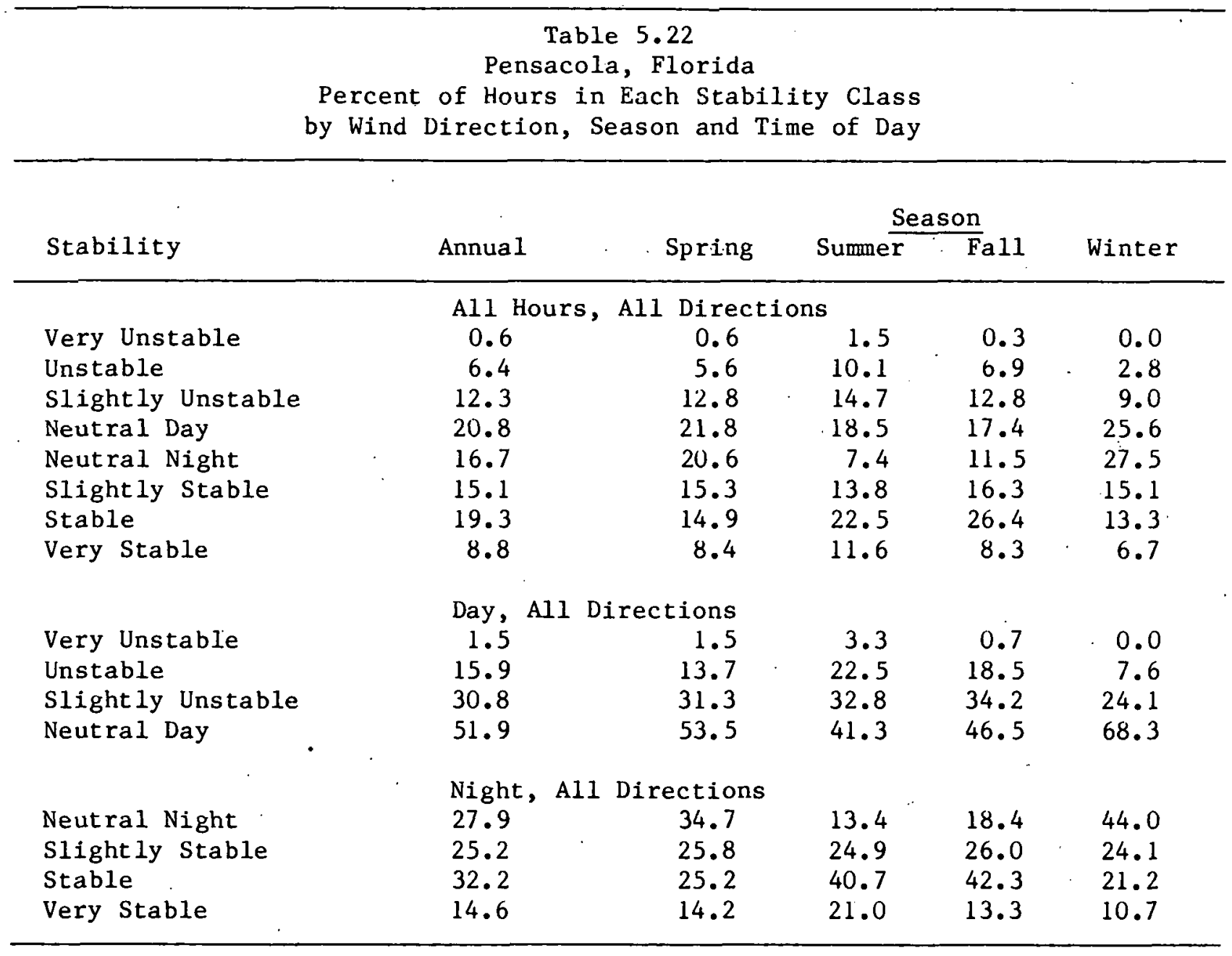


Table 5.22 (continued)

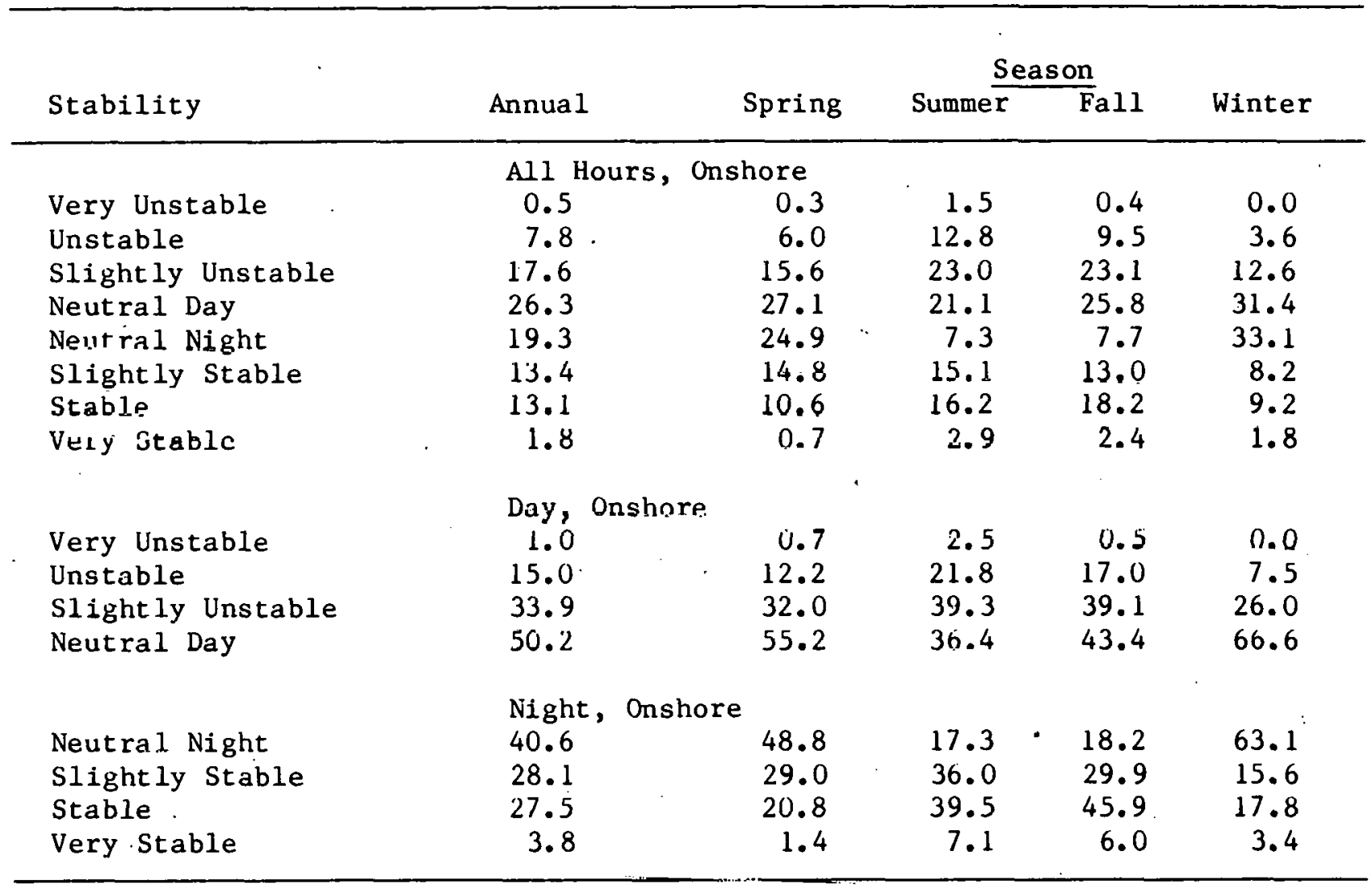


Table 5.22 (continued)

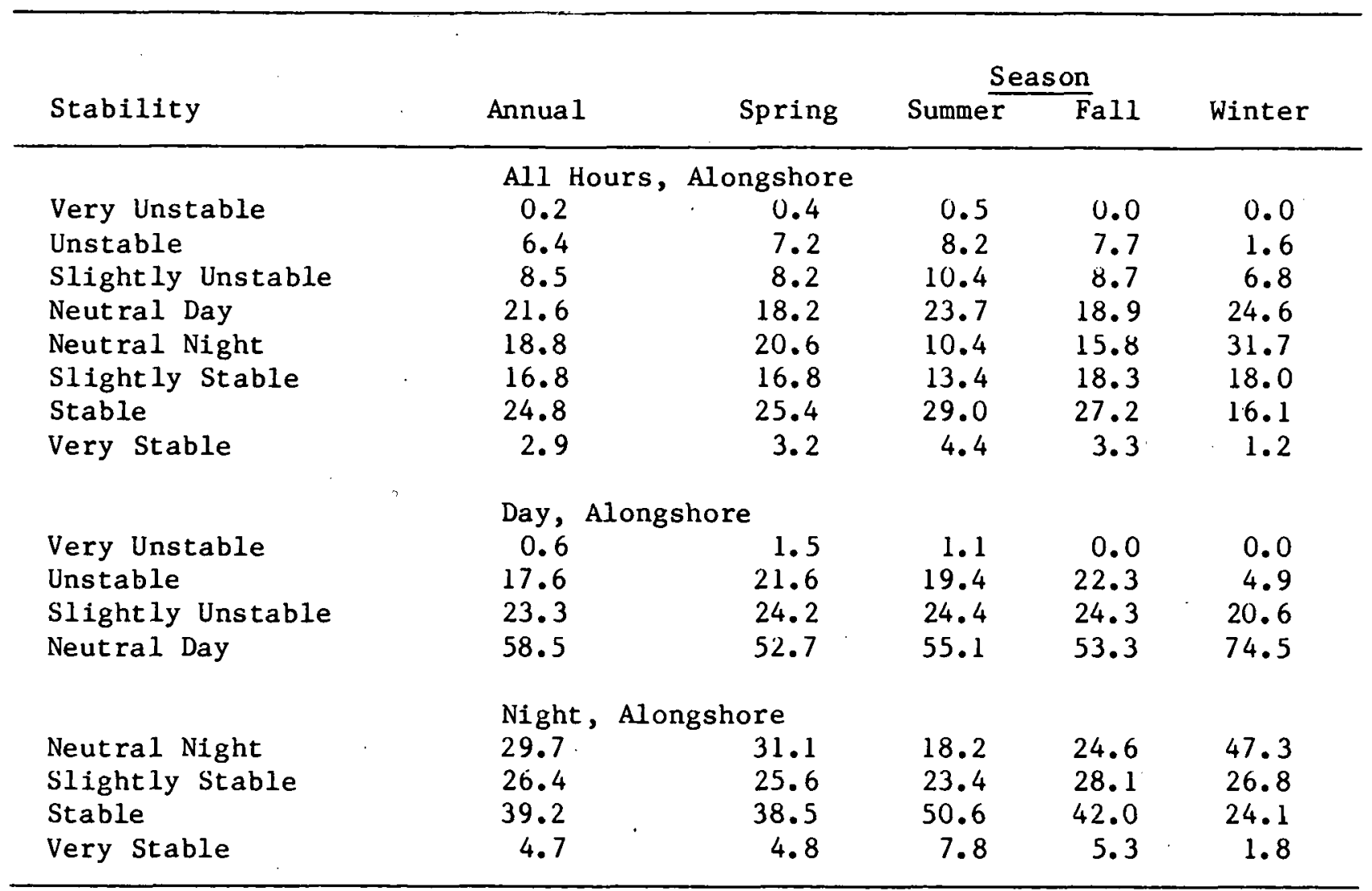


Table 5.22 (continued)

\begin{tabular}{|c|c|c|c|c|c|}
\hline \multirow[b]{2}{*}{ Stability } & \multirow[b]{2}{*}{ Annual } & \multirow[b]{2}{*}{ Spring } & \multicolumn{2}{|c|}{ Season } & \multirow[b]{2}{*}{ Winter } \\
\hline & & & Summe $\bar{r}$ & Fall & \\
\hline \multicolumn{6}{|c|}{ All Hours, of $f$ shore } \\
\hline Very Unstable & 0.4 & 1.1 & 0.7 & 0.1 & 0.0 \\
\hline Unstable & 5.6 & 5.6 & 13.4 & 4.6 & 2.7 \\
\hline Slightly Unstable & 10.8 & 11.9 & 10.9 & 11.4 & 9.4 \\
\hline Neutral Day & 20.0 & 20.7 & 16.0 & 15.2 & 26.4 \\
\hline Neutral Night & 16.9 & 20.2 & 6.7 & 12.9 & 25.6 \\
\hline Slightly Stable & 15.3 & 15.5 & 11.0 & 17.0 & 15.9 \\
\hline SLable & 26.7 & 19.9 & 36.8 & 34.2 & 17.1 \\
\hline Vory Stahle & 4.2 & 5.0 & 4.5 & 4.5 & 2.8 \\
\hline \multicolumn{6}{|c|}{ Day, offshore } \\
\hline Very Unstable & 1.0 & $2 . y$ & 1.8 & 0.3 & 0.0 \\
\hline Unstable & 15.2 & 14.4 & 32.7 & 14.7 & 6.9 \\
\hline Slightly Unstable & 29.3 & 30.3 & 26.7 & 36.4 & 24.5 \\
\hline Neutral Day & 54.4 & 52.4 & 38.7 & 48.4 & 68.6 \\
\hline \multicolumn{6}{|c|}{ N1ght, Orfsliore } \\
\hline Neutral Night & 26.8 & 33.1 & 11.3 & 18.9 & 41.7 \\
\hline Slightly Stable & 24.2 & 25.4 & 18.5 & 24.8 & 25.9 \\
\hline Stable & 42.3 & 33.0 & 62.5 & 49.7 & 27.9 \\
\hline Very Stable & 6.7 & 8.5 & 7.7 & 6.6 & 4.6 \\
\hline
\end{tabular}


Table 5.23

Mobile, Alabama

Percent of Hours in Each Stability Class

by Wind Direction, Season and Time of Day

\begin{tabular}{|c|c|c|c|c|c|}
\hline \multirow[b]{2}{*}{ Stability } & \multirow[b]{2}{*}{ Annua 1} & \multicolumn{4}{|c|}{ Season } \\
\hline & & Spring & Summer & $\mathrm{Fall}$ & Winter \\
\hline \multicolumn{6}{|c|}{ All Hours, All Directions } \\
\hline Very Unstable & 0.6 & 0.5 & 1.0 & 0.5 & 0.1 \\
\hline Unstable & 4.7 & 3.8 & 8.2 & 5.0 & 1.8 \\
\hline Slightly Unstable & 10.7 & 9.2 & 14.0 & 11.9 & 7.6 \\
\hline Neutral Day & 24.1 & 26.5 & 22.1 & 20.1 & 27.9 \\
\hline Neutral Night & 19.5 & 24.9 & 7.4 & 13.1 & 32.8 \\
\hline Slight ly Stable & 16.2 & 17.9 & 16.8 & 15.5 & 14.7 \\
\hline Stable & 18.7 & 14.1 & 24.9 & 23.8 & 11.7 \\
\hline Very Stable & 5.5 & 2.9 & 5.6 & 10.1 & 3.2 \\
\hline \multicolumn{6}{|c|}{ Day, All Directions } \\
\hline Very Unstable & 1.4 & 1.4 & 2.3 & 1.5 & 0.4 \\
\hline Unstable & 11.7 & 9.5 & 18.0 & 13.4 & 4.8 \\
\hline Slightly Unstable & 26.7 & 23.1 & 30.9 & 31.7 & 20.4 \\
\hline Neutral Day & 60.2 & 66.1 & 48.8 & 53.5 & 74.4 \\
\hline \multicolumn{6}{|c|}{ Night, All Directions } \\
\hline Neutral Night & 32.6 & 41.6 & 13.5 & 21.0 & 52.6 \\
\hline Slightly Stable & 27.1 & 29.9 & 30.7 & 24.7 & 23.6 \\
\hline Stable & 31.2 & 23.6 & 45.5 & 38.1 & 18.8 \\
\hline Very Stable & 9.1 & 4.9 & 10.3 & 16.2 & 5.1 \\
\hline
\end{tabular}


Table 5.23 (continued)

\begin{tabular}{|c|c|c|c|c|c|}
\hline \multirow[b]{2}{*}{ Stability } & \multirow[b]{2}{*}{ Annual } & & \multicolumn{2}{|c|}{ Season } & \multirow[b]{2}{*}{ Winter } \\
\hline & & Spring & Summer & Fall & \\
\hline & \multicolumn{2}{|c|}{ All Hours, Onshore } & & & \\
\hline Very Unstable & 0.3 & 0.4 & 0.6 & 0.0 & 0.0 \\
\hline Unstable & 3.1 & 3.1 & 4.8 & 2.5 & 1.8 \\
\hline Slightly Unstable & 9.7 & 8.1 & 12.8 & 12.2 & 7.3 \\
\hline Neutral Day & 26.6 & 26.5 & 23.8 & 27.5 & 29.6 \\
\hline Neutral Night & 21.9 & 28.9 & 7.7 & 11.7 & 33.7 \\
\hline Slightly stable & 17.1 & 17.7 & 21.4 & 13.7 & 13.2 \\
\hline Stable & 18.9 & 13.4 & 25.7 & 27.9 & 13.8 \\
\hline Verý Stable & 2.3 & 1.9 & 3.3 & 4.4 & 0.7 \\
\hline & \multicolumn{2}{|c|}{ Day, Onshore } & & & \\
\hline Very Unstable & 0.8 & 1.1 & 1.5 & 0.0 & 0.0 \\
\hline Unstable & 7.8 & 8.2 & 11.3 & 5.9 & 4.5 \\
\hline Slightly Unstable & 24.4 & 21.0 & 30.5 & 29.0 & 18.8 \\
\hline \multirow[t]{2}{*}{ Neutral Day } & 67.0 & 69.7 & 56.7 & 65.1 & 76.7 \\
\hline & Night, & , Onshore & & & \\
\hline Neutral Night & 36.3 & 46.5 & 13.2 & 20.3 & 54.9 \\
\hline Slightly Stable & 28.4 & 28.6 & 36.8 & 23.4 & 21.5 \\
\hline Stable & 31.5 & 21.8 & 44.3 & 48.4 & 22.5 \\
\hline Very Stable & 3.8 & 3.1 & 5.7 & 7.9 & 1.1 \\
\hline
\end{tabular}


Table 5.23 (continued)

\begin{tabular}{|c|c|c|c|c|c|}
\hline \multirow[b]{2}{*}{ Stability } & \multirow[b]{2}{*}{ - Annual } & \multirow[b]{2}{*}{ Spring } & \multicolumn{2}{|c|}{ Season } & \multirow[b]{2}{*}{ Winter } \\
\hline & & & Summer & Fall & \\
\hline \multicolumn{6}{|c|}{ All Hours, Alongshore } \\
\hline Very Unstable & 1.1 & 1.6 & 1.2 & 1.2 & 0.0 \\
\hline Unstable & 7.5 & 6.2 & 9.6 & 9.8 &, 1.5 \\
\hline Slightly Unstable & 14.2 & 11.6 & 16.8 & 1.4 .1 & 12.8 \\
\hline Neutral Day. & 24.6 & 24.6 & 25.4 & 22.3 & 27.1 \\
\hline Neutral Night & 15.4 & 17.3 & 5.6 & 16.5 & 30.0 \\
\hline Slightly Stable & 13.3 & 16.2 & 11.5 & 12.5 & 14.2 \\
\hline Stable & 21.3 & 20.0 & 27.2 & 20.3 & 12.8 \\
\hline Very Stable & 2.6 & 2.5 & 2.7 & 3.4 & 1.5 \\
\hline \multicolumn{6}{|c|}{ Day, Alongshore } \\
\hline Very Unstable & 2.3 & 3.7 & 2.4 & 2.6 & 0.0 \\
\hline Unstable & 15.9 & 14.0 & 18.2 & 21.0 & 3.4 \\
\hline Slightly Unstable & 29.7 & 26.4 & 31.6 & 29.0 & 31.0 \\
\hline \multirow[t]{2}{*}{ Neutral Day } & 52.0 & 55.9 & 47.8 & .47 .3 & 65.6 \\
\hline & Night & , Alongshore & & & \\
\hline Neutra1 Night & 29.4 & 30.9 & 12.2 & 31.8 & 51.4 \\
\hline Slightly Stable & 25.3 & 28.9 & 24.5 & 23.9 & 24.1 \\
\hline $\begin{array}{l}\text { Stable } \\
\text { State }\end{array}$ & 40.3 & 35.7 & 57.6 & 37.8 & 21.9 \\
\hline Very Stable & $5: 0$ & 4.5 & 5.7 & 6.5 & 2.6 \\
\hline
\end{tabular}


Table 5.23 (continued)

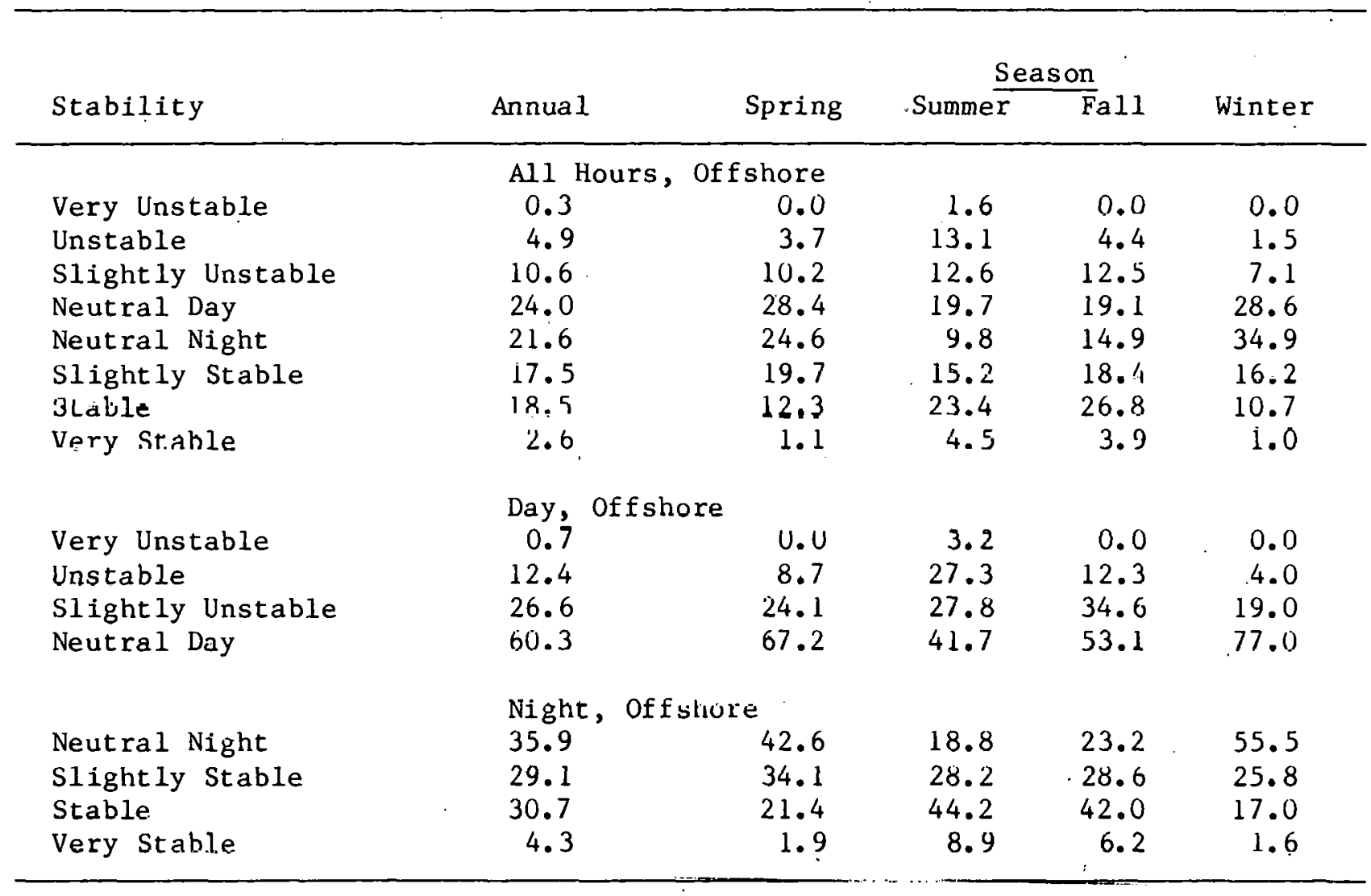




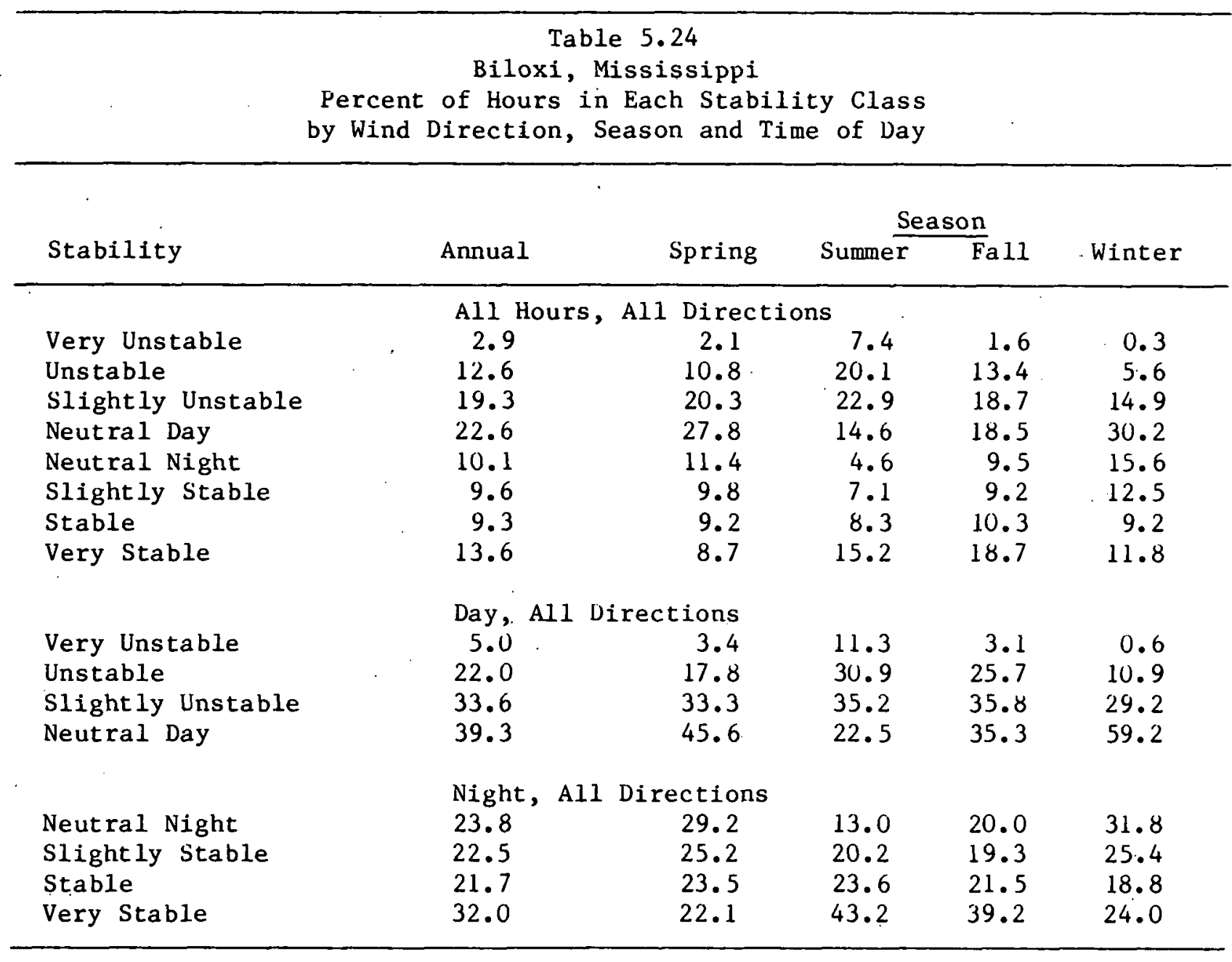


Table 5.24 (continued)

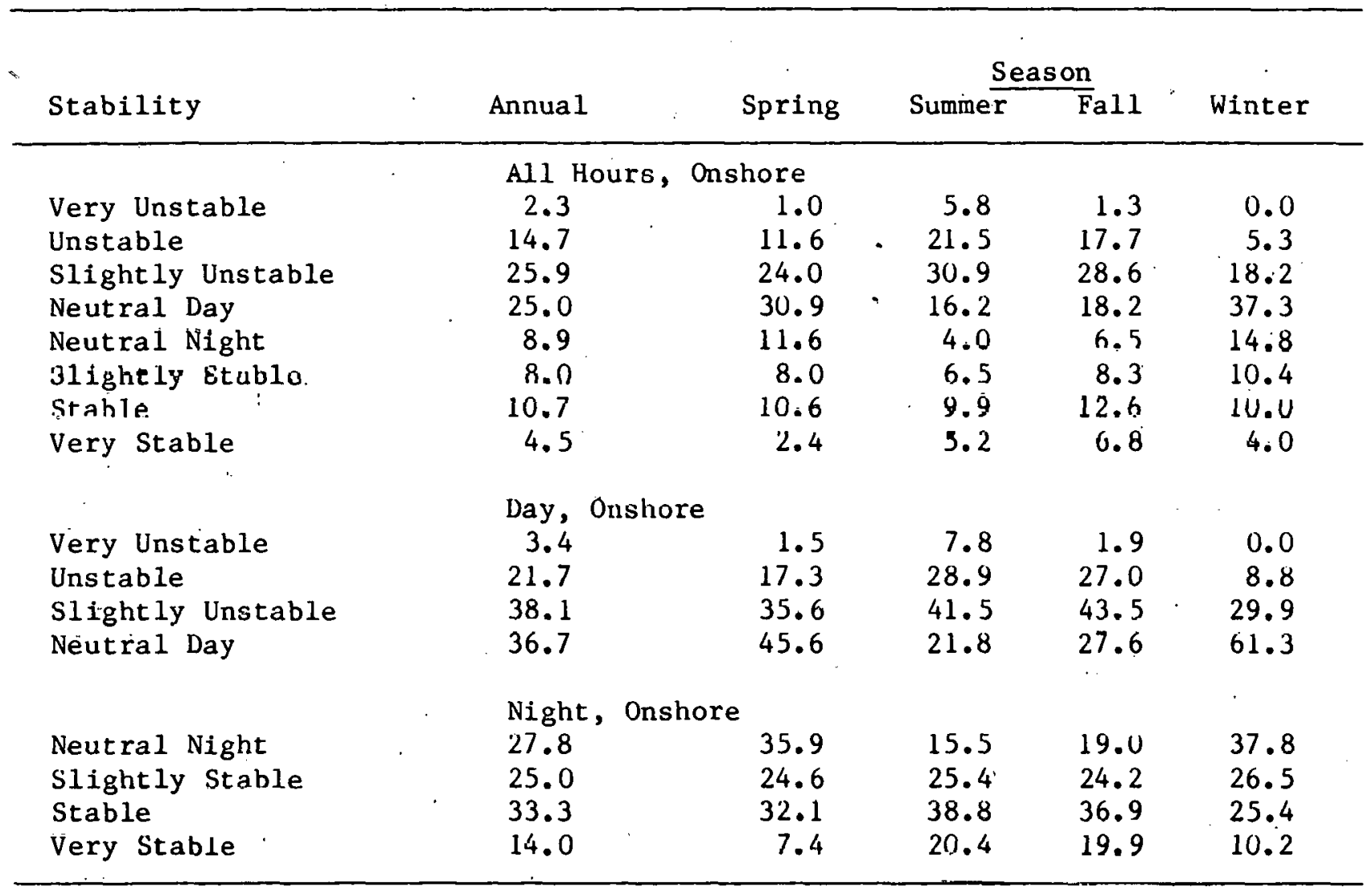


Table 5.24 (continued)

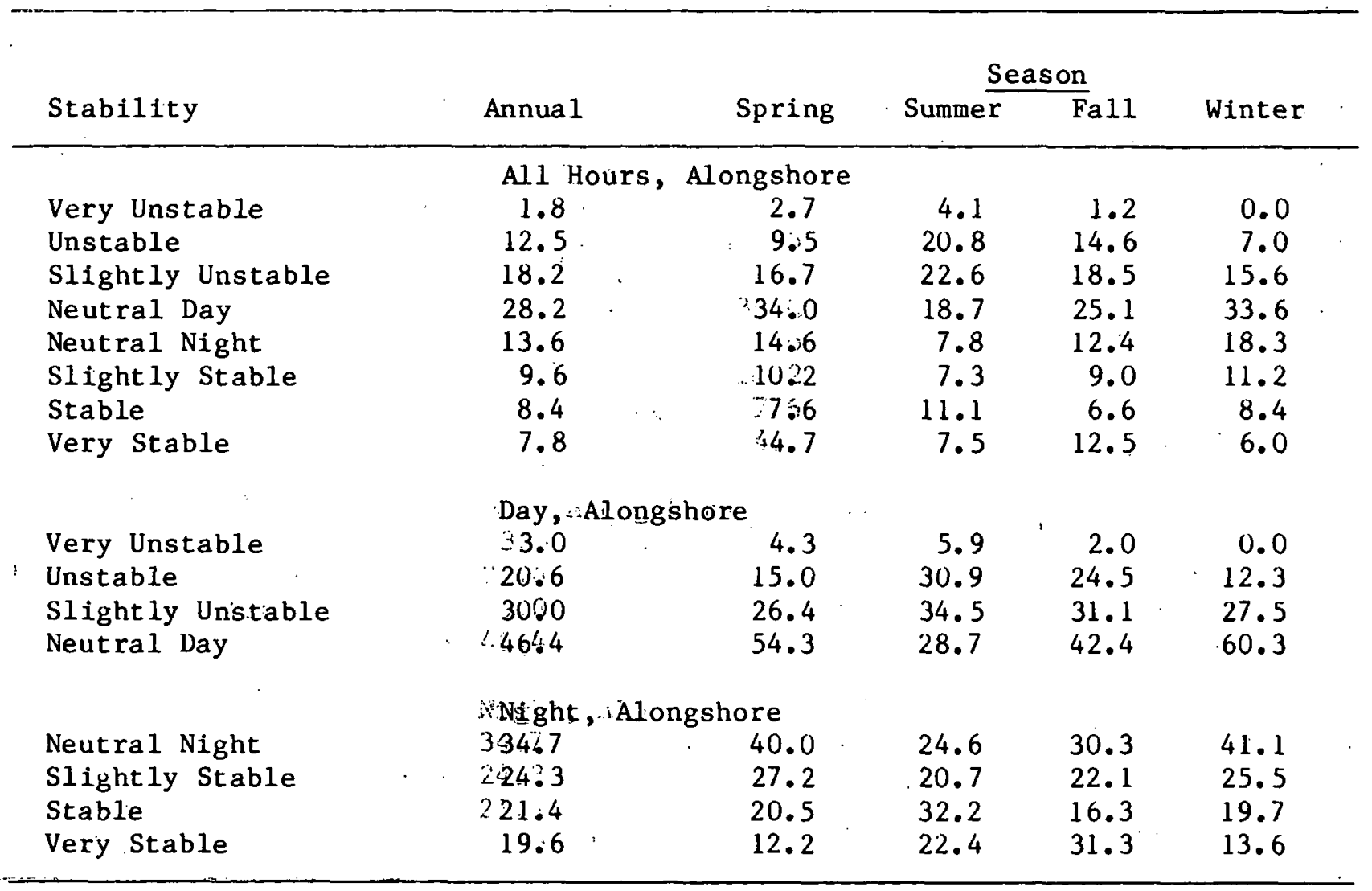


Table 5.24 (continued)

\begin{tabular}{|c|c|c|c|c|c|}
\hline \multirow[b]{2}{*}{ Stability } & \multirow[b]{2}{*}{ Annual } & \multirow[b]{2}{*}{ Spring } & \multicolumn{2}{|c|}{ Season } & \multirow[b]{2}{*}{ Winter } \\
\hline & & & Summer & Fall & \\
\hline $\begin{array}{l}\text { Very Unstable } \\
\text { Unstable } \\
\text { Slightly Unstable } \\
\text { Neutral Day } \\
\text { Neutral Night } \\
\text { Slight ly Stable } \\
\text { Stable } \\
\text { Very Stable }\end{array}$ & $\begin{array}{l}\text { A11 H } \\
1.6 \\
11.8 \\
16.8 \\
27.2 \\
14.2 \\
10.7 \\
10.9 \\
6.8\end{array}$ & $\begin{array}{r}\text { Hours, of fshore } \\
1.8 \\
11.1 \\
17.7 \\
30.9 \\
13.3 \\
11.3 \\
9.6 \\
4.3\end{array}$ & $\begin{array}{r}5.8 \\
23.9 \\
19.4 \\
19.1 \\
5.9 \\
6.8 \\
8.4 \\
10.6\end{array}$ & $\begin{array}{r}0.5 \\
11.6 \\
17.3 \\
24.1 \\
14.2 \\
11.1 \\
12.8 \\
8.5\end{array}$ & $\begin{array}{r}0.1 \\
5.1 \\
14.4 \\
33.1 \\
19.5 \\
12.1 \\
11.6 \\
4.2\end{array}$ \\
\hline $\begin{array}{l}\text { Very Unstable } \\
\text { Unstable } \\
\text { Slightly Unstable } \\
\text { Neutral Day }\end{array}$ & $\begin{array}{r}\text { Day, } \\
2.8 \\
20.5 \\
29.2 \\
47.4\end{array}$ & $\begin{array}{r}\text { Offehore } \\
\quad 3.0 \\
18.0 \\
28.7 \\
50.3\end{array}$ & $\begin{array}{r}8.6 \\
35.2 \\
28.5 \\
27.8\end{array}$ & $\begin{array}{r}0.9 \\
21.8 \\
32.3 \\
45.1\end{array}$ & $\begin{array}{r}0.2 \\
9.6 \\
27.2 \\
63.1\end{array}$ \\
\hline $\begin{array}{l}\text { Neutral Night } \\
\text { Slightly Stable } \\
\text { Stable } \\
\text { Very Stable }\end{array}$ & $\begin{array}{l}\text { Night } \\
33.3 \\
25.1 \\
25.6 \\
15.9\end{array}$ & $\begin{array}{r}t, \text { of fshore } \\
34.6 \\
29.2 \\
25.0 \\
11.2\end{array}$ & $\begin{array}{l}18.8 \\
21.4 \\
26.7 \\
33.2\end{array}$ & $\begin{array}{l}30.5 \\
23.8 \\
27.4 \\
18.3\end{array}$ & $\begin{array}{r}41.0 \\
25.6 \\
24.6 \\
8.8\end{array}$ \\
\hline
\end{tabular}




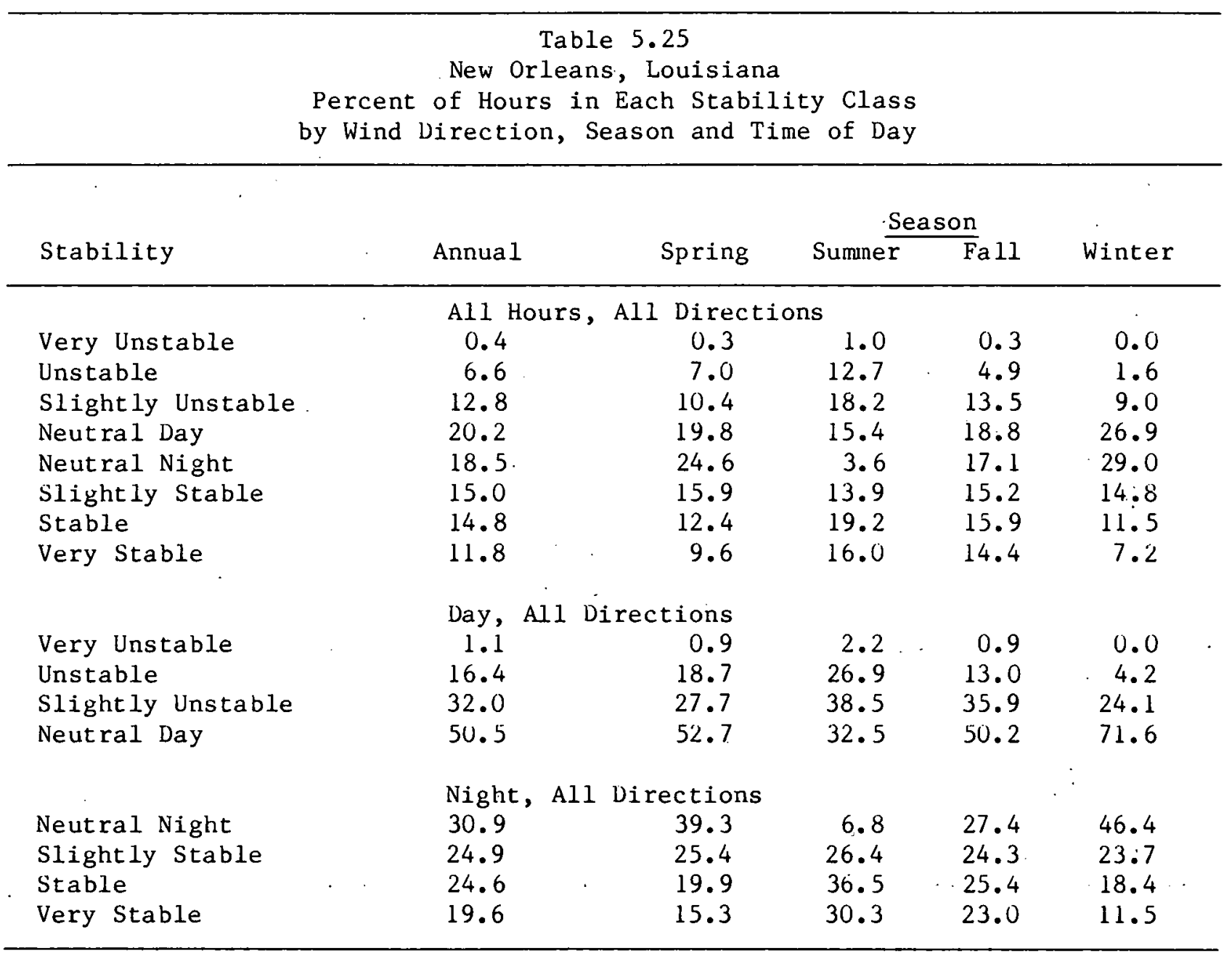


Table 5.25 (continued)

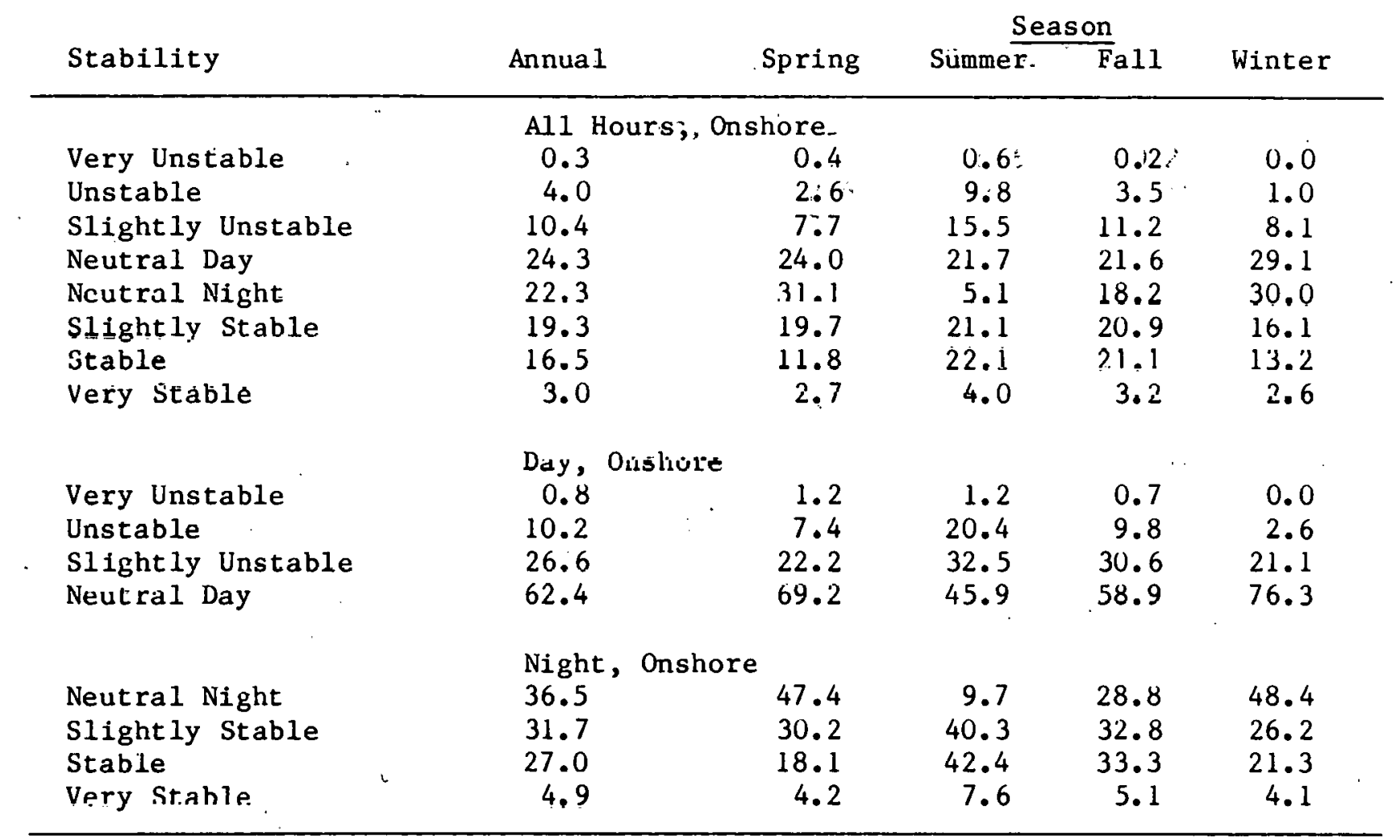


Table 5.25 (continued)

\begin{tabular}{|c|c|c|c|c|c|}
\hline \multirow[b]{2}{*}{ Stability. } & \multirow[b]{2}{*}{ Annual } & \multirow[b]{2}{*}{ Spring } & \multicolumn{2}{|c|}{ Season } & \multirow[b]{2}{*}{ Winter } \\
\hline & & & Summer & Fall & \\
\hline \multicolumn{6}{|c|}{ All Hours, Alongshore } \\
\hline Very Unstable & 0.1 & 0.0 & 0.4 & 0.0 & 0.0 \\
\hline Unstable & 6.0 & 4.5 & 9.7 & 6.3 & 2.1 \\
\hline Slightly Unstable & 11.6 & 9.7 & 14.2 & 12.0 & 9.8 \\
\hline Neutral Day & 22.6 & 22.0 & 21.8 & 20.9 & 23.5 \\
\hline Neutral Night & 14.5 & 18.1 & 3.3 & 16.6 & 23.1 \\
\hline Slight ly Stable & 13.6 & 15.1 & 11.8 & 14.4 & 13.9 \\
\hline Stable & 22.3 & 18.9 & 27.1 & 21.7 & 22.3 \\
\hline Very Stab.le & 9.2 & 11.6 & 11.8 & 8.1 & 5.5 \\
\hline \multicolumn{6}{|c|}{ Day, Alongshore... } \\
\hline Very Unstable. & 0.3 & 0.0 & 0.7 & 0.0 & 0.0 \\
\hline Unstable & 14.9 & 13.3 & 21.4 & 16.3 & 5.7 \\
\hline Slight ly Unstable & 28.8 & 25.0 & 31.9 & 30.6 & 28.9 \\
\hline \multirow[t]{2}{*}{ Neutral Day } & 56.0 & 61.7 & 46.0 & 53.2 & $65.5^{\circ}$ \\
\hline & Night & , Alongshore & & & \\
\hline Neutral Night & 24.3 & 29.6 & 6.8 & 27.2 & 35.2 \\
\hline S1ightly Stable & 22.9 & 22.7 & 22.5 & 23.6 & 21.7 \\
\hline Stable & 37.3 & 30.3 & 48.3 & 35.9 & 34.6 \\
\hline Very Stable & 15.5 & 17.4 & 22.5 & 13.3 & 8.5 \\
\hline
\end{tabular}


Table 5.25 (continued)

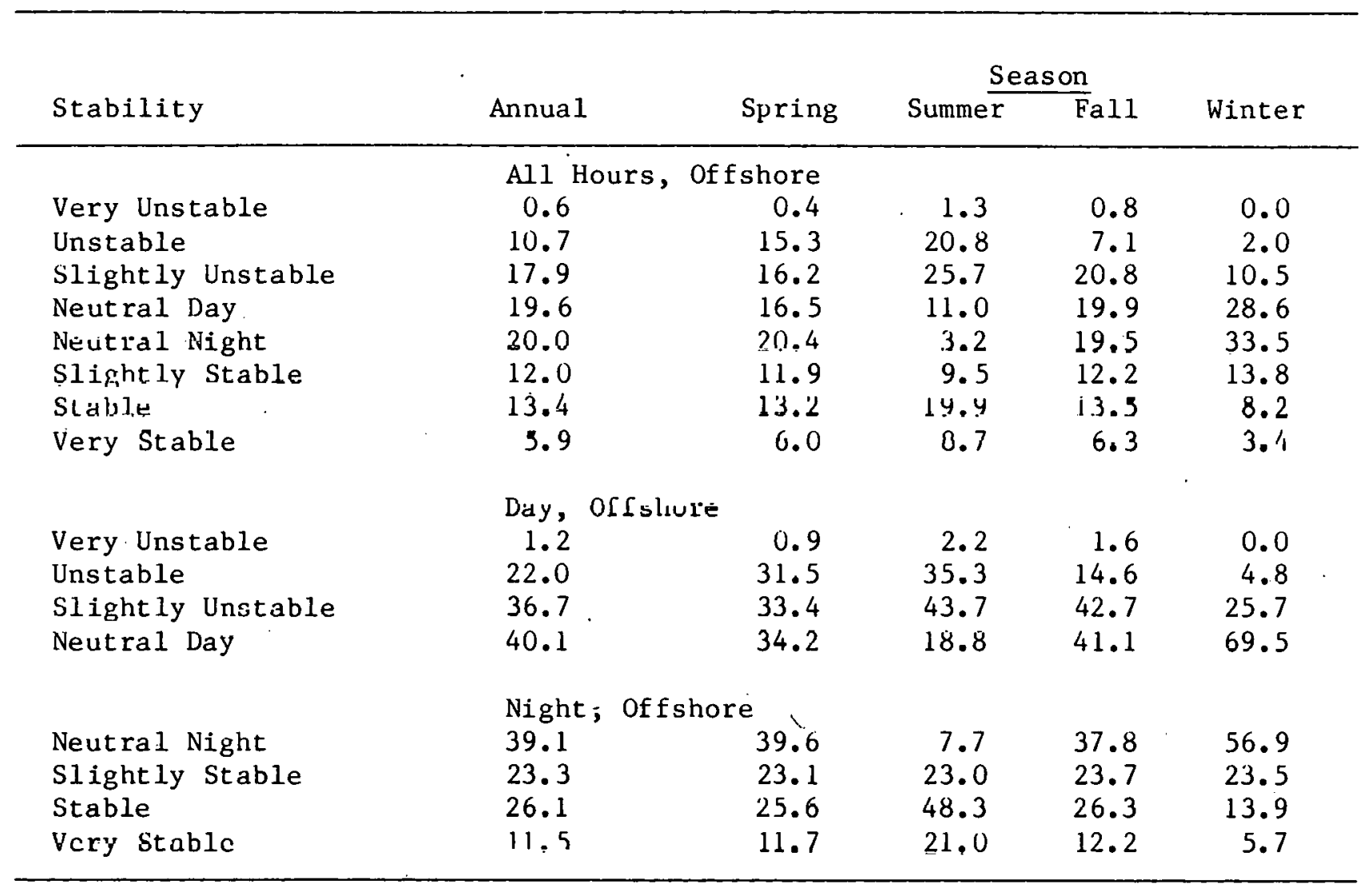


Table 5.26

Boothville, Louisiana

Percent of Hours in Each Stability Class

by Wind Direction, Season and Time of Day

\begin{tabular}{|c|c|c|c|c|c|}
\hline \multirow[b]{2}{*}{ Stability } & \multirow[b]{2}{*}{ Annual } & \multirow[b]{2}{*}{ Spring } & \multicolumn{2}{|c|}{ Season } & \multirow[b]{2}{*}{ Winter } \\
\hline & & & Summer & $\bar{F} a l l$ & \\
\hline · & \multicolumn{5}{|c|}{ All Hours, All Directions } \\
\hline Very Unstable & 0.8 & 0.6 & 1.9 & 0.8 & 0.0 \\
\hline Unstable & 7.0 & 6.8 & 13.4 & 6.0 & 1.6 \\
\hline Slightly Unstable & 12.4 & 12.7 & 13.9 & 12.4 & 10.4 \\
\hline Neutral Day. & 19.5 & 18.2 & 15.6 & 18.3 & 26.2 \\
\hline Neutral Night & 18.3 & 23.2 & 5.5 & 16.9 & 27.6 \\
\hline Slightly Stable & 13.8 & 16.6 & 11.5 & 12.3 & 14.6 \\
\hline Stable & 16.5 & 16.0 & 20.6 & 16.5 & 12.7 \\
\hline \multirow[t]{2}{*}{ Very Stable } & 11.8 & 5.8 & 17.6 & 16.8 & 6.9 \\
\hline & \multicolumn{5}{|c|}{ Day, All Directions } \\
\hline Very Unstable & 2.1 & 1.6 & 4.3 & 2.2 & 0.0 \\
\hline Unstable & 17.6 & 17.7 & 29.9 & 15.9 & 4.3 \\
\hline Slightly Unstable & 31.2 & 33.2 & 31.1 & 33.2 & 27.2 \\
\hline Neutral Day & 49.1 & 47.5 & 34.7 & 48.7 & 68.5 \\
\hline 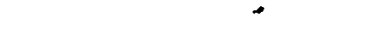 & \multicolumn{5}{|c|}{ Night, All Directions } \\
\hline Neutral Night & 30.3 & 37.6 & 10.1 & 27.0 & 44.7 \\
\hline Slightly Stable & 22.8 & 27.0 & 20.9 & 19.7 & 23.6 \\
\hline Stable & 27.3 & 26.0 & 37.2 & 26.4 & 20.5 \\
\hline Very Stable & 19.6 & 9.5 & 31.8 & 26.9 & 11.2 \\
\hline
\end{tabular}


Table 5.26 (continued)

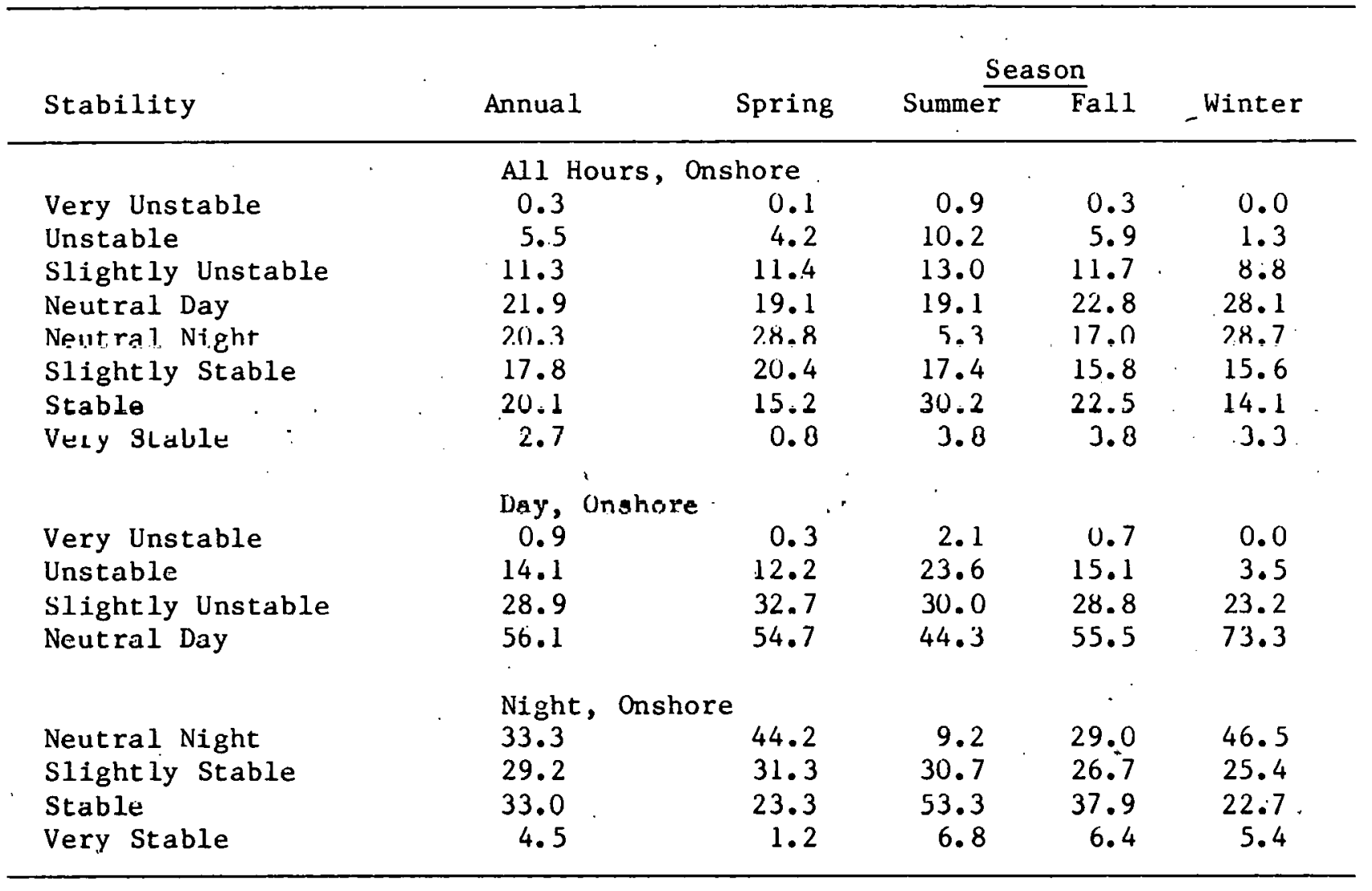


Table 5.26 (continued)

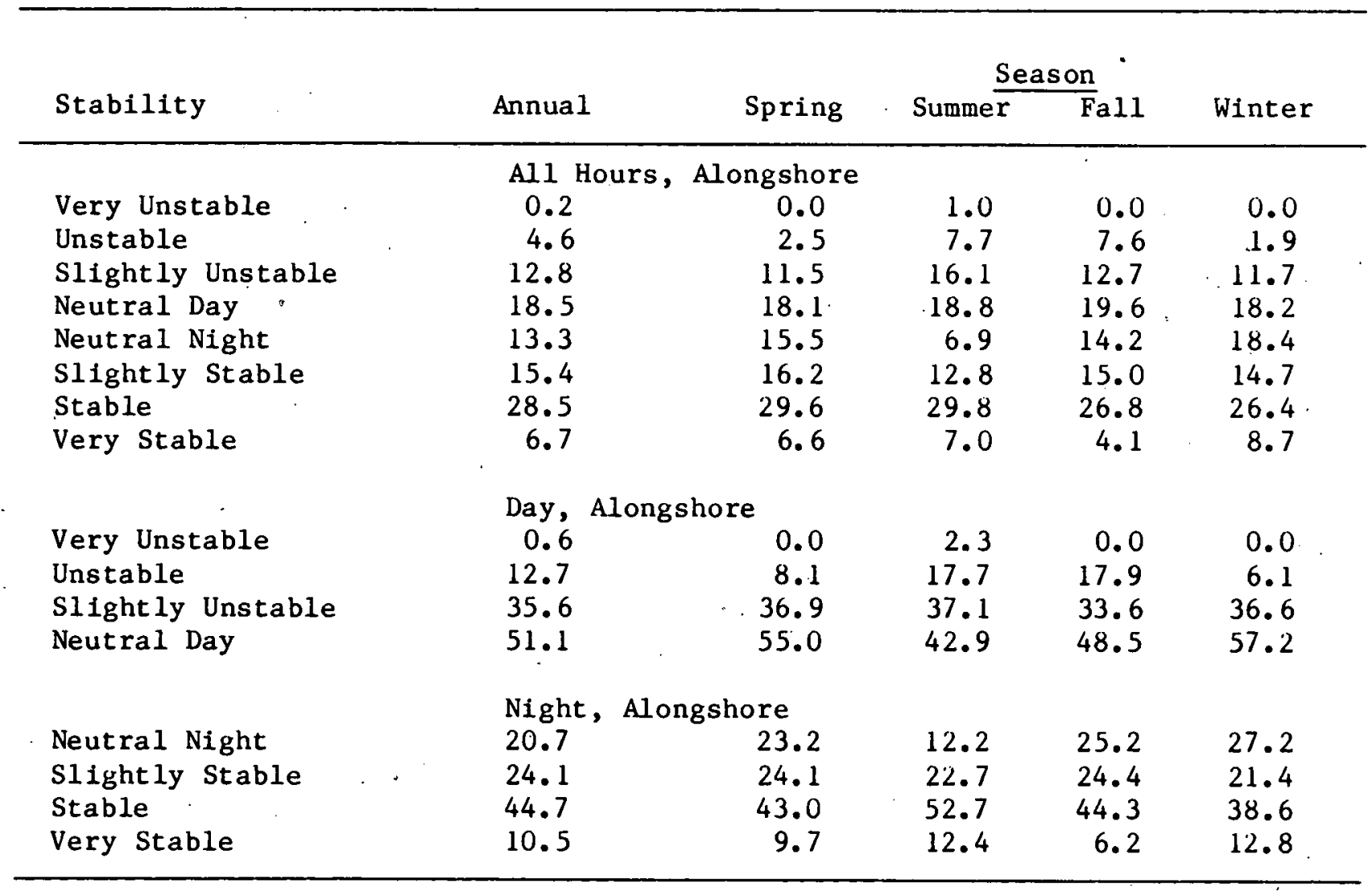


Table 5.26 (continued)

\begin{tabular}{|c|c|c|c|c|c|}
\hline \multirow[b]{2}{*}{ Stability } & \multirow[b]{2}{*}{ Annua 1} & \multirow[b]{2}{*}{ Spring } & \multicolumn{2}{|c|}{ Season } & \multirow[b]{2}{*}{ Winter } \\
\hline & & & Summer & Fall & \\
\hline \multicolumn{6}{|c|}{ All Hours, Offshore } \\
\hline Very Unstable & 0.7 & 1.1 & 2.2 & 0.3 & 0.0 \\
\hline Unstable & 11.1 & 14.8 & 28.6 & 7.6 & 1.4 \\
\hline Slightly Unstable & 15.4 & 16.4 & 18.3 & 17.7 & 11.3 \\
\hline Neutral Day & 23.2 & 18.9 & 17.4 & 22.2 & 30.1 \\
\hline Neutral Night & 23.4 & 21.5 & 7.6 & 24.4 & .32 .6 \\
\hline Slightly Stable & 11.0 & 11.6 & 7.6 & 11.7 & 12.5 \\
\hline Stable & 12.4 & 10.1 & 15.2 & 14.1 & 10.3 \\
\hline Very Stable & 2.0 & 5.0 & 3.2 & 1.9 & 1.8 \\
\hline \multicolumn{6}{|c|}{ Day, off $\mathbf{f}$ hore } \\
\hline Very Unstable & 1.5 & 2.1 & 3.3 & 0.7 & 0.0 \\
\hline Unstable & 21.9 & 28.9 & 43.1 & 15.9 & 3.2 \\
\hline Slightly Unstable & 30.6 & 32.0 & 27.6 & 36.4 & 26.3 \\
\hline Neutral Day & 46.0 & 37.0 & 26.0 & 46.9 & 70.5 \\
\hline \multicolumn{6}{|c|}{ Night, of fshore } \\
\hline Neutral Night & 47.2 & 44.1 & 22.7 & 46.1 & 57.0 \\
\hline Slight ly Stable & 22.1 & 23.3 & 22.6 & 22.8 & 21.8 \\
\hline Stable & 25.1 & 22.2 & 45.0 & 27.3 & 18.0 \\
\hline Very Stable & 5.6 & 10.4 & 9.6 & 3.8 & 3.2 \\
\hline
\end{tabular}




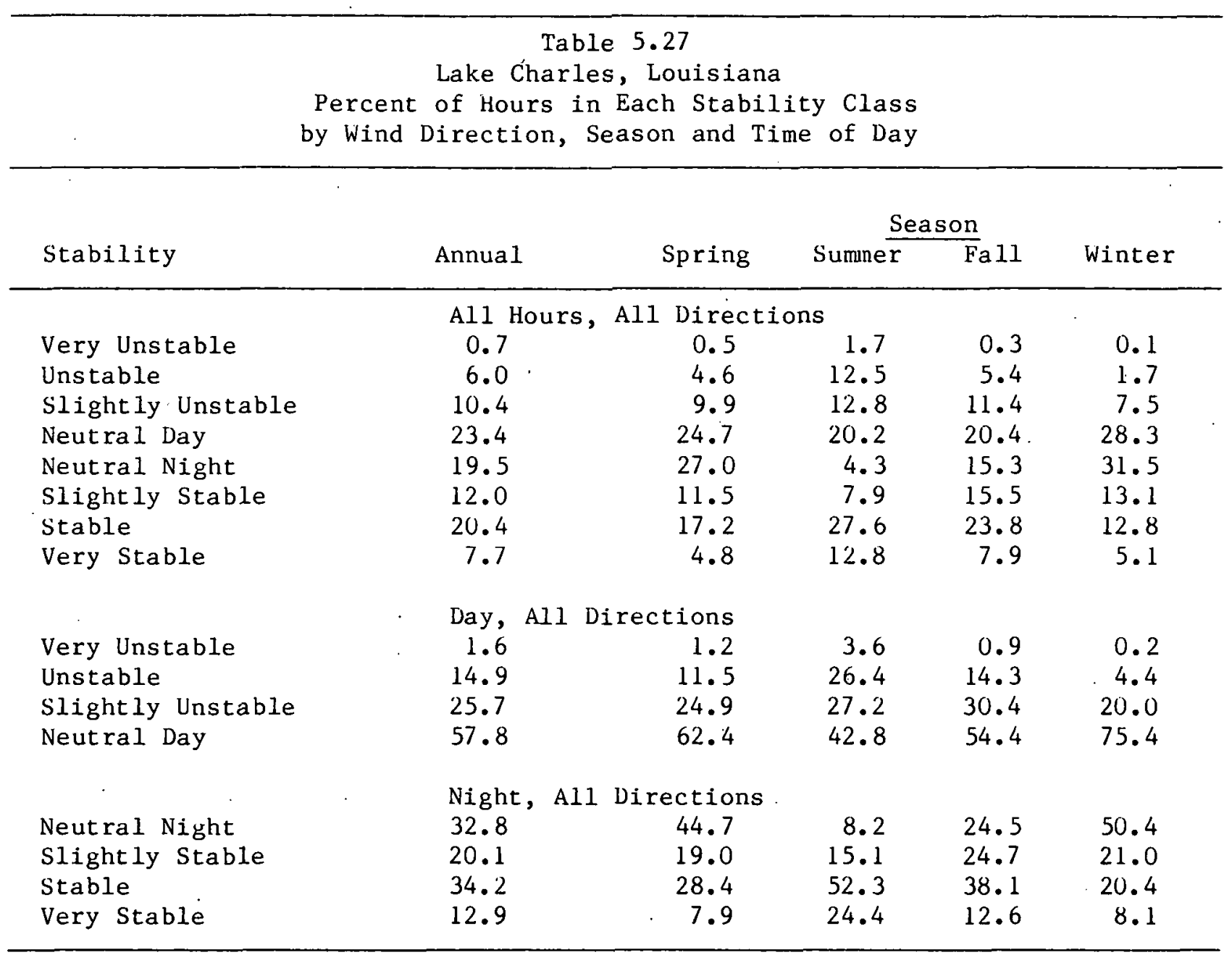


Table 5.27 (continued)

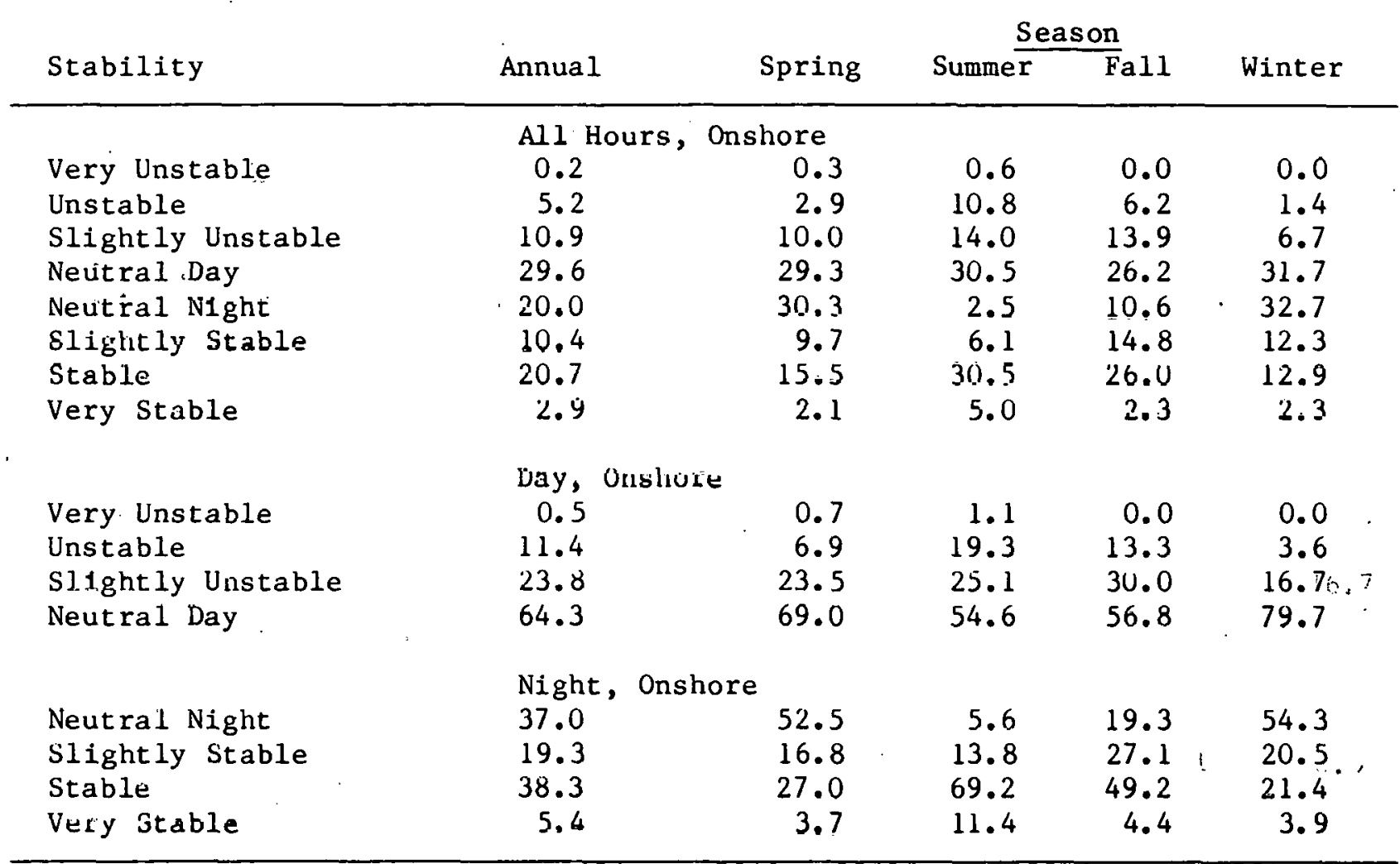


Table 5.27 (continued)

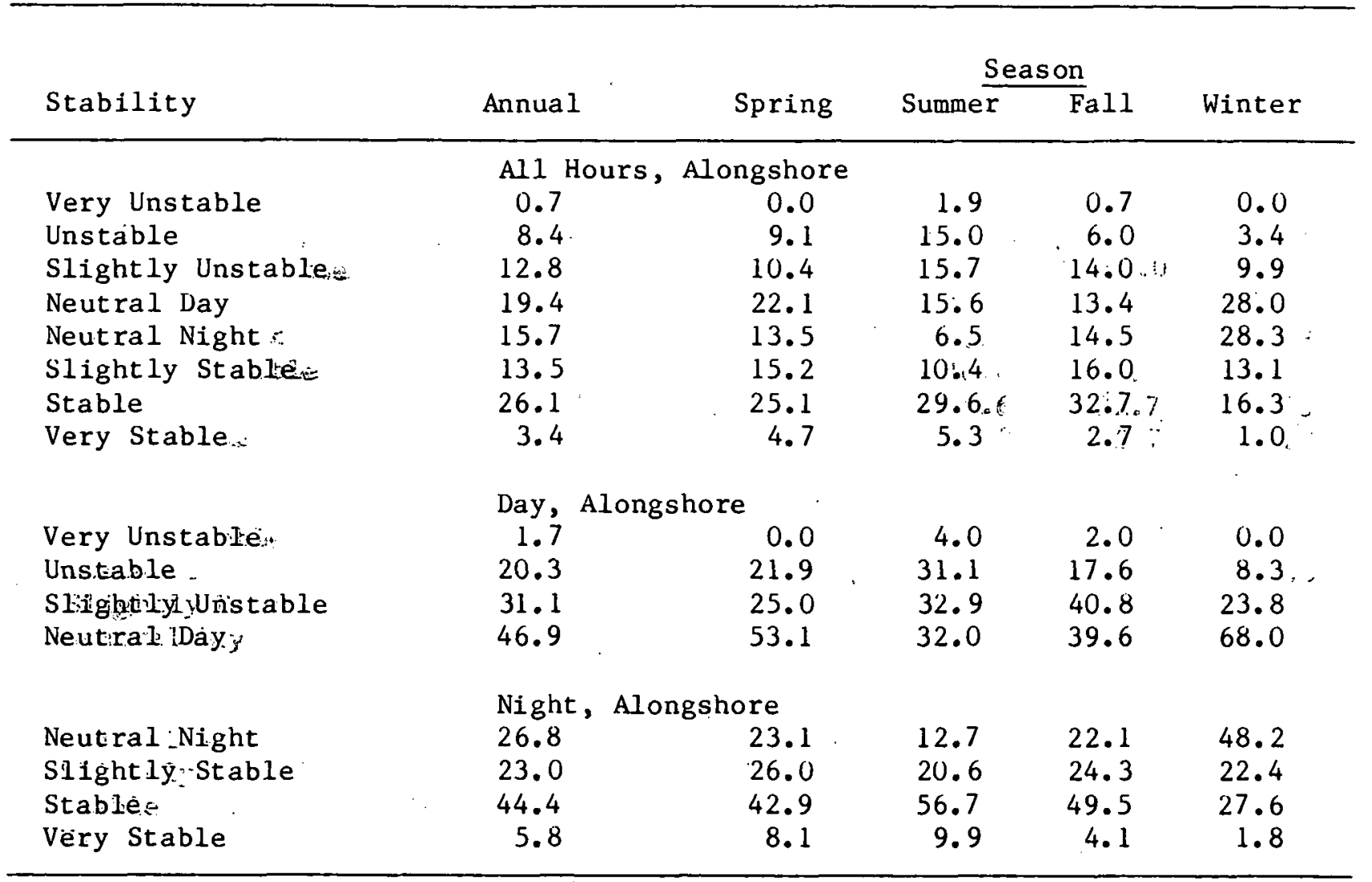


Table 5.27 (continued)

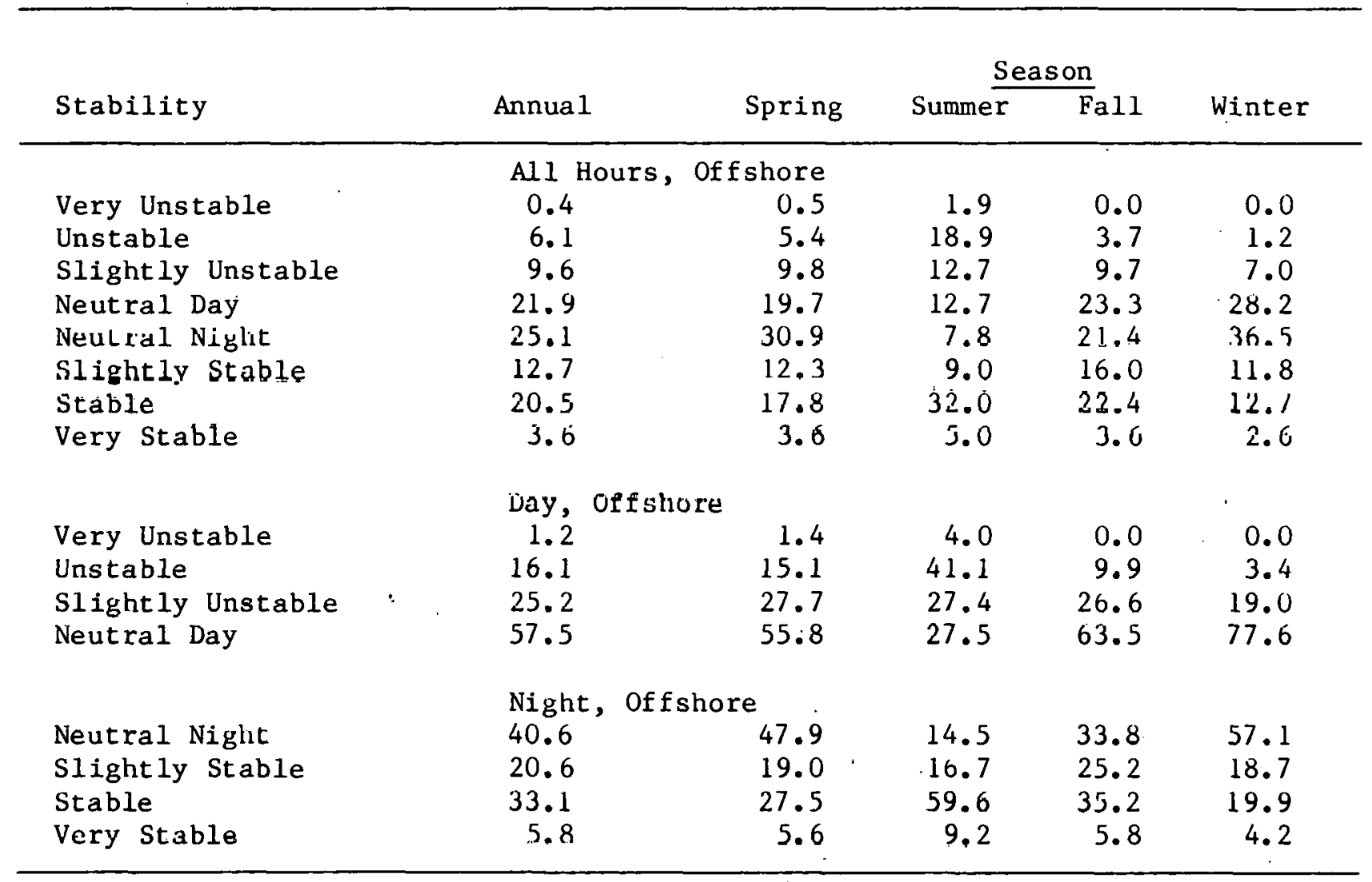




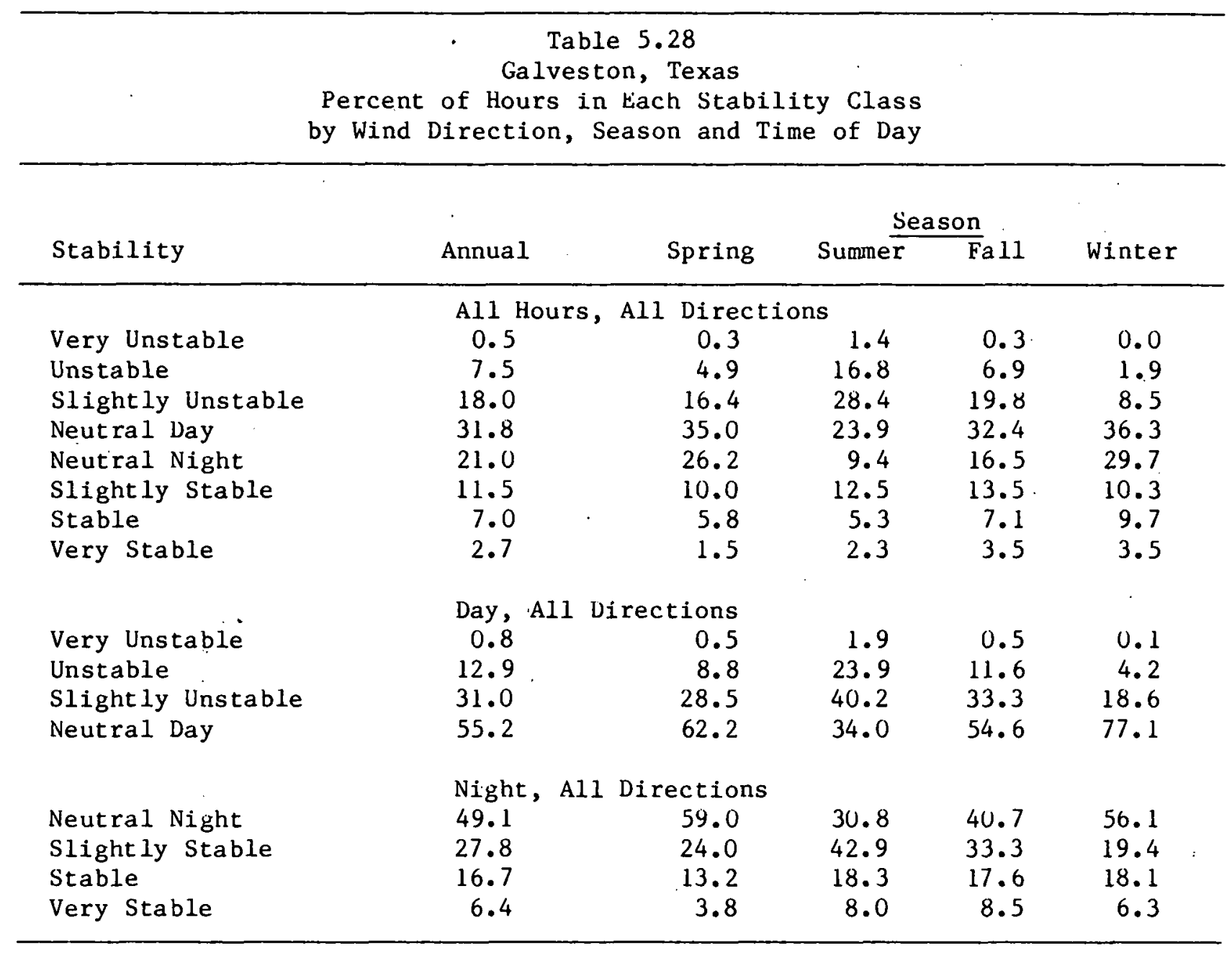


Table 5.28 (continued)

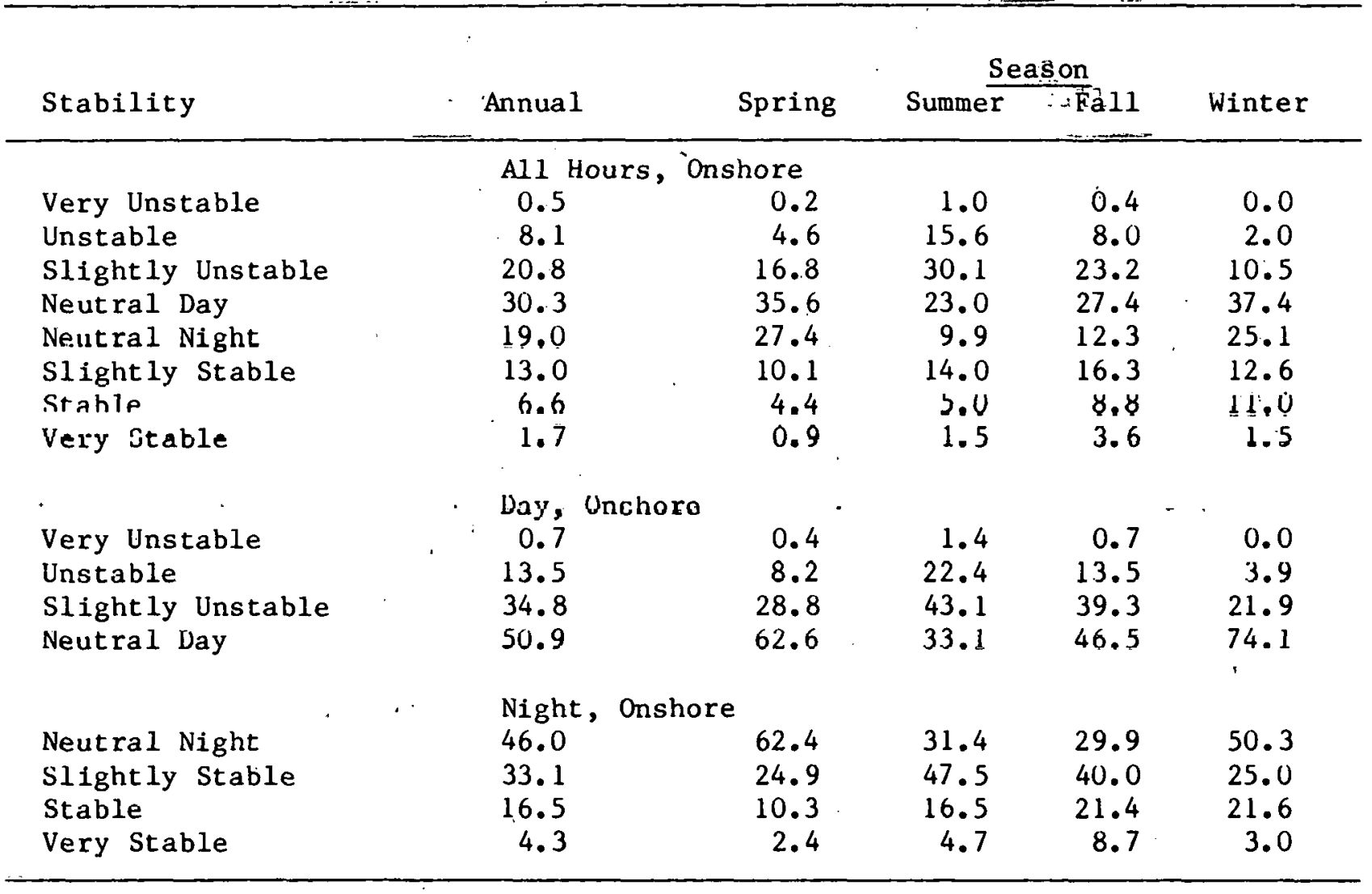


Table 5.28 (continued)

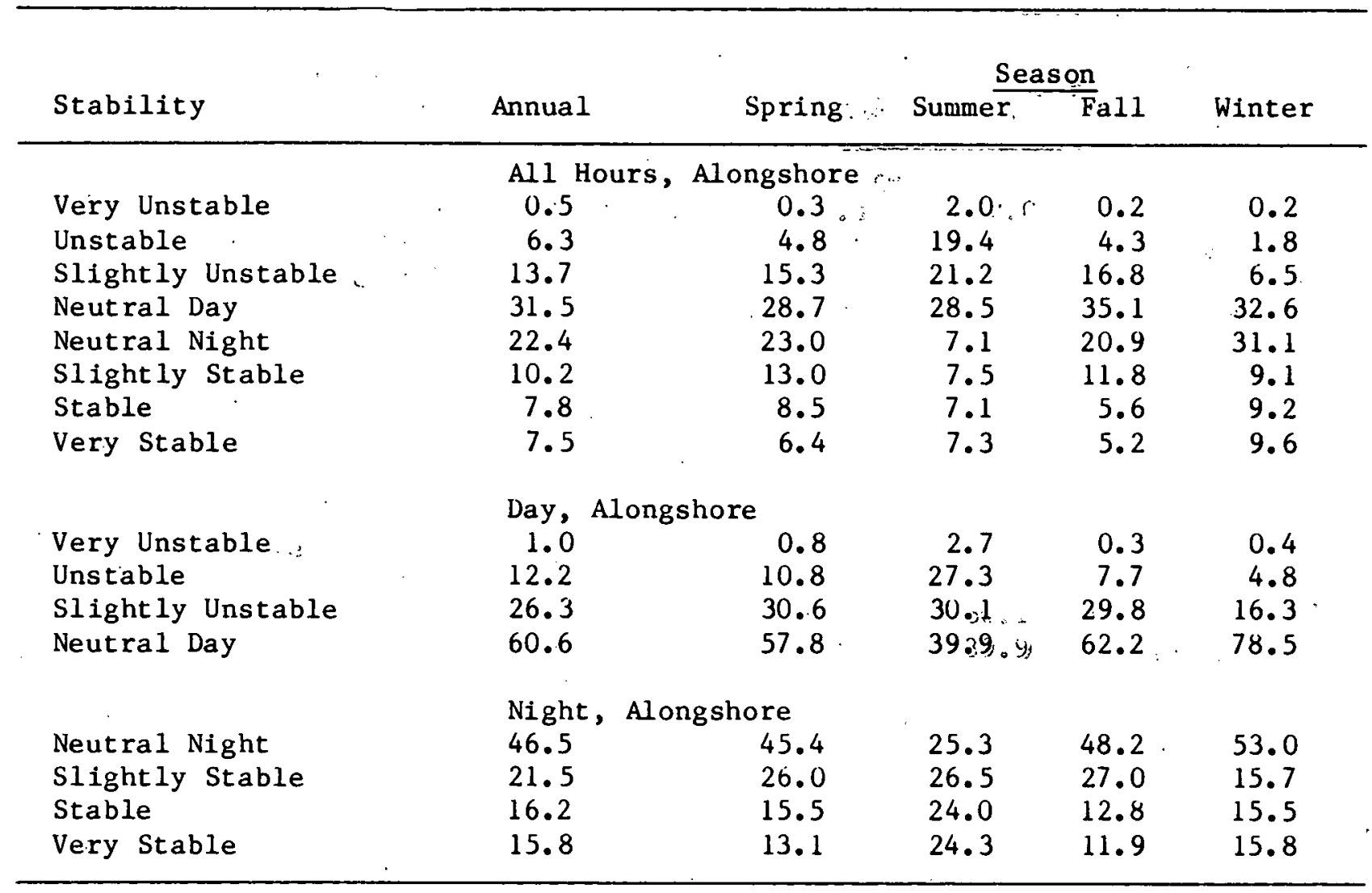


Table 5.28 (continued)

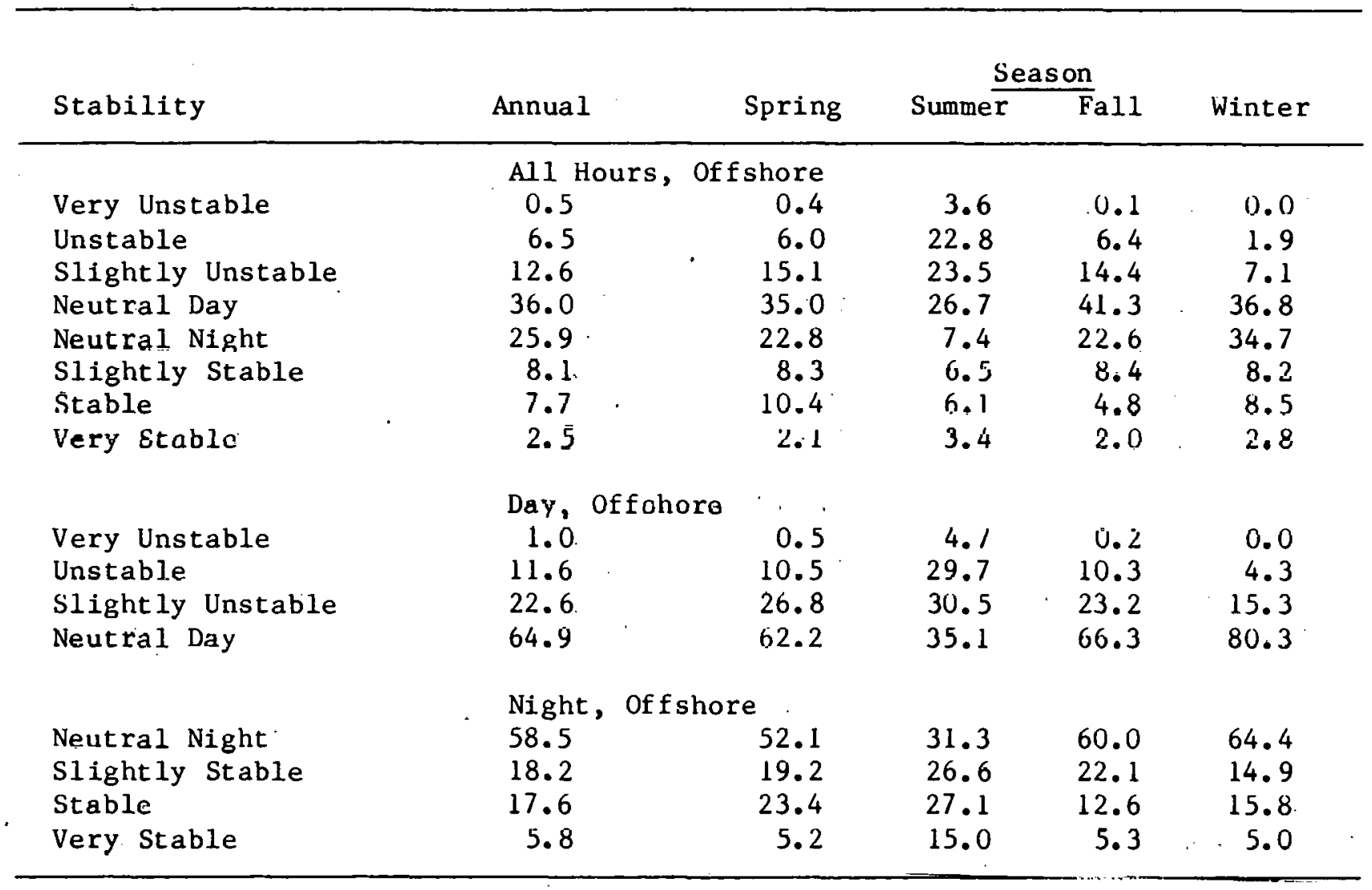


Table 5.29

Houston, Texas

Percent of Hours in Each Stability Class

by Wind Direction, Season and Time of Day

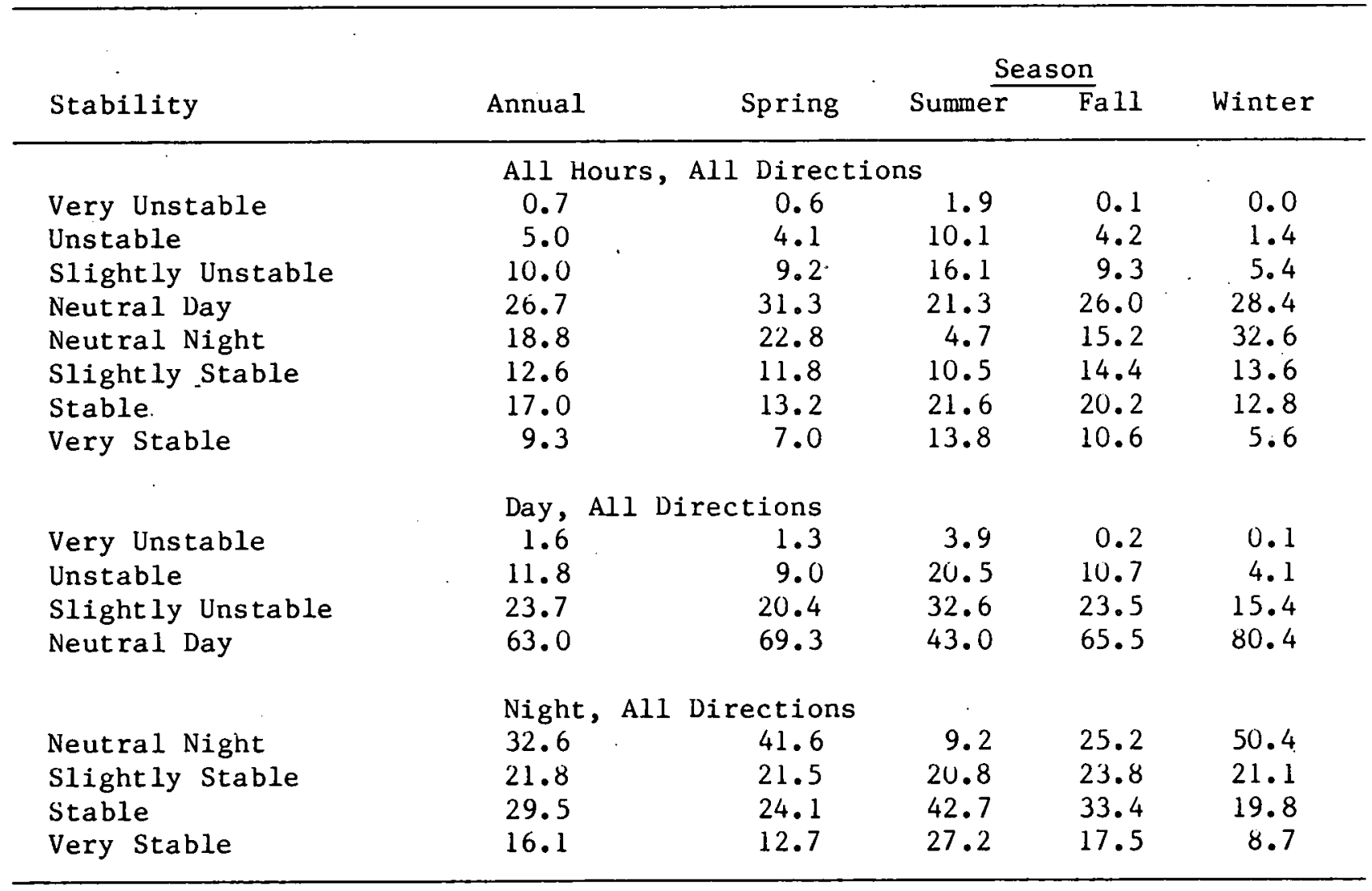


Table 5.29 (continued)

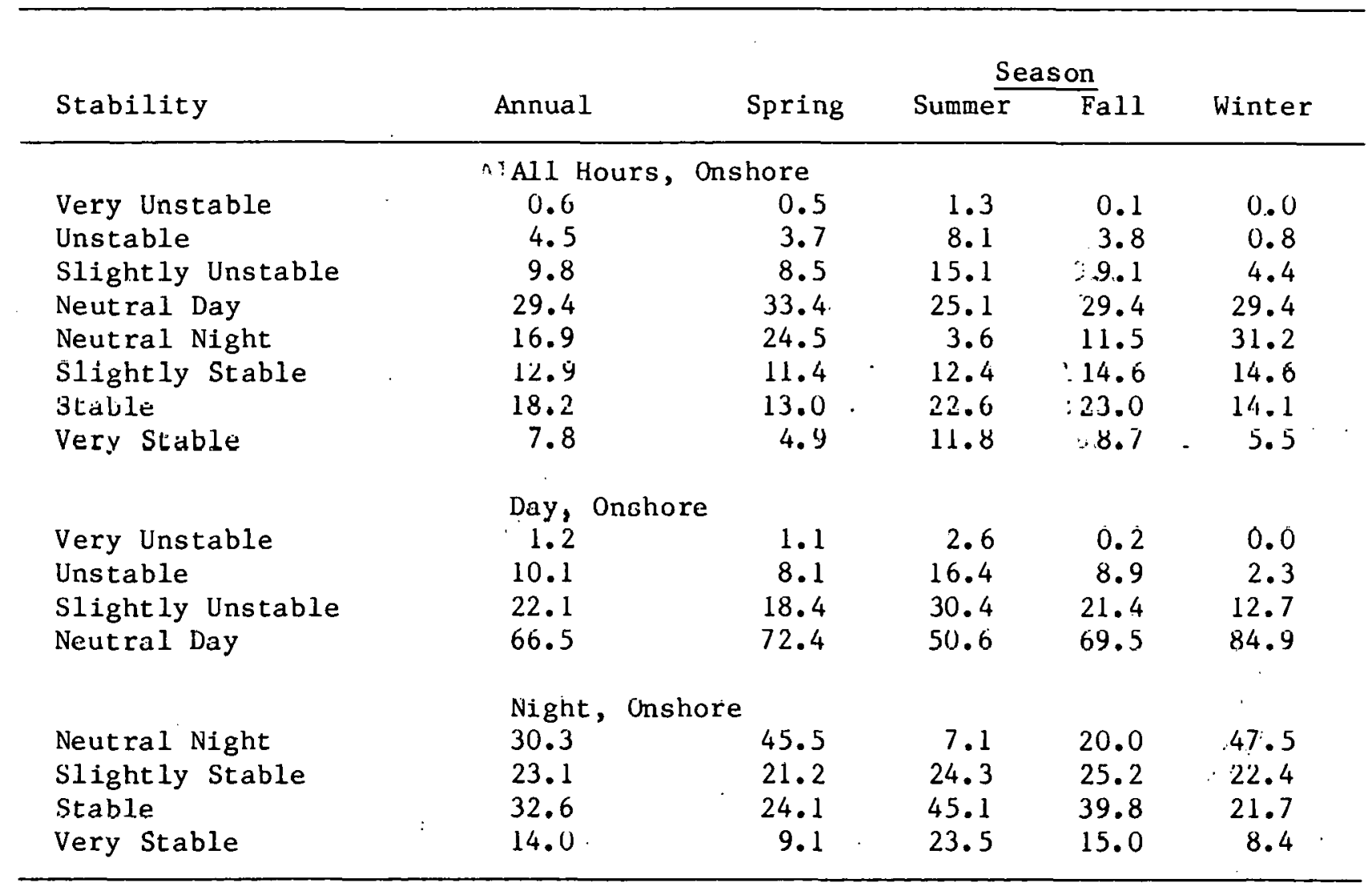


Table 5.29 (continued)

\begin{tabular}{|c|c|c|c|c|c|}
\hline \multirow[b]{2}{*}{ Stability } & \multirow[b]{2}{*}{ Annua 1} & \multicolumn{4}{|c|}{ Season } \\
\hline & & Spring & Summe $\bar{r}$ & Fall & Winter \\
\hline \multicolumn{6}{|c|}{ All Hours, Alongshore } \\
\hline Very Unstable & 1.2 & 1.0 & 3.7 & 0.1 & 0.0 \\
\hline Unstable & 7.5 & 8.0 & 14.1 & 5.3 & 2.8 \\
\hline Slightly Unstable & 13.8 & 13.1 & 18.9 & 14.1 & 8.4 \\
\hline Neutral Day & 21.8 & 24.1 & 15.5 & 23.1 & 26.2 \\
\hline Neutral Night & 17.1 & 18.7 & 7.2 & 14.6 & 29.0 \\
\hline Slightly Stable & 11.6 & 11.8 & 8.5 & 14.2 & 12.2 \\
\hline Stable & 18.4 & 14.4 & 21.1 & 19.8 & 15.6 \\
\hline Very Stable . & 8.6 & 8.9 & 10.9 & 8.7 & 5.8 \\
\hline \multicolumn{6}{|c|}{ Day, Alongshore } \\
\hline Very Unstable & 2.8 & 2.1 & 7.1 & 0.3 & 0.0 \\
\hline Unstable & 16.9 & 17.3 & 27.0 & 12.5 & 7.6 \\
\hline Slight1y Unstable & 31.1 & 28.4 & 36.2 & 33.1 & 23.0 \\
\hline Neutral Day & 49.2 & 52.2 & 29.7 & 54.2 & 69.5 \\
\hline \multicolumn{6}{|c|}{ Night, Alongshore } \\
\hline Neuțral Night & 30.5 & 34.7 & 15.2 & 25.4 & 46.3 \\
\hline Slightly Stable & 20.9 & 22.0 & 17.8 & 24.7 & 19.7 \\
\hline Stable & 33.2 & 26.7 & 44.1 & 34.6 & 25.0 \\
\hline Very Stable & 15.4 & 16.6 & 22.9 & 15.3 & 9.1 \\
\hline
\end{tabular}


Table 5.29 (continued)

\begin{tabular}{|c|c|c|c|c|c|}
\hline \multirow[b]{2}{*}{ Stability } & \multirow[b]{2}{*}{ Annua 1} & \multirow[b]{2}{*}{ Spring } & \multicolumn{2}{|c|}{ Season } & \multirow[b]{2}{*}{ Winter } \\
\hline & & & Summer & $\bar{F} a 11$ & \\
\hline \multicolumn{6}{|c|}{ All Hours, Of fshore } \\
\hline Very Unstable & 0.5 & 0.6 & 2.3 & 0.0 & 0.0 \\
\hline Unstable & 4.5 & 3.5 & 13.4 & 4.5 & 1.4 \\
\hline Slightly Unstable & 8.7 & 10.3 & 18.0 & 7.4 & 4.9 \\
\hline Neutral Day & 26.7 & 31.1 & 17.7 & 24.3 & 29.9 \\
\hline Neutral Night & 24.9 & 21.4 & 7.1 & 22.4 & 36.5 \\
\hline Slightly Stable & 1.2 .3 & 12.7 & 5.8 & 15.0 & 12.7 \\
\hline Stahle & 14.9 & 14.4 & 20.9 & 17.3 & 10.7 \\
\hline Veryy stable & 1.5 & 6.1 & 14,8 & 9.2 & 3.9 \\
\hline \multicolumn{6}{|c|}{ Day, offshore } \\
\hline Very Unstable & 1.1 & 1.3 & 4.4 & 0.0 & 0.0 \\
\hline Unstable & 11.1 & 7.6 & 26.1 & 12.4 & 3.9 \\
\hline Slightly Unstable & 21.5 & 22.6 & 34.7 & 20.3 & 13.5 \\
\hline Neutral Day & 66.3 & 68.5 & 34.8 & 67.3 & 82.6 \\
\hline \multicolumn{6}{|c|}{ Night, offshore } \\
\hline Neutral Night & 41.8 & 39.2 & 14.3 & 35.0 & 57.2 \\
\hline Slightly Stable & 20.7 & 23.2 & 11.6 & 23.4 & 19.9 \\
\hline Stable & 24.9 & 26.4 & 42.8 & 27.1 & 16.7 \\
\hline Very Stable & 12.6 & 11.2 & 31.3 & 14.4 & 6.1 \\
\hline
\end{tabular}




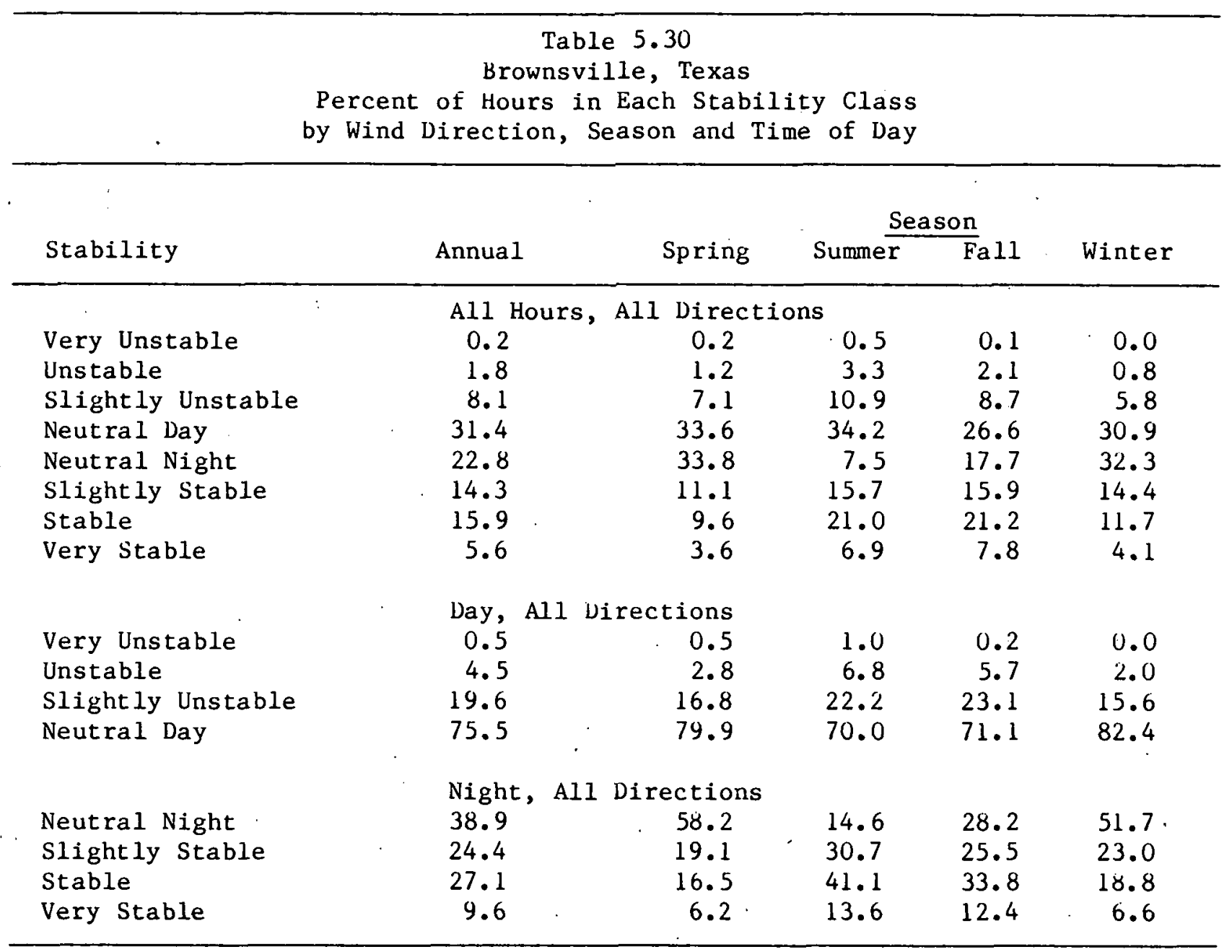


Table 5.30 (continued)

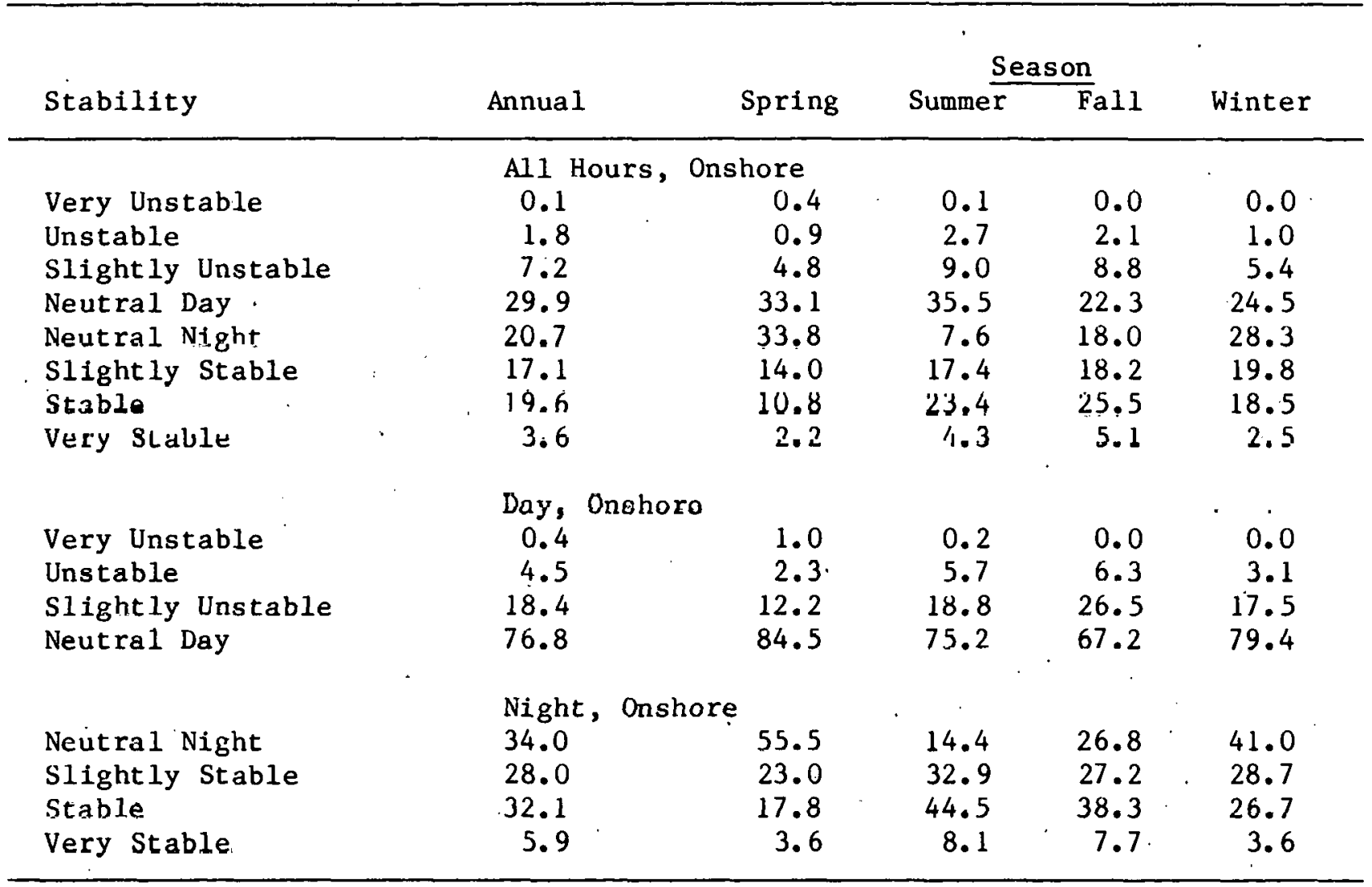


Table 5.30 (continued)

\begin{tabular}{|c|c|c|c|c|c|}
\hline \multirow[b]{2}{*}{ Stability } & \multirow[b]{2}{*}{ Annual } & \multicolumn{4}{|c|}{ Season } \\
\hline & & Spring & Summer & Fall & Winter \\
\hline \multicolumn{6}{|c|}{ Al1 Hours, Alongshore } \\
\hline Very Unstable & 0.0 & 0.0 & 0.0 & 0.0 & 0.0 \\
\hline Unstable & 1.5 & 1.3 & 3.3 & 1.8 & 0.3 \\
\hline Slightly Unstable & 10.0 & 10.8 & 16.8 & 9.2 & 5.3 \\
\hline Neutral Day & 37.2 & 37.2 & 36.9 & 37.7 & 37.2 \\
\hline Neutral Night & 25.6 & 37.5 & 8.1 & 16.2 & 34.8 \\
\hline Slightly Stable & 11.6 & 6.8 & 14.5 & 15.0 & 11.1 \\
\hline Stable & 10.6 & 4.8 & 15.6 & 16.2 & 7.8 \\
\hline Very Stable & 3.4 & 1.7 & 4.8 & 4.0 & 3.5 \\
\hline \multicolumn{6}{|c|}{ Day, Alongshore } \\
\hline Very Unstable & 0.0 & 0.0 & 0.0 & 0.0 & 0.0 \\
\hline Unstable & 3.1 & 2.6 & 5.7 & 3.6 & 0.7 \\
\hline Slightly Unstable & 20.6 & 22.0 & 29.5 & 19.0 & 12.4 \\
\hline Neutral Day & 76.3 & 75.3 & 64.8 & 77.4 & 86.9 \\
\hline \multicolumn{6}{|c|}{ Night, Alongshore } \\
\hline Neutral Night & 49.9 & 74.0 & 18.9 & 31.5 & 60.8 \\
\hline Slightly Stable & 22.7 & 13.4 & 33.8 & 29.1 & 19.4 \\
\hline Stable & 20.7 & 9.4 & 36.1 & 31.5 & 13.7 \\
\hline Very Stable & 6.7 & 3.3 & 11.2 & 7.8 & 6.2 \\
\hline
\end{tabular}


Table 5.30 (continued)

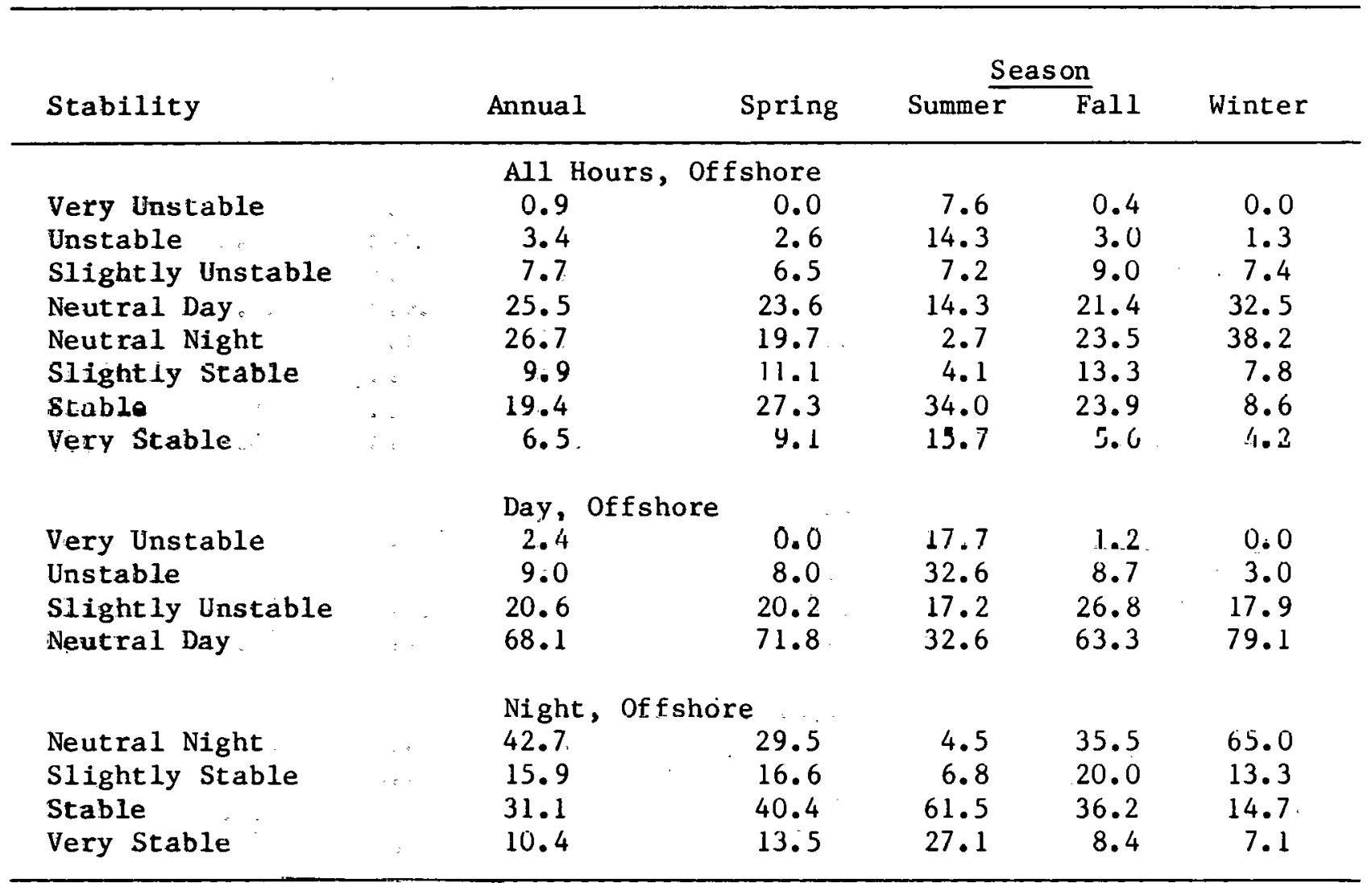


Table 6.1

Portland, Maine

Percent of Hours in Each Wind Speed Class

by Wind Direction, Season and Time of Day

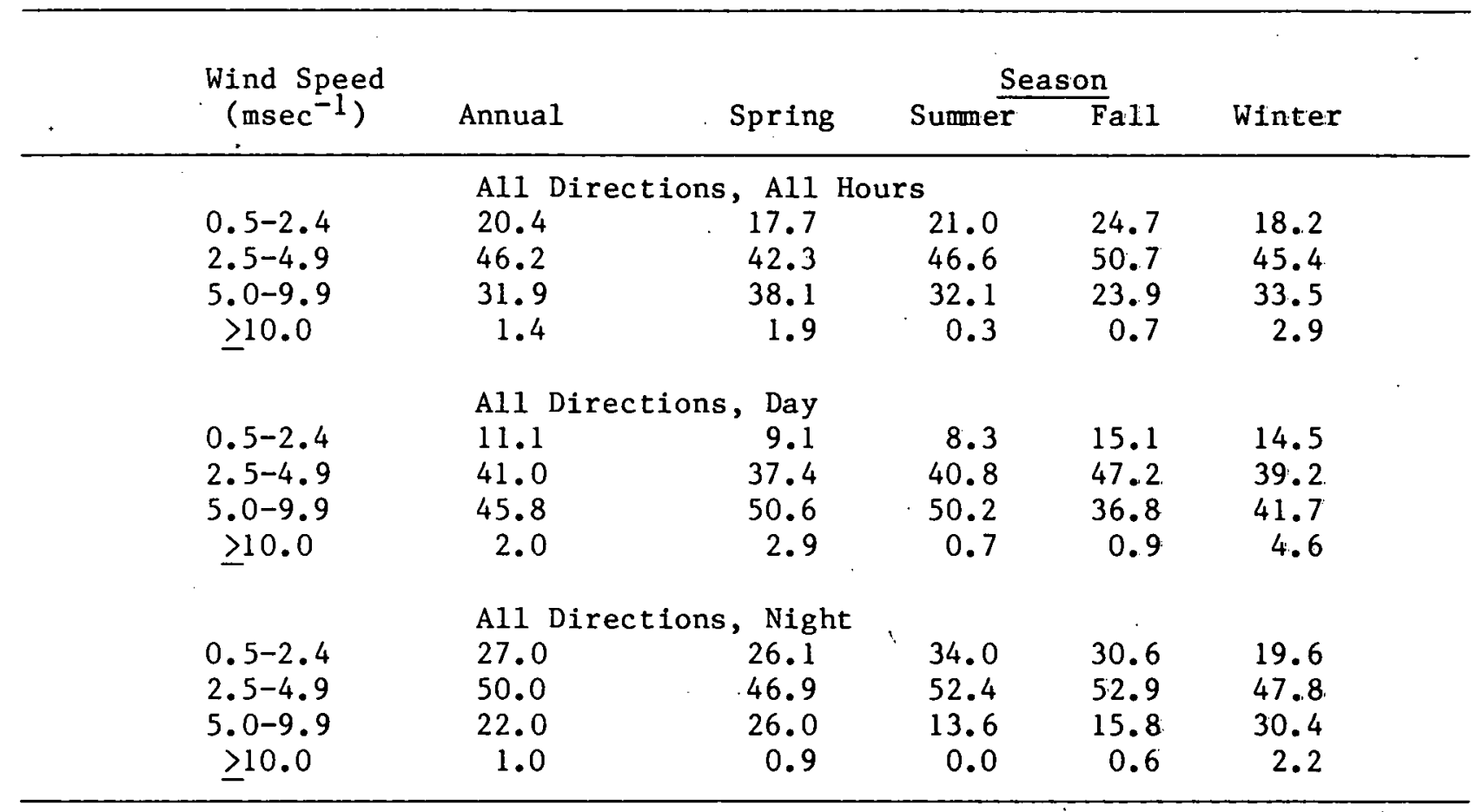




\section{Table 6.1 (continued)}

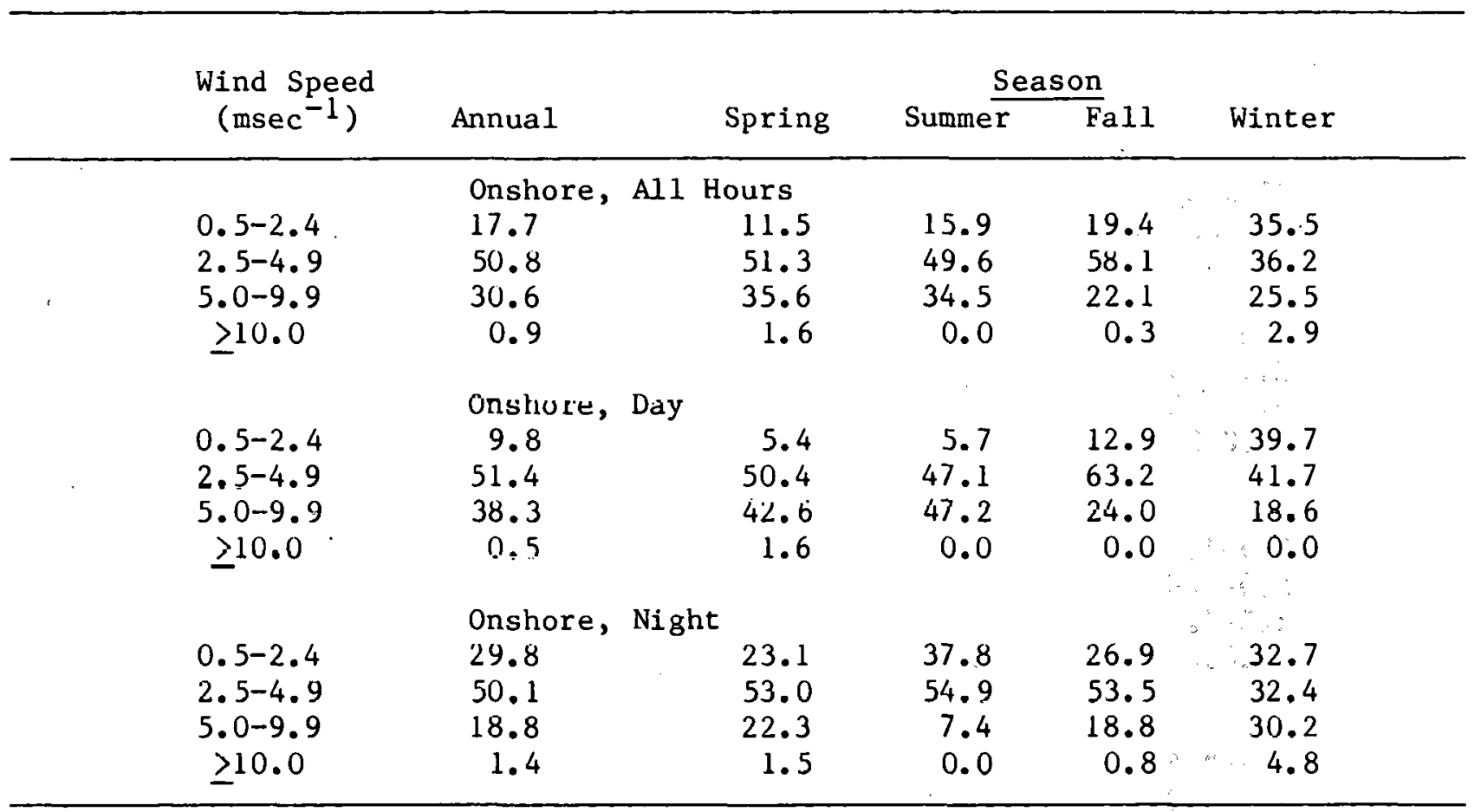


Table: $6 \pi$ do) (continued)

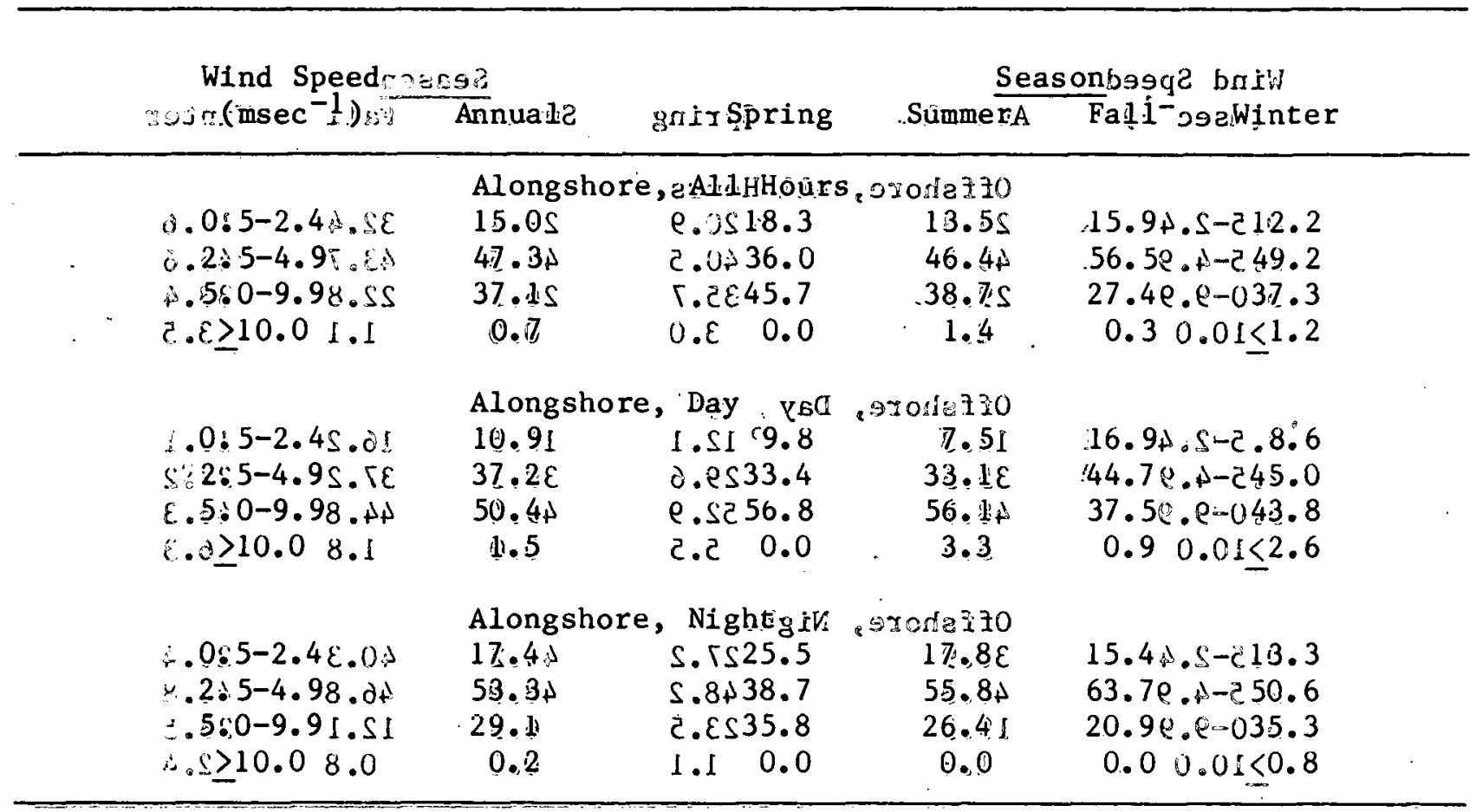




\section{Tábleí 6rdo) (continủed)}

\begin{tabular}{|c|c|c|c|c|}
\hline \multicolumn{2}{|c|}{ Wind Speedmozss } & \multirow[b]{2}{*}{ gnixsp̀ng } & \multicolumn{2}{|c|}{ Seasonbaga' briv. } \\
\hline 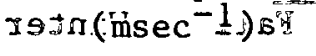 & Añnualid & & SümmerA & Fail sqawinter \\
\hline \multicolumn{5}{|c|}{ 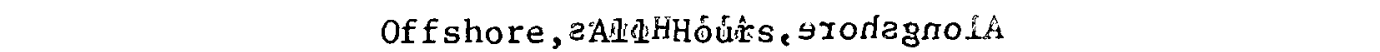 } \\
\hline$S .0 ! 5-2.49 .25$ & 24.31 & $\varepsilon .8120 .9$ & 29.41 & $32.4 .9-217.6$ \\
\hline$s .2 s 5-4.9 z . \partial z$ & 43.78 & $0 . \partial \varepsilon 40.5$ & 44.5 & 43.79 .15 .6 \\
\hline$\varepsilon .5 .0-9.9 \mathrm{H} . \mathrm{is}$ & $29.9 \varepsilon$ & $\Gamma .2+35.7$ & $26 . \overline{I E}$ & $22.89 .2-033.4$ \\
\hline$S .1 \geq 10.0 \varepsilon .0$ & 2.1 & $0.0 \quad 3.0$ & 0.0 & $1.10 .01 \leq 3.5$ \\
\hline & offshore, & \multicolumn{3}{|c|}{ Day Y.6 , gronegnoLA } \\
\hline$a_{0} 0.52 .4 \bar{q} \cdot d i$ & $1 \overline{2} \cdot \overline{3}$ & K.t 12.1 & 19. & $16.21 .5-8-810.1$ \\
\hline $0.245-4.9 T .+4$ & $35.5 \mathrm{C}$ & $+. C<29.6$ & $39.1 \varepsilon$ & $37.2+.+-37.2$ \\
\hline $8.5 \$ 0-9.92 .1 \mathcal{E}$ & 48.72 & 8.0252 .9 & $49.4 \mathrm{C}$ & $44.8 e . e-046.3$ \\
\hline $0.5 \geq 10.0 \quad 0.0$ & 3.4 & $0.0 \quad 5.5$ & 0.0 & 1.84 .0186 .3 \\
\hline \multicolumn{5}{|c|}{ offshore, Nightizin , эrodagnoLA } \\
\hline$\varepsilon .0 .5-2.4+.21$ & $3 \hat{0} . \overline{9 l}$ & ट.टS 27.2 & 44.21 & $40.3+.5-\tau 20.4$ \\
\hline$\partial .27 .5-4.9 \Gamma . \varepsilon \partial$ & $4 \overline{8} \cdot \overline{3} \mathrm{C}$ & $\Gamma .8 \varepsilon 48.2$ & $48 . \hat{g} \mathrm{C}$ & 46.8 e. +-248.8 \\
\hline$\varepsilon .58 .0-9.9 e .0 S$ & $19.5 \mathrm{~s}$ & $8.2 \varepsilon 23.5$ & 6.95 & $12.1 e . e-028.5$ \\
\hline $8.0>10.00 .0$ & 9.3 & $0.0 \quad 1.1$ & 0.0 & $0.8 \quad 0.01 \leq 2.4$ \\
\hline
\end{tabular}


(bounitiabile 6..2) $91 d 8 \mathrm{I}$

boston,-Massachusett-s-

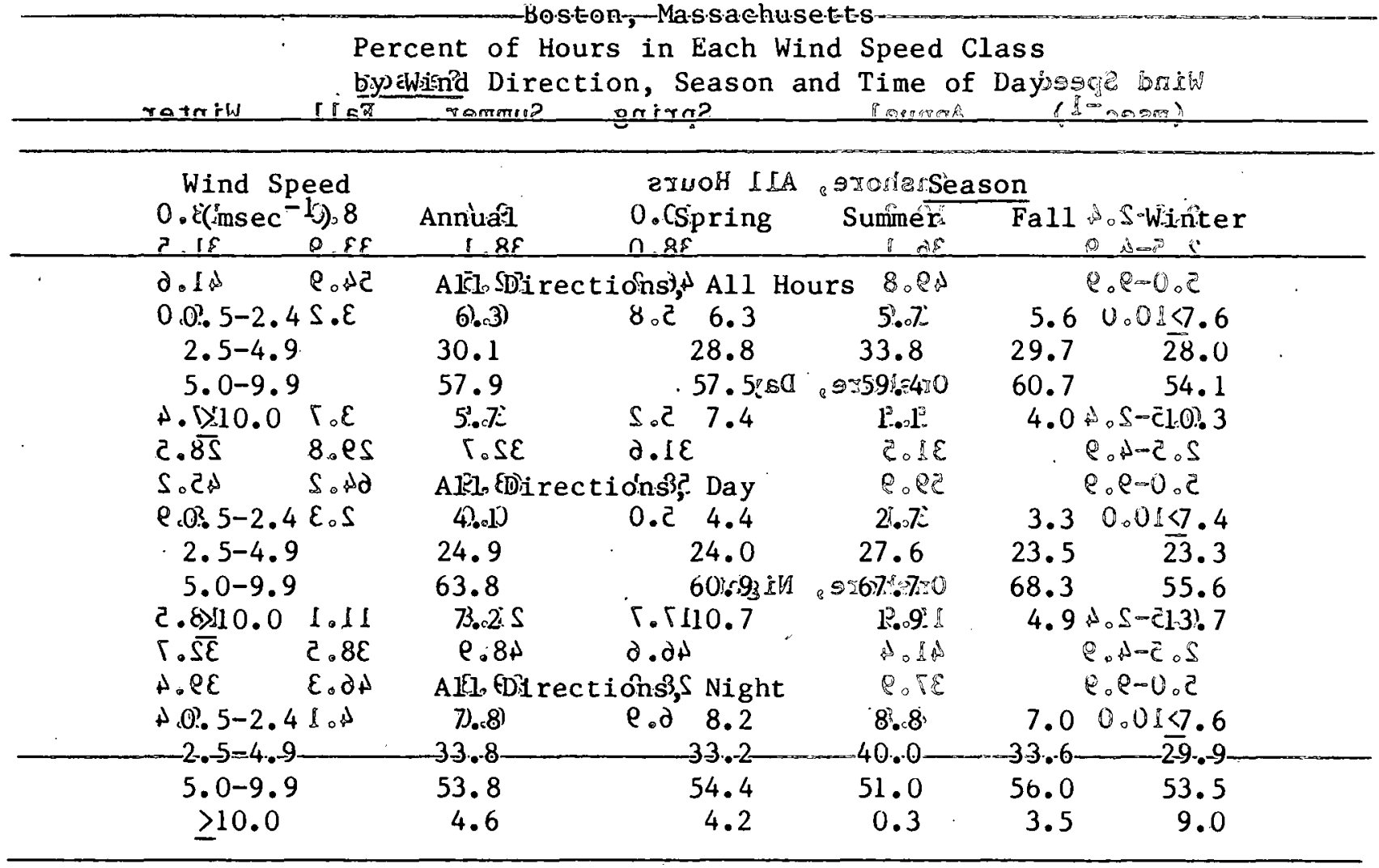


Table 6.2: s(continued)

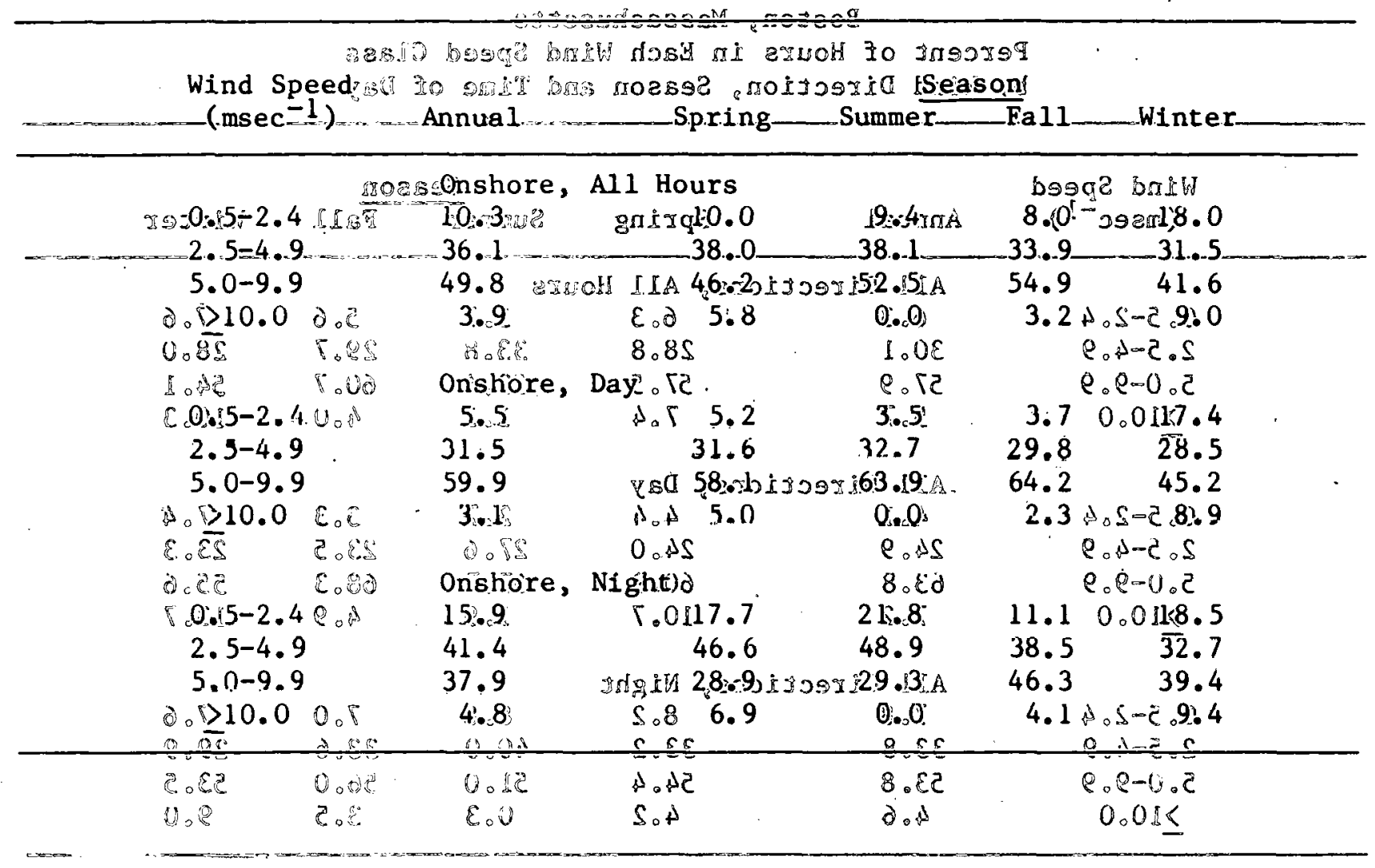


Táblei 6:220) (côntinuéd)

\begin{tabular}{|c|c|c|c|c|}
\hline \multicolumn{2}{|c|}{ Wind Speedroesse? } & \multirow{2}{*}{ 3nirsŝpring } & \multicolumn{2}{|c|}{ Seasond 939 g } \\
\hline ว๑งก.(Imsec -1 -1)s & Annuarle & & Summer & Fall sewinter \\
\hline \multicolumn{5}{|c|}{ Alongshore, a*Allit Hoürse } \\
\hline$e .00 .5-2.42 .5$ & 9.2 & $\mathcal{E} \cdot \mathcal{E} \quad 8.7$ & 7.0 & $8.2+9-212.2$ \\
\hline $5.25-4.93 \cdot 15$ & 39.165 & 0.0540 .7 & 36.5 & 41.5 , $92=39.0$ \\
\hline$c \cdot 570-9.9 \% .0 T$ & 47.78 & $\partial . \partial \partial 48.8$ & 55.7 & 48.400 .0 \\
\hline$I . I D 10.0+. c$ & 83.5 & $0.01: 1.8$ & 0.9 & 2.00008 .8 \\
\hline & Alongs & e, Day Per & $8+20090$ & \\
\hline $10.0 .5-2.45 .1$ & .6 .5 & $0 . \varepsilon \quad 7.3$ & 2.8 & $8.5 \cdot 6.2$ \\
\hline$S .2 ! 5-4.9 \bar{c} \cdot 2 !$ & 33.35 & S.EI 37.6 & $28.2 \%$ & $30.0-40.9$ \\
\hline ट. $5 \geqslant 0-9.9 \varepsilon . \partial \Gamma$ & 55.65 & $\Gamma .0 \partial 50.9$ & 67.1 & $55.8,45.6$ \\
\hline$I . P 10.00 . T$ & 4.6 & 8.21 .4 .2 & 1.9 & $5.6001<7.3$ \\
\hline & Alongs & e, NAghtg & atsotis? & \\
\hline d.0.5-2.4E.E & $10.6^{\circ}$ & $0.8 \quad 99.5$ & 10.1 & 8.1 . \\
\hline $5.2 .5-4.9 \varepsilon . \Omega S$ & 42.78 & $\varepsilon_{\theta} 2 \$ 43.1$ & 42.5 & 46.1 .39 .2 \\
\hline d. $570-9.98 . d 0$ & 443.72 & 2.2847 .4 & 47.4 & $45.4 \circ-37.9$ \\
\hline$e .8 \geq 10.0 \partial . \mathrm{s}$ & 300 & $\left.\partial_{\theta}\right\rangle \quad 0.0$ & 0.0 & $0.504: 9.2$ \\
\hline
\end{tabular}


Table 6r.25) (cônt inúéd)

\begin{tabular}{|c|c|c|c|c|}
\hline \multicolumn{2}{|c|}{ Wind Speedros } & \multirow[b]{2}{*}{ 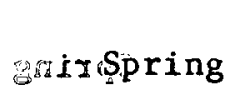 } & \multicolumn{2}{|c|}{ Seasonb9gq" baik } \\
\hline $393\left(\mathrm{msec}^{-1}\right)$ & Annua 1 & & Sümme $\overline{r A}$ & Fali- sagwinter \\
\hline \multicolumn{5}{|c|}{ Of $f$ shore, Ald H Hoürs groragro LA } \\
\hline$S 00.5-2.4 .8$ & 3.4 & 5.83 .3 & 3.6 & $2.5 \$ . S-2.3 .9$ \\
\hline $2.5-4.9$ & 23.9 & $\Gamma .0+20.0$ & $30.8 \varepsilon$ & $21.62+c 23.4$ \\
\hline $5.0-9.9$ & 65.5 & $8.8+66.6$ & $63.9 \mathrm{~s}$ & $70.49 \cdot 9-0.61 .5$ \\
\hline 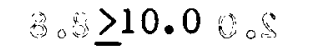 & 7.3 & 8.110 .0 & 1.8 & 5.40 .0111 .1 \\
\hline & Off shore, & Day $\operatorname{Yad}$ & EgroLA & \\
\hline$\therefore 0.5-2.4$ & 2.6 & e.r $3.0^{8}$ & 2,2 & $1.2+03-3.4 .1$ \\
\hline $2 \cdot 5-4.9$ & 17.7. & $0 . \Gamma<13.1$ & $23.8 \varepsilon$ & 15.50 od $\log 17.2$ \\
\hline $5.0-9.9$ & 68.9 & e.02 66.7 & 70.6 & $76.39 .8-0.1 .5$ \\
\hline$\geq \geq 10.0$ & 10.9 & $S_{0}>16.8$ & 3.4 & 7.00 .0117 .1 \\
\hline & offishore, & Nightgils & JagnolA & \\
\hline $0.5-2.4$ & 3.8 & $\pi^{2} 3.6^{8}$ & 4.81 & $3.3 \beta . \Omega-2.3 .8$ \\
\hline $5.2,5-4.9$ & $27 \cdot 7$ & L. E\& 25.3 & $36.3 \mathrm{~A}$ & $25.32 .-25.7$ \\
\hline $5: 0-9.9$ & 63.4 & 的.「\$66.5 & 58.45 & $66.80 \cdot Q-061.6$ \\
\hline$\because \geq 10.0$ & 5.1 & $0.0 \quad 4.6$ & 0.5 & $4.60 .01 \leq 8.9$ \\
\hline
\end{tabular}




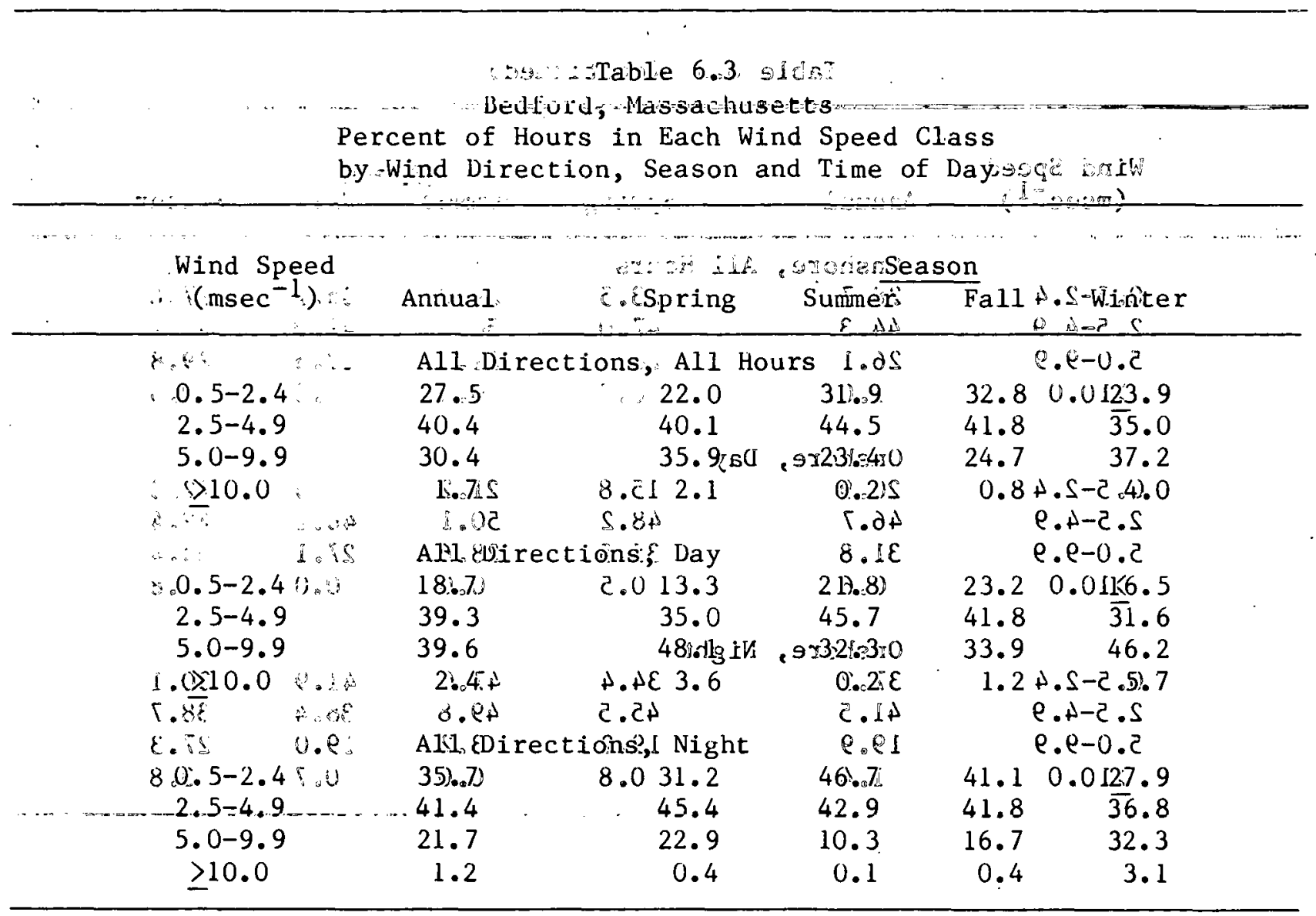


Table 6..3: s(ficontinued)

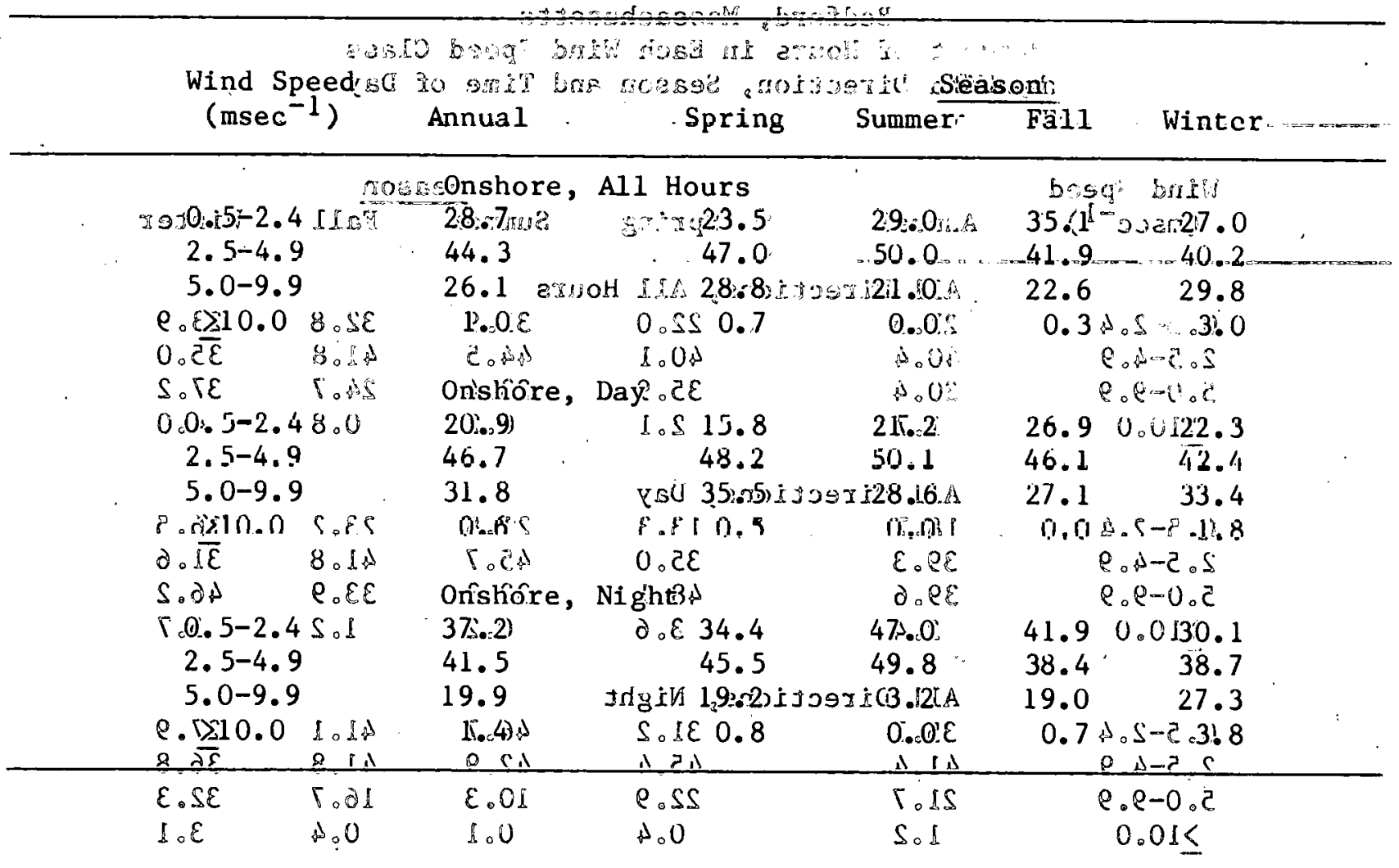


Table 6.3 : (continued)

\begin{tabular}{|c|c|c|c|c|c|c|}
\hline \multirow{2}{*}{$\begin{array}{l}\text { Wind Speed } \\
\left(\mathrm{msec}^{-1}\right)\end{array}$} & \multirow[b]{2}{*}{ Annual } & \multicolumn{4}{|c|}{ Season } & \\
\hline & & Spring & Summe $\bar{r}$ & $\bar{F} a 11$ & Winter & $\therefore$ \\
\hline $\begin{array}{c}0.5-2.4 \\
2.5-4.9 \\
5.0-9.9 \\
\geq 10.0\end{array}$ & $\begin{array}{l}\text { Alongshore, } \\
30.7 \\
40.6 \\
26.8 \\
1.9\end{array}$ & $\begin{array}{c}\text { All Hours } \\
27.9 \\
42.2 \\
29.4 \\
0.5\end{array}$ & $\begin{array}{r}34.6 \\
47.3 \\
17.6 \\
0.5\end{array}$ & $\begin{array}{r}37.1 \\
42.1 \\
20.4 \\
0.4\end{array}$ & $\begin{array}{r}24.5 \\
31.6 \\
38.1 \\
\quad 5.8\end{array}$ & \\
\hline $\begin{array}{c}0.5-2.4 \\
2.5-4.9 \\
5.0-9.9 \\
\geq 10.0\end{array}$ & $\begin{array}{l}\text { Alongshore, } \\
23.6 \\
42.6 \\
32.3 \\
1.5\end{array}$ & $\begin{array}{c}\text { Day. } \\
18.1 \\
42.9 \\
38.3 \\
0.7\end{array}$ & $\begin{array}{r}26.0 \\
48.3 \\
24.7 \\
0.9\end{array}$ & $\begin{array}{r}28.9 \\
43.5 \\
27.2 \\
0.4\end{array}$ & $\begin{array}{r}22.0 \\
33.3 \\
40.1 \\
4.6\end{array}$ & \\
\hline $\begin{array}{l}0.5-2.4 \\
2.5-4.9 \\
5.0-9.9 \\
\geq 10.0\end{array}$ & $\begin{array}{l}\text { Alongshore, } \\
37.5 \\
38.6 \\
21.6 \\
2.3\end{array}$ & $\begin{array}{r}\text { Night } \\
39.5 \\
41.6 \\
18.6 \\
0.3\end{array}$ & $\begin{array}{r}46.6 \\
45.8 \\
7.7 \\
0.0\end{array}$ & $\begin{array}{r}44.4 \\
40.9 \\
14.3 \\
0.3\end{array}$ & $\begin{array}{r}25.9 \\
30.7 \\
37.0 \\
6.4\end{array}$ & \\
\hline
\end{tabular}


Table 6.3, (continued)

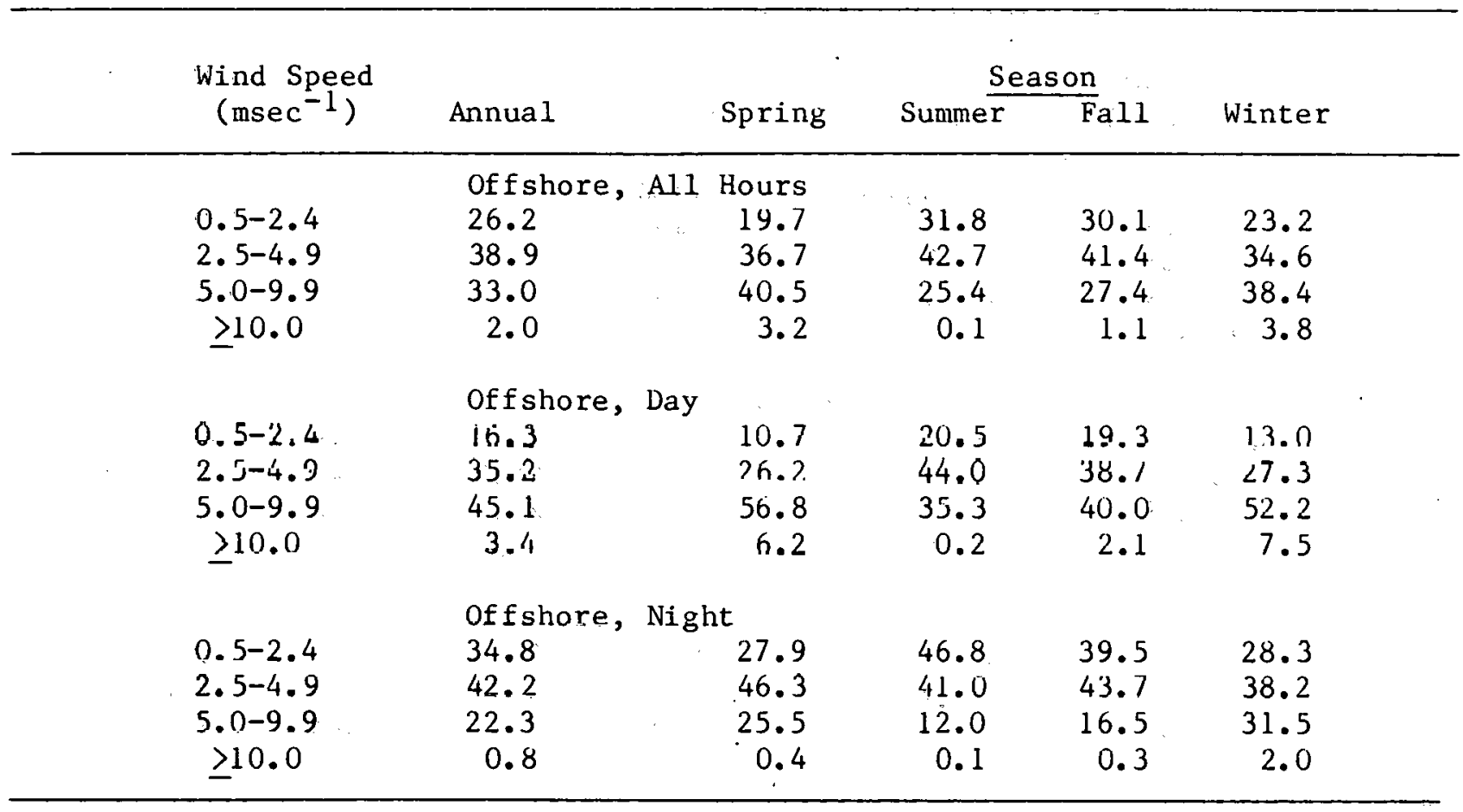


Table 6.4

Falmouth, Massachusetts

Percent of Hours in Each Wind Speed Class

by Wind Direction, Season and Time of Day

\begin{tabular}{|c|c|c|c|c|c|c|}
\hline & \multirow{2}{*}{$\begin{array}{l}\text { Wind Speed } \\
\quad\left(\mathrm{msec}^{-1}\right)\end{array}$} & \multirow[b]{2}{*}{ Annual } & \multirow[b]{2}{*}{ Spring } & \multicolumn{2}{|c|}{ Season } & \multirow[b]{2}{*}{ Winter } \\
\hline & & & & Summer & Fall & \\
\hline & $\begin{array}{l}0.5-2.4 \\
2.5-4.9 \\
5.0-9.9 \\
\geq 10.0\end{array}$ & $\begin{array}{r}\text { A1. } \\
18.5 \\
34.8 \\
41.3 \\
5.3\end{array}$ & $\begin{array}{r}\text { A11 } \\
14.5 \\
32.0 \\
45.6 \\
8.0\end{array}$ & $\begin{array}{r}\text { es } \\
21.6 \\
40.6 \\
37.1 \\
0.6\end{array}$ & $\begin{array}{r}22.2 \\
37.8 \\
36.9 \\
3.1\end{array}$ & $\begin{array}{r}16.2 \\
29.2 \\
45.2 \\
9.4\end{array}$ \\
\hline & $\begin{array}{c}0.5-2.4 \\
2.5-4.9 \\
5.0-9.9 \\
\geq 10.0\end{array}$ & $\begin{array}{l}\text { Al1 I } \\
10.1 \\
32.4 \\
50.7 \\
6.7\end{array}$ & $\begin{array}{r}\text { say } \\
7.0 \\
26.3 \\
55.7 \\
10.9\end{array}$ & $\begin{array}{r}14.0 \\
37.2 \\
47.8 \\
0.9\end{array}$ & $\begin{array}{r}10.1 \\
38.6 \\
47.1 \\
4.2\end{array}$ & $\begin{array}{l}8.3 \\
26.3 \\
52.4 \\
13.0\end{array}$ \\
\hline & $\begin{array}{c}0.5-2.4 \\
2.5-4.9 \\
5.0-9.9 \\
\geq 10.0\end{array}$ & $\begin{array}{l}\text { A11 I } \\
25.6 \\
36.8 \\
33.4 \\
4.2\end{array}$ & $\begin{array}{c}\text {, Night } \\
21.6 \\
37.3 \\
35.9 \\
5.2\end{array}$ & $\begin{array}{r}31.6 \\
45.2 \\
23.0 \\
0.2\end{array}$ & $\begin{array}{r}31.1 \\
37.2 \\
29.5 \\
2.2\end{array}$ & $\begin{array}{r}20.3 \\
30.7 \\
41.5 \\
7.6\end{array}$ \\
\hline
\end{tabular}




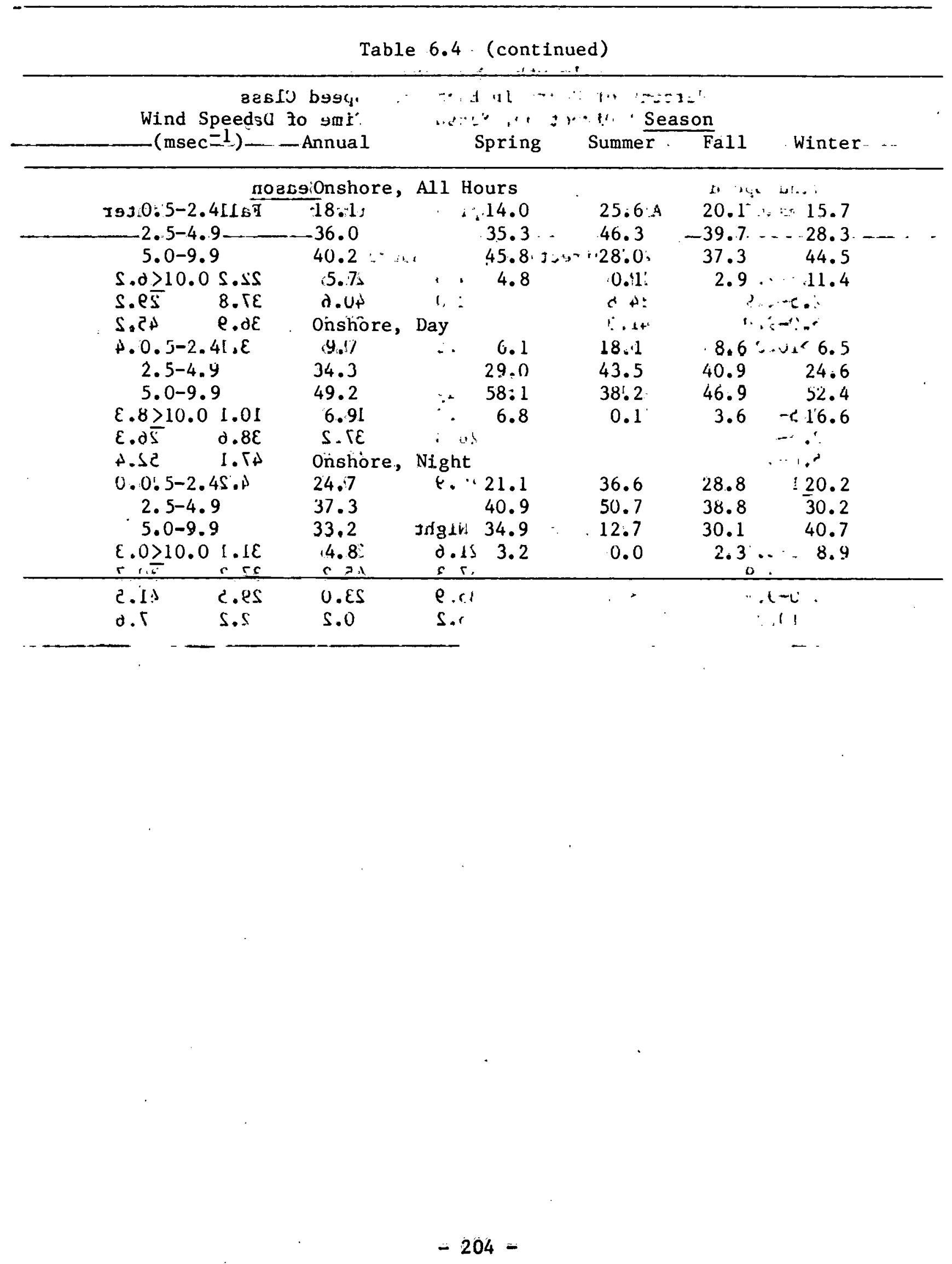


(Tablei $=6 \% 4 ;)$ (cont inued)

\begin{tabular}{|c|c|c|c|c|}
\hline $\begin{array}{l}\text { Wind Speed } \\
\text { trs (msec-litis }\end{array}$ & Annua 1 & incspring & \multicolumn{2}{|c|}{ Seasonjogqe' bis } \\
\hline $\begin{array}{l}0.5-2.4 \\
2.5-4.9 .95 \\
5.0-9.93 .58 \\
\geq 10.08\end{array}$ & $\begin{array}{l}\text { Alongst } \\
15 . .8: \\
30.80 \\
49.0 \\
5.6\end{array}$ & 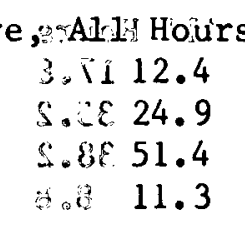 & 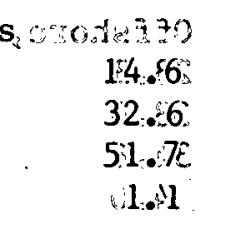 & $\begin{array}{r}18.8,50.34 .3 \\
37.2,0.26 .5 \\
40.6 .00 .1 .3 \\
3.40,0157.9\end{array}$ \\
\hline $\begin{array}{l}0.5-2.4: 31 \\
2.5-4.9: 0.8 \\
5.0-9.9 \\
\geq 10.0\end{array}$ & $\begin{array}{c}\text { Alongst } \\
8.05 \\
23.8 \\
59.8 \\
7.8\end{array}$ & 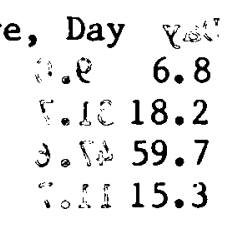 & $\begin{array}{r}10.82 \\
23.52 \\
65.0 .0 \\
81.6\end{array}$ & 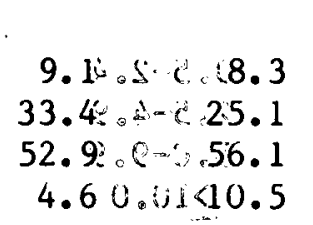 \\
\hline $\begin{array}{c}0.5-2.4 \\
2.5-4.9 \\
5.0-9.9 \\
\geq 10.0\end{array}$ & $\begin{array}{c}\text { Alongst } \\
20.0 \\
35.0 \\
40.44 \\
4.3\end{array}$ & 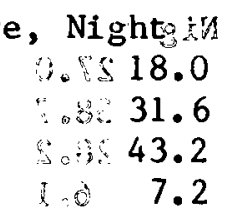 & $\begin{array}{r}0.01200 \\
19.8 \\
43.8 \\
35.89 \\
40.55\end{array}$ & 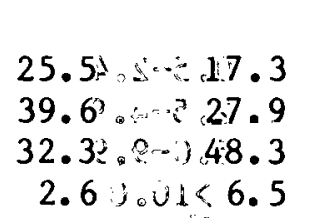 \\
\hline
\end{tabular}




\section{Table 6.4 (continued)}

\begin{tabular}{|c|c|c|c|c|c|c|}
\hline \multirow{2}{*}{$\begin{array}{l}\text { Wind Speed } \\
\left(\mathrm{msec}^{-1}\right)\end{array}$} & \multirow{2}{*}{ Annual. } & \multicolumn{5}{|c|}{ Season } \\
\hline & & & Spring & Summer & Fall & Winter \\
\hline $\begin{array}{l}0.5-2.4 \\
2.5-4.9 \\
5.0-9.9 \\
\geq 10.0\end{array}$ & $\begin{array}{l}\text { Off shore, } \\
23.3 \\
38.0 \\
34.6 \\
4.0\end{array}$ & A11 & $\begin{array}{r}\text { Hours } \\
17.8 \\
35.2 \\
38.2 \\
8.8\end{array}$ & $\begin{array}{c}24.5 \\
43.0 \\
3.1 .9 \\
0.7\end{array}$ & $\begin{array}{r}29.3 \\
35.3 \\
32.2 \\
3.2\end{array}$ & $\begin{array}{r}2.1 .2 \\
37.3 \\
37.6 \\
3.9\end{array}$ \\
\hline $\begin{array}{l}0.5-2.4 \\
2.5-4.9 \\
5.0-9.9 \\
\geq 10.0\end{array}$ & $\begin{array}{l}\text { Offshore, } \\
1.2 .5 \\
38.4 \\
43.8 \\
5 . .3\end{array}$ & $\begin{array}{c}\text { Day } \\
\therefore \\
\therefore \\
\therefore \therefore\end{array}$ & $\begin{array}{r}9.0 \\
31.7 \\
47.6 \\
11.7\end{array}$ & $\begin{array}{r}13.6 \\
43.2 \\
42.0 \\
1.1\end{array}$ & $\begin{array}{c}14.0 \\
39.8 \\
41.1: \\
5.1\end{array}$ & $\begin{array}{r}14.6 \\
35.7 \\
46.2 \\
3.6\end{array}$ \\
\hline $\begin{array}{l}0.5-2.4 \\
2.5-4.9 \\
5.0-9.9 \\
\geq 10.0\end{array}$ & $\begin{array}{l}\text { Off shore, } \\
33.6 \\
37.7 \\
25.8 \\
2.9\end{array}$ & , & $\begin{array}{r}27.0 \\
38.7 \\
28.2 \\
6.1\end{array}$ & $\begin{array}{c}38.4 \\
42.7 \\
18.7 \\
0.2\end{array}$ & $\begin{array}{r}40.5 \\
31.9 \\
25.7 \\
1.9\end{array}$ & $\begin{array}{r}25.4 \\
38.3 \\
32.2 \\
31.4\end{array}$ \\
\hline
\end{tabular}


Table 6.5

Nantucket, Massachusetts

Percent of Hours in Each Wind Speed Class

by Wind Direction, Season and Time of Day

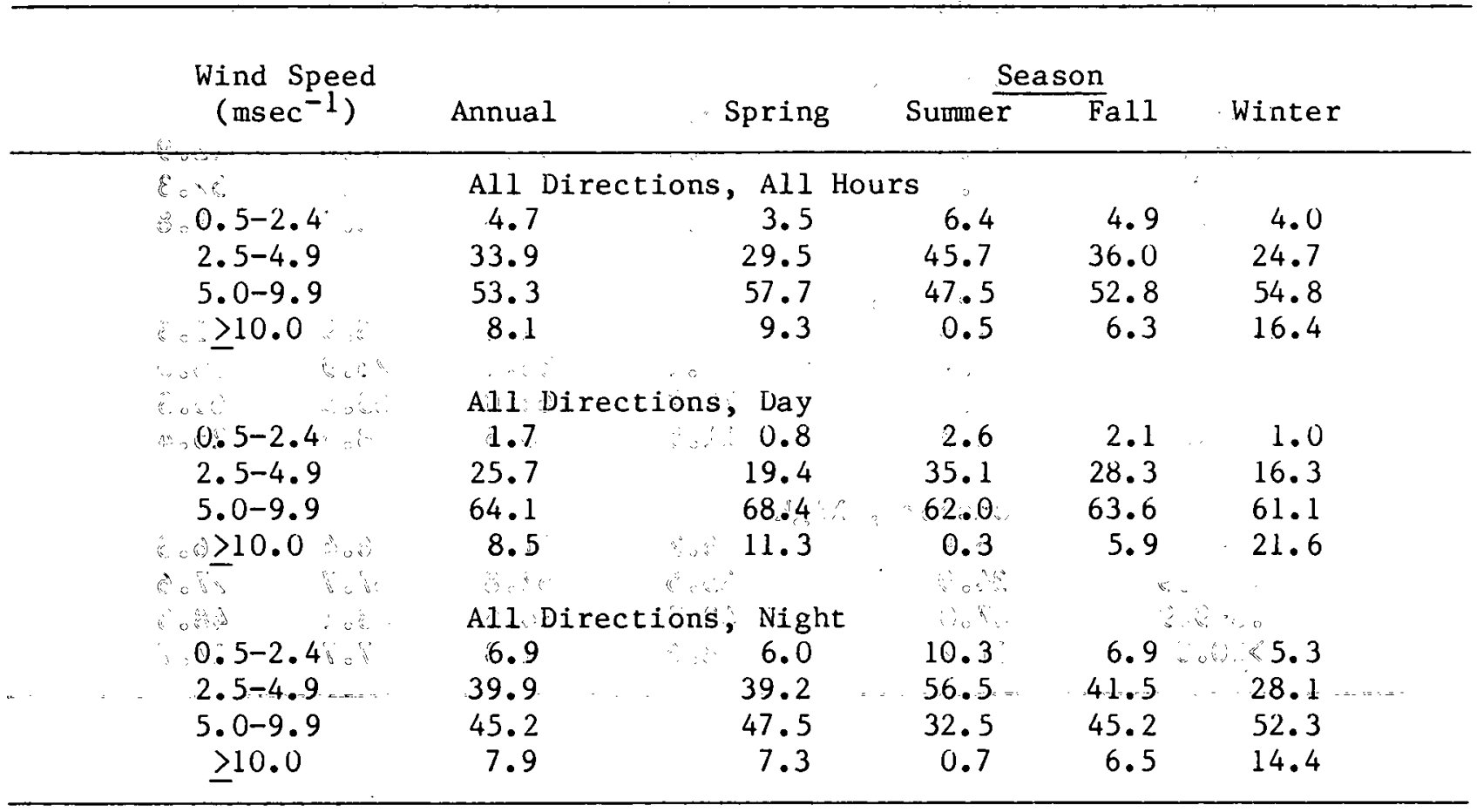


Table 6.5 (continued)

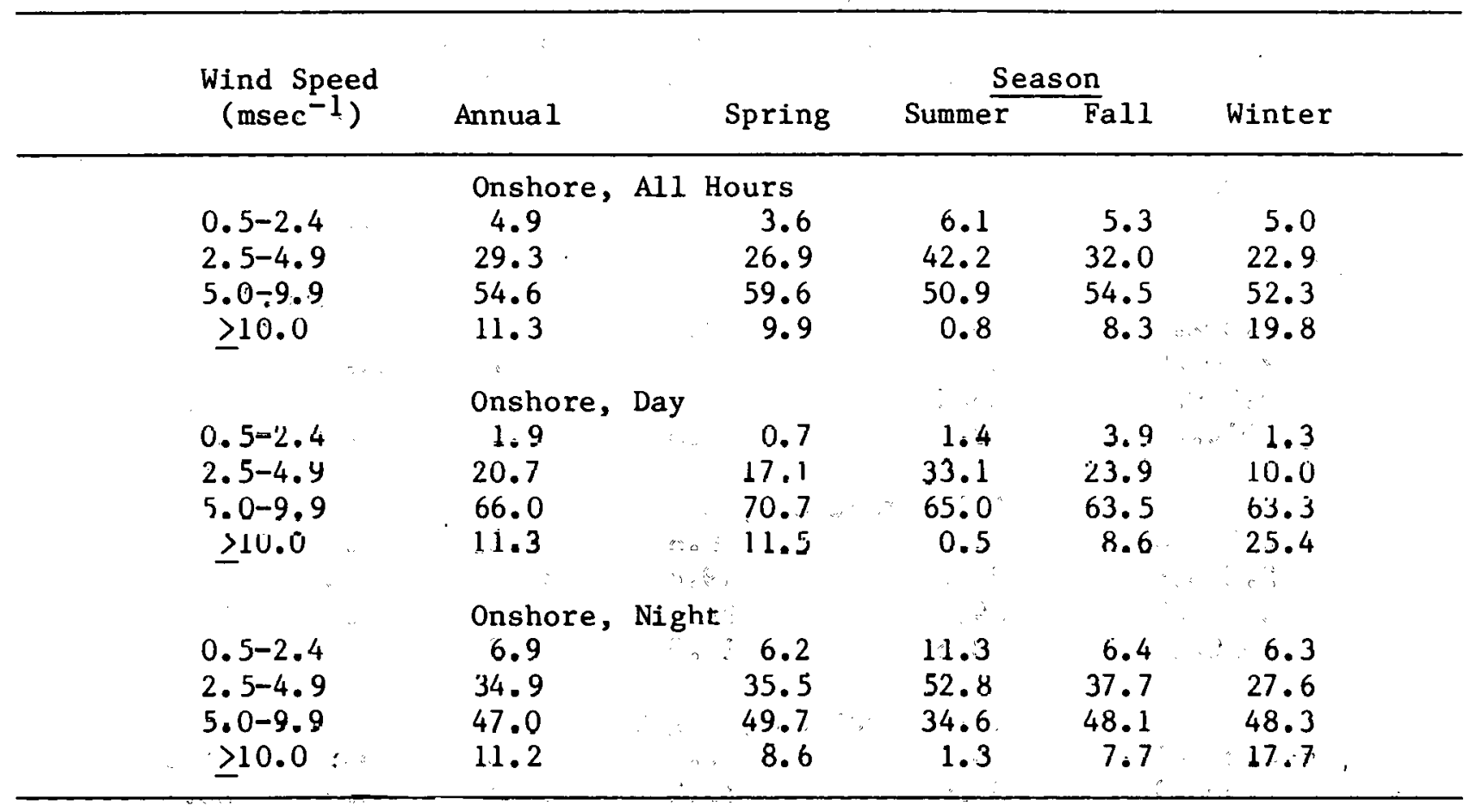


Table 6.5, (continued)

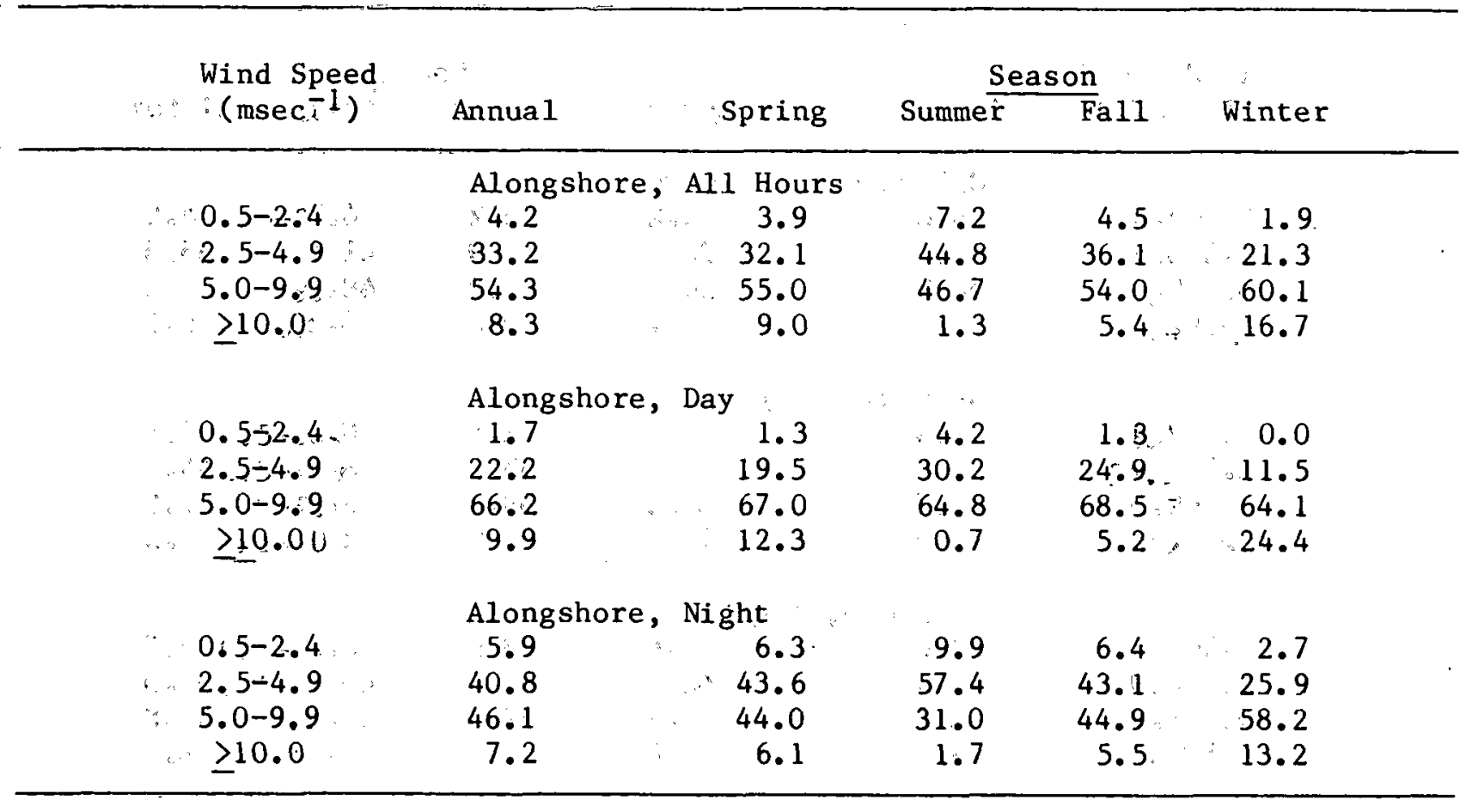


Table 6.5 (continued)

\begin{tabular}{|c|c|c|c|c|c|c|}
\hline \multirow{2}{*}{$\begin{array}{l}\text { Wind Speed } \\
\left(\mathrm{msec}^{-1}\right)\end{array}$} & \multirow[b]{2}{*}{ Annual } & \multirow{2}{*}{\multicolumn{2}{|c|}{ Spring }} & \multicolumn{2}{|c|}{ Season } & \multirow[b]{2}{*}{ Winter } \\
\hline & & & & Summer & Fall & \\
\hline $\begin{array}{c}0.5-2.4 \\
2.5-4.9 \\
5.0-9.9 \\
\geq 10.0\end{array}$ & $\begin{array}{l}\text { Off shore, } \\
4.8 \\
40.1 \\
50.8 \\
4.3\end{array}$ & Al1 & $\begin{array}{r}\text { Hours } \\
3.2 \\
32.0 \\
56.1 \\
8.7\end{array}$ & $\begin{array}{r}6.2 \\
47.9 \\
45.9 \\
0.0\end{array}$ & $\begin{array}{r}4.7 \\
41.9 \\
48.8 \\
4.6\end{array}$ & $\begin{array}{r}4.0 \\
35.5 \\
53.8 \\
6.7\end{array}$ \\
\hline $\begin{array}{c}0.52 .4 \\
2.3-4.9 \\
5.0-9.9 \\
\geq 10.0\end{array}$ & $\begin{array}{l}\text { Of f shore, } \\
1.4 \\
33.4 \\
60.7 \\
4.5\end{array}$ & Day & $\begin{array}{r}0.8 \\
27.4 \\
66.3 \\
10.5\end{array}$ & $\begin{array}{r}2.5 \\
38.1 \\
59.4 \\
0.0\end{array}$ & $\begin{array}{r}0.0 \\
38.3 \\
58.7 \\
.3 .1\end{array}$ & $\begin{array}{r}1.1 \\
44.4 \\
47.0 \\
7.5\end{array}$ \\
\hline $\begin{array}{c}0.5-2.4 \\
2.5-4.9 \\
5.0-9.9 \\
\geq 10.0\end{array}$ & $\begin{array}{l}\text { Of fshore, } \\
7.8 \\
45.9 \\
42.2 \\
4.0\end{array}$ & $\begin{array}{c}\text { Ni.gh } \\
\text {. }\end{array}$ & $\begin{array}{r}5.8 \\
42.1 \\
45.3 \\
6.8\end{array}$ & $\begin{array}{r}9.9 \\
57.0 \\
32.3 \\
0.0\end{array}$ & $\begin{array}{r}8.2 \\
45.0 \\
40.9 \\
5.8\end{array}$ & $\begin{array}{r}5.3 \\
+\quad 31.5 \\
56.8 \\
6.4\end{array}$ \\
\hline
\end{tabular}


Table 6.6

Belinar, New Jersey

Percent of Hours in Each Wind Speed Class

by Wind Direction, Season and Time of Day.

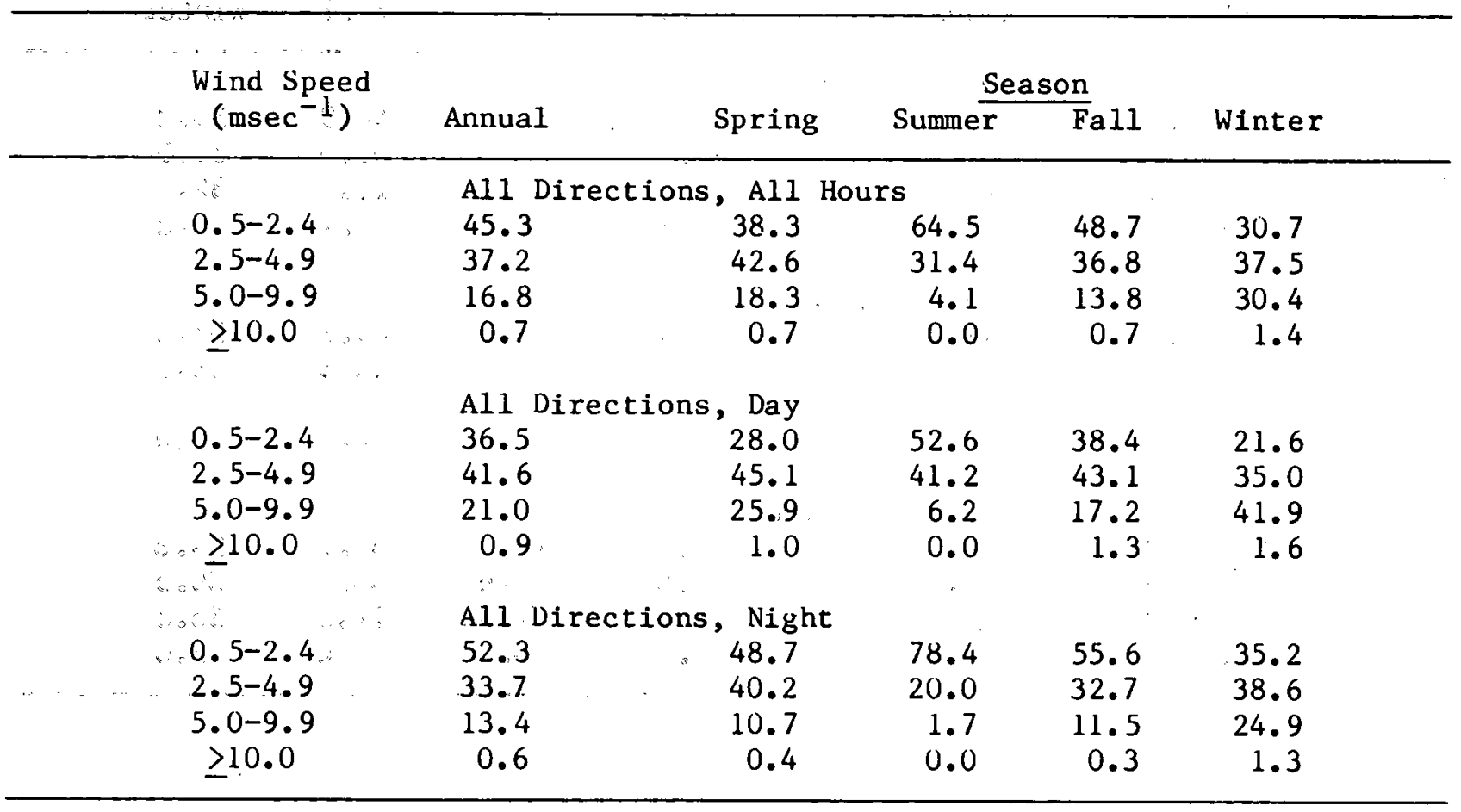


Table 6.6 (continued)

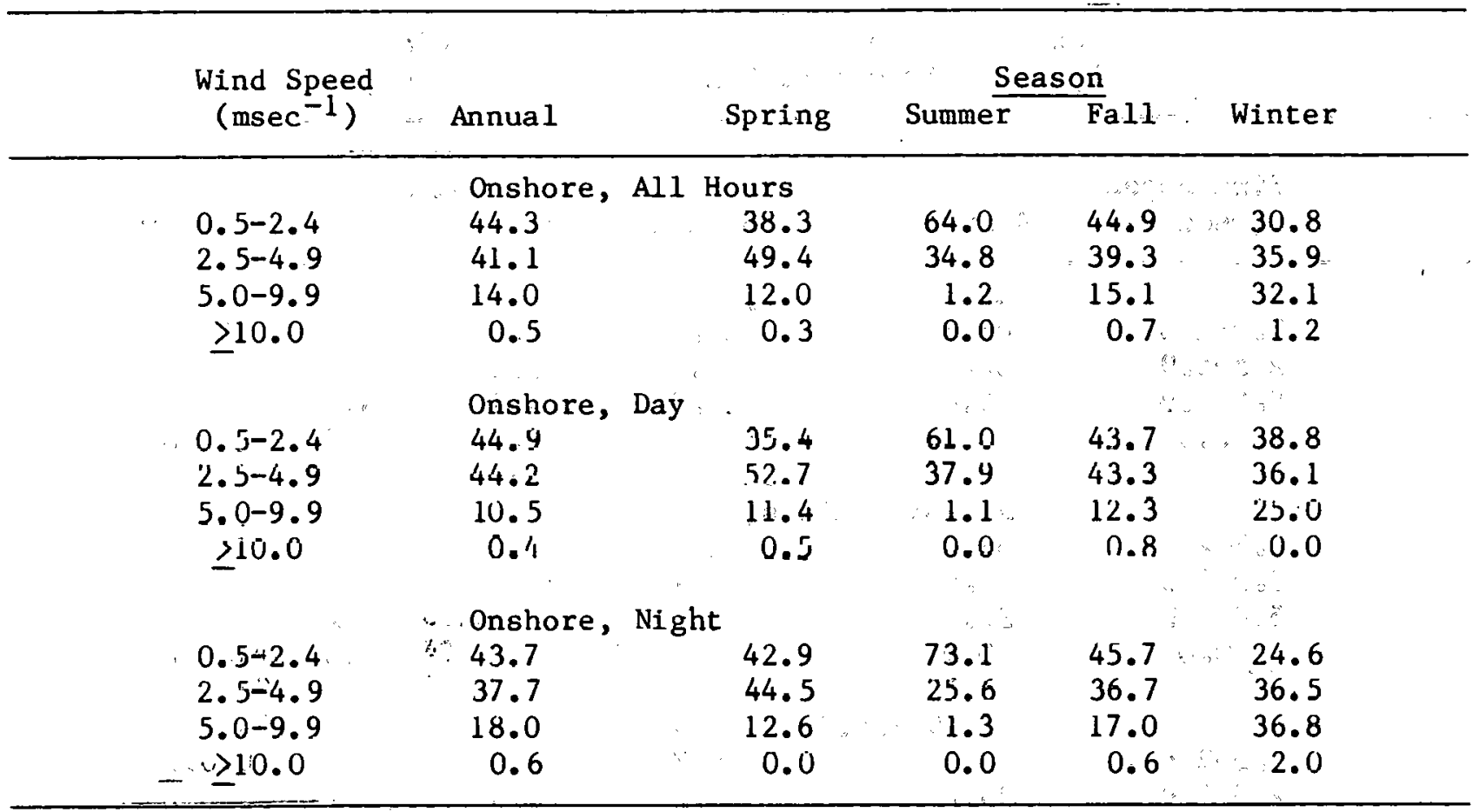


(Table 6.6 (continued)

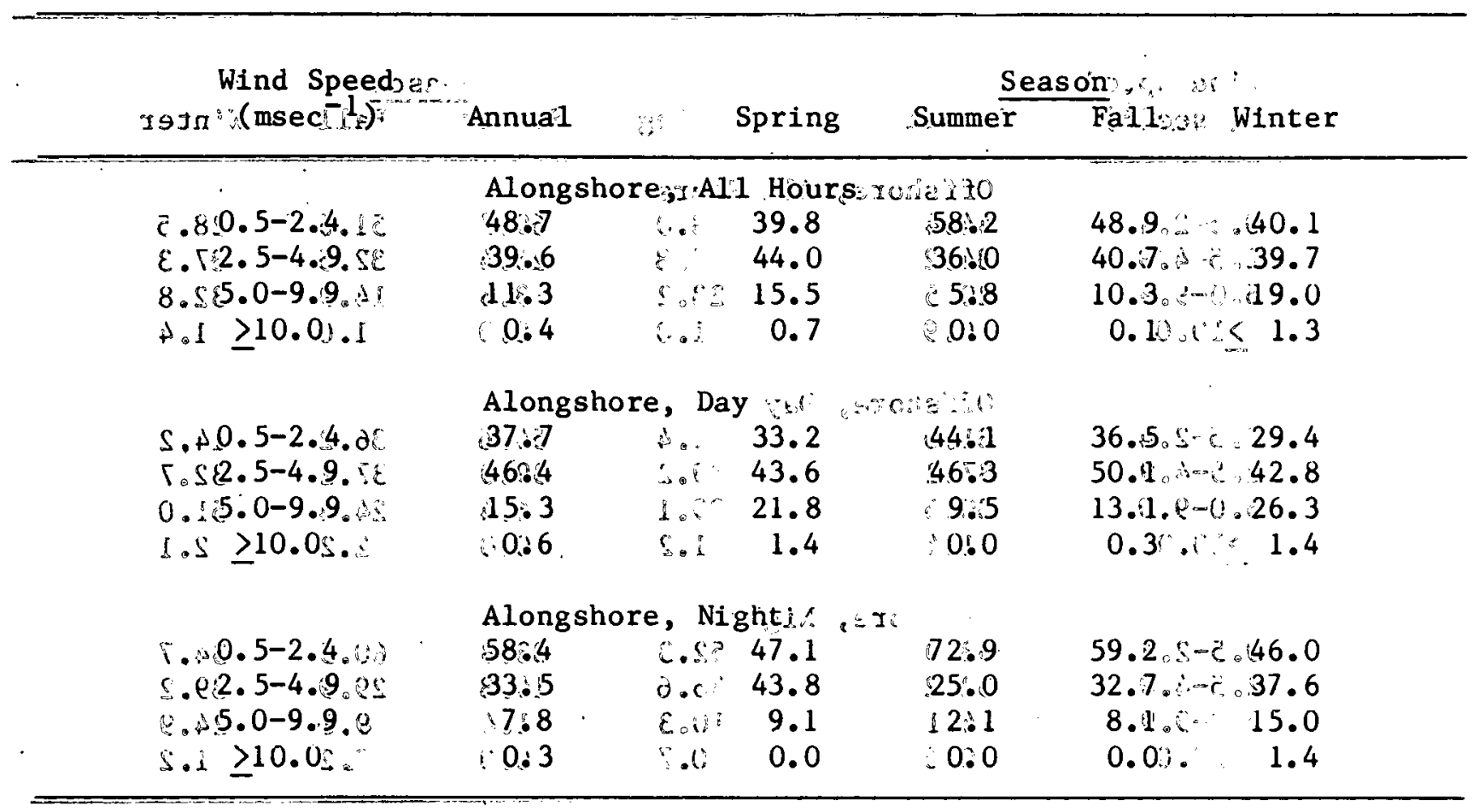




\section{(Table $: 6.6 \quad$ (continued)}

\begin{tabular}{|c|c|c|c|c|}
\hline \multirow{2}{*}{$\therefore \quad$ Wind Speed } & \multirow{2}{*}{ Annua1 } & \multirow[b]{2}{*}{ Soring } & \multicolumn{2}{|c|}{ Seasonyers } \\
\hline & & & Sùmmer & Fa11 Winter \\
\hline & \multicolumn{3}{|c|}{ Off shore, $9 \mathrm{All}$ l Hours ansio } & \\
\hline$\therefore 0.5-2.4 \ldots$ & .44 .04 & $\therefore 838.0$ & 69.7 & $51.6, \cdots .28 .5$ \\
\hline$\because 2.5-4.9 \ldots$ & .34 .62 & $\therefore \quad 37.8$ & 26.4 & $32.5 \cdot 3+37 \cdot 3$ \\
\hline$\therefore 5.0-9.9 \ldots$ & 20.5 & $\therefore \therefore \quad 23.2$ & 3.8 & $14.9 \cdot-1.32 .8$ \\
\hline$\therefore \geq 10.0$ & .0 .9 & $i$. & 0.0 & $1.0 \cdot 101.4$ \\
\hline \multirow{5}{*}{ 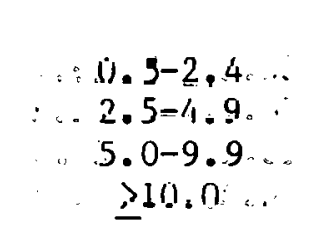 } & \multicolumn{3}{|c|}{ Offshore, Day in a s } & \\
\hline & 31.0 & 5.420 .4 & 54.86 & $36.2 \cdot g-c \cdot 0.14 .2$ \\
\hline & 37.2 & औ. 39.2 & 3900 & $37.0+0.32 .7$ \\
\hline & 30.5 & $\because 39.1$ & 6.4 & $24.43-8=81.0$ \\
\hline & .1 .4 & $\therefore \quad 1.2$ & $\therefore 0.0$ & $2.20 .01 \leq 2.1$ \\
\hline & Offshore, & Night:" "x & $\therefore \therefore \therefore$ & \\
\hline$\ldots 0.5-2.4$. & 53.0 & 52.3 & $83 \% 8$ & $60.5 \cdot s-\vec{c} \cdot 34.7$ \\
\hline$\therefore 2.5-4.9 \ldots$ & 32.2 & $\therefore \therefore \quad 36.6$ & $14: 8$ & $29.9 .3-2.39 .2$ \\
\hline$\therefore 5.0-9.9 .:$ & $14: 1$ & $\therefore \quad 10.3$ & 31.4 & $9.4 \cdot 0-0.24 .9$ \\
\hline$\ldots \geq 10.0 \ldots$ & .0 .6 & $\therefore$ & 0.0 & 0.2201 .2 \\
\hline
\end{tabular}




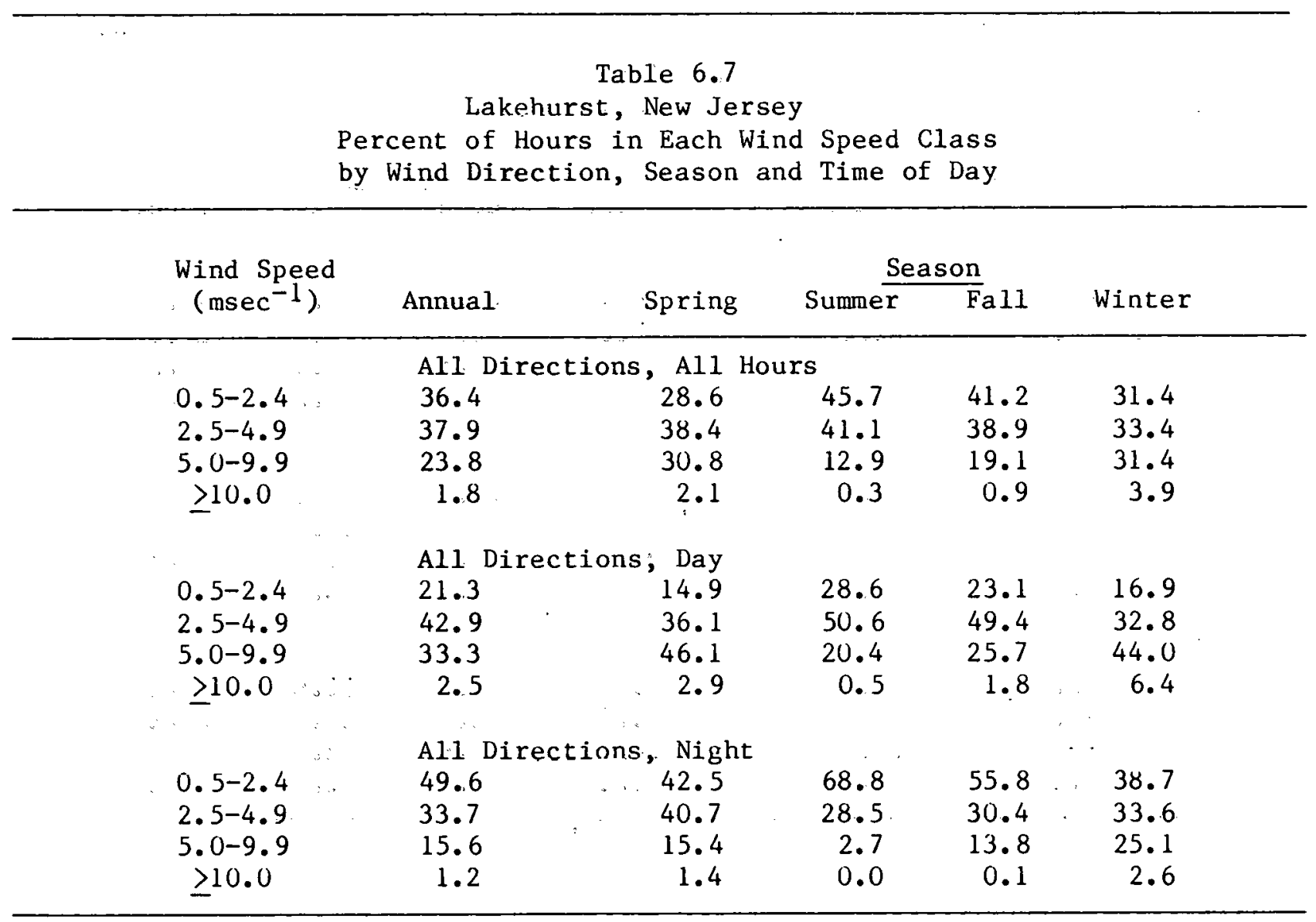


Table 6.7 (continued)

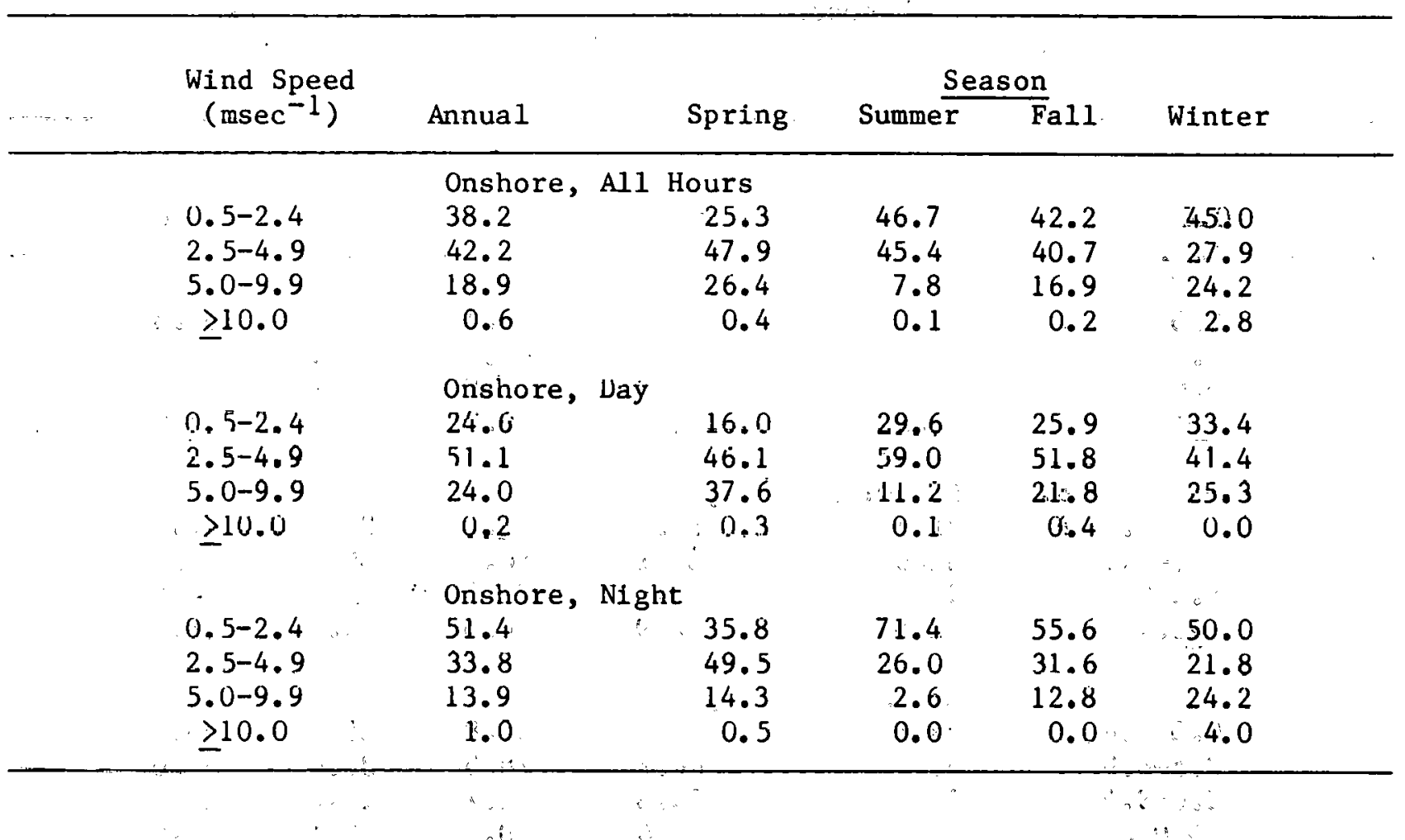


(Tablè 6.0) (continued)

\begin{tabular}{|c|c|c|c|c|}
\hline $\begin{array}{l}\text { Wind Speed } \\
\text { ys }\end{array}$ & Annual & whospring & \multicolumn{2}{|c|}{$\therefore$ Seasonoce } \\
\hline 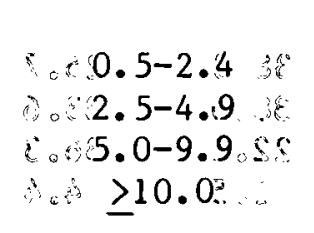 & $\begin{array}{l}\text { Alongs } \\
44: 7 \\
38: .4 \\
16.0 \\
0.0 .9\end{array}$ & 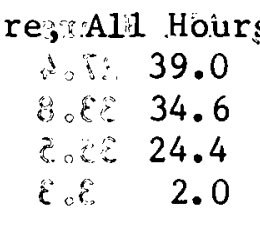 & $\begin{array}{r}\operatorname{sic} 130 \\
47.0 \\
40 \% 8 \\
12.2 \\
60.0\end{array}$ & $\begin{array}{r}45.2 \\
39.0 .0 .35 .3 \\
15.4 \\
0.3\end{array}$ \\
\hline $\begin{array}{l}0.5-2.4 \\
32.5-4.9 \\
555.0-9.9 \\
\therefore 3 \geq 10.0\end{array}$ & $\begin{array}{l}\text { Alongs } \\
27.9 \\
46: 7 \\
24 \% .1 \\
21: 4\end{array}$ & 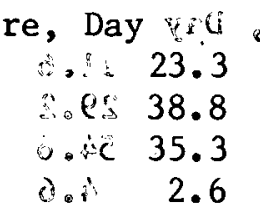 & $\begin{array}{r}3253 \\
46: 11 \\
21 ! 5 \\
80.0\end{array}$ & $\begin{array}{r}24.9 .5 .26 .1 \\
55.1, \\
19.11 .0 .21 .1 \\
0.90 .3 .1\end{array}$ \\
\hline 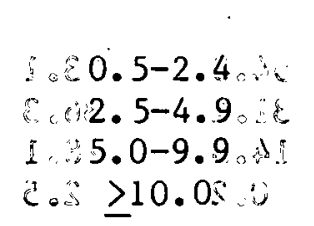 & $\begin{array}{l}\text { Alongs } \\
1578.1 \\
32: 8 \\
10: 1 \\
00: 6\end{array}$ & $\begin{array}{rr}\text { re, Night } \\
\text {. } & 52.2 \\
0.8 & 31.3 \\
8.81 & 15.1 \\
4.8 & 1.4\end{array}$ & $\begin{array}{r}.520898 \% \\
60 \% 6 \\
35 \% 9 \\
13 \% 4 \\
20.0\end{array}$ & $\begin{array}{r}58.5 .5-8.54 .5 \\
28.4 .33 .3 \\
13.0 .2-10.9 \\
0.00 .0 \leq 1.3\end{array}$ \\
\hline
\end{tabular}


Table 6.7 (continued)

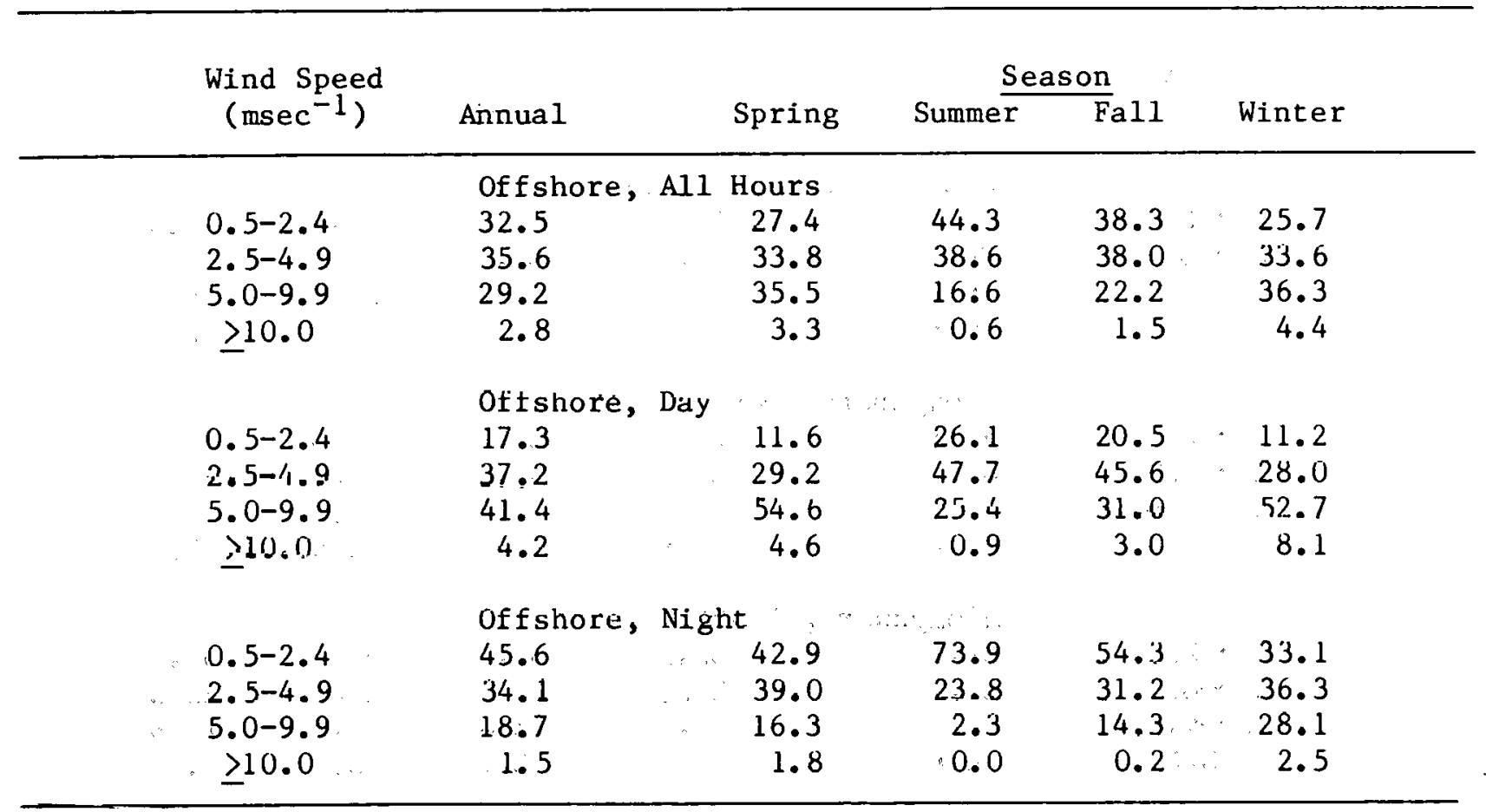




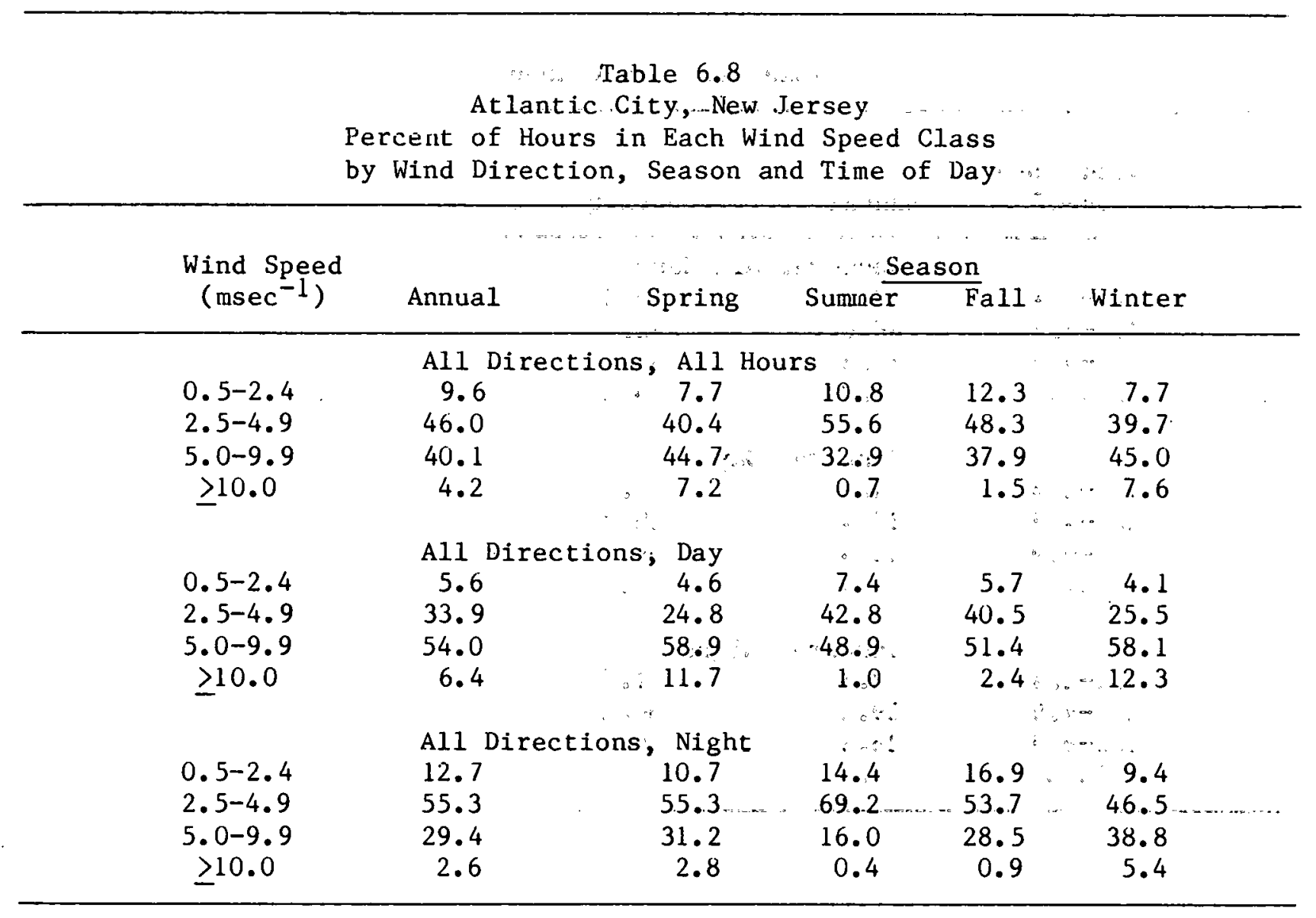


Table 6.8 (continued)

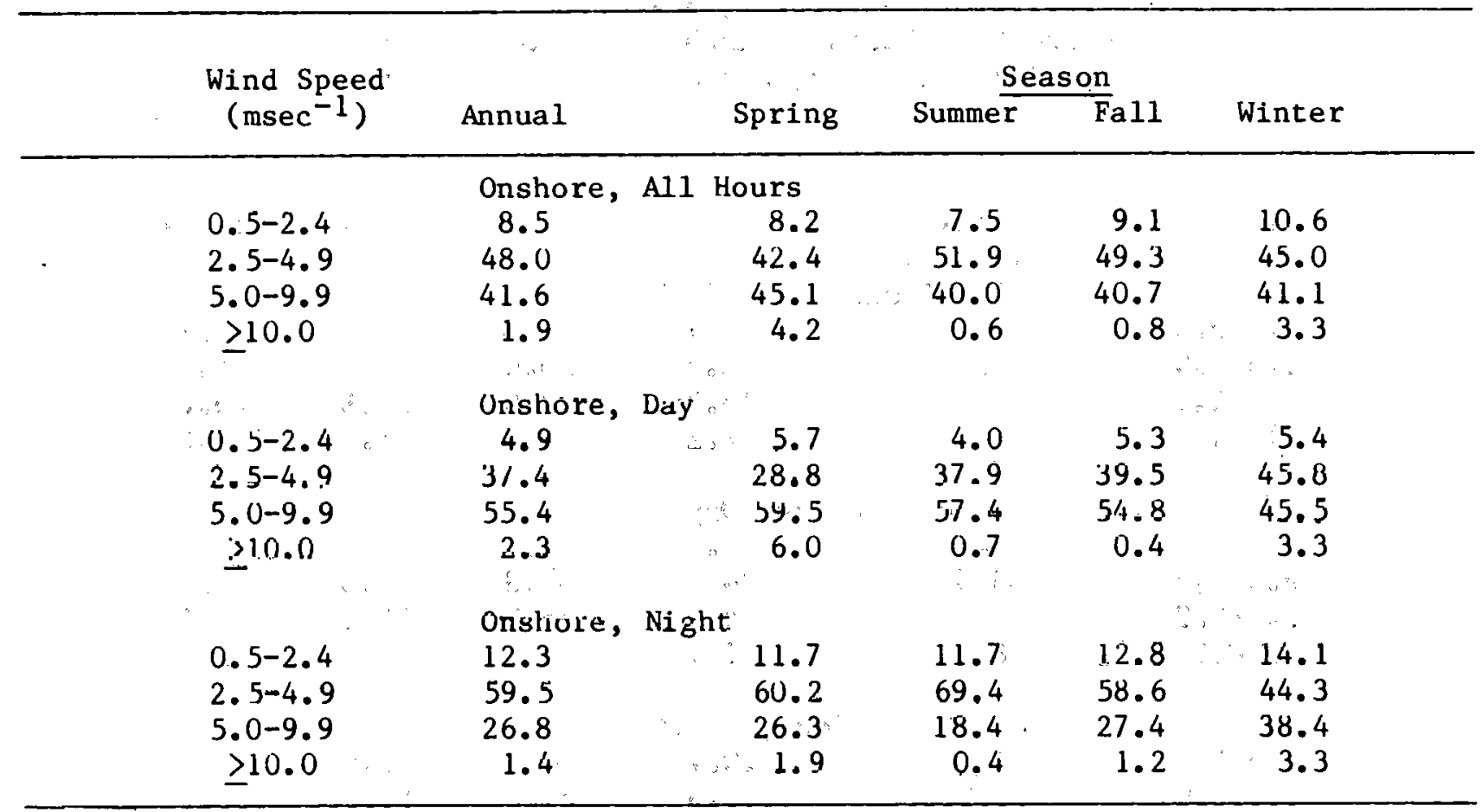

- $220-$ 
Table 6.8 (continued)

\begin{tabular}{|c|c|c|c|c|c|c|}
\hline \multirow{2}{*}{\multicolumn{2}{|c|}{$\begin{array}{l}\text { Wind Speed } \\
\text { (msec }^{-1} \text { ) }\end{array}$}} & \multirow[b]{2}{*}{ Annual } & \multicolumn{4}{|c|}{ Season } \\
\hline & & & Spring & Summer & $\overline{F a l l}$ & Winter \\
\hline , & $\begin{array}{c}0.5-2.4 \\
2.5-4.9 \\
5.0-9.9 \\
\geq 10.0\end{array}$ & $\begin{array}{l}\text { Alongshore, } \\
10.2 \\
48.1 \\
38.6 \\
3.1\end{array}$ & $\begin{array}{c}\text { All Hours } \\
9.8 \\
39.9 \\
43.7 \\
6.6\end{array}$ & $\begin{array}{r}11.1 \\
55.5 \\
32.9 \\
0.5\end{array}$ & $\begin{array}{r}11.6 \\
51.3 \\
36.8 \\
0.3\end{array}$ & $\begin{array}{r}7.8 \\
42.9 \\
43.3 \\
6.0\end{array}$ \\
\hline & $\begin{array}{l}. \\
0.5-2.4 \\
2.5-4.9 \\
5.0-9.9 \\
\geq 10.0\end{array}$ & $\begin{array}{l}\text { Alongshore, } \\
8.2 \\
34.4 \\
52.3 \\
5.0\end{array}$ & $\begin{array}{r}\text { Day } \\
8.6 \\
24.2 \\
53.9 \\
13.3\end{array}$ & $\begin{array}{r}10.3 \\
36.8 \\
51.7 \\
1.2\end{array}$ & $\begin{array}{r}6.8 \\
46.3 \\
46.1 \\
0.8\end{array}$ & $\begin{array}{r}5.3 \\
26.3 \\
62.1 \\
6.4\end{array}$ \\
\hline & $\begin{array}{c}0.5-2.4 \\
2.5-4.9 \\
5.0-9.9 \\
\geq 10.0\end{array}$ & $\begin{array}{l}\text { Along shore, } \\
11.5 \\
56.4 \\
30.1 \\
2.0\end{array}$ & $\begin{array}{r}\text { Night } \\
10.5 \\
50.9 \\
36.5 \\
2.1\end{array}$ & $\begin{array}{r}12.0 \\
68.8 \\
19.2 \\
0.0\end{array}$ & $\begin{array}{r}14.5 \\
55.1 \\
30.4 \\
0.0\end{array}$ & $\begin{array}{r}8.6 \\
49.1 \\
36.6 \\
5.7\end{array}$ \\
\hline
\end{tabular}


Table 6.8 (continued)

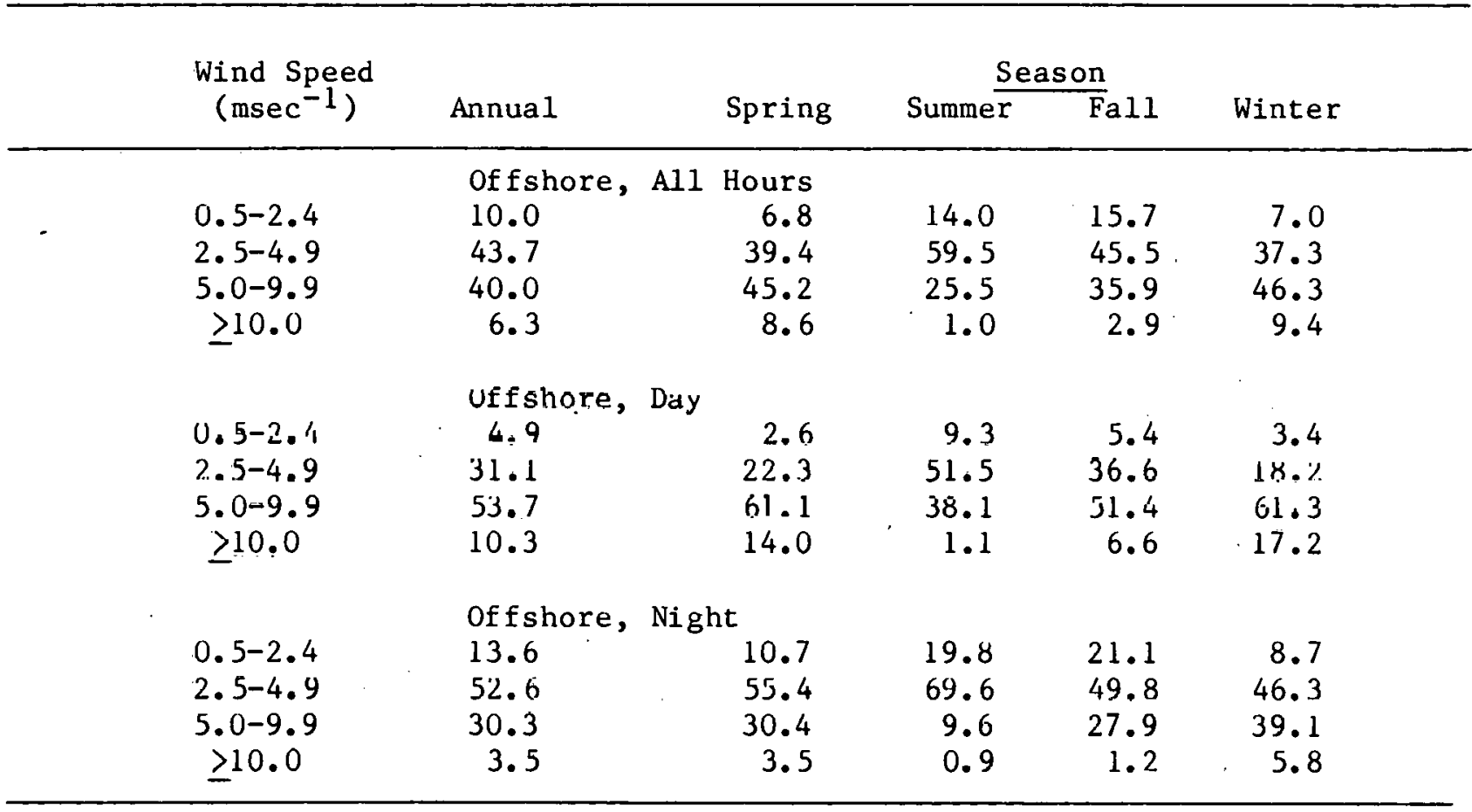


Table 6.9

Wilmington, Delaware

Percent of Hours in Each Wind Speed Class

by Wind Direction, Season and Time of Day

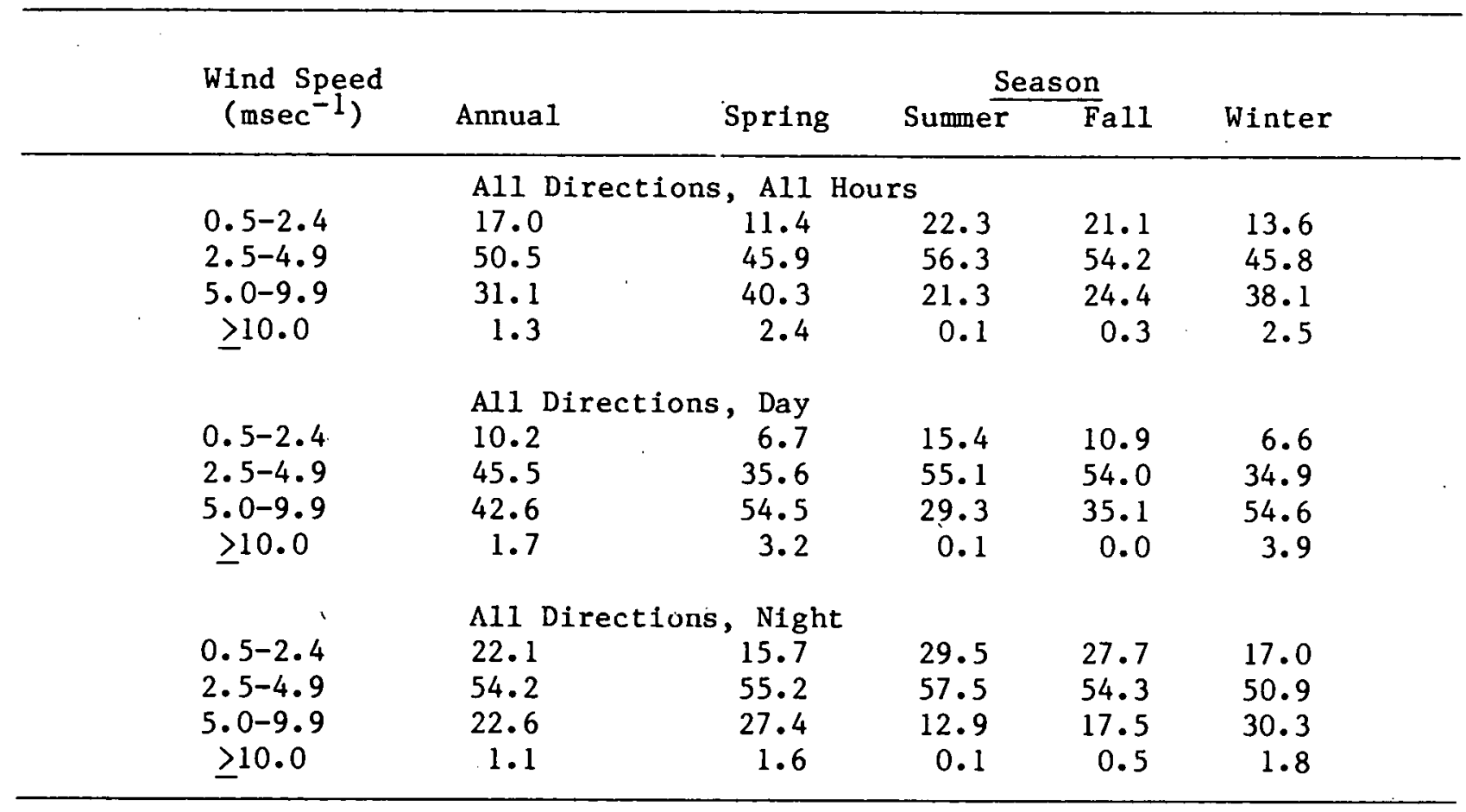


Table 6.9 (continued)

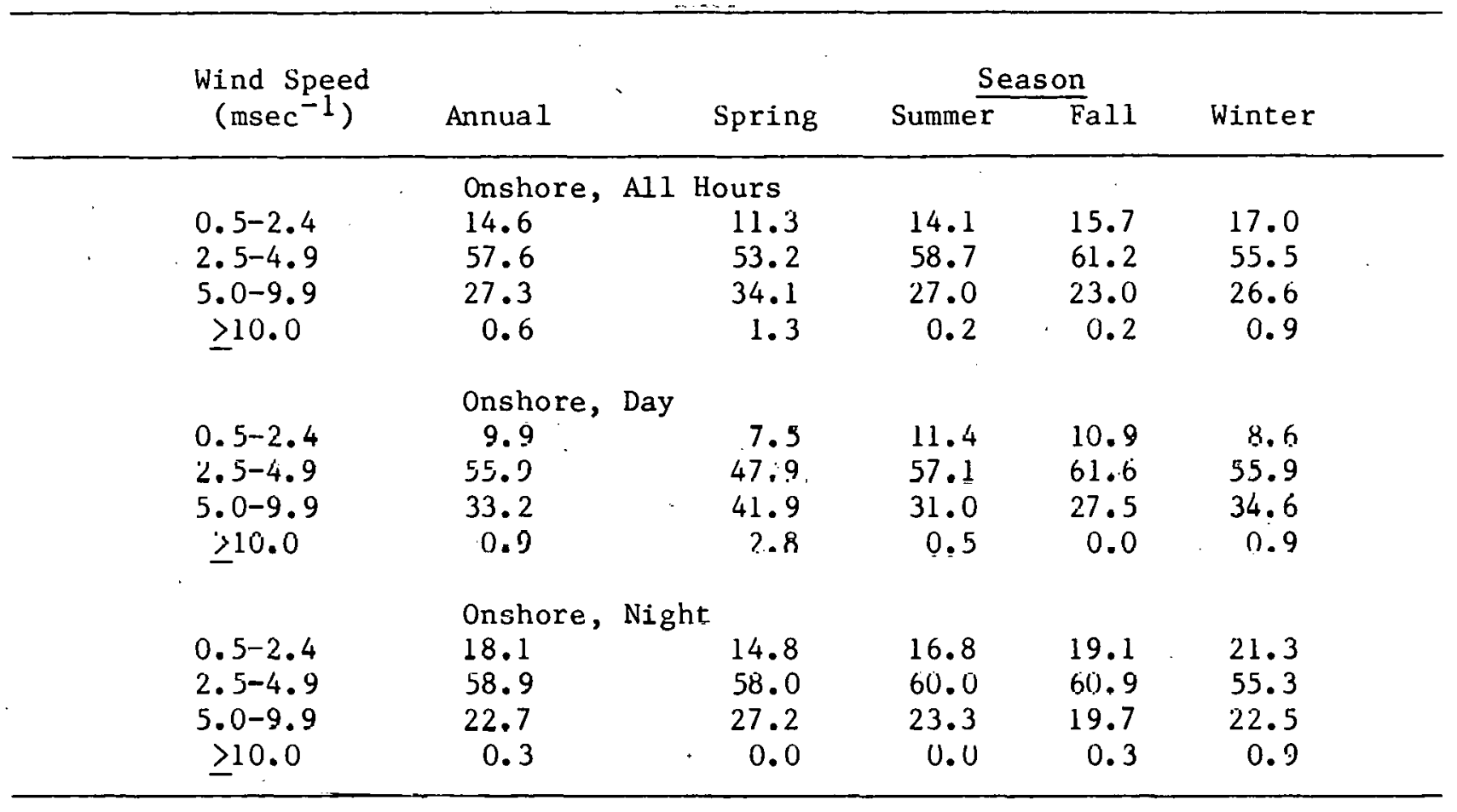


Table 6.9 (continuedi)

\begin{tabular}{|c|c|c|c|c|c|}
\hline \multirow{2}{*}{$\begin{array}{l}\text { Wind Speed } \\
\left(\mathrm{msec}^{-1}\right)\end{array}$} & \multirow[b]{2}{*}{ Annual } & \multirow[b]{2}{*}{ Spring } & \multicolumn{2}{|c|}{ Season } & \multirow[b]{2}{*}{ Winter } \\
\hline & & & Summer & $\bar{F}$ all & \\
\hline $\begin{array}{c}0.5-2.4 \\
2.5-4.9 \\
5.0-9.9 \\
\geq 10.0\end{array}$ & $\begin{array}{l}\text { Alongshore, } \\
: 20.9 \\
48.3 \\
2.9 .4 \\
: 1.3\end{array}$ & $\begin{array}{c}\text { Al1 Hours } \\
10.3 \\
44.4 \\
4.2 .4 \\
2.9\end{array}$ & $\begin{array}{l}28.1 \\
47.9 \\
24.0 \\
.0 .0\end{array}$ & $\begin{array}{r}26.3 \\
51.4 \\
21.5 \\
0.9\end{array}$ & $\begin{array}{r}17.3 \\
49.0 \\
31.5 \\
2.2\end{array}$ \\
\hline $\begin{array}{c}0.5-2.4 \\
2.5-4.9 \\
5.0-9.9 \\
\geq 10.0\end{array}$ & $\begin{array}{l}\text { Alongshore, } \\
11.9 \\
45.1 \\
42.3 \\
0.8\end{array}$ & $\begin{array}{r} \\
5.7 \\
35.6 \\
55.7 \\
3.0\end{array}$ & $\begin{array}{r}18.4 \\
46.9 \\
34.7 \\
0.0\end{array}$ & $\begin{array}{r}8.7 \\
52.9 \\
38.4 \\
0.0\end{array}$ & $\begin{array}{r}11.9 \\
44.1 \\
44.1 \\
0.0\end{array}$ \\
\hline $\begin{array}{c}0.5-2.4 \\
2.5-4.9 \\
5.0-9.9 \\
\geq 10.0\end{array}$ & $\begin{array}{l}\text { Alongshore, } \\
28.5 \\
51.1 \\
18.7 \\
1.8\end{array}$ & $\begin{array}{r}\text { Night } \\
14.8 \\
52.7 \\
29.8 \\
2.8\end{array}$ & $\begin{array}{r}40.1 \\
49.1 \\
10.8 \\
0.10\end{array}$ & $\begin{array}{r}40.5 \\
50.5 \\
7.5 \\
1.5\end{array}$ & $\begin{array}{r}19.5 \\
51.1 \\
26.4 \\
3.0\end{array}$ \\
\hline
\end{tabular}




\section{Table 6.9 (continued)}

\begin{tabular}{|c|c|c|c|c|c|c|}
\hline \multirow{2}{*}{$\begin{array}{l}\text { Wind Speed } \\
\left(\mathrm{msec}^{-1}\right)\end{array}$} & \multirow[b]{2}{*}{ Annual } & & \multirow[b]{2}{*}{ Spring } & \multicolumn{2}{|c|}{ Season } & \multirow[b]{2}{*}{ Winter } \\
\hline & & & & Summer & $\overline{F a l l}$ & \\
\hline $\begin{array}{c}0.5-2.4 \\
2.5-4.9 \\
5.0-9.9 \\
\geq 10.0\end{array}$ & $\begin{array}{l}\text { Off fhore, } \\
17.4 \\
46.7 \\
34.1 \\
1.8\end{array}$ & All & $\begin{array}{r}\text { Hours } \\
11.6 \\
42.6 \\
43.1 \\
2.8\end{array}$ & $\begin{array}{r}5.7 \\
57.8 \\
16.3 \\
0.1\end{array}$ & $\begin{array}{r}24.6 \\
48.3 \\
27.0 \\
0.2\end{array}$ & $\begin{array}{r}11.5 \\
40.9 \\
44.3 \\
3.2\end{array}$ \\
\hline $\begin{array}{c}0.5-2.1 \\
2.5-4.9 \\
5.0-9.9 \\
\geq 10.0\end{array}$ & $\begin{array}{l}\text { of f shore, } \\
9.8 \\
38.9 \\
48.8 \\
2.5\end{array}$ & Day & $\begin{array}{r}6.4 \\
29.0 \\
61.2 \\
3.4\end{array}$ & $\begin{array}{r}6.6 \\
57.3 \\
26.2 \\
0.0\end{array}$ & $\begin{array}{r}11.4 \\
45.6 \\
43.0 \\
0.0\end{array}$ & $\begin{array}{r}4.5 \\
23.5 \\
65.9 \\
6.0\end{array}$ \\
\hline $\begin{array}{c}0.5-2.4 \\
2.5-4.9 \\
5.0-9.9 \\
>10.0\end{array}$ & $\begin{array}{l}\text { Of f shore, } \\
22.8 \\
52.2 \\
23.7 \\
1.3\end{array}$ & $\mathrm{Nigh}$ & $\begin{array}{l}\text { ht } \\
16.3 \\
55.0 \\
26.6 \\
2.2\end{array}$ & $\begin{array}{r}6.0 \\
58.2 \\
5.5 \\
0.3\end{array}$ & $\begin{array}{r}31.4 \\
49.6 \\
18.7 \\
0.3\end{array}$ & $\begin{array}{r}14.7 \\
49.1 \\
34.3 \\
1.9\end{array}$ \\
\hline
\end{tabular}


Table 6.10

Wallops Is land, Virginla

Percent of Hours in Each Wind Speed Class

by Wind Direction, Season and Time of Day

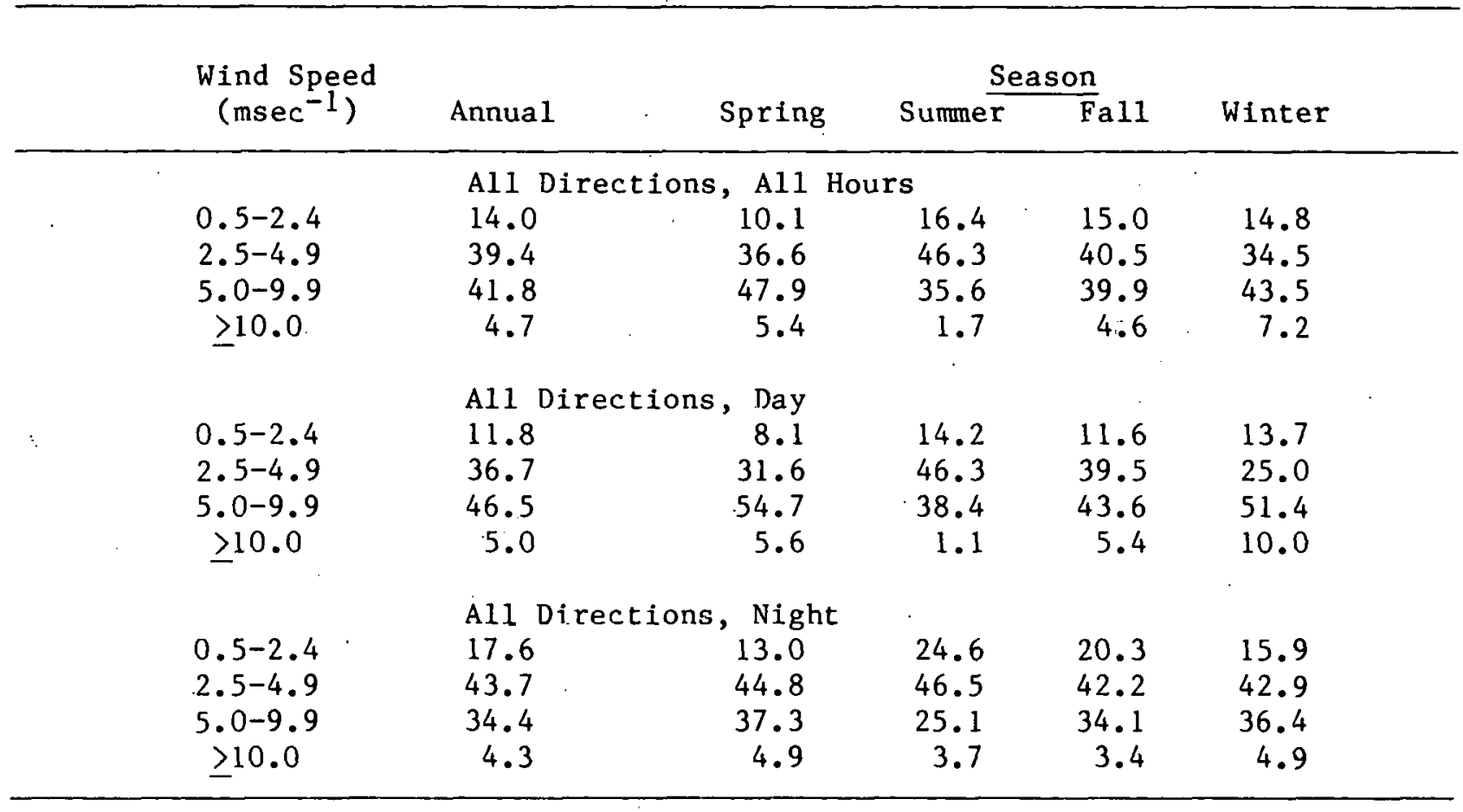


Table 6.10 (continued)

\begin{tabular}{|c|c|c|c|c|c|c|}
\hline \multirow{2}{*}{$\begin{array}{l}\text { Wind Speed } \\
\left(\mathrm{msec}^{-1}\right)\end{array}$} & \multirow[b]{2}{*}{ Annual } & & \multirow[b]{2}{*}{ Spring } & \multicolumn{2}{|c|}{ Season } & \multirow{2}{*}{ Winter } \\
\hline & & & & Summer & $\bar{F} a l 1$ & \\
\hline $\begin{array}{c}0.5-2.4 \\
2.5-4.9 \\
5.0-9.9 \\
\geq 10.0\end{array}$ & $\begin{array}{l}\text { Onshore, } \\
1.4 .6 \\
48.6 \\
35.3 \\
1.5\end{array}$ & All & $\begin{array}{r}\text { Hours } \\
\begin{array}{r}10.1 \\
51.7 \\
37.3 \\
0.9\end{array}\end{array}$ & $\begin{array}{r}14.9 \\
52.1 \\
32.0 \\
1.0\end{array}$ & $\begin{array}{r}12.4 \\
48.9 \\
37.5 \\
1.3\end{array}$ & $\begin{array}{r}27.3 \\
32.3 \\
35.6 \\
4.8\end{array}$ \\
\hline $\begin{array}{c}0.5-2.4 \\
2.5=4.9 \\
5.0-9.9 \\
\geq 10.0\end{array}$ & $\begin{array}{l}\text { Onshore, } \\
12.9 \\
19.4 \\
36.6 \\
1.1\end{array}$ & Day & $\begin{array}{r}10.1 \\
47.8 \\
40.9 \\
1.2\end{array}$ & $\begin{array}{r}11.5 \\
53.9 \\
34.4 \\
0.3\end{array}$ & $\begin{array}{r}10.6 \\
52.1 \\
36.6 \\
0.7\end{array}$ & $\begin{array}{r}30.7 \\
29.6 \\
35.0 \\
4.7\end{array}$ \\
\hline $\begin{array}{c}0.5-2.4 \\
2.5-4.9 \\
5.0-9.9 \\
\geq 10.0\end{array}$ & $\begin{array}{l}\text { Onshore, } \\
18.8 \\
46.2 \\
32.4 \\
2.7\end{array}$ & Nigh & $\begin{array}{r}9.0 \\
62.5 \\
28.5 \\
0.0\end{array}$ & $\begin{array}{r}28.6 \\
44.6 \\
22.8 \\
4.0\end{array}$ & $\begin{array}{r}17.2 \\
40.5 \\
39.6 \\
2.7\end{array}$ & $\begin{array}{r}21.7 \\
36.3 \\
37.1 \\
4.9\end{array}$ \\
\hline
\end{tabular}


Table 6.10 (continued)

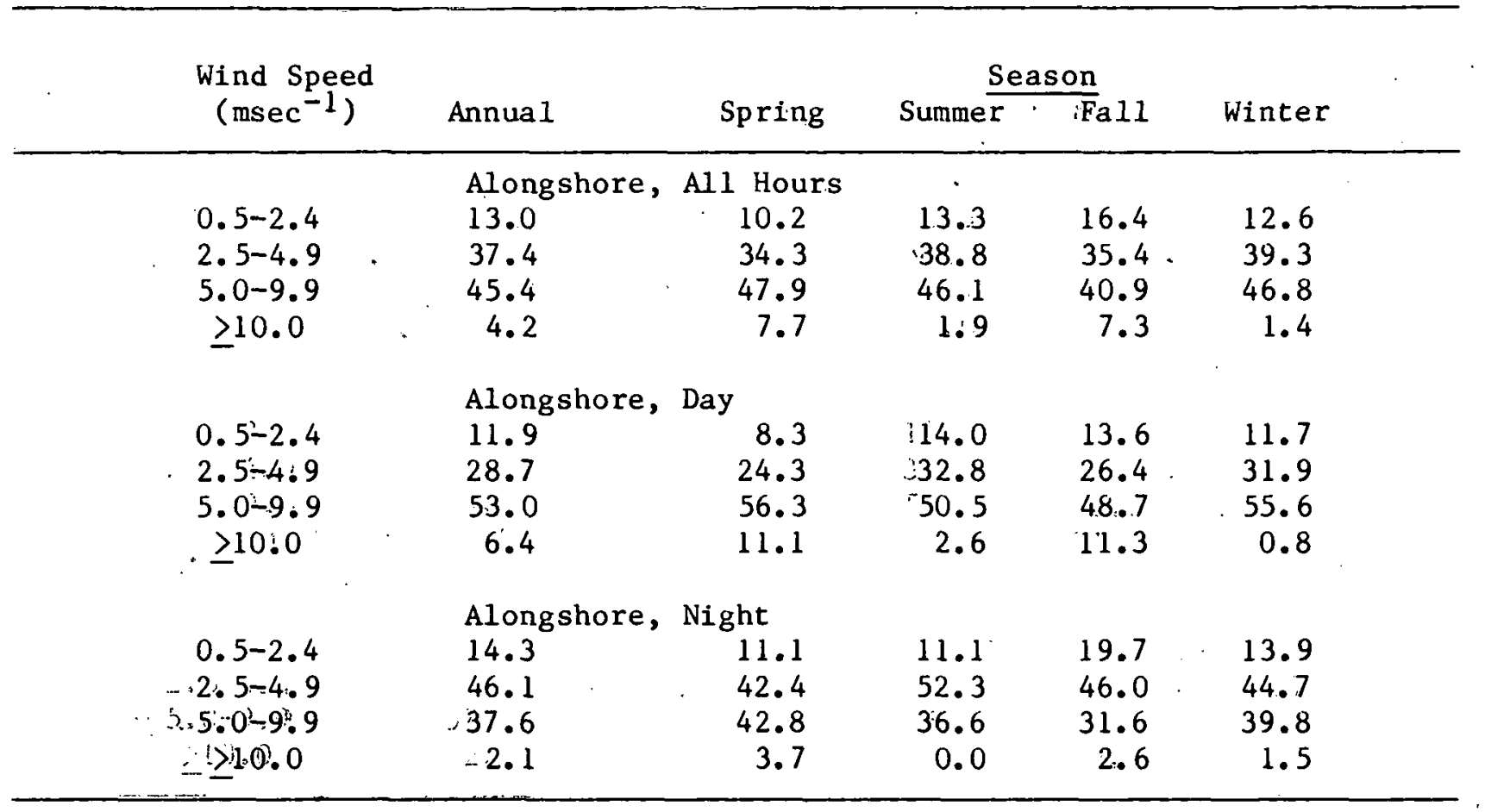


Table 6.10 (continued)

\begin{tabular}{|c|c|c|c|c|c|}
\hline \multirow{2}{*}{$\begin{array}{l}\text { Wind Speed } \\
\left(\mathrm{msec}^{-1}\right)\end{array}$} & \multirow[b]{2}{*}{ Annual } & \multicolumn{4}{|c|}{ Season } \\
\hline & & Spring & Summer & Fall & Winter \\
\hline $\begin{array}{c}0.5-2.4 \\
2.5-4.9 \\
5.0-9.9 \\
\geq 10.0\end{array}$ & $\begin{array}{l}\text { Offshore, } \\
14.2 \\
30.9 \\
46.4 \\
8.5\end{array}$ & $\begin{array}{r}\text { Al1 Hours } \\
10.3 \\
25.1 \\
56.6 \\
7.9\end{array}$ & $\begin{array}{r}23.8 \\
39.1 \\
33.3 \\
3.8\end{array}$ & $\begin{array}{r}17.8 \\
32.1 \\
43.1 \\
7.1\end{array}$ & $\begin{array}{l}11.6 \\
32.6 \\
44.2 \\
11.6\end{array}$ \\
\hline $\begin{array}{l}11.5-2.4 \\
2.5-4.9 \\
5.0-9.9 \\
\geqslant 10.0 .\end{array}$ & $\begin{array}{l}\text { Of f shore, } \\
10.4 \\
24.9 \\
55.3 \\
9.4\end{array}$ & $\begin{array}{r}6.2 \\
20.4 \\
66.0 \\
7.4\end{array}$ & $\begin{array}{r}2.1 .8 \\
39.3 \\
36.3 \\
2.5\end{array}$ & $\begin{array}{r}11.7 \\
25.3 \\
54.2 \\
8.7\end{array}$ & $\begin{array}{r}6.7 \\
20.2 \\
36.5 \\
16.6\end{array}$ \\
\hline $\begin{array}{c}0.5-2.4 \\
2.5-4.9 \\
5.0-9.9 \\
\geq 10.0\end{array}$ & $\begin{array}{l}\text { Offshore, } \\
19.6 \\
39.7 \\
33.2 \\
7.5\end{array}$ & $\begin{array}{l}\text { Night } \\
17.7 \\
33.7 \\
39.1 \\
9.5\end{array}$ & $\begin{array}{l}37.4 \\
38.1 \\
12.2 \\
12.2\end{array}$ & $\begin{array}{r}25.2 \\
40.1 \\
30.0 \\
4.8\end{array}$ & $\begin{array}{r}16.0 \\
43.5 \\
33.1 \\
7.3\end{array}$ \\
\hline
\end{tabular}


Táble 6.11

Norfolk, Virginia

Percent of Hours in Each Wind Speed Class

by Wind Direction, Season and Time of Day

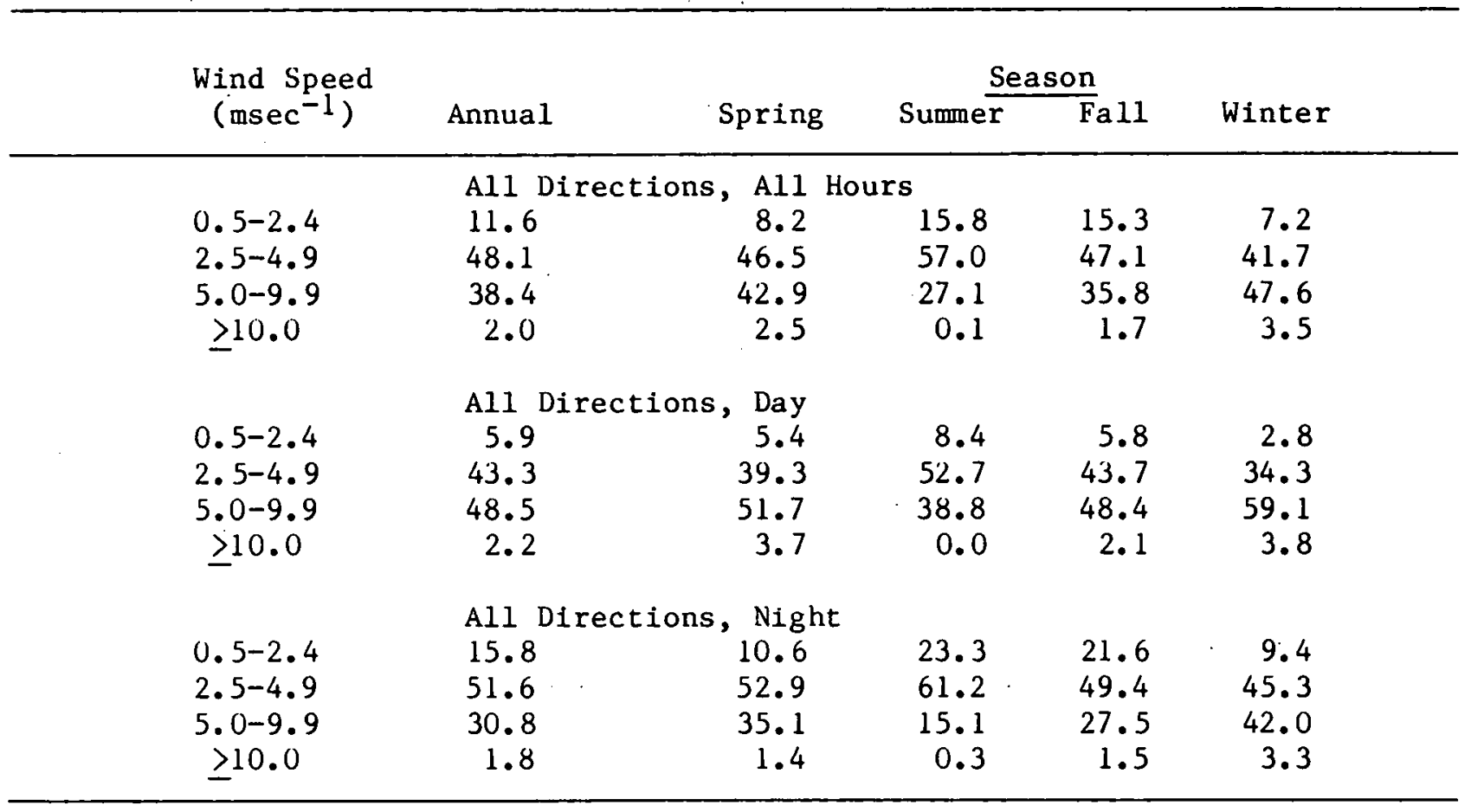


Table 6.1ll (icontinuedi))

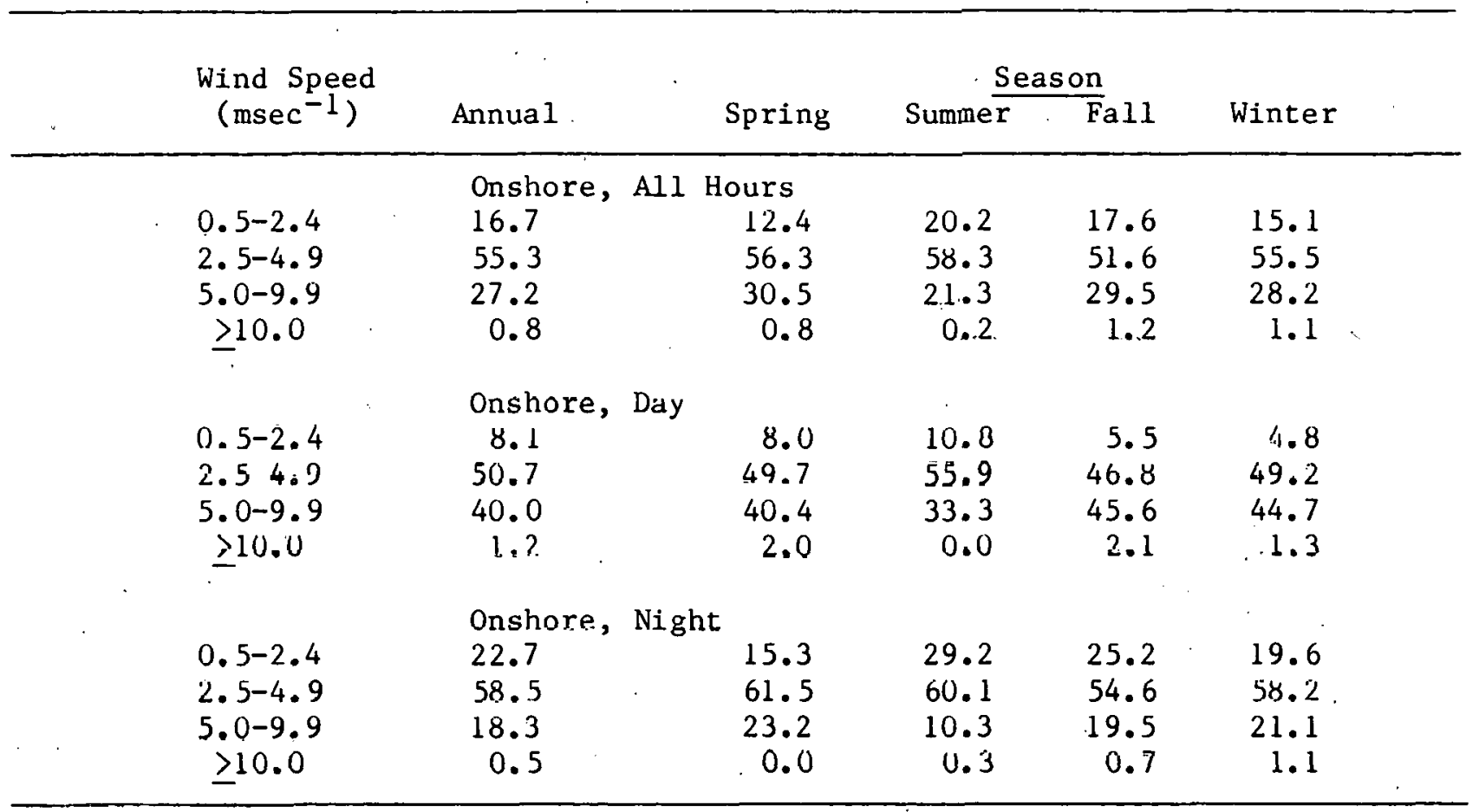


Table 6.11 (continued)

\begin{tabular}{|c|c|c|c|c|c|}
\hline \multirow{2}{*}{$\begin{array}{l}\text { Wind Speed } \\
\left(\mathrm{msec}^{-1}\right)\end{array}$} & \multirow[b]{2}{*}{ Annual } & \multirow[b]{2}{*}{ Spring } & \multicolumn{2}{|c|}{ Season } & \multirow[b]{2}{*}{ Winter } \\
\hline & & & Summer & Fall & \\
\hline $\begin{array}{c}0.5-2.4 \\
2.5-4.9 \\
5.0-9.9 \\
\geq 10.0\end{array}$ & $\begin{array}{l}\text { Alongshore, } \\
9.7 \\
46.3 \\
41.9 \\
2.1\end{array}$ & $\begin{array}{c}\text { All Hours } \\
7.3 \\
41.3 \\
48.1 \\
3.3\end{array}$ & $\begin{array}{r}12.1 \\
55.3 \\
32.6 \\
0.0\end{array}$ & $\begin{array}{r}13.0 \\
47.5 \\
36.1 \\
3.4\end{array}$ & $\begin{array}{r}6.6 \\
41.1 \\
50.3 \\
2.0\end{array}$ \\
\hline $\begin{array}{l}0.5-2.4 \\
2.5-4.9 \\
5.0-9.9 \\
\geq 10.0\end{array}$ & $\begin{array}{l}\text { Alongshore, } \\
5.4 \\
41.2 \\
51.1 \\
2.3\end{array}$ & $\begin{array}{r}\text { Day } \\
5.2 \\
35.9 \\
54.0 \\
4.8\end{array}$ & $\begin{array}{r}6.5 \\
46.7 \\
46.8 \\
0.0\end{array}$ & $\begin{array}{r}6.9 \\
42.5 \\
47.9 \\
2.7\end{array}$ & $\begin{array}{r}2.4 \\
38.6 \\
57.0 \\
2.0\end{array}$ \\
\hline $\begin{array}{c}0.5-2.4 \\
2.5-4.9 \\
5.0-9.9 \\
\geq 10.0\end{array}$ & $\begin{array}{l}\text { Alongshore, } \\
12.6 \\
50.1 \\
35.4 \\
1.9\end{array}$ & $\begin{array}{r}\text { Night } \\
8.9 \\
45.8 \\
43.2 \\
2.0\end{array}$ & $\begin{array}{r}17.1 \\
63.0 \\
19.9 \\
0.0\end{array}$ & $\begin{array}{r}17.4 \\
51.1 \\
27.6 \\
3.8\end{array}$ & $\begin{array}{r}8.4 \\
42.4 \\
47.1 \\
2.1\end{array}$ \\
\hline
\end{tabular}


Table 6.11 (continued)

\begin{tabular}{|c|c|c|c|c|c|c|}
\hline \multirow{2}{*}{$\begin{array}{l}\text { Wind Speed } \\
\left(\mathrm{msec}^{-1}\right)\end{array}$} & \multirow[b]{2}{*}{ Annual } & \multicolumn{5}{|c|}{ Season } \\
\hline & & Spring & Summer & $\overline{F a l l}$ & Winter & \\
\hline $\begin{array}{c}0.5-2.4 \\
2.5-4.9 \\
5.0-9.9 \\
\geq 10.0\end{array}$ & $\begin{array}{c}\text { of } f \text { shore, } \\
8.2 \\
42.0 \\
46.7 \\
3.1\end{array}$ & $\begin{array}{r}\text { All Hours } \\
4.8 \\
43.4 \\
48.7 \\
3.1\end{array}$ & $\begin{array}{r}15.1 \\
57.8 \\
26.8 \\
0.3\end{array}$ & $\begin{array}{r}13.9 \\
37.0 \\
48.8 \\
0.3\end{array}$ & $\begin{array}{r}3.9 \\
35.4 \\
54.2 \\
6.4\end{array}$ & \\
\hline $\begin{array}{c}0.5=2.4 \\
2.5-4.9 \\
5.0-9.9 \\
\geq 10.0\end{array}$ & $\begin{array}{l}\text { Offshore, } \\
4.1 \\
38.2 \\
54.4 \\
3.3\end{array}$ & Day $\begin{array}{r}2.1 \\
35.4 \\
58.0 \\
3.8\end{array}$ & $\begin{array}{r}7.8 \\
57.0 \\
35.2 \\
0.0\end{array}$ & $\begin{array}{r}4.4 \\
39.6 \\
55.1 \\
0.9\end{array}$ & $\begin{array}{r}2.1 \\
2.3 .7 \\
67.2 \\
7.0\end{array}$ & $\cdot$ \\
\hline $\begin{array}{c}0.5-2.4 \\
2.5-4.9 \\
5.0-9.9 \\
\geq 10.0\end{array}$ & $\begin{array}{l}\text { Off fshore, } \\
11.6 \\
45.1 \\
40.2 \\
3.1\end{array}$ & $\begin{array}{r}\text { Night } \\
7.0 \\
52.4 \\
38.2 \\
2.3\end{array}$ & $\begin{array}{r}25.8 \\
59.2 \\
14.2 \\
0.8\end{array}$ & $\begin{array}{r}20.1 \\
34.7 \\
45.1 \\
0.0\end{array}$ & $\begin{array}{r}4.9 \\
41.8 \\
47.1 \\
6.1\end{array}$ & \\
\hline
\end{tabular}


Table 6.12

Cape Hatteras, North Carolina

Percent of Hours in Each Wind Speed Class

by Wind Direction, Season and Time of Day

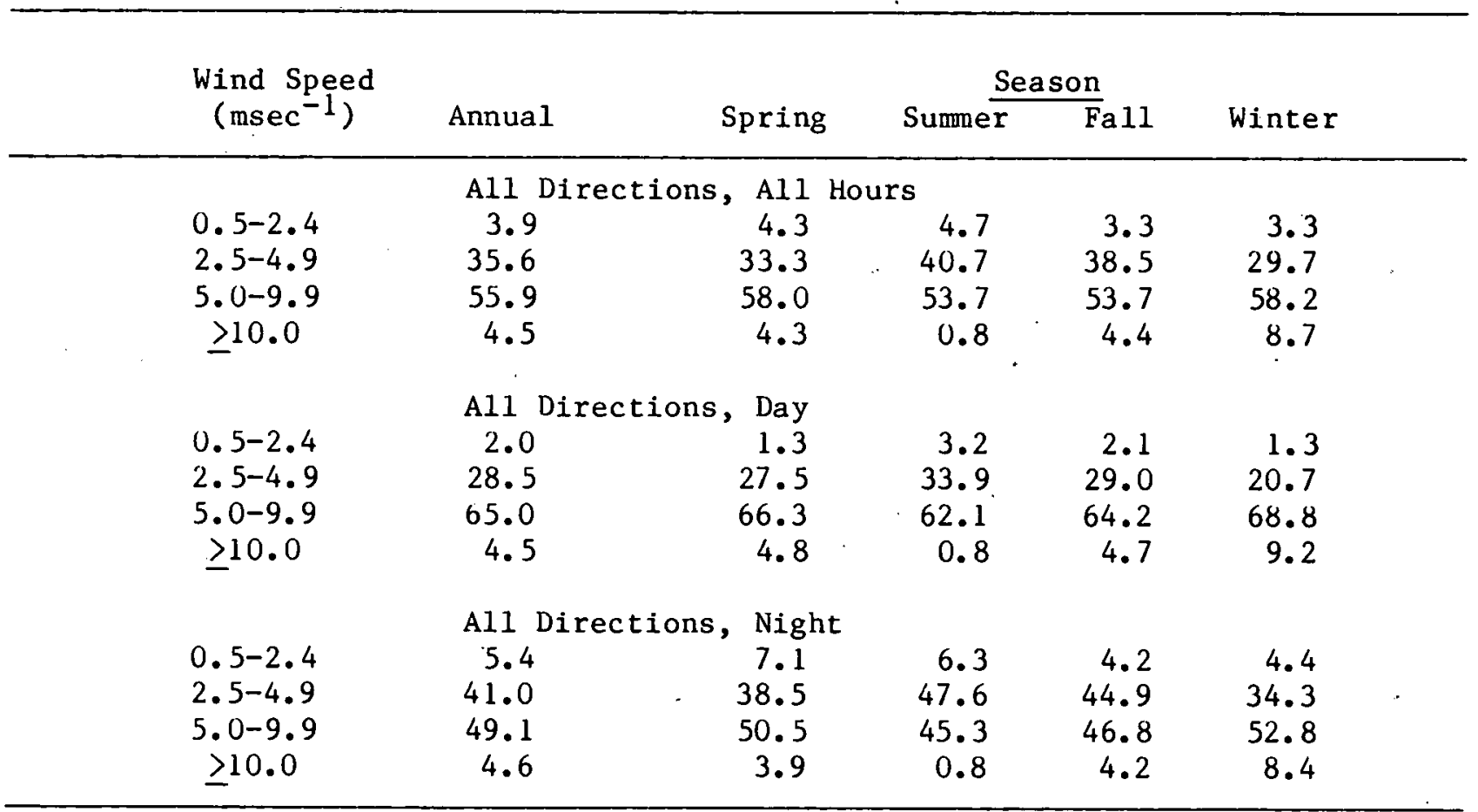


Table 6.12 (continued)

\begin{tabular}{|c|c|c|c|c|c|}
\hline \multirow{2}{*}{$\begin{array}{l}\text { Wind Speed } \\
\left(\mathrm{msec}^{-1}\right)\end{array}$} & \multirow[b]{2}{*}{ Annual } & \multicolumn{4}{|c|}{ Season } \\
\hline & & Spring & Summer & $\overline{F a l l}$ & Winter \\
\hline $\begin{array}{c}0.5-2.4 \\
2.5-4.9 \\
5.0-9.9 \\
\geq 10.0\end{array}$ & $\begin{array}{l}\text { Onshore, } \\
6.5 \\
47.2 \\
43.6 \\
2.8\end{array}$ & $\begin{array}{r}\text { All Hours } \\
8.2 \\
45.8 \\
44.5 \\
1.5\end{array}$ & $\begin{array}{r}7.7 \\
52.2 \\
39.1 \\
0.9\end{array}$ & $\begin{array}{r}4.0 \\
46.7 \\
45.4 \\
3.8\end{array}$ & $\begin{array}{r}6.3 \\
41.9 \\
43.3 \\
8.5\end{array}$ \\
\hline $\begin{array}{l}0.5-2.4 \\
2.5-4.4 \\
5.0-9.9 \\
\geq 10.0\end{array}$ & $\begin{array}{l}\text { Onshore, } \\
2.9 \\
43.5 \\
50.6 \\
2.9\end{array}$ & $\begin{array}{r}2.3 \\
38.8 \\
56.1 \\
3.3\end{array}$ & $\begin{array}{r}3.8 \\
48.0 \\
46.5 \\
1.7\end{array}$ & $\begin{array}{r}1.5 \\
42.6 \\
51.9 \\
3.9\end{array}$ & $\begin{array}{r}1.6 \\
45.3 \\
44.6 \\
5.6\end{array}$ \\
\hline $\begin{array}{c}0.5-2.4 \\
2.5-4.9 \\
5.0-9.9 \\
\geq 10.0\end{array}$ & $\begin{array}{l}\text { Onshore, } \\
9.7 \\
50.6 \\
37.2 \\
2.6\end{array}$ & $\begin{array}{r}\text { Night } \\
13.8 \\
52.8 \\
32.9 \\
0.5\end{array}$ & $\begin{array}{r}12.7 \\
56.6 \\
30.7 \\
0.0\end{array}$ & $\begin{array}{r}6.0 \\
50.2 \\
40.2 \\
3.7\end{array}$ & $\begin{array}{r}7.5 \\
39.9 \\
41.7 \\
10.9\end{array}$ \\
\hline
\end{tabular}


Table 6.12 (continued)

\begin{tabular}{|c|c|c|c|c|c|c|}
\hline & \multirow{2}{*}{$\begin{array}{l}\text { Wind Speed } \\
\left(\mathrm{msec}^{-1}\right)\end{array}$} & \multirow{2}{*}{ Annual } & \multicolumn{4}{|c|}{ Season } \\
\hline & & & Spring & Summer & $\bar{F} a 11$ & Winter \\
\hline & $\begin{array}{c}0.5-2.4 \\
2.5-4.9 \\
5.0-9.9 \\
\geq 10.0\end{array}$ & $\begin{array}{l}\text { Alongshore, } \\
2.0 \\
28.4 \\
64.8 \\
4.8\end{array}$ & $\begin{array}{c}\text { All Hours } \\
2.6 \\
28.4 \\
66.0 \\
3.0\end{array}$ & $\begin{array}{r}1.5 \\
31.3 \\
66.2 \\
0.9\end{array}$ & $\begin{array}{r}2.0 \\
30.9 \\
61.8 \\
5.4\end{array}$ & $\begin{array}{r}2.0 \\
21.7 \\
63.9 \\
12.4\end{array}$ \\
\hline . & $\begin{array}{c}0.5-2.4 \\
2.5-4.9 \\
5.0-9.9 \\
\geq 10.0\end{array}$ & $\begin{array}{l}\text { Alongshore, } \\
1.2 \\
20.4 \\
74.1 \\
4.4\end{array}$ & $\begin{array}{r}\text { Day } \\
0.6 \\
21.9 \\
74.8 \\
2.7\end{array}$ & $\begin{array}{r}0.8 \\
23.0 \\
75.7 \\
0.5\end{array}$ & $\begin{array}{r}2.6 \\
19.3 \\
72.1 \\
6.0\end{array}$ & $\begin{array}{l}1.0 \\
14.4 \\
71.3 \\
13.3\end{array}$ \\
\hline & $\begin{array}{c}0.5-2.4 \\
2.5-4.9 \\
5.0-9.9 \\
\geq 10.0\end{array}$ & $\begin{array}{l}\text { Alongshore, } \\
2.7 \\
35.0 \\
57.2 \\
5.1\end{array}$ & $\begin{array}{r}\text { Night } \\
4.5 \\
34.3 \\
57.9 \\
3.3\end{array}$ & $\begin{array}{r}2.3 \\
40.0 \\
56.3 \\
1.4\end{array}$ & $\begin{array}{r}1.5 \\
39.4 \\
54.1 \\
4.9\end{array}$ & $\begin{array}{r}2.6 \\
25.8 \\
59.7 \\
11.9\end{array}$ \\
\hline
\end{tabular}


Table 6.12 (continued)

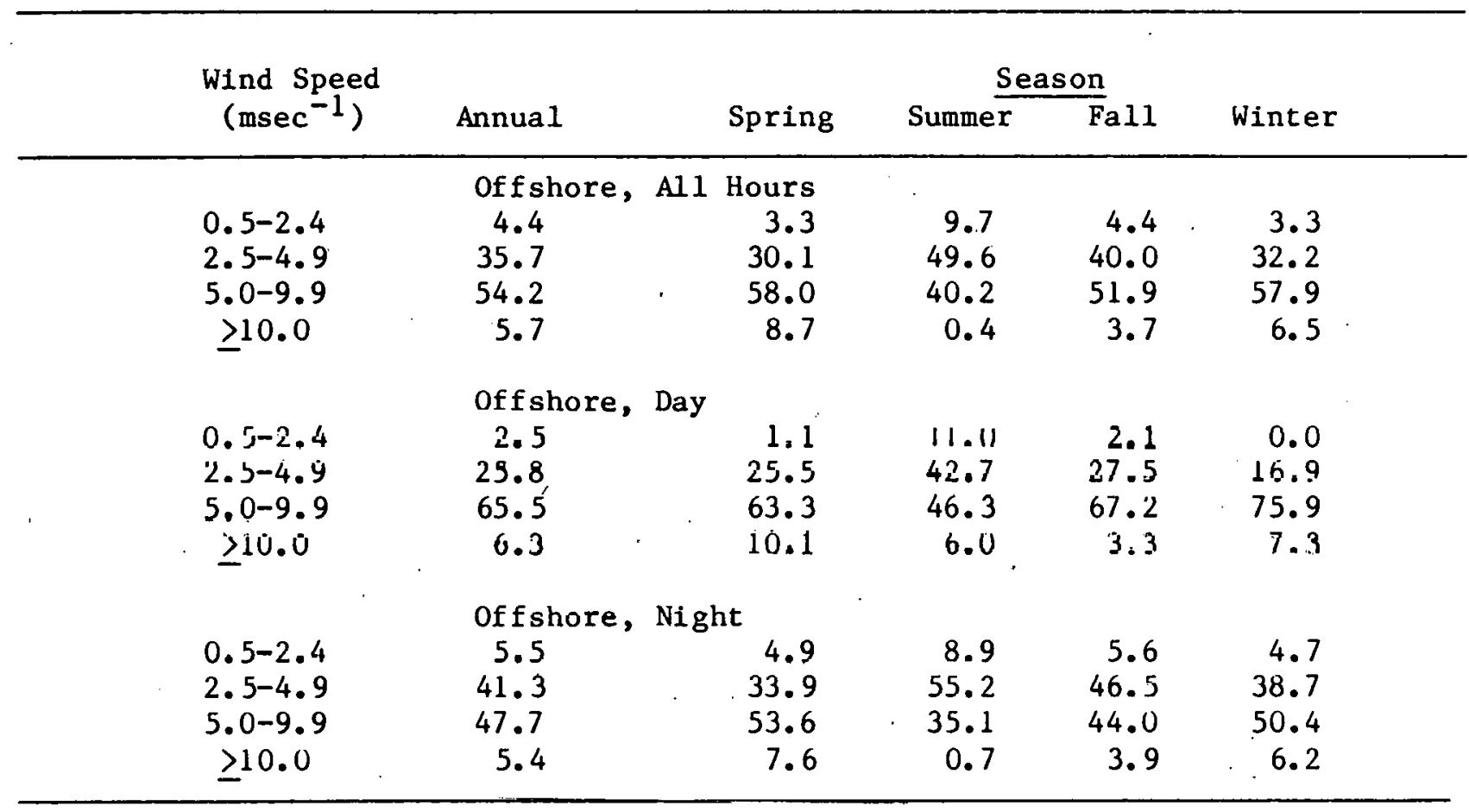


Table 6.13

Wilmington, North Carolina

Percent of Hours in Each Wind Speed Class

by Wind Direction, Season and Time of Day

\begin{tabular}{|c|c|c|c|c|c|}
\hline \multirow{2}{*}{$\begin{array}{l}\text { Wind Speed } \\
\left(\mathrm{msec}^{-1}\right)\end{array}$} & \multirow[b]{2}{*}{ Annual } & \multirow[b]{2}{*}{ Spring } & \multicolumn{2}{|c|}{ Season } & \multirow[b]{2}{*}{ Winter } \\
\hline & & & Sumner & Fa 11 & \\
\hline \multicolumn{6}{|c|}{ All Directions, Al1 Hours } \\
\hline $0.5-2.4$ & 17.6 & 13.9 & 24.1 & 19.0 & 13.6 \\
\hline $2.5-4.9$ & 55.0 & 51.1 & 59.5 & 55.6 & 54.1 \\
\hline $5.0-9.9$ & 26.9 & 34.3 & 16.5 & 24.8 & 31.8 \\
\hline$\geq 10.0$ & 0.5 & 0.7 & 0.0 & 0.6 & 0.6 \\
\hline \multicolumn{6}{|c|}{ All Directions, Day } \\
\hline $0.5-2.4$ & 9.4 & 6.4 & 15.0 & 8.7 & 6.3 \\
\hline $2.5-4.9$ & 52.1 & 44.1 & 60.3 & 55.5 & 47.9 \\
\hline $5.0-9.9$ & 37.8 & 48.3 & 24.7 & 35.1 & 44.7 \\
\hline$\geq 10.0$ & 0.7 & 1.2 & 0.0 & 0.7 & 1.1 \\
\hline \multicolumn{6}{|c|}{ All Directions, Night } \\
\hline $0.5-2.4$ & 25.3 & 21.2 & 34.9 & 27.9 & 19.1 \\
\hline $2.5-4.9$ & 57.6 & 57.8 & 58.5 & 55.5 & 58.7 \\
\hline $5.0-9.9$ & 16.9 & 20.9 & 6.6 & 16.0 & 22.1 \\
\hline$\geq 10.0$ & 0.2 & 0.1 & 0.0 & 0.6 & 0.1 \\
\hline
\end{tabular}


Table 6.13 (continued)

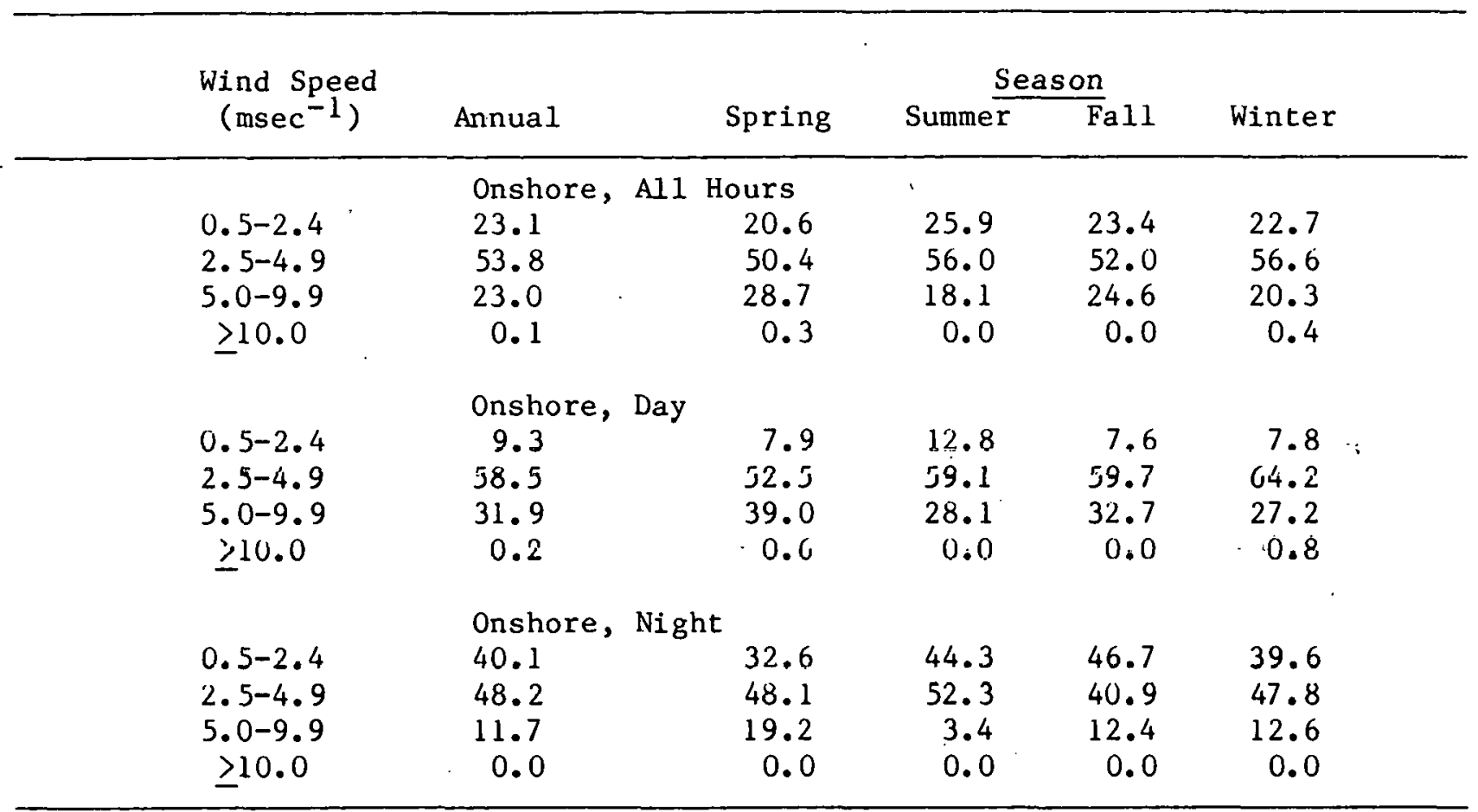


Table 6.13 (continued)

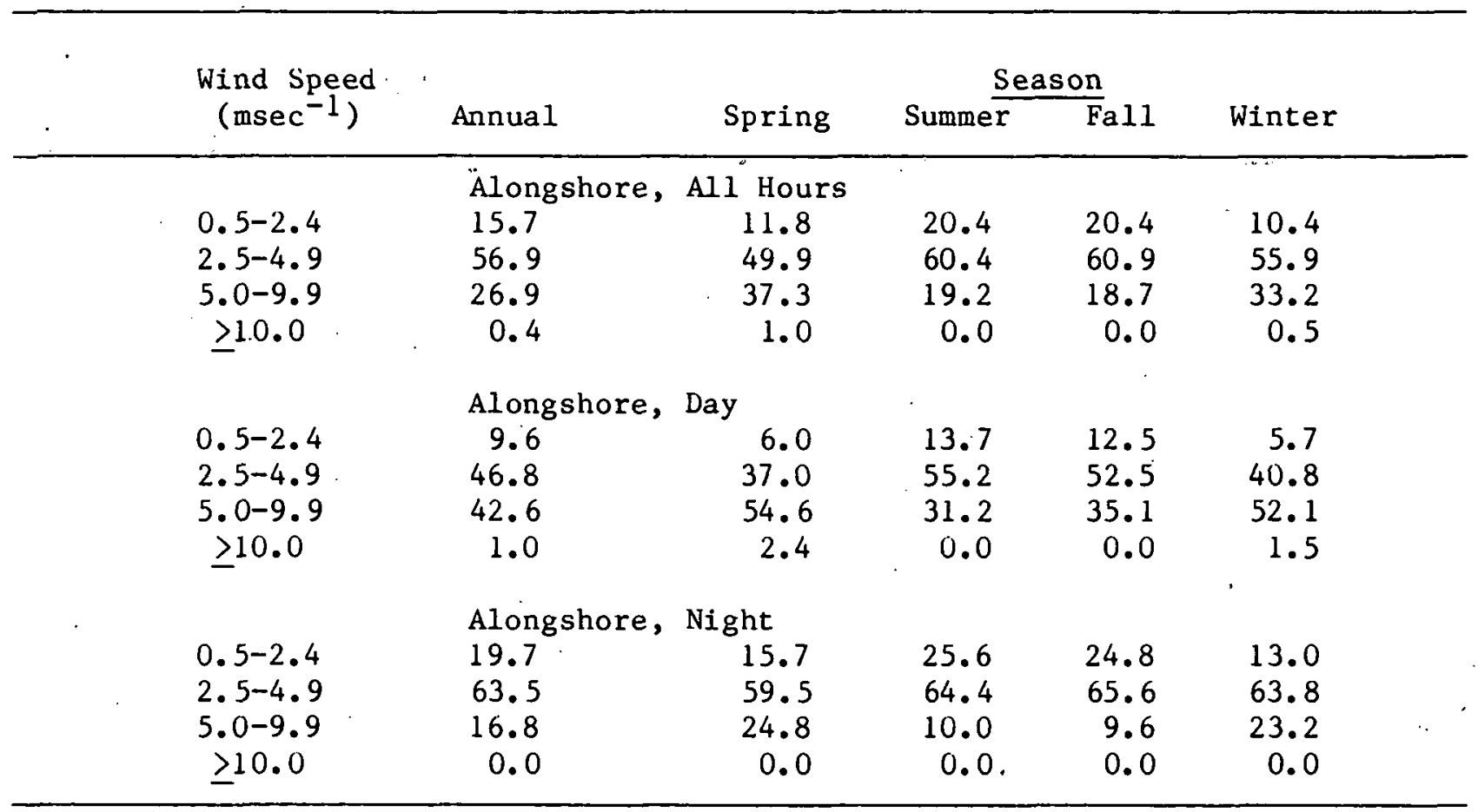


Table 6.13 (continued)

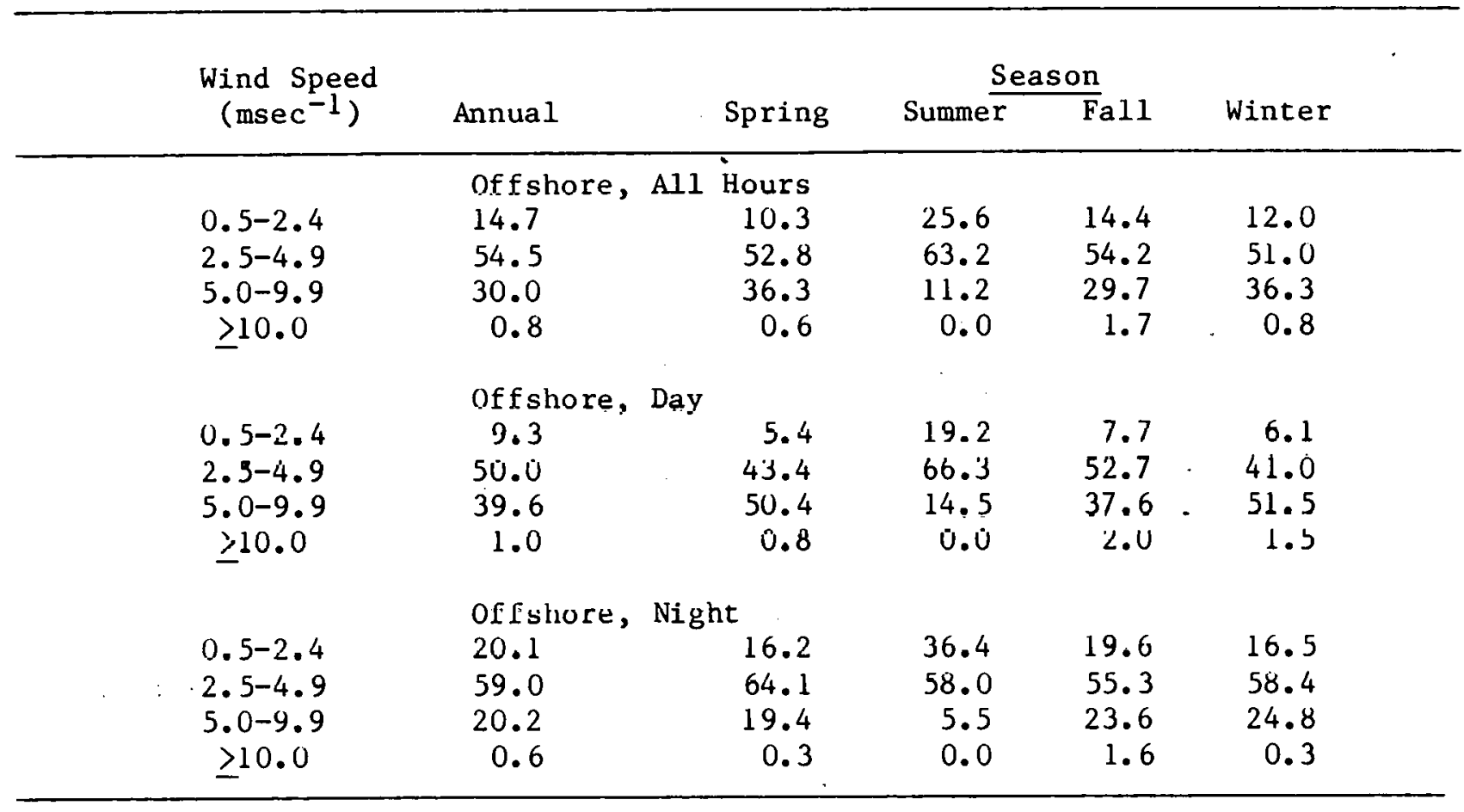


Table 6.14

Charleston, South Carolina

Percent of Hours, in Each Wind Speed Class

by Wind Direction, Season and Time of Day

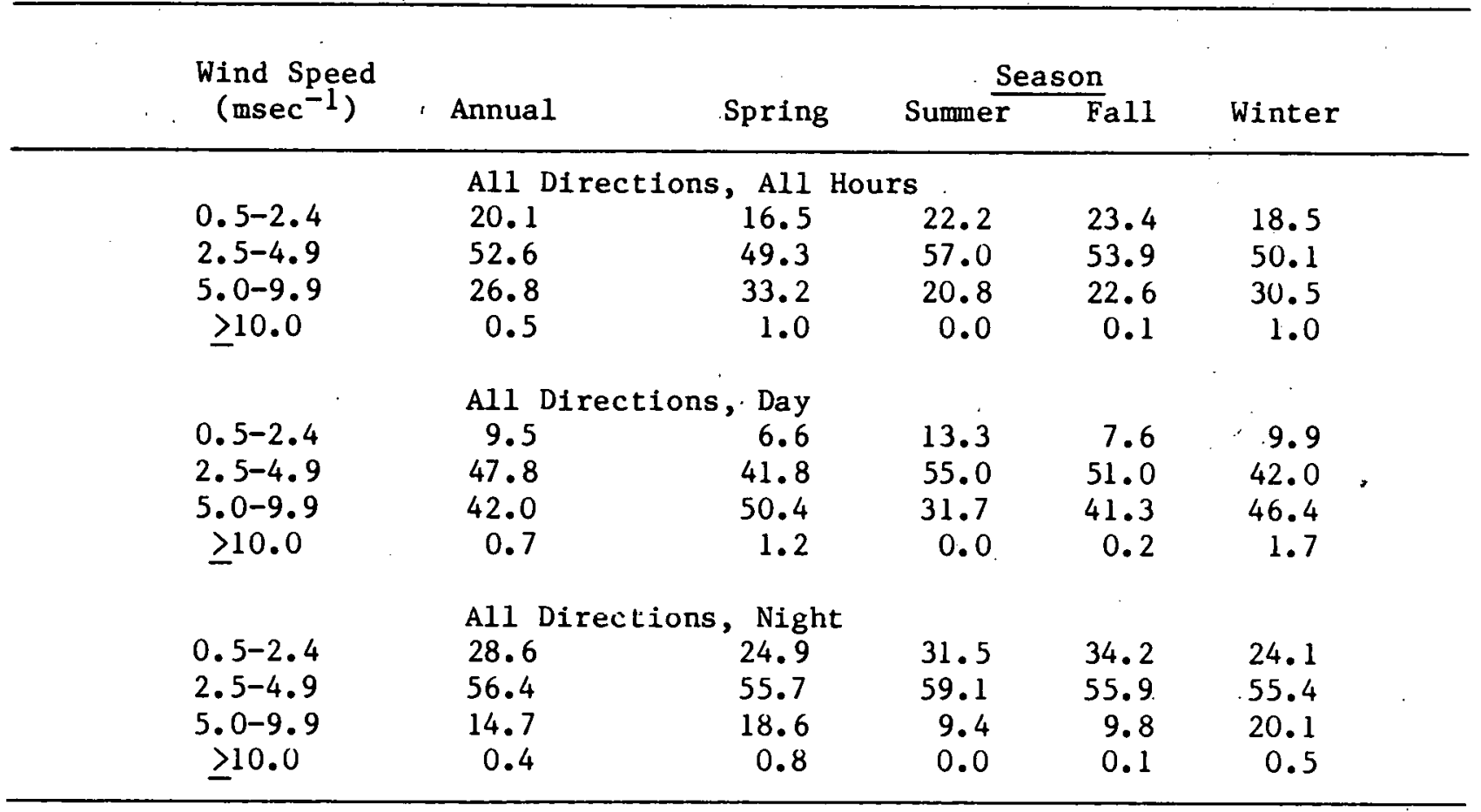


Table 6.14 (continued)

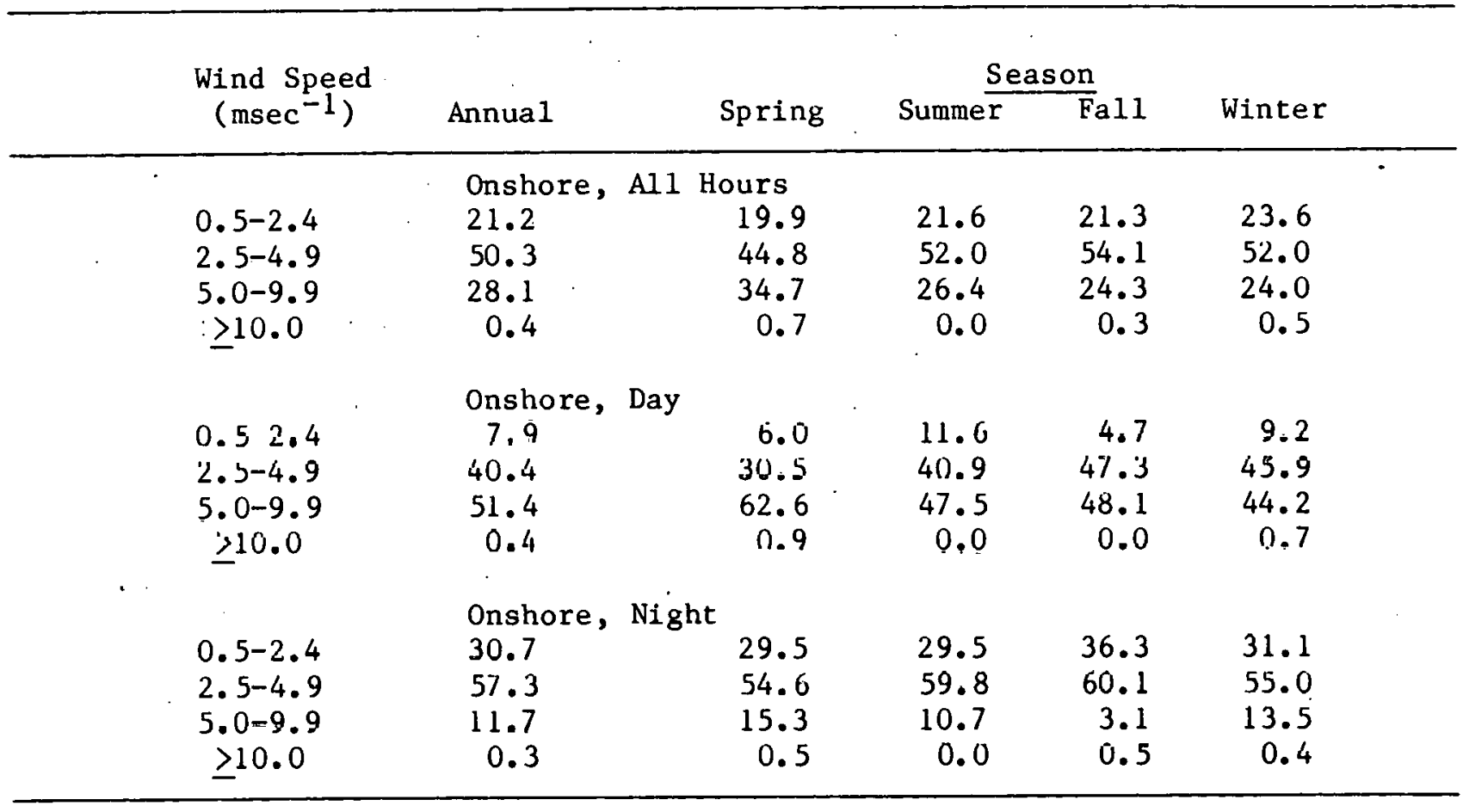


Table 6.14 (continued)

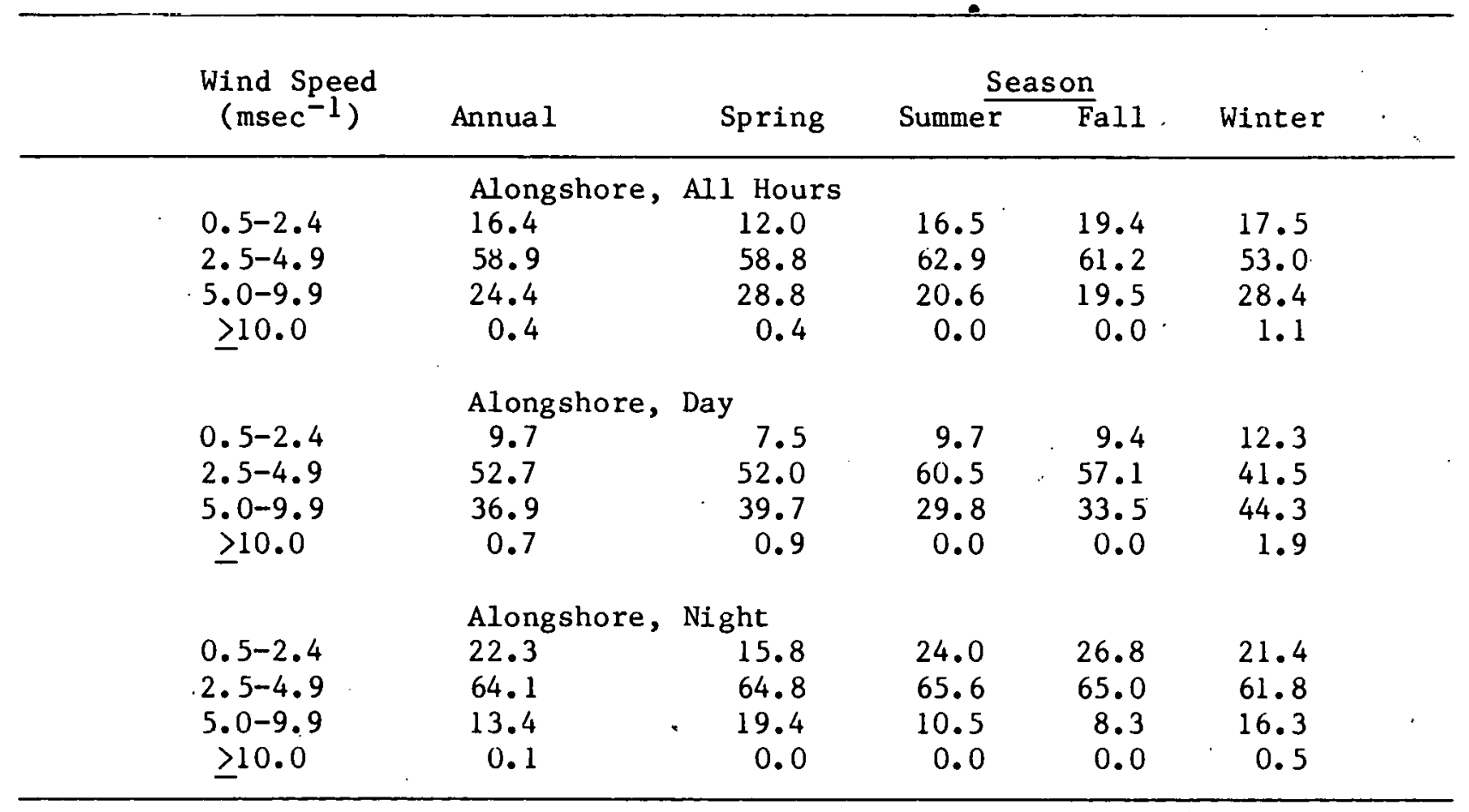


Table 6.14 (continued)

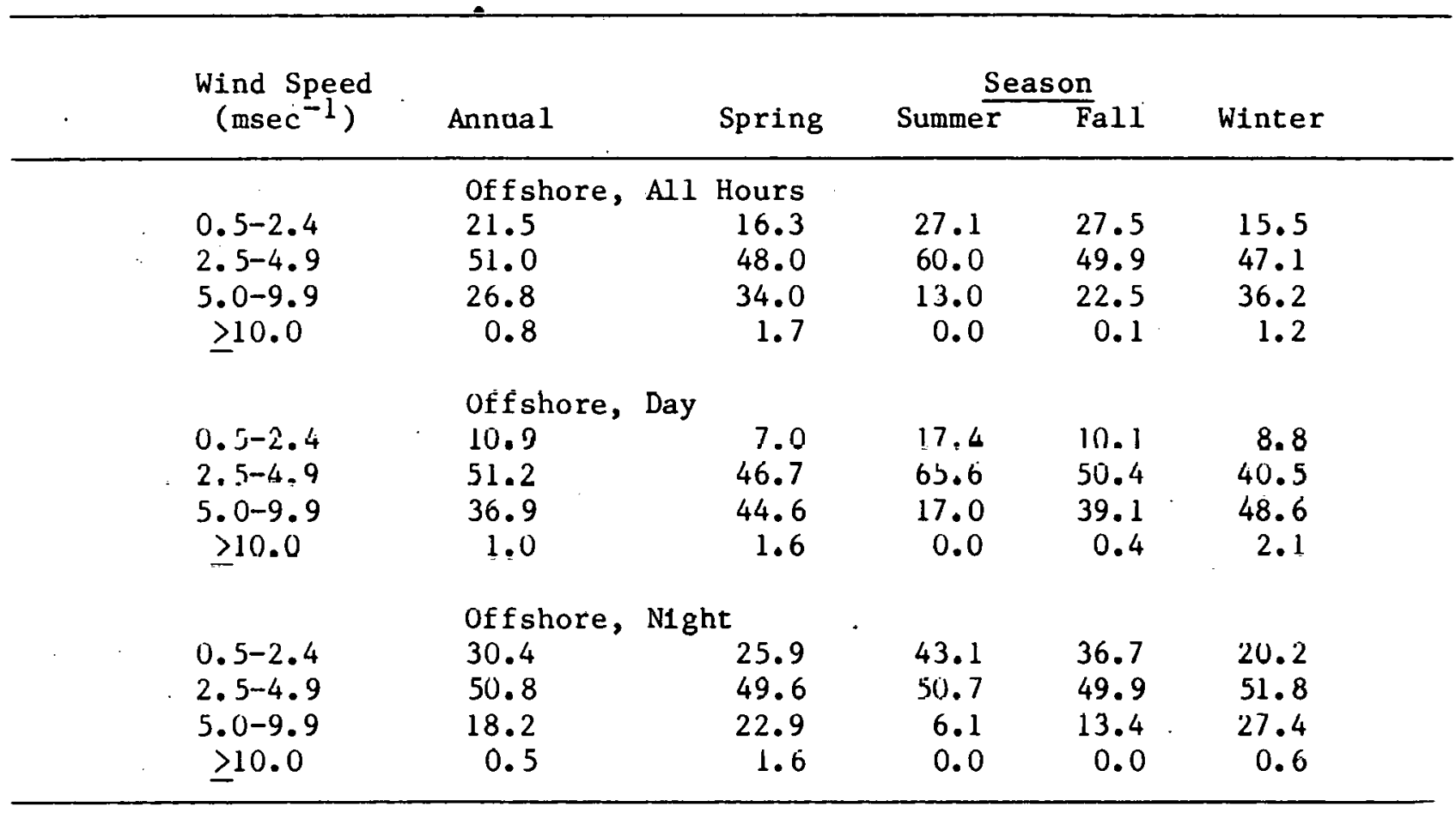


Table 6.15

Brunswick, Georgia

Percent of Hours in Each Wind Speed Class

by Wind Direction, Season and Time of Day

\begin{tabular}{|c|c|c|c|c|c|}
\hline \multirow{2}{*}{$\begin{array}{l}\text { Wind Speed } \\
\left(\mathrm{msec}^{-1}\right)\end{array}$} & \multirow[b]{2}{*}{ Annual } & \multicolumn{4}{|c|}{ Season } \\
\hline & & Spring & Summer & Fall & Winter \\
\hline \multicolumn{6}{|c|}{ All Directions, All Hours } \\
\hline $0.5-2.4$ & 45.3 & 40.9 & 52.9 & 43.0 & 45.1 \\
\hline $2.5-4.9$ & 43.7 & 45.8 & 42.8 & 44.7 & 41.2 \\
\hline $5.0-9.9$ & 10.9 & 13.2 & 4.1 & 12.3 & 13.6 \\
\hline$\geq 10.0$ & 0.1 & 0.1 & 0.1 & 0.0 & 0.1 \\
\hline \multicolumn{6}{|c|}{ All Directions, Day } \\
\hline $0.5-2.4$ & 31.3 & 24.8 & 43.0 & 23.6 & 32.9 \\
\hline $2.5-4.9$ & 52.6 & 54.7 & 51.2 & 55.7 & 48.7 \\
\hline $5.0-9.9$ & 16.0 & 20.5 & 5.7 & 20.7 & 18.4 \\
\hline$\geq 10.0$ & 0.0 & 0.0 & 0.2 & 0.0 & 0.0 \\
\hline \multicolumn{6}{|c|}{ All Directions, Night } \\
\hline $0.5-2.4$ & 59.9 & 57.3 & 66.7 & 62.1 & 55.7 \\
\hline $2.5-4.9$ & 34.6 & 36.6 & 31.3 & 34.3 & 34.7 \\
\hline $5.0-9.9$ & 5.5 & 5.9 & 2.1 & 3.6 & 9.4 \\
\hline$\geq 10.0$ & 0.1 & 0.2 & 0.0 & 0.0 & 0.2 \\
\hline
\end{tabular}

3 
Table 6.15 (continued)

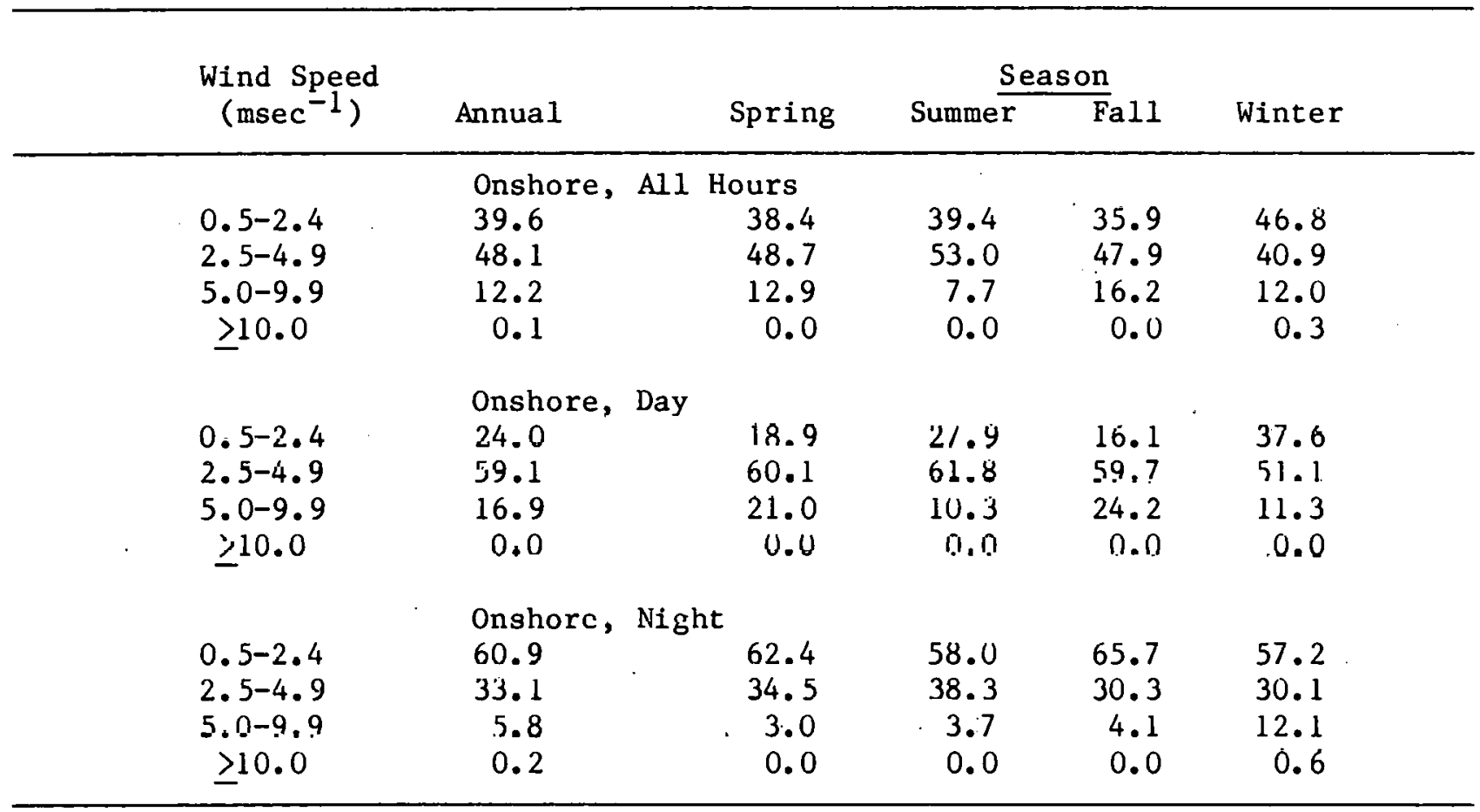


Table 6.15 (continued)

\begin{tabular}{|c|c|c|c|c|c|}
\hline \multirow{2}{*}{$\begin{array}{l}\text { Wind Speed } \\
\left(\mathrm{msec}^{-1}\right)\end{array}$} & \multirow[b]{2}{*}{ Annual } & \multirow[b]{2}{*}{ Spring } & \multicolumn{2}{|c|}{ Season } & \multirow[b]{2}{*}{ Winter } \\
\hline & & & Summer & $\bar{F} a 11$ & \\
\hline $\begin{array}{c}0.5-2.4 \\
2.5-4.9 \\
5.0-9.9 \\
\geq 10.0\end{array}$ & $\begin{array}{l}\text { Alongshore, } \\
45.3 \\
44.9 \\
9.6 \\
0.1\end{array}$ & $\begin{array}{c}\text { Al1 Hours } \\
34.8 \\
51.4 \\
13.8 \\
0.0\end{array}$ & $\begin{array}{r}63.3 \\
34.4 \\
1.8 \\
0.5\end{array}$ & $\begin{array}{r}39.4 \\
48.7 \\
11.9 \\
0.0\end{array}$ & $\begin{array}{r}45.8 \\
42.9 \\
11.3 \\
0.0\end{array}$ \\
\hline $\begin{array}{c}0.5-2.4 \\
2.5-4.9 \\
5.0-9.9 \\
\geq 10.0\end{array}$ & $\begin{array}{l}\text { Alongshore, } \\
33.8 \\
51.3 \\
14.6 \\
0.2\end{array}$ & $\begin{array}{l}\text { Day } \\
\begin{array}{r}26.7 \\
58.0 \\
15.3 \\
0.0\end{array}\end{array}$ & $\begin{array}{r}54.2 \\
42.1 \\
2.5 \\
1.1\end{array}$ & $\begin{array}{r}26.3 \\
51.0 \\
22.6 \\
0.0\end{array}$ & $\begin{array}{r}31.1 \\
52.8 \\
16.1 \\
0.0\end{array}$ \\
\hline $\begin{array}{c}0.5-2.4 \\
2.5-4.9 \\
5.0-9.9 \\
\geq 10.0\end{array}$ & $\begin{array}{l}\text { Alongshore, } \\
53.3 \\
40.6 \\
6.1 \\
0.0\end{array}$ & $\begin{array}{r}\text { Night } \\
41.3 \\
47.4 \\
11.3 \\
0.0\end{array}$ & $\begin{array}{r}69.6 \\
29.0 \\
1.4 \\
0.0\end{array}$ & $\begin{array}{r}48.4 \\
47.1 \\
4.4 \\
0.0\end{array}$ & $\begin{array}{r}55.9 \\
36.2 \\
8.0 \\
0.0\end{array}$ \\
\hline
\end{tabular}


Table 6.15 (continued)

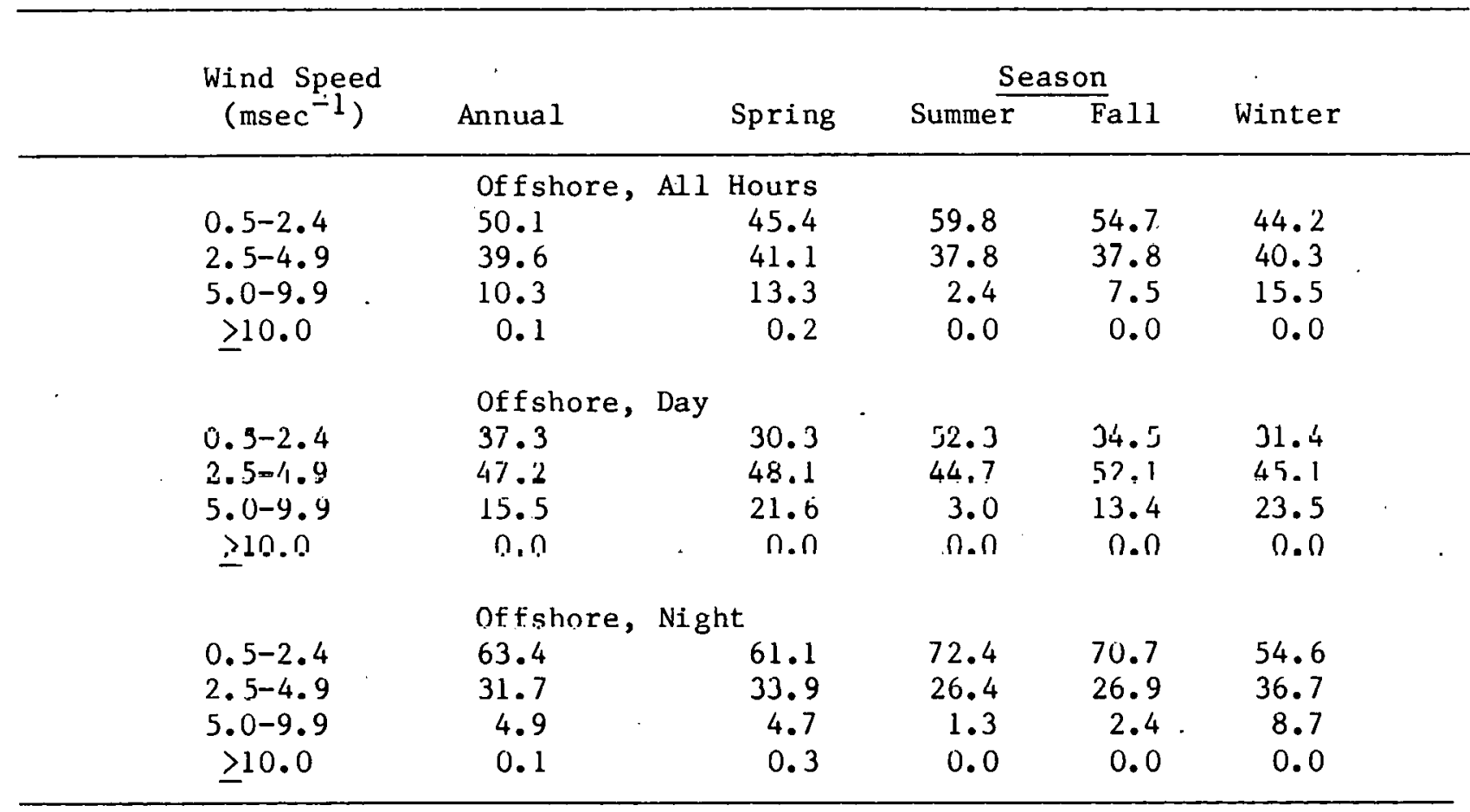


Table 6.16

Cape Kennedy, Florida

Percent of Hours in Each Wind Speed Class

by Wind Direction, Season and Time of Day

\begin{tabular}{|c|c|c|c|c|c|}
\hline \multirow{2}{*}{$\begin{array}{l}\text { Wind Speed } \\
\left(\mathrm{msec}^{-1}\right)\end{array}$} & \multirow[b]{2}{*}{ Annual } & \multicolumn{4}{|c|}{ Season } \\
\hline & & Spring & Summer & Fall & Winter \\
\hline \multicolumn{6}{|c|}{ All Directions, All Hours } \\
\hline $0.5-2.4$ & 34.9 & 27.9 & 40.0 & 39.8 & 32.4 \\
\hline $2.5-4.9$ & 42.1 & 41.4 & 46.6 & 41.8 & 38.6 \\
\hline $5.0-9.9$ & 22.7 & 30.3 & 13.4 & 18.3 & 28.3 \\
\hline$\geq 10.0$ & 0.3 & 0.4 & 0.0 & 0.1 & 0.7 \\
\hline \multicolumn{6}{|c|}{ All Directions, Day } \\
\hline $0.5-2.4$ & 15.3 & 11.7 & 22.0 & 14.8 & 11.7 \\
\hline $2.5-4 \cdot 9$ & 50.4 & 42.6 & 56.3 & 58.3 & 44.3 \\
\hline $5.0-9.9$ & 33.9 & 45.3 & 21.7 & 26.7 & 43.5 \\
\hline$\geq 10.0$ & 0.3 & 0.5 & 0.0 & 0.2 & 0.6 \\
\hline \multicolumn{6}{|c|}{ A11 Directions, Night } \\
\hline $0.5-2.4$ & 50.1 & 41.0 & 58.9 & 56.4 & 45.7 \\
\hline $2.5-4.9$ & 35.6 & 40.4 & 36.4 & 30.8 & 35.0 \\
\hline $5.0-9.9$ & 14.0 & 18.2 & 4.7 & 12.8 & 18.6 \\
\hline$\geq 10.0$ & 0.3 & 0.4 & 0.0 & 0.0 & 0.7 \\
\hline
\end{tabular}




\section{Table 6.16 (continued)}

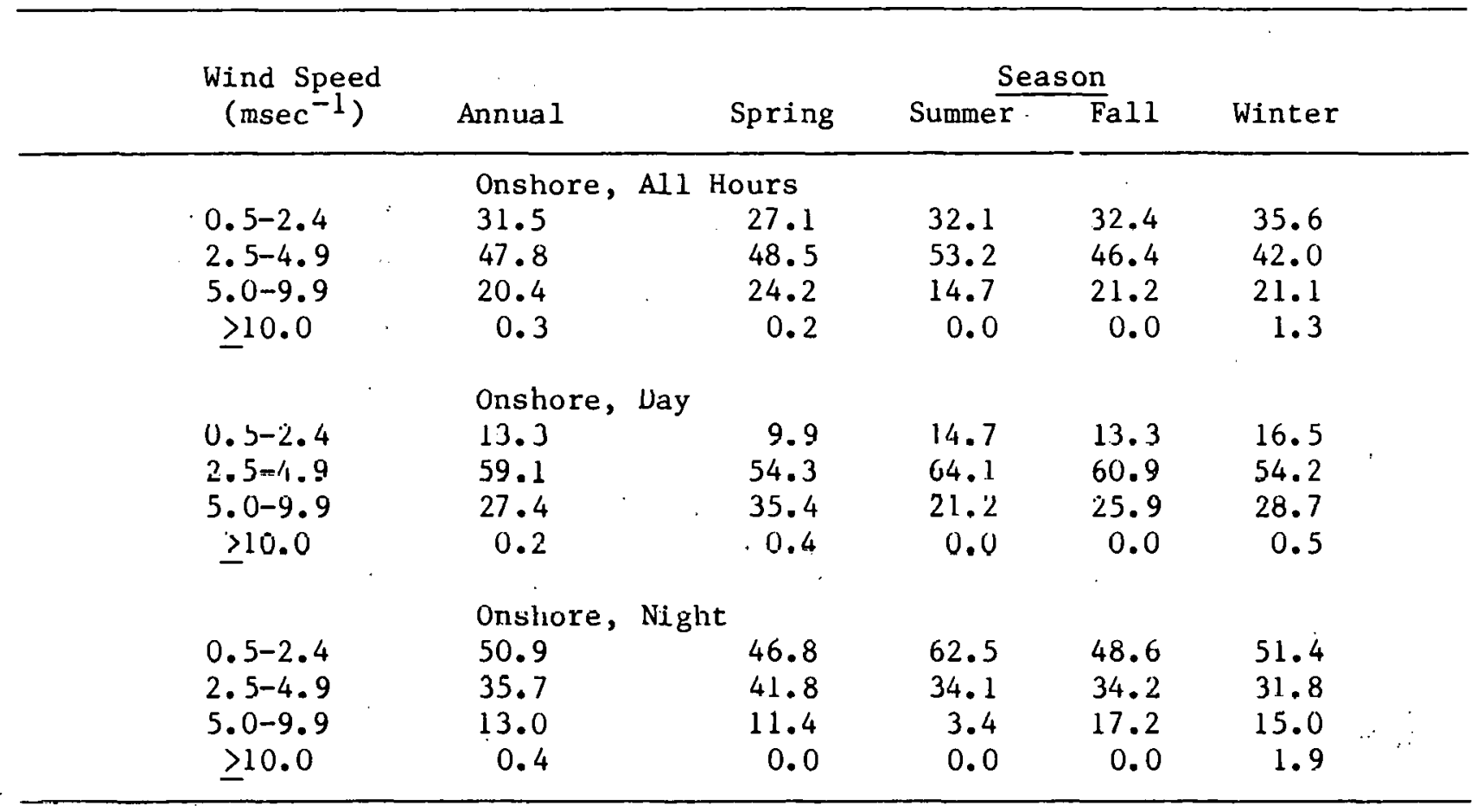


Table 6.16 (continued)

\begin{tabular}{|c|c|c|c|c|c|c|}
\hline & \multirow{2}{*}{$\begin{array}{l}\text { Wind Speed } \\
\left(\mathrm{msec}^{-1}\right)\end{array}$} & \multirow[b]{2}{*}{ Annual } & \multirow[b]{2}{*}{ Spring } & \multicolumn{2}{|c|}{ Season } & \multirow[b]{2}{*}{ Winter } \\
\hline & & & & Summer & Fall & \\
\hline & $\begin{array}{c}0.5-2.4 \\
2.5-4.9 \\
5.0-9.9 \\
\geq 10.0\end{array}$ & $\begin{array}{l}\text { Alongshore, } \\
32.4 \\
38.8 \\
28.5 \\
0.3\end{array}$ & $\begin{array}{c}\text { Al1 Hours } \\
27.4 \\
36.9 \\
35.6 \\
0.2\end{array}$ & $\begin{array}{r}38.3 \\
45.6 \\
16.0 \\
0.0\end{array}$ & $\begin{array}{r}42.4 \\
35.9 \\
21.3 \\
0.3\end{array}$ & $\begin{array}{r}26.6 \\
37.6 \\
35.2 \\
0.6\end{array}$ \\
\hline$\cdot$ & $\begin{array}{c}0.5-2.4 \\
2.5-4.9 \\
5.0-9.9 \\
\geq 10.0\end{array}$ & $\begin{array}{l}\text { Alongshore, } \\
11.3 \\
42.1 \\
46.3 \\
0.3\end{array}$ & $\begin{array}{r}\text { Day } \\
9.6 \\
35.0 \\
55.4 \\
0.0\end{array}$ & $\begin{array}{r}20.1 \\
49.6 \\
30.3 \\
0.0\end{array}$ & $\begin{array}{r}9.8 \\
53.4 \\
35.9 \\
0.8\end{array}$ & $\begin{array}{r}7.4 \\
36.9 \\
55.2 \\
0.5\end{array}$ \\
\hline & $\begin{array}{c}0.5-2.4 \\
2.5-4.9 \\
5.0-9.9 \\
\geq 10.0\end{array}$ & $\begin{array}{l}\text { Alongshore, } \\
46.5 \\
36.7 \\
16.6 \\
0.3\end{array}$ & $\begin{array}{r}\text { Night } \\
39.5 \\
38.1 \\
22.0 \\
0.3\end{array}$ & $\begin{array}{r}52.2 \\
42.6 \\
5.3 \\
0.0\end{array}$ & $\begin{array}{r}60.1 \\
26.5 \\
13.4 \\
0.0\end{array}$ & $\begin{array}{r}39.6 \\
38.4 \\
21.4 \\
0.7\end{array}$ \\
\hline
\end{tabular}


Table 6.16 (continued)

\begin{tabular}{|c|c|c|c|c|c|}
\hline \multirow{2}{*}{$\begin{array}{l}\text { Wind Speed } \\
\text { (msec }\end{array}$} & \multirow[b]{2}{*}{ Annual } & \multicolumn{4}{|c|}{ Season } \\
\hline & & Spring & Summer & Fall & Winter \\
\hline $\begin{array}{c}0.5-2.4 \\
2.5-4.9 \\
5.0-9.9 \\
\geq 10.0\end{array}$ & $\begin{array}{l}\text { Off shore, } \\
42.5 \\
37.5 \\
19.7 \\
0.3\end{array}$ & $\begin{array}{r}\text { Ail Hours } \\
31.0 \\
36.5 \\
31.3 \\
1.1\end{array}$ & $\begin{array}{r}51.8 \\
38.9 \\
9.4 \\
0.0\end{array}$ & $\begin{array}{r}54.7 \\
37.0 \\
8.3 \\
0.0\end{array}$ & $\begin{array}{r}37.5 \\
37.0 \\
25.2 \\
0.3\end{array}$ \\
\hline $\begin{array}{c}0.5-2.4 \\
2.5-4.4 .9 \\
5.0-9.9 \\
\geq 10.0\end{array}$ & $\begin{array}{l}\text { Offshore, } \\
24.3 \\
43.1 \\
32.1 \\
0.5\end{array}$ & $\begin{array}{r}\text { Day } \\
19.8 \\
30.3 \\
48.8 \\
1.0\end{array}$ & $\begin{array}{r}37.9 \\
47.5 \\
14.6 \\
0.0\end{array}$ & $\begin{array}{r}28.3 \\
53.7 \\
18.0 \\
0.0\end{array}$ & $\begin{array}{r}13.2 \\
44.0 \\
42.0 \\
0.8\end{array}$ \\
\hline $\begin{array}{c}0.5-2.4 \\
2.5-4.9 \\
5.0-9.9 \\
>10.0\end{array}$ & $\begin{array}{l}\text { Offshore, } \\
52.9 \\
34.2 \\
12.7 \\
0.2\end{array}$ & $\begin{array}{r}\text { Night } \\
38.2 \\
40.4 \\
20.3 \\
1.1\end{array}$ & $\begin{array}{r}62.2 \\
32.7 \\
5.1 \\
0.0\end{array}$ & $\begin{array}{r}66.2 \\
29.7 \\
4.1 \\
0.0\end{array}$ & $\begin{array}{r}49.2 \\
33.7 \\
17.1 \\
0.0\end{array}$ \\
\hline
\end{tabular}




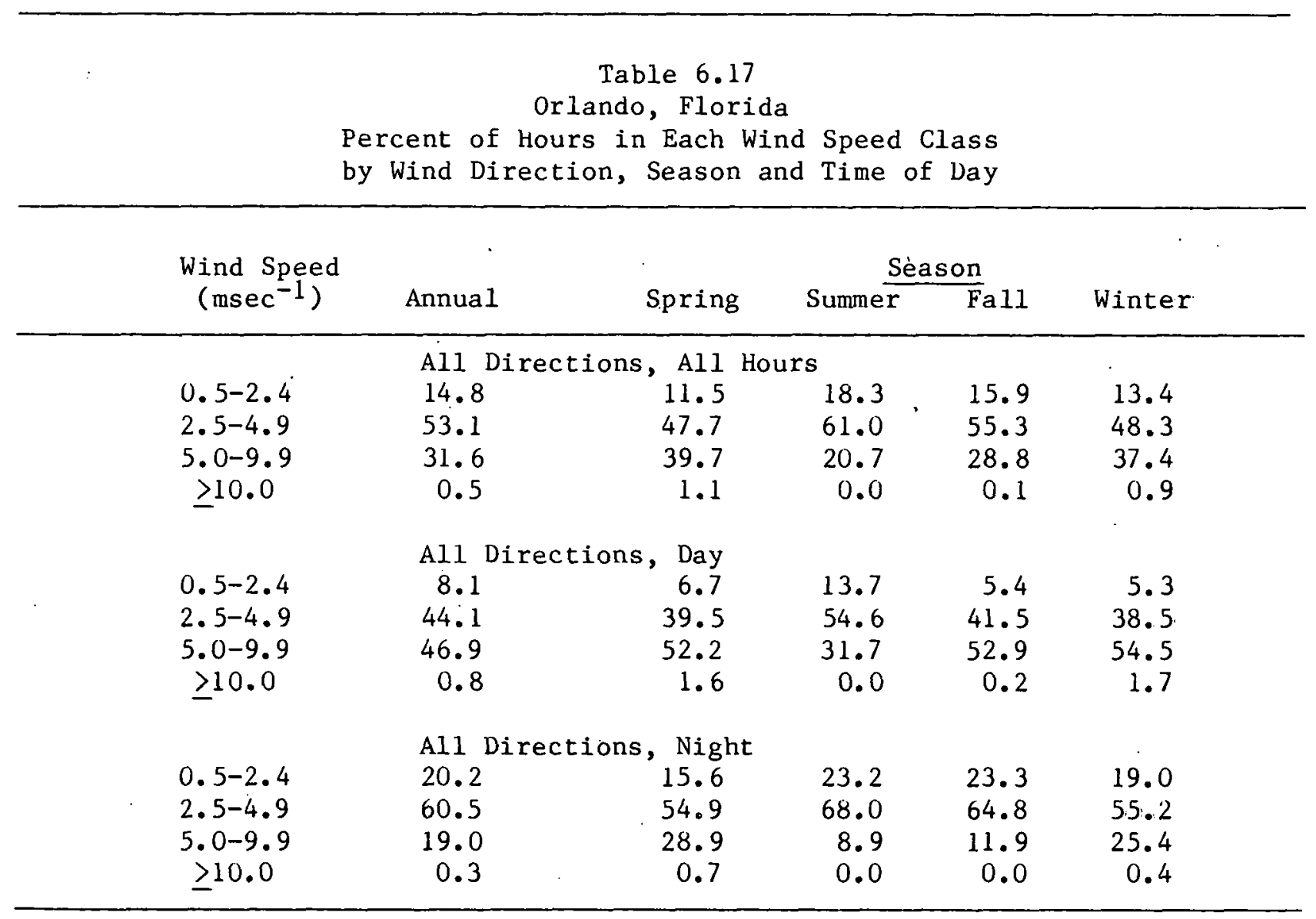


Table 6.17' (continued)

\begin{tabular}{|c|c|c|c|c|c|c|}
\hline \multirow{2}{*}{$\begin{array}{l}\text { Wind Speed } \\
\quad\left(\mathrm{msec}^{-1}\right)\end{array}$} & \multirow[b]{2}{*}{ Annual } & \multicolumn{5}{|c|}{ Season } \\
\hline & & & Spring & Summer & Fall & Winter \\
\hline $\begin{array}{c}0.5-2.4 \\
2.5-4.9 \\
5.0-9.9 \\
\geq 10.0\end{array}$ & $\begin{array}{c}\text { Onshore, } \\
15.2 \\
53.2 \\
31.5 \\
0.1\end{array}$ & All & $\begin{array}{l}\text { Hours } \\
11.4 \\
49.0 \\
39.6 \\
0.0\end{array}$ & $\begin{array}{r}18.1 \\
56.8 \\
25.1 \\
0.0\end{array}$ & $\begin{array}{r}15.5 \\
54.1 \\
30.4 \\
0.0\end{array}$ & $\begin{array}{r}15.7 \\
51.7 \\
31.9 \\
0.6\end{array}$ \\
\hline $\begin{array}{c}0.5-2.4 \\
2.5-4.9 \\
5.0-9.9 \\
\geq 10.0\end{array}$ & $\begin{array}{l}\text { Onshore, } \\
7.8 \\
40.0 \\
51.9 \\
0.3\end{array}$ & Day & $\begin{array}{r}8.0 \\
38.9 \\
53.1 \\
0.0\end{array}$ & $\begin{array}{r}13.0 \\
48.0 \\
39.1 \\
0.0\end{array}$ & $\begin{array}{r}3.9 \\
33.6 \\
62.5 \\
0.0\end{array}$ & $\begin{array}{r}7.6 \\
41.7 \\
49.2 \\
1.5\end{array}$ \\
\hline $\begin{array}{c}0.5-2.4 \\
2.5-4.9 \\
5.0-9.9 \\
\geq 10.0\end{array}$ & $\begin{array}{c}\text { Onshore, } \\
21.0 \\
63.5 \\
15.4 \\
0.0\end{array}$ & $\mathrm{Nigl}$ & ht $\begin{array}{r}14.3 \\
57.4 \\
28.3 \\
0.0\end{array}$ & $\begin{array}{c}23.7 \\
63.6 \\
10.7 \\
0.0\end{array}$ & $\begin{array}{r}23.8 \\
68.5 \\
7.7 \\
0.0\end{array}$ & $\begin{array}{r}21.4 \\
50.5 \\
20.2 \\
0.0\end{array}$ \\
\hline
\end{tabular}


Table 6.17 (continued)

\begin{tabular}{|c|c|c|c|c|c|c|}
\hline & \multirow{2}{*}{$\begin{array}{l}\text { Wind Speed } \\
\left(\mathrm{msec}^{-1}\right)\end{array}$} & \multirow[b]{2}{*}{ Annual } & \multirow[b]{2}{*}{ Spring } & \multicolumn{2}{|c|}{ Season } & \multirow[b]{2}{*}{ Winter } \\
\hline & & & & Summer & $\overline{F a l 1}$ & \\
\hline & $\begin{array}{c}0.5-2.4 \\
2.5-4.9 \\
5.0-9.99 \\
\geq 10.0\end{array}$ & $\begin{array}{l}\text { Alongshore, } \\
16.7 \\
55.3 \\
27.8 \\
0.22\end{array}$ & $\begin{array}{c}\text { Al1 Hours } \\
116.9 \\
51.5 \\
31.3 \\
0.3\end{array}$ & $\begin{array}{r}21.2 \\
63.8 \\
15.0 \\
0.0\end{array}$ & $\begin{array}{r}13.6 \\
58.7 \\
27.7 \\
0.0\end{array}$ & $\begin{array}{r}15.1 \\
48.0 \\
36.3 \\
0.6\end{array}$ \\
\hline & $\begin{array}{c}0.5-2.4 \\
2.5-4.9 \\
5.0-9.9 \\
\geq 10.0\end{array}$ & $\begin{array}{l}\text { Alongshore, } \\
10.0 \\
49.9 \\
40.1 \\
0.0\end{array}$ & $\begin{array}{l}\text { Day } \\
\begin{array}{r}11.6 \\
43.4 \\
45.0 \\
0.0\end{array}\end{array}$ & $\begin{array}{l}17.1 \\
57.7 \\
25.3 \\
0.0\end{array}$ & $\begin{array}{r}5.6 \\
52.5 \\
41.9 \\
0.0\end{array}$ & $\begin{array}{r}2.3 \\
44.5 \\
53.1 \\
0.0\end{array}$ \\
\hline . & $\begin{array}{c}0.5-2.4 \\
2.5-4.9 \\
5.0-9.9 \\
\geq 10.0\end{array}$ & $\begin{array}{l}\text { Alongshore, } \\
21.0 \\
58.7 \\
19.8 \\
0.4\end{array}$ & $\begin{array}{r}\text { Night } \\
20.9 \\
57.5 \\
21.0 \\
0.6\end{array}$ & $\begin{array}{r}25.0 \\
69.7 \\
5.3 \\
0.0\end{array}$ & $\begin{array}{r}17.8 \\
62.0 \\
20.3 \\
0.0\end{array}$ & $\begin{array}{r}21.2 \\
49.8 \\
28.2 \\
0.8\end{array}$ \\
\hline
\end{tabular}


Table 6.17 (continued)

\begin{tabular}{|c|c|c|c|c|c|}
\hline \multirow{2}{*}{$\begin{array}{l}\text { Wind Speed } \\
\left(\mathrm{msec}^{-1}\right)\end{array}$} & \multirow[b]{2}{*}{ Annual } & \multirow[b]{2}{*}{ Spring } & \multicolumn{2}{|c|}{ Season } & \multirow[b]{2}{*}{ Winter } \\
\hline & & & Summer & Fall & \\
\hline $\begin{array}{c}0.5-2.4 \\
2.5-4.9 \\
5.0-9.9 \\
\geq 10.0\end{array}$ & $\begin{array}{l}\text { Of } \mathrm{f} \text { shore, } \\
12.8 \\
51.3 \\
34.7 \\
1.2\end{array}$ & $\begin{array}{r}\text { A11. Hours } \\
9.1 \\
43.9 \\
44.7 \\
2.4\end{array}$ & $\begin{array}{r}16.5 \\
63.3 \\
20.2 \\
0.0\end{array}$ & $\begin{array}{r}19.4 \\
53.7 \\
26.6 \\
0.4\end{array}$ & $\begin{array}{r}9.3 \\
44.4 \\
44.8 \\
1.6\end{array}$ \\
\hline $\begin{array}{c}0.5-2.4 \\
2.5-4.9 \\
5.0-9.9 \\
\geq 10.0\end{array}$ & $\begin{array}{l}\text { Offshore, } \\
7.3 \\
43.3 \\
45.5 \\
1.9\end{array}$ & $\begin{array}{r}3.3 \\
38.8 \\
54.4 \\
3.5\end{array}$ & $\begin{array}{r}12.6 \\
50.6 \\
28.8 \\
0.0\end{array}$ & $\begin{array}{r}8.6 \\
50.2 \\
40.4 \\
1.1 .8\end{array}$ & $\begin{array}{r}4.7 \\
32.2 \\
60.3 \\
2.8\end{array}$ \\
\hline $\begin{array}{c}0.5-2.4 \\
2.5-4.9 \\
5.0-9.9 \\
\geq 10.0\end{array}$ & $\begin{array}{l}\text { Offshore, } \\
18.4 \\
57.5 \\
23.5 \\
0.6\end{array}$ & $\begin{array}{l}\text { Night } \\
\begin{array}{r}14.8 \\
48.7 \\
35.3 \\
1.2\end{array}\end{array}$ & $\begin{array}{r}21.5 \\
69.3 \\
9.2 \\
0.0\end{array}$ & $\begin{array}{r}29.6 \\
57.0 \\
13.4 \\
0.0\end{array}$ & $\begin{array}{r}13.3 \\
56.6 \\
29.7 \\
0.4\end{array}$ \\
\hline
\end{tabular}


Table 6.18

Miami, Florida

Percent of Hours in Each Wind Speed Class

by Wind Direction, Season and Time of Day

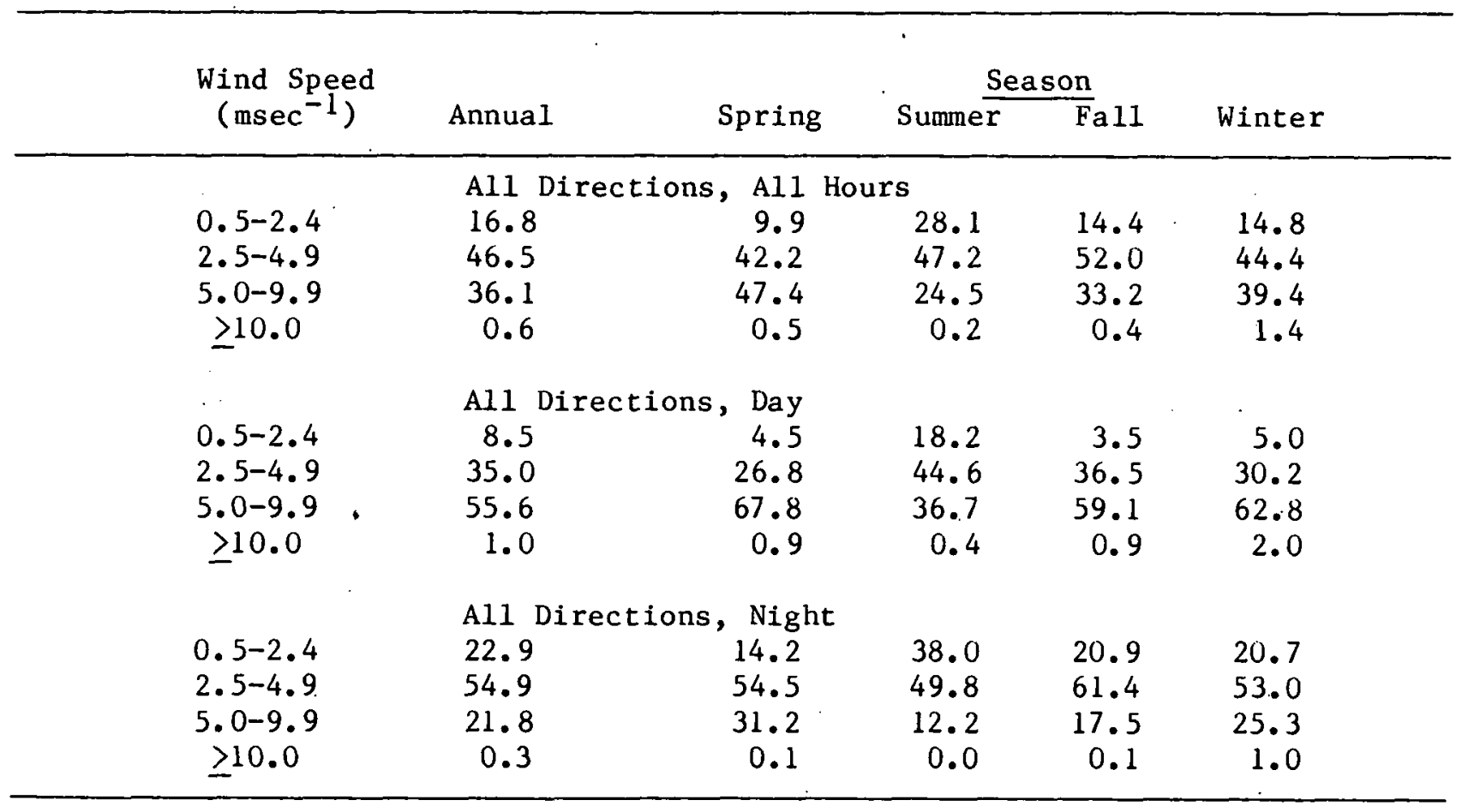




\section{- Table 6.18 (continued)}

\begin{tabular}{|c|c|c|c|c|c|c|c|}
\hline & \multirow{2}{*}{\multicolumn{2}{|c|}{$\begin{array}{l}\text { Wind Speed } \\
\left(\mathrm{msec}^{-1}\right)\end{array}$}} & \multirow[b]{2}{*}{ Annual } & \multirow[b]{2}{*}{ Spring } & \multicolumn{2}{|c|}{ Season } & \multirow[b]{2}{*}{ Winter } \\
\hline & & & & & Summe $\bar{r}$ & Fall & \\
\hline & $\begin{array}{c}0.5-2.4 \\
2.5-4.9 \\
5.0-9.9 \\
\geq 10.0\end{array}$ & $i$ & $\begin{array}{l}\text { Onshore, } \\
9.4 \\
44.9 \\
45.3 \\
0.3\end{array}$ & $\begin{array}{r}\text { Al1 Hours } \\
4.9 \\
39.7 \\
55.3 \\
0.1\end{array}$ & $\begin{array}{r}14.6 \\
51.3 \\
33.8 \\
0.2\end{array}$ & $\begin{array}{r}9.5 \\
46.9 \\
43.1 \\
0.5\end{array}$ & $\begin{array}{r}9.1 \\
42.1 \\
48.1 \\
0.7\end{array}$ \\
\hline & $\begin{array}{c}0.5-2.4 \\
2.5-4.9 \\
5.0=9.9 \\
\geq 10.0\end{array}$ & & $\begin{array}{l}\text { Onshore, } \\
3.0 \\
32.4 \\
64.0 \\
0.6\end{array}$ & $\begin{array}{r}1.6 \\
23.3 \\
74.9 \\
0.2\end{array}$ & $\begin{array}{r}6.1 \\
46.2 \\
47.3 \\
0.4\end{array}$ & $\begin{array}{r}1.4 \\
2 \dot{y} .5 \\
68.0 \\
1.1\end{array}$ & $\begin{array}{r}1.8 \\
28.0 \\
69.6 \\
0.6\end{array}$ \\
\hline - & $\begin{array}{c}0.5-2.4 \\
2.5-4.9 \\
5.0-9.9 \\
\geq 10.0\end{array}$ & & $\begin{array}{l}\text { Onshore, } \\
15.1 \\
56.1 \\
28.7 \\
0.2\end{array}$ & $\begin{aligned} & \text { Night } \\
& 8.1 \\
& 54.3 \\
& 37.6 \\
& 0.0\end{aligned}$ & $\begin{array}{r}25.1 \\
57.6 \\
17.3 \\
0.0\end{array}$ & $\begin{array}{r}15.5 \\
59.9 \\
24.6 \\
0.0\end{array}$ & $\begin{array}{r}14.5 \\
52.6 \\
32.0 \\
0.8\end{array}$ \\
\hline
\end{tabular}


Table 6.18 (continued)

\begin{tabular}{|c|c|c|c|c|c|c|}
\hline & \multirow{2}{*}{$\begin{array}{l}\text { Wind Speed } \\
\quad\left(\mathrm{msec}^{-1}\right)\end{array}$} & \multirow[b]{2}{*}{ Annual } & \multicolumn{4}{|c|}{ Season } \\
\hline & & & Spring & Summer & Fall & Winter \\
\hline ' & $\begin{array}{c}0.5-2.4 \\
2.5-4.9 \\
5.0-9.9 \\
\geq 10.0\end{array}$ & $\begin{array}{l}\text { Alongshore, } \\
24.1 \\
47.5 \\
27.1 \\
1.3\end{array}$ & $\begin{array}{c}\text { Al1 Hours } \\
15.9 \\
47.7 \\
34.7 \\
1.7\end{array}$ & $\begin{array}{r}41.4 \\
39.3 \\
19.3 \\
0.0\end{array}$ & $\begin{array}{r}19.4 \\
52.5 \\
28.1 \\
0.0\end{array}$ & $\begin{array}{r}20.1 \\
48.6 \\
26.7 \\
4.6\end{array}$ \\
\hline & $\begin{aligned} & 0.5-2.4 \\
& 2.5-4.9 \\
& 5.0-9.9 \\
& \therefore \geq 10.0\end{aligned}$ & $\begin{array}{l}\text { Alongshore, } \\
16.2 \\
36.9 \\
44.5 \\
2.4\end{array}$ & $\begin{array}{l}\text { Day } \\
8.2 \\
27.4 \\
60.4 \\
4.1\end{array}$ & $\begin{array}{r}33.3 \\
40.2 \\
26.5 \\
0.0\end{array}$ & $\begin{array}{r}6.9 \\
47.3 \\
45.8 \\
0.0\end{array}$ & $\begin{array}{r}19.9 \\
30.5 \\
42.3 \\
7.4\end{array}$ \\
\hline & $\begin{array}{c}0.5-2.4 \\
2.5-4.9 \\
5.0-9.9 \\
>10.0\end{array}$ & $\begin{array}{l}\text { Alongshore, } \\
29.8 \\
55.3 \\
14.4 \\
0.5\end{array}$ & $\begin{array}{r}\text { Night } \\
21.4 \\
64.6 \\
14.0 \\
0.0\end{array}$ & $\begin{array}{r}46.6 \\
39.4 \\
14.0 \\
0.0\end{array}$ & $\begin{array}{r}29.0 \\
57.4 \\
13.6 \\
0.0\end{array}$ & $\begin{array}{r}20.2 \\
60.7 \\
16.7 \\
2.4\end{array}$ \\
\hline
\end{tabular}


Table 6.18 (continued)

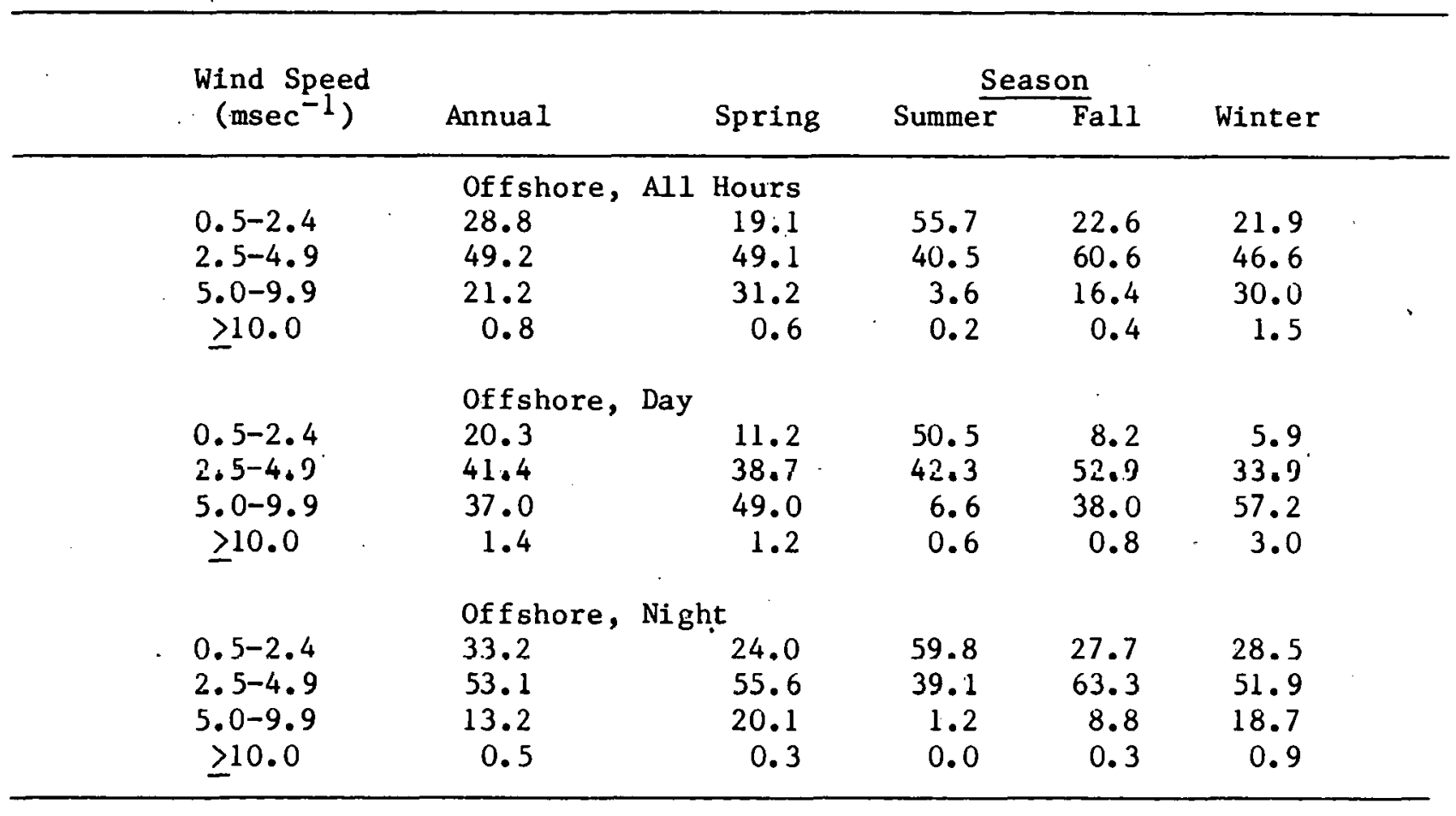


Table 6.19

Fort Myers, Florida

Hours in Each Wind Speed Class

by Wind Direction, Season and Time of Day

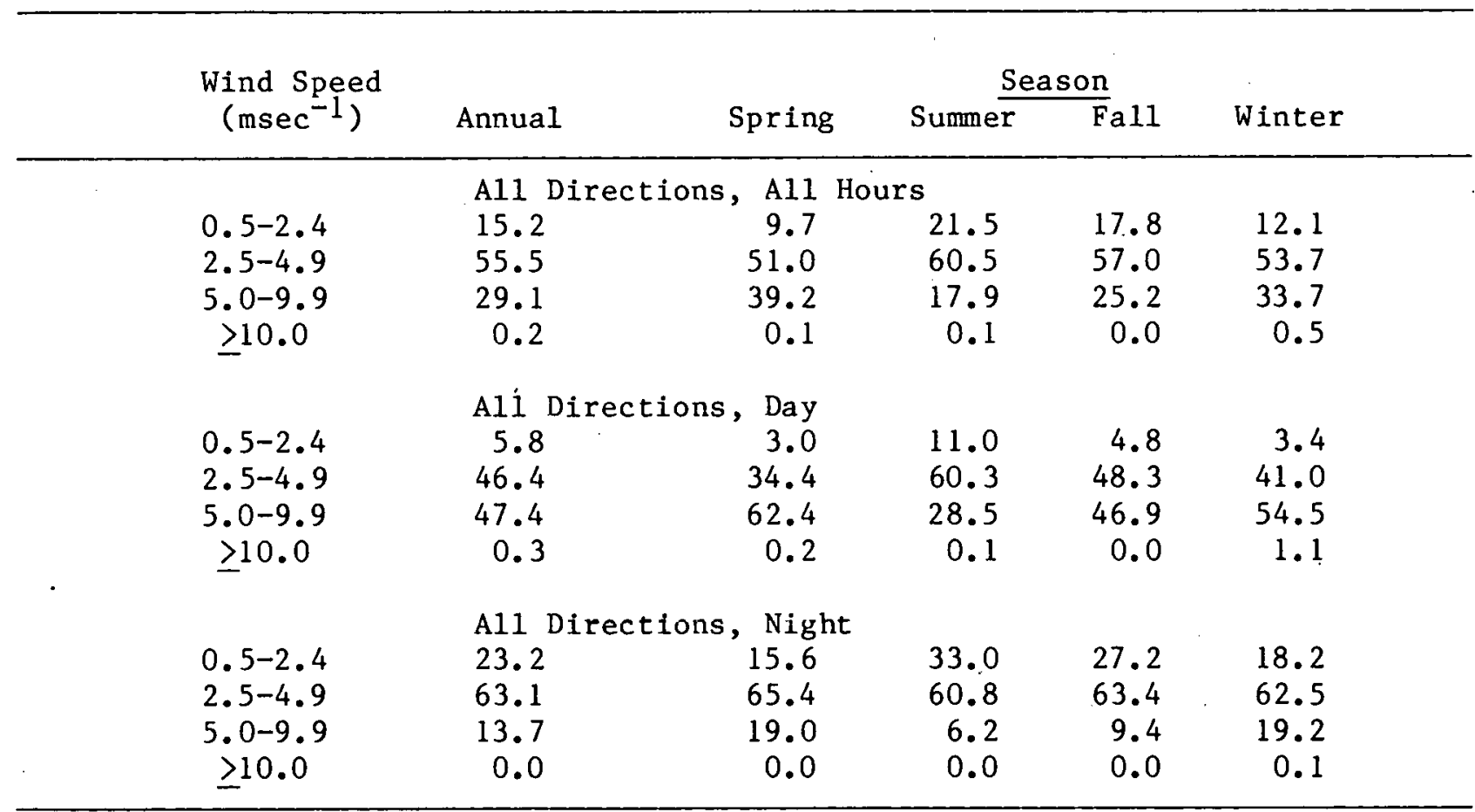


Table 6.194 (icontinuedi))

\begin{tabular}{|c|c|c|c|c|c|c|}
\hline \multirow{2}{*}{$\begin{array}{l}\text { Wind Speed } \\
\left(\mathrm{msec}^{-1}\right)\end{array}$} & \multirow[b]{2}{*}{ Annual } & \multirow{2}{*}{\multicolumn{2}{|c|}{ Spring }} & \multicolumn{2}{|c|}{ Season } & \multirow[b]{2}{*}{ Winter } \\
\hline & & & & Summer & Fall & \\
\hline $\begin{array}{c}0.5-2.4 \\
2.5-4.9 \\
5.0-9.9 \\
\geq 10.0\end{array}$ & $\begin{array}{l}\text { Onshore, } \\
7.5 \\
51.8 \\
40.2 \\
0.5\end{array}$ & All & $\begin{array}{r}\text { Hours } \\
3.0 \\
46.7 \\
50.2 \\
0.2\end{array}$ & $\begin{array}{r}10.2 \\
57.6 \\
31.9 \\
0.3\end{array}$ & $\begin{array}{r}12.8 \\
59.3 \\
28.0 \\
0.0\end{array}$ & $\begin{array}{r}8.3 \\
49.6 \\
40.4 \\
1.7\end{array}$ \\
\hline $\begin{array}{l}0.5-2.4 \\
2.5-4.9 \\
5.0-9.9 \\
\geq 10.0\end{array}$ & $\begin{array}{c}\text { Onshore, } \\
.3 .0 \\
41.4 \\
54.8 \\
0.8\end{array}$ & Day & $\begin{array}{r}1.1 \\
28.0 \\
70.6 \\
0.3\end{array}$ & $\begin{array}{r}4.8 \\
52.0 \\
42.8 \\
0.4\end{array}$ & $\begin{array}{c}3.3 \\
58.9 \\
37.9 \\
0.0\end{array}$ & $\begin{array}{r}3.7 \\
34.7 \\
58.6 \\
3.0\end{array}$ \\
\hline $\begin{array}{c}0.5-2.4 \\
2.5-4.9 \\
5.0-9.9 \\
\geq 10.0\end{array}$ & $\begin{array}{l}\text { Onshore, } \\
14.5 \\
67.7 \\
17.8 \\
0.0\end{array}$ & $\mathrm{Nigh}$ & $\begin{array}{r}5.4 \\
69.6 \\
25.0 \\
0.0\end{array}$ & $\begin{array}{r}22.3 \\
69.7 \\
8.0 \\
0.0\end{array}$ & $\begin{array}{r}32.0 \\
60.3 \\
7.6 \\
0.0\end{array}$ & $\begin{array}{r}14.0 \\
67.9 \\
18.1 \\
0.0\end{array}$ \\
\hline
\end{tabular}


Table 6.19 (continued)

\begin{tabular}{|c|c|c|c|c|c|}
\hline \multirow{2}{*}{$\begin{array}{l}\text { Wind Speed } \\
\left(\mathrm{msec}^{-1}\right)\end{array}$} & \multirow[b]{2}{*}{ Annual } & \multirow[b]{2}{*}{ Spring } & \multicolumn{2}{|c|}{ Season } & \multirow{2}{*}{ Winter } \\
\hline & & & Summer & $\bar{F} a 11$ & \\
\hline $\begin{array}{c}0.5-2.4 \\
2.5-4.9 \\
5.0-9.9 \\
\geq 10.0\end{array}$ & $\begin{array}{l}\text { Alongshore, } \\
15.6 \\
55.9 \\
28.4 \\
0.1\end{array}$ & $\begin{array}{c}\text { Al1. Hours } \\
11.7 \\
56.4 \\
32.0 \\
0.0\end{array}$ & $\begin{array}{r}25.0 \\
59.4 \\
15.6 \\
0.0\end{array}$ & $\begin{array}{r}16.5 \\
60.3 \\
23.2 \\
0.0\end{array}$ & $\begin{array}{r}10.8 \\
49.8 \\
39.0 \\
0.3\end{array}$ \\
\hline $\begin{array}{c}0.5-2.4 \\
2.5-4.9 \\
5.0-9.9 \\
\geq 10.0\end{array}$ & $\begin{array}{l}\text { Alongshore, } \\
6.5 \\
51.8 \\
41.4 \\
0.2\end{array}$ & $\begin{array}{r}\text { Day } \\
6.0 \\
45.9 \\
48.1 \\
0.0\end{array}$ & $\begin{array}{r}12.6 \\
64.5 \\
22.9 \\
0.0\end{array}$ & $\begin{array}{r}4.5 \\
56.7 \\
38.8 \\
0.0\end{array}$ & $\begin{array}{r}2.6 \\
39.8 \\
56.6 \\
0.9\end{array}$ \\
\hline $\begin{array}{c}0.5-2.4 \\
2.5-4.9 \\
5.0-9.9 \\
\geq 10.0\end{array}$ & $\begin{array}{l}\text { Alongshore, } \\
22.5 \\
59.1 \\
18.4 \\
0.0\end{array}$ & $\begin{array}{r}\text { Night } \\
15.1 \\
62.7 \\
22.2 \\
0.0\end{array}$ & $\begin{array}{c}36.9 \\
54.5 \\
8.6 \\
0.0\end{array}$ & $\begin{array}{r}27.8 \\
63.1 \\
9.1 \\
0.0\end{array}$ & $\begin{array}{r}16.4 \\
57.9 \\
25.8 \\
0.0\end{array}$ \\
\hline
\end{tabular}


Table 6.19 (continued)

\begin{tabular}{|c|c|c|c|c|c|c|}
\hline & \multirow{2}{*}{$\begin{array}{l}\text { Wind Speed } \\
\left(\mathrm{msec}^{-1}\right)\end{array}$} & \multirow{2}{*}{ Annual } & \multicolumn{4}{|c|}{ Season } \\
\hline & & & Spring & Summer & $\overline{F a} 11$ & Winter \\
\hline . & $\begin{array}{c}0.5-2.4 \\
2.5-4.9 \\
5.0-9.9 \\
\geq 10.0\end{array}$ & $\begin{array}{l}\text { Of fshore, } \\
18.8 \\
57.4 \\
23.8 \\
0.0\end{array}$ & $\begin{array}{r}\text { All Hours } \\
15.2 \\
54.2 \\
30.6 \\
0.0\end{array}$ & $\begin{array}{r}26.4 \\
62.5 \\
11.1 \\
0.0\end{array}$ & $\begin{array}{r}19.0 \\
56.2 \\
24.9 \\
0.0\end{array}$ & $\begin{array}{r}14.0 \\
57.1 \\
28.8 \\
0.1\end{array}$ \\
\hline & $\begin{array}{c}0.5-2.4 \\
2.5-4.9 \\
5.0-9.9 \\
\geq 10.0\end{array}$ & $\begin{array}{l}\text { Of f shore, } \\
7.6 \\
48.6 \\
43.8 \\
0.0\end{array}$ & $\begin{array}{r}\text { Day } \quad 4.0 \\
38.5 \\
57.4 \\
0.0\end{array}$ & $\begin{array}{r}15.0 \\
6.5 .4 \\
18.8 \\
0.0\end{array}$ & $\begin{array}{r}5.5 \\
42.7 \\
51.8 \\
0.0\end{array}$ & $\begin{array}{r}3.4 \\
45.3 \\
51.3 \\
0.0\end{array}$ \\
\hline$\cdot$ & $\begin{array}{c}0.5-2.4 \\
2.5-4.9 \\
5.0-9.9 \\
\geqslant 10.0\end{array}$ & $\begin{array}{l}\text { Offshore, } \\
26.0 \\
63.1 \\
10.9 \\
0.1\end{array}$ & $\begin{array}{l}\text { Night } \\
\begin{array}{r}23.0 \\
65.3 \\
11.8 \\
0.0\end{array}\end{array}$ & $\begin{array}{r}35.0 \\
60.1 \\
4.9 \\
0.0\end{array}$ & $\begin{array}{r}26.6 \\
63.8 \\
9.6 \\
0.0\end{array}$ & $\begin{array}{r}19.5 \\
63.4 \\
16.9 \\
0.2\end{array}$ \\
\hline
\end{tabular}


Table 6.20

Tampa, Florida

Percent of Hours in Each Wind Speed Class

by Wind Direction, Season and Time of Day

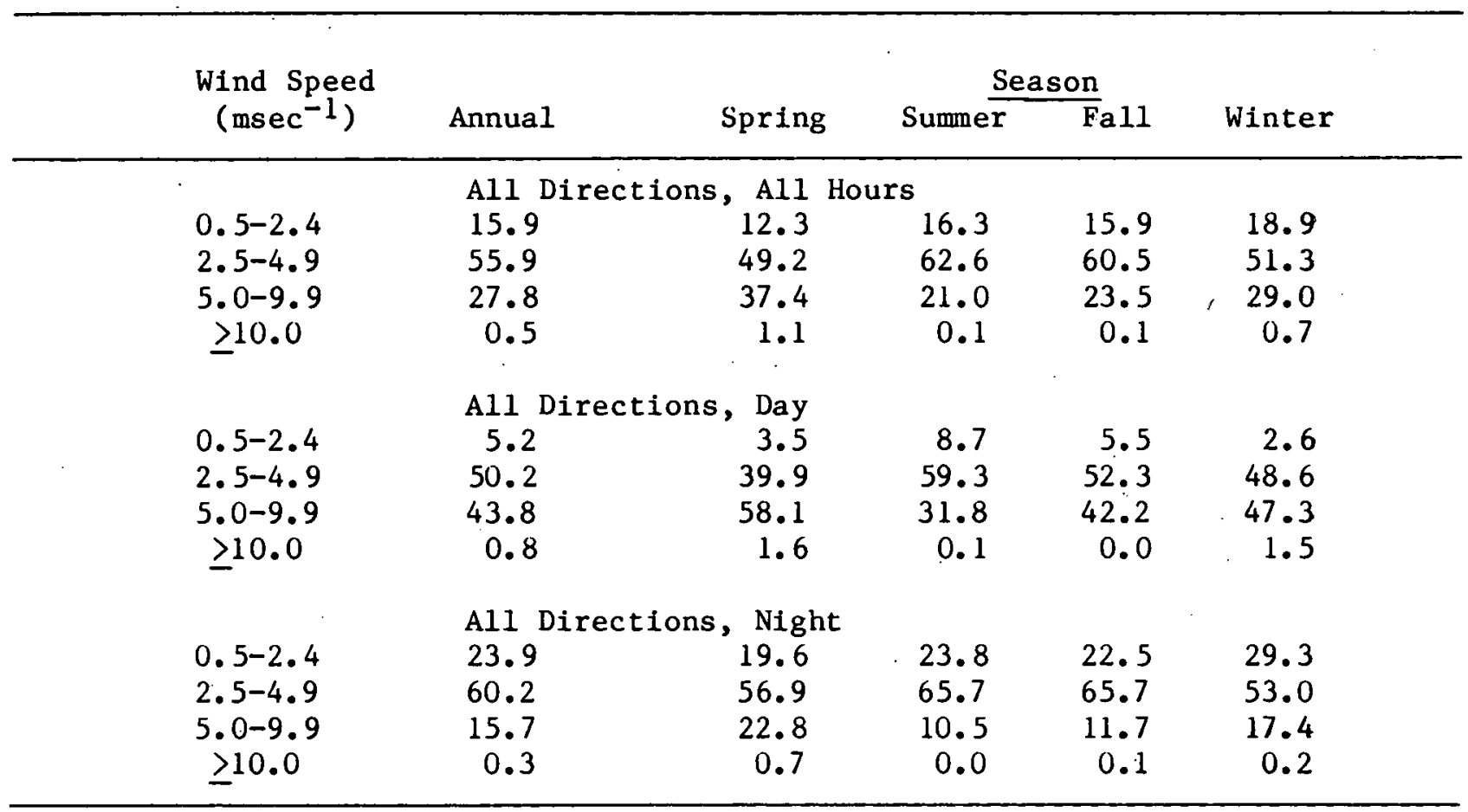


Table 6.20 (continued)

\begin{tabular}{|c|c|c|c|c|c|c|}
\hline \multirow{2}{*}{$\begin{array}{l}\text { Wind Speed } \\
\left(\mathrm{msec}^{-1}\right)\end{array}$} & \multirow[b]{2}{*}{ Annual } & \multicolumn{5}{|c|}{ Season } \\
\hline & & & Spring & Summer & Fall & Winter \\
\hline \multicolumn{7}{|c|}{ Onshore, Ali Hours } \\
\hline $0.5-2.4$ & 10.6 & & 7.1 & 7.5 & 15.6 & 17.0 \\
\hline $2.5-4.9$ & 52.8 & & 44.9 & 59.6 & 62.2 & 49.9 \\
\hline $5.0-9.9$ & 35.7 & & 46.1 & 33.0 & 22.1 & 31.6 \\
\hline$\geq 10.0$ & 1.0 & & 1.9 & 0.0 & 0.0 & .1 .4 \\
\hline \multicolumn{7}{|c|}{ Onshore, Day } \\
\hline $0.5-2.1$ & 2.8 & & 0.7 & 3.9 & 4.7 & 3.0 \\
\hline $2.5=4.9$ & 50.0 & & 36.0 & 51.0 & 65.3 & 53.3 \\
\hline $5.0-9.9$ & 46.0 & & 61.0 & 42.1 & 30.0 & 41.7 \\
\hline$\geq 10.0$ & 1.2 & & 2.3 & (1) & 0.0 & $2 . n$ \\
\hline \multicolumn{7}{|c|}{ Onshore, Night } \\
\hline $0.5-2.4$ & 21.7 & & 14.3 & 13.9 & 39.0 & 33.9 \\
\hline $2.5-4.9$ & 56.8 & & 55.3 & $69: 7$ & 55.8 & 45.9 \\
\hline $5.0-9.9$ & 20.8 & & 28.8 & 16.4 & 5.3 & 19.5 \\
\hline$\geq 10.0$ & 0.7 & & 1.5 & 0.0 & 0.0 & 0.7 \\
\hline
\end{tabular}




\section{Table 6.20 (continued)}

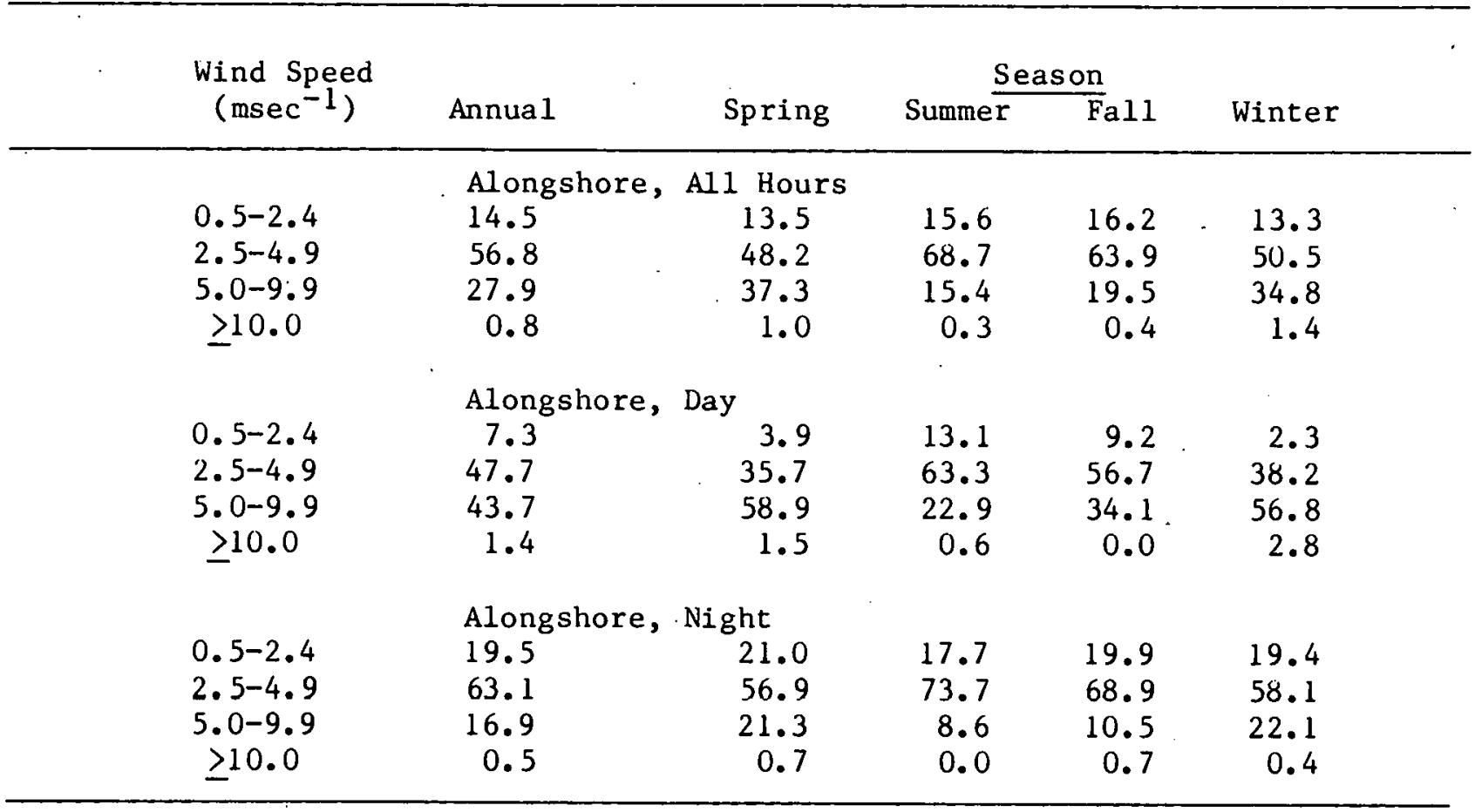




\section{Table 6.20 (continued)}

\begin{tabular}{|c|c|c|c|c|c|c|}
\hline & \multirow{2}{*}{$\begin{array}{l}\text { Wind Speed } \\
\quad\left(\mathrm{msec}^{-1}\right)\end{array}$} & \multirow[b]{2}{*}{ Annual } & \multirow[b]{2}{*}{ Spring } & \multicolumn{2}{|c|}{ Season } & \multirow[b]{2}{*}{ Winter } \\
\hline & & & & Summer & Fall & \\
\hline & $\begin{array}{c}0.5-2.4 \\
2.5-4.9 \\
5.0-9.9 \\
\geq 10.0\end{array}$ & $\begin{array}{l}\text { Of f shore, } \\
19.9 \\
57.4 \\
22.7 \\
0.0\end{array}$ & $\begin{array}{r}\text { Al1 Hours } \\
18.2 \\
55.6 \\
26.2 \\
0.0\end{array}$ & $\begin{array}{r}24.4 \\
62.3 \\
13.2 \\
0.0\end{array}$ & $\begin{array}{r}16.1 \\
59.2 \\
24.8 \\
0.0\end{array}$ & $\begin{array}{r}23.1 \\
52.3 \\
24.6 \\
0.0\end{array}$ \\
\hline & $\begin{array}{c}0.5-2.4 \\
2.5-4.9 \\
5.0-9.9 \\
\geq 10.0\end{array}$ & $\begin{array}{l}\text { offohore, } \\
6.8 \\
52.0 \\
41.2 \\
0.0\end{array}$ & $\begin{array}{r}\text { Day } \\
7.5 \\
50.9 \\
41.6 \\
0.0\end{array}$ & $\begin{array}{r}13.2 \\
65.2 \\
21.6 \\
0.0\end{array}$ & $\begin{array}{r}4.7 \\
44.8 \\
50.5 \\
0.0\end{array}$ & $\begin{array}{r}2.4 \\
50.5 \\
47.1 \\
0.0\end{array}$ \\
\hline & $\begin{array}{c}0.5-2.4 \\
2.5-4.9 \\
5.0-9.9 \\
\geq 10.0\end{array}$ & $\begin{array}{l}\text { of fshore, } \\
26.7 \\
60.2 \\
13.1 \\
0.0\end{array}$ & $\begin{array}{r}\text { Night } \\
24.4 \\
58.5 \\
17.1 \\
0.0\end{array}$ & $\begin{array}{r}31.0 \\
60.7 \\
8.4 \\
0.0\end{array}$ & $\begin{array}{r}21.5 \\
65.9 \\
12.6 \\
0.0\end{array}$ & $\begin{array}{r}32.5 \\
53.0 \\
14.4 \\
0.0\end{array}$ \\
\hline
\end{tabular}


Table 6.21

Tallahassee, Florida

Percent of Hours in Each Wind Speed Class

by Wind Direction, Season and Time of Day

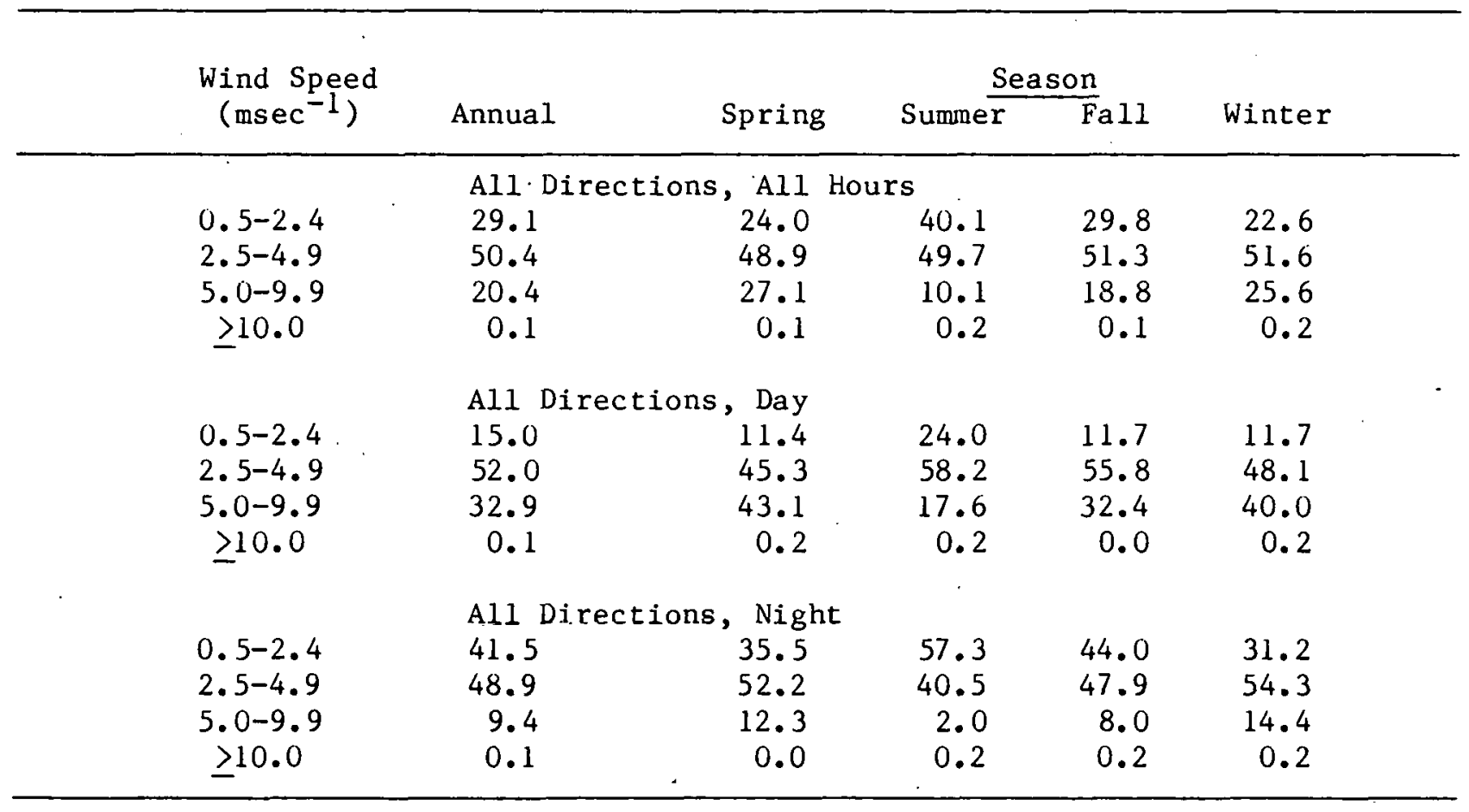


Table 6.21 (continued)

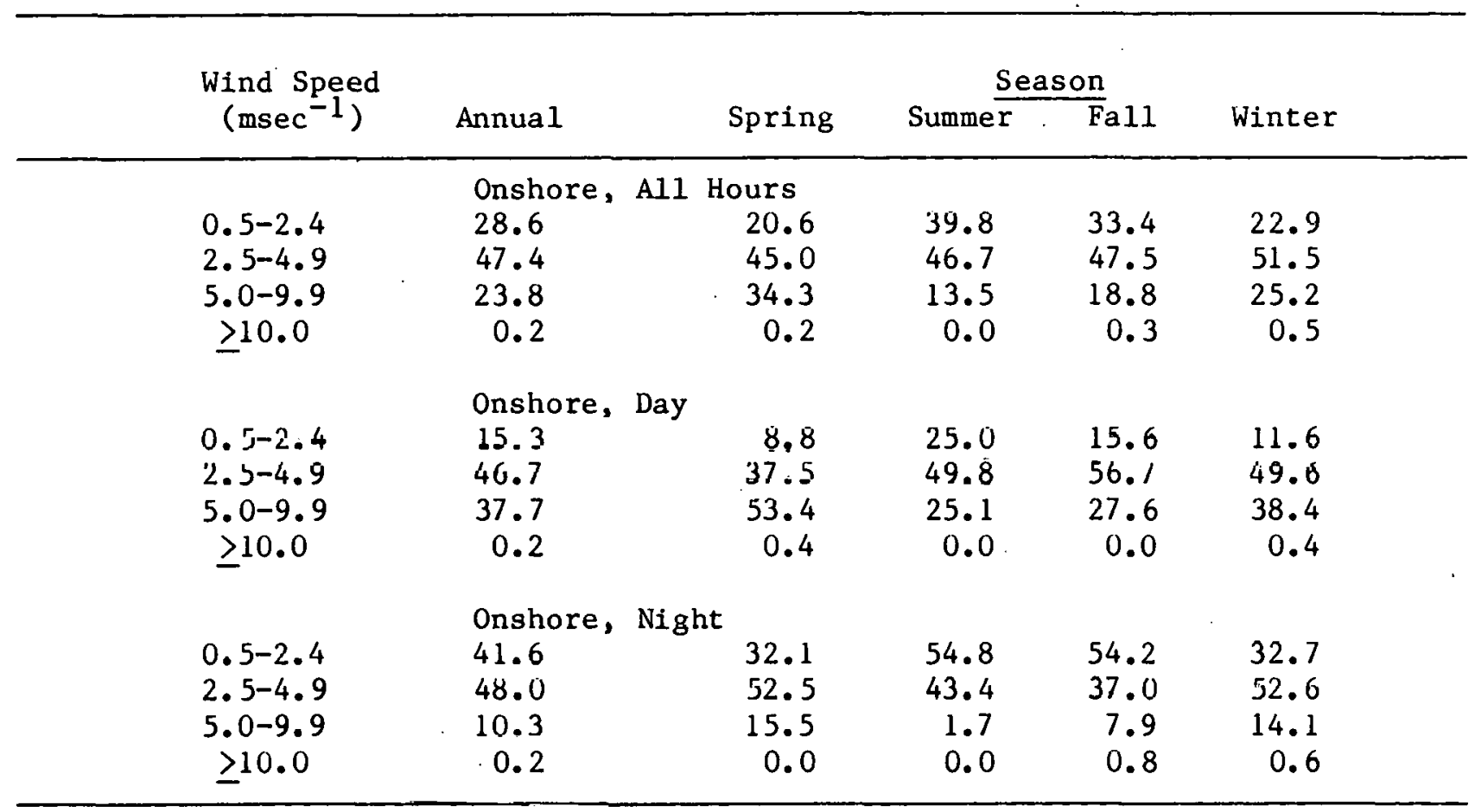




\begin{tabular}{|c|c|c|c|c|c|}
\hline \multirow{2}{*}{$\begin{array}{l}\text { Wind Speed } \\
\left(\mathrm{msec}^{-1}\right)\end{array}$} & \multirow[b]{2}{*}{ Annual } & \multirow[b]{2}{*}{ Spring } & \multicolumn{2}{|c|}{ Season } & \multirow[b]{2}{*}{ Winter } \\
\hline & & & Summer & . Fall & \\
\hline $\begin{array}{c}0.5-2.4 \\
2.5-4.9 \\
5.0-9.9 \\
\geq 10.0\end{array}$ & $\begin{array}{l}\text { Alongshore, } \\
32.3 \\
53.7 \\
13.8 \\
0.2\end{array}$ & $\begin{array}{c}\text { Al1 Hours } \\
29.6 \\
58.3 \\
12.1 \\
0.0\end{array}$ & $\begin{array}{r}40.6 \\
51.5 \\
7.1 \\
0.7\end{array}$ & $\begin{array}{r}27.8 \\
50.7 \\
21.5 \\
0.0\end{array}$ & $\begin{array}{r}29.6 \\
57.2 \\
13.2 \\
0.0\end{array}$ \\
\hline $\begin{array}{l}0.5-2.4 \\
2.5-4.9 \\
5.0-9.9 \\
\geq 10.0\end{array}$ & $\begin{array}{l}\text { Alongshore, } \\
16.2 \\
59.5 \\
24.1 \\
0.3\end{array}$ & $\begin{array}{l}\text { Day } \\
15.8 \\
62.2 \\
21.9 \\
0.0\end{array}$ & $\begin{array}{r}23.4 \\
60.8 \\
14.8 \\
0.9\end{array}$ & $\begin{array}{r}7.7 \\
53.9 \\
38.4 \\
0.0\end{array}$ & $\begin{array}{r}18.3 \\
62.1 \\
19.6 \\
0.0\end{array}$ \\
\hline $\begin{array}{c}0.5-2.4 \\
2.5-4.9 \\
5.0-9.9 \\
\geq 10.0\end{array}$ & $\begin{array}{l}\text { Alongshore, } \\
44.0 \\
49.5 \\
6.3 \\
0.2\end{array}$ & $\begin{array}{r}\text { Night } \\
39.2 \\
55.5 \\
5.3 \\
0.0\end{array}$ & $\begin{array}{r}53.3 \\
44.7 \\
1.4 \\
0.6\end{array}$ & $\begin{array}{r}41.0 \\
48.3 \\
10.7 \\
0.0\end{array}$ & $\begin{array}{r}40.0 \\
53.1 \\
7.0 \\
0.0\end{array}$ \\
\hline
\end{tabular}


Table 6.21 (continued)

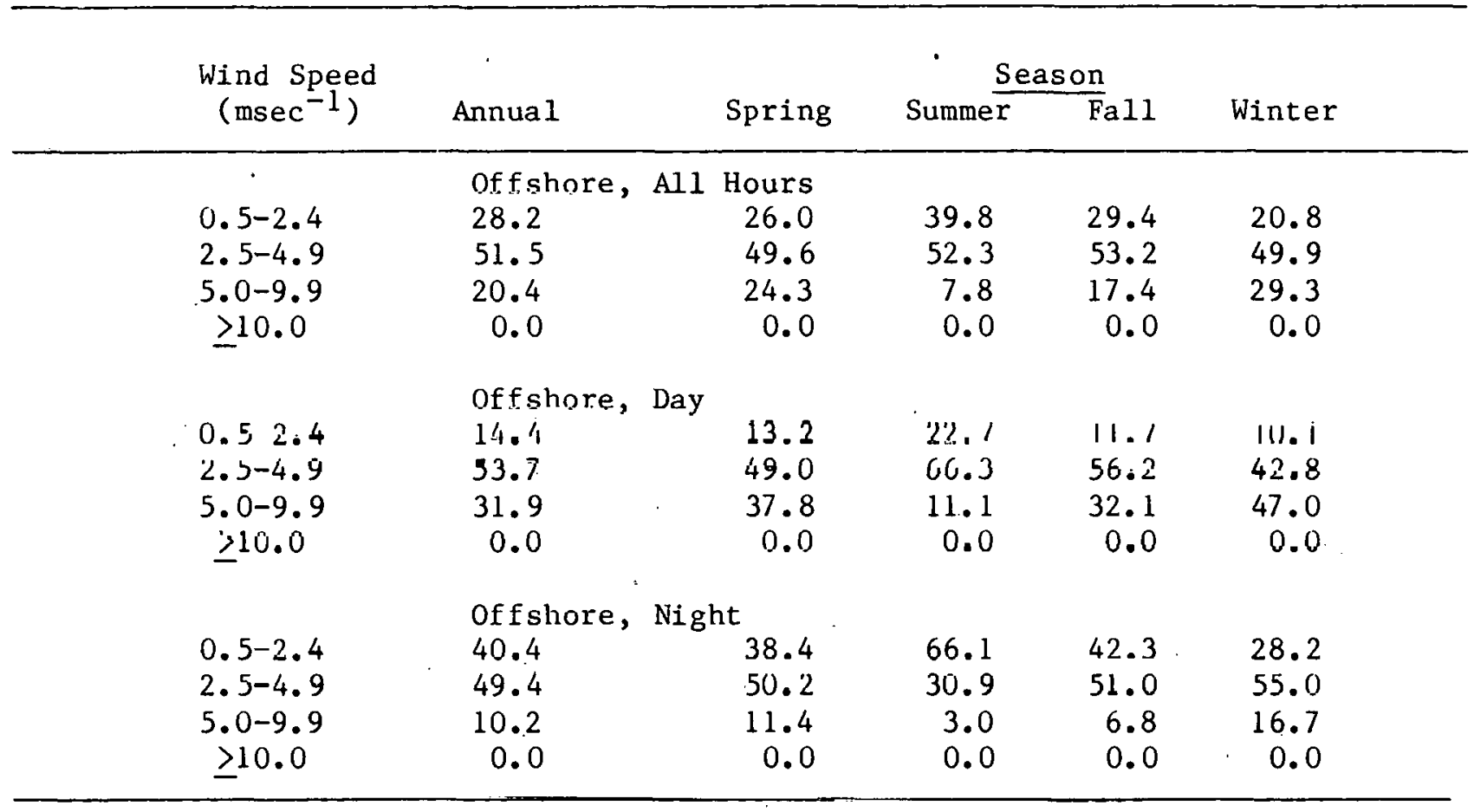


Table 6.22

Pensacola, Florida

Percent of Hours in Each Wind Speed Class

by Wind Direction, Season and Time of Day

\begin{tabular}{|c|c|c|c|c|c|}
\hline \multirow{2}{*}{$\begin{array}{l}\text { Wind Speed } \\
\left(\mathrm{msec}^{-1}\right)\end{array}$} & \multirow[b]{2}{*}{ Annual } & \multirow[b]{2}{*}{ Spring } & \multicolumn{2}{|c|}{ Season } & \multirow[b]{2}{*}{ Winter } \\
\hline & & & Summe $\bar{x}$ & Fall & \\
\hline $\begin{array}{c}0.5-2.4 \\
2.5-4.9 \\
5.0-9.9 \\
\geq 10.0\end{array}$ & $\begin{array}{c}\text { Al1 D } \\
15.8 \\
55.3 \\
28.7 \\
0.2\end{array}$ & $\begin{array}{r}\text { All } \\
12.1 \\
49.0 \\
38.5 \\
0.4\end{array}$ & $\begin{array}{l}\text { rs } \\
22.0 \\
58.8 \\
19.1 \\
0.1\end{array}$ & $\begin{array}{r}16.4 \\
62.3 \\
21.3 \\
0.0\end{array}$ & $\begin{array}{r}13.0 \\
51.0 \\
35.7 \\
0.4\end{array}$ \\
\hline $\begin{array}{c}0.5-2.4 \\
2.5-4.9 \\
5.0-9.9 \\
\geq 10.0\end{array}$ & $\begin{array}{l}\text { Al1 D } \\
8.5 \\
49.5 \\
41.6 \\
0.4\end{array}$ & $\begin{array}{r}\text { say } \\
5.1 \\
40.2 \\
53.9 \\
0.9\end{array}$ & $\begin{array}{r}13.4 \\
56.1 \\
30.3 \\
0.2\end{array}$ & $\begin{array}{r}8.4 \\
54.9 \\
36.7 \\
0.0\end{array}$ & $\begin{array}{r}6.8 \\
46.6 \\
46.2 \\
0.4\end{array}$ \\
\hline $\begin{array}{c}0.5-2.4 \\
2.5-4.9 \\
5.0-9.9 \\
\geq 10.0\end{array}$ & $\begin{array}{l}\text { A11 D } \\
21.3 \\
59.6 \\
19.0 \\
0.1\end{array}$ & $\begin{array}{c}\text { sight } \\
17.6 \\
55.9 \\
26.5 \\
0.0\end{array}$ & $\begin{array}{r}30.2 \\
61.4 \\
8.4 \\
0.0\end{array}$ & $\begin{array}{r}21.5 \\
67.1 \\
11.4 \\
0.0\end{array}$ & $\begin{array}{r}17.1 \\
53.9 \\
28.7 \\
0.4\end{array}$ \\
\hline
\end{tabular}


Table 6.22 (continued)

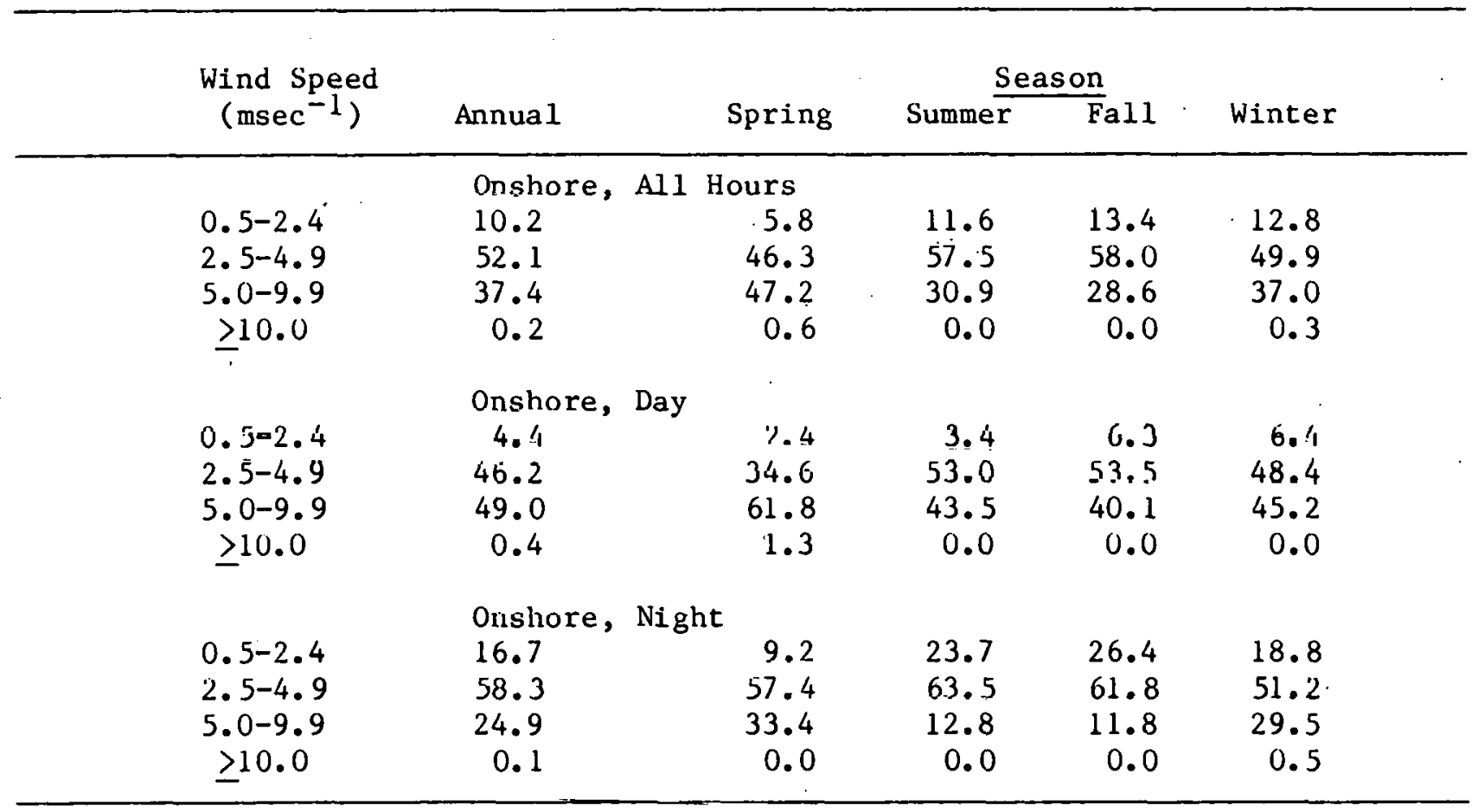


Table 6.22 (continued)

\begin{tabular}{|c|c|c|c|c|c|}
\hline \multirow{2}{*}{$\begin{array}{l}\text { Wind Speed } \\
\left(\mathrm{msec}^{-1}\right)\end{array}$} & \multirow[b]{2}{*}{ Annual } & \multirow[b]{2}{*}{ Spring } & \multicolumn{2}{|c|}{ Season } & \multirow[b]{2}{*}{ Winter } \\
\hline & & & Summer & Fall & \\
\hline $\begin{array}{c}0.5-2.4 \\
2.5-4.9 \\
5.0-9.9 \\
\geq 10.0\end{array}$ & $\begin{array}{l}\text { Alongshore, } \\
18.8 \\
62.8 \\
18.4 \\
0.0\end{array}$ & $\begin{array}{c}\text { Al1 Hours } \\
21.8 \\
58.6 \\
19.7 \\
0.0\end{array}$ & $\begin{array}{r}23.3 \\
62.9 \\
13.8 \\
0.0\end{array}$ & $\begin{array}{r}18.0 \\
65.7 \\
16.3 \\
0.0\end{array}$ & $\begin{array}{r}13.4 \\
60.6 \\
25.9 \\
0.0\end{array}$ \\
\hline $\begin{array}{c}0.5-2.4 \\
2.5-4.9 \\
5.0-9.9 \\
\geq 10.0\end{array}$ & $\begin{array}{l}\text { Alongshore, } \\
13.6 \\
58.8 \\
27.5 \\
0.0\end{array}$ & $\begin{array}{l}\text { Day } \\
14.9 \\
57.0 \\
28.1 \\
0.0\end{array}$ & $\begin{array}{r}19.3 \\
60.1 \\
20.6 \\
0.0\end{array}$ & $\begin{array}{r}11.7 \\
59.9 \\
28.4 \\
0.0\end{array}$ & $\begin{array}{r}7.2 \\
55.1 \\
37.7 \\
0.0\end{array}$ \\
\hline $\begin{array}{c}0.5-2.4 \\
2.5-4.9 \\
5.0-9.9 \\
\geq 10.0\end{array}$ & $\begin{array}{l}\text { Alongshore, } \\
21.8 \\
65.2 \\
13.0 \\
0.0\end{array}$ & $\begin{array}{r}\text { Night } \\
25.1 \\
59.5 \\
15.3 \\
0.0\end{array}$ & $\begin{array}{r}26.4 \\
64.9 \\
8.7 \\
0.0\end{array}$ & $\begin{array}{r}21.6 \\
.68 .8 \\
9.6 \\
0.0\end{array}$ & $\begin{array}{r}16.5 \\
63.3 \\
20.1 \\
0.0\end{array}$ \\
\hline
\end{tabular}


Table 6.22 (continued)

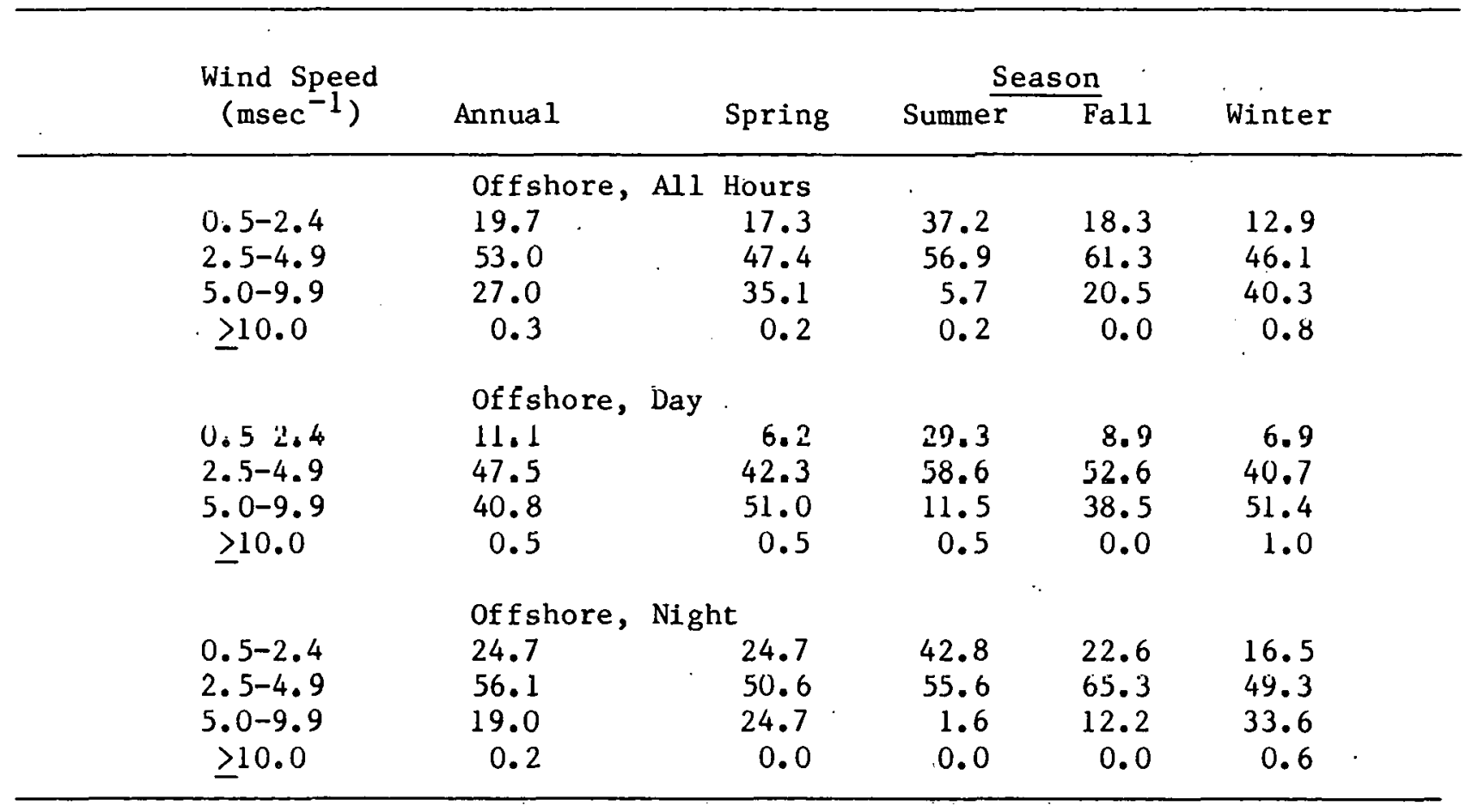


Table 6.23

Mobile, Alabama

Percent of Hours in Each Wind Speed Class

by Wind Direction, Season and Time of Day

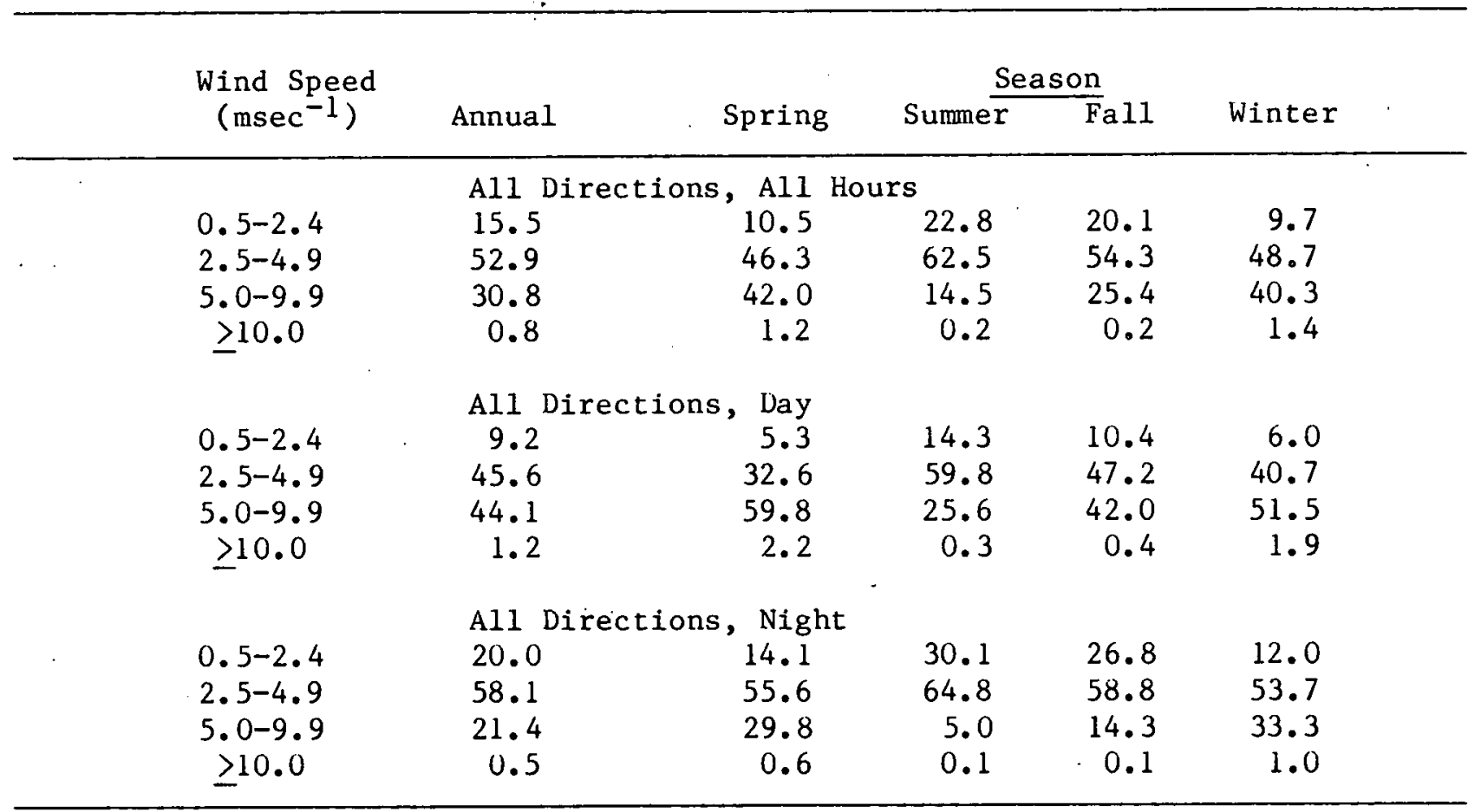


Table 6.23 (continued)

\begin{tabular}{|c|c|c|c|c|c|c|}
\hline & \multirow{2}{*}{$\begin{array}{l}\text { Wind Speed } \\
\left(\mathrm{msec}^{-1}\right)\end{array}$} & \multirow[b]{2}{*}{ Annual } & \multirow[b]{2}{*}{ Spring } & \multicolumn{2}{|c|}{ Season } & \multirow[b]{2}{*}{ Winter } \\
\hline & & & & Summer & $\bar{F} a 11$ & \\
\hline$\cdot$ & $\begin{array}{l}0.5-2.4 \\
2.5-4.9 \\
5.0-9.9 \\
\geq 10.0\end{array}$ & $\begin{array}{l}\text { Onshore, } \\
14.0 . \\
51.9 \\
33.1 \\
1.0\end{array}$ & $\begin{array}{r}\text { All Hours } \\
\cdots \quad 8.9 \\
43.9 \\
45.4 \\
1.8\end{array}$ & $\begin{array}{r}18.6 \\
61.9 \\
19.4 \\
0.0\end{array}$ & $\begin{array}{r}23.1 \\
53.6 \\
22.9 \\
0.4\end{array}$ & $\begin{array}{r}11.1 \\
51.0 \\
36.6 \\
1.3\end{array}$ \\
\hline & $\begin{array}{c}0.5-2.4 \\
2.5-4.9 \\
5.0-9.9 \\
\geq 10.0\end{array}$ & $\begin{array}{l}\text { Onshore, } \\
0.2 \\
40.3 \\
49.8 \\
1.7\end{array}$ & $\begin{array}{r}3.9 \\
26.3 \\
66.3 \\
3.6\end{array}$ & $\begin{array}{r}10.5 \\
55.3 \\
34.2 \\
0.0\end{array}$ & $\begin{array}{r}11.0 \\
45.3 \\
42.9 \\
0.0\end{array}$ & $\begin{array}{r}0.0 \\
38.9 \\
50.3 \\
2.1\end{array}$ \\
\hline & $\begin{array}{c}0.5-2.4 \\
2.5-4.9 \\
5.0-9.9 \\
\geq 10.0\end{array}$ & $\begin{array}{l}\text { Onshore, } \\
17.9 \\
59.5 \\
22.0 \\
0.5\end{array}$ & $\begin{array}{r}\text { Night } \\
12.0 \\
54.8 \\
32.5 \\
0.7\end{array}$ & $\begin{array}{r}24.5 \\
66.7 \\
8.8 \\
0.0\end{array}$ & $\begin{array}{r}31.6 \\
59.1 \\
8.5 \\
0.8\end{array}$ & $\begin{array}{r}12.5 \\
58.7 \\
28.0 \\
0.8\end{array}$ \\
\hline
\end{tabular}


Table 6.23 (continued)

\begin{tabular}{|c|c|c|c|c|c|}
\hline \multirow{2}{*}{$\begin{array}{l}\text { Wind Speed } \\
\left(\mathrm{msec}^{-1}\right)\end{array}$} & \multirow[b]{2}{*}{ Annual } & \multicolumn{4}{|c|}{ Season } \\
\hline & & Spring & Summer & $\bar{F} a 11$ & Winter \\
\hline $\begin{array}{c}0.5-2.4 \\
2.5-4.9 \\
5.0-9.9 \\
\geq 10.0\end{array}$ & $\begin{array}{l}\text { Alongshore, } \\
19.9 \\
56.5 \\
23.2 \\
0.3\end{array}$ & $\begin{array}{c}\text { Al1 Hours } \\
.14 .7 \\
54.6 \\
30.2 \\
0.5\end{array}$ & $\begin{array}{r}28.2 \\
58.6 \\
13.0 \\
0.2\end{array}$ & $\begin{array}{c}20.0 . \\
55.0 \\
24.9 \\
0.0\end{array}$ & $\begin{array}{r}10.3 \\
57.2 \\
31.9 \\
0.5\end{array}$ \\
\hline $\begin{array}{c}0.5-2.4 \\
2.5-4.9 \\
5.0-9.9 \\
\geq 10.0\end{array}$ & $\begin{array}{l}\text { Alongshore, } \\
12.8 \\
54.2 \\
32.6 \\
0.4\end{array}$ & Day $\begin{array}{r} \\
8.7 \\
45.5 \\
44.7 \\
1.1\end{array}$ & $\begin{array}{r}18.5 \\
57.7 \\
23.3 \\
0.5\end{array}$ & $\begin{array}{r}12.5 \\
52.6 \\
34.8 \\
0.0\end{array}$ & $\begin{array}{r}4.8 \\
58.1 \\
37.2 \\
0.0\end{array}$ \\
\hline $\begin{array}{c}0.5-2.4 \\
2.5-4.9 \\
5.0-9.9 \\
\geq 10.0\end{array}$ & $\begin{array}{l}\text { Alongshore, } \\
26.3 \\
58.7 \\
14.8 \\
0.2\end{array}$ & $\begin{array}{r}\text { Night } \\
19.4 \\
61.9 \\
18.8 \\
0.0\end{array}$ & $\begin{array}{r}39.1 \\
59.7 \\
1.2 \\
0.0\end{array}$ & $\begin{array}{r}26.8 \\
56.9 \\
16.4 \\
0.0\end{array}$ & $\begin{array}{r}14.3 \\
56.6 \\
28.3 \\
0.8\end{array}$ \\
\hline
\end{tabular}


Table 6.23 (continued)

\begin{tabular}{|c|c|c|c|c|c|}
\hline \multirow{2}{*}{$\begin{array}{l}\text { Wind Speed } \\
\left(\mathrm{msec}^{-1}\right)\end{array}$} & \multirow[b]{2}{*}{ Annual } & \multicolumn{4}{|c|}{ Season } \\
\hline & & Spring & Summer & $\bar{F}$ all & Winter \\
\hline $\begin{array}{c}0.5-2.4 \\
2.5-4.9 \\
5.0-9.9 \\
\geq 10.0\end{array}$ & $\begin{array}{l}\text { Off shore, } \\
14.6 \\
52.0 \\
32.5 \\
0.8\end{array}$ & $\begin{array}{r}\text { Al1 Hours } \\
10.7 \\
45.6 \\
43.0 \\
0.7\end{array}$ & $\begin{array}{r}24.0 \\
66.5 \\
9.0 \\
0.5\end{array}$ & $\begin{array}{r}18.7 \\
53.4 \\
27.5 \\
0.3\end{array}$ & $\begin{array}{r}7.8 \\
44.5 \\
46.1 \\
1.7\end{array}$ \\
\hline $\begin{array}{c}0.5-2.4 \\
2.5-4.9 \\
5.0-9.9 \\
\geqslant 10.0\end{array}$ & $\begin{array}{c}\text { Off shore, } \\
7.9 \\
45.4 \\
45.6 \\
1.1\end{array}$ & $\begin{array}{r}5.6 \\
34.5 \\
59.1 \\
0.8\end{array}$ & $\begin{array}{r}15.0 \\
67.7 \\
16.6 \\
0.6\end{array}$ & $\begin{array}{r}8.4 \\
44.4 \\
46.3 \\
0.9\end{array}$ & $\begin{array}{r}3.5 \\
37.0 \\
57.2 \\
2.2\end{array}$ \\
\hline $\begin{array}{c}0.5-2.4 \\
2.5-4.9 \\
5.0-9.9 \\
\geq 10.0\end{array}$ & $\begin{array}{l}\text { Offshore, } \\
19.1 \\
56.3 \\
23.9 \\
0.6\end{array}$ & $\begin{array}{l}\text { Night } \\
\begin{array}{r}14.4 \\
53.8 \\
31.1 \\
0.6\end{array}\end{array}$ & $\begin{array}{r}32.3 \\
64.8 \\
2.6 \\
0.3\end{array}$ & $\begin{array}{r}24.7 \\
58.3 \\
17.0 \\
0.0\end{array}$ & $\begin{array}{r}10.3 \\
48.8 \\
39.5 \\
1.3\end{array}$ \\
\hline
\end{tabular}


Table 6.24

Biloxi, Mississippi

Percent of Hours in Each Wind Speed Class

by Wind Direction, Season and Time of Day

\begin{tabular}{|c|c|c|c|c|c|}
\hline \multirow{2}{*}{$\begin{array}{l}\text { Wind Speed } \\
\left(\mathrm{msec}^{-1}\right)\end{array}$} & \multirow[b]{2}{*}{ Annual } & \multirow[b]{2}{*}{ Spring } & \multicolumn{2}{|c|}{ Season } & \multirow[b]{2}{*}{ Winter } \\
\hline & & & Summer & Fa 11 & \\
\hline $\begin{array}{c}0.5-2.4 \\
2.5-4.9 \\
5.0-9.9 \\
\geq 10.0\end{array}$ & $\begin{array}{l}\text { All I } \\
25.1 \\
48.1 \\
26.2 \\
0.6\end{array}$ & $\begin{array}{r}\text { Al1 } \\
16.4 \\
50.3 \\
32.4 \\
0.9\end{array}$ & $\begin{array}{r}\text { rs } \\
30.3 \\
50.0 \\
18.9 \\
0.8\end{array}$ & $\begin{array}{r}29.7 \\
45.7 \\
24.3 \\
0.3\end{array}$ & $\begin{array}{r}24.5 \\
46.2 \\
28.7 \\
0.6\end{array}$ \\
\hline $\begin{array}{c}0.5-2.4 \\
2.5-4.9 \\
5.0-9.9 \\
\geq 10.0\end{array}$ & $\begin{array}{l}\text { A11 D } \\
18.9 \\
49.5 \\
31.0 \\
0.7\end{array}$ & $\begin{array}{r}\text { Day } \\
11.0 \\
49.5 \\
38.4 \\
1.1\end{array}$ & $\begin{array}{r}23.6 \\
52.8 \\
23.2 \\
0.5\end{array}$ & $\begin{array}{r}22.1 \\
48.0 \\
29.5 \\
0.4\end{array}$ & $\begin{array}{r}19.2 \\
47.1 \\
33.1 \\
0.6\end{array}$ \\
\hline $\begin{array}{c}0.5-2.4 \\
2.5-4.9 \\
5.0-9.9 \\
\geq 10.0\end{array}$ & $\begin{array}{l}\text { A11 D } \\
35.5 \\
45.7 \\
18.2 \\
0.6\end{array}$ & $\begin{array}{c}\text { Night } \\
26.3 \\
51.8 \\
21.4 \\
0.4\end{array}$ & $\begin{array}{r}47.1 \\
43.0 \\
8.4 \\
1.6\end{array}$ & $\begin{array}{r}40.5 \\
42.4 \\
16.8 \\
0.3\end{array}$ & $\begin{array}{r}31.2 \\
45.2 \\
23.1 \\
0.5\end{array}$ \\
\hline
\end{tabular}


Table 6.24 (continued)

\begin{tabular}{|c|c|c|c|c|c|}
\hline \multirow{2}{*}{$\begin{array}{l}\text { Wind Speed } \\
\left(\mathrm{msec}^{-1}\right)\end{array}$} & \multirow[b]{2}{*}{ Annual } & \multicolumn{4}{|c|}{ Season } \\
\hline & & Spring & Summer & Fall & Winter \\
\hline $\begin{array}{c}0.5-2.4 \\
2.5-4.9 \\
5.0-9.9 \\
\geq 10.0\end{array}$ & $\begin{array}{l}\text { Onshore, } \\
20.0 \\
51.7 \\
27.4 \\
0.9\end{array}$ & $\begin{array}{r}\text { Al1 Hours } \\
12.6 \\
51.0 \\
34.7 \\
1.7\end{array}$ & $\begin{array}{r}20.6 \\
53.7 \\
24.9 \\
0.9\end{array}$ & $\begin{array}{r}27.5 \\
51.6 \\
20.8 \\
0.1\end{array}$ & $\begin{array}{r}22.8 \\
50.0 \\
26.9 \\
0.3\end{array}$ \\
\hline $\begin{array}{c}0.5-2.4 \\
2.5-4.9 \\
5.0-9.9 \\
\geq 10.0\end{array}$ & $\begin{array}{l}\text { Onshore, } \\
13.3 \\
53.2 \\
32.6 \\
0.9\end{array}$ & $\begin{array}{r}6.3 \\
49.9 \\
41.6 \\
2.1\end{array}$ & $\begin{array}{r}14.5 \\
55.1 \\
29.9 \\
0.5\end{array}$ & $\begin{array}{r}19.0 \\
55.2 \\
25.7 \\
0.1\end{array}$ & $\begin{array}{r}16.4 \\
53.6 \\
29.5 \\
0.5\end{array}$ \\
\hline $\begin{array}{c}0.5-2.4 \\
2.5-4.9 \\
5.0-9.9 \\
\geq 10.0\end{array}$ & $\begin{array}{l}\text { Onshore, } \\
34.3 \\
48.6 \\
16.4 \\
0.8\end{array}$ & $\begin{array}{r}\text { Night } \\
25.5 \\
53.4 \\
20.3 \\
0.9\end{array}$ & $\begin{array}{r}38.1 \\
49.6 \\
10.3 \\
1.9\end{array}$ & $\begin{array}{r}43.8 \\
44.8 \\
11.4 \\
0.0\end{array}$ & $\begin{array}{r}32.6 \\
44.6 \\
22.9 \\
0.0\end{array}$ \\
\hline
\end{tabular}


Table 6.24 (continued)

\begin{tabular}{|c|c|c|c|c|c|c|}
\hline & \multirow{2}{*}{$\begin{array}{l}\text { Wind Speed } \\
\left(\mathrm{msec}^{-1}\right)\end{array}$} & \multirow[b]{2}{*}{ Annual } & \multicolumn{4}{|c|}{ Season } \\
\hline & & & Spring & Summer & Fall & Winter \\
\hline v & $\begin{array}{c}0.5-2.4 \\
2.5-4.9 \\
5.0-9.9 \\
\geq 10.0\end{array}$ & $\begin{array}{l}\text { Alongshore, } \\
31.4 \\
43.7 \\
24.6 \\
0.4\end{array}$ & $\begin{array}{c}\text { All Hours } \\
22.1 \\
45.6 \\
32.3 \\
0.0\end{array}$ & $\begin{array}{r}36.2 \\
47.0 \\
15.3 \\
1.4\end{array}$ & $\begin{array}{r}33.6 \\
44.7 \\
21.7 \\
0.0\end{array}$ & $\begin{array}{r}33.2 \\
38.7 \\
27.9 \\
0.2\end{array}$ \\
\hline . & $\begin{array}{c}0.5-2.4 \\
2.5-4.9 \\
5.0-9.9 \\
\geq 10.0\end{array}$ & $\begin{array}{l}\text { Alongshore, } \\
25.0 \\
45.7 \\
29.2 \\
0.0\end{array}$ & $\begin{array}{l}\text { Day } \\
\begin{array}{r}18.4 \\
47.3 \\
34.3 \\
0.0\end{array}\end{array}$ & $\begin{array}{r}28.3 \\
52.4 \\
19.3 \\
0.0\end{array}$ & $\begin{array}{r}24.4 \\
46.5 \\
29.2 \\
0.0\end{array}$ & $\begin{array}{r}28.1 \\
37.8 \\
34.1 \\
0.0\end{array}$ \\
\hline & $\begin{array}{c}0.5-2.4 \\
2.5-4.9 \\
5.0-9.9 \\
\geq 10.0\end{array}$ & $\begin{array}{l}\text { Alongshore, } \\
41.1 \\
40.6 \\
17.4 \\
0.9\end{array}$ & $\begin{array}{l}\text { Night } \\
28.1 \\
43.0 \\
28.9 \\
0.0\end{array}$ & $\begin{array}{r}49.9 \\
36.2 \\
8.8 \\
5.1\end{array}$ & $\begin{array}{r}47.2 \\
41.9 \\
10.8 \\
0.0\end{array}$ & $\begin{array}{r}39.6 \\
39.9 \\
20.0 \\
0.5\end{array}$ \\
\hline
\end{tabular}


Table 6.24 (continued)

\begin{tabular}{|c|c|c|c|c|c|c|}
\hline & \multirow{2}{*}{$\begin{array}{l}\text { Wind Speed } \\
\left.\text { (msec }^{-1}\right)\end{array}$} & \multirow[b]{2}{*}{ Annual } & \multicolumn{4}{|c|}{ Season } \\
\hline & & & Spring & Summer & $\bar{F} a 11$ & Winter \\
\hline & $\begin{array}{c}0.5-2.4 \\
2.5-4.9 \\
5.0-9.9 \\
\geq 10.0\end{array}$ & $\begin{array}{l}\text { Of f shore, } \\
28.9 \\
44.8 \\
25.7 \\
0.5\end{array}$ & $\begin{array}{r}\text { All Hours } \\
20.8 \\
49.5 \\
29.6 \\
0.0\end{array}$ & $\begin{array}{r}46.7 \\
43.9 \\
9.0 \\
0.4\end{array}$ & $\begin{array}{r}29.8 \\
41.6 \\
28.0 \\
0.5\end{array}$ & $\begin{array}{r}23.7 \\
45.8 \\
29.7 \\
0.8\end{array}$ \\
\hline ' & $\begin{array}{c}0.5-2.4 \\
2.5-4.9 \\
3.0-9.9 \\
210.0\end{array}$ & $\begin{array}{l}\text { Off fhore, } \\
24.3 \\
45.3 \\
30.0 \\
0.5\end{array}$ & $\begin{array}{r}\text { Day } 16.0 \\
48.6 \\
34.7 \\
0.0\end{array}$ & $\begin{array}{r}40.0 \\
47.9 \\
10.9 \\
0.5\end{array}$ & $\begin{array}{r}23.7 \\
41.5 \\
34.2 \\
0.7\end{array}$ & $\begin{array}{r}19.2 \\
44.5 \\
35.5 \\
0.9\end{array}$ \\
\hline & $\begin{array}{c}0.5-2.4 \\
2.5-4.9 \\
5.0-9.9 \\
\geq 10.0\end{array}$ & $\begin{array}{l}\text { Off shore, } \\
35.1 \\
44.4 \\
20.1 \\
0.5\end{array}$ & $\begin{array}{l}\text { Night } \\
\begin{array}{r}27.3 \\
51.1 \\
21.5 \\
0.0\end{array}\end{array}$ & $\begin{array}{r}59.9 \\
34.9 \\
4.9 \\
0.3\end{array}$ & $\begin{array}{r}36.9 . \\
41.8 \\
20.9 \\
0.4\end{array}$ & $\begin{array}{r}28.8 \\
.47 .1 \\
23.4 \\
0.8\end{array}$ \\
\hline
\end{tabular}


Table 6.25

New Orleans, Louisiana

Percent of Hour's in Each Wind Speed Class

by Wind Direction, Season and Time of Day

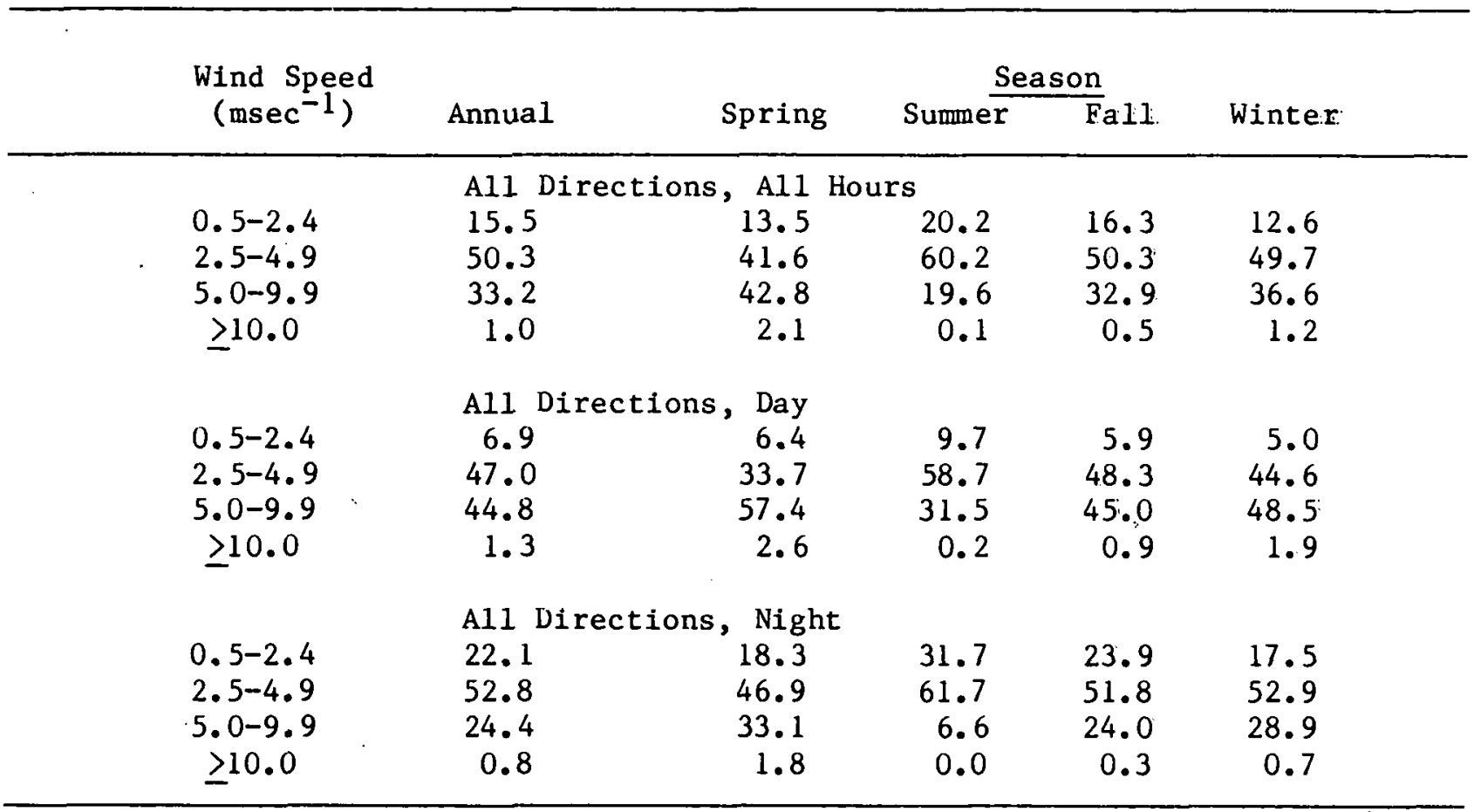


Table 6.25 (continued)

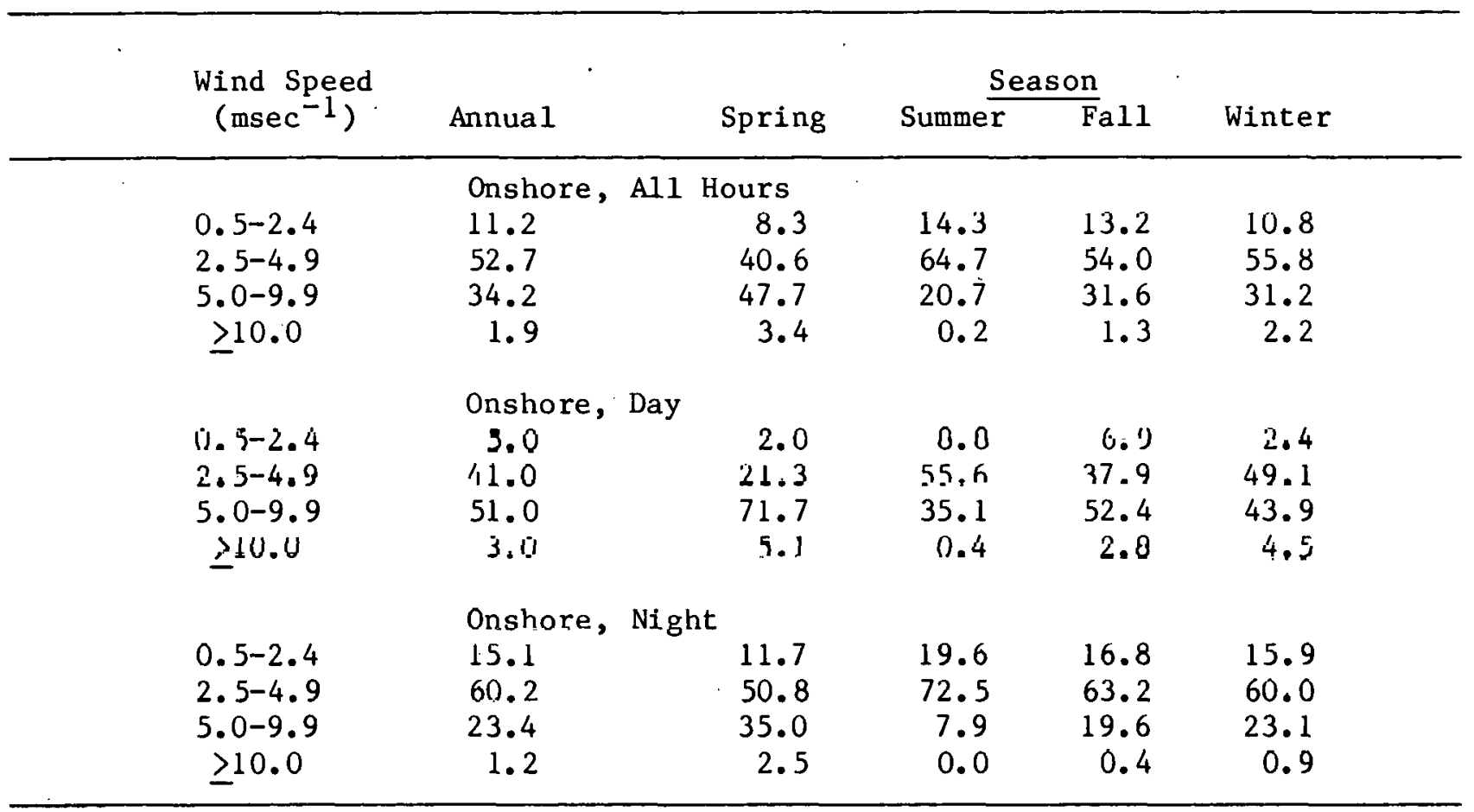


Table 6.25 (continued)

\begin{tabular}{|c|c|c|c|c|c|}
\hline \multirow{2}{*}{$\begin{array}{l}\text { Wind Speed } \\
\left(\mathrm{msec}^{-1}\right)\end{array}$} & \multirow[b]{2}{*}{ Annual } & \multirow[b]{2}{*}{ Spring } & \multicolumn{2}{|c|}{ Season } & \multirow[b]{2}{*}{ Winter } \\
\hline & & & Summer & Fall & \\
\hline $\begin{array}{c}0.5-2.4 \\
2.5-4.9 \\
5.0-9.9 \\
\geq 10.0\end{array}$ & $\begin{array}{l}\text { Alongshore, } \\
26.3 \\
46.3 \\
27.4 \\
0.0\end{array}$ & $\begin{array}{c}\text { A.1 Hours } \\
25.7 \\
39.9 \\
34.4 \\
0.0\end{array}$ & $\begin{array}{r}32.7 \\
46.0 \\
21.3 \\
0.0\end{array}$ & $\begin{array}{r}22.7 \\
47.4 \\
30.0 \\
0.0\end{array}$ & $\begin{array}{r}26.5 \\
52.9 \\
20.6 \\
0.0\end{array}$ \\
\hline $\begin{array}{c}0.5-2.4 \\
2.5-4.9 \\
5.0-9.9 \\
\geq 10.0\end{array}$ & $\begin{array}{l}\text { Alongshore, } \\
9.1 \\
42.44 \\
48.4 \\
.0: 0\end{array}$ & $\begin{array}{r}\text { Day } \\
6.7 \\
33.8 \\
59.5 \\
0.0\end{array}$ & $\begin{array}{r}14.6 \\
46.3 \\
39.1 \\
0.0\end{array}$ & $\begin{array}{r}1.1 \\
46.5 \\
52.4 \\
0.0\end{array}$ & $\begin{array}{r}19.7 \\
42.4 \\
37.9 \\
0.0\end{array}$ \\
\hline $\begin{array}{c}0.5-2.4 \\
2.5-4.9 \\
5.0-9.9 \\
\geq 10.0\end{array}$ & $\begin{array}{l}\text { Alongshore, } \\
38.0 \\
48.9 \\
13.1 \\
0.0\end{array}$ & $\begin{array}{r}\text { Night } \\
36.0 \\
42.2 \\
21.8 \\
0.0\end{array}$ & $\begin{array}{r}47.7 \\
46.3 \\
6.0 \\
0.0\end{array}$ & $\begin{array}{r}36.7 \\
47.8 \\
15.5 \\
0.0\end{array}$ & $\begin{array}{r}31.1 \\
59.2 \\
9.8 \\
0.0\end{array}$ \\
\hline
\end{tabular}


Table 6.25 (continued)

\begin{tabular}{|c|c|c|c|c|c|c|}
\hline \multirow{2}{*}{$\begin{array}{l}\text { Wind Speed } \\
\left.\text { (msec }^{-1}\right)\end{array}$} & \multirow[b]{2}{*}{ Annual } & \multicolumn{5}{|c|}{ Season } \\
\hline & & & Spring & Summer & Fall & Winter \\
\hline $\begin{array}{c}0.5-2.4 \\
2.5-4.9 \\
5.0-9.9 \\
\geq 10.0\end{array}$ & $\begin{array}{l}\text { Offshore, } \\
16.0 \\
49.4 \\
34.2 \\
0.4\end{array}$ & Al1 & $\begin{array}{r}\text { Hours } \\
17.1 \\
44.4 \\
37.9 \\
0.6\end{array}$ & $\begin{array}{r}20.8 \\
61.6 \\
17.6 \\
0.0\end{array}$ & $\begin{array}{r}17.0 \\
48.8 \\
34.1 \\
0.2\end{array}$ & $\begin{array}{r}10.6 \\
43.5 \\
45.2 \\
0.8\end{array}$ \\
\hline $\begin{array}{l}0.5-2.4 \\
2.5-4.9 \\
5.0-9.9 \\
\geq 10.0\end{array}$ & $\begin{array}{l}\text { Off shore, } \\
7.7 \\
33.3 \\
38.7 \\
0.3\end{array}$ & Day & $\begin{array}{r}10.5 \\
47.1 \\
42.0 \\
0.4\end{array}$ & $\begin{array}{r}8.8 \\
65.0 \\
26.2 \\
0.0\end{array}$ & $\begin{array}{r}7.0 \\
56.1 \\
36.9 \\
0.0\end{array}$ & $\begin{array}{r}4.5 \\
42.1 \\
52.7 \\
0.7\end{array}$ \\
\hline $\begin{array}{c}0.5-2.4 \\
2.5-4.9 \\
5.0-9.9 \\
\geq 10.0\end{array}$ & $\begin{array}{l}\text { Off shore, } \\
23.9 \\
45.6 \\
30.0 \\
0.5\end{array}$ & $\cdot$ & $\begin{array}{r}23.3 \\
42.0 \\
33.9 \\
0.8\end{array}$ & $\begin{array}{r}37.9 \\
56.6 \\
5.5 \\
0.0\end{array}$ & $\begin{array}{r}26.6 \\
41.7 \\
31.4 \\
0.3\end{array}$ & $\begin{array}{r}14.9 \\
44.4 \\
39.9 \\
0.8\end{array}$ \\
\hline
\end{tabular}


Table 6.26

Boothville, Louisiana

Percent of Hours in Each Wind Speed Class

by Wind Direction, Season and Time of Day

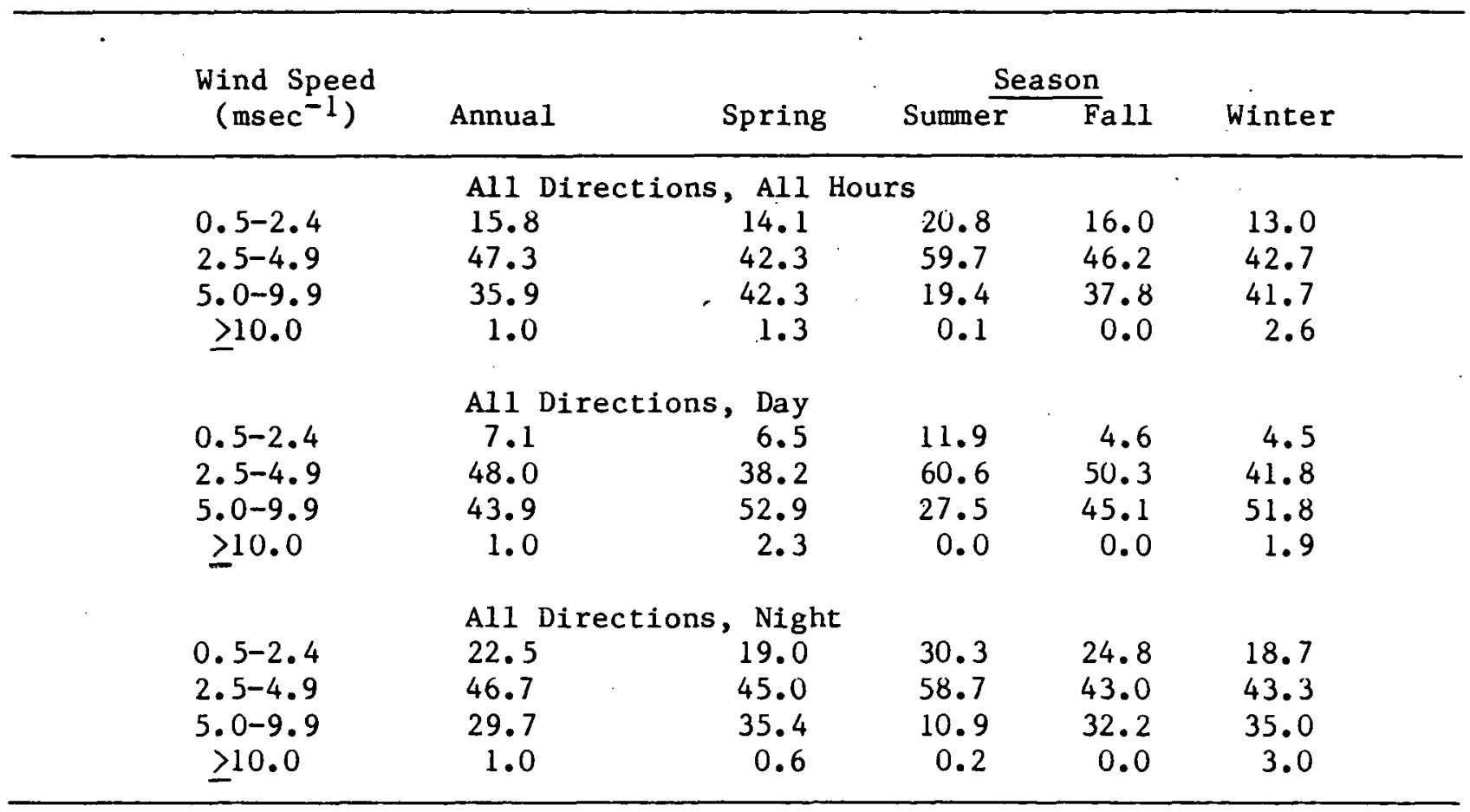


Table 6:26 (continued)

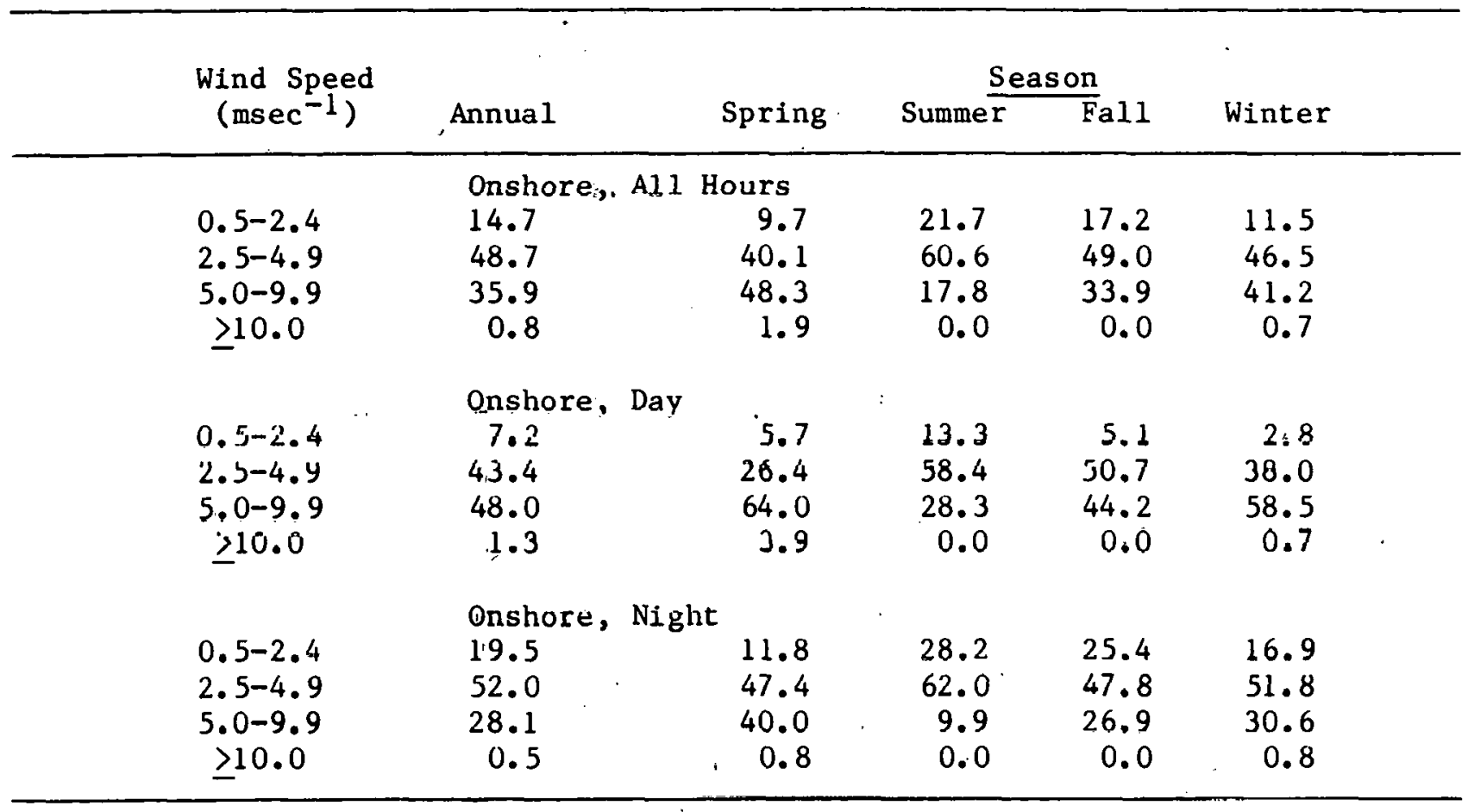


Table 6.26 (continued)

\begin{tabular}{|c|c|c|c|c|c|}
\hline \multirow{2}{*}{$\begin{array}{l}\text { Wind Speed } \\
\text { (msec }\end{array}$} & \multirow[b]{2}{*}{ Annual } & \multirow[b]{2}{*}{ Spring } & \multicolumn{2}{|c|}{ Season } & \multirow{2}{*}{ Winter } \\
\hline & & & Summer & $\overline{F a l l}$ & \\
\hline $\begin{array}{c}0.5-2.4 \\
2.5-4.9 \\
5.0-9.9 \\
\geq 10.0\end{array}$ & $\begin{array}{l}\text { Alongshore, } \\
24.9 \\
49.9 \\
25.1 \\
0.0\end{array}$ & $\begin{array}{c}\text { All Hours } \\
23.3 \\
49.1 \\
27.6 \\
0.0\end{array}$ & $\begin{array}{r}22.2 \\
57.4 \\
20.4 \\
0.0\end{array}$ & $\begin{array}{r}19.7 \\
47.1 \\
33.2 \\
0.0\end{array}$ & $\begin{array}{r}36.3 \\
47.4 \\
16.4 \\
0.0\end{array}$ \\
\hline $\begin{array}{c}0.5-2.4 \\
2.5-4.9 \\
5.0-9.9 \\
\geq 10.0\end{array}$ & $\begin{array}{l}\text { Alongshore, } \\
9.0 \\
48.7 \\
42.3 \\
0.0\end{array}$ & $\begin{array}{l}\text { Day } \\
10.4 \\
40.3 \\
49.3 \\
0.0\end{array}$ & $\begin{array}{r}11.0 \\
52.5 \\
36.6 \\
0.0\end{array}$ & $\begin{array}{r}4.5 \\
47.7 \\
47.8 \\
0.0\end{array}$ & $\begin{array}{r}11.9 \\
63.0 \\
25.2 \\
0.0\end{array}$ \\
\hline $\begin{array}{c}0.5-2.4 \\
2.5-4.9 \\
5.0-9.9 \\
\geq 10.0\end{array}$ & $\begin{array}{l}\text { Alongshore, } \\
33.9 \\
50.8 \\
15.2 \\
0.0\end{array}$ & $\begin{array}{r}\text { Night } \\
29.3 \\
53.4 \\
17.3 \\
0.0\end{array}$ & $\begin{array}{r}30.9 \\
61.3 \\
7.8 \\
0.0\end{array}$ & $\begin{array}{r}27.9 \\
49.5 \\
22.6 \\
0.0\end{array}$ & $\begin{array}{r}47.4 \\
40.2 \\
12.4 \\
0.0\end{array}$ \\
\hline
\end{tabular}


Table 6.26 (continued)

\begin{tabular}{|c|c|c|c|c|c|c|}
\hline & \multirow{2}{*}{$\begin{array}{l}\text { Wind Speed } \\
\left(\text { msec }^{-1}\right)\end{array}$} & \multirow[b]{2}{*}{ Annua 1 } & \multicolumn{4}{|c|}{ Season } \\
\hline & & & Spring & Summer & $\bar{F} a 11$ & Winter \\
\hline & $\begin{array}{c}0.5-2.4 \\
2.5-4.9 \\
5.0-9.9 \\
\geq 10.0\end{array}$ & $\begin{array}{l}\text { Of f shore, } \\
13.1 \\
45.1 \\
40.2 \\
1.7\end{array}$ & $\begin{array}{r}\text { Al1 Hours } \\
14.8 \\
42.0 \\
42.4 \\
0.8\end{array}$ & $\begin{array}{r}18.8 \\
59.9 \\
21.1 \\
0.2\end{array}$ & $\begin{array}{r}13.0 \\
44.5 \\
42.5 \\
0.0\end{array}$ & $\begin{array}{r}8.9 \\
38.8 \\
47.8 \\
4.5\end{array}$ \\
\hline & $\begin{array}{c}0.5-1.1 \\
2.5-4.9 \\
5.0-9.9 \\
\geq 10.0\end{array}$ & $\begin{array}{l}\text { Off shore, } \\
6.3 \\
51.6 \\
41.0 \\
1.1\end{array}$ & $\begin{array}{r}\text { Day } \quad 5.0 \\
4 y .8 \\
43.2 \\
1.1\end{array}$ & $\begin{array}{r}11.0 \\
65.6 \\
23.4 \\
0.0\end{array}$ & $\begin{array}{r}4.4 \\
51.4 \\
44.3 \\
0.0\end{array}$ & $\begin{array}{r}4.3 \\
39.7 \\
52.9 \\
3.1\end{array}$ \\
\hline r. & $\begin{array}{l}0.5-2.4 \\
2.5-4.9 \\
5.0-9.9 \\
\geq 10.0\end{array}$ & $\begin{array}{l}\text { of f shore, } \\
20.0 \\
38.4 \\
39.3 \\
2.2\end{array}$ & $\begin{array}{l}\text { Night } \\
24.2 \\
33.5 \\
41.8 \\
0.4\end{array}$ & $\begin{array}{r}34.5 \\
48.4 \\
16.3 \\
0.8\end{array}$ & $\begin{array}{r}21.0 \\
38.2 \\
40.8 \\
0.0\end{array}$ & $\begin{array}{r}12.3 \\
38.1 \\
44.0 \\
5.6\end{array}$ \\
\hline
\end{tabular}


Table 6.27

Lake Charles, Louisiana

Percent of Hours in Each Wind Speed Class

by 'Wind Direction, Season and Time of Day

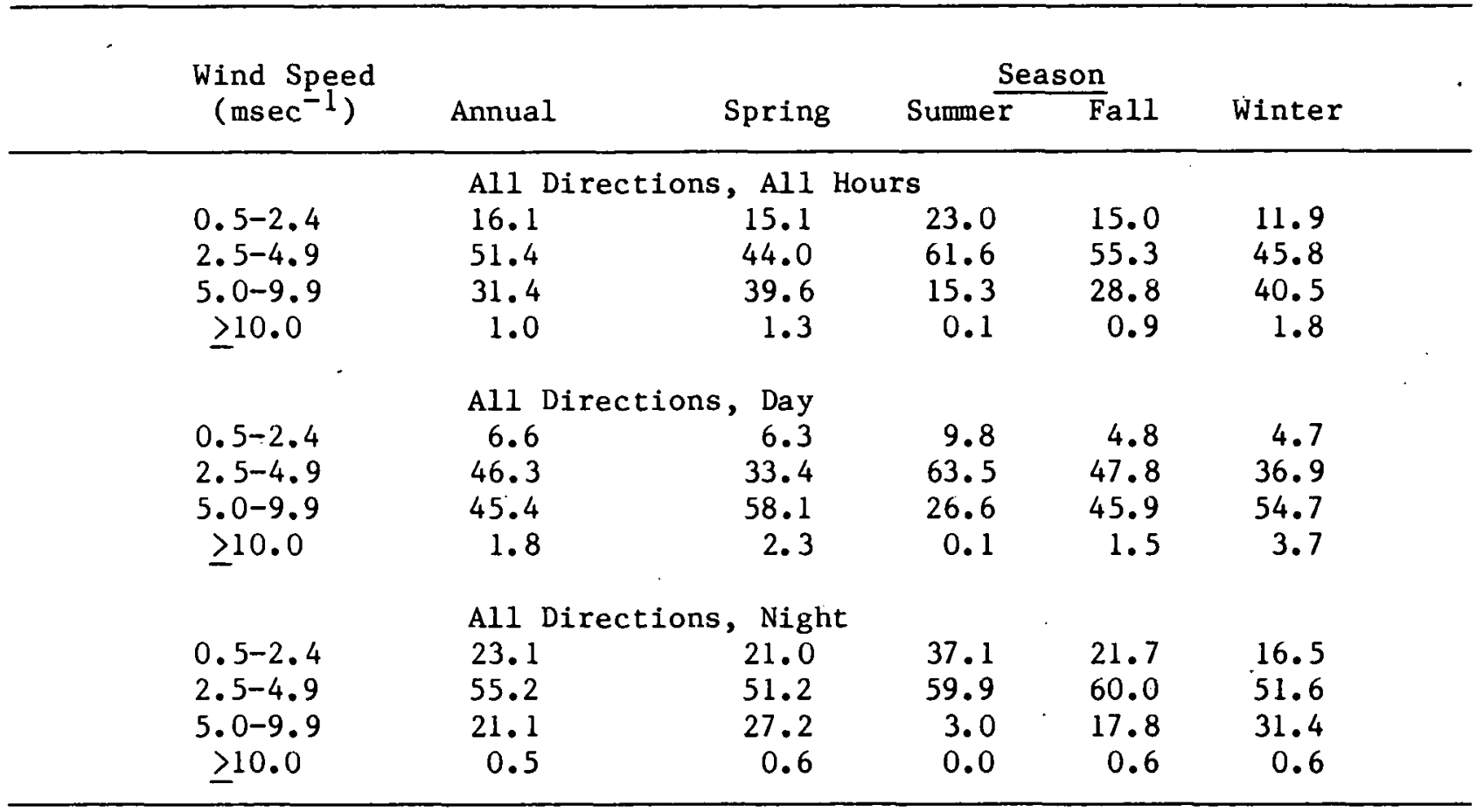


Table 6.27 (continued).

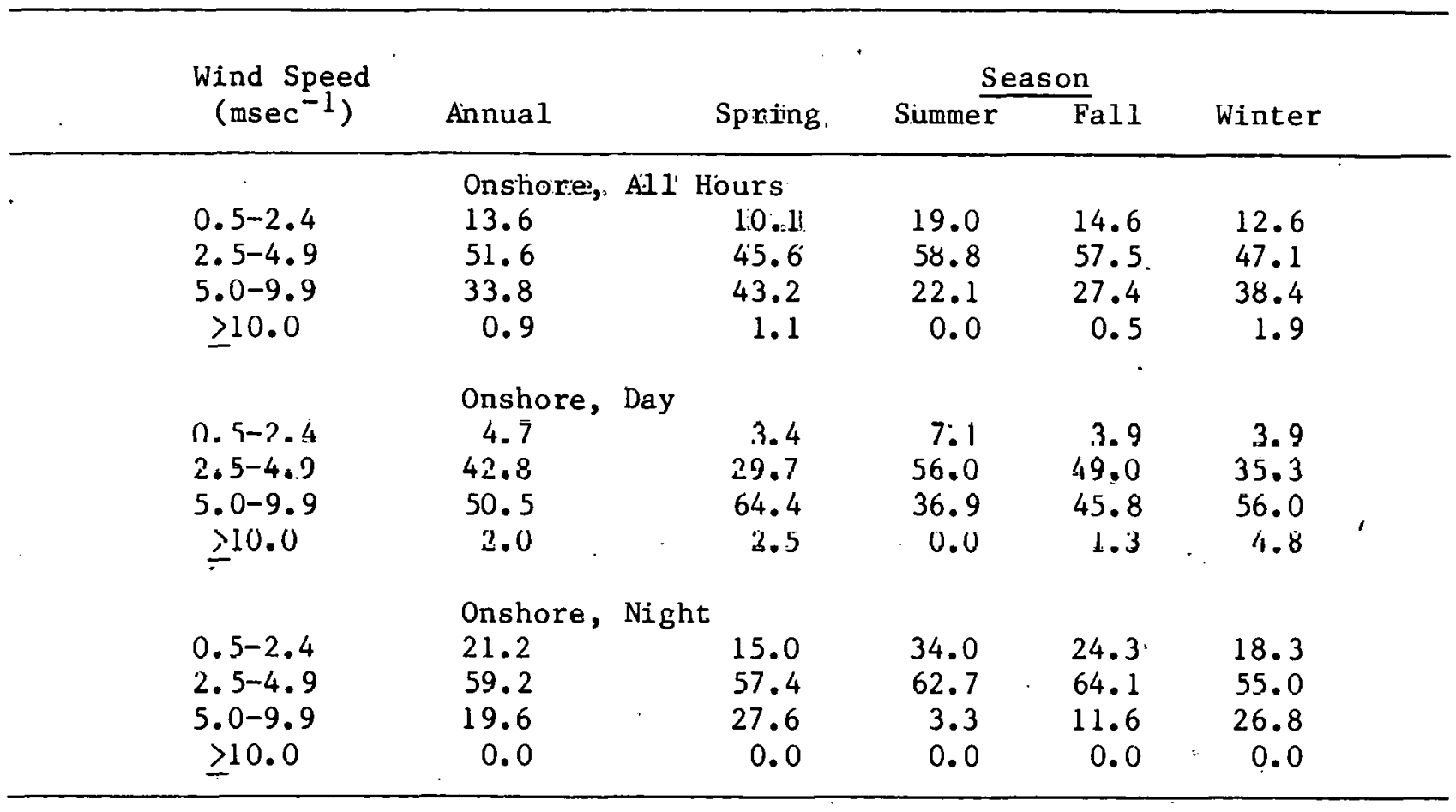


Table 6.27 (continued)

\begin{tabular}{|c|c|c|c|c|c|c|}
\hline & \multirow{2}{*}{$\begin{array}{l}\text { Wind Speed } \\
\left(\mathrm{msec}^{-1}\right)\end{array}$} & \multirow[b]{2}{*}{ Annual } & \multicolumn{4}{|c|}{ Season } \\
\hline & & & Spring & Summe $\bar{r}$ & $\overline{F a l l}$ & Winter \\
\hline & $\begin{array}{c}0.5-2.4 \\
2.5-4.9 \\
5.0-9.9 \\
\geq 10.0\end{array}$ & $\begin{array}{l}\text { Alongshore, } \\
20.2 \\
61.7 \\
17.8 \\
0.4\end{array}$ & $\begin{array}{c}\text { Al1 Hours } \\
28.5 \\
45.9 \\
25.2 \\
0.4\end{array}$ & $\begin{array}{r}25.2 \\
68.1 \\
6.7 \\
0.0\end{array}$ & $\begin{array}{r}15.7 \\
72.1 \\
11.9 \\
0.4\end{array}$ & $\begin{array}{r}12.7 \\
56.9 \\
29.7 \\
0.7\end{array}$ \\
\hline & $\begin{array}{c}0.5-2.4 \\
2.5-4.9 \\
5.0-9.9 \\
\geq 10.0\end{array}$ & $\begin{array}{l}\text { Alongshore, } \\
7.7 \\
63.6 \\
28.3 \\
0.4\end{array}$ & $\begin{array}{r}\text { Day } \\
8.3 \\
44.8 \\
45.8 \\
1.0\end{array}$ & $\begin{array}{r}9.5 \\
79.6 \\
10.8 \\
0.0\end{array}$ & $\begin{array}{r}6.0 \\
71.4 \\
22.6 \\
0.0\end{array}$ & $\begin{array}{r}6.4 \\
52.5 \\
40.4 \\
0.8\end{array}$ \\
\hline & $\begin{array}{c}0.5-2.4 \\
2.5-4.9 \\
5.0-9.9 \\
\geq 10.0\end{array}$ & $\begin{array}{l}\text { Alongshore, } \\
28.9 \\
60.3 \\
10.5 \\
0.3\end{array}$ & $\begin{array}{r}\text { Night } \\
42.8 \\
46.6 \\
10.5 \\
0.0\end{array}$ & $\begin{array}{r}39.7 \\
57.3 \\
2.9 \\
0.0\end{array}$ & $\begin{array}{r}20.7 \\
72.4 \\
6.3 \\
0.5\end{array}$ & $\begin{array}{r}17.1 \\
60.0 \\
22.4 \\
0.6\end{array}$ \\
\hline
\end{tabular}




\section{Table 6.27 (continued)}

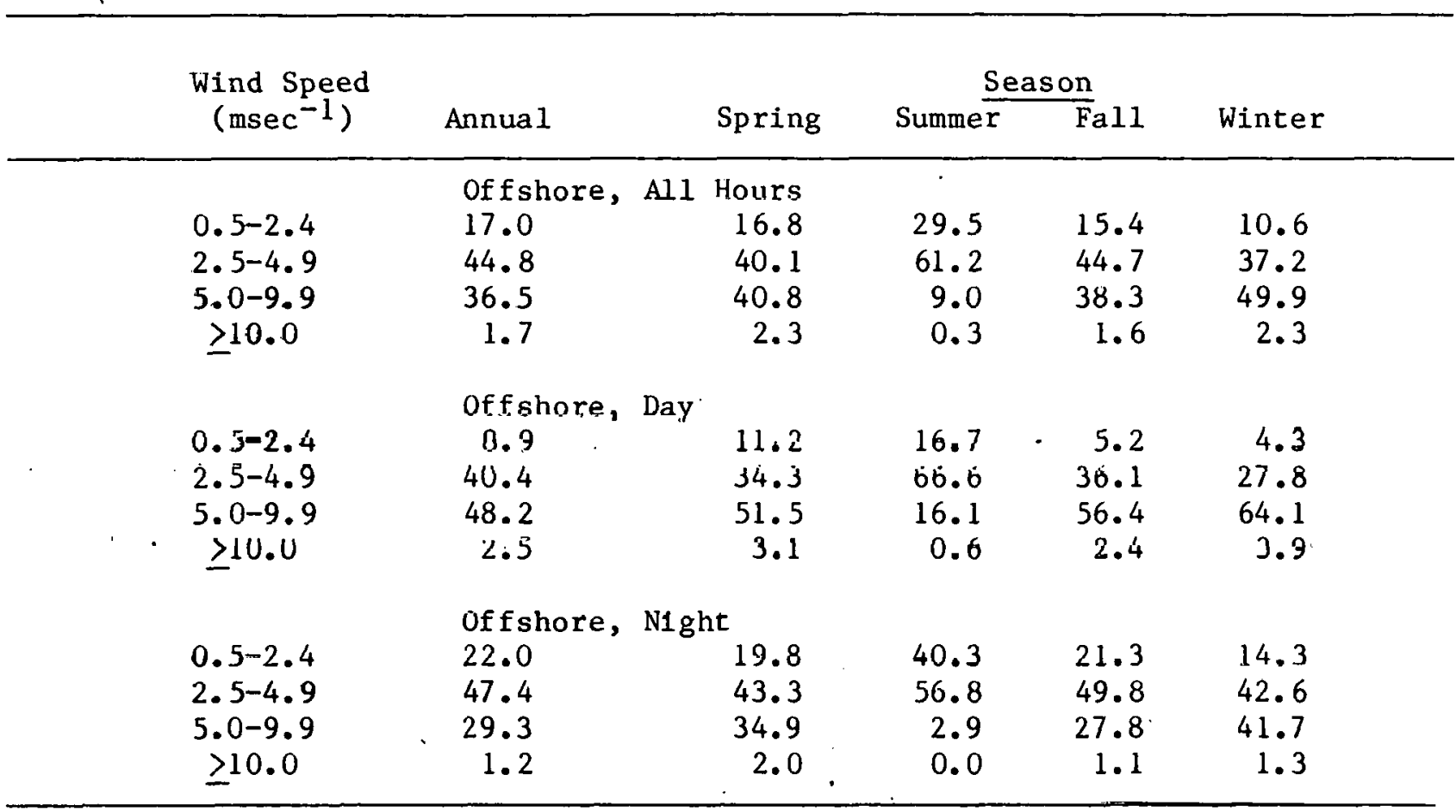




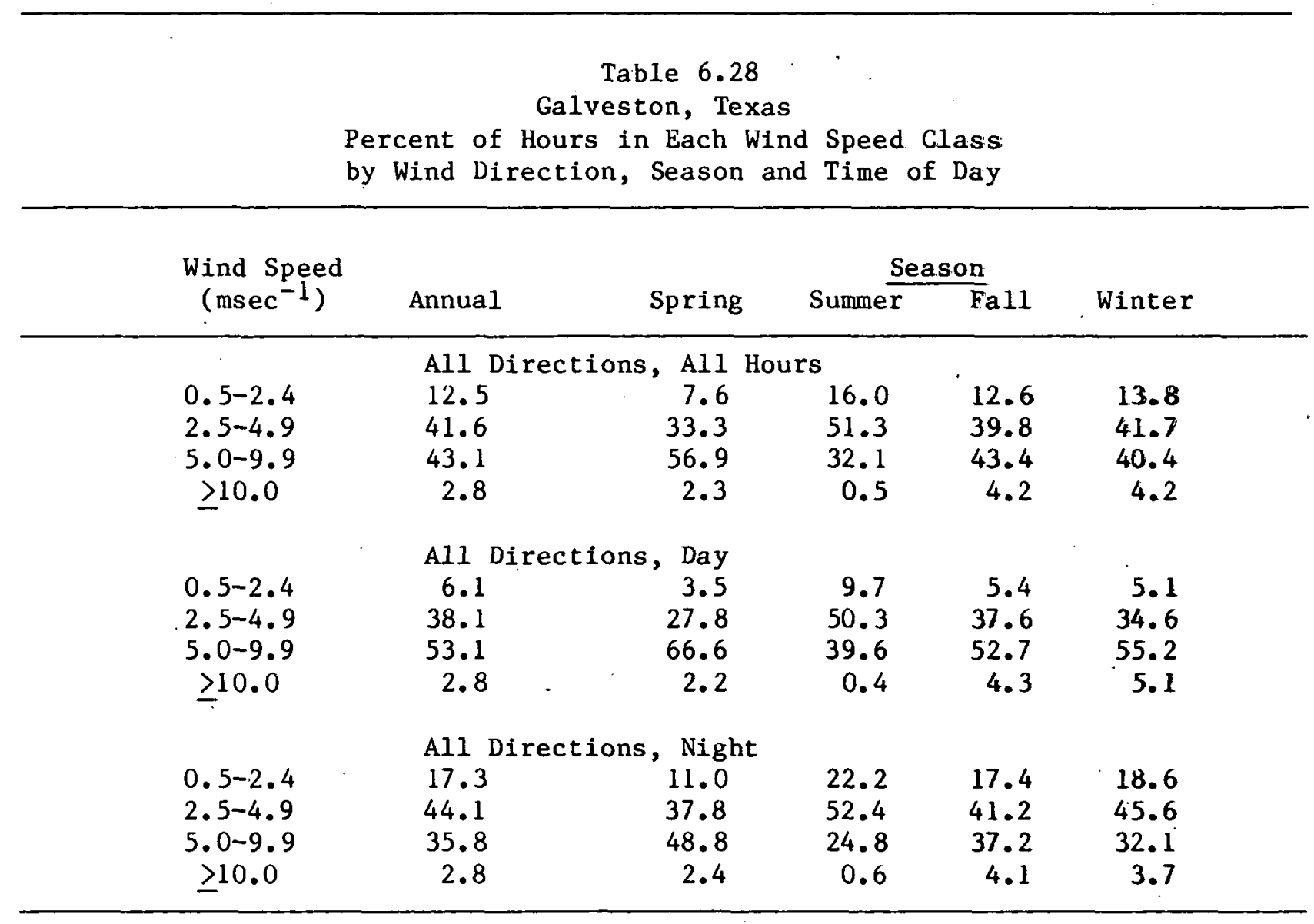


Table 6.28 (continued)

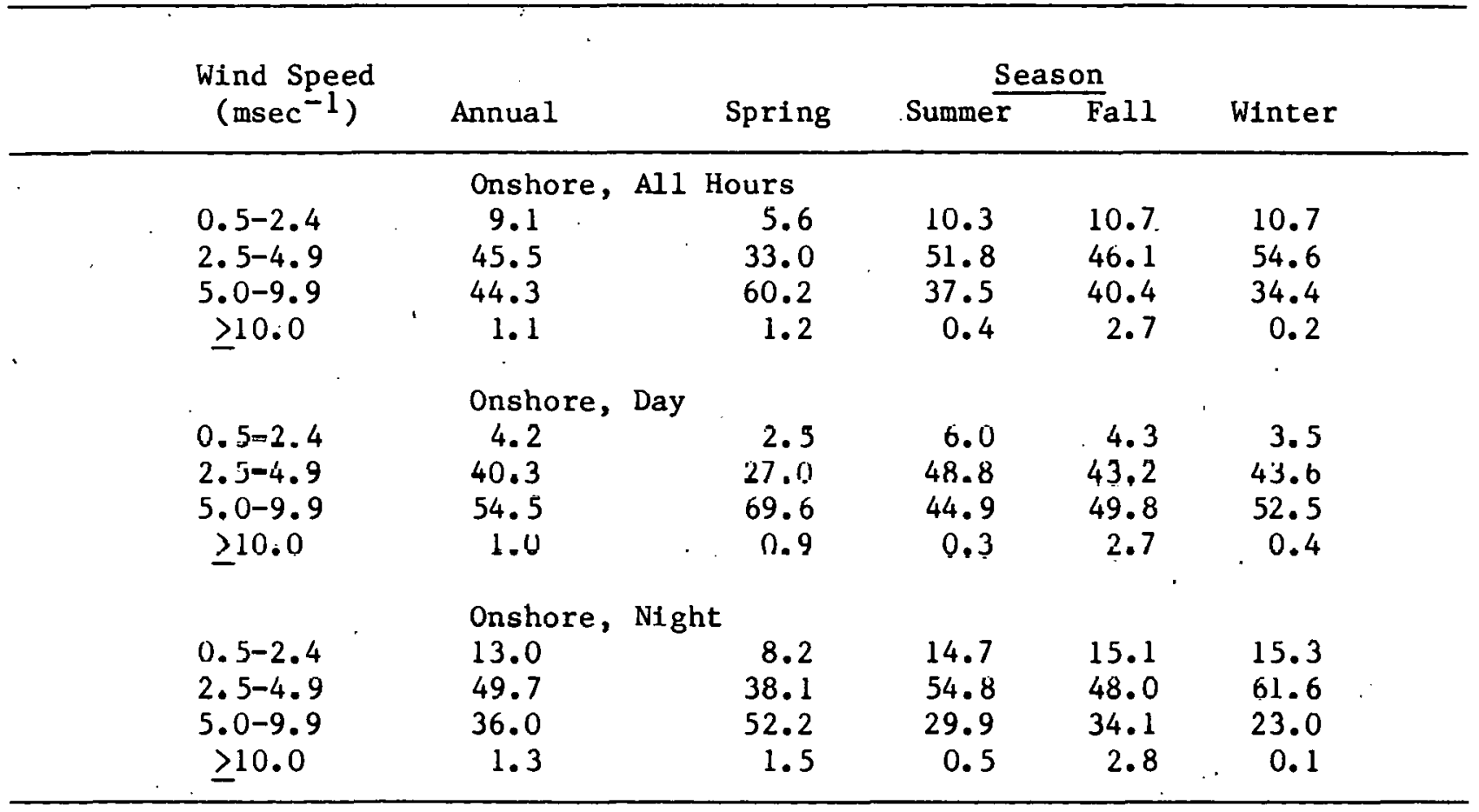


Table 6.28 (continued)

\begin{tabular}{|c|c|c|c|c|c|}
\hline \multirow{2}{*}{$\begin{array}{l}\text { Wind Speed } \\
\left(\mathrm{msec}^{-1}\right)\end{array}$} & \multirow[b]{2}{*}{ Annual } & \multicolumn{4}{|c|}{ Season } \\
\hline & & Spring & Summer & $\overline{F a l l}$ & Winter \\
\hline $\begin{array}{c}0.5-2.4 \\
2.5-4.9 \\
5.0-9.9 \\
\geq 10.0\end{array}$ & $\begin{array}{l}\text { Alongshore, } \\
24.3 \\
37.8 \\
35.4 \\
2.6\end{array}$ & $\begin{array}{c}\text { Al1 Hours } \\
21.4 \\
33.6 \\
43.2 \\
1.7\end{array}$ & $\begin{array}{r}40.2 \\
45.6 \\
13.7 \\
0.6\end{array}$ & $\begin{array}{r}16.3 \\
36.1 \\
43.4 \\
4.2\end{array}$ & $\begin{array}{r}23.1 \\
36.1 \\
38.3 \\
2.5\end{array}$ \\
\hline $\begin{array}{c}0.5-2.4 \\
2.5-4.9 \\
5.0-9.9 \\
\geq 10.0\end{array}$ & $\begin{array}{l}\text { Alongshore, } \\
11.0 \\
35.7 \\
50.5 \\
2.8\end{array}$ & $\begin{array}{r}\text { Day } \\
7.4 \\
31.4 \\
60.3 \\
0.8\end{array}$ & $\begin{array}{r}24.1 \\
50.5 \\
25.0 \\
0.5\end{array}$ & $\begin{array}{r}6.7 \\
30.8 \\
56.6 \\
5.9\end{array}$ & $\begin{array}{r}6.5 \\
30.5 \\
61.0 \\
2.0\end{array}$ \\
\hline $\begin{array}{c}0.5-2.4 \\
2.5-4.9 \\
5.0-9.9 \\
\geq 10.0\end{array}$ & $\begin{array}{l}\text { Alongshore, } \\
31.0 \\
38.8 \\
27.7 \\
2.5\end{array}$ & $\begin{array}{r}\text { Night } \\
29.0 \\
34.8 \\
33.9 \\
2.3\end{array}$ & $\begin{array}{r}49.9 \\
43.2 \\
6.2 \\
0.7\end{array}$ & $\begin{array}{c}21.2 \\
38.8 \\
36.6 \\
3.3\end{array}$ & $\begin{array}{r}30.2 \\
38.4 \\
28.8 \\
2.6\end{array}$ \\
\hline
\end{tabular}


Table 6.28 (continued)

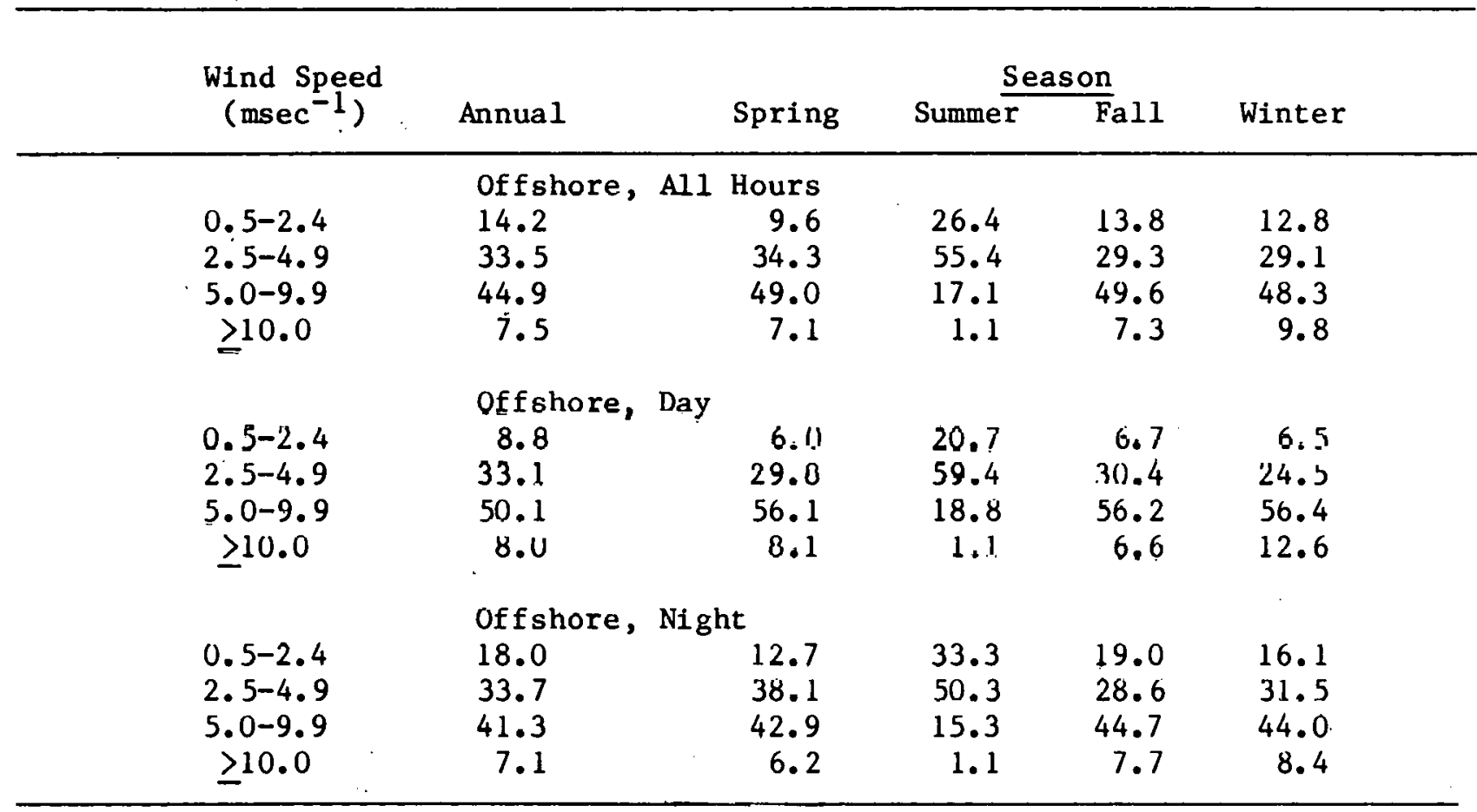


Table 6.29

Houston, Texas

Percent of Hours in Each Wind Speed Class

by Wind Direction, Season and Time of Day

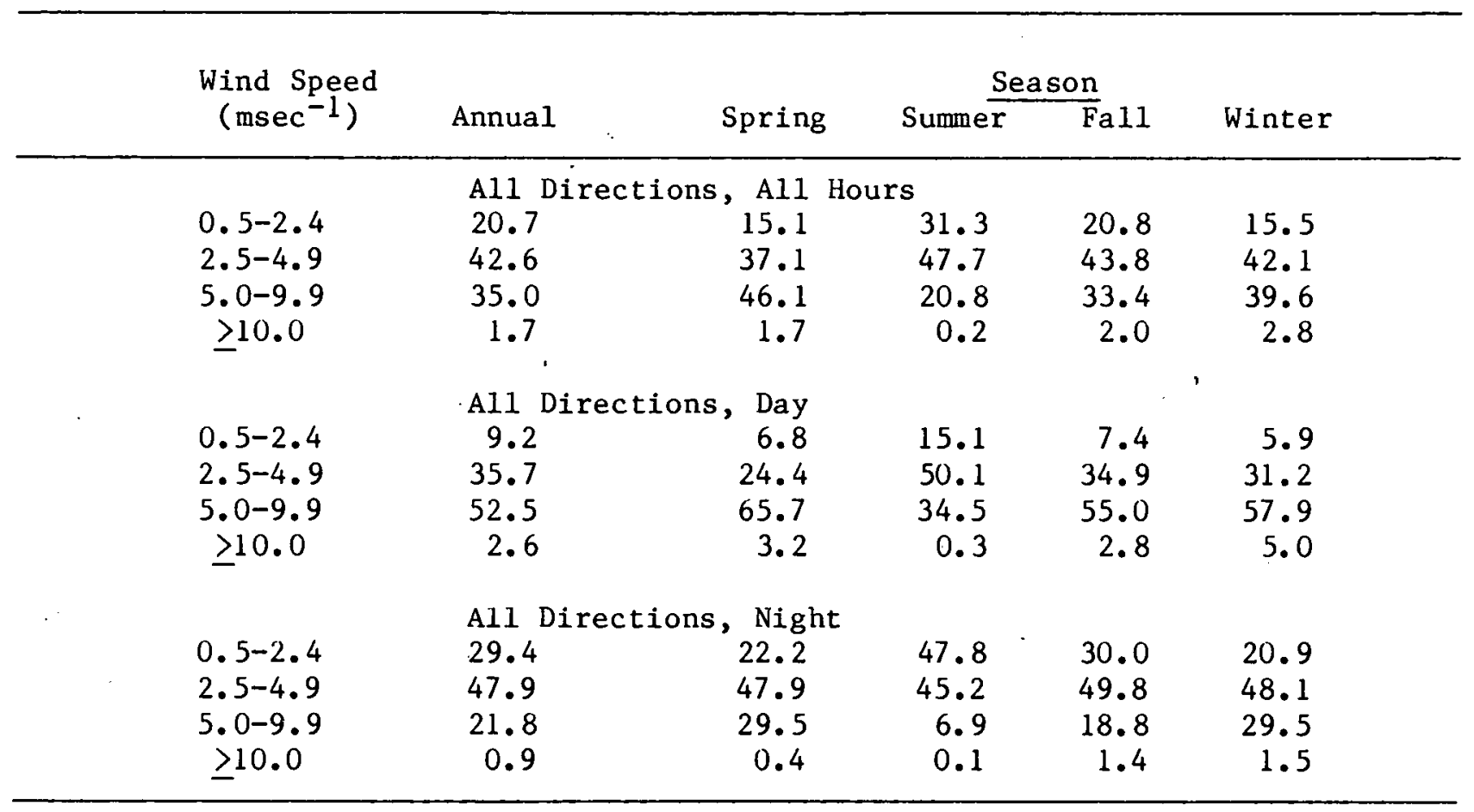


Table 6.29 (continued)

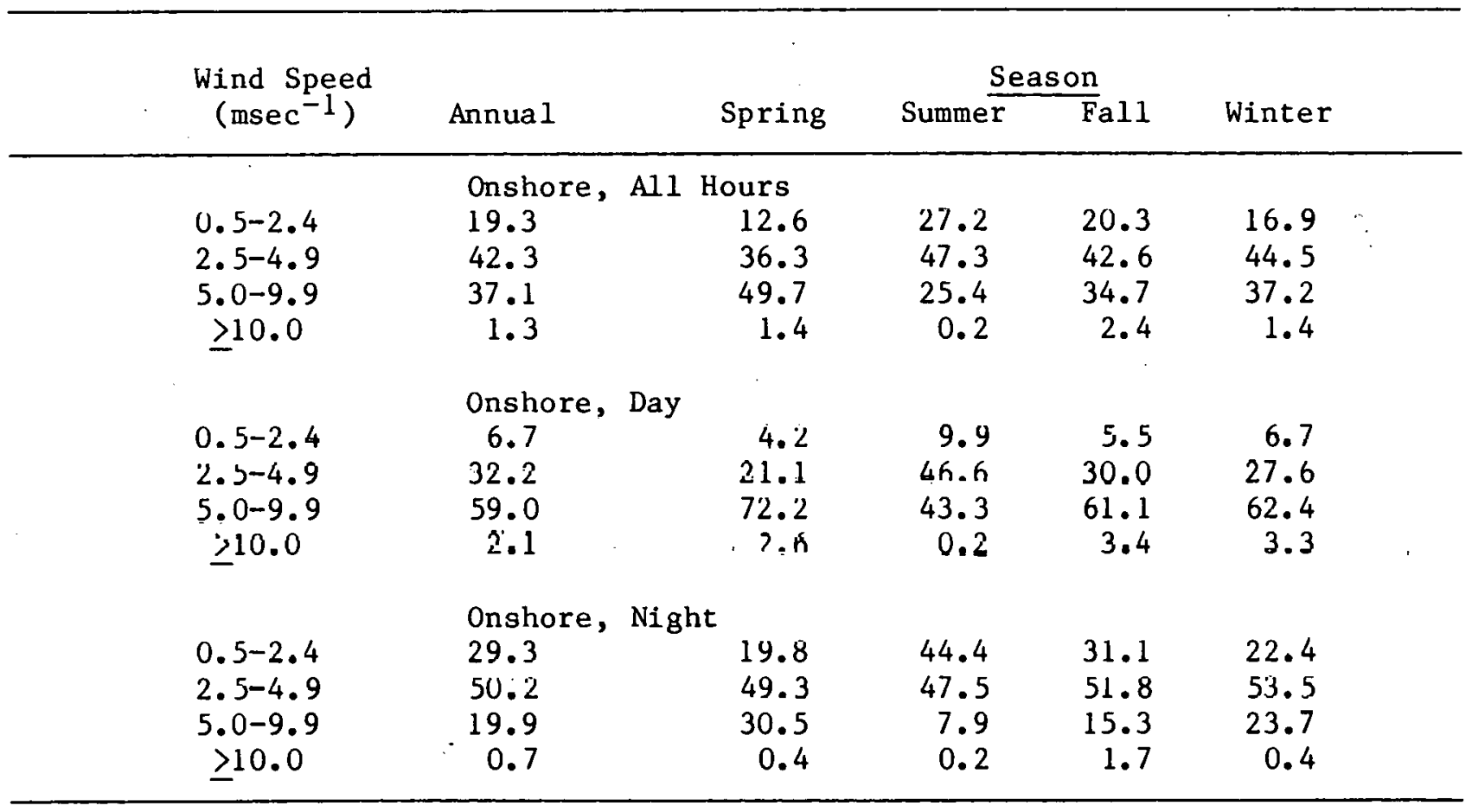


Table 6.29 (continued)

\begin{tabular}{|c|c|c|c|c|c|}
\hline \multirow{2}{*}{$\begin{array}{l}\text { Wind Speed } \\
\quad\left(\mathrm{msec}^{-1}\right)\end{array}$} & \multirow[b]{2}{*}{ Annual } & \multirow[b]{2}{*}{ Spring } & \multicolumn{2}{|c|}{ Season } & \multirow[b]{2}{*}{ Winter } \\
\hline & & & Summer & $\bar{F}$ all & \\
\hline $\begin{array}{c}0.5-2.4 \\
2.5-4.9 \\
5.0-9.9 \\
\geq 10.0\end{array}$ & $\begin{array}{l}\text { Alongshore, } \\
27.2 \\
48.9 \\
23.2 \\
0.7\end{array}$ & $\begin{array}{c}\text { Al1. Hours } \\
26.1 \\
48.7 \\
24.7 \\
0.5\end{array}$ & $\begin{array}{r}36.6 \\
50.7 \\
12.7 \\
0.0\end{array}$ & $\begin{array}{r}24.2 \\
49.1 \\
24.8 \\
1.8\end{array}$ & $\begin{array}{r}21.5 \\
46.6 \\
31.6 \\
0.3\end{array}$ \\
\hline $\begin{array}{c}0.5-2.4 \\
2.5-4.9 \\
5.0-9.9 \\
\geq 10.0\end{array}$ & $\begin{array}{l}\text { Alongshore, } \\
14.7 \\
49.6 \\
35.1 \\
0.6\end{array}$ & $\begin{array}{l}\text { Day } \\
15.2 \\
50.2 \\
33.5 \\
1.1\end{array}$ & $\begin{array}{r}21.7 \\
58.1 \\
20.2 \\
0.0\end{array}$ & $\begin{array}{r}11.1 \\
47.2 \\
40.2 \\
1.4\end{array}$ & $\begin{array}{r}9.1 \\
39.3 \\
51.2 \\
0.3\end{array}$ \\
\hline $\begin{array}{c}0.5-2.4 \\
2.5-4.9 \\
5.0-9.9 \\
\geq 10.0\end{array}$ & $\begin{array}{l}\text { Alongshore, } \\
37.2 \\
48.4 \\
13.6 \\
0.7\end{array}$ & $\begin{array}{r}\text { Night } \\
35.4 \\
47.5 \\
17.0 \\
0.0\end{array}$ & $\begin{array}{r}53.0 \\
42.5 \\
4.5 \\
0.0\end{array}$ & $\begin{array}{r}33.9 \\
50.5 \\
13.4 \\
2.1\end{array}$ & $\begin{array}{r}28.6 \\
51.4 \\
19.8 \\
0.3\end{array}$ \\
\hline
\end{tabular}




\section{Table 6.29 (continued)}

\begin{tabular}{|c|c|c|c|c|c|c|}
\hline \multirow{2}{*}{$\begin{array}{l}\text { Wind Speed } \\
\quad\left(\mathrm{msec}^{-1}\right)\end{array}$} & \multirow[b]{2}{*}{ Annual } & \multicolumn{5}{|c|}{ Season } \\
\hline & & & Spring & Summer & Fall & Winter \\
\hline $\begin{array}{c}0.5-2.4 \\
2.5-4.9 \\
5.0-9.9 \\
\geq 10.0\end{array}$ & $\begin{array}{l}\text { of fshore, } \\
19.5 \\
39.4 \\
38.1 \\
3.0\end{array}$ & Al1 & $\begin{array}{r}\text { Hours } \\
18.0 \\
34.4 \\
44.7 \\
2.9\end{array}$ & $\begin{array}{r}40.9 \\
45.0 \\
13.5 \\
0.6\end{array}$ & $\begin{array}{r}19.6 \\
42.4 \\
36.6 \\
1.4\end{array}$ & $\begin{array}{r}11.2 \\
37.6 \\
45.9 \\
5.3\end{array}$ \\
\hline $\begin{array}{c}u . j-2.4 \\
2.5-4.9 \\
5.0-9.9 \\
\geq 10.0\end{array}$ & $\begin{array}{l}\text { Offshore, } \\
10.8 \\
33.9 \\
50.2 \\
5.1\end{array}$ & Day & $\begin{array}{r}11.2 \\
23.0 \\
60.0 \\
5.8\end{array}$ & $\begin{array}{r}27.1 \\
51.6 \\
20.2 \\
1.1\end{array}$ & $\begin{array}{r}8.0 \\
34.1 \\
54.7 \\
2.5\end{array}$ & $\begin{array}{r}3.4 \\
30.6 \\
57.0 \\
8.9\end{array}$ \\
\hline $\begin{array}{c}0.5-2.4 \\
2.5-4.9 \\
5.0-9.9 \\
>10.0\end{array}$ & $\begin{array}{l}\text { of fshore, } \\
25.3 \\
43.2 \\
30.0 \\
1.6\end{array}$ & $\mathrm{Nigh}$ & $\begin{array}{r}23.7 \\
43.8 \\
31.9 \\
0.6\end{array}$ & $\begin{array}{r}56.4 \\
37.5 \\
6.0 \\
0.0\end{array}$ & $\begin{array}{r}26.2 \\
46.7 \\
26.4 \\
0.7\end{array}$ & $\begin{array}{r}15.7 \\
41.5 \\
39.5 \\
3.3\end{array}$ \\
\hline
\end{tabular}


Table 6.30

Brownsville, Texas

Percent of Hours in Each Wind Speed Class

by Wind Direction, Season and Time of Day

\begin{tabular}{|c|c|c|c|c|c|}
\hline \multirow{2}{*}{$\begin{array}{l}\text { Wind Speed } \\
\left(\mathrm{msec}^{-1}\right)\end{array}$} & \multirow[b]{2}{*}{ Annual } & \multirow[b]{2}{*}{ Spring } & \multicolumn{2}{|c|}{ Season } & \multirow[b]{2}{*}{ Winter } \\
\hline & & & Summer & $\bar{F} a 11$ & \\
\hline $\begin{array}{c}0.5-2.4 \\
2.5-4.9 \\
5.0-9.9 \\
\geq 10.0\end{array}$ & $\begin{array}{l}\text { All Directions } \\
12.7 \\
34.5 \\
49.2 \\
3.6\end{array}$ & $\begin{array}{r}\text { A } 11 \\
6.5 \\
24.5 \\
62.0 \\
7.1\end{array}$ & $\begin{array}{l}\text { rs } \\
\begin{array}{r}15.2 \\
36.4 \\
47.1 \\
1.3\end{array}\end{array}$ & $\begin{array}{r}17.0 \\
39.8 \\
41.2 \\
2.0\end{array}$ & $\begin{array}{r}12.5 \\
37.8 \\
45.9 \\
3.9\end{array}$ \\
\hline $\begin{array}{c}0.5-2.4 \\
2.5-4.9 \\
5.0-9.9 \\
\geq 10.0\end{array}$ & $\begin{array}{l}\text { Al1 Directions } \\
2.9 \\
19.5 \\
70.8 \\
6.8\end{array}$ & $\begin{array}{r}\text { Day } \\
1.8 \\
9.4 \\
76.7 \\
12.1\end{array}$ & $\begin{array}{r}2.2 \\
18.0 \\
77.3 \\
2.5\end{array}$ & $\begin{array}{r}3.7 \\
25.9 \\
65.4 \\
5.0\end{array}$ & $\begin{array}{r}4.1 \\
26.6 \\
60.9 \\
8.4\end{array}$ \\
\hline $\begin{array}{c}0.5-2.4 \\
2.5-4.9 \\
5.0-9.9 \\
\geq 10.0\end{array}$ & $\begin{array}{l}\text { A11 Directions } \\
20.0 \\
45.6 \\
33.2 \\
1.2\end{array}$ & $\begin{array}{c}\text { Nigh } \\
9.9 \\
35.6 \\
51.1 \\
3.3\end{array}$ & $\begin{array}{r}28.2 \\
54.7 \\
17.0 \\
0.0\end{array}$ & $\begin{array}{r}25.4 \\
48.6 \\
25.9 \\
0.1\end{array}$ & $\begin{array}{r}17.6 \\
44.7 \\
36.7 \\
1.0\end{array}$ \\
\hline
\end{tabular}




\section{Table 6.30 (continued)}

\begin{tabular}{|c|c|c|c|c|c|c|c|}
\hline & \multirow{2}{*}{$\begin{array}{l}\text { Wind Speed } \\
\left(\mathrm{msec}^{-1}\right)\end{array}$} & \multirow[b]{2}{*}{ Annual } & \multicolumn{5}{|c|}{ Season } \\
\hline & & & & Spring & Summer & Fall & Winter \\
\hline & $\begin{array}{c}0.5-2.4 \\
2.5-4.9 \\
5.0-9.9 \\
\geq 10.0\end{array}$ & $\begin{array}{l}\text { Onshore, } \\
12.3 \\
38.4 \\
47.7 \\
1.7\end{array}$ & Al1 & $\begin{array}{r}\text { Hours } \\
5.5 \\
27.9 \\
62.5 \\
4.1\end{array}$ & $\begin{array}{r}14.8 \\
37.1 \\
47.5 \\
0.6\end{array}$ & $\begin{array}{r}16.1 \\
44.3 \\
39.2 \\
0.4\end{array}$ & $\begin{array}{r}13.1 \\
48.9 \\
36.6 \\
1.4\end{array}$ \\
\hline & $\begin{array}{c}0.5-2.4 \\
2.5-4.9 \\
5.0-9.9 \\
\geq 10.0\end{array}$ & $\begin{array}{c}\text { Onshore, } \\
2.4 \\
18.4 \\
75.7 \\
3.5\end{array}$ & Day & $\begin{array}{r}1.3 \\
8.6 \\
82.2 \\
7.8\end{array}$ & $\begin{array}{r}2.3 \\
16.2 \\
80.2 \\
1.3\end{array}$ & $\begin{array}{r}1.8 \\
27.9 \\
68.9 \\
1.3\end{array}$ & $\begin{array}{r}5.6 \\
30.0 \\
60.0 \\
1.4\end{array}$ \\
\hline - & $\begin{array}{c}0.5-2.4 \\
2.5-4.9 \\
5.0-9.9 \\
\geq 10.0\end{array}$ & $\begin{array}{l}\text { Onshore, } \\
18.6 \\
51.1 \\
29.8 \\
0.5\end{array}$ & $\mathrm{~N} 1 \mathrm{gh}$ & $\begin{array}{r}8.2 \\
40.3 \\
49.7 \\
1.8\end{array}$ & $\begin{array}{r}26.0 \\
55.8 \\
18.2 \\
0.0\end{array}$ & $\begin{array}{r}23.2 \\
52.4 \\
24.4 \\
0.0\end{array}$ & $\begin{array}{r}16.4 \\
57.4 \\
26.2 \\
0.0\end{array}$ \\
\hline
\end{tabular}


Table 6.30 (continued)

\begin{tabular}{|c|c|c|c|c|c|}
\hline \multirow{2}{*}{$\begin{array}{l}\text { Wind Speed } \\
\left(\mathrm{msec}^{-1}\right)\end{array}$} & \multirow[b]{2}{*}{ Annual } & \multirow[b]{2}{*}{ Spring } & \multicolumn{2}{|c|}{ Season } & \multirow[b]{2}{*}{ Winter } \\
\hline & & & Summer & $\bar{F}$ Fall & \\
\hline $\begin{array}{c}0.5-2.4 \\
2.5-4.9 \\
5.0-9.9 \\
\geq 10.0\end{array}$ & $\begin{array}{l}\text { Alongshore, } \\
9.8 \\
26.9 \\
56.6 \\
6.8\end{array}$ & $\begin{array}{c}\text { All Hours } \\
4.2 \\
14.9 \\
68.5 \\
12.4\end{array}$ & $\begin{array}{r}12.5 \\
32.5 \\
52.1 \\
2.8\end{array}$ & $\begin{array}{r}13.9 \\
34.4 \\
48.8 \\
2.9\end{array}$ & $\begin{array}{r}9.6 \\
27.1 \\
56.0 \\
7.3\end{array}$ \\
\hline $\begin{array}{c}0.5-2.4 \\
2.5-4.9 \\
5.0-9.9 \\
\geq 10.0\end{array}$ & $\begin{array}{l}\text { Alongshore, } \\
1.5 \\
15.7 \\
71.5 \\
11.2\end{array}$ & $\begin{array}{r}\text { Day } \\
0.4 \\
8.6 \\
72.8 \\
18.2\end{array}$ & $\begin{array}{r}0.8 \\
15.2 \\
79.0 \\
.5 .0\end{array}$ & $\begin{array}{r}3.6 \\
20.0 \\
70.4 \\
6.1\end{array}$ & $\begin{array}{r}1.5 \\
19.5 \\
65.2 \\
13.8\end{array}$ \\
\hline $\begin{array}{c}0.5-2.4 \\
2.5-4.9 \\
5.0-9.9 \\
\geq 10.0\end{array}$ & $\begin{array}{l}\text { Alongshore, } \\
17.7 \\
37.4 \\
42.4 \\
2.6\end{array}$ & $\begin{array}{r}\text { Night } \\
8.0 \\
21.0 \\
64.4 \\
6.7\end{array}$ & $\begin{array}{r}28.1 \\
55.4 \\
16.6 \\
0.0\end{array}$ & $\begin{array}{r}23.7 \\
48.2 \\
28.1 \\
0.0\end{array}$ & $\begin{array}{r}15.6 \\
32.8 \\
49.0 \\
2.5\end{array}$ \\
\hline
\end{tabular}


Table 6.30 (continued)

\begin{tabular}{|c|c|c|c|c|c|c|}
\hline \multirow{2}{*}{$\begin{array}{l}\text { Wind Speed } \\
\left(\mathrm{msec}^{-1}\right)\end{array}$} & \multirow[b]{2}{*}{ Annual } & \multicolumn{5}{|c|}{ Season } \\
\hline & & & Spring & Summer & Fall & Winter \\
\hline $\begin{array}{c}0.5-2.4 \\
2.5-4.9 \\
5.0-9.9 \\
\geq 10.0\end{array}$ & $\begin{array}{l}\text { Of f shore, } \\
24.2 \\
42.6 \\
31.3 \\
1.9\end{array}$ & Al1 & $\begin{array}{r}\text { Hours } \\
23.5 \\
48.9 \\
25.6 \\
1.9\end{array}$ & $\begin{array}{r}41.3 \\
54.2 \\
4.5 \\
0.0\end{array}$ & $\begin{array}{r}27.0 \\
38.4 \\
30.8 \\
3.8\end{array}$ & $\begin{array}{r}18.2 \\
40.7 \\
40.7 \\
0.4\end{array}$ \\
\hline $\begin{array}{l}0.5-2.4 \\
2.5-4.4 \\
5.0-9.9 \\
\geq 10.0\end{array}$ & $\begin{array}{l}\text { Off shore, } \\
10.9 \\
40.0 \\
45.0 \\
4.0\end{array}$ & Day & $\begin{array}{r}16.0 \\
22.1 \\
59.9 \\
1.9\end{array}$ & $\begin{array}{r}14.4 \\
13.1 \\
10.5 \\
0.0\end{array}$ & $\begin{array}{l}10.0 \\
31.8 \\
42.1 \\
10.0\end{array}$ & $\begin{array}{r}8.7 \\
38.1 \\
51.6 \\
1.0\end{array}$ \\
\hline $\begin{array}{c}0.5-2.4 \\
2.5-4.9 \\
5.0-9.9 \\
\geq 10.0\end{array}$ & $\begin{array}{l}\text { Off shore, } \\
32.1 \\
44.2 \\
23.1 \\
0.5\end{array}$ & $\mathrm{~N} 1 \mathrm{~g}$ & $\begin{array}{r}27.2 \\
61.7 \\
9.2 \\
1.9\end{array}$ & $\begin{array}{r}61.8 \\
38.2 \\
0.0 \\
0.0\end{array}$ & $\begin{array}{r}35.4 \\
38.8 \\
25.1 \\
0.6\end{array}$ & $\begin{array}{r}24.7 \\
42.1 \\
33.2 \\
0.0\end{array}$ \\
\hline
\end{tabular}




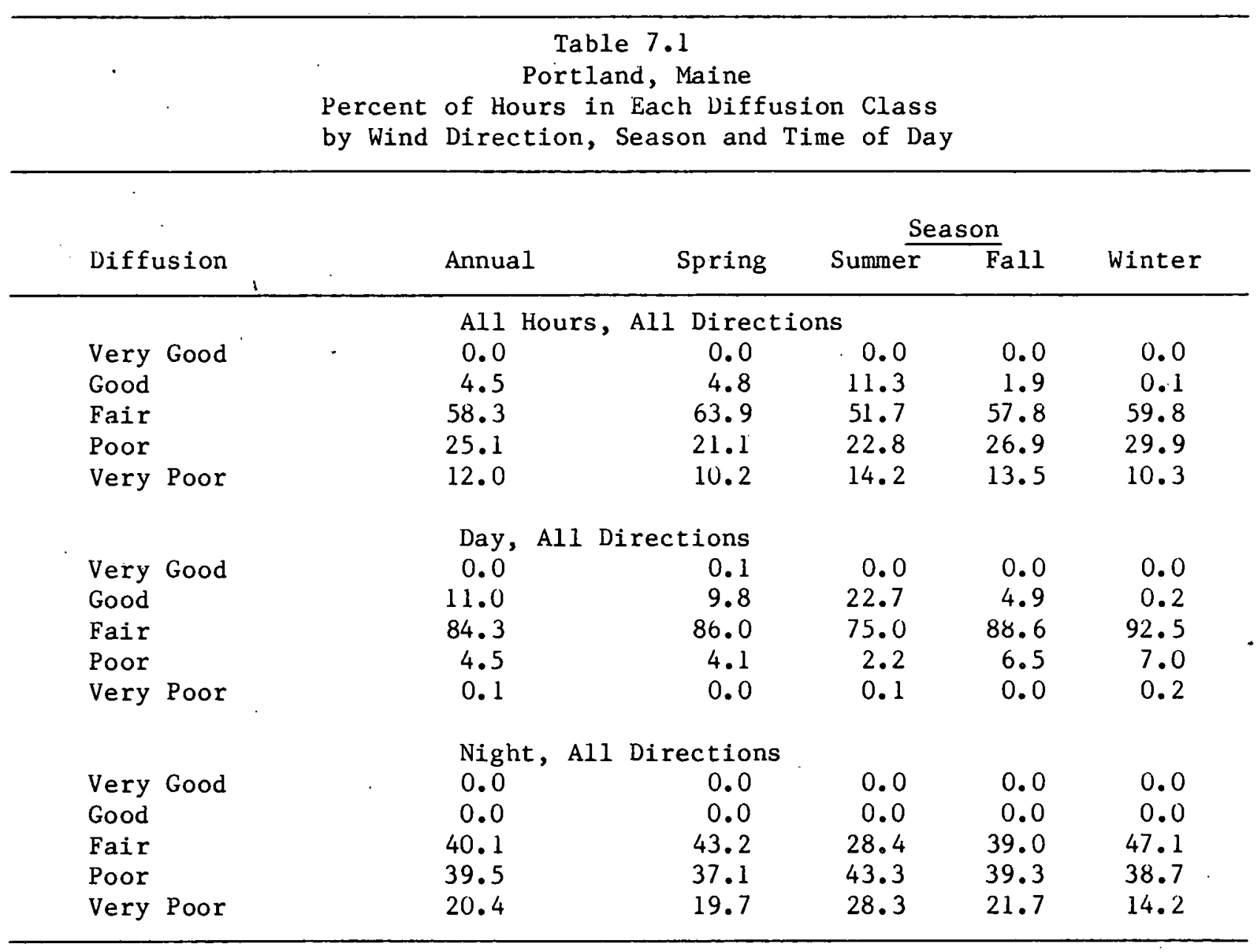


Table 7.1 (continued)

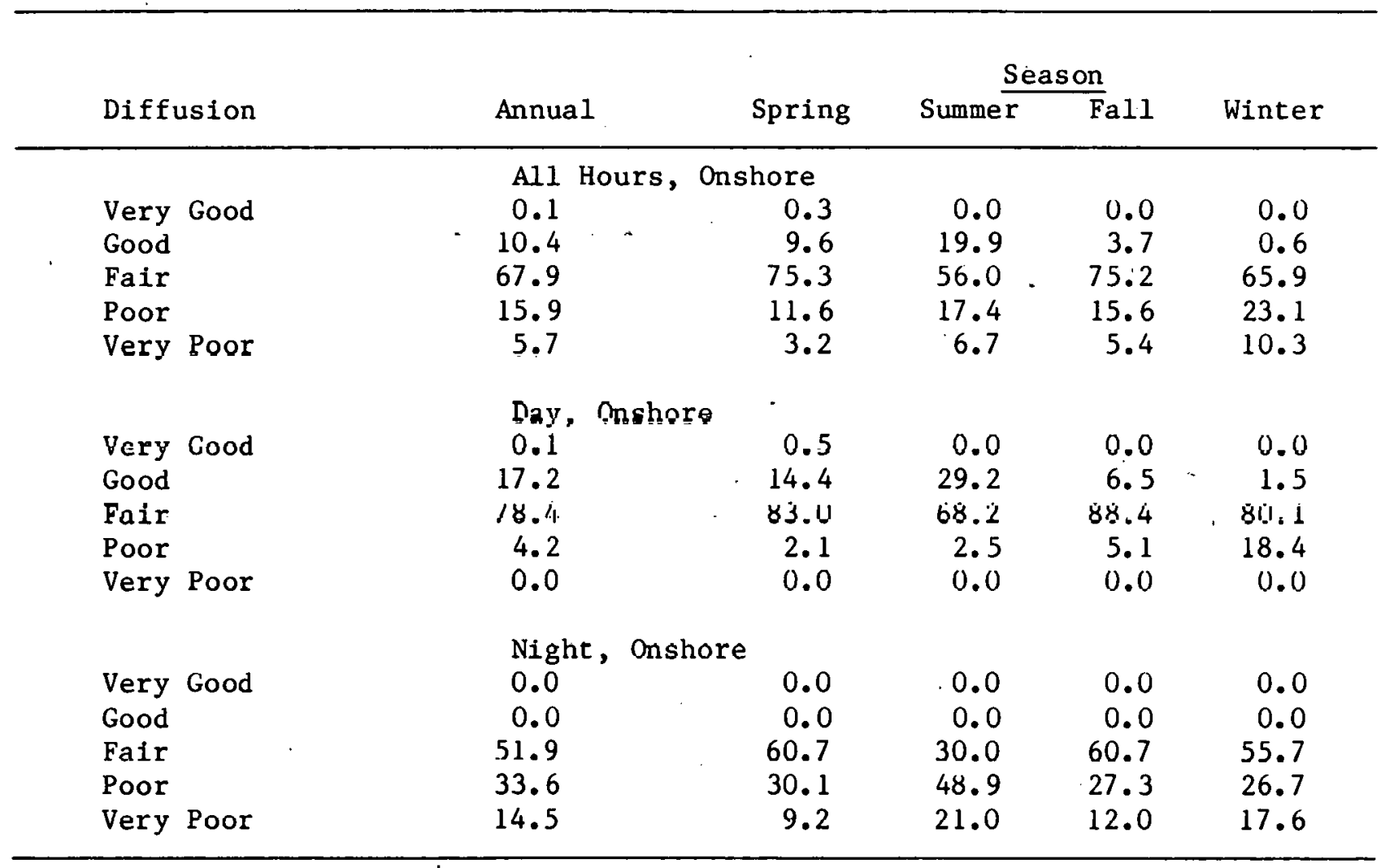


Table 7.1 (continued)

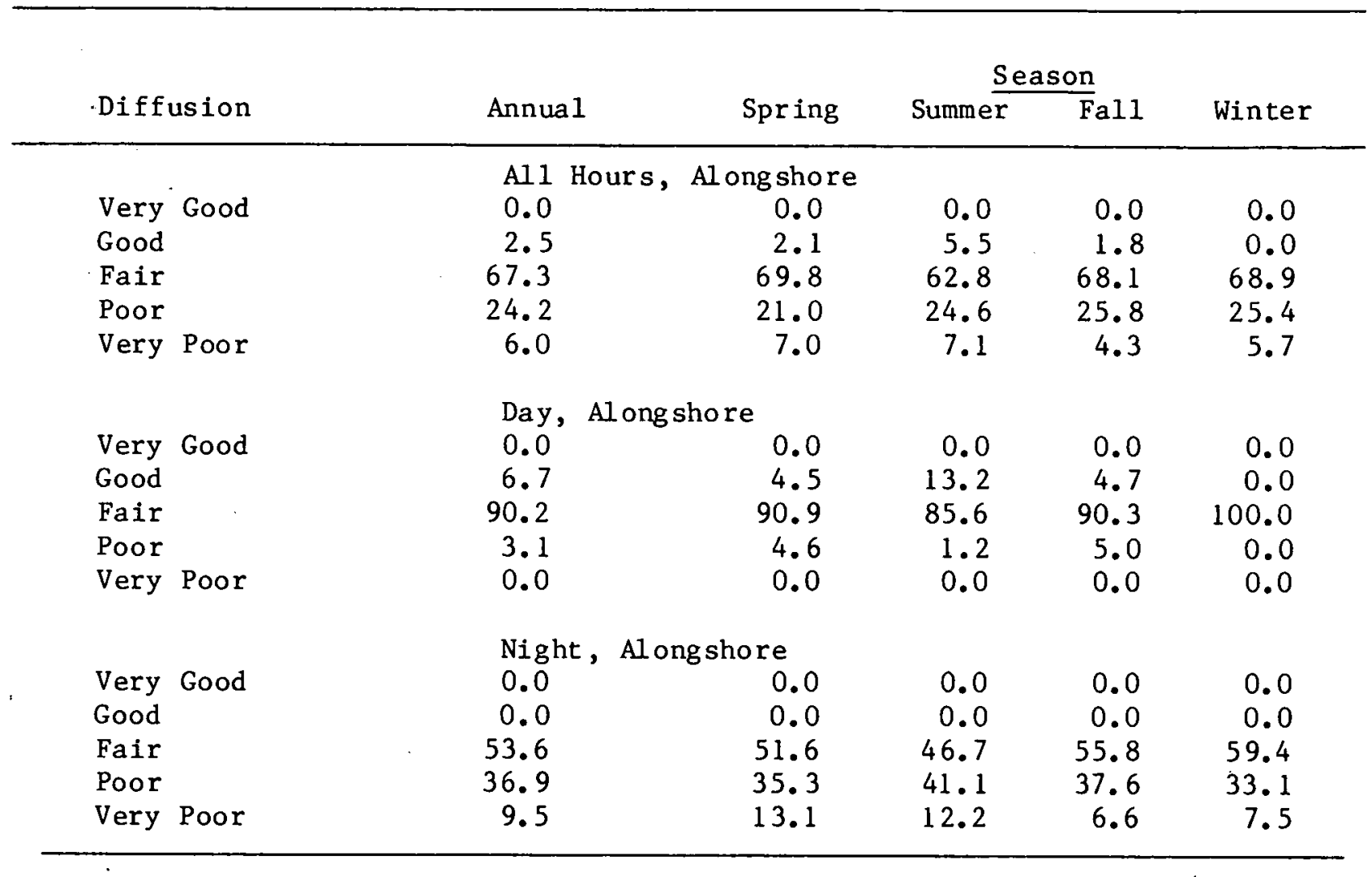


Table 7.1 (continued)

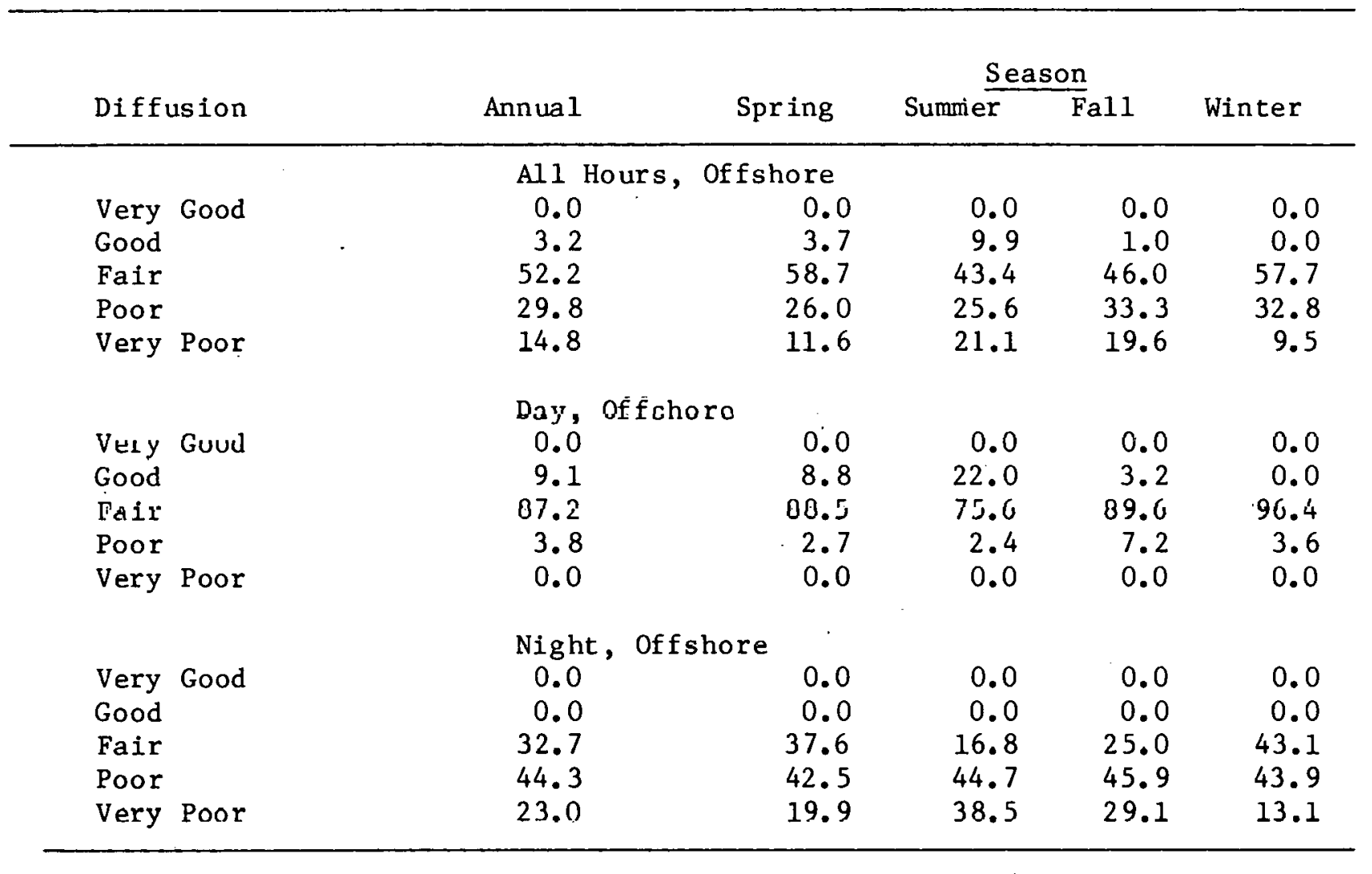


Table 7.2

Boston, Massachusetts

Percent of Hours in Each Diffusion Class

by Wind Direction, Season and Time of Day

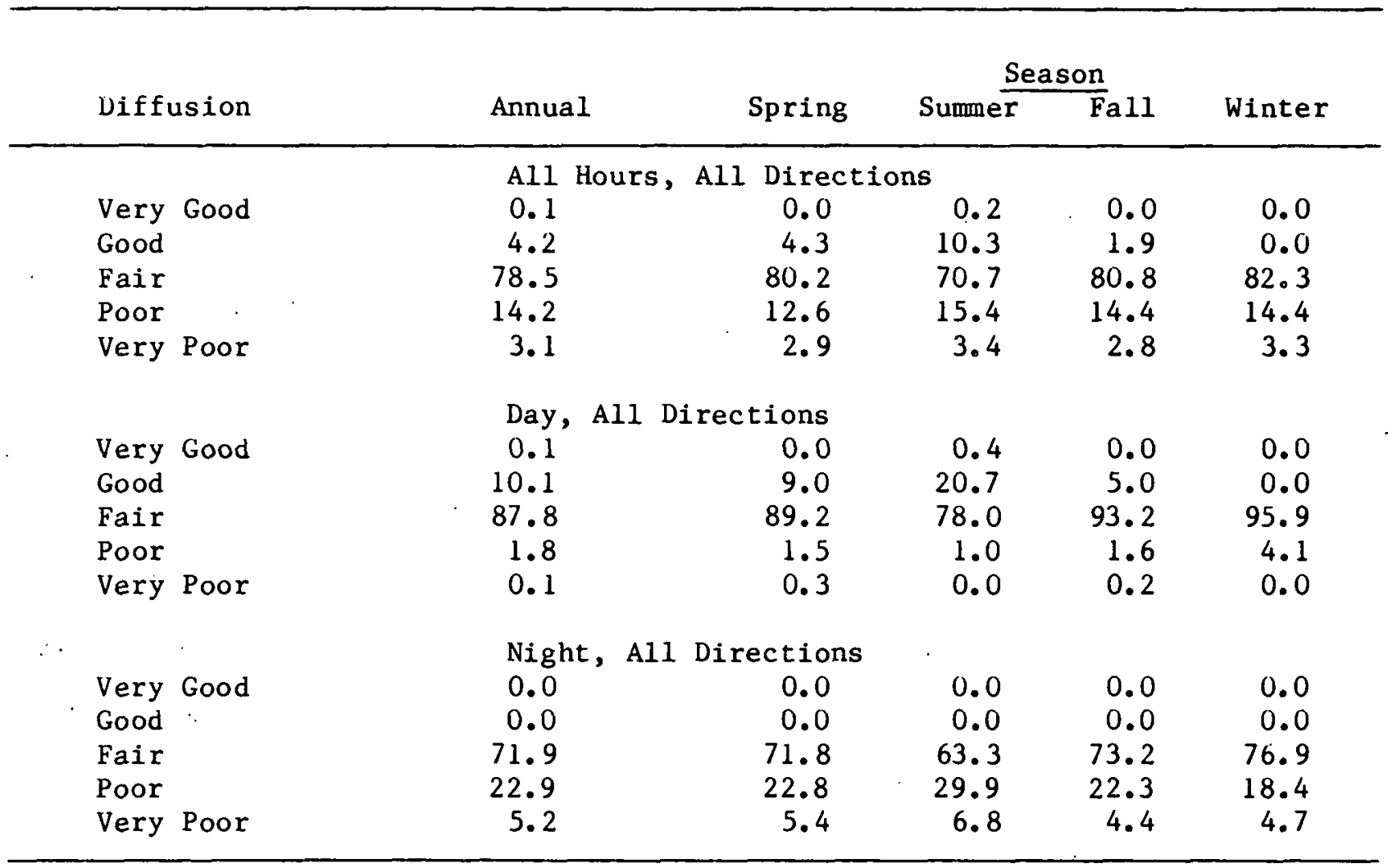


Table 7.2 (continued)

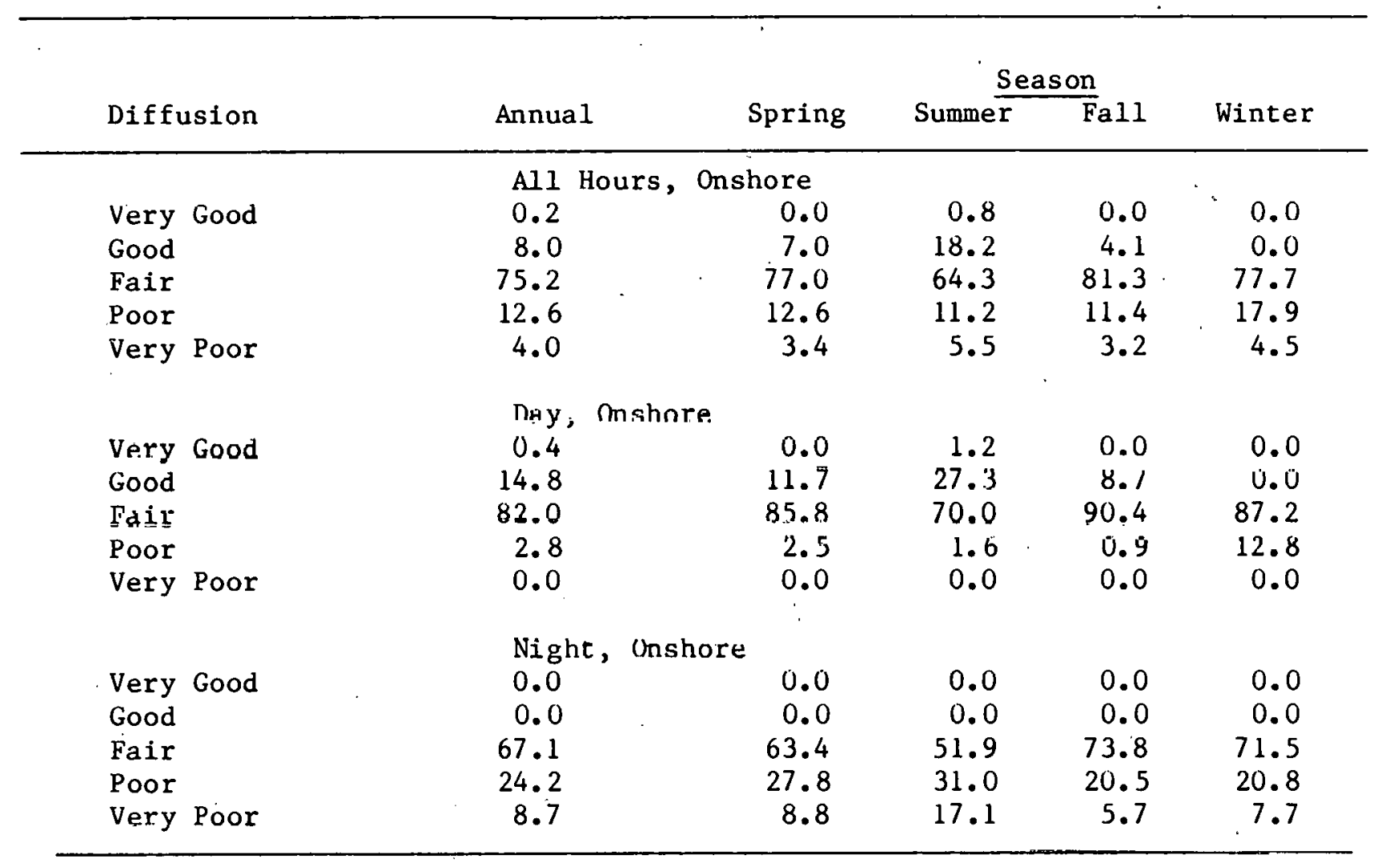


Table 7.2 (continued)

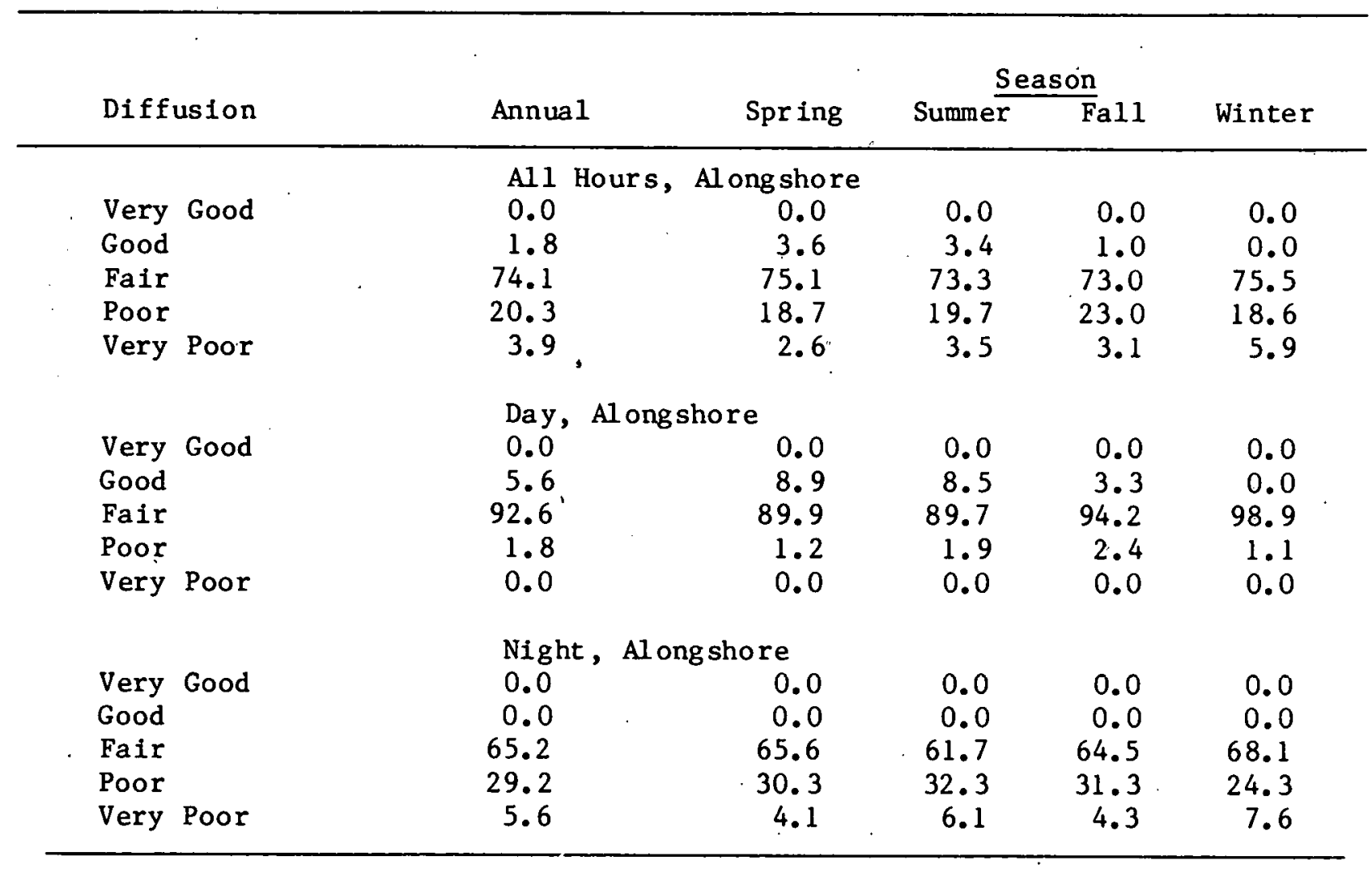


Table 7.2 (continued)

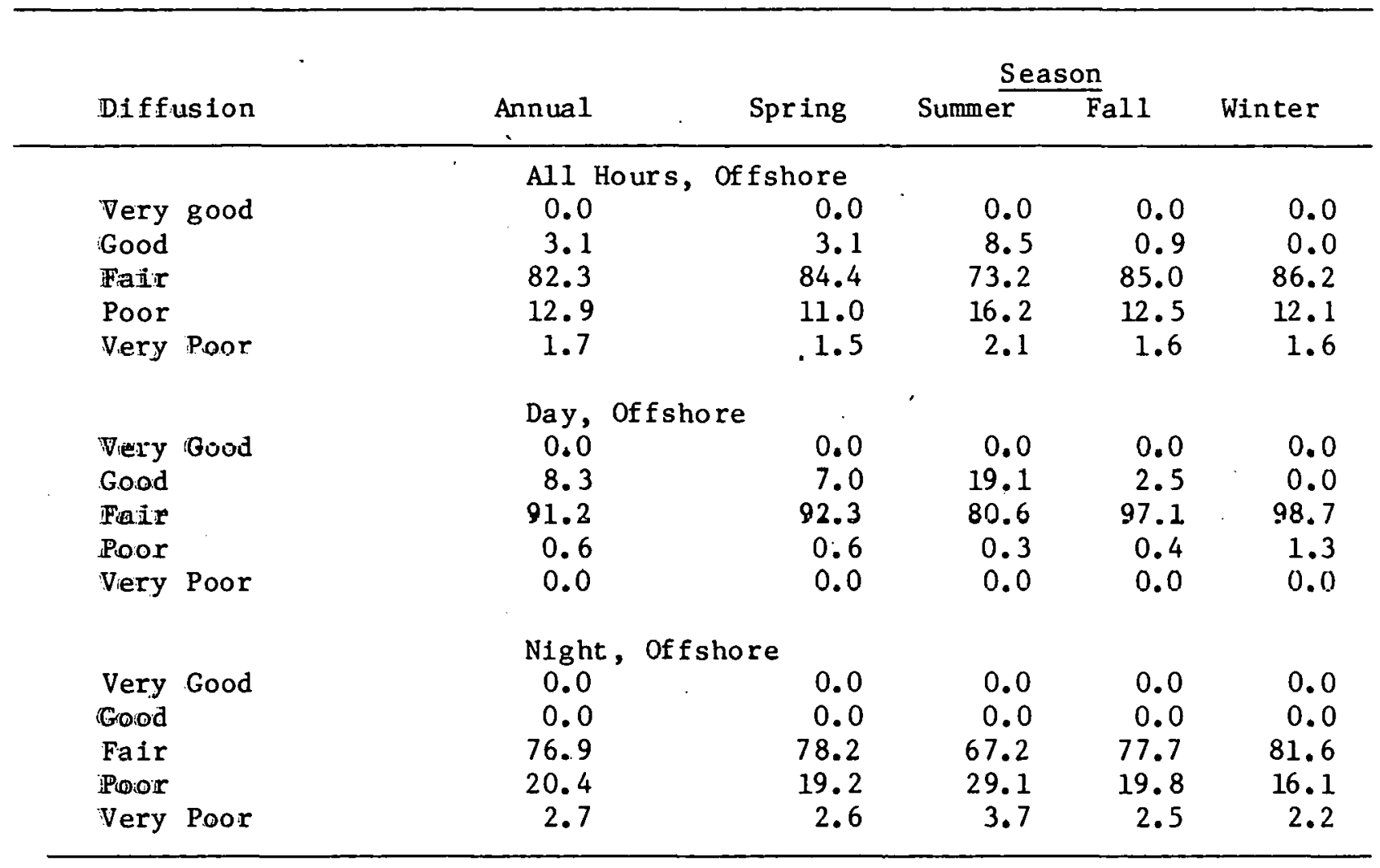


Table 7.3

Bedford, Massachusetts

Percent of Hours in Each Diffusion Class

by Wind Direction, Season and Time of Day

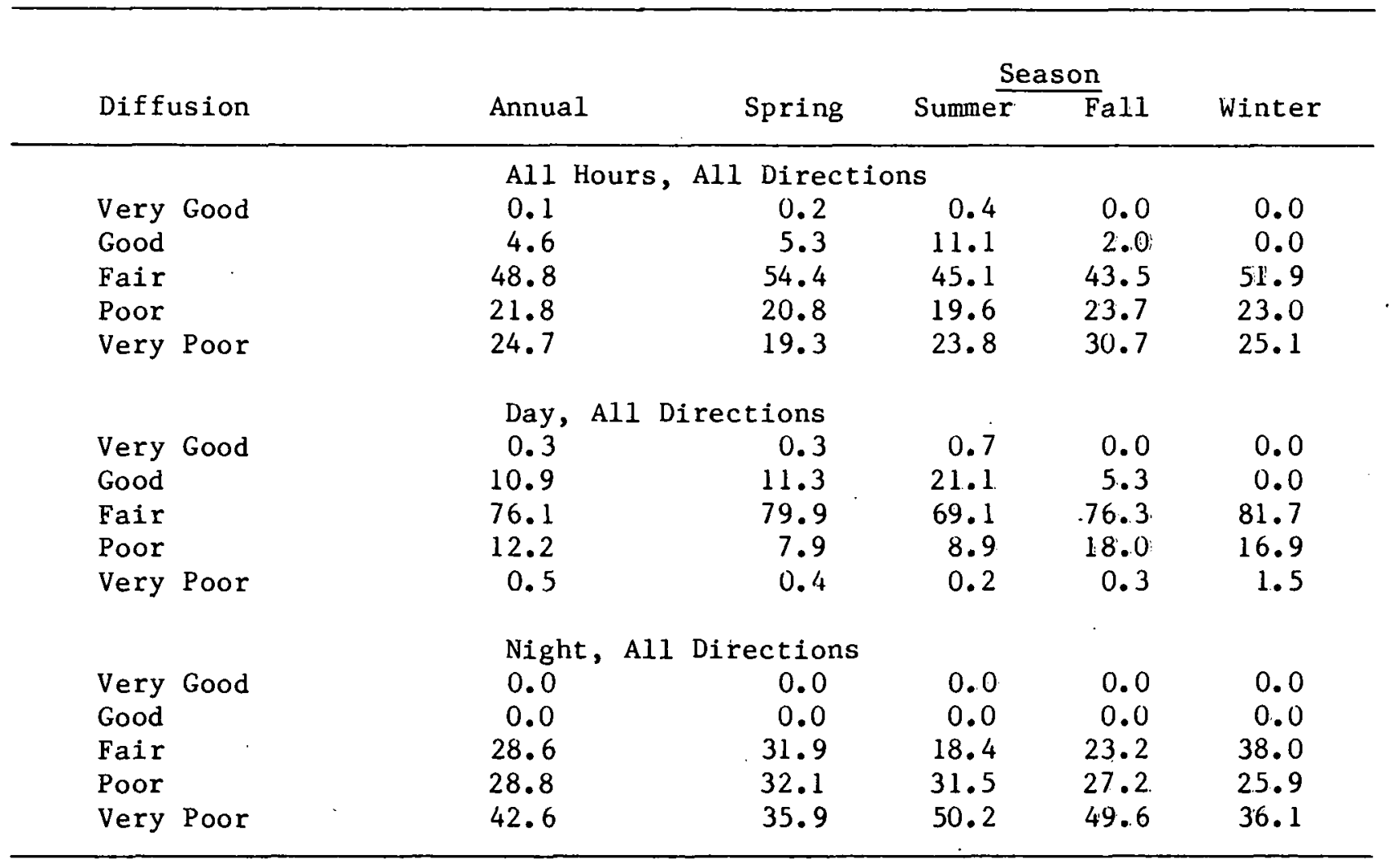


Table 7.3 (continued)

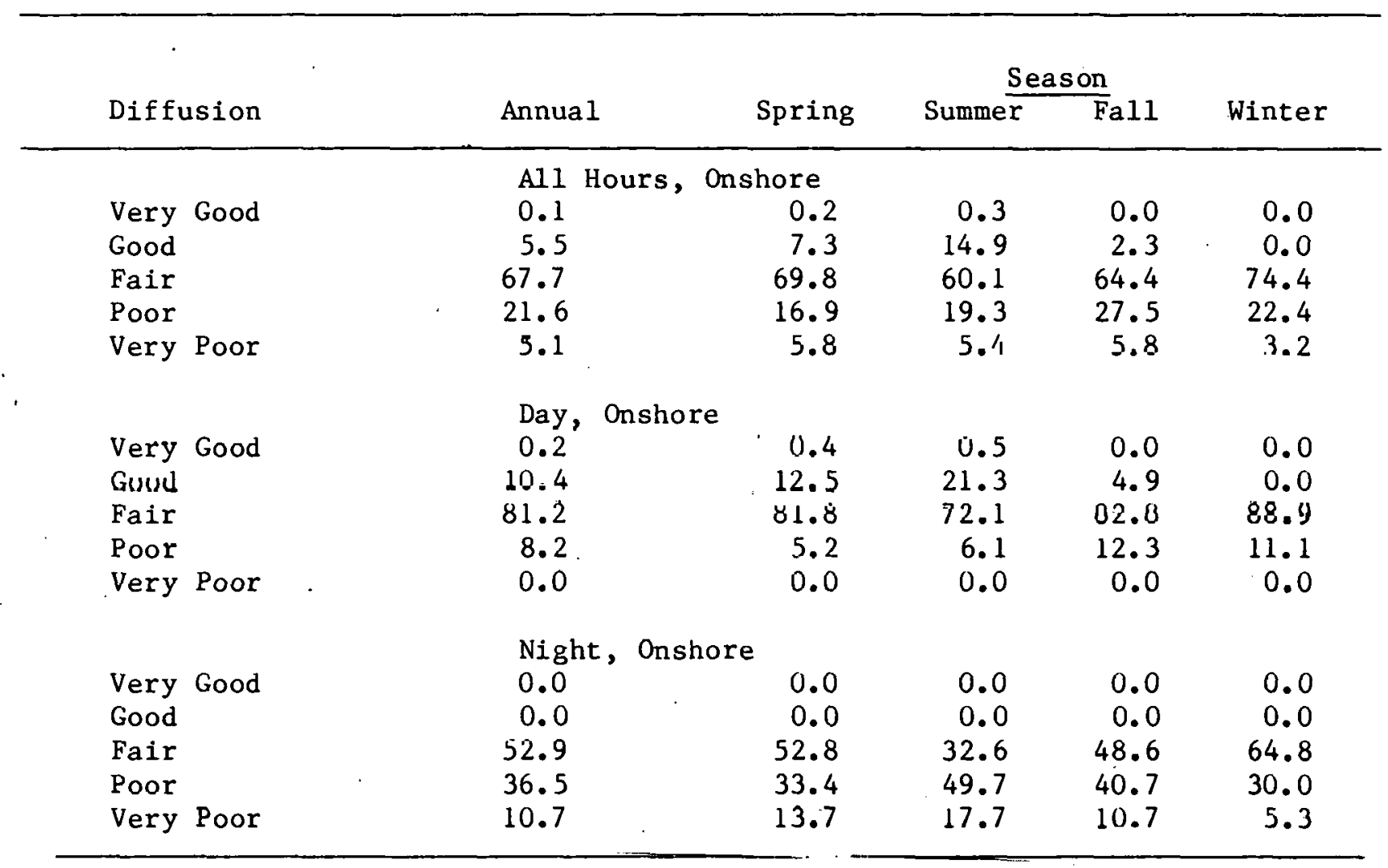


Table 7.3 (continued)

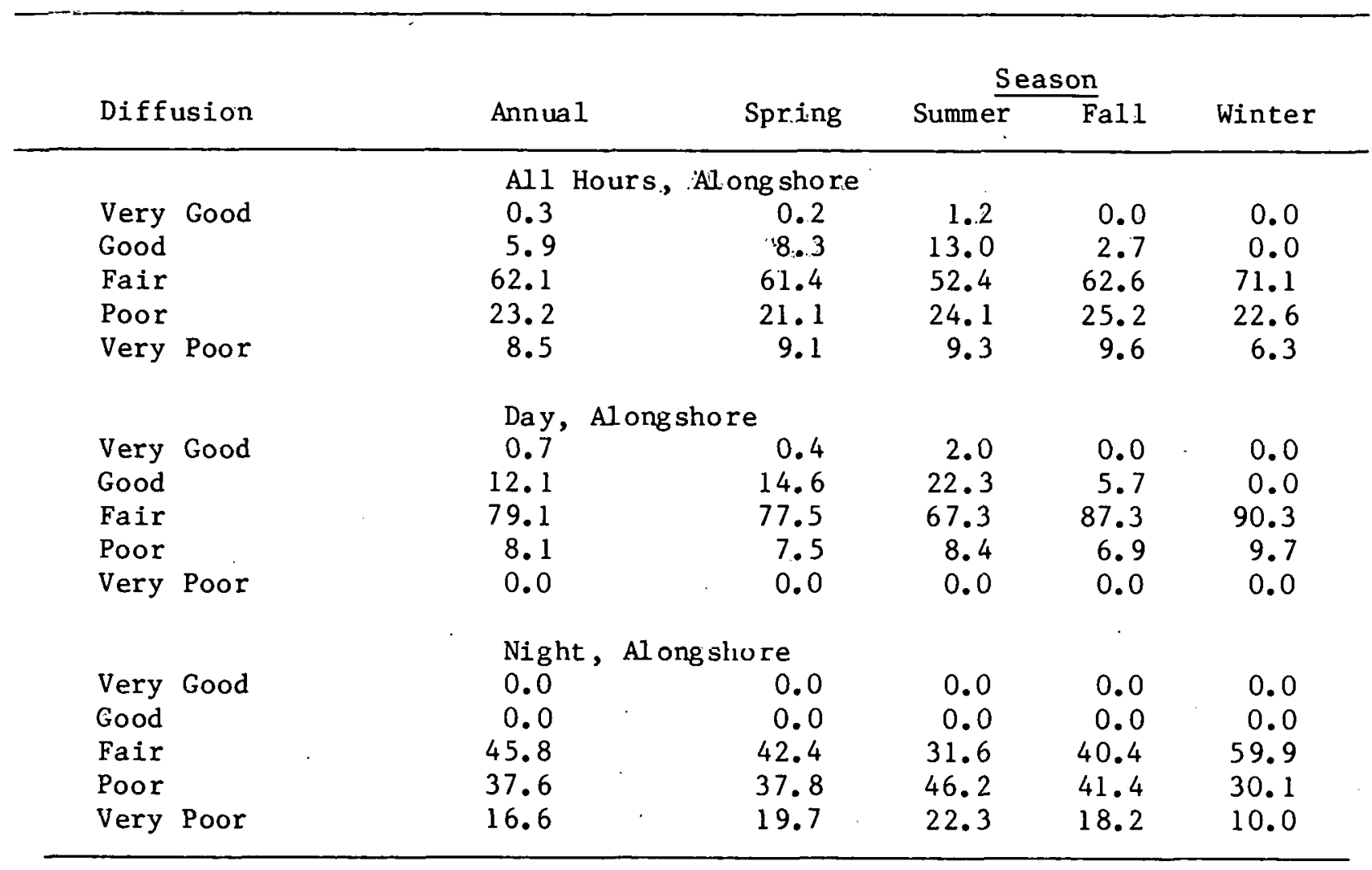


Table 7.3 (continued)

\begin{tabular}{|c|c|c|c|c|c|}
\hline \multirow[b]{2}{*}{ Diffusion } & \multirow[b]{2}{*}{ Annual } & \multirow[b]{2}{*}{ Spring } & \multicolumn{2}{|c|}{ Season } & \multirow[b]{2}{*}{ Winter } \\
\hline & & & Summer & Fall & \\
\hline $\begin{array}{l}\text { Very Good } \\
\text { Good } \\
\text { Fair } \\
\text { Poor } \\
\text { Very Poor }\end{array}$ & $\begin{array}{l}\text { All H } \\
0.1 \\
5.9 \\
57.2 \\
24.0 \\
12.8\end{array}$ & $\begin{array}{r}\text { Hours, Off shore } \\
0.2 \\
5.2 \\
60.8 \\
24.2 \\
9.7\end{array}$ & $\begin{array}{r}0.3 \\
13.1 \\
52.0 \\
19.7 \\
14.9\end{array}$ & $\begin{array}{r}0.0 \\
3.2 \\
56.3 \\
26.6 \\
13.9\end{array}$ & $\begin{array}{r}0.0 \\
0.0 \\
60.4 \\
26.9 \\
12.7\end{array}$ \\
\hline $\begin{array}{l}\text { Very Good } \\
\text { Good } \\
\text { Fair } \\
\text { Poor } \\
\text { Very Poor }\end{array}$ & $\begin{array}{r}\text { Ilay, } \\
0.3 \\
12.5 \\
83.8 \\
\quad 3.4 \\
0.0\end{array}$ & $\begin{array}{r}\text { Uffsliuse } \\
0.4 \\
10.7 \\
86.9 \\
2.1 \\
0.0\end{array}$ & $\begin{array}{r}0.3 \\
22.8 \\
73.9 \\
2.9 \\
0.0\end{array}$ & $\begin{array}{r}0.0 \\
7.0 \\
88.4 \\
4.6 \\
0.0\end{array}$ & $\begin{array}{r}0.0 \\
U . U \\
95.1 \\
4.9 \\
0.0\end{array}$ \\
\hline $\begin{array}{l}\text { Very Good } \\
\text { Good } \\
\text { Fair } \\
\text { Poor } \\
\text { Very Poor }\end{array}$ & $\begin{array}{l}\text { Night } \\
0.0 \\
0.0 \\
33.7 \\
42.3 \\
24.0\end{array}$ & $\begin{array}{r}\text {, Offshore } \\
\\
0.0 \\
0.0 \\
36.8 \\
44.6 \\
18.6\end{array}$ & $\begin{array}{r}0.0 \\
0.0 \\
22.6 \\
42.4 \\
35.0\end{array}$ & $\begin{array}{r}0.0 \\
0.0 \\
28.6 \\
45.4 \\
26.0\end{array}$ & $\begin{array}{r}0.0 \\
0.0 \\
43.0 \\
38.0 \\
19.0\end{array}$ \\
\hline
\end{tabular}




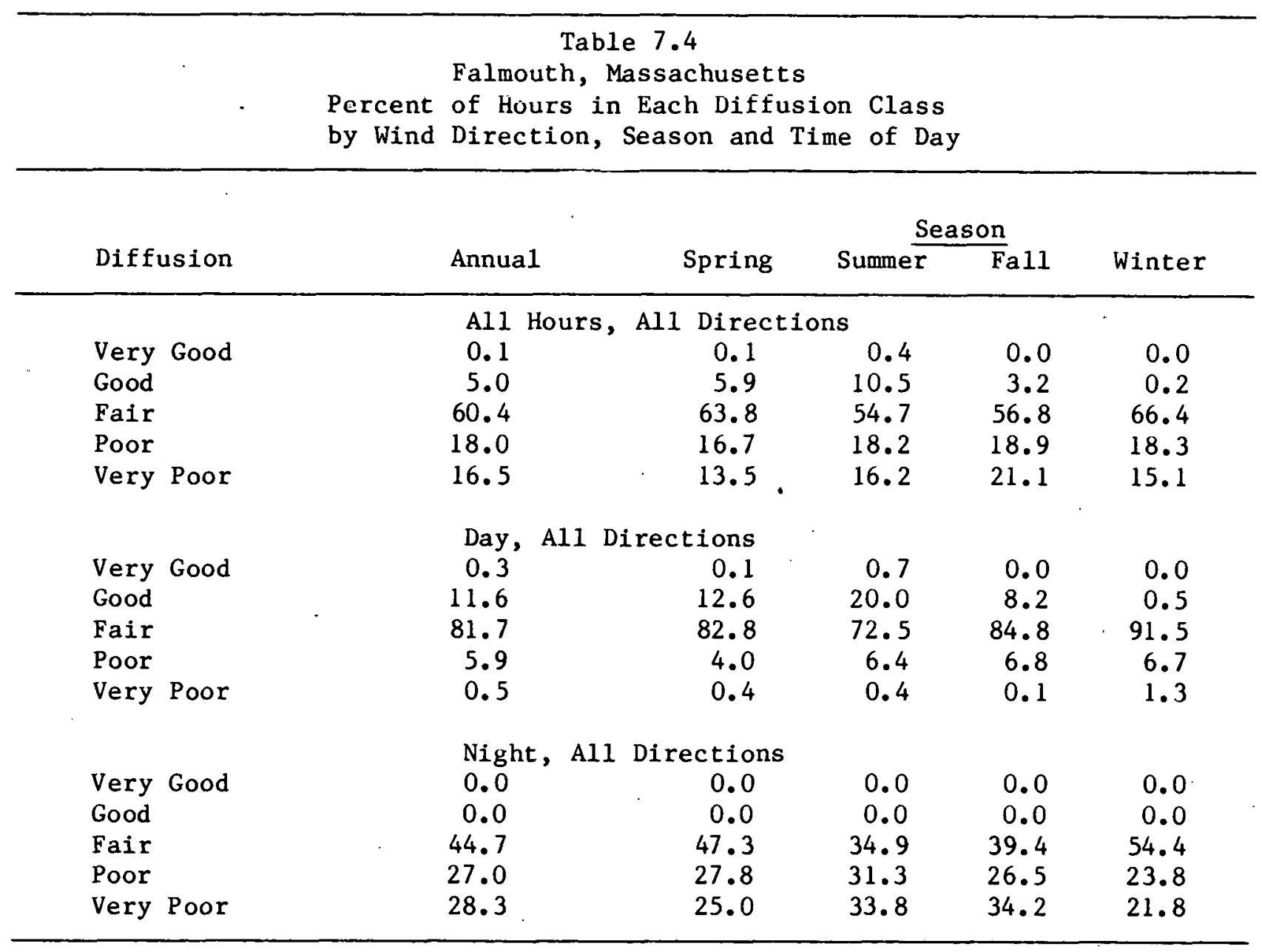


Table 7.4 (continued)

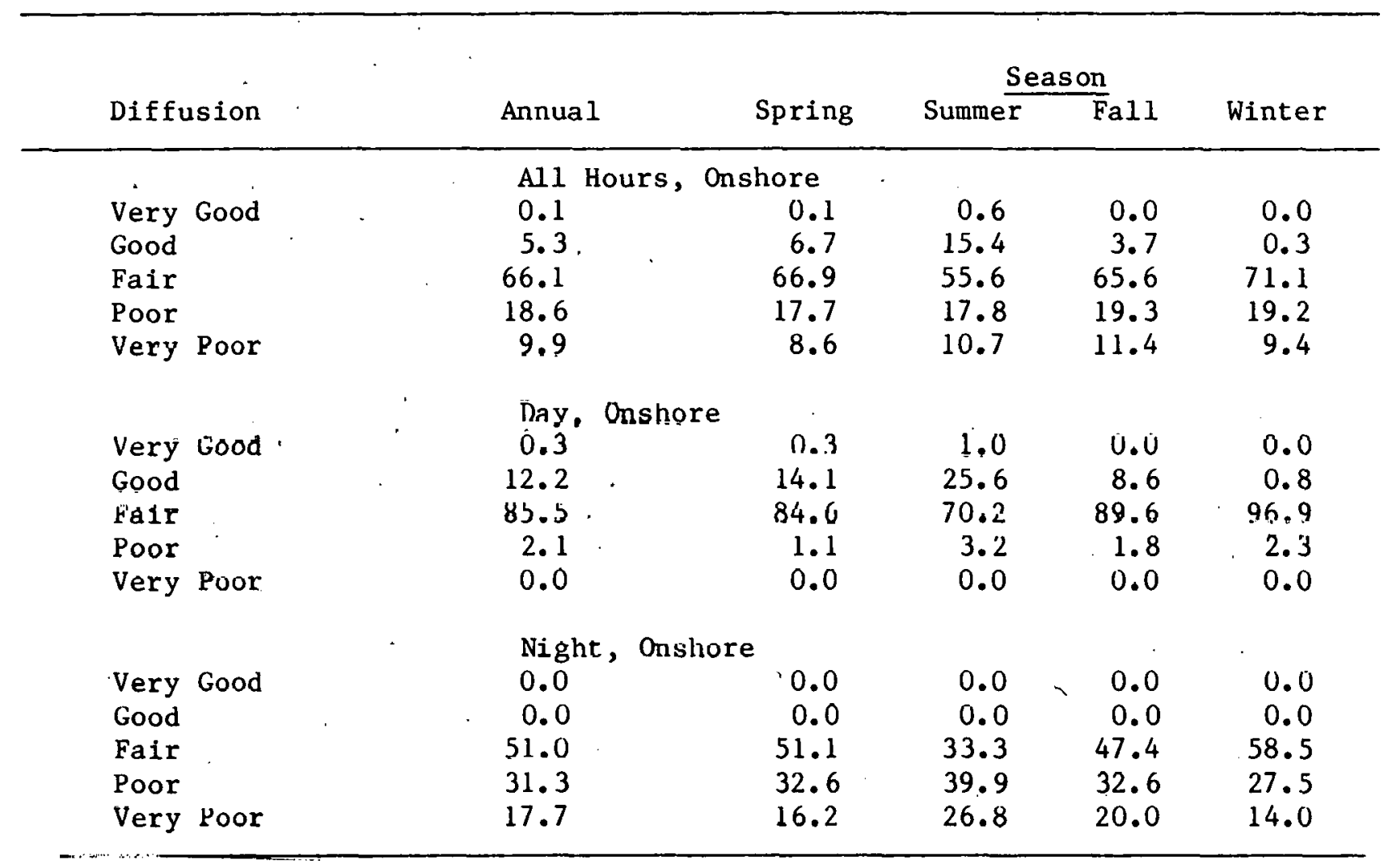


-Table 7.4 (continued)

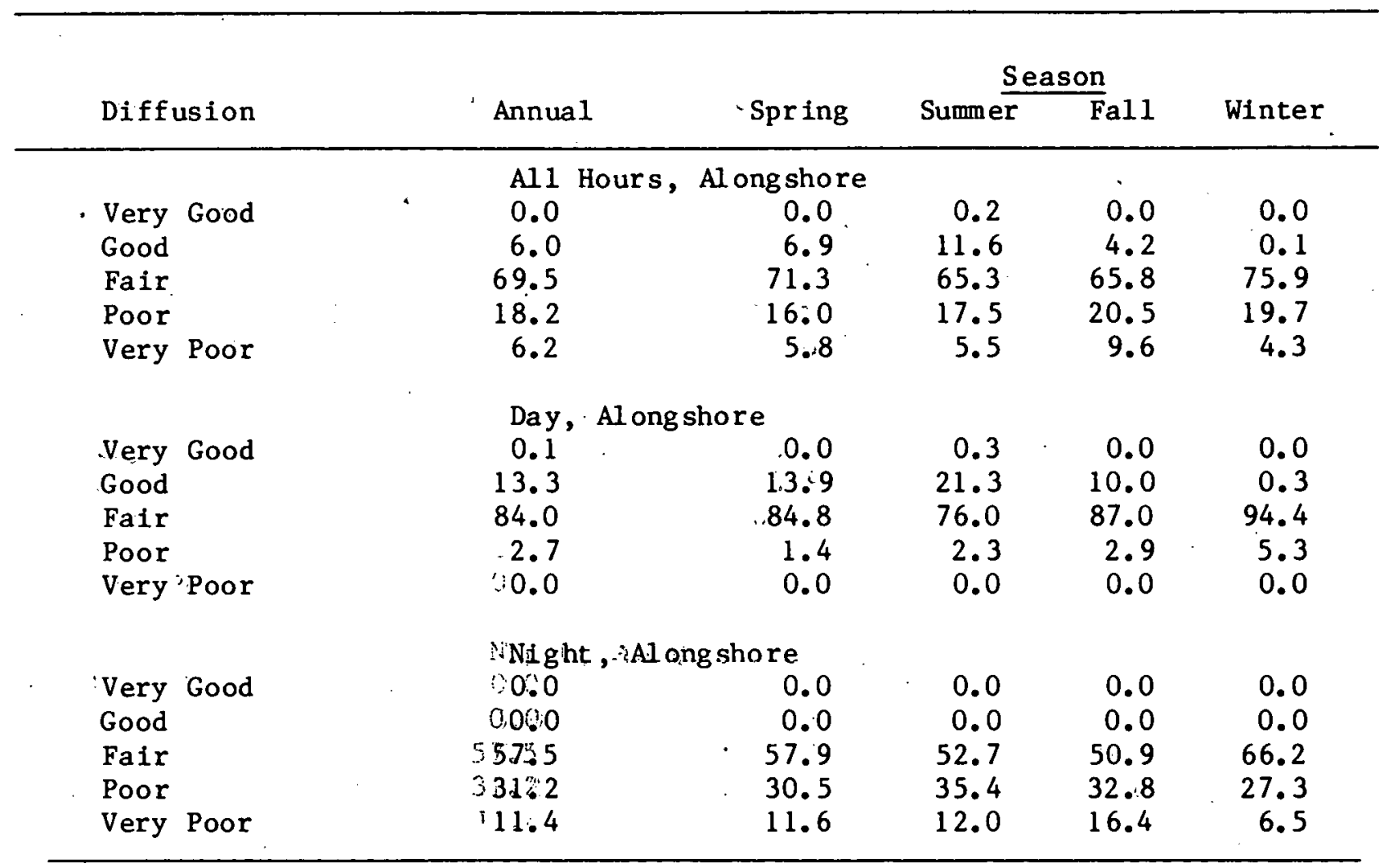


Table 7.4 (continued)

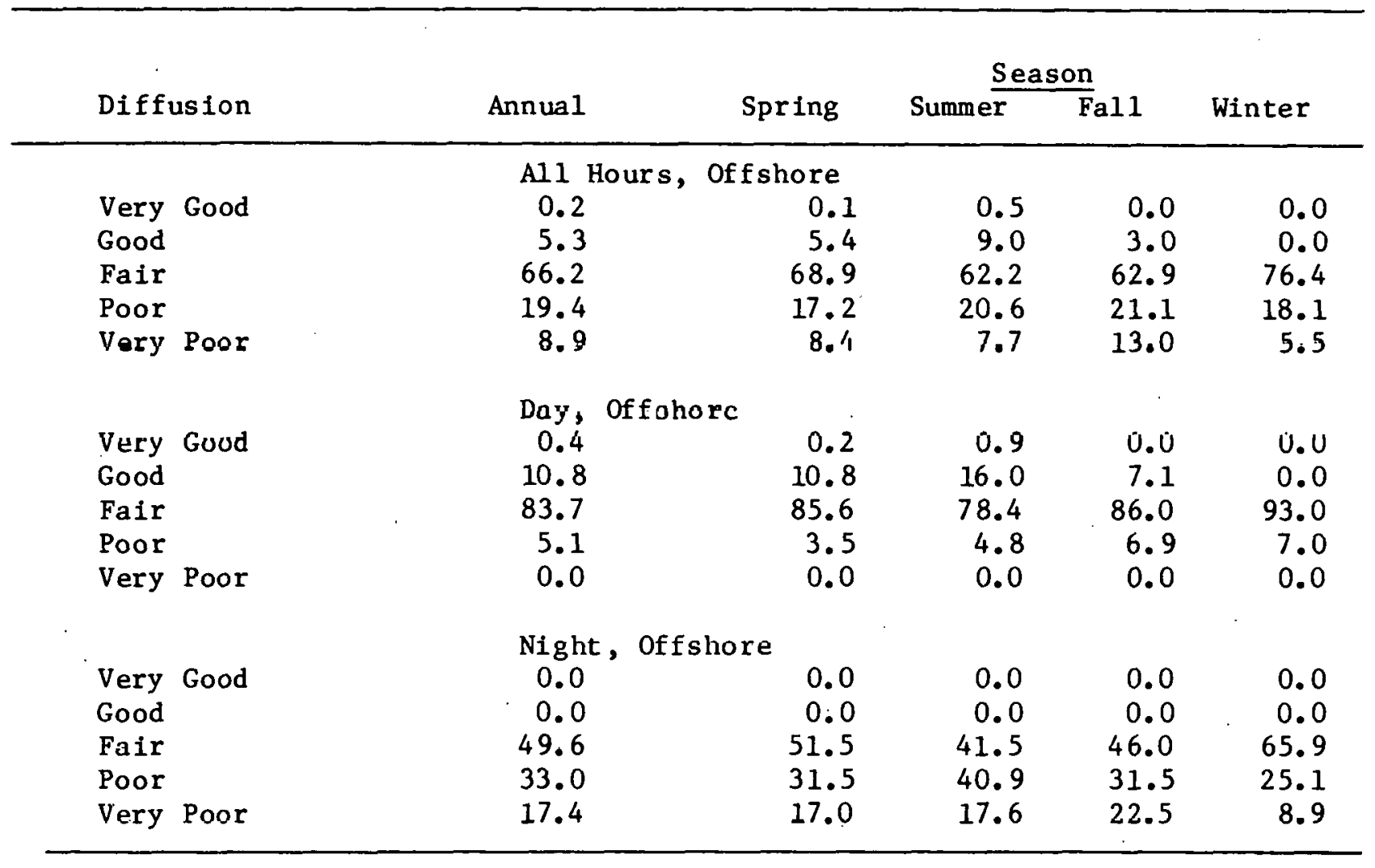


Table 7.5

Nantucket, Massachusetts

Percent of Hours in Each Diffusion Class

by Wind Direction; Season and Time of Day

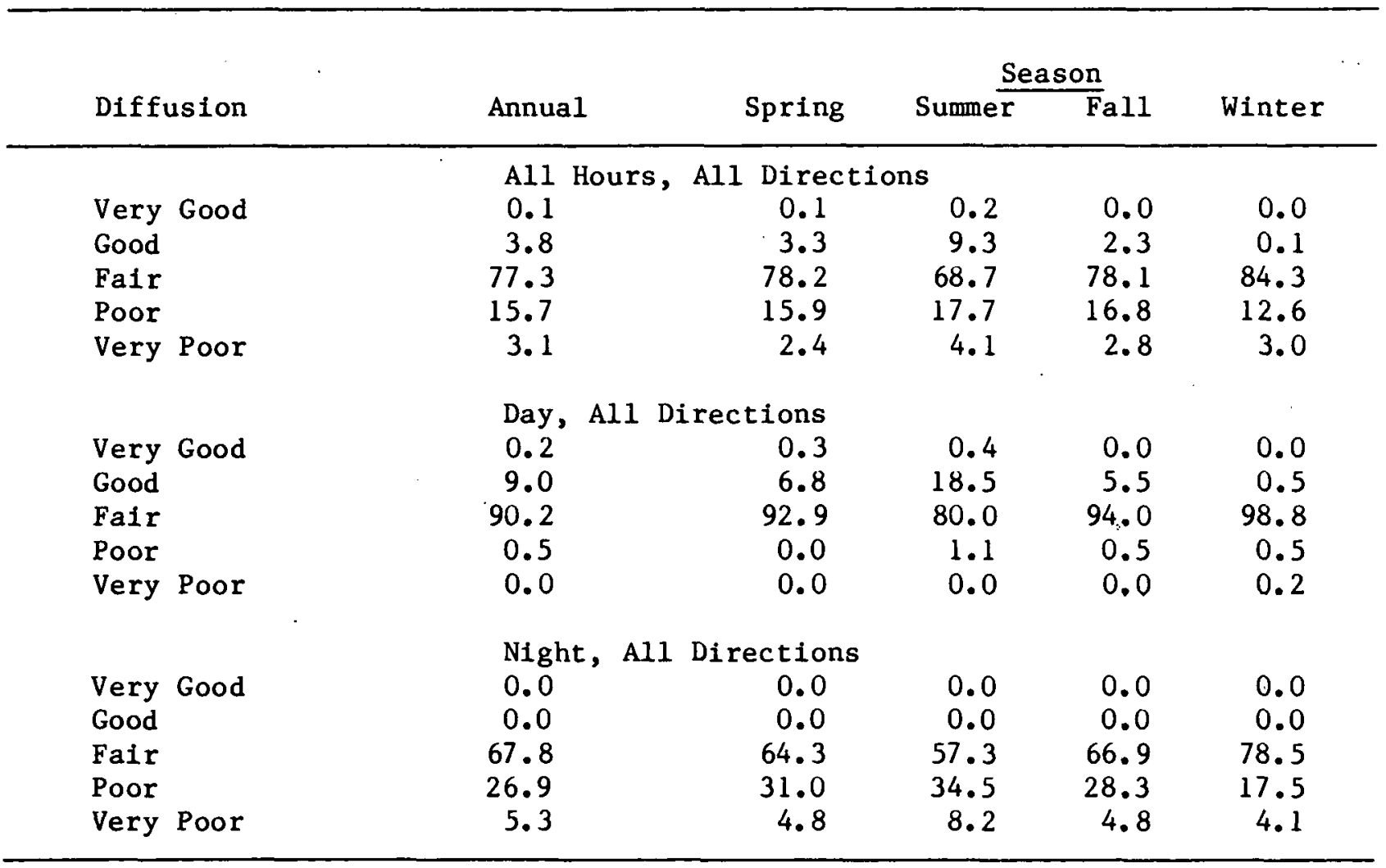


Table 7\%.5: (icontinued):

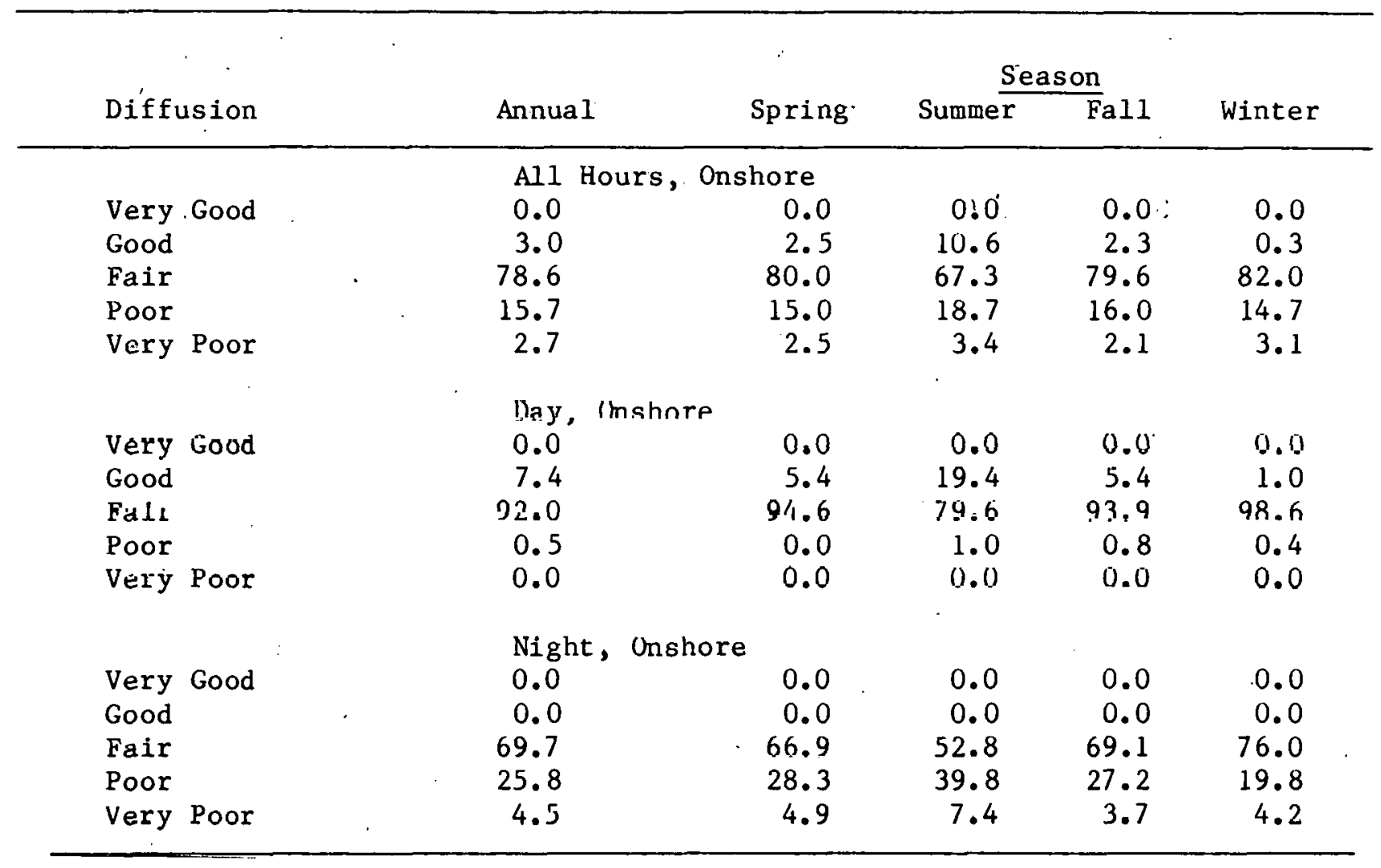


Table 7.5 (continued)

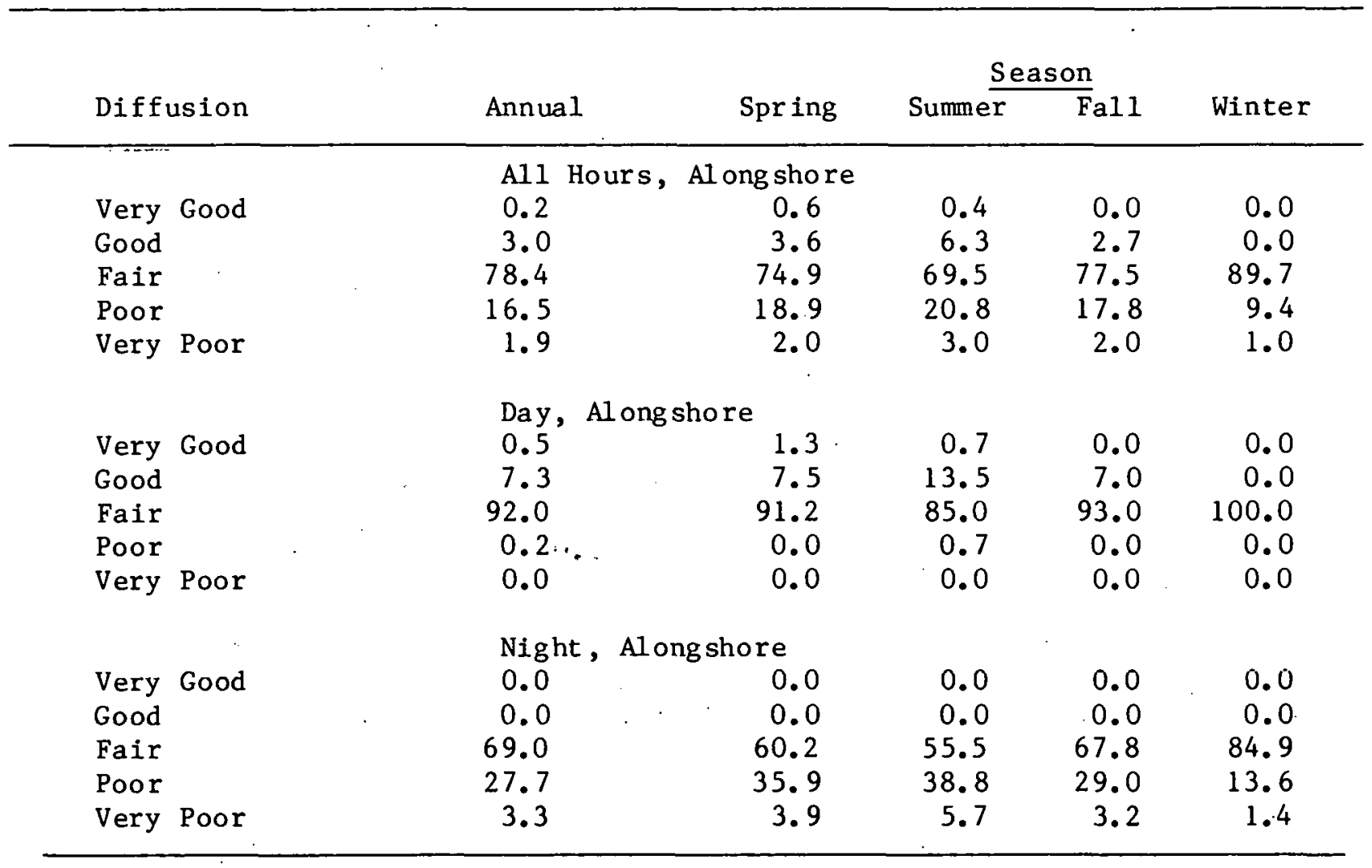


Table 7.5 (continued)

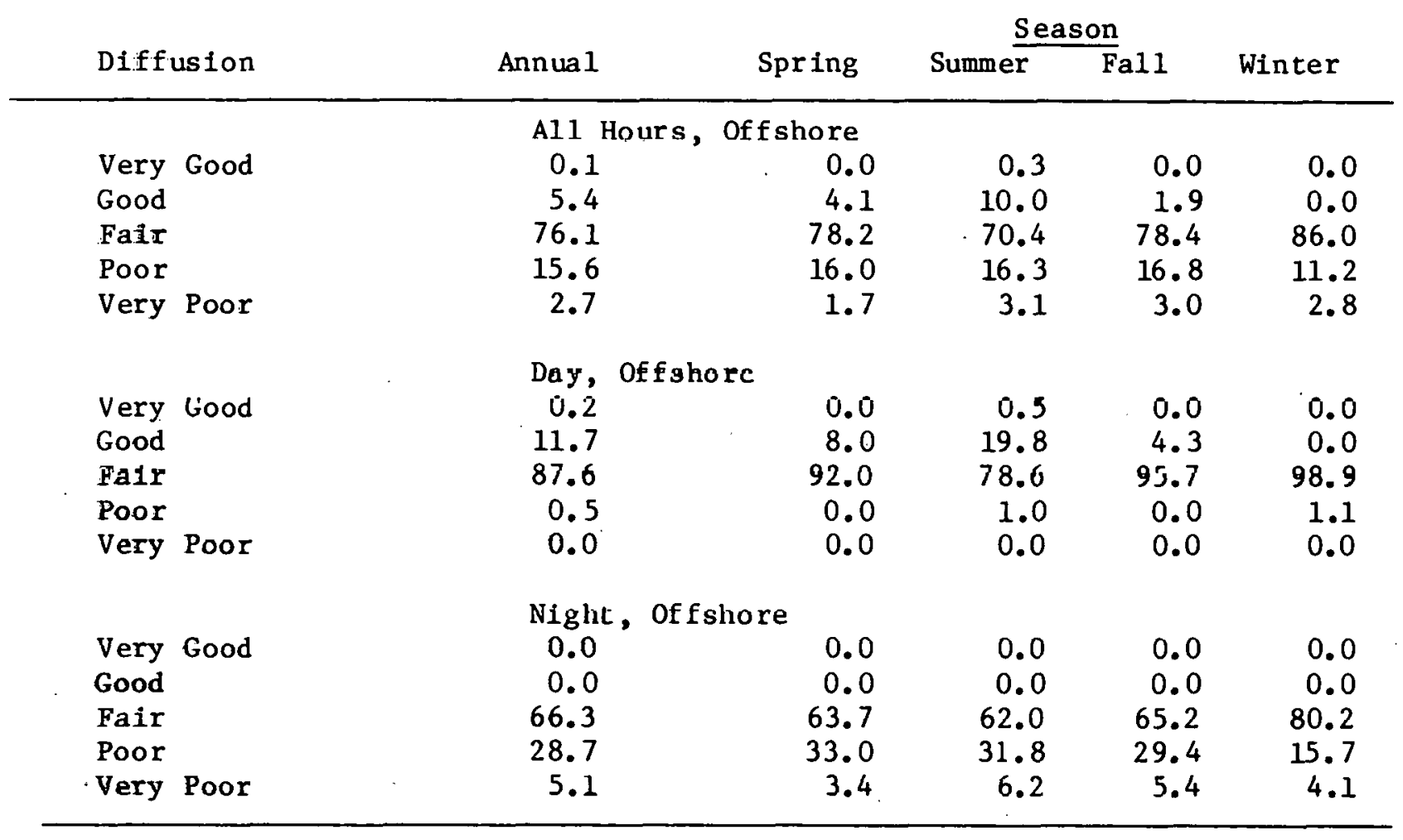




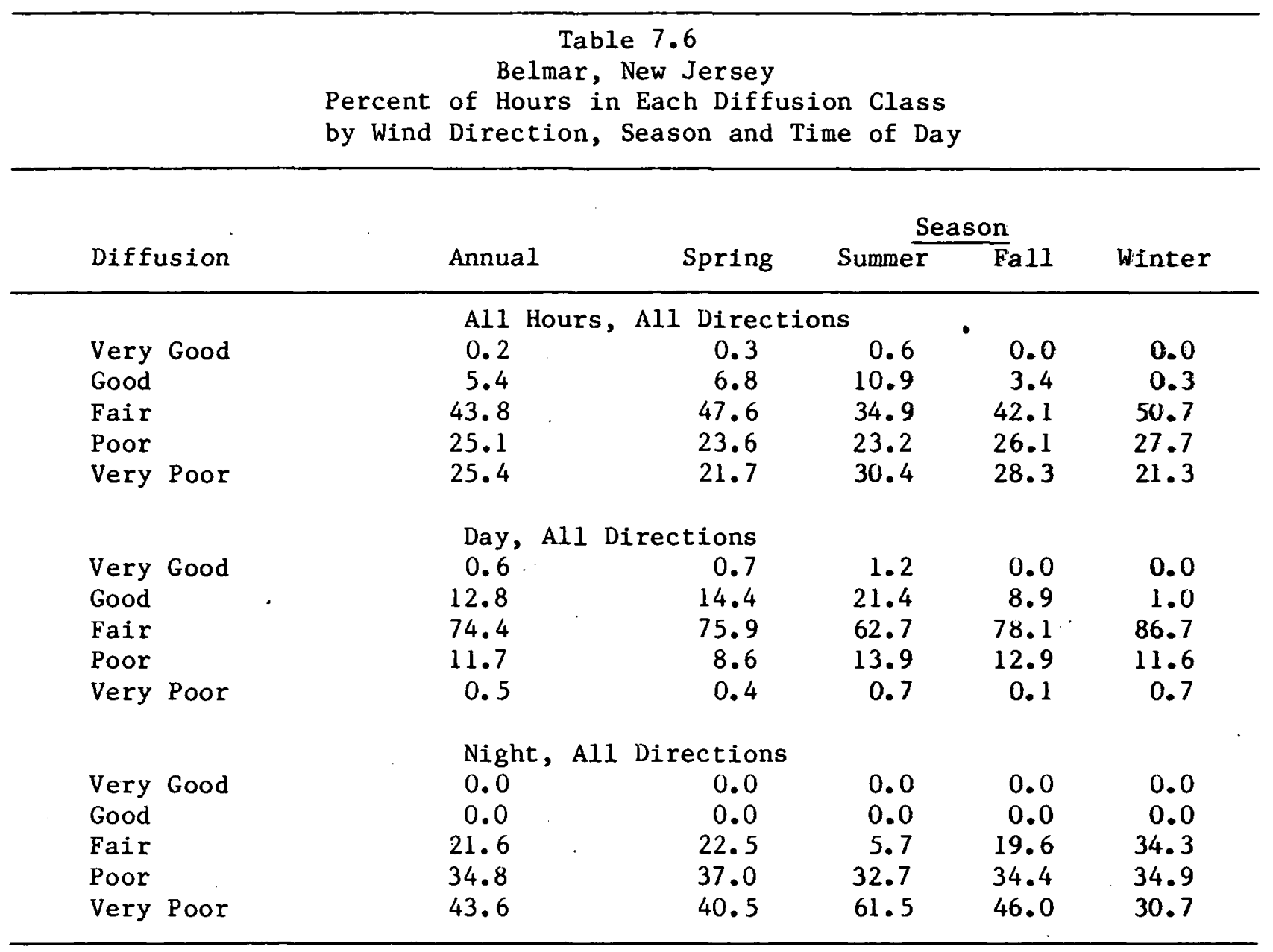


Table 7.6 (continued)

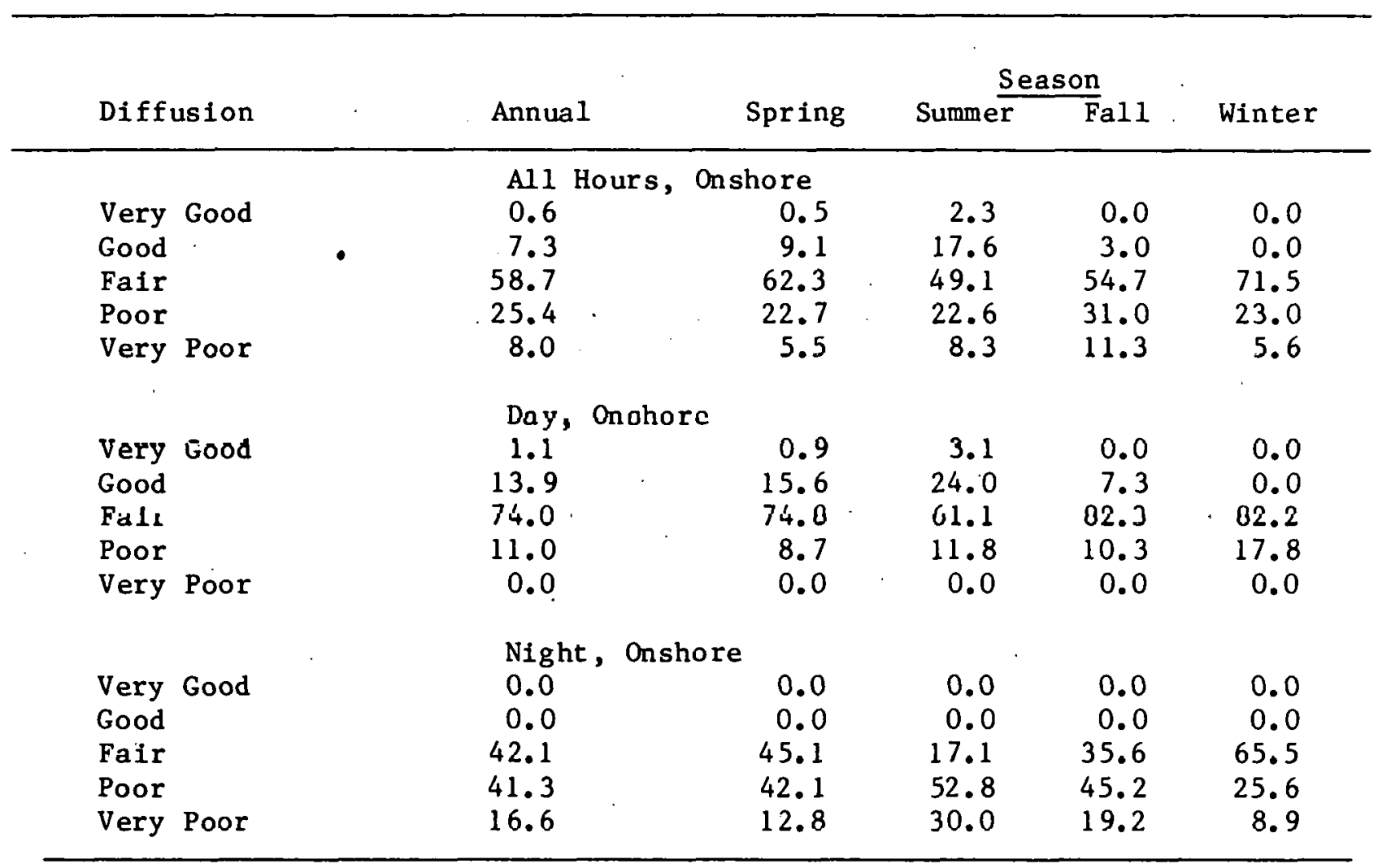


Table 7.6 (continued)

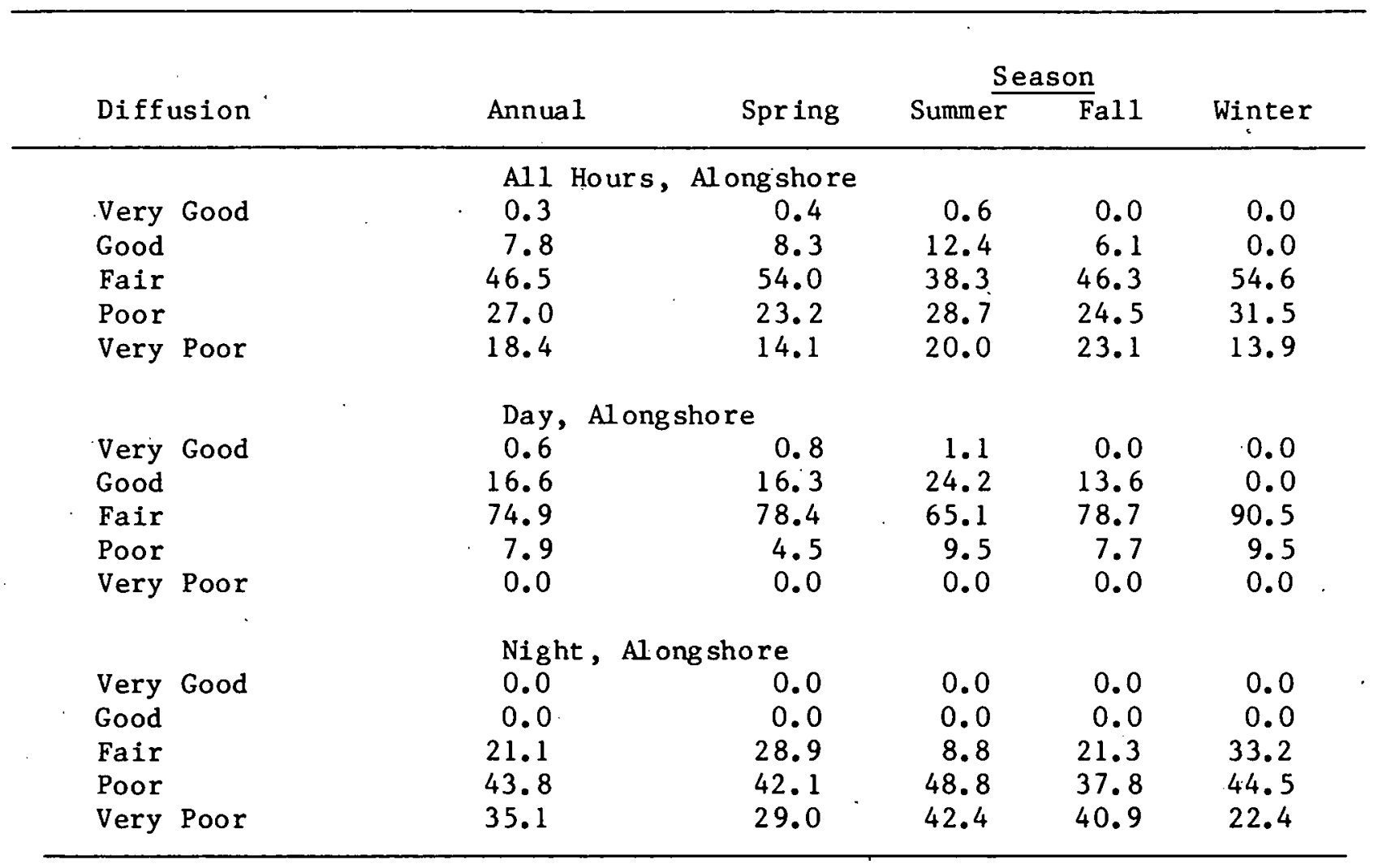


Table 7.6 (continued)

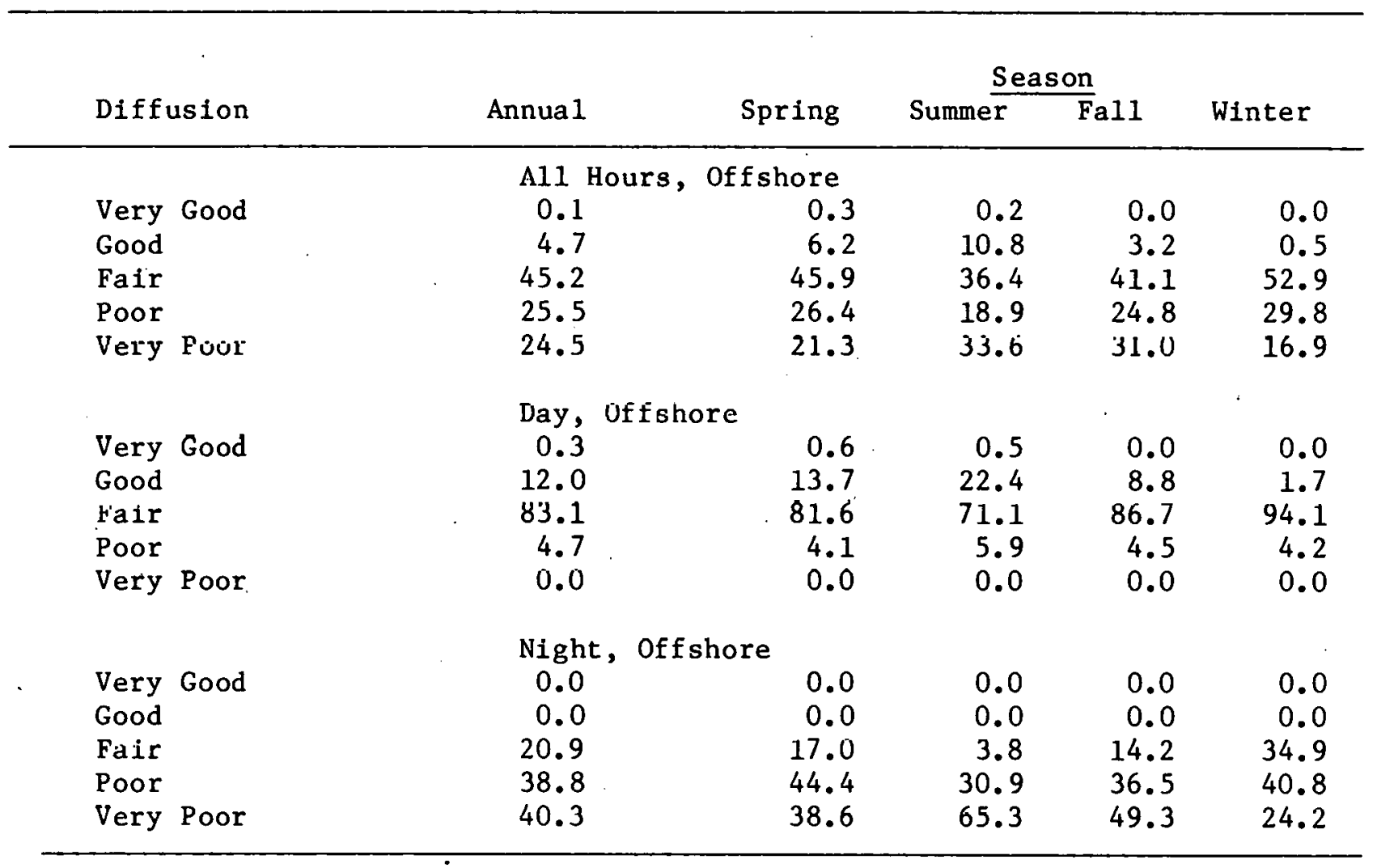


Table 7.7

Lakehurst, New Jersey

Percent of Hours in Each Diffusion Class

by Wind Direction, Season and Time of Day

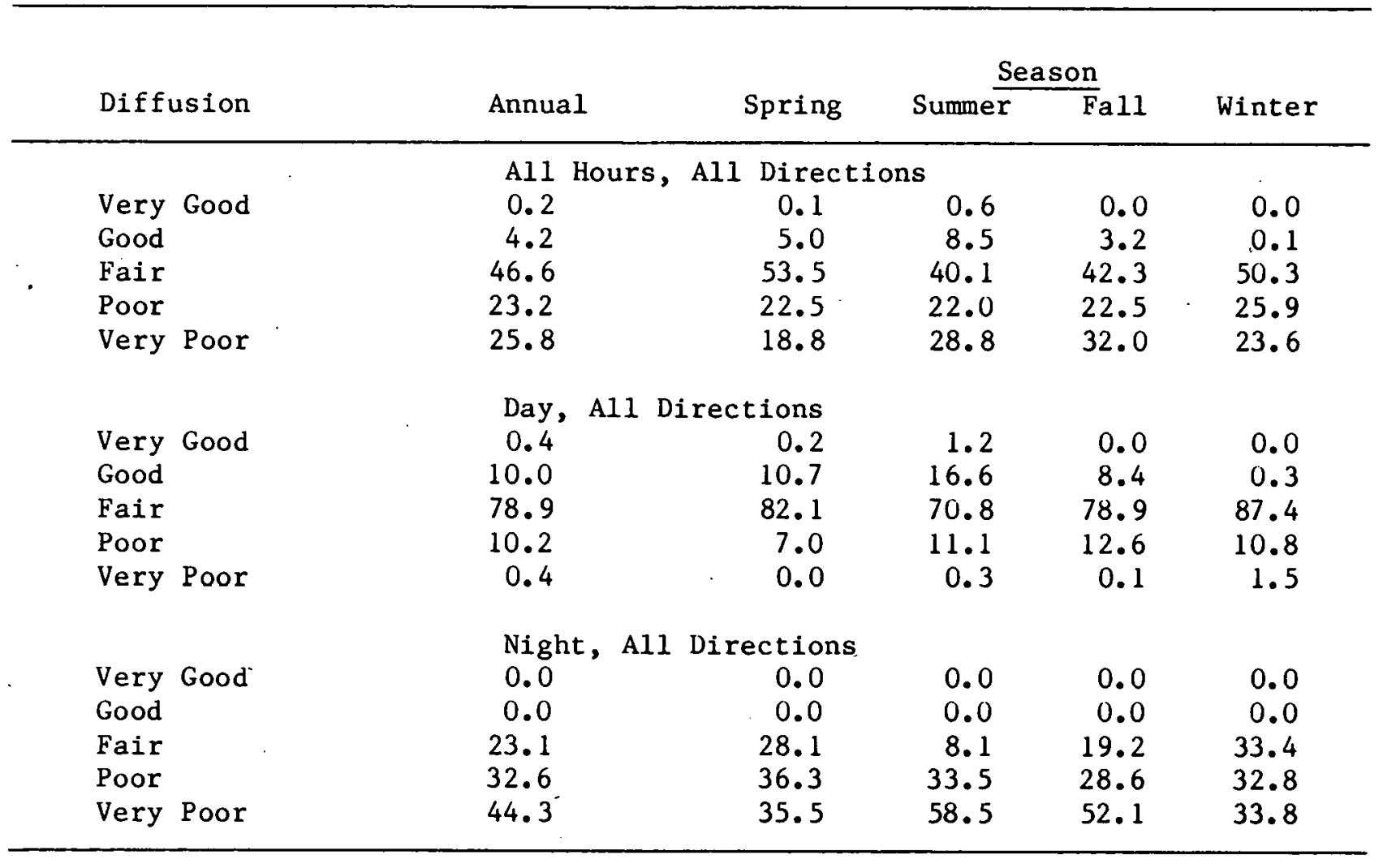


Table 7.7 (continued)

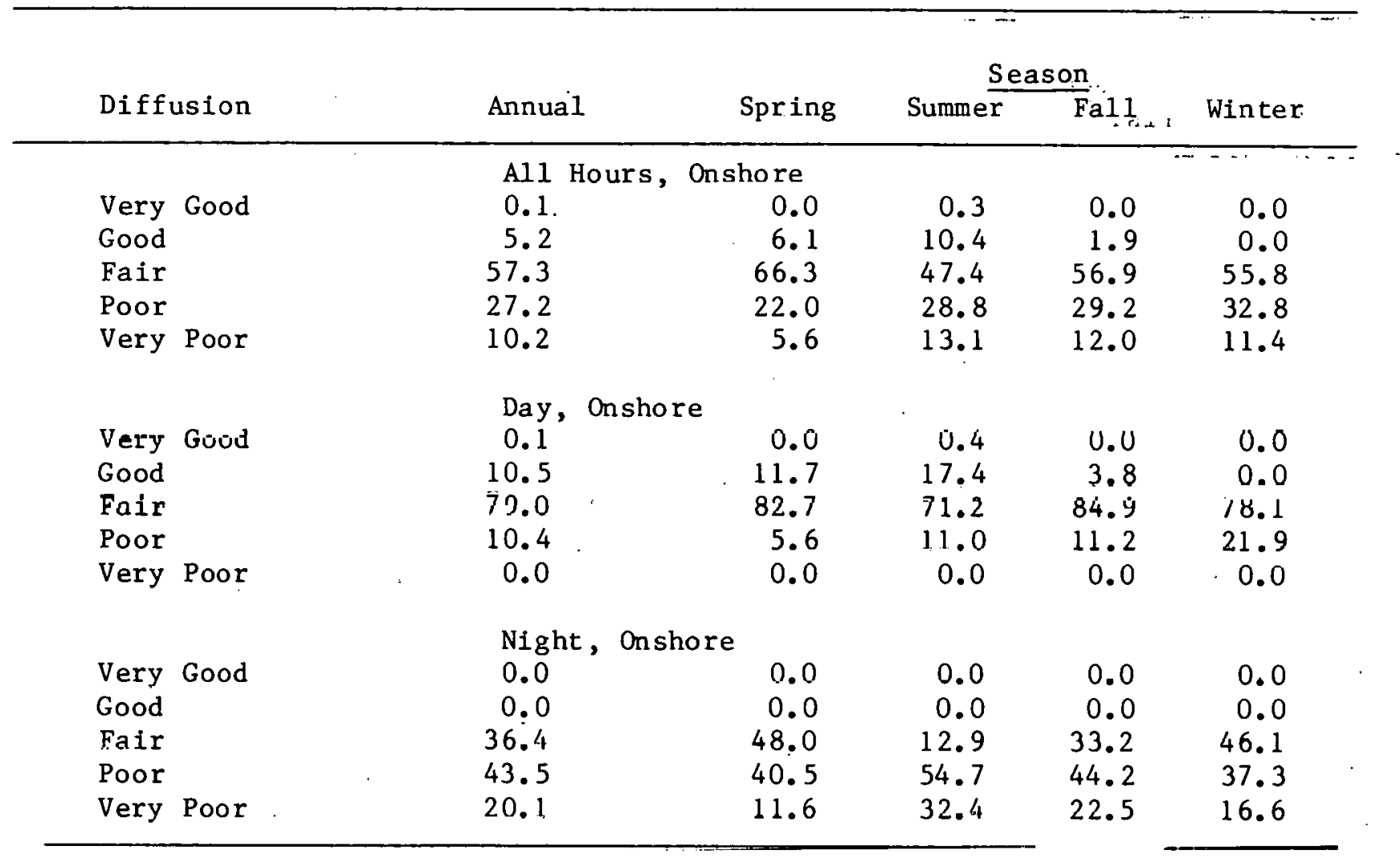


Table 7.7 (continued)

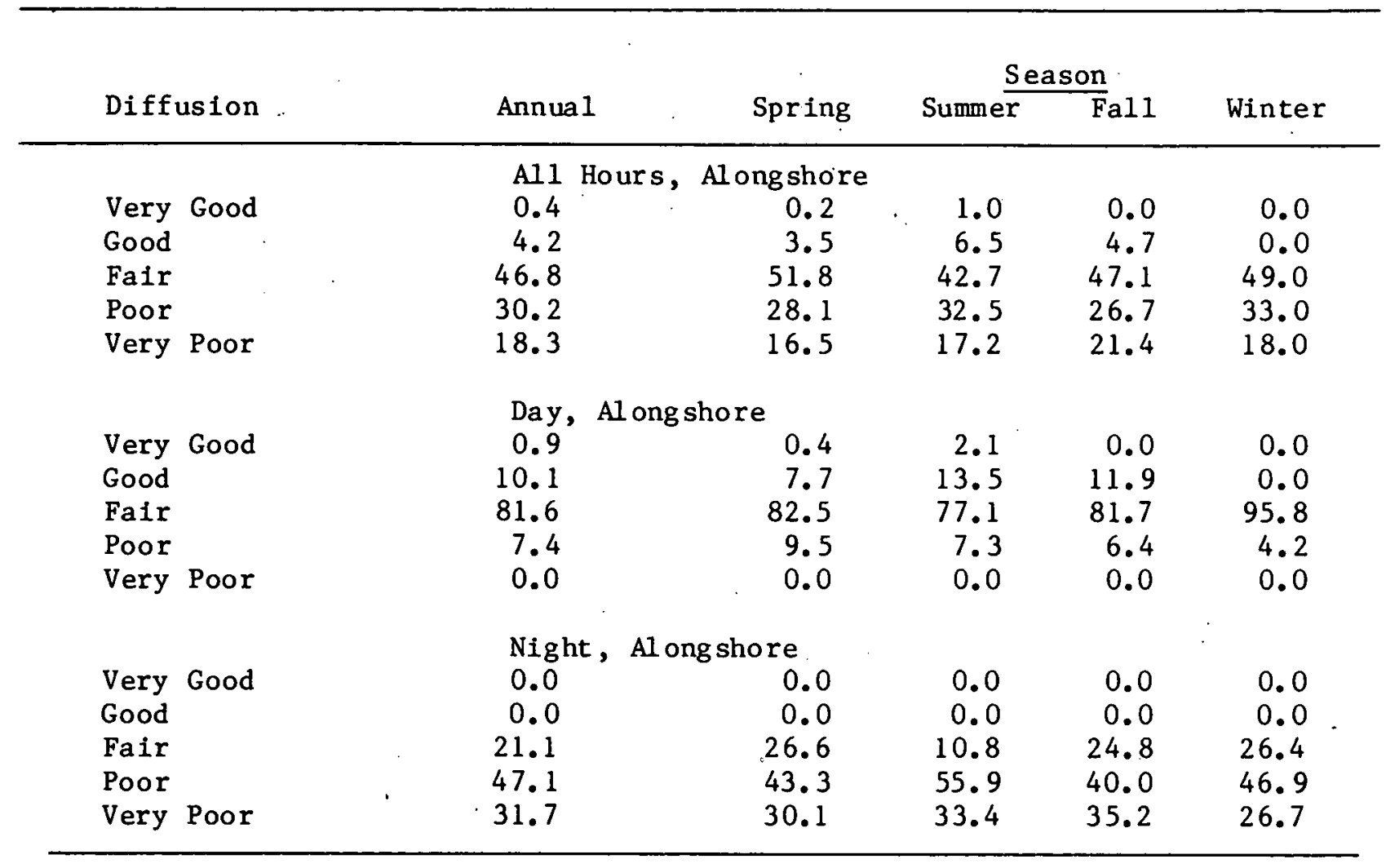


Table 7.7 (continued)

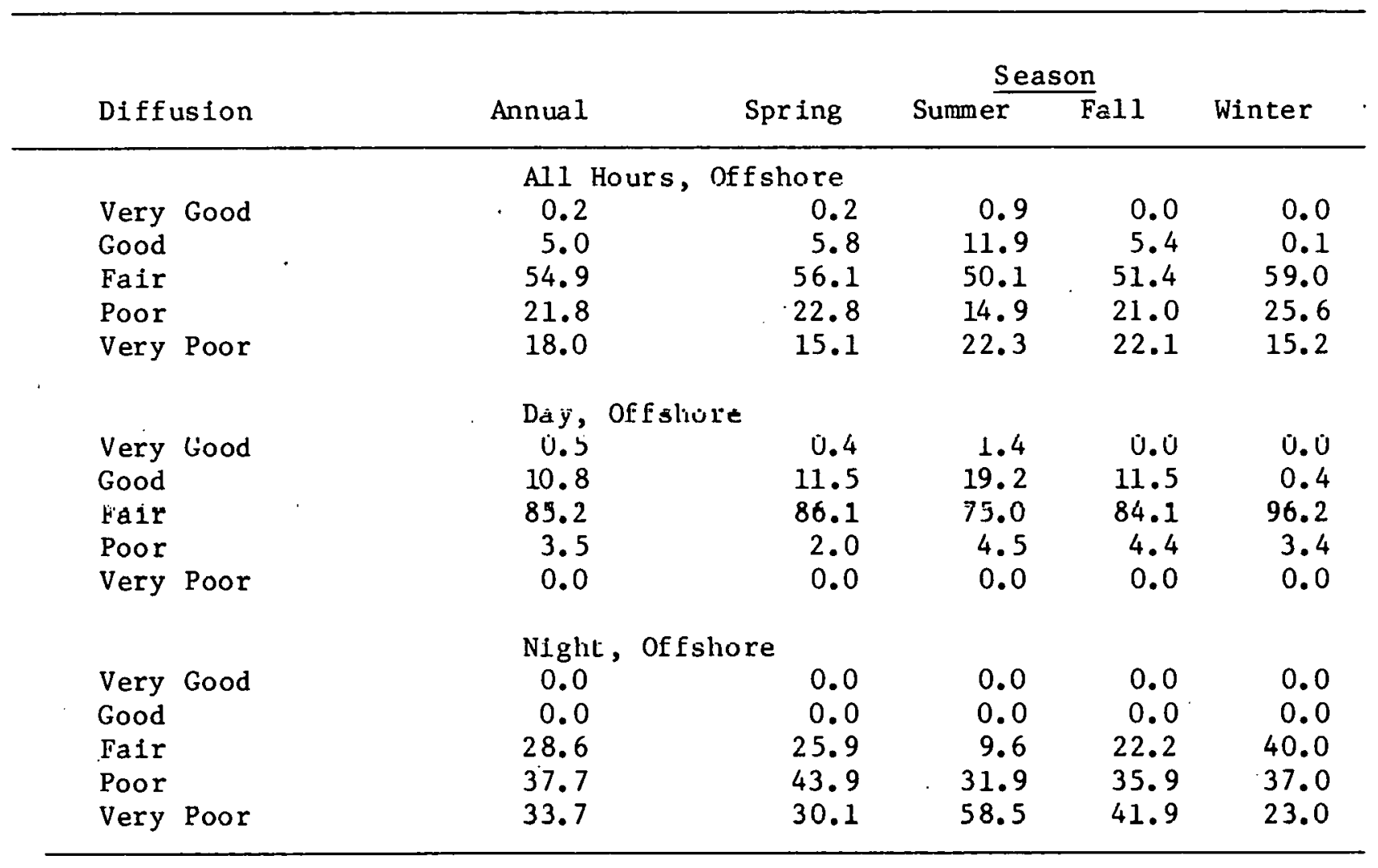


Table 7.8

$\Lambda$ tlantic City, New Jersey

Percent of Hours in Each Diffusion Class.

by Wind Direction, Season and Time of Day

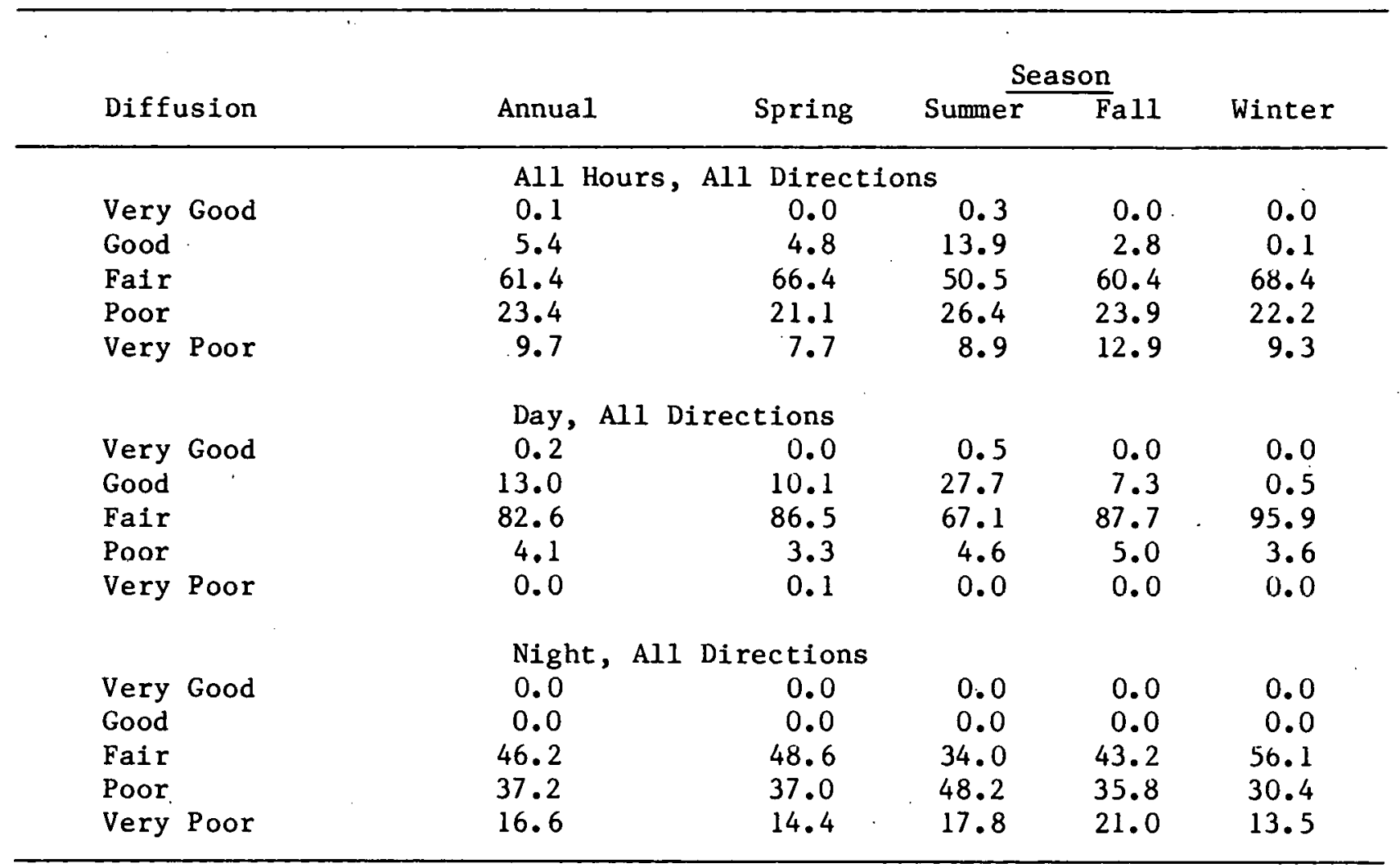


Table 7.8 (continued)

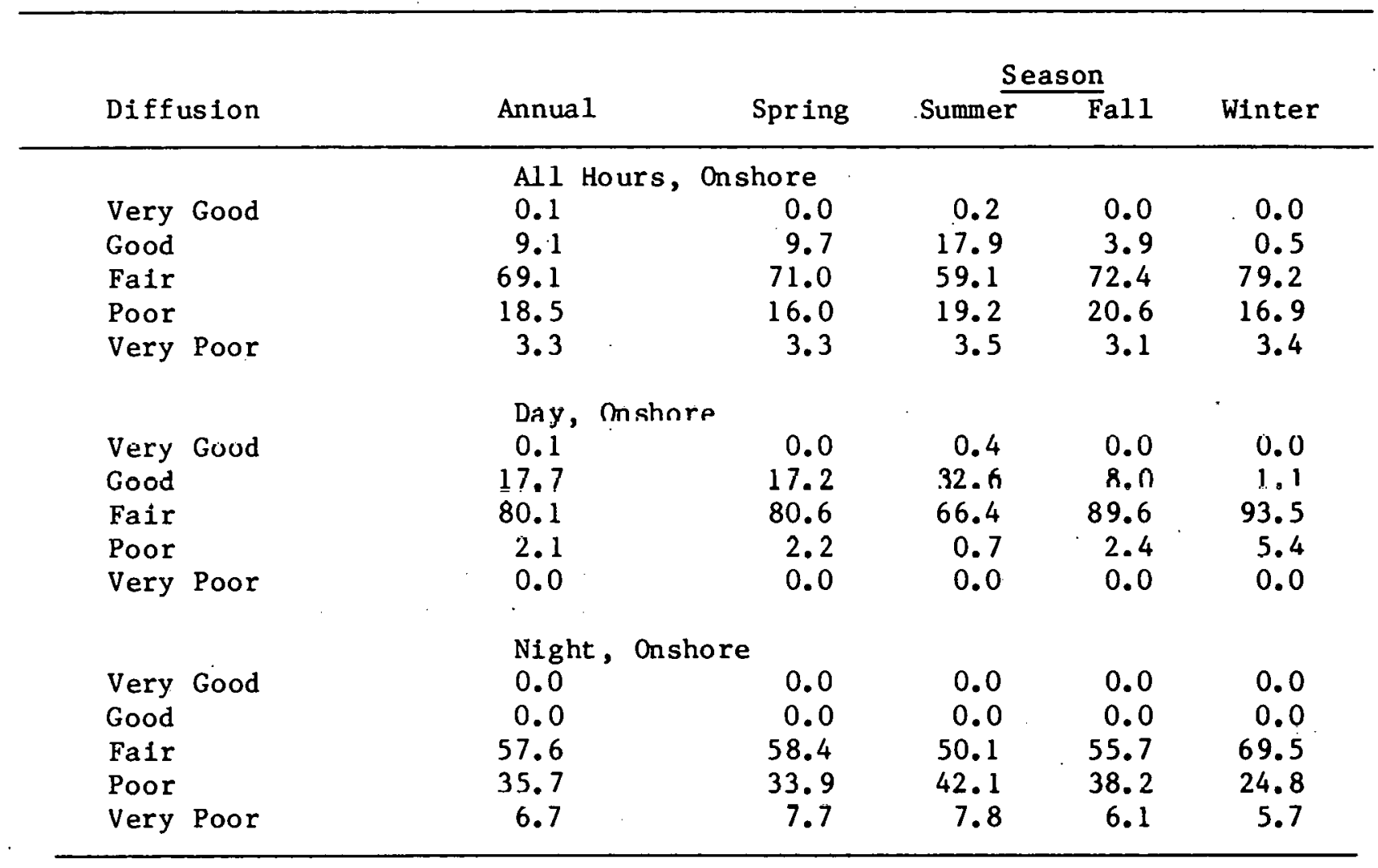


Table 7.8 (continued)

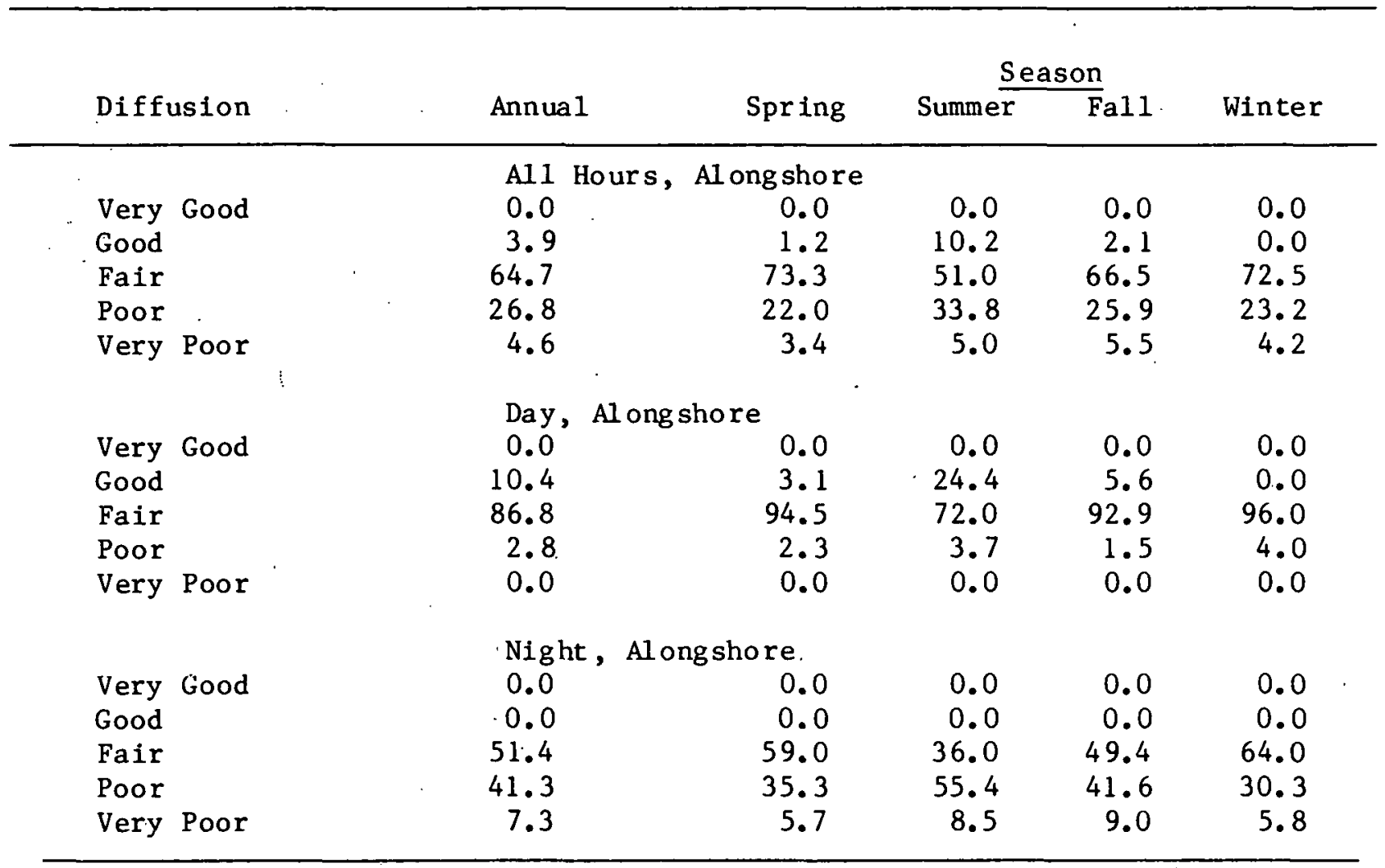


Table 7.8 (continued)

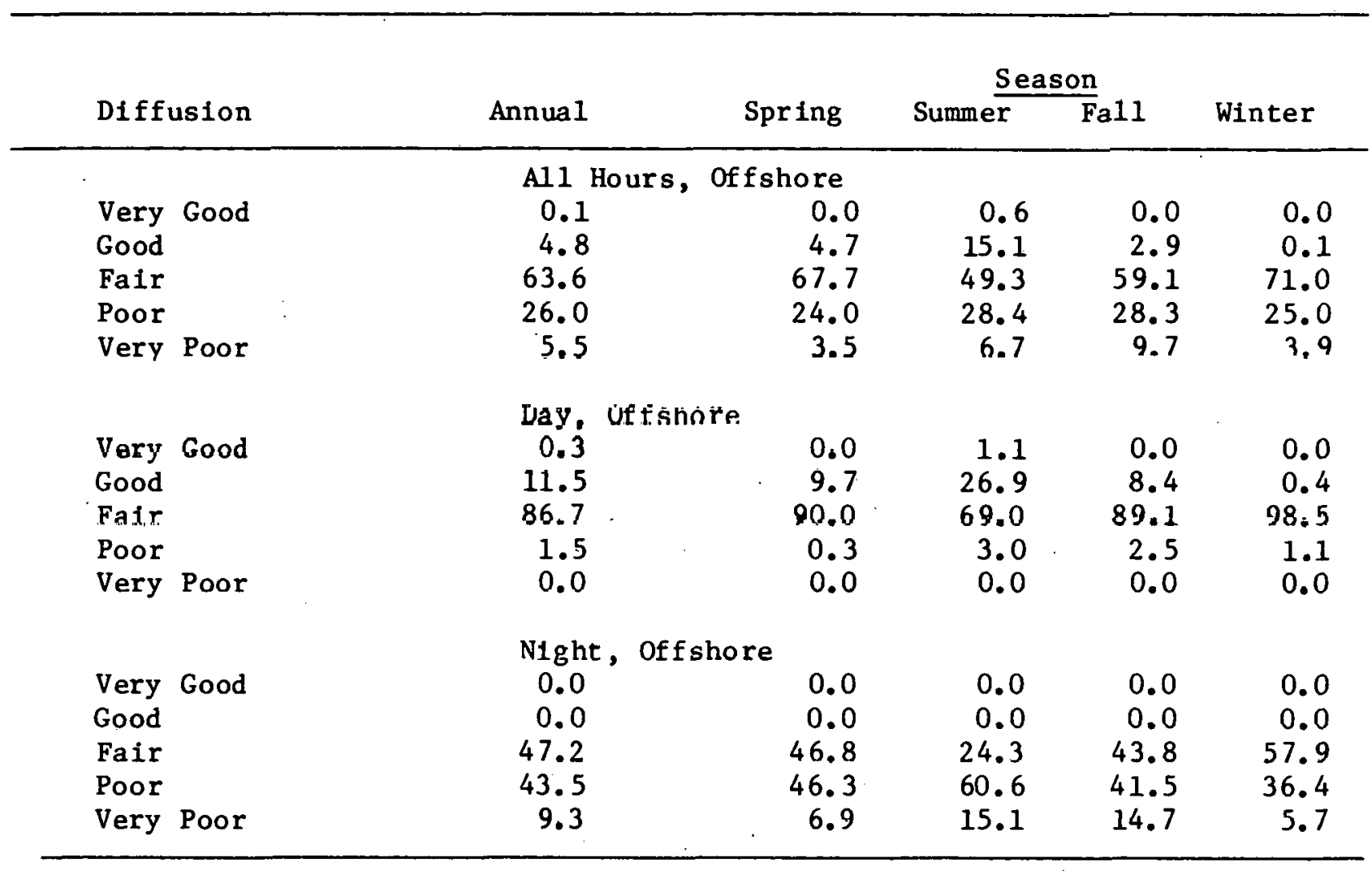


Table 7.9

Wilmington, Delaware

Percent of Hours in Each Diffusion Class

by Wind Direction, Season and Time of Day

\begin{tabular}{lccrrr}
\hline & \multicolumn{5}{c}{ Season } \\
Diffusion & Annual & Spring & Sumer & Fall & Winter \\
\hline & All Hours, All Directions & & \\
Very Good & 0.1 & 0.1 & 0.3 & 0.0 & 0.0 \\
Good & 5.4 & 4.8 & 13.5 & 3.2 & 0.1 \\
Fair & 55.8 & 64.2 & 44.4 & 54.0 & 60.6 \\
Poor & 26.7 & 22.4 & 27.6 & 28.0 & 29.1 \\
Very Poor & 12.0 & 8.6 & 14.2 & 14.9 & 10.1 \\
& & & & & \\
Very Good & Day, All Directions & & & \\
Good & 0.2 & 0.1 & 0.7 & 0.0 & 0.0 \\
Fair & 13.0 & 10.2 & 26.9 & 8.2 & 0.4 \\
Poor & 80.8 & 85.8 & 66.2 & 84.3 & 92.9 \\
Very Poor & 5.6 & 3.8 & 5.7 & 7.1 & 6.2 \\
& 0.4 & 0.1 & 0.5 & 0.4 & 0.4 \\
Very Good & Night, All Directions & & & \\
Good & 0.0 & 0.0 & 0.0 & 0.0 & 0.0 \\
Fair & 0.0 & 0.0 & 0.0 & 0.0 & 0.0 \\
Poor & 37.9 & 45.3 & 22.7 & 35.0 & 45.9 \\
Very Poor & 41.9 & 38.8 & 49.5 & 41.0 & 39.6 \\
\hline
\end{tabular}


Table 7.9 (continued)

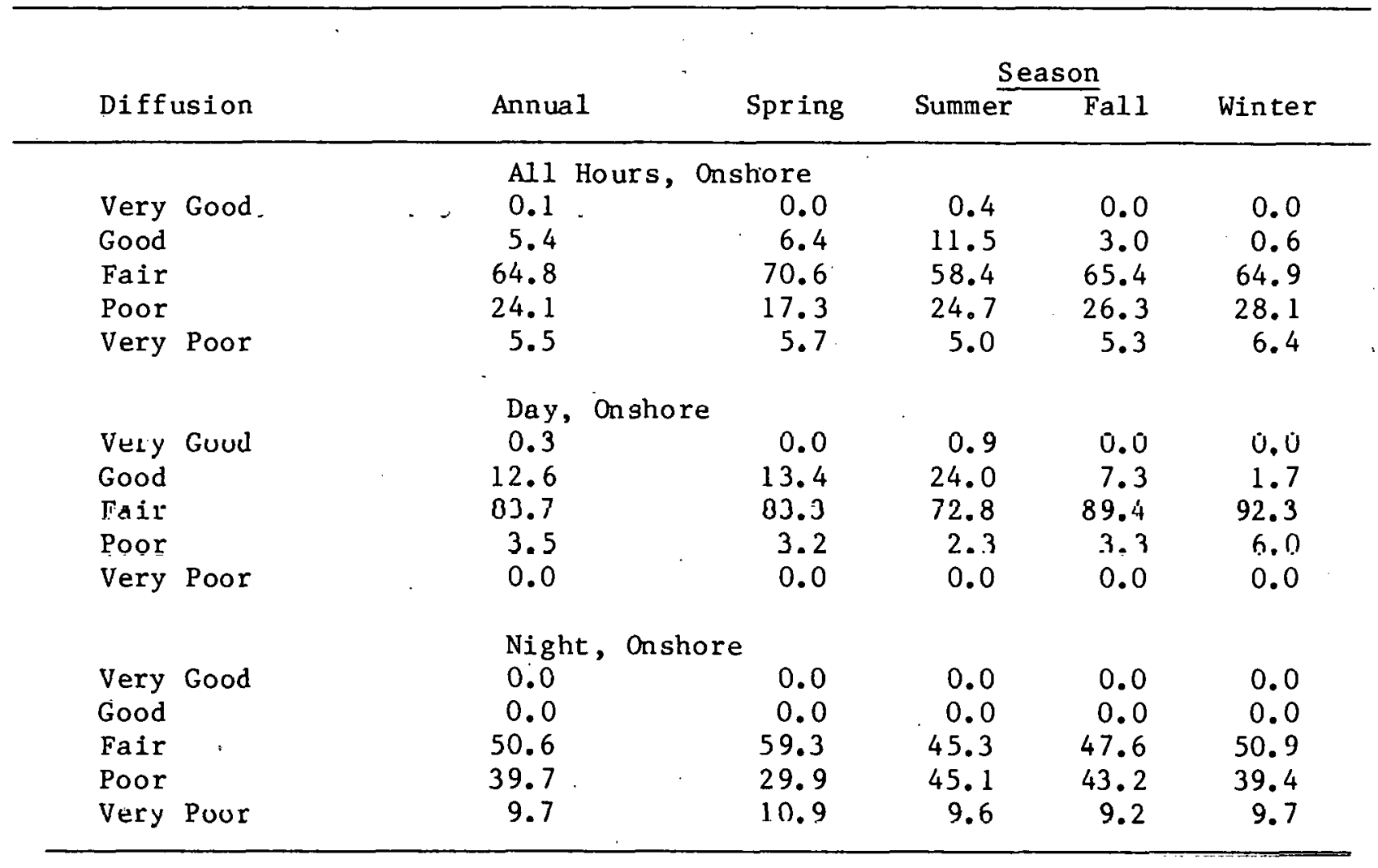


Table 7.9 (continued)

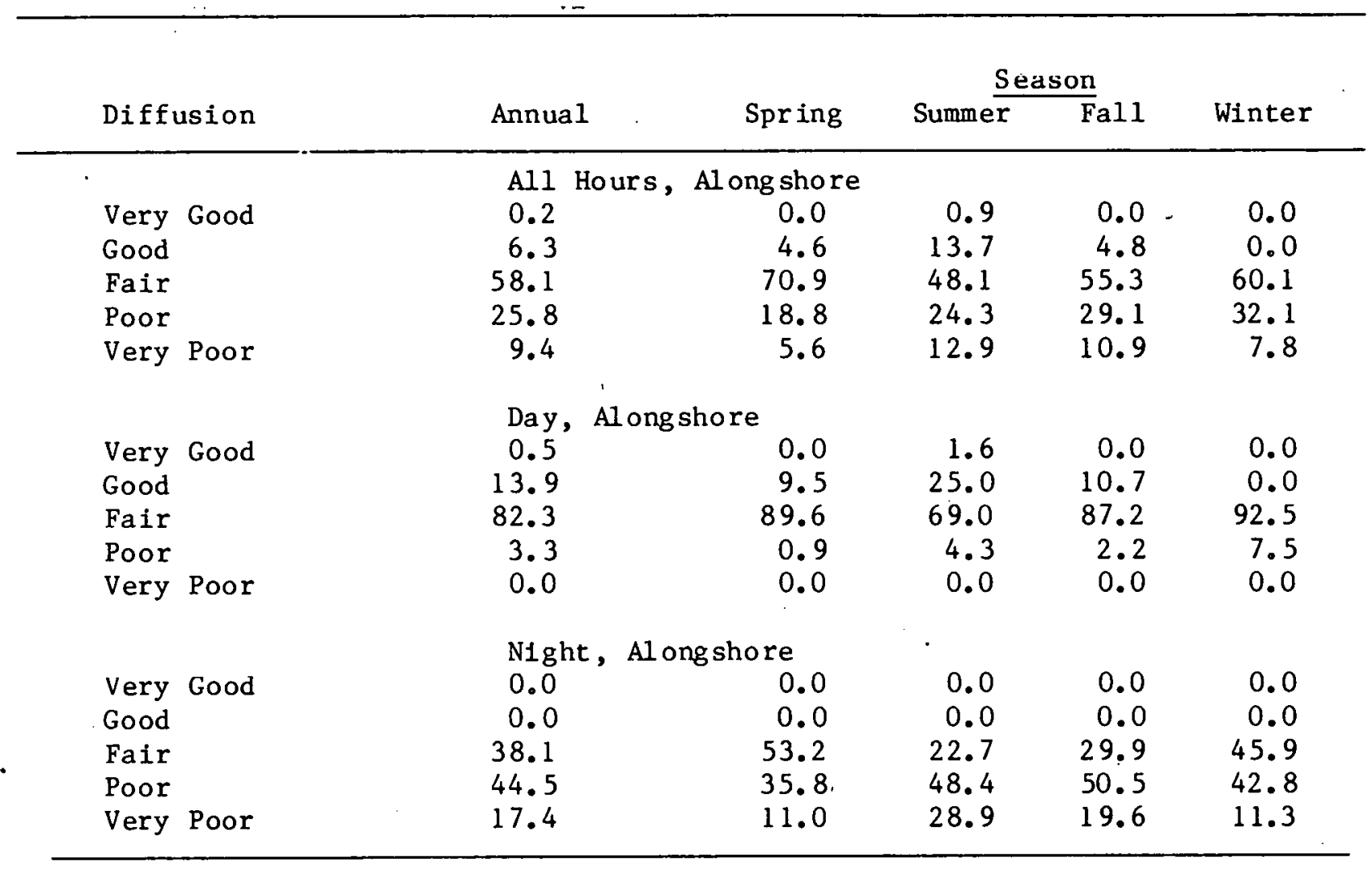


Table 7.9 (continued)

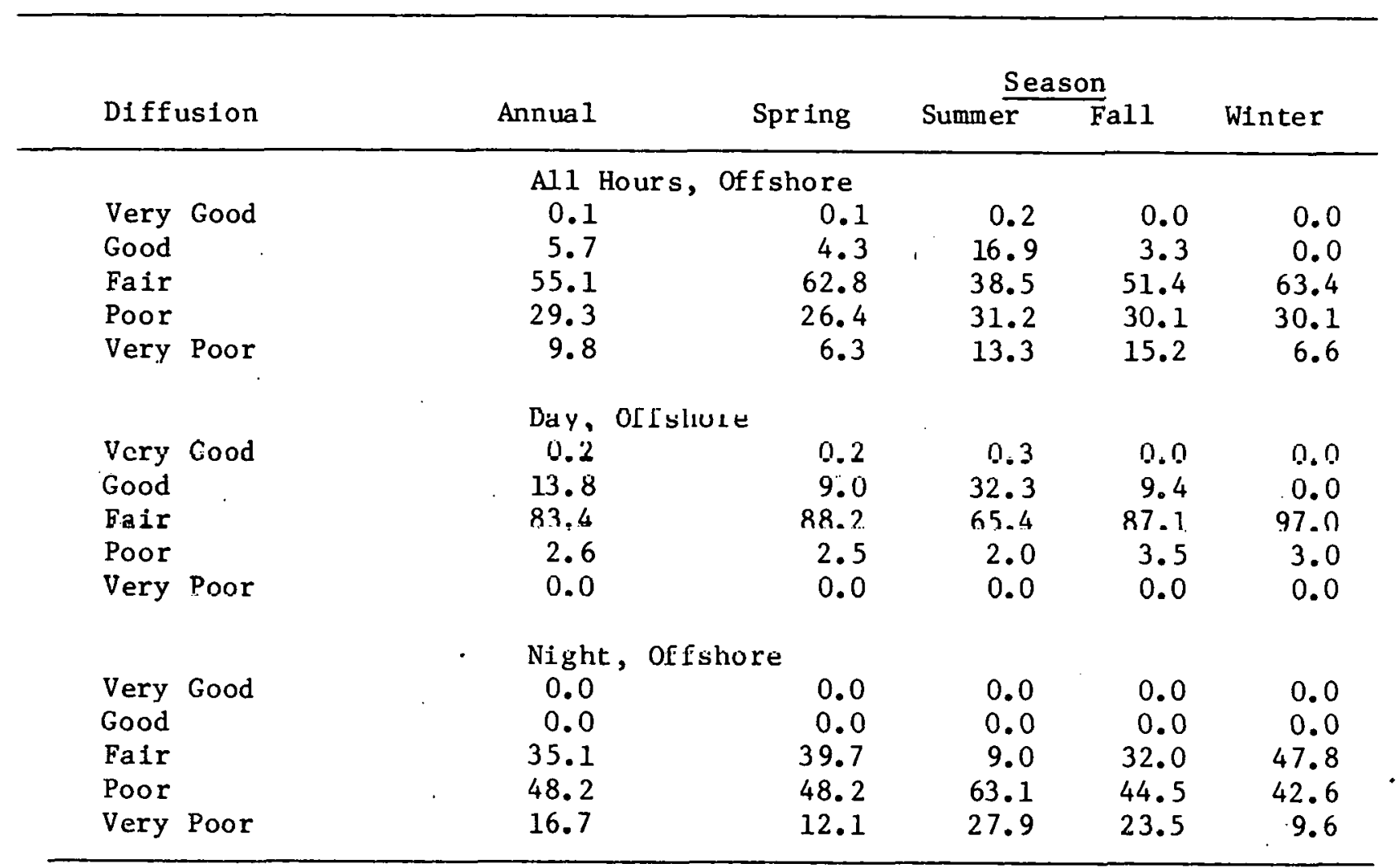




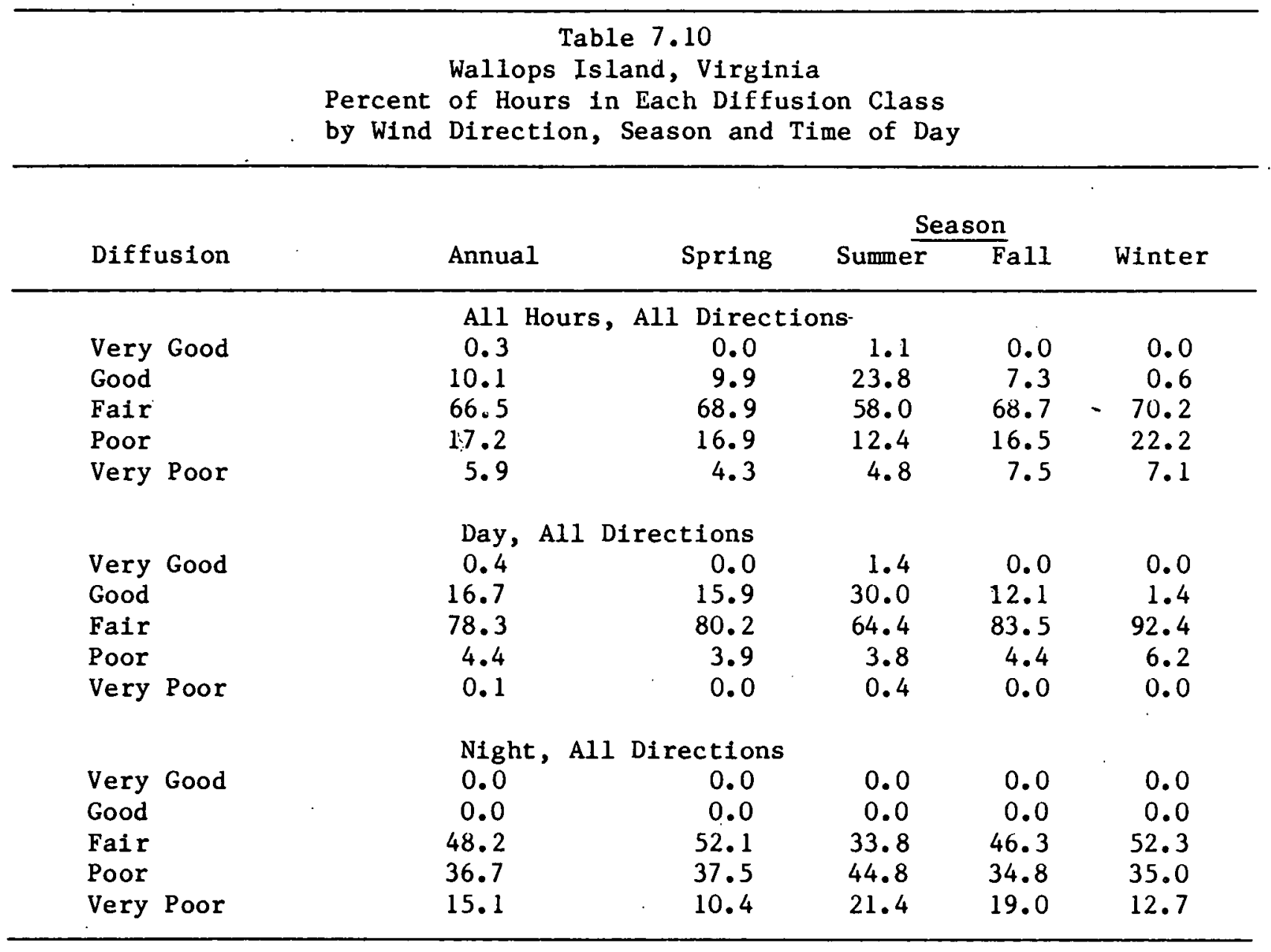


Table 7.10 (continued)

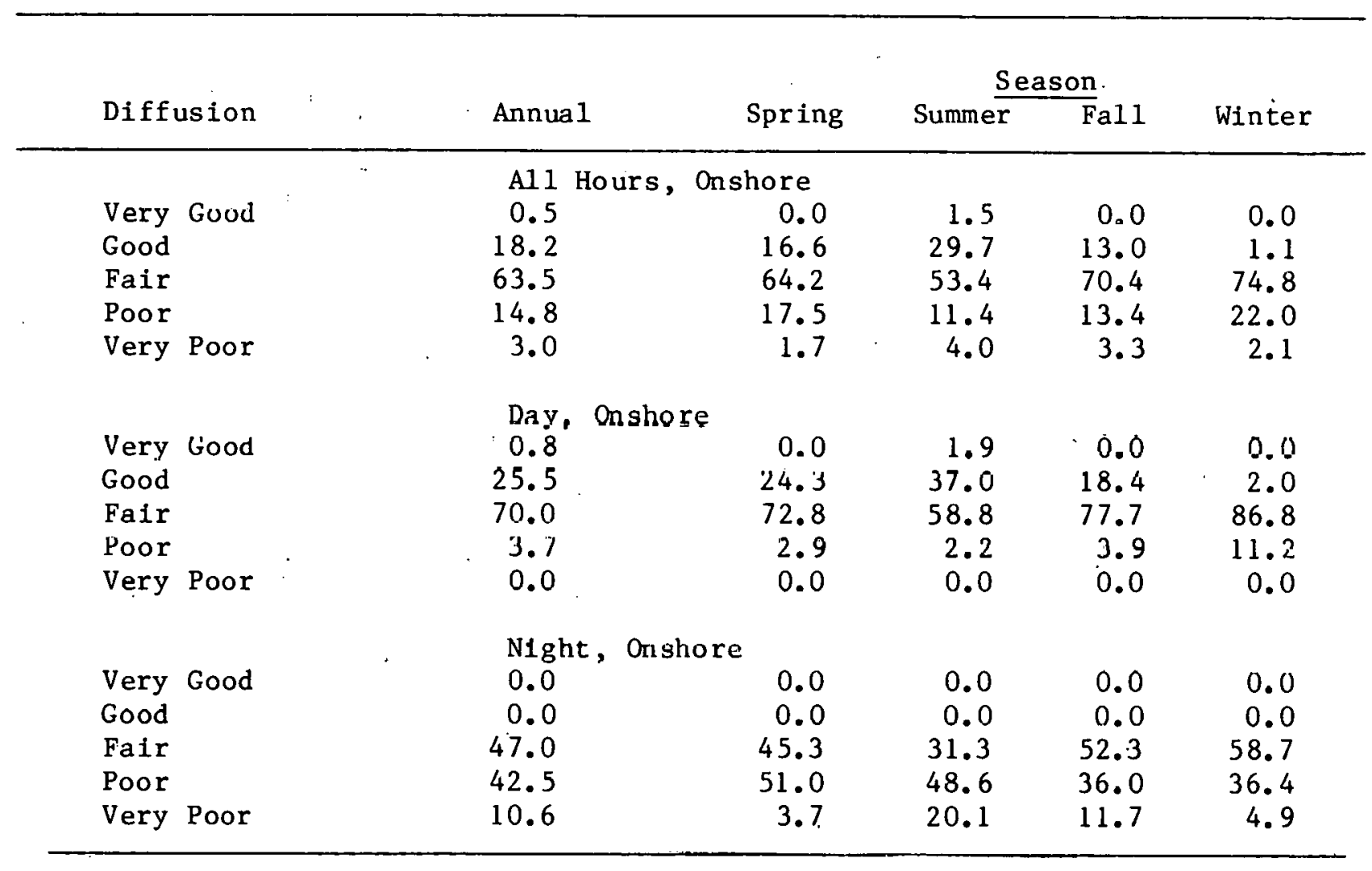


Table 7.10 (continued)

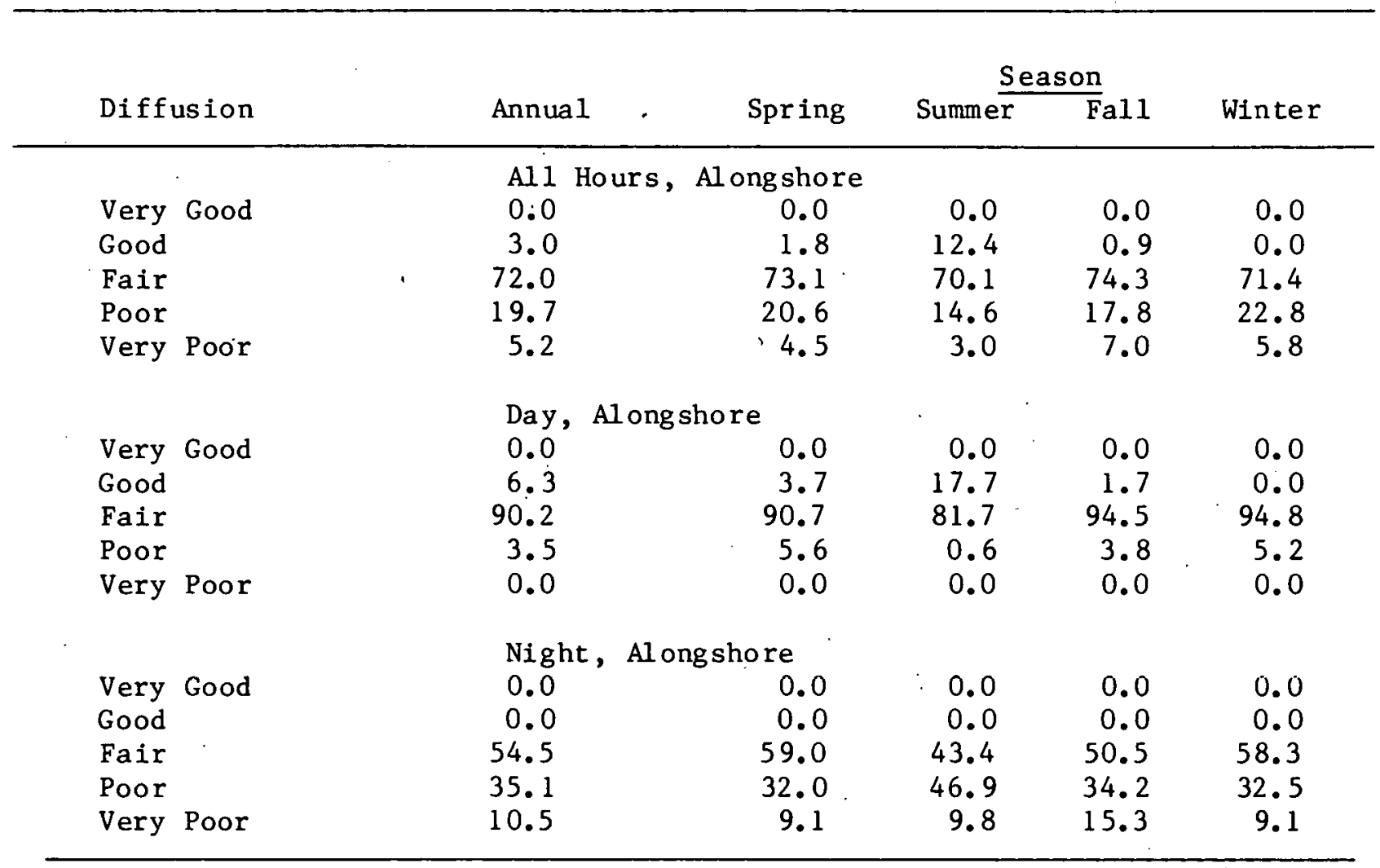




\section{Table 7.10 (continued)}

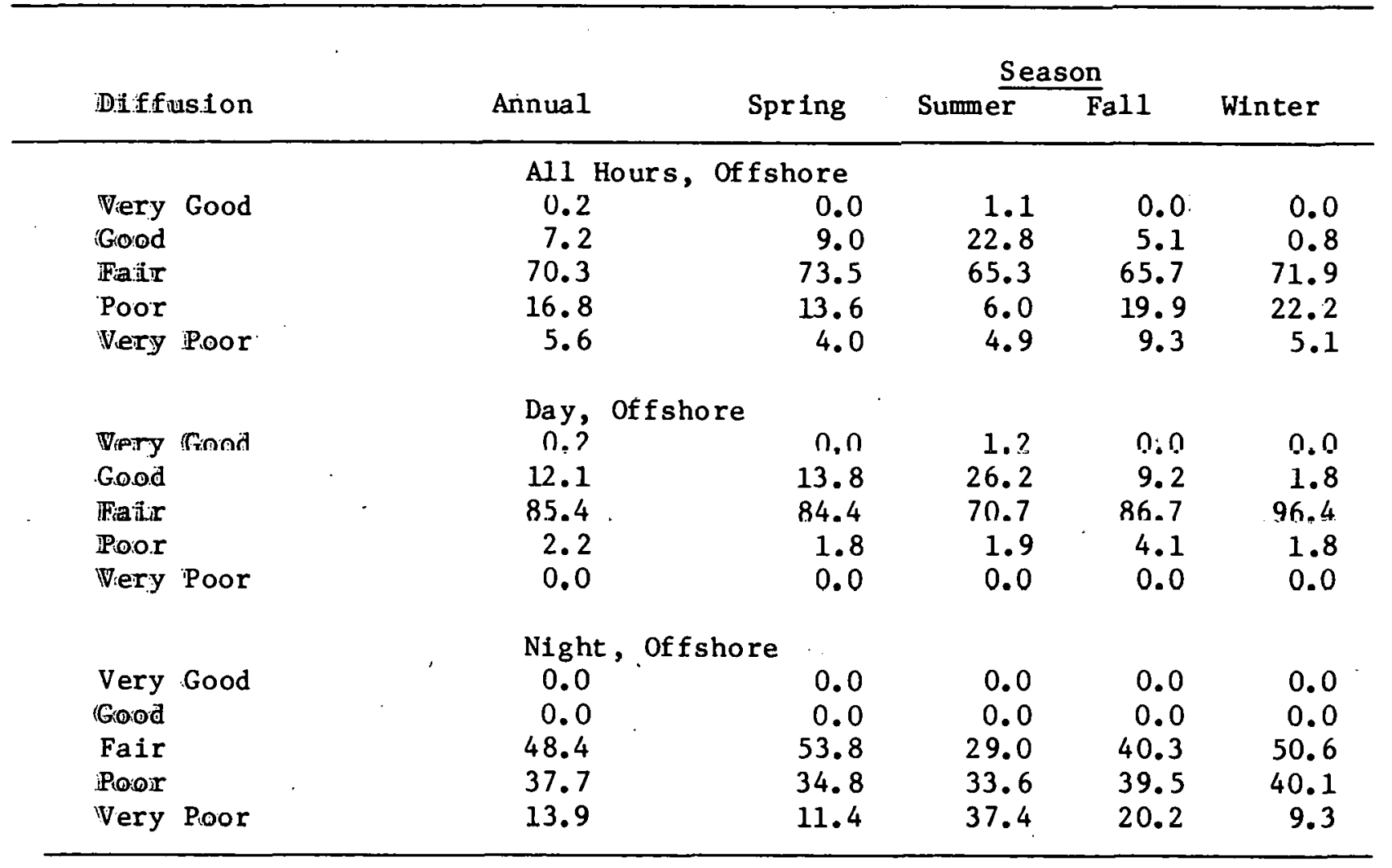




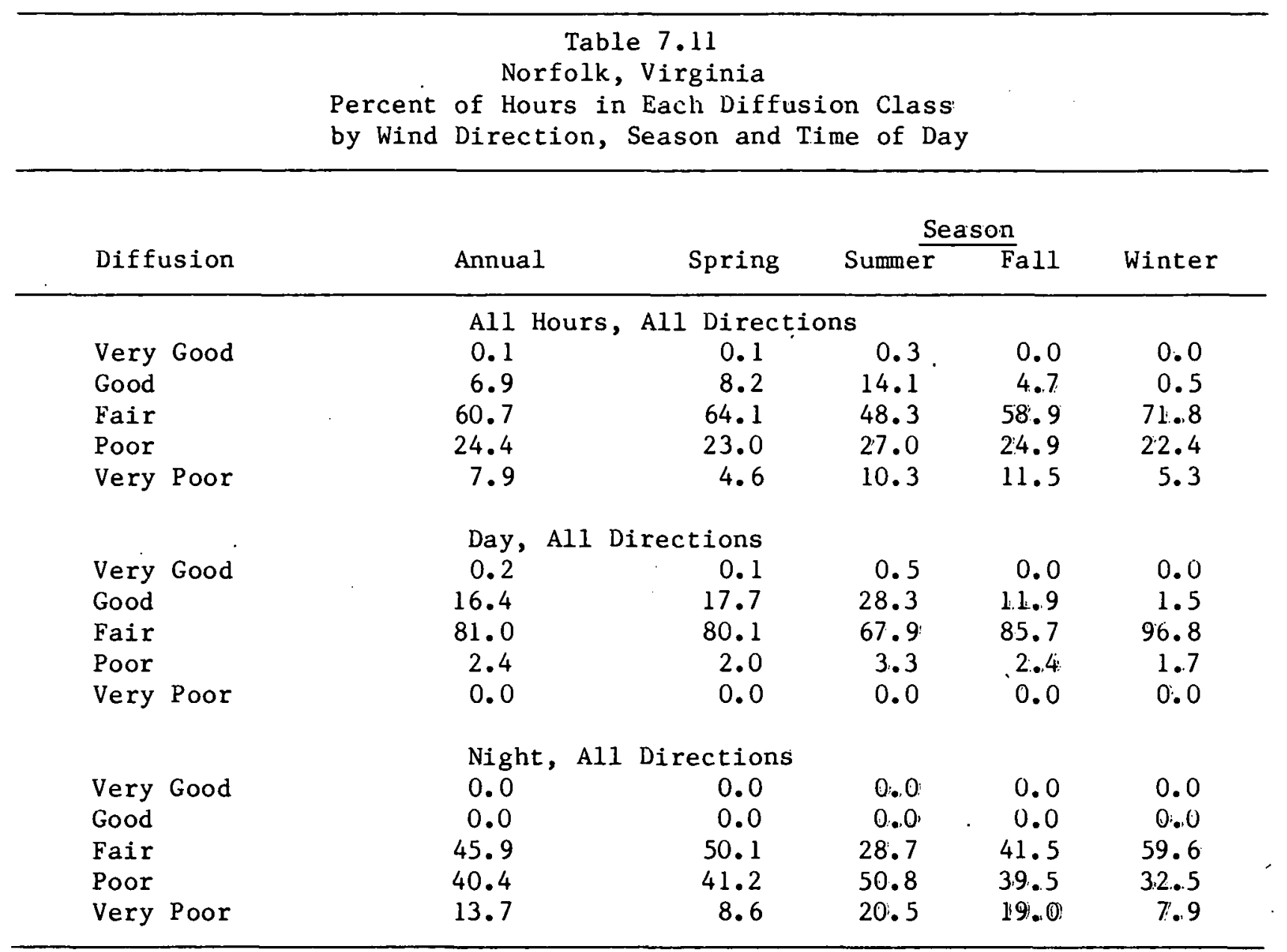


Table 7.11 (continued)

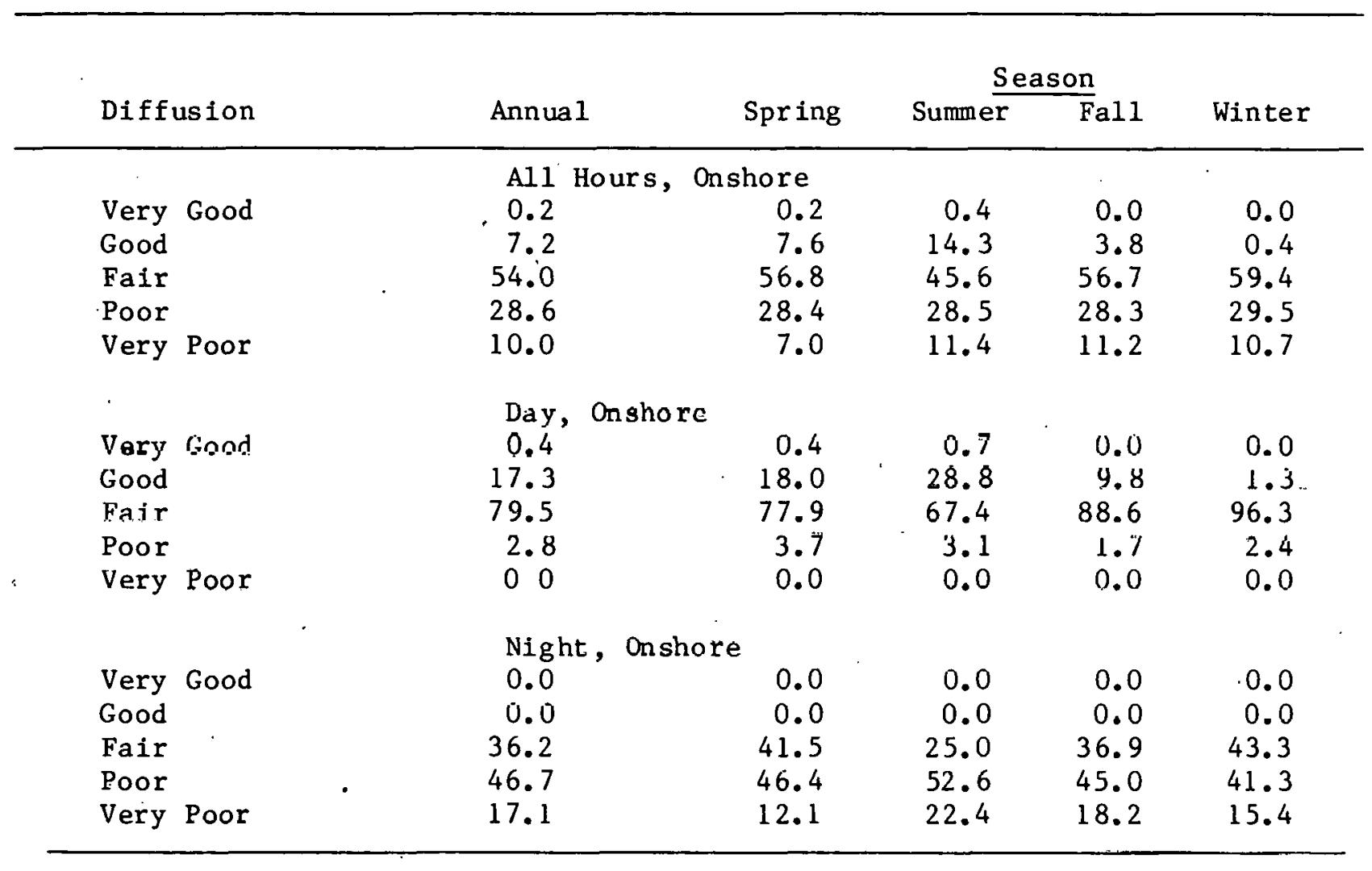


Table 7.11 (continued)

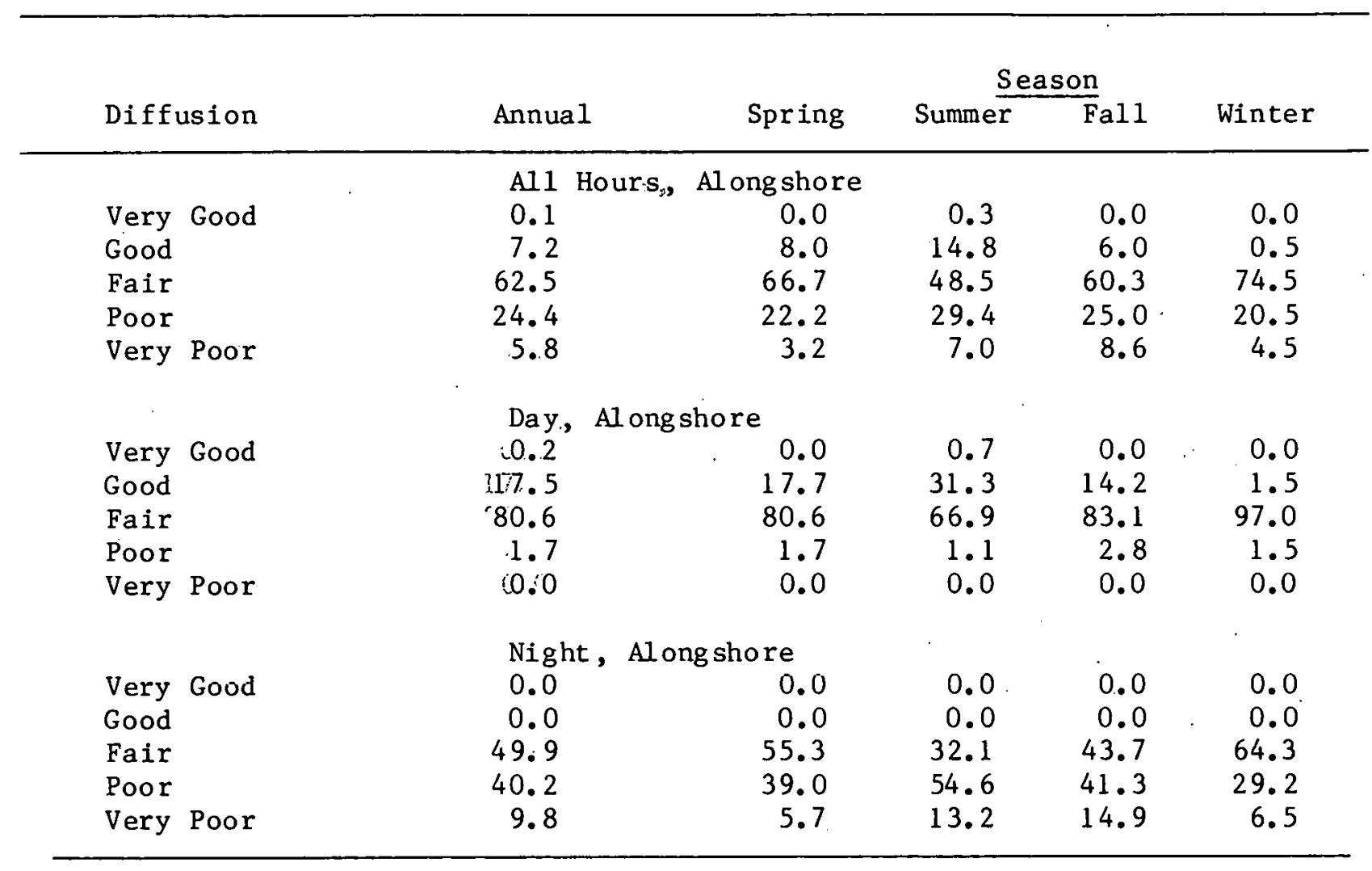


Table 7.11 (continued)

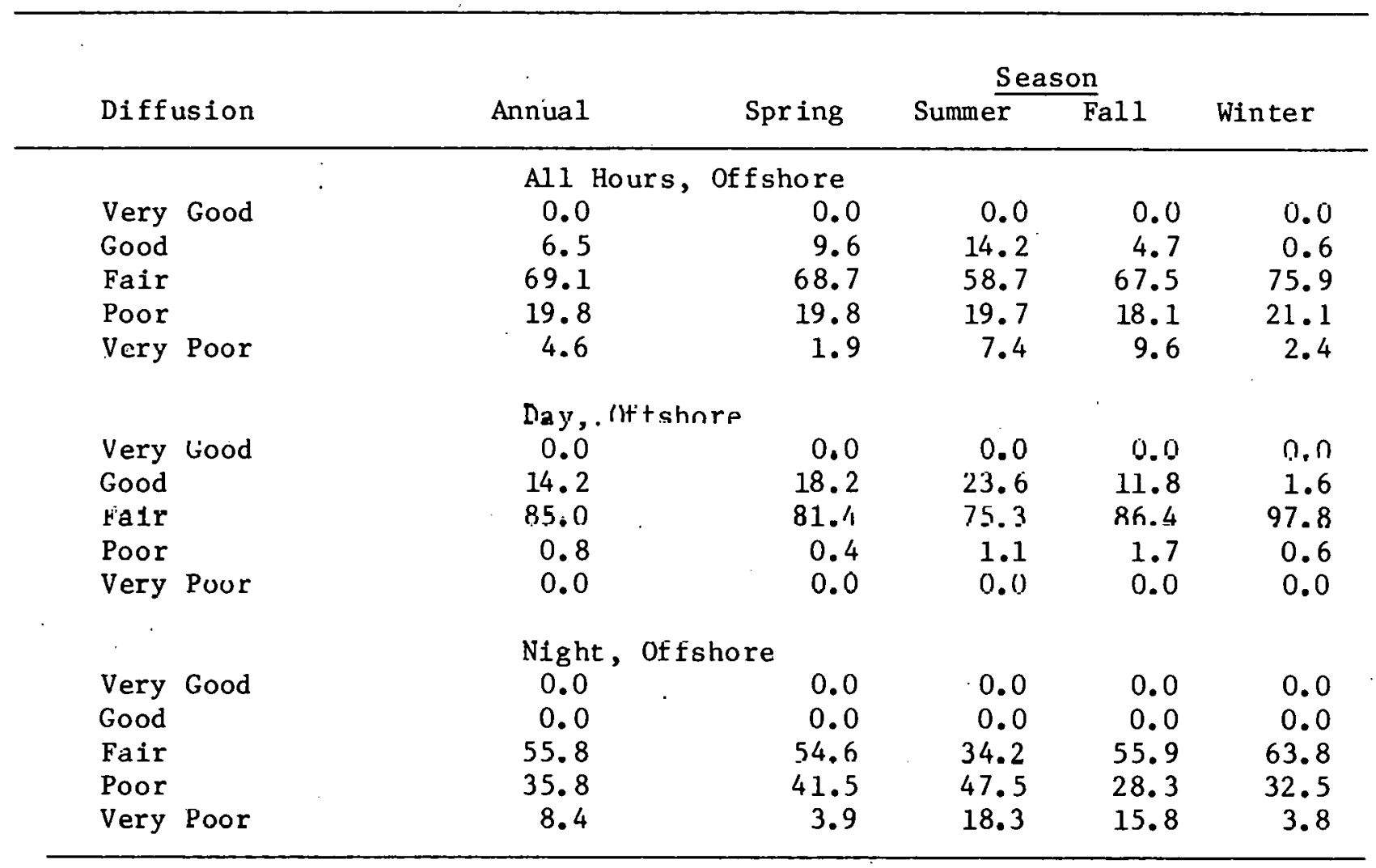




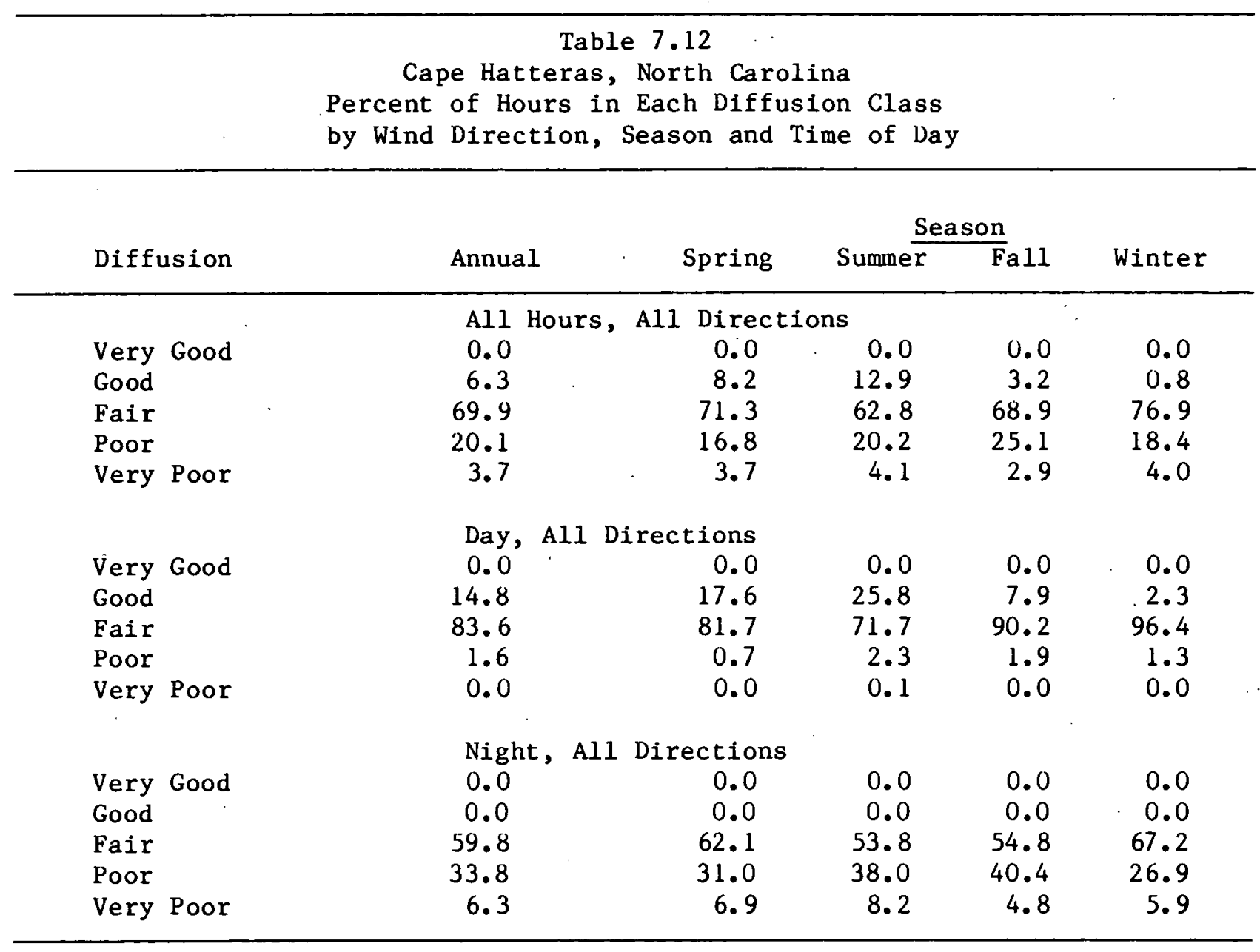


Tablee 7.12 (continued)

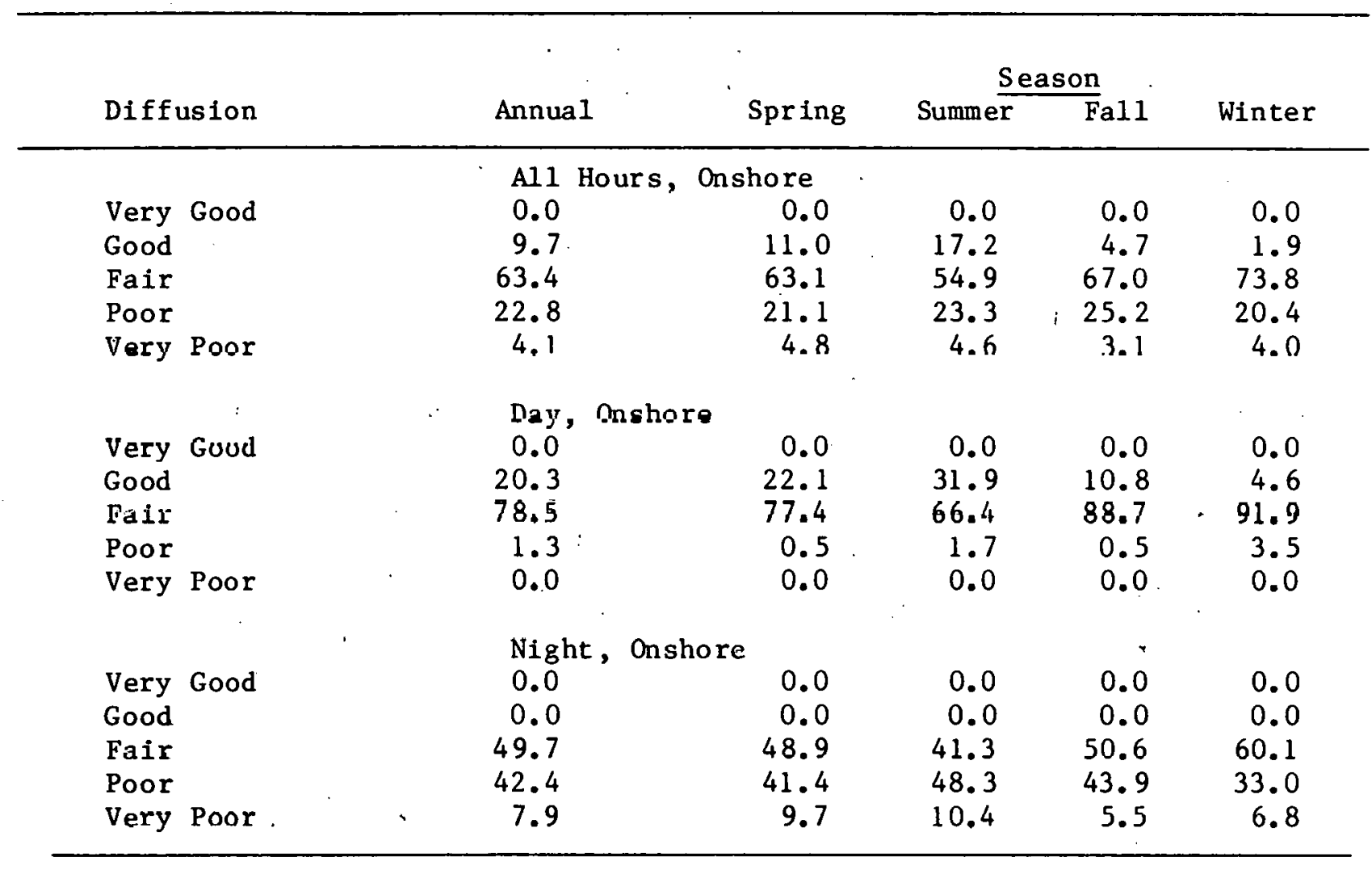


Table 7.12 (continued)

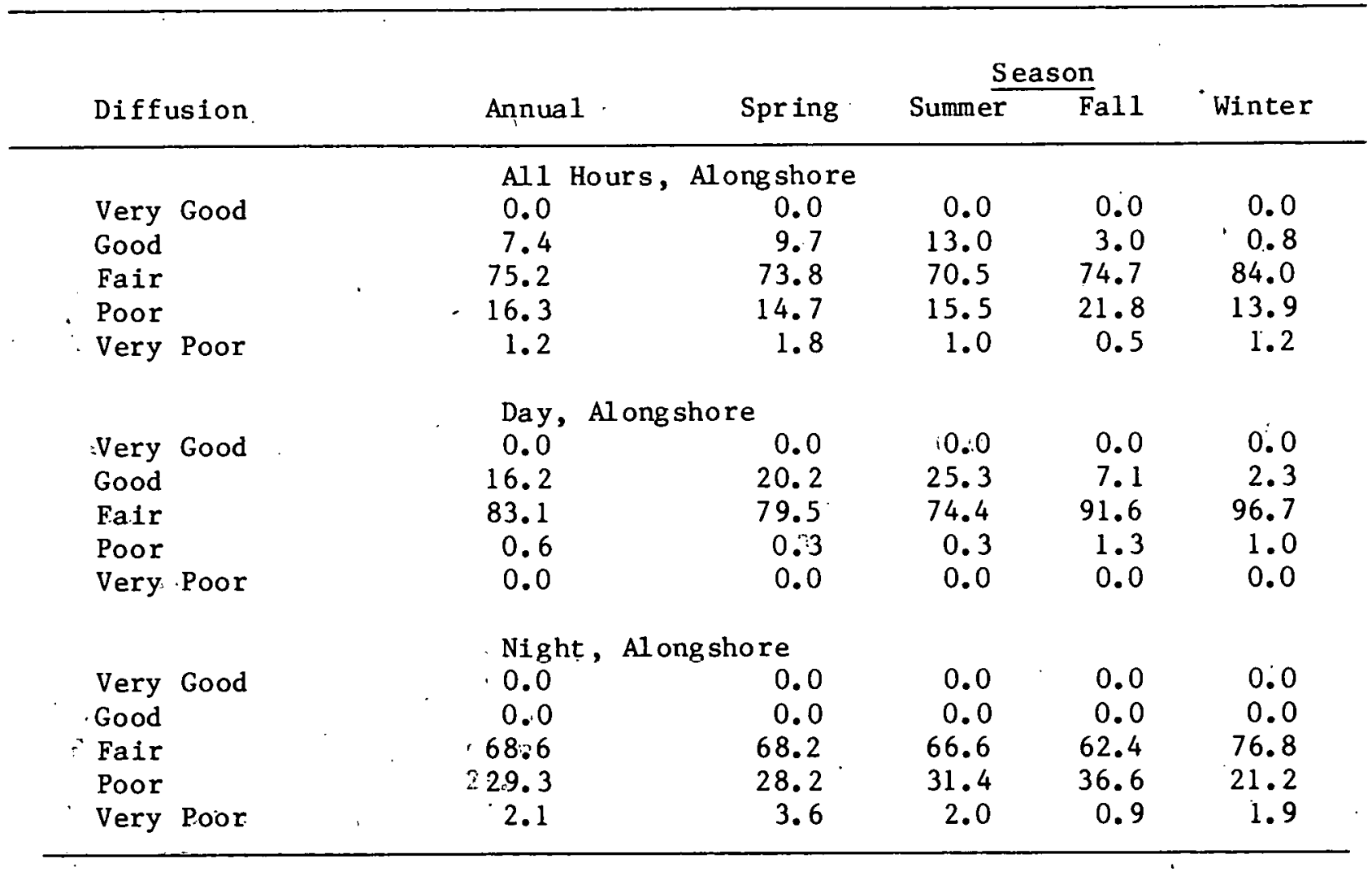


Table 7.12 (continued)

\begin{tabular}{|c|c|c|c|c|c|}
\hline \multirow[b]{2}{*}{ Diffusion } & \multirow[b]{2}{*}{ Annual } & \multirow[b]{2}{*}{ Spring } & \multicolumn{2}{|c|}{ Season } & \multirow[b]{2}{*}{ Winter } \\
\hline & & & Summer & $\bar{F}$ al1 & \\
\hline $\begin{array}{l}\text { Very Good } \\
\text { Good } \\
\text { Fair } \\
\text { Poor } \\
\text { Very Poor }\end{array}$ & $\begin{array}{r}\text { A11 } \\
0.0 \\
2.5 \\
72.0 \\
23.0 \\
2.4\end{array}$ & $\begin{array}{r}\text { Hours, of f shore } \\
0.0 \\
3.8 \\
77.8 \\
16.9 \\
1.5\end{array}$ & $\begin{array}{r}0.0 \\
7.5 \\
60.8 \\
27.4 \\
4.3\end{array}$ & $\begin{array}{r}0.0 \\
1.8 \\
66.5 \\
29.1 \\
2.6\end{array}$ & $\begin{array}{r}0.0 \\
0.4 \\
75.6 \\
21.8 \\
2.2\end{array}$ \\
\hline $\begin{array}{l}\text { Very Goud } \\
\text { Good } \\
\text { Fair } \\
\text { Poor } \\
\text { Very Poor }\end{array}$ & $\begin{array}{r}\text { Day, } \\
0.0 \\
7.0 \\
92.2 \\
0.8 \\
0.0\end{array}$ & $\begin{array}{r}0.1 \\
8.7 \\
90.7 \\
0.6 \\
0.0\end{array}$ & $\begin{array}{r}0.0 \\
17.1 \\
80.8 \\
2.1 \\
0.0\end{array}$ & $\begin{array}{r}0.0 \\
5.3 \\
93.2 \\
1.4 \\
0.0\end{array}$ & $\begin{array}{r}0.0 \\
1.5 \\
98.5 \\
0.0 \\
0.0\end{array}$ \\
\hline $\begin{array}{l}\text { Very Good. } \\
\text { Good } \\
\text { Fair } \\
\text { Poor } \\
\text { Very Poor }\end{array}$ & $\begin{array}{l}\text { Night } \\
0.0 \\
0.0 \\
60.7 \\
35.6 \\
3.7\end{array}$ & $\begin{array}{r}\text {, Offshore } \\
0.0 \\
0.0 \\
67.6 \\
29.8 \\
2.6\end{array}$ & $\begin{array}{r}0.0 \\
0.0 \\
45.6 \\
47.0 \\
7.4\end{array}$ & $\begin{array}{r}0.0 \\
0.0 \\
52.4 \\
43.7 \\
3.9\end{array}$ & $\begin{array}{r}0.0 \\
0.0 \\
66.0 \\
30.9 \\
3.1\end{array}$ \\
\hline
\end{tabular}




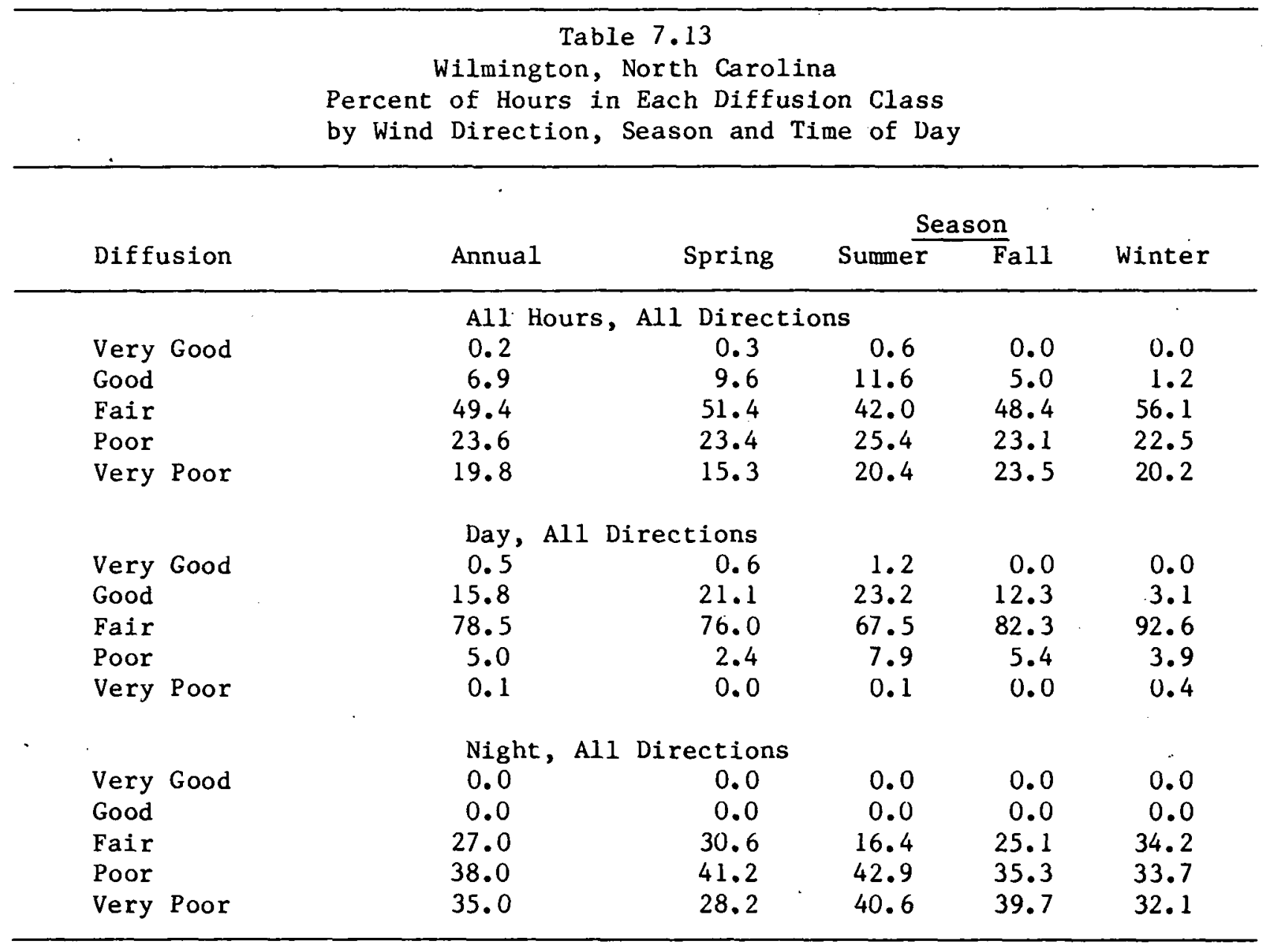


Tablie: 7.13 (continued)

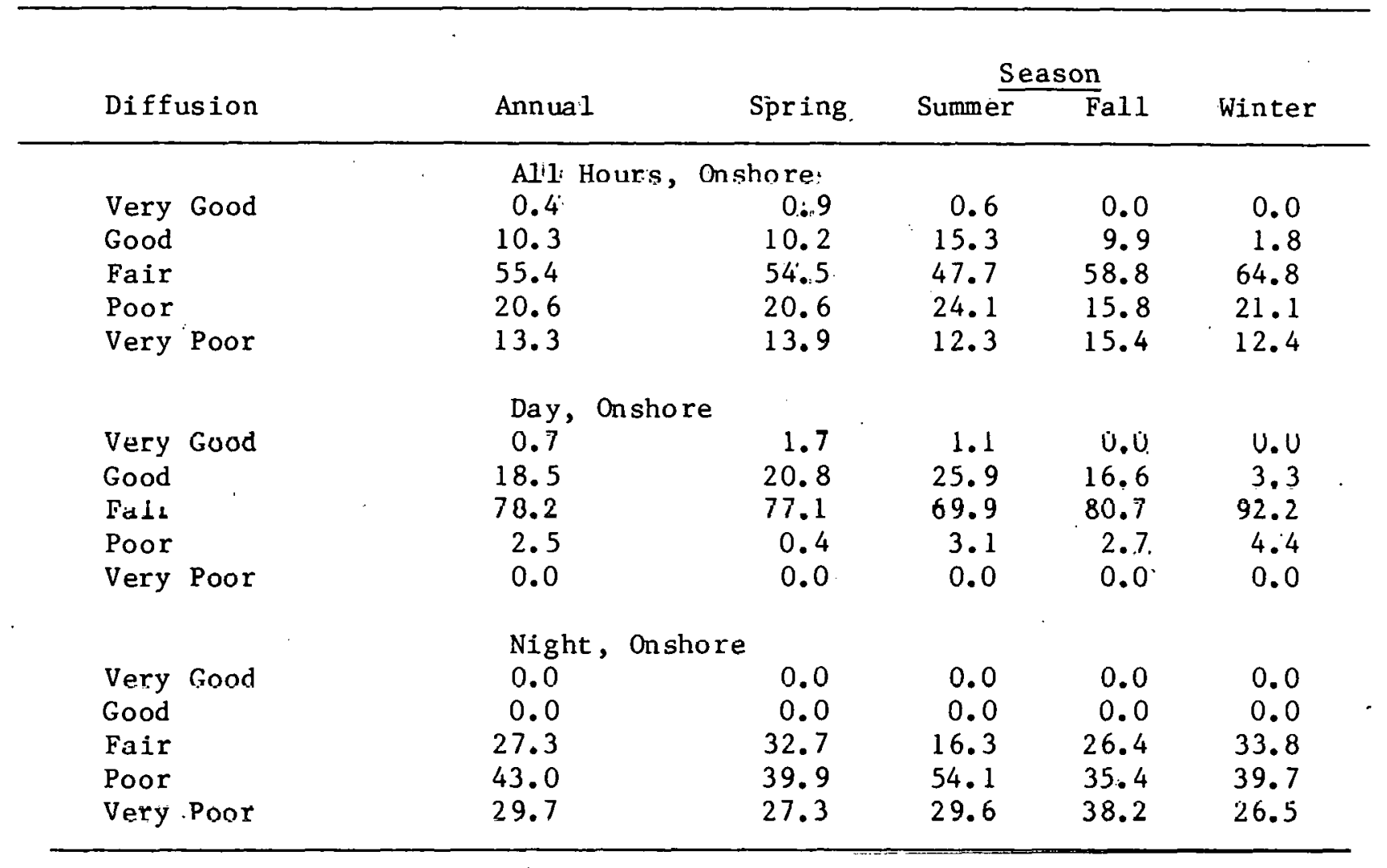


Table 7.13 (continued)

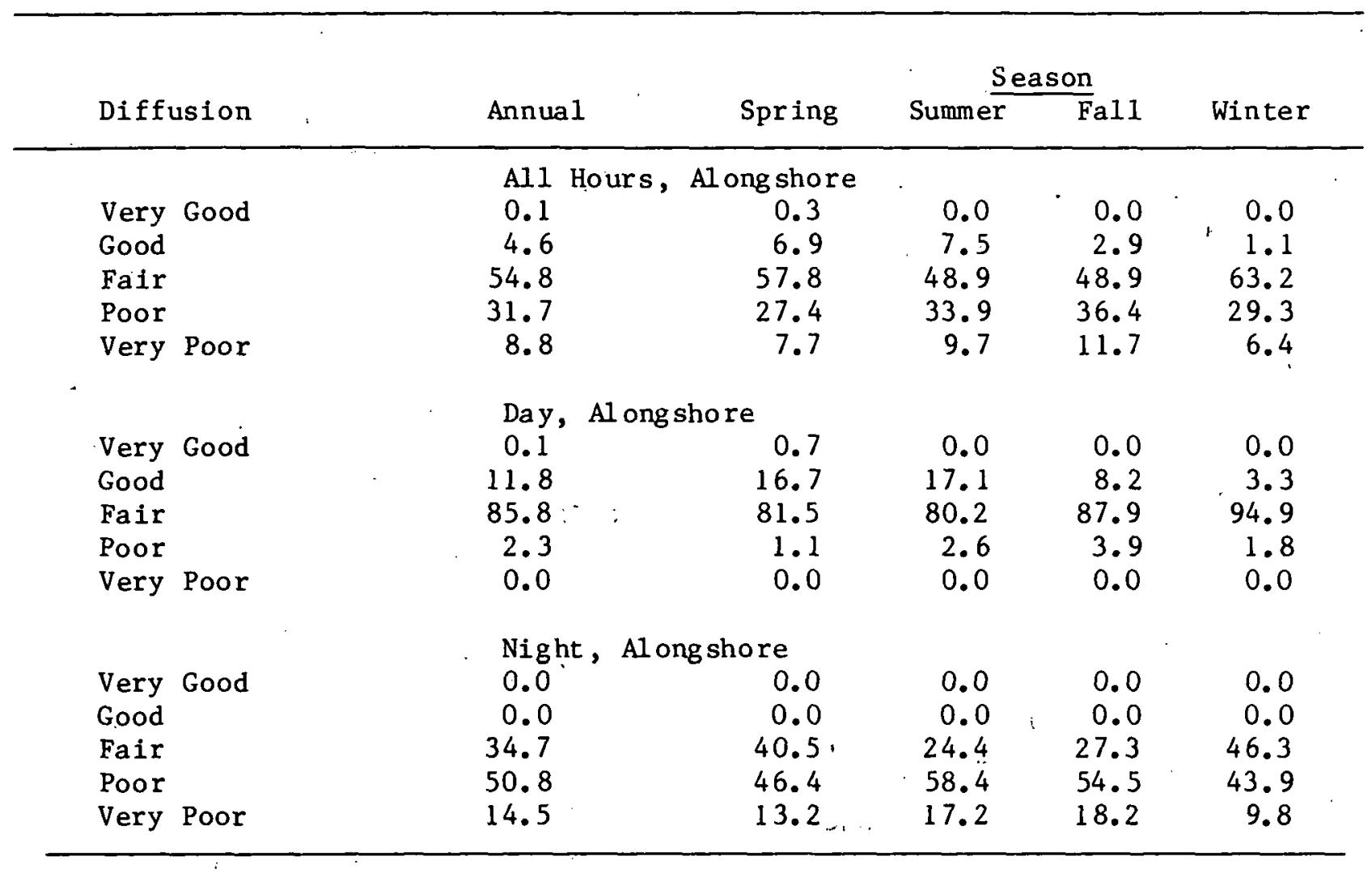


Table 7.13 (continued)

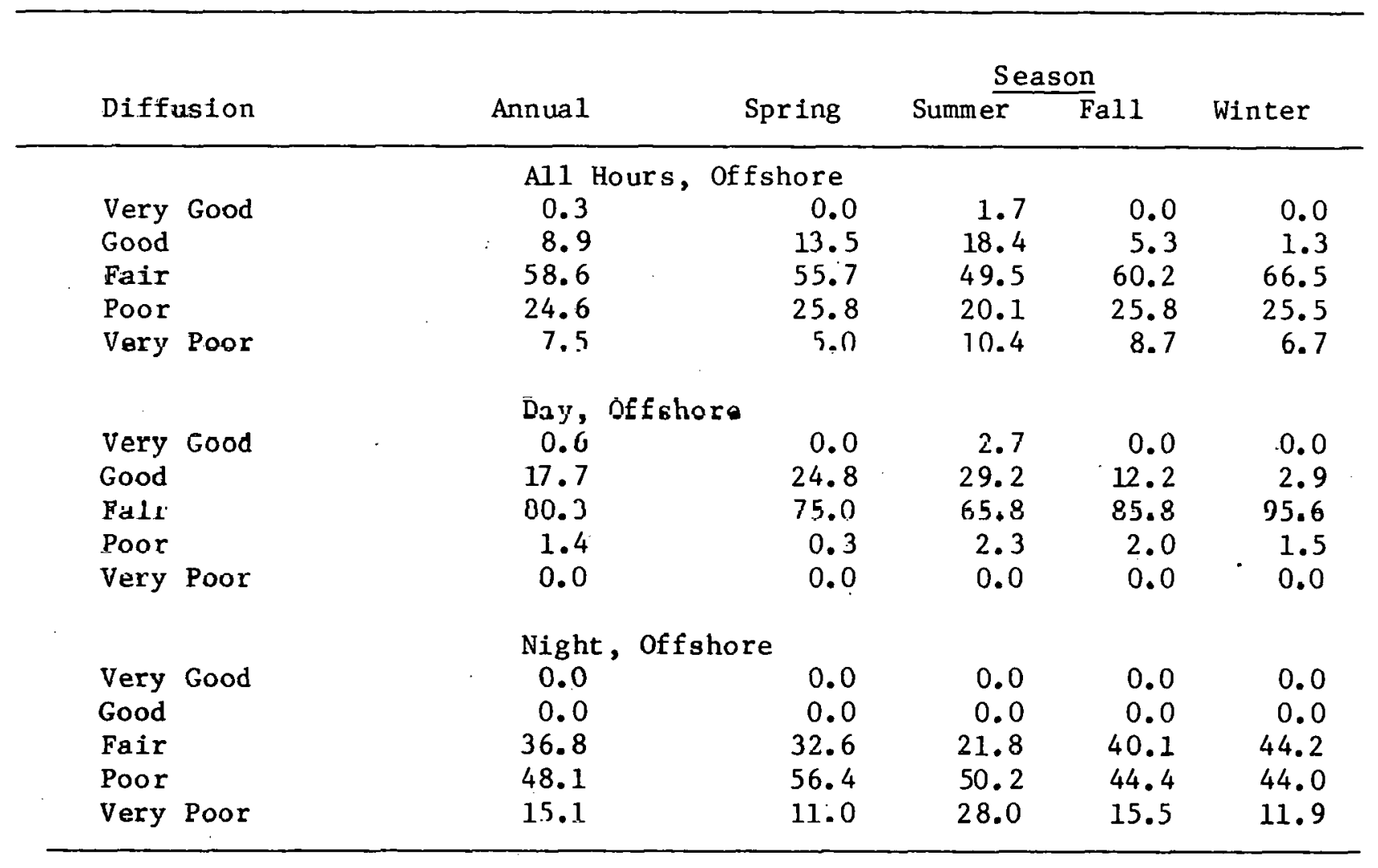




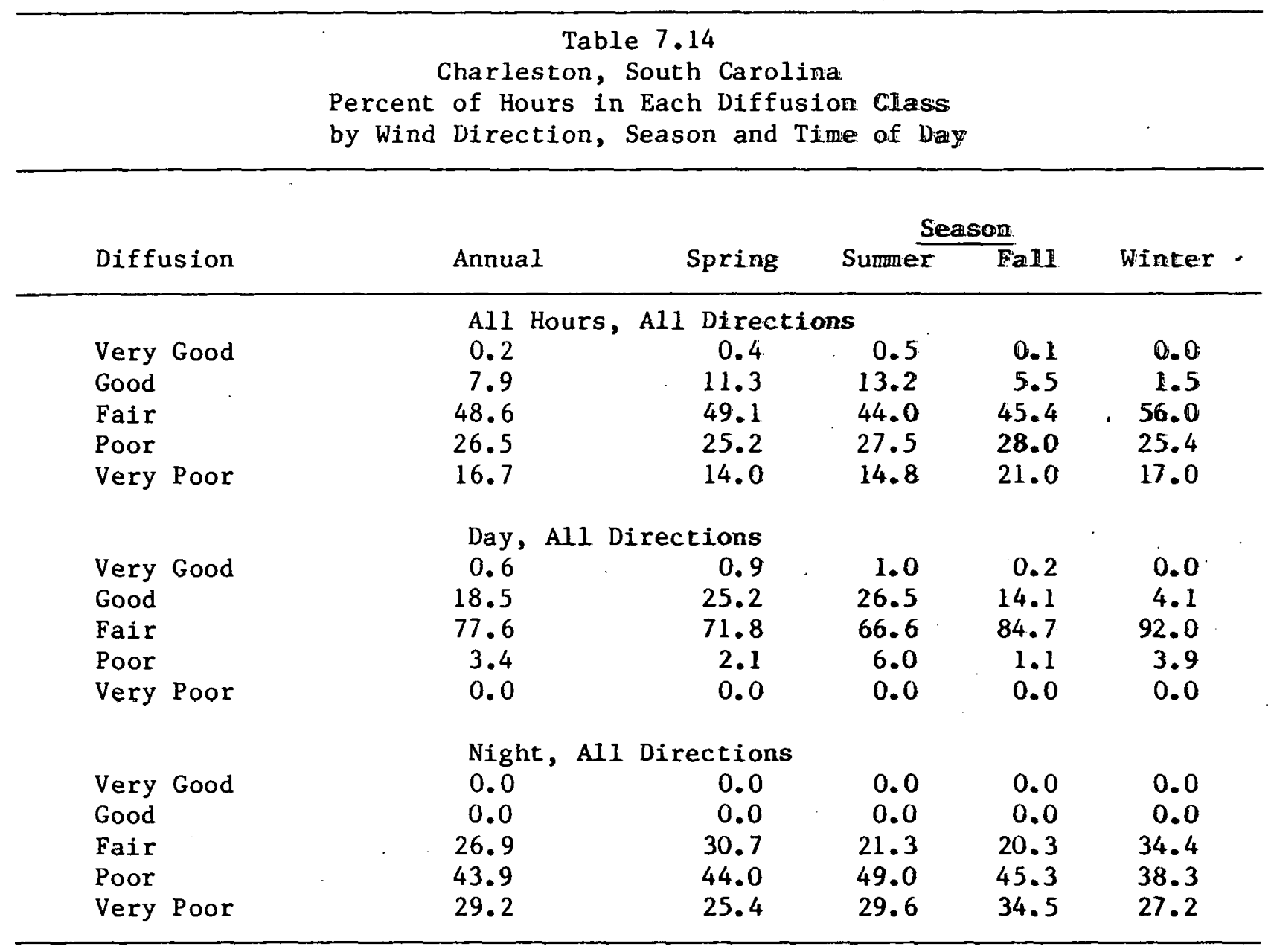


Table 7.14 (continued)

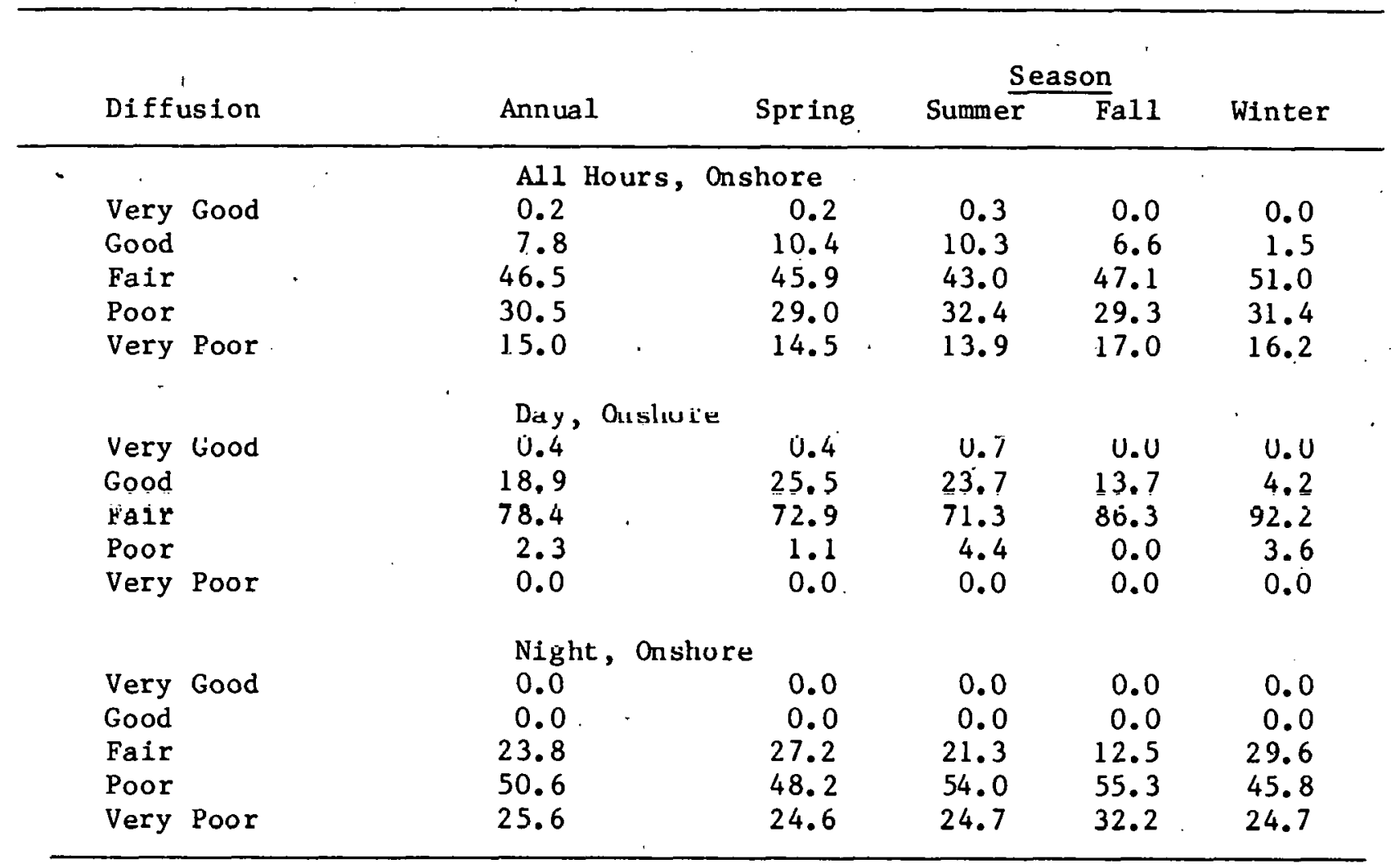


Table 7.14 (continued)

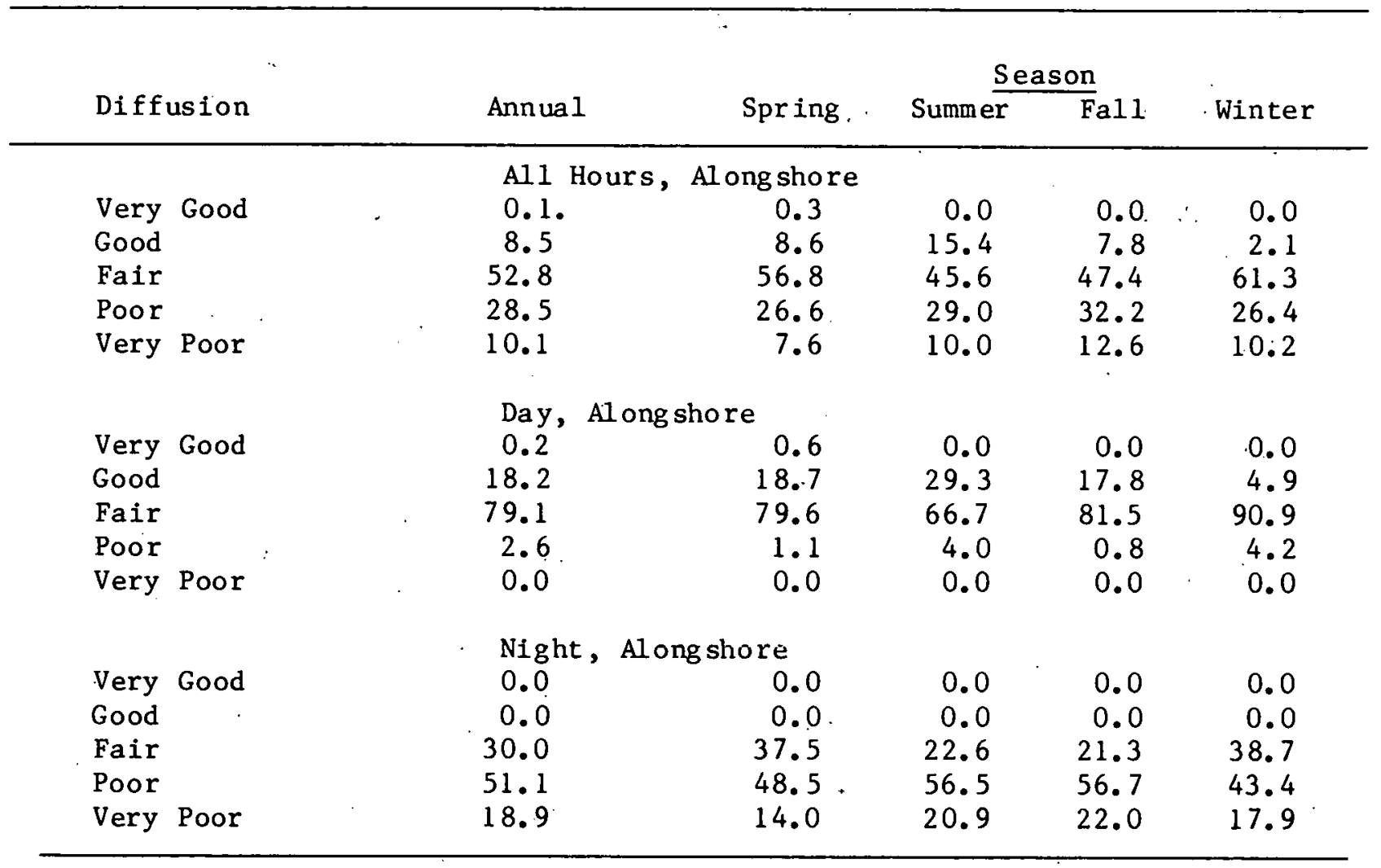


Table 7.14 (continued)

\begin{tabular}{|c|c|c|c|c|c|}
\hline \multirow[b]{2}{*}{ Diffusion } & \multirow[b]{2}{*}{ Annual } & \multicolumn{4}{|c|}{ Season } \\
\hline & & Spring & Summer & $\bar{F} a 11$ & Winter \\
\hline $\begin{array}{l}\text { Very Good } \\
\text { Good } \\
\text { Fair } \\
\text { Ponr } \\
\text { Very Poor }\end{array}$ & $\begin{array}{r}\text { A11 } \\
0.4 \\
8.5 \\
53.5 \\
23.6 \\
14.0\end{array}$ & $\begin{array}{r}\text { lours, Offshore } \\
0.8 \\
14.9 \\
53.3 \\
20.3 \\
10.8\end{array}$ & $\begin{array}{l}1.1 \\
17.6 \\
48.8 \\
20.4 \\
12.1\end{array}$ & $\begin{array}{r}0.2 \\
4.0 \\
47.0 \\
27.8 \\
21.0\end{array}$ & $\begin{array}{r}0.0 \\
1.5 \\
65.0 \\
23.2 \\
10.3\end{array}$ \\
\hline $\begin{array}{l}\text { Very Good } \\
\text { Cood } \\
\text { Fair } \\
\text { Poor } \\
\text { Very Poor }\end{array}$ & $\begin{array}{r}\text { Day, } \\
1.0 \\
18.6 \\
77.8 \\
2.6 \\
0.0\end{array}$ & $\begin{array}{r}1.5 \\
28.9 \\
69.6 \\
0.0 \\
0.0\end{array}$ & $\begin{array}{r}1.8 \\
28.3 \\
63.8 \\
6.2 \\
0.0\end{array}$ & $\begin{array}{r}0.5 \\
11.4 \\
86.4 \\
1.7 \\
0.0\end{array}$ & $\begin{array}{r}0.0 \\
3.7 \\
94.3 \\
2.0 \\
0.0\end{array}$ \\
\hline $\begin{array}{l}\text { Very Good } \\
\text { Good } \\
\text { Fair } \\
\text { Poor } \\
\text { Very Poor }\end{array}$ & $\begin{array}{l}\text { N1ght } \\
0.0 \\
0.0 \\
33.1 \\
41.3 \\
25.7\end{array}$ & $\begin{array}{r}\text {, Offshore } \\
\quad 0.0 \\
0.0 \\
36.3 \\
41.7 \\
22.0\end{array}$ & $\begin{array}{r}0.0 \\
0.0 \\
24.5 \\
43.8 \\
31.8\end{array}$ & $\begin{array}{r}0.0 \\
0.0 \\
26.1 \\
41.9 \\
32.0\end{array}$ & $\begin{array}{l}0.0 \\
0.0 \\
44.5 \\
38.1 \\
17.5\end{array}$ \\
\hline
\end{tabular}




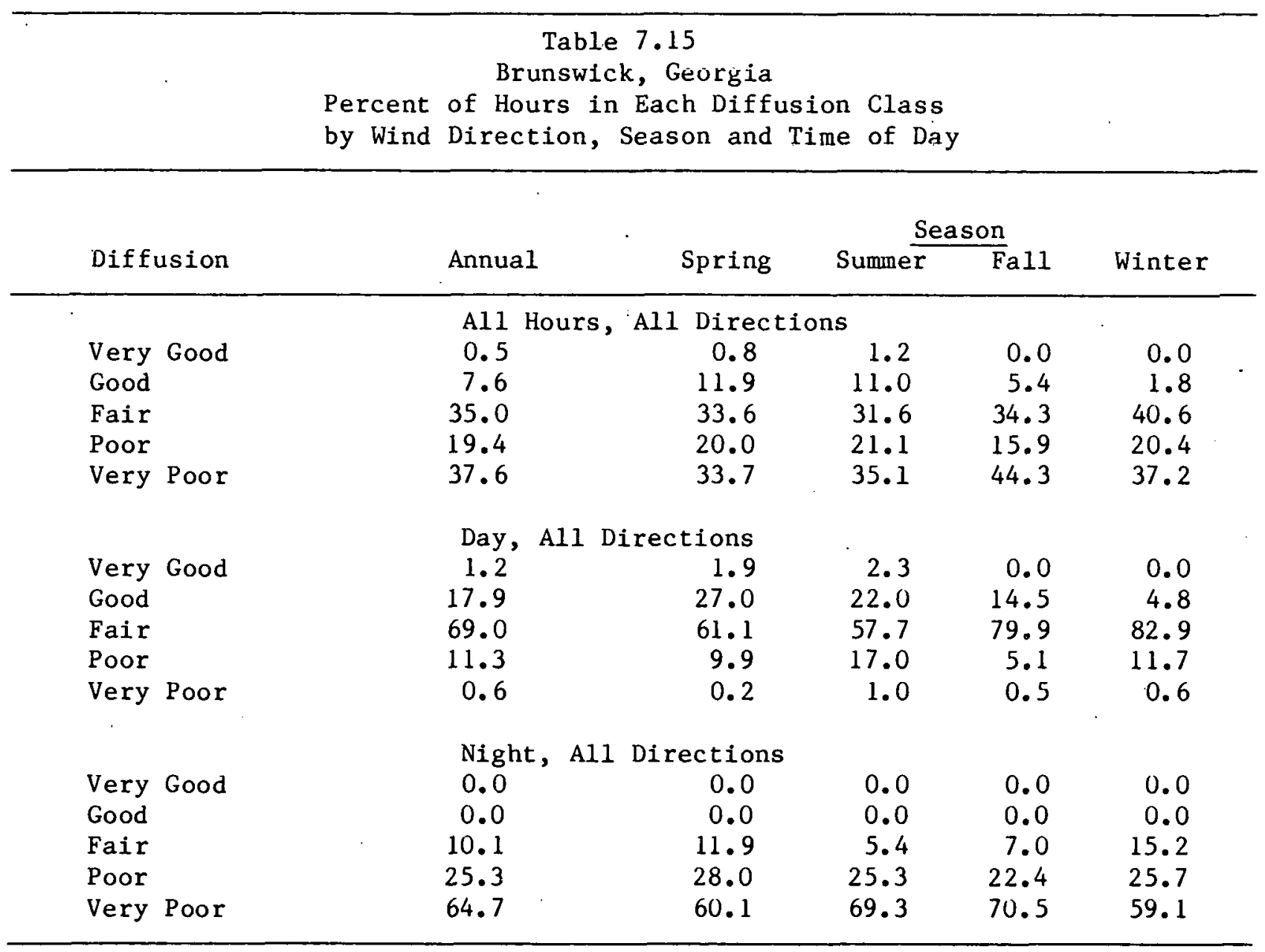


Table 7.15 (continued)

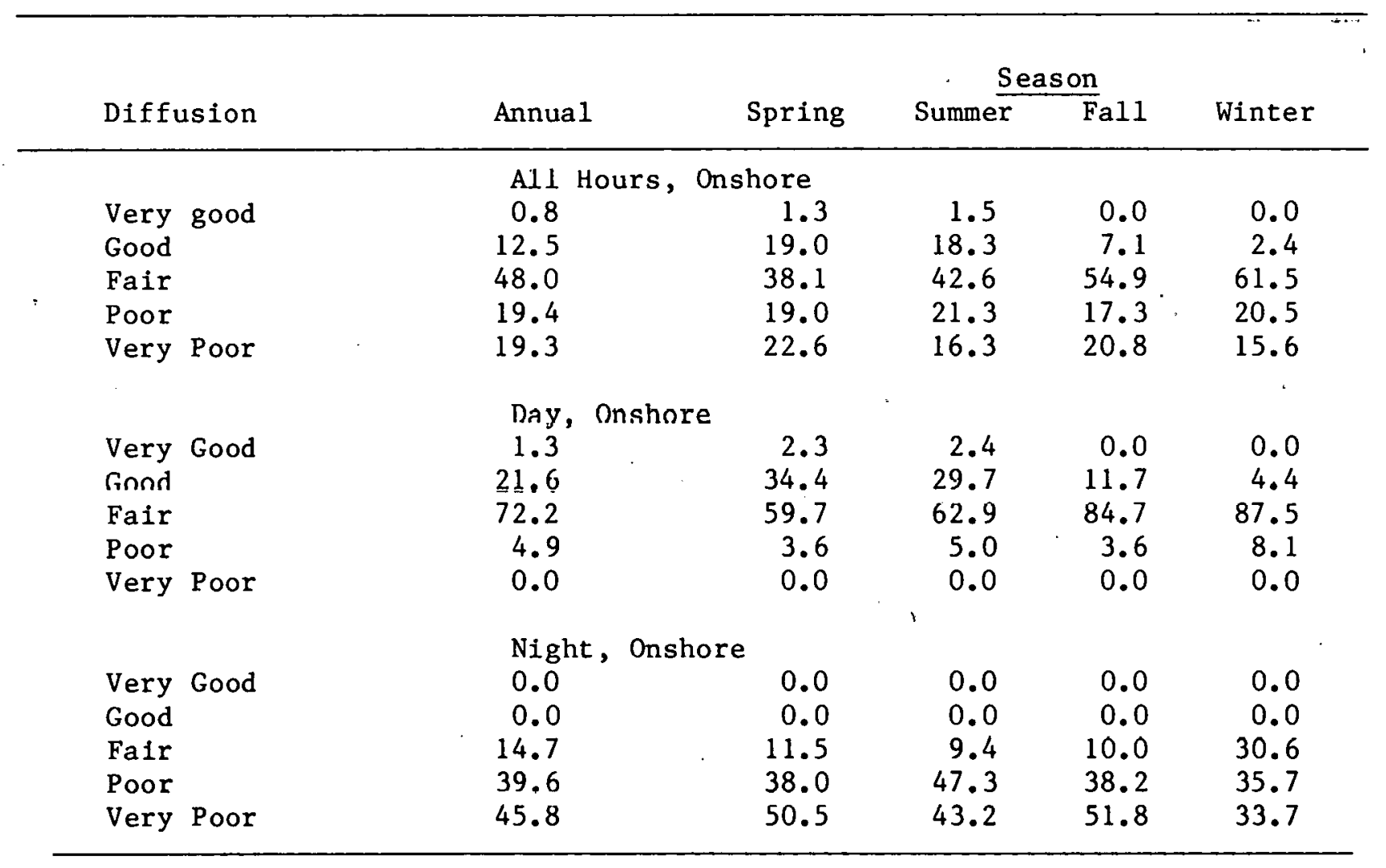


Table 7.15 (continued)

\begin{tabular}{|c|c|c|c|c|c|}
\hline \multirow[b]{2}{*}{ Diffusion } & \multirow[b]{2}{*}{ Annual } & \multirow[b]{2}{*}{ Spring } & \multicolumn{2}{|c|}{ Season } & \multirow[b]{2}{*}{ Winter } \\
\hline & & & Summer & $\bar{F} a 11$ & \\
\hline $\begin{array}{l}\text { Very Good } \\
\text { Good } \\
\text { Fair } \\
\text { Poor } \\
\text { Very Poor }\end{array}$ & $\begin{array}{r}\text { A11 } \\
0.6 \\
8.1 \\
40.7 \\
26.3 \\
24.3\end{array}$ & $\begin{array}{c}\text { Hours, Alongshore } \\
1.6 \\
12.2 \\
42.3 \\
27.4 \\
16.5\end{array}$ & $\begin{array}{r}1.1 \\
9.3 \\
30.4 \\
24.8 \\
34.4\end{array}$ & $\begin{array}{r}0.0 \\
8.6 \\
42.9 \\
26.8 \\
21.7\end{array}$ & $\begin{array}{r}0.0 \\
2.7 \\
47.6 \\
24.5 \\
25.1\end{array}$ \\
\hline $\begin{array}{l}\text { Very Good } \\
\text { Good } \\
\text { Fair } \\
\text { Poor } \\
\text { Very Poor }\end{array}$ & $\begin{array}{l}\text { Day, } \\
1.5 \\
20.1 \\
71.8 \\
6.6 \\
0.0\end{array}$ & $\begin{array}{r}\text { Al ong shore } \\
3.5 \\
29.8 \\
64.2 \\
2.5 \\
0.0\end{array}$ & $\begin{array}{r}2.8 \\
22.8 \\
64.6 \\
9.8 \\
0.0\end{array}$ & $\begin{array}{r}0.0 \\
21.0 \\
75.3 \\
3.7 \\
0.0\end{array}$ & $\begin{array}{r}0.0 \\
6.7 \\
82.8 \\
10.6 \\
0.0\end{array}$ \\
\hline $\begin{array}{l}\text { Very Good } \\
\text { Good } \\
\text { Fair } \\
\text { Poor } \\
\text { Very Poor }\end{array}$ & $\begin{array}{l}\text { Night } \\
0.0 \\
0.0 \\
19.4 \\
39.7 \\
40.3\end{array}$ & $\begin{array}{r}\text { t, Alongshore } \\
0.0 \\
0.0 \\
28.4 \\
43.6 \\
28.0\end{array}$ & $\begin{array}{r}0.0 \\
0.0 \\
6.8 \\
35.2 \\
58.1\end{array}$ & $\begin{array}{r}0.0 \\
0.0 \\
20.1 \\
43.1 \\
36.8\end{array}$ & $\begin{array}{r}0.0 \\
0.0 \\
23.7 \\
34.1 \\
42.2\end{array}$ \\
\hline
\end{tabular}


Table 7.15 (continued)

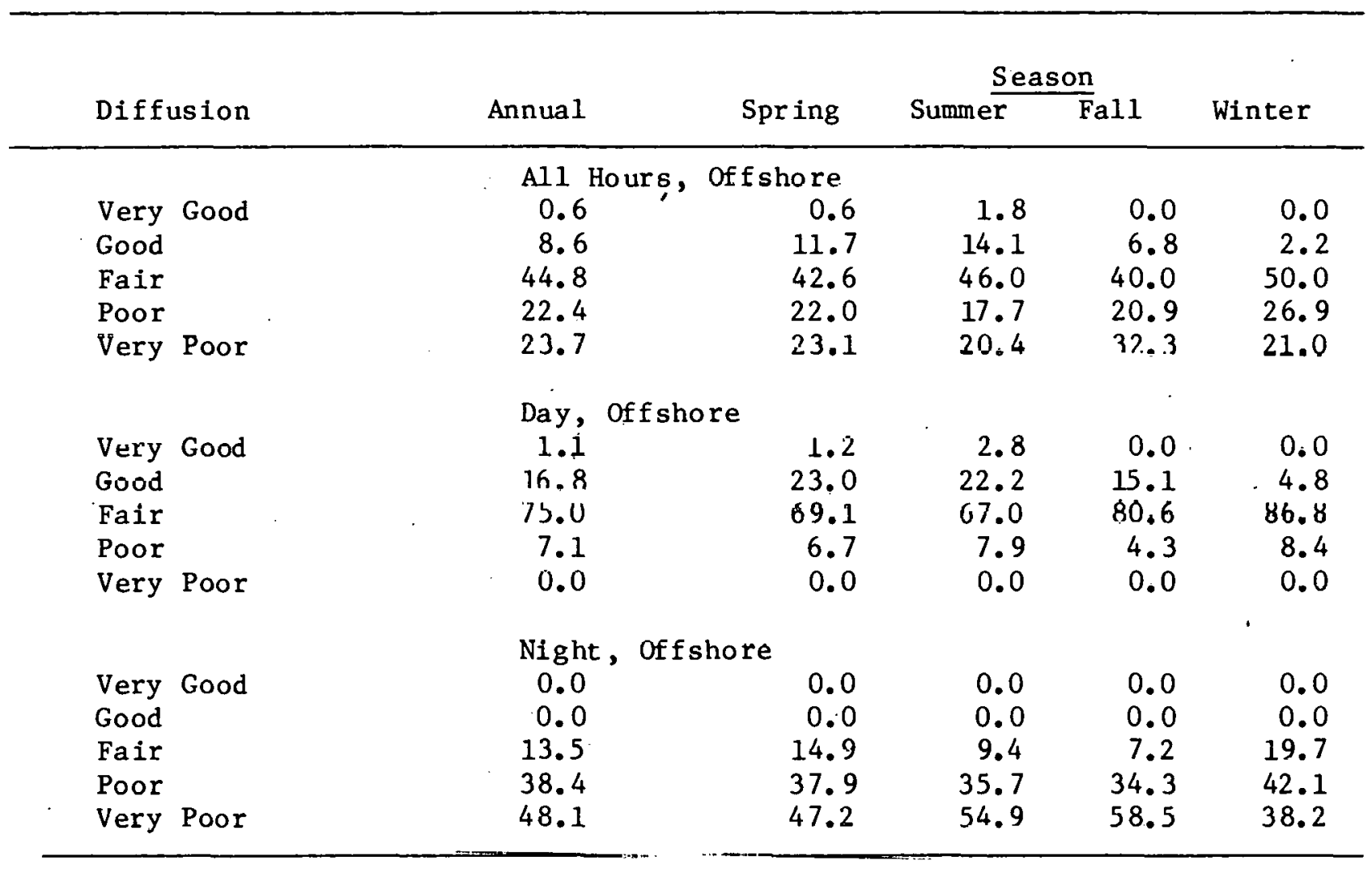




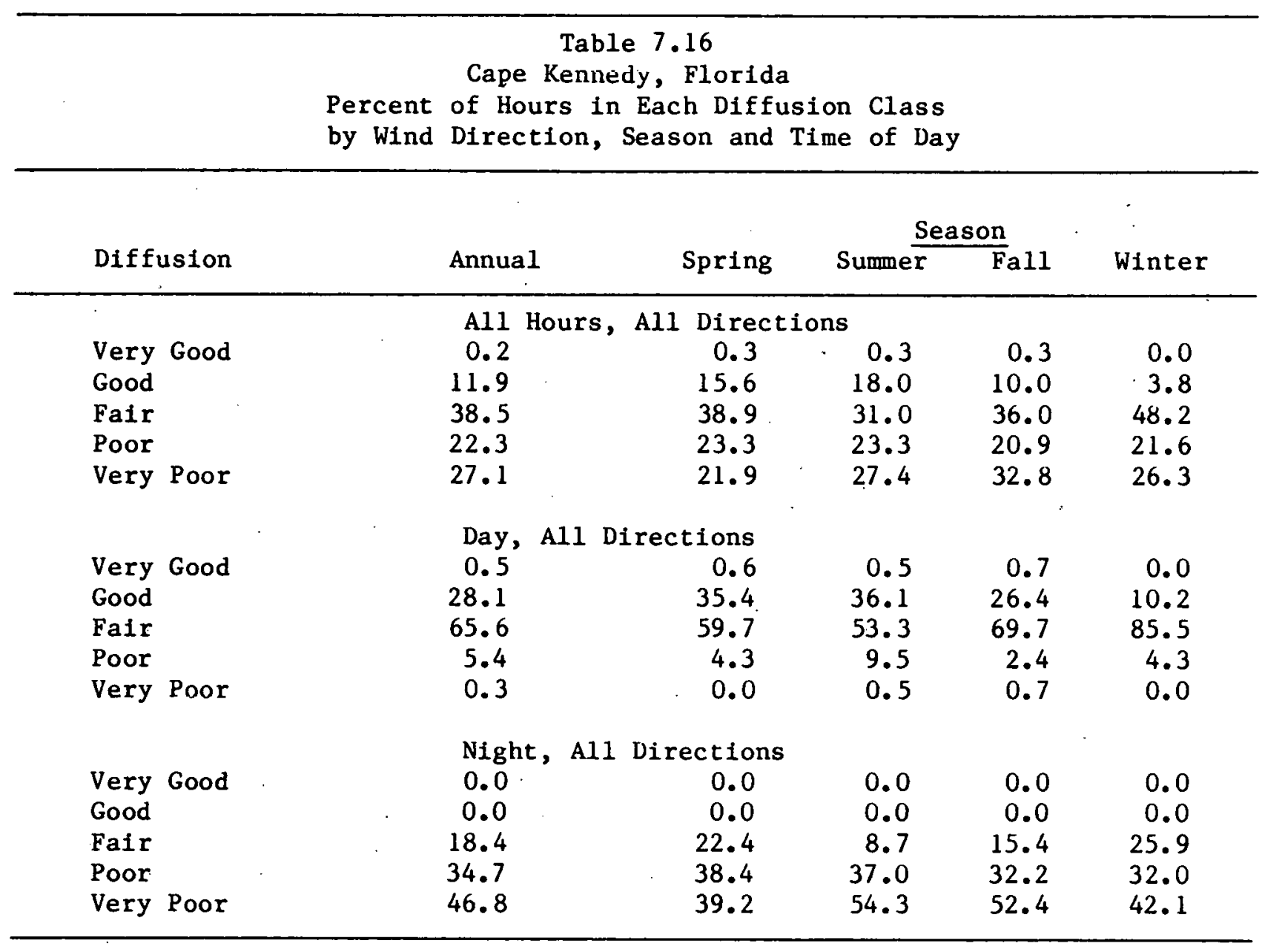


Tab1e 7.16 (continued)

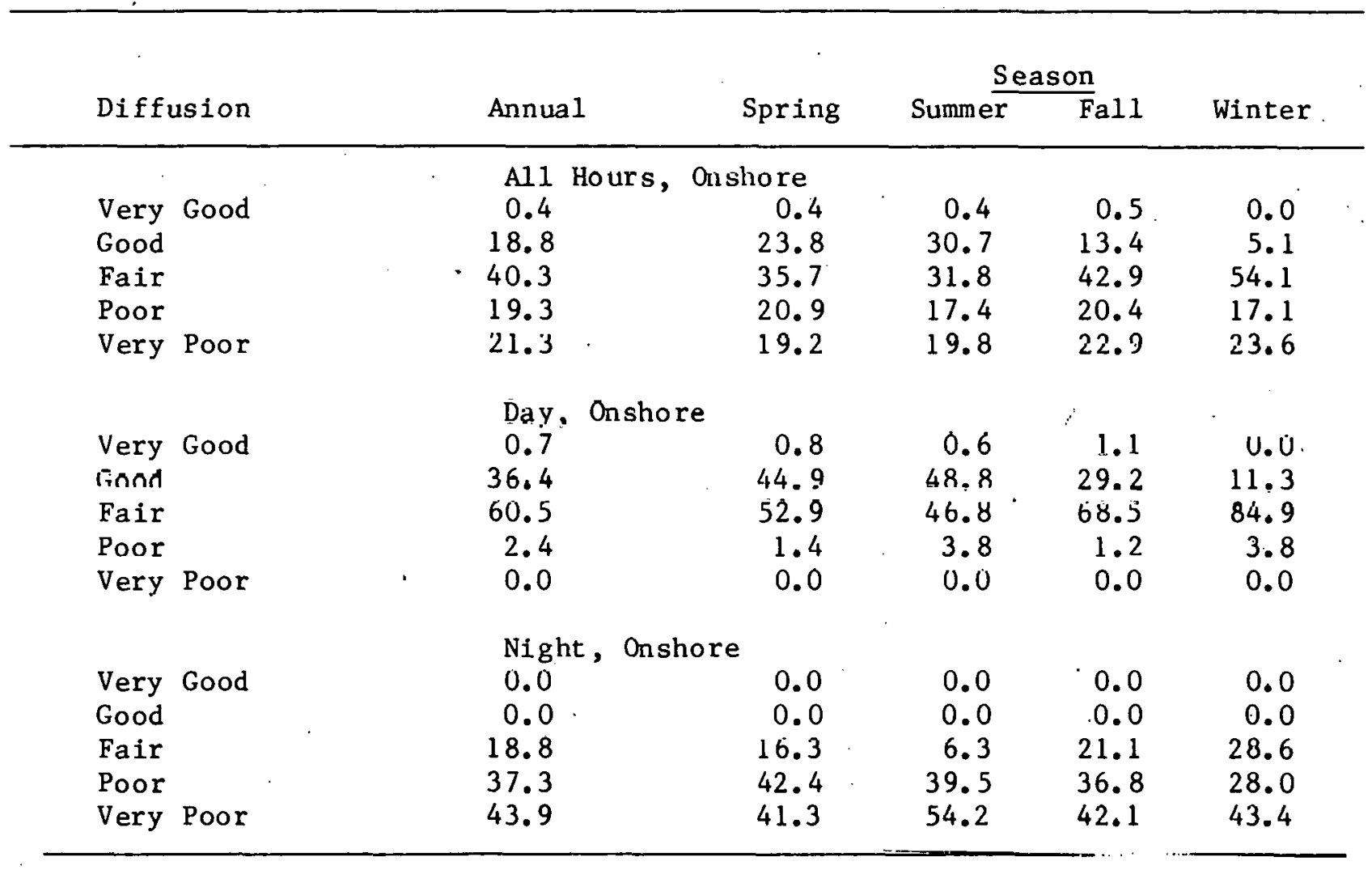


Table 7.16 (continued)

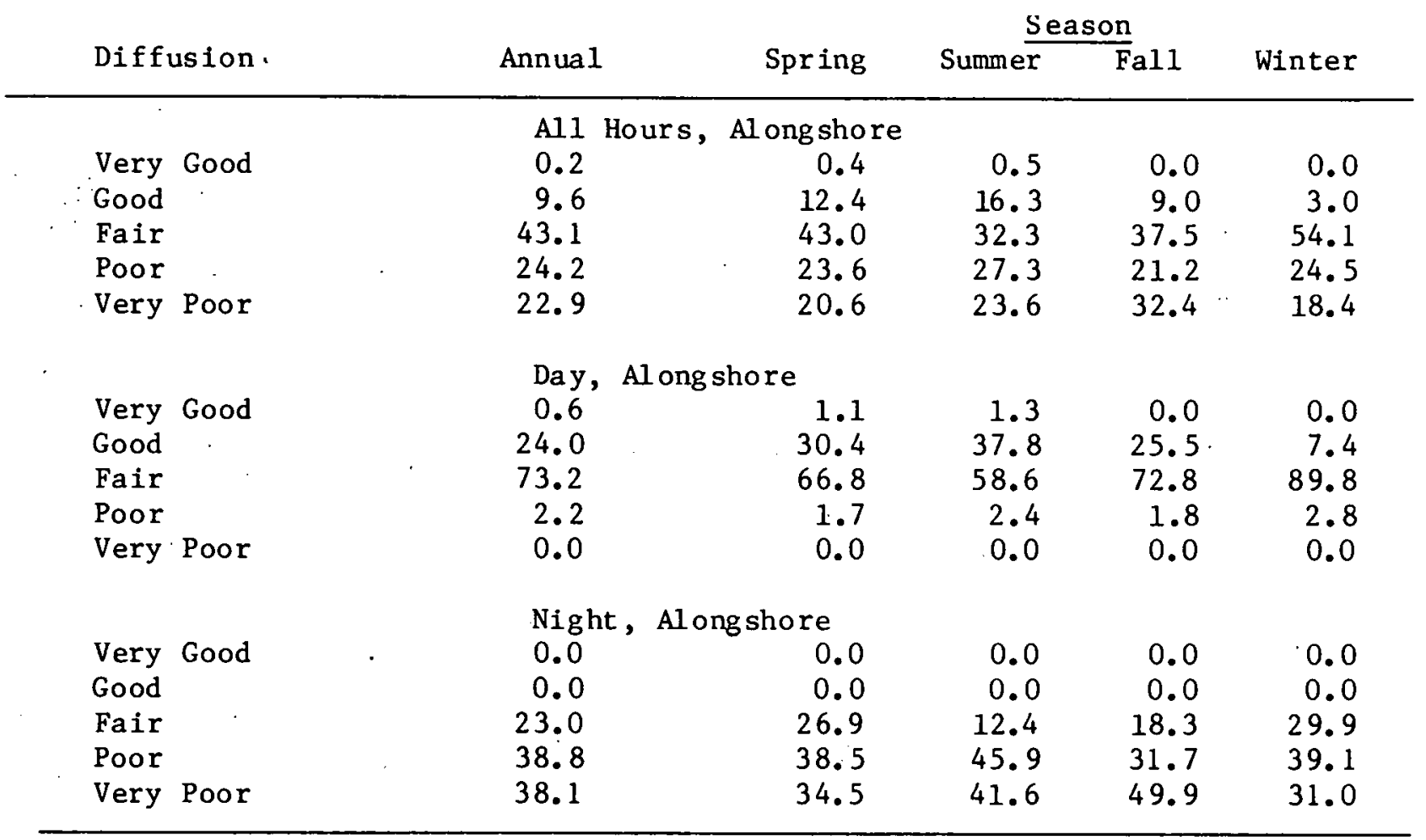


Tabie 7.16 (continued)

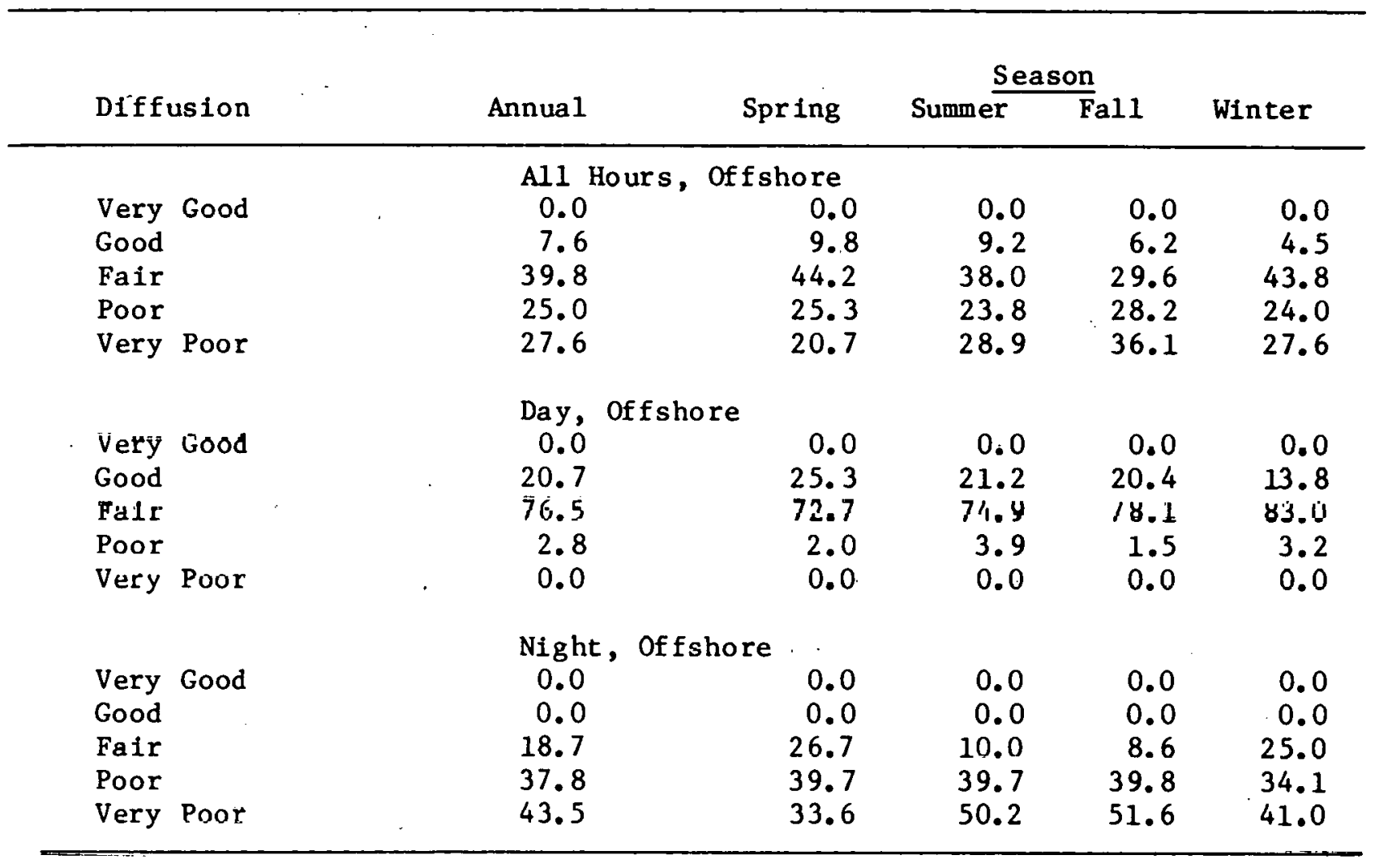




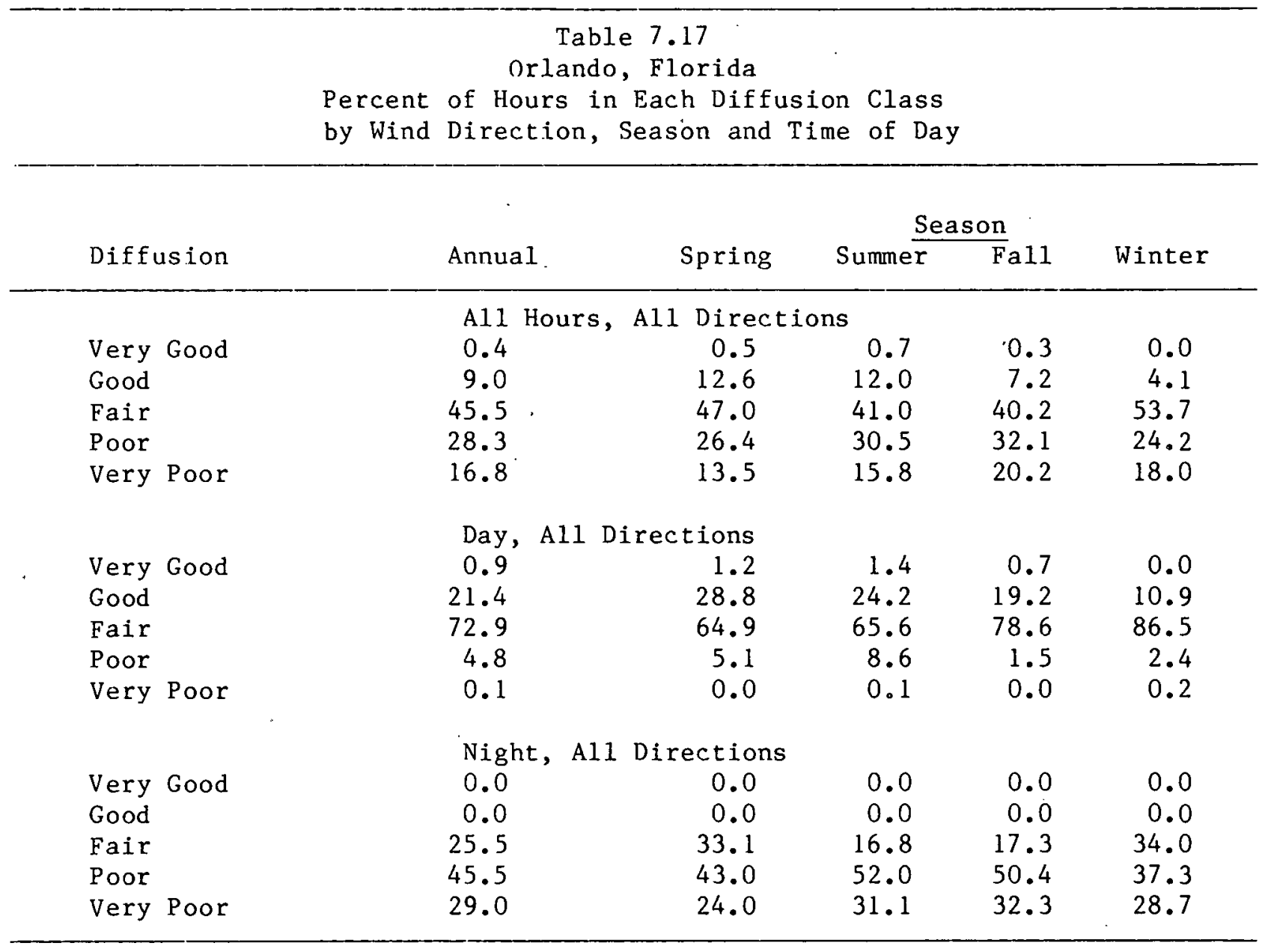


Table 7.17 (continued)

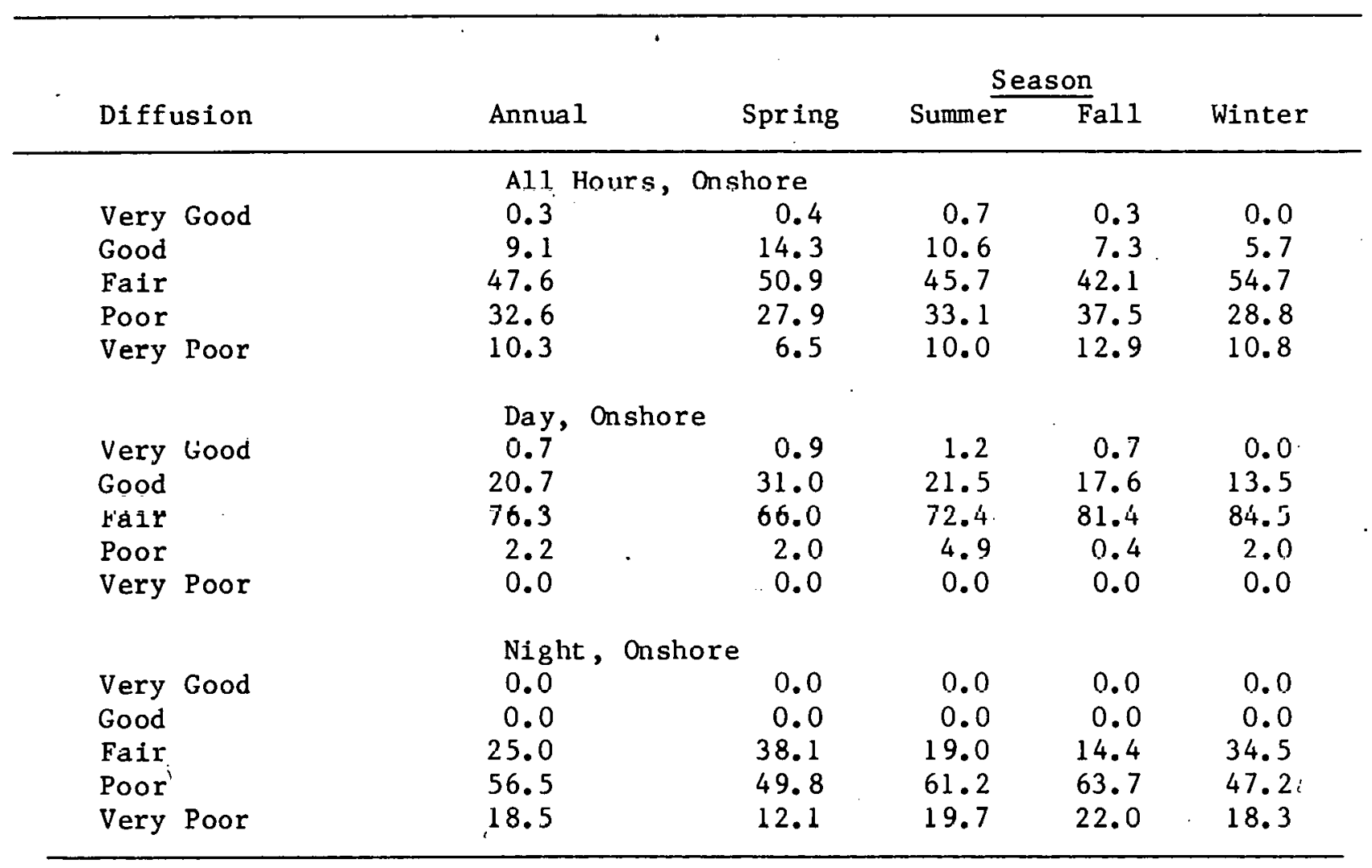


Table 7.17 (continued)

\begin{tabular}{|c|c|c|c|c|c|}
\hline \multirow[b]{2}{*}{ Diffusion } & \multirow[b]{2}{*}{ Annual } & - & \multicolumn{2}{|c|}{ Season } & \multirow[b]{2}{*}{ Winter } \\
\hline & & Spring & Summer & Fall & \\
\hline \multicolumn{6}{|c|}{ Al1 Hours, Alongshore } \\
\hline Very Good & $\therefore \quad 0.7$ & 0.6 & 1.8 & 0.4 & 0.0 \\
\hline Good & 8.5 & 9.2 & 13.9 & 8.7 & 2.8 \\
\hline Fair & 47.1 & 46.3 & 38.5 & 43.5 & 57.8 \\
\hline Poor & 33.4 & 34.0 & 34.3 & 38.5 & 27.8 \\
\hline Very Poor & 10.4 & 9.8 & 11.4 & 8.8 & 11.6 \\
\hline \multicolumn{6}{|c|}{ Day, Al ong shore } \\
\hline Very Good & 1.7 & 1.4 & 3.8 & 1.1 & 0.0 \\
\hline Good & 21.6 & 21.7 & 28.9 & 25.5 & 8.8 \\
\hline Fair & 73.5 & 71.8 & 63.6 & 70.5 & 91.2 \\
\hline Poor & 3.1 & 5.1 & 3.7 & 2.8 & 0.0 \\
\hline Very Poor & 0.0 & 0.0 & 0.0 & 0.0 & 0.0 \\
\hline \multicolumn{6}{|c|}{ Night, Al ong shore } \\
\hline Very Good & 0.0 & 0.0 & 0.0 & 0.0 & 0.0 \\
\hline Good & 0.0 & 0.0 & 0.0 & 0.0 & 0.0 \\
\hline Fair & 29.9 & 27.4 & 14.9 & 29.4 & 41.7 \\
\hline Poor & 52.9 & 55.4 & 63.1 & 57.1 & 41.2 \\
\hline Very Poor & 17.2 & 17.1 & 22.0 & 13.4 & 17.1 \\
\hline
\end{tabular}


Table 7.17 (continued)

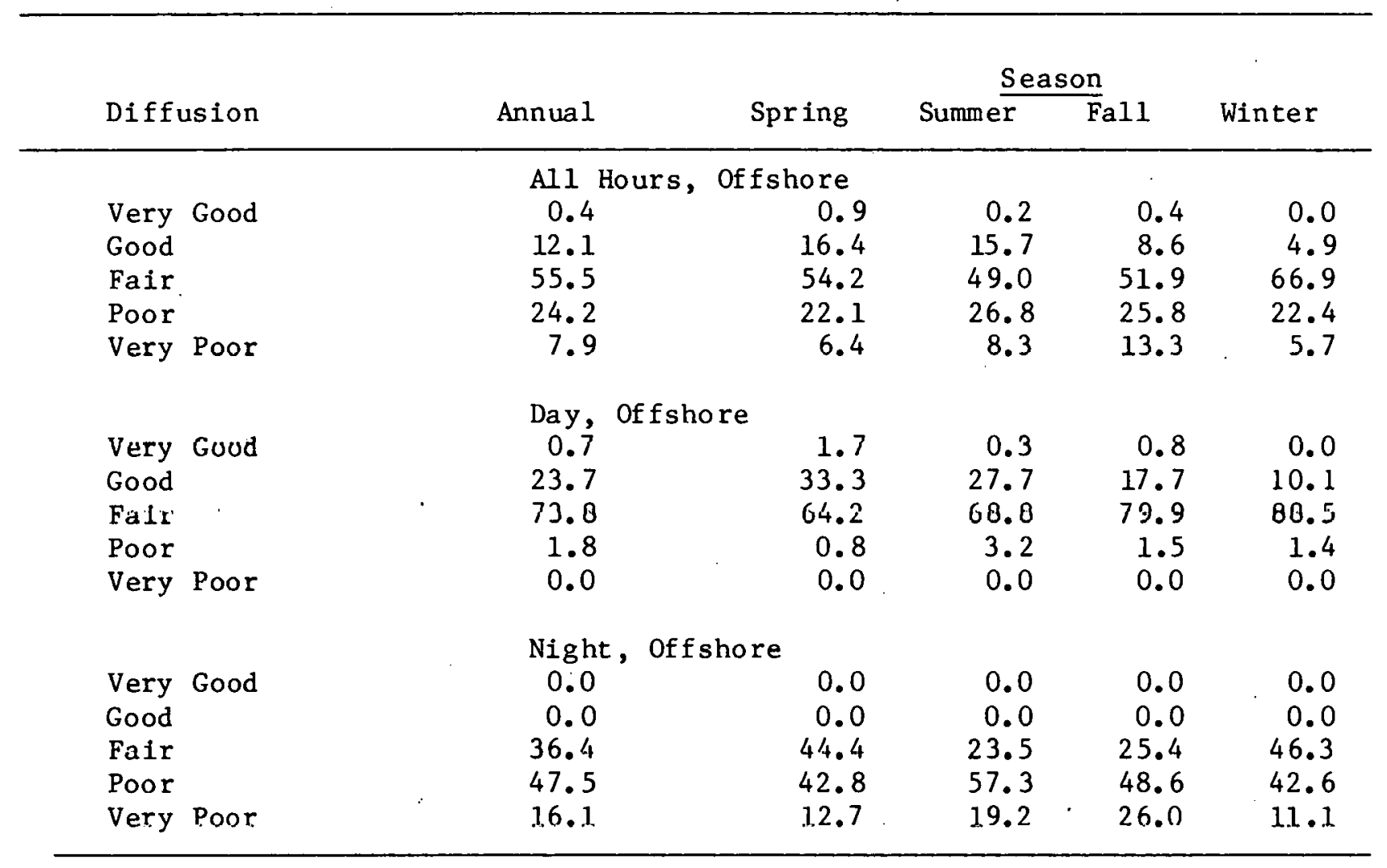




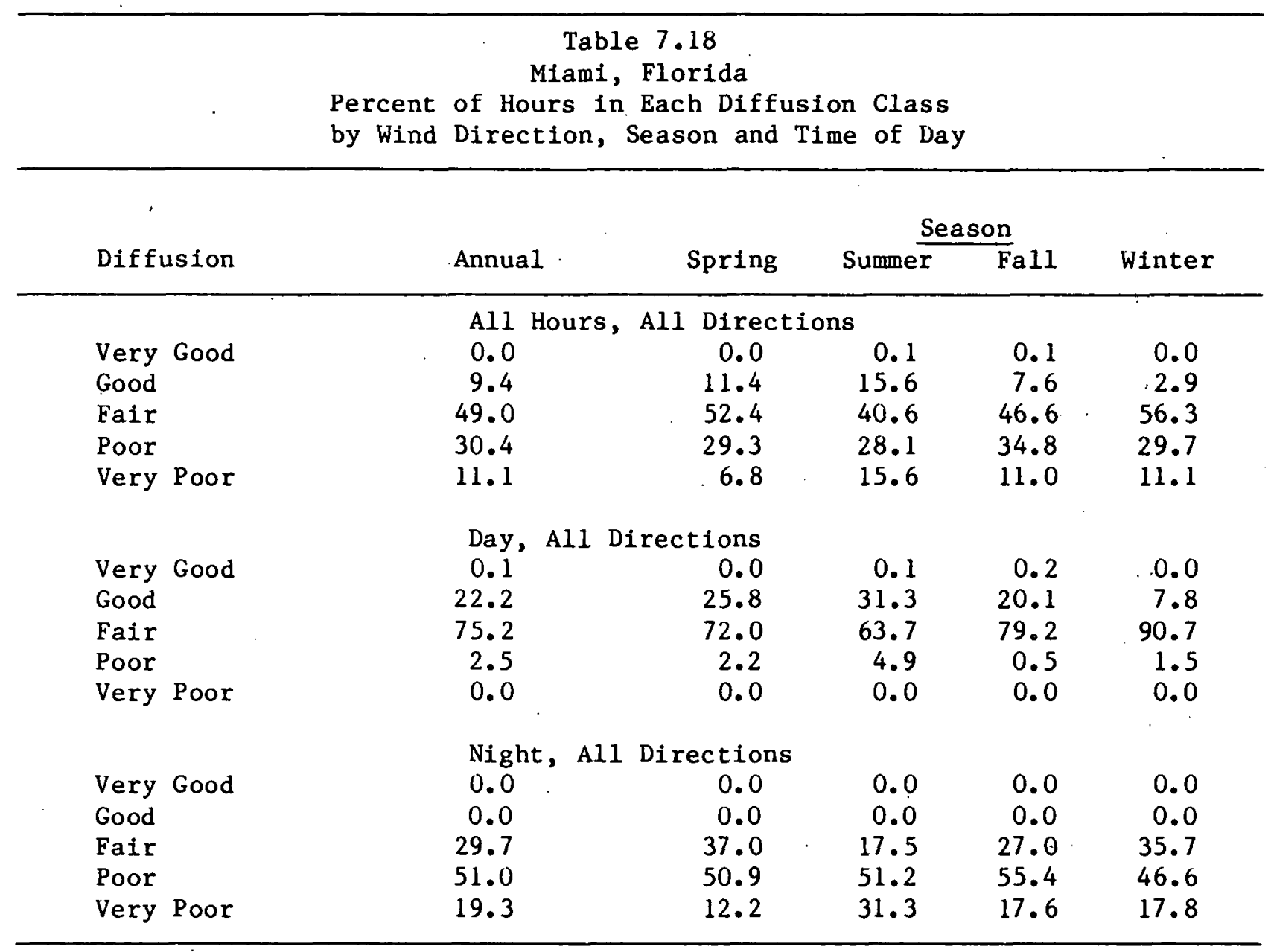


Table 7.18 (continued)

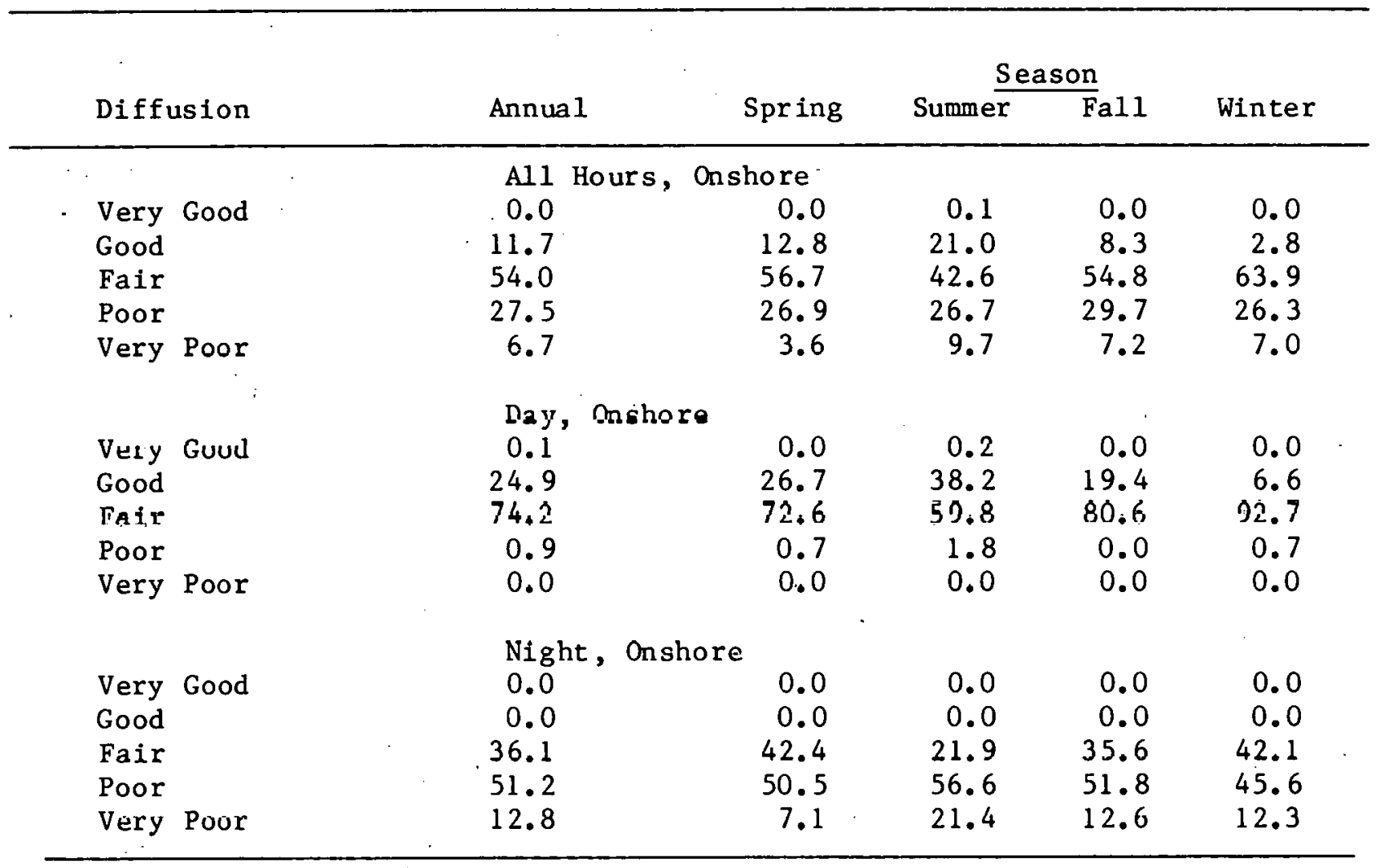


Table 7.18 (continued)

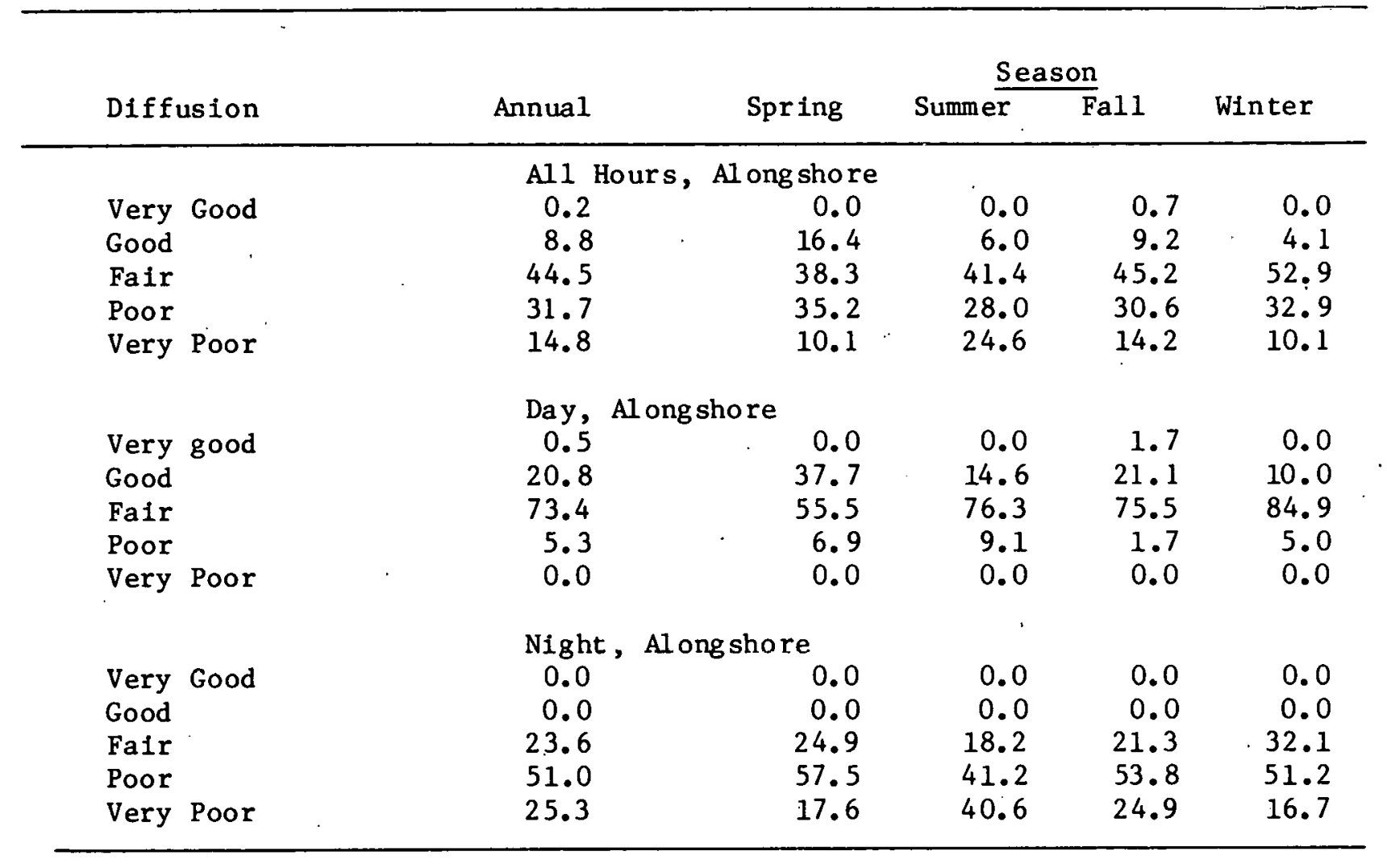


Table 7.18 (continued)

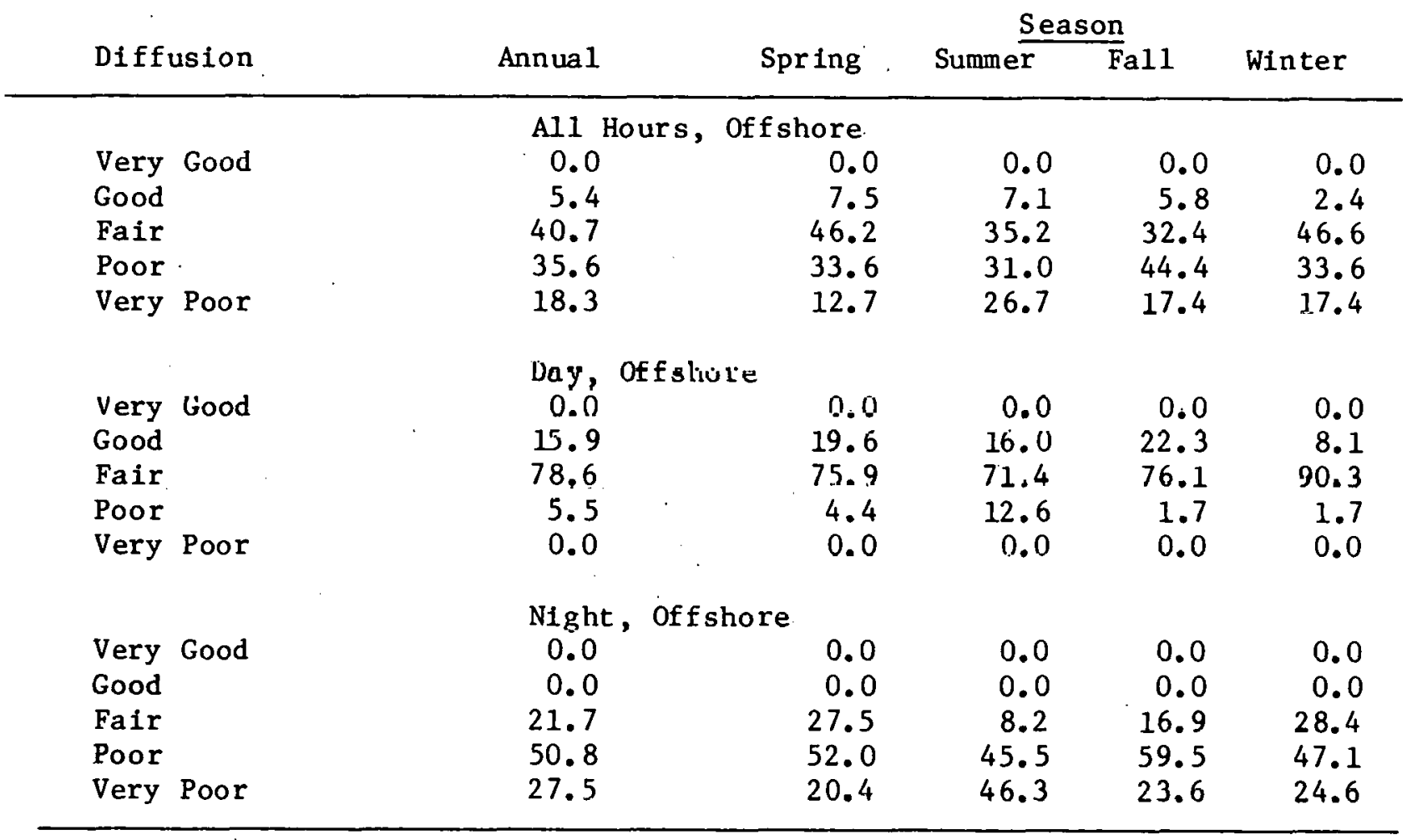




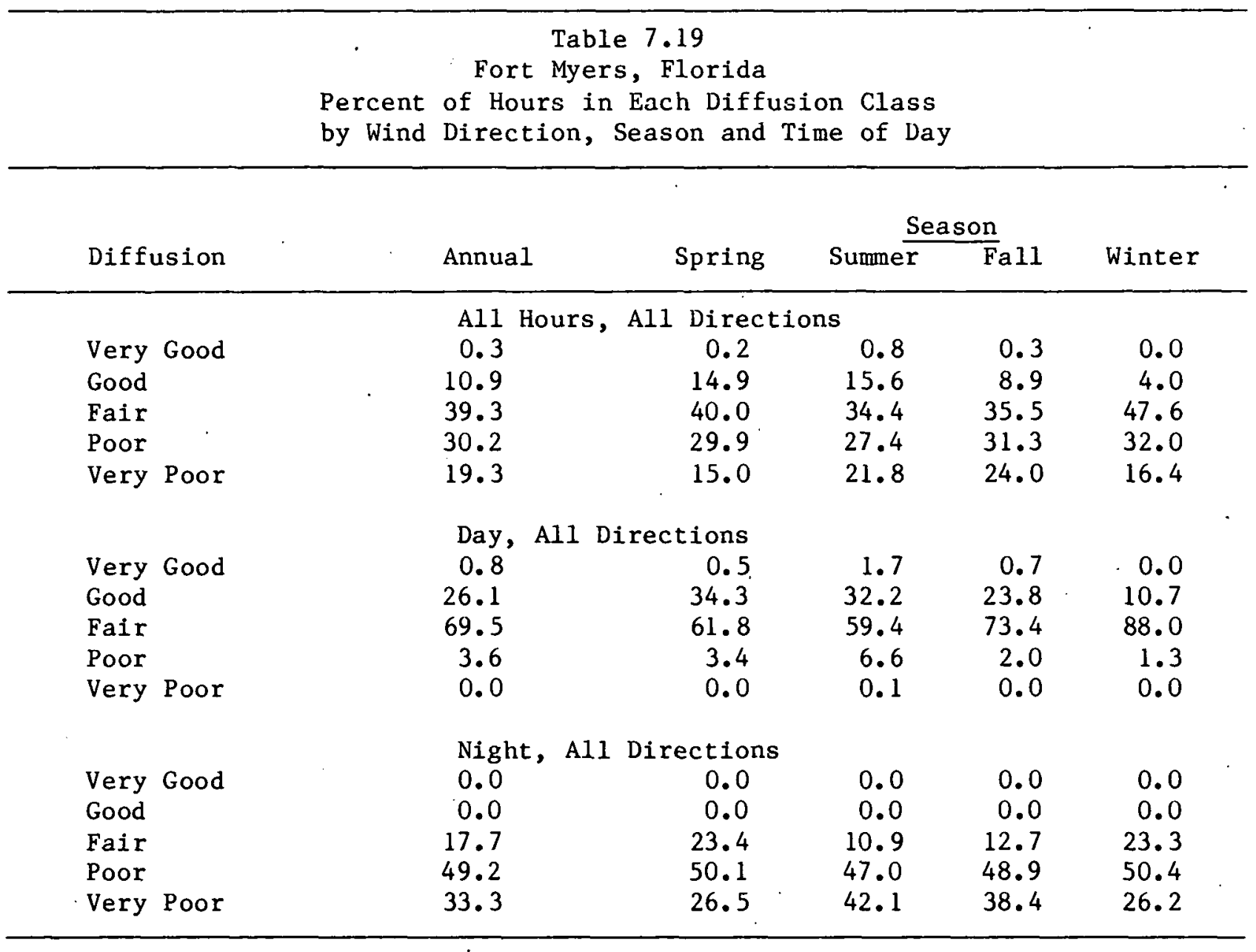


Table 7.19 (continued)

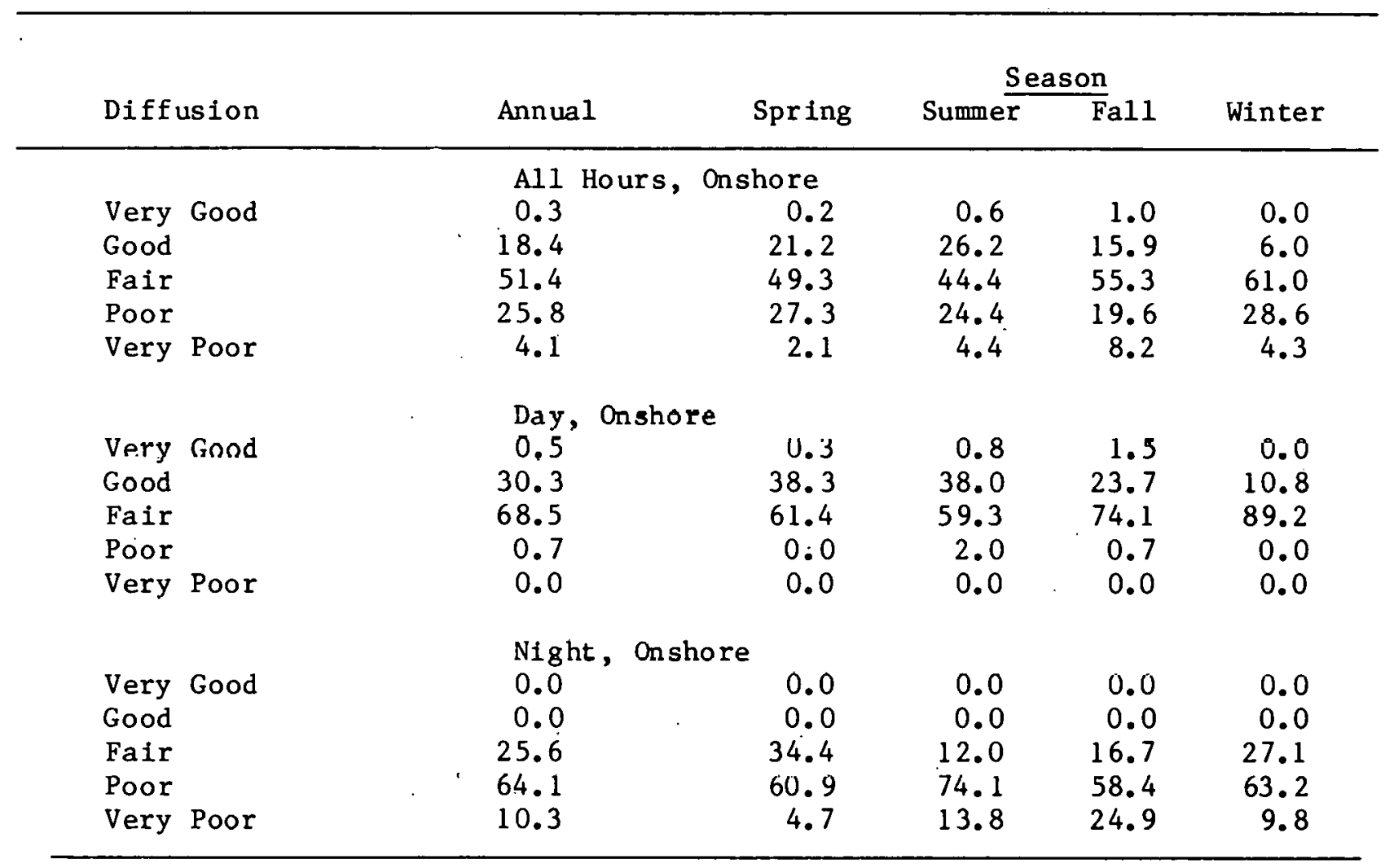


Table 7.19 (continued)

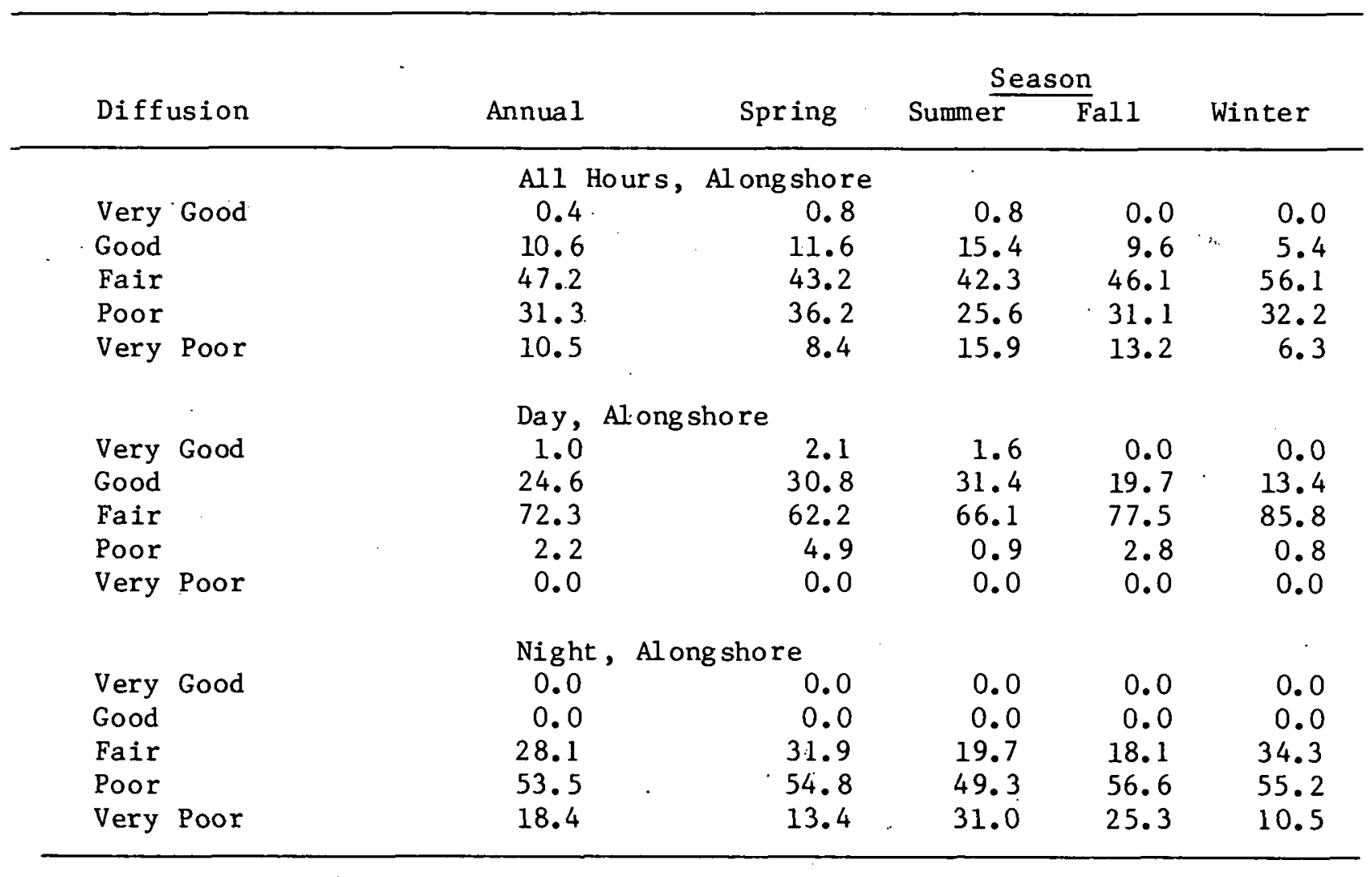


Table 7.19 (continued)

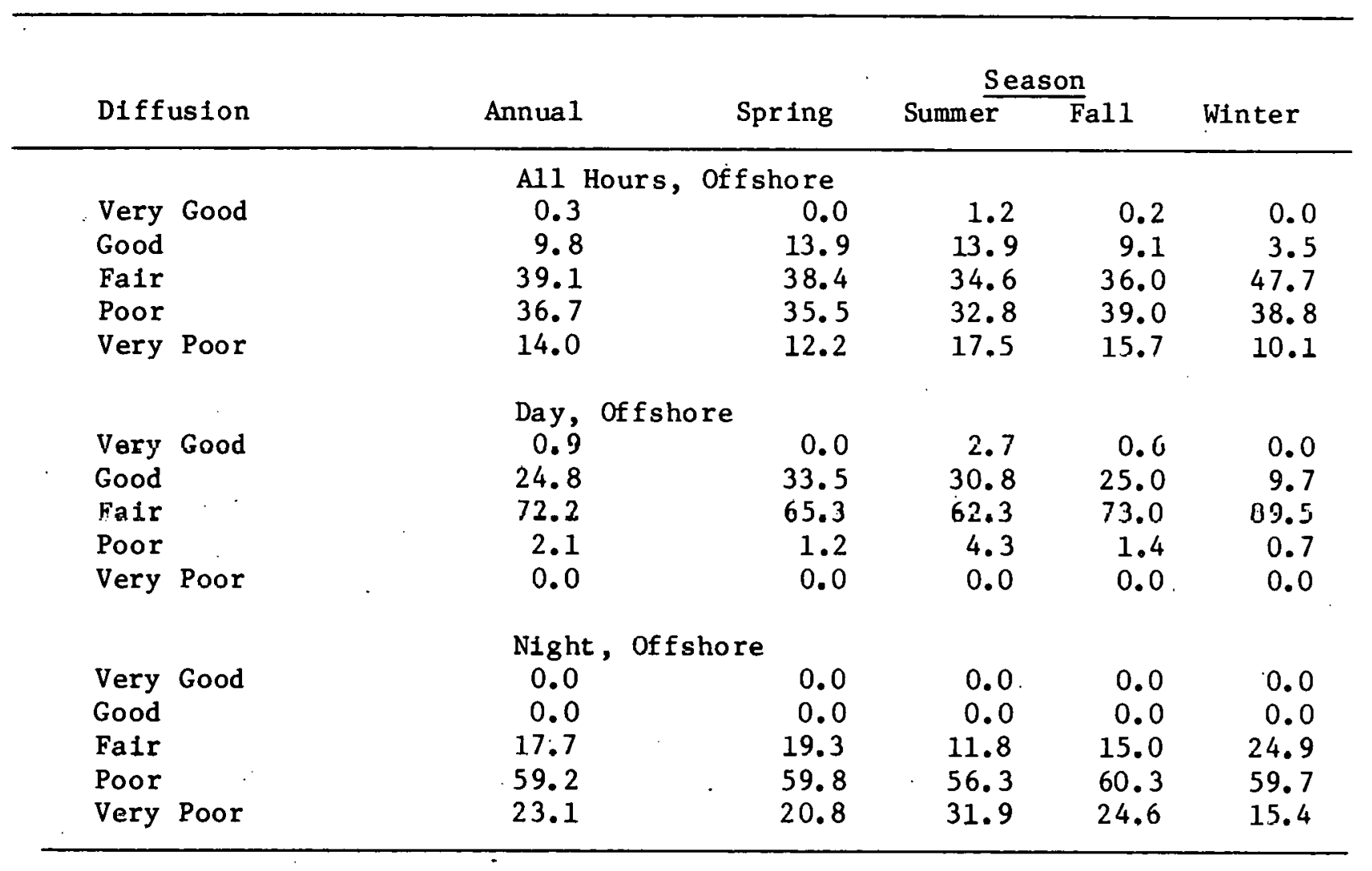




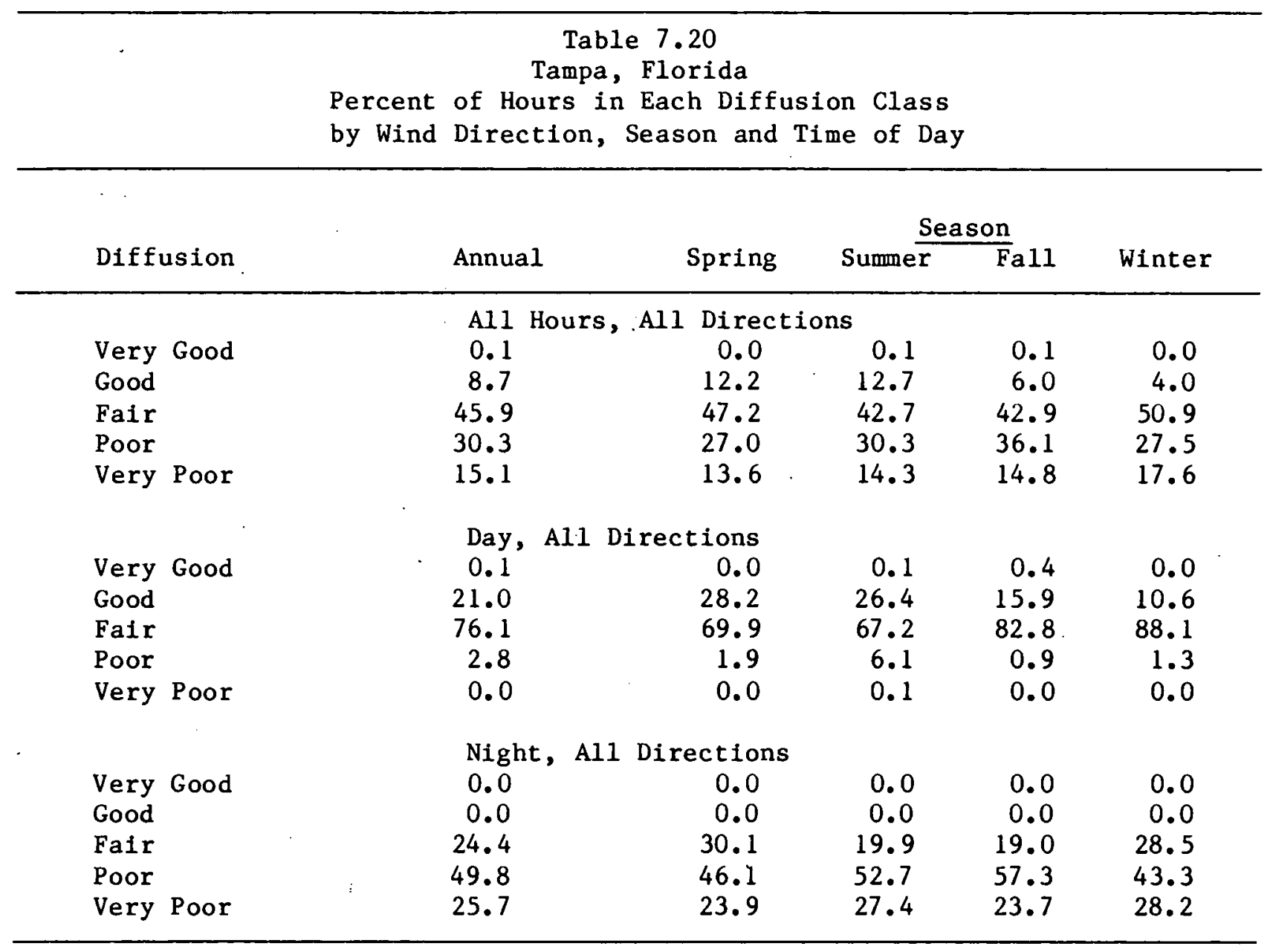


Table 7.20 (continued)

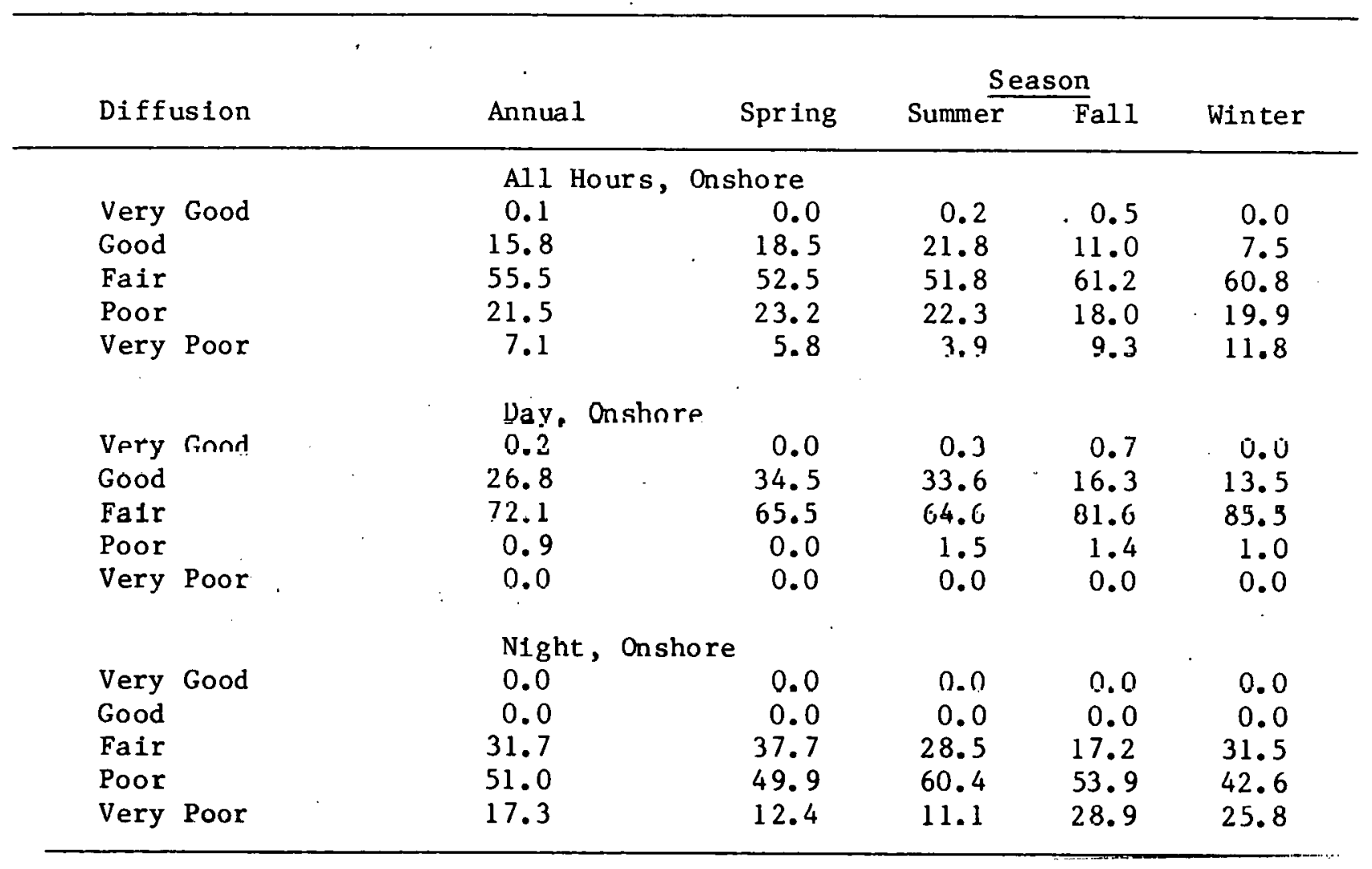


Table 7.20 (continued)

\begin{tabular}{|c|c|c|c|c|c|}
\hline \multirow{2}{*}{ Diffusion } & \multirow[b]{2}{*}{ Annual } & \multicolumn{4}{|c|}{ Season } \\
\hline & & Spring & Summer & Fal1 & Winter \\
\hline $\begin{array}{l}\text { Very Good } \\
\text { Good } \\
\text { Fair } \\
\text { Poor } \\
\text { Very Poor }\end{array}$ & $\begin{array}{l}\text { Al1 H } \\
0.1 \\
7.4 \\
50.2 \\
33.3 \\
9.0\end{array}$ & $\begin{array}{r}\text { lours, Alongshore } \\
0.0 \\
11.3 \\
49.6 \\
29.0 \\
10.1\end{array}$ & $\begin{array}{r}0.0 \\
12.1 \\
46.2 \\
34.4 \\
7.2\end{array}$ & $\begin{array}{r}0.4 \\
4.8 \\
46.0 \\
39.5 \\
9.3\end{array}$ & $\begin{array}{r}0.0 \\
2.5 \\
56.4 \\
32.0 \\
9.1\end{array}$ \\
\hline $\begin{array}{l}\text { Very Good } \\
\text { Good } \\
\text { Fair } \\
\text { Foor } \\
\text { Very Poor }\end{array}$ & $\begin{array}{r}\text { Day, } \\
0.2 \\
18.1 \\
79.7 \\
2.0 \\
\therefore 0.0\end{array}$ & $\begin{array}{rr}\text { Al ong sho re } & \\
0.0 \\
26.1 \\
71.6 \\
2.3 \\
0.0\end{array}$ & $\begin{array}{r}0.0 \\
25.3 \\
70.2 \\
4.5 \\
0.0\end{array}$ & $\begin{array}{r}0.9 \\
12.9 \\
86.2 \\
0.0 \\
0.0\end{array}$ & $\begin{array}{r}0.0 \\
6.9 \\
93.1 \\
0.0 \\
0.0\end{array}$ \\
\hline $\begin{array}{l}\text { Very Good } \\
\text { Good } \\
\text { Fair } \\
\text { Poor } \\
\text { Very Poor }\end{array}$ & $\begin{array}{l}\text { N1ght } \\
0.0 \\
00.0 \\
29.7 \\
55.2 \\
15.2\end{array}$ & $\begin{array}{r}\text {, Al ong shore } \\
0.0 \\
0.0 \\
33.8 \\
48.7 \\
17.5\end{array}$ & $\begin{array}{r}0.0 \\
0.0 \\
24.3 \\
61.8 \\
13.9\end{array}$ & $\begin{array}{r}0.0 \\
0.0 \\
21.6 \\
63.6 \\
.14 .8\end{array}$ & $\begin{array}{r}0.0 \\
0.0 \\
35.4 \\
50.5 \\
14.0\end{array}$ \\
\hline
\end{tabular}


Table 7.20 (continued)

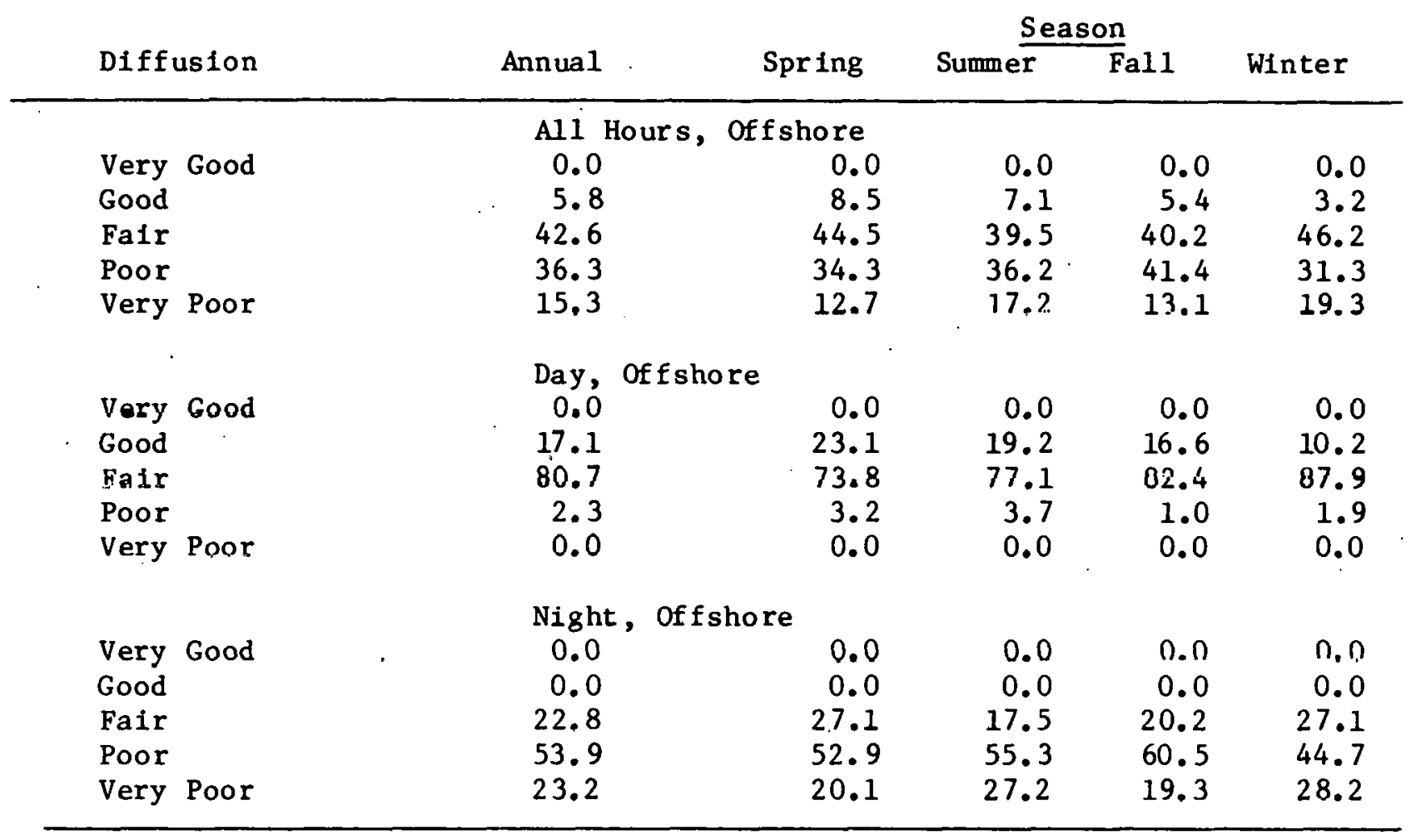




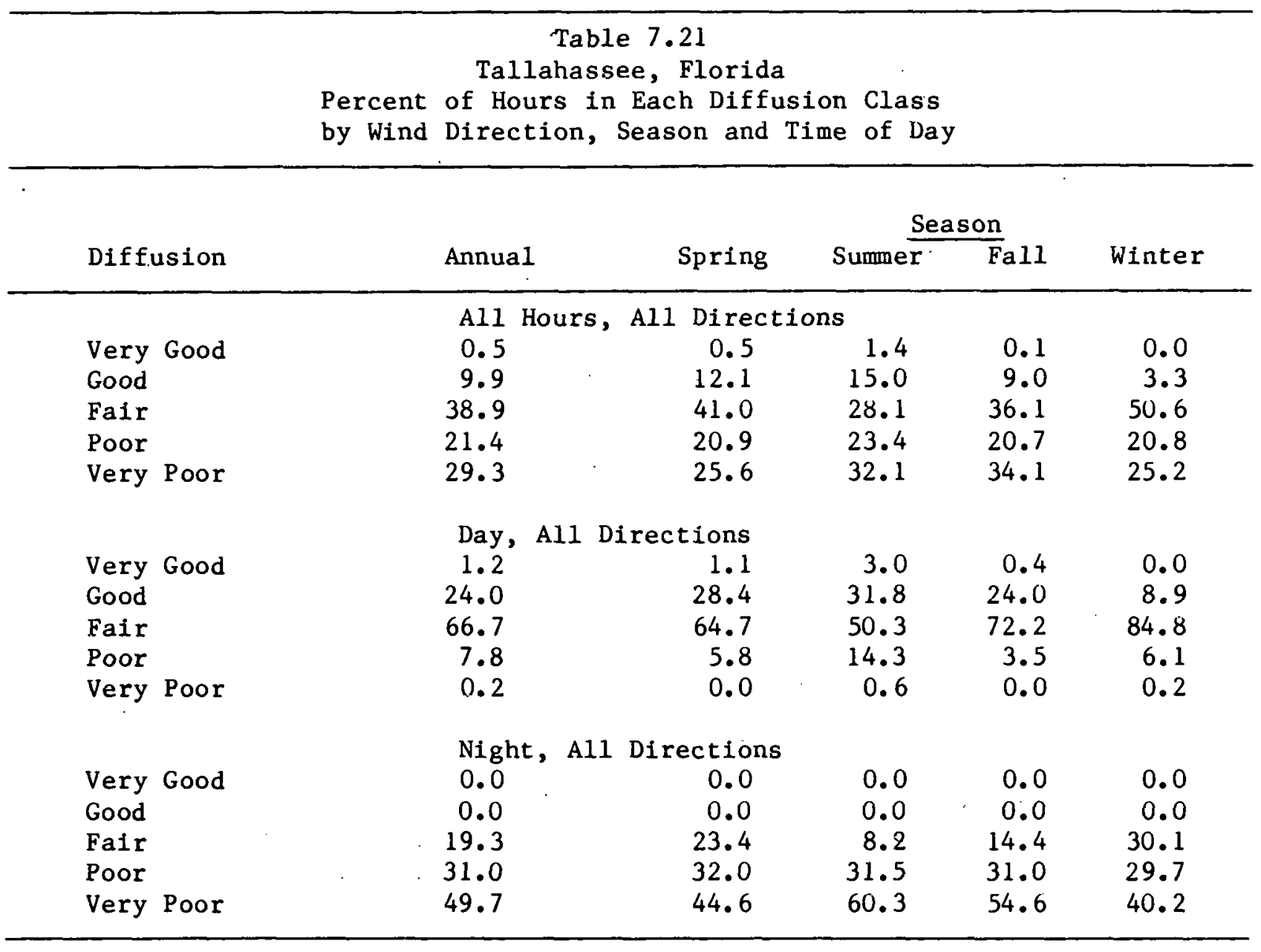


Table 7.21. (continued)

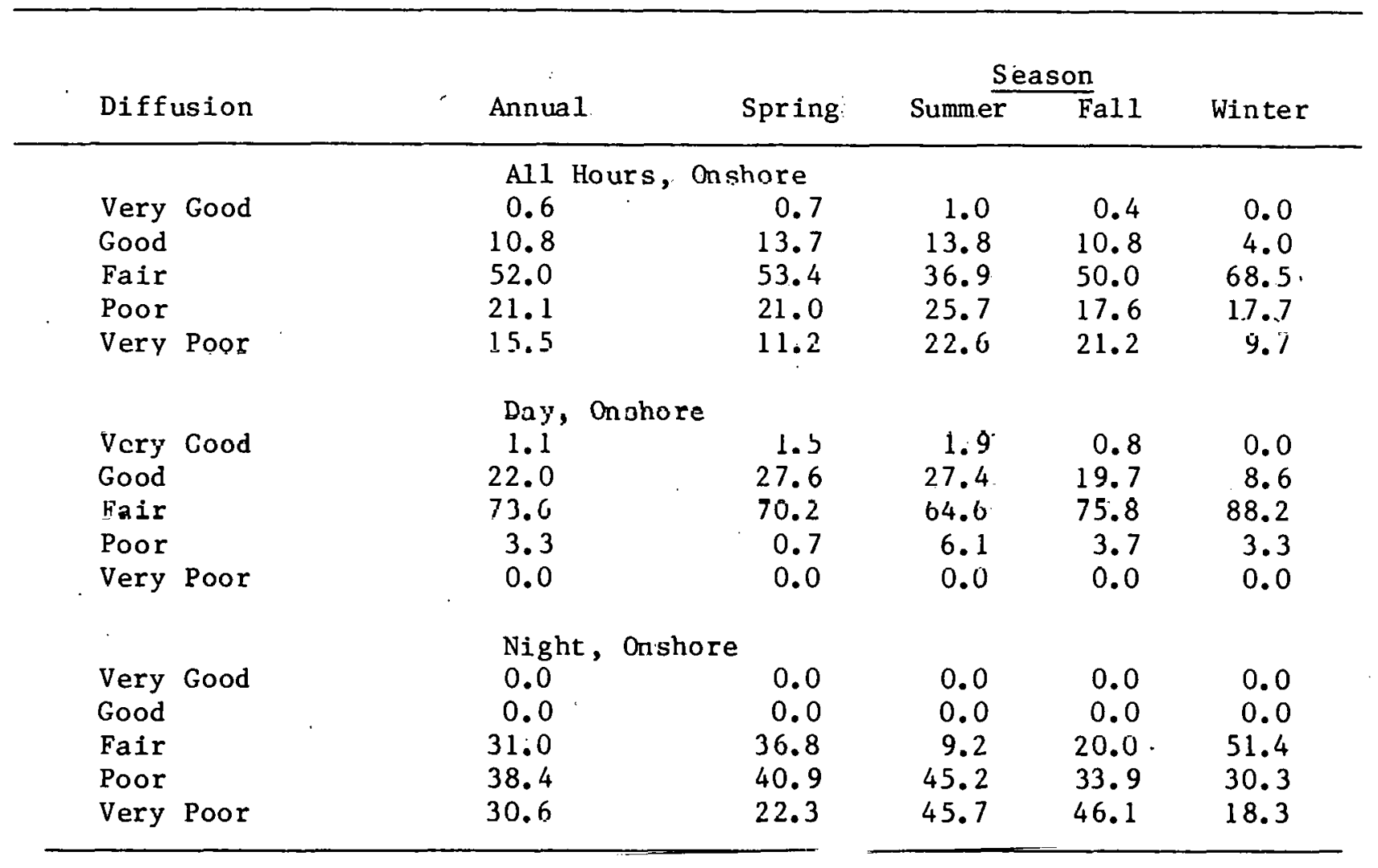




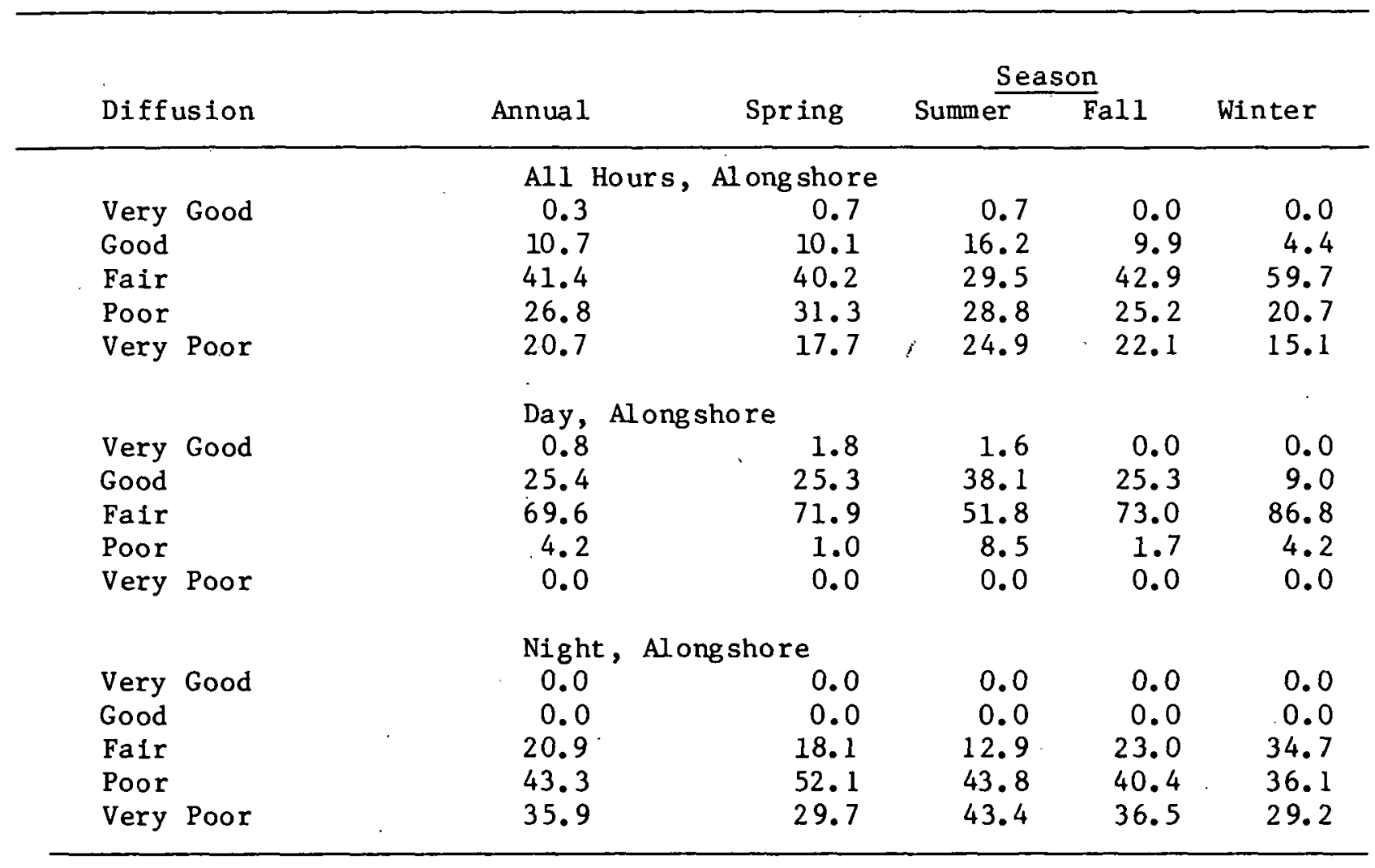


Table 7.21 (continued)

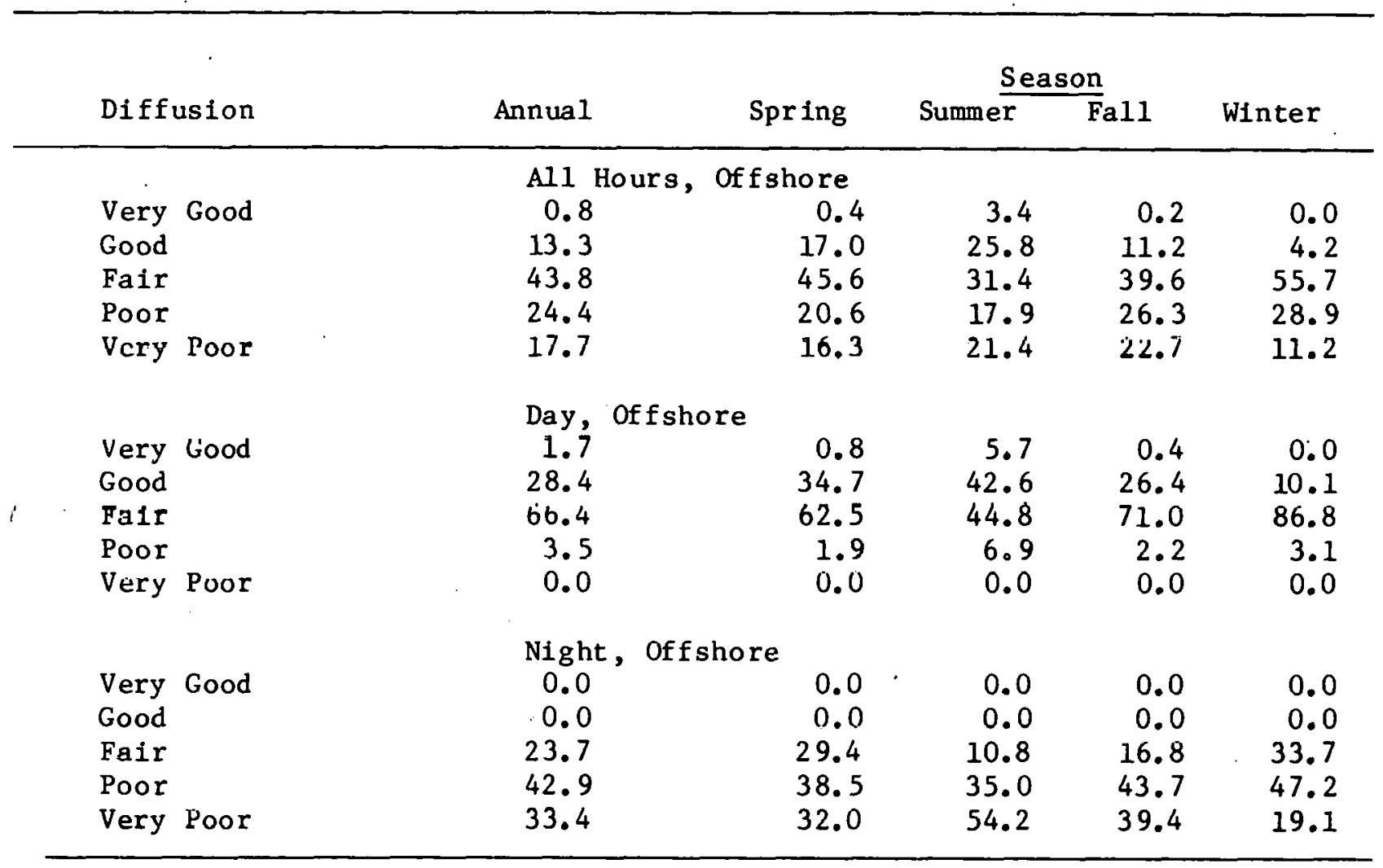




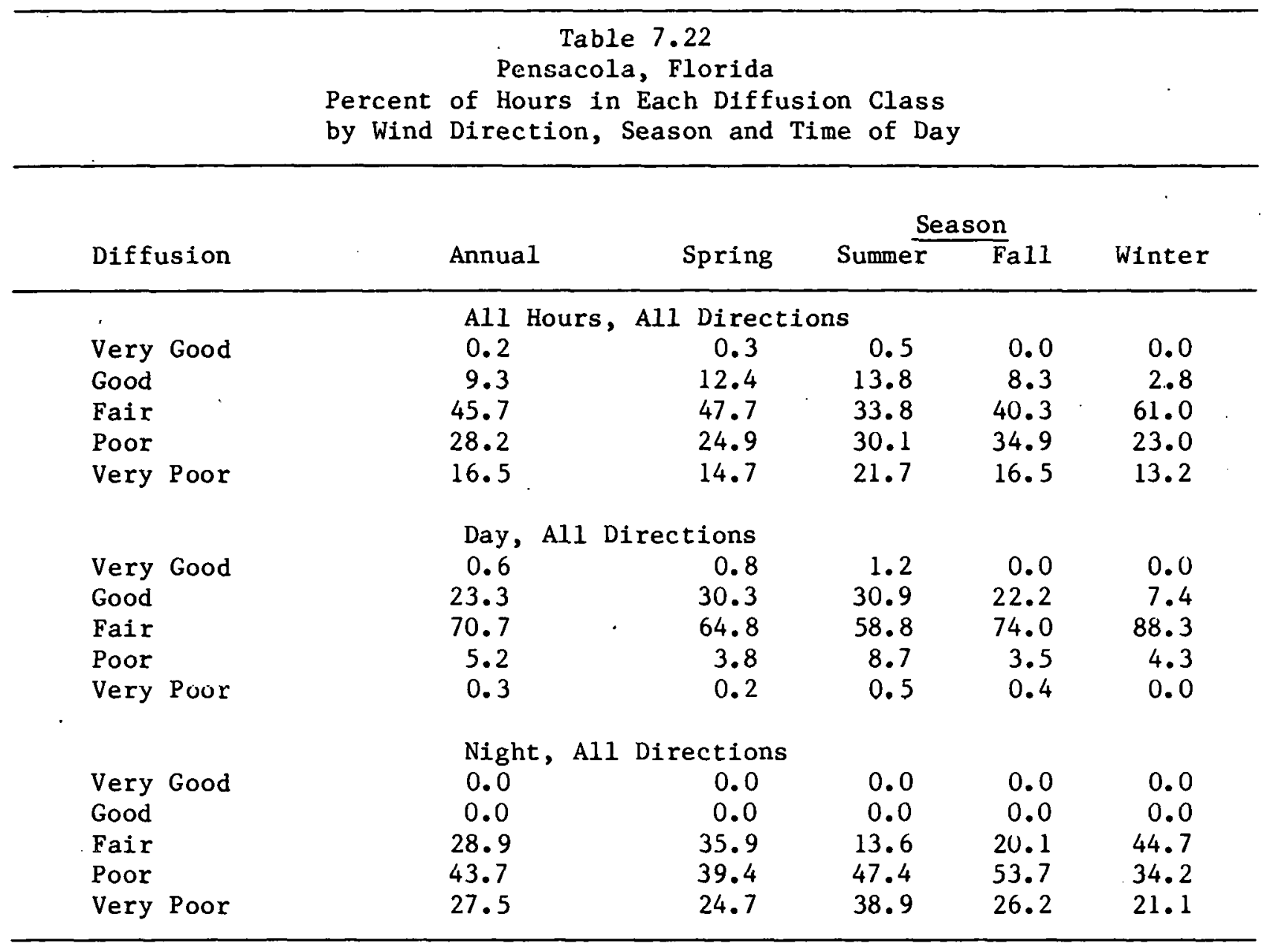


Table 7.22 (continued)

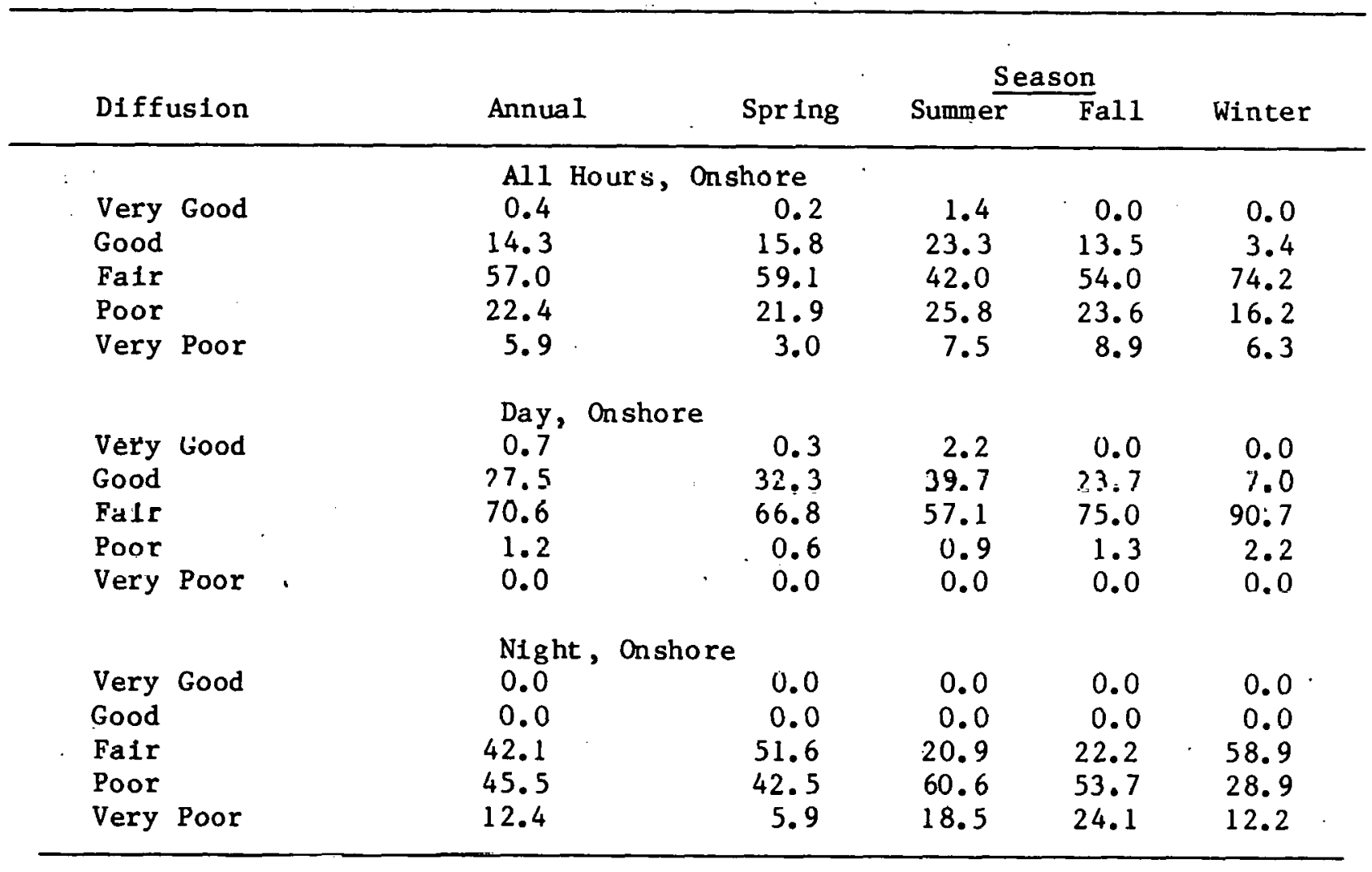


Table 7.22 (continued)

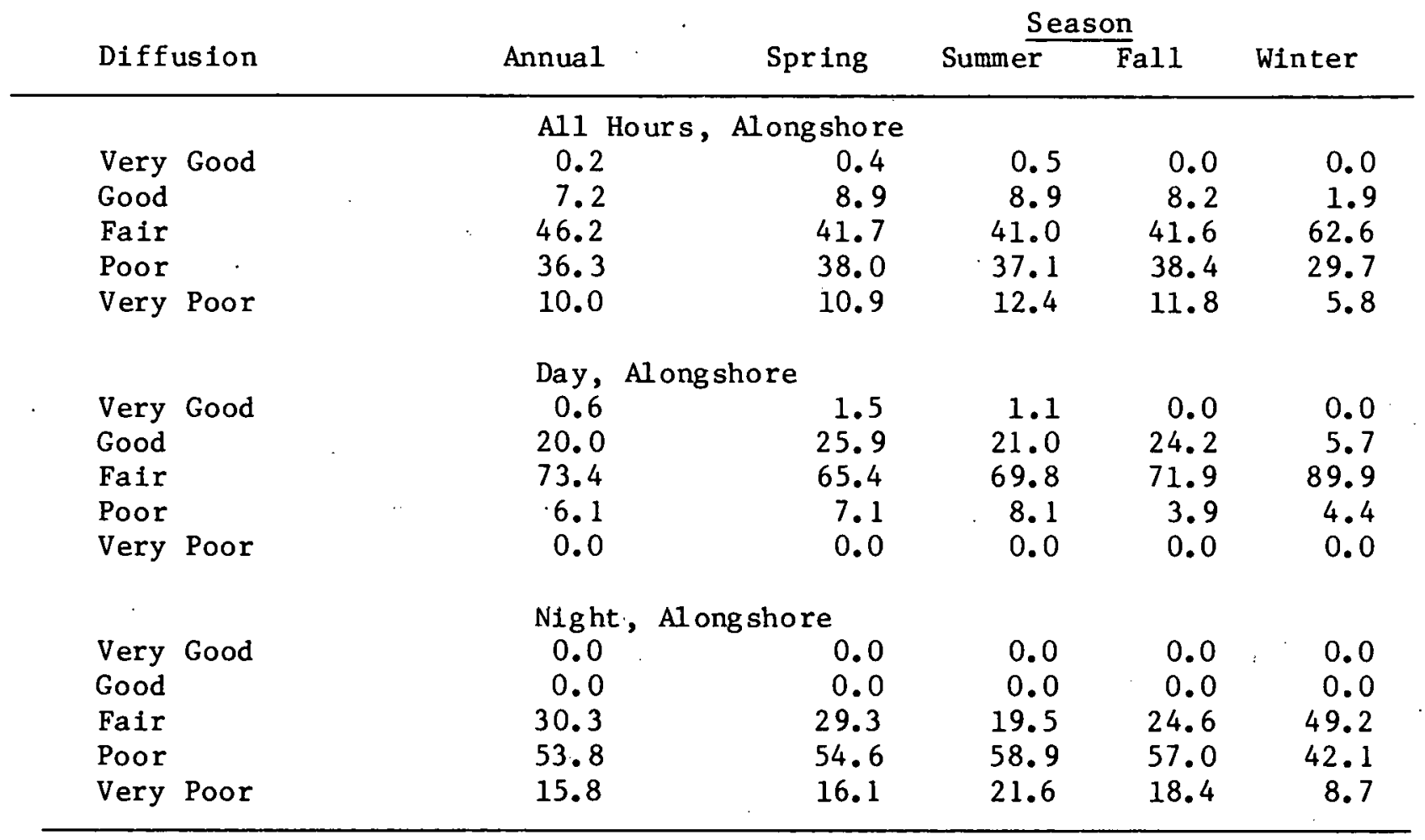


Table 7.22 (continued)

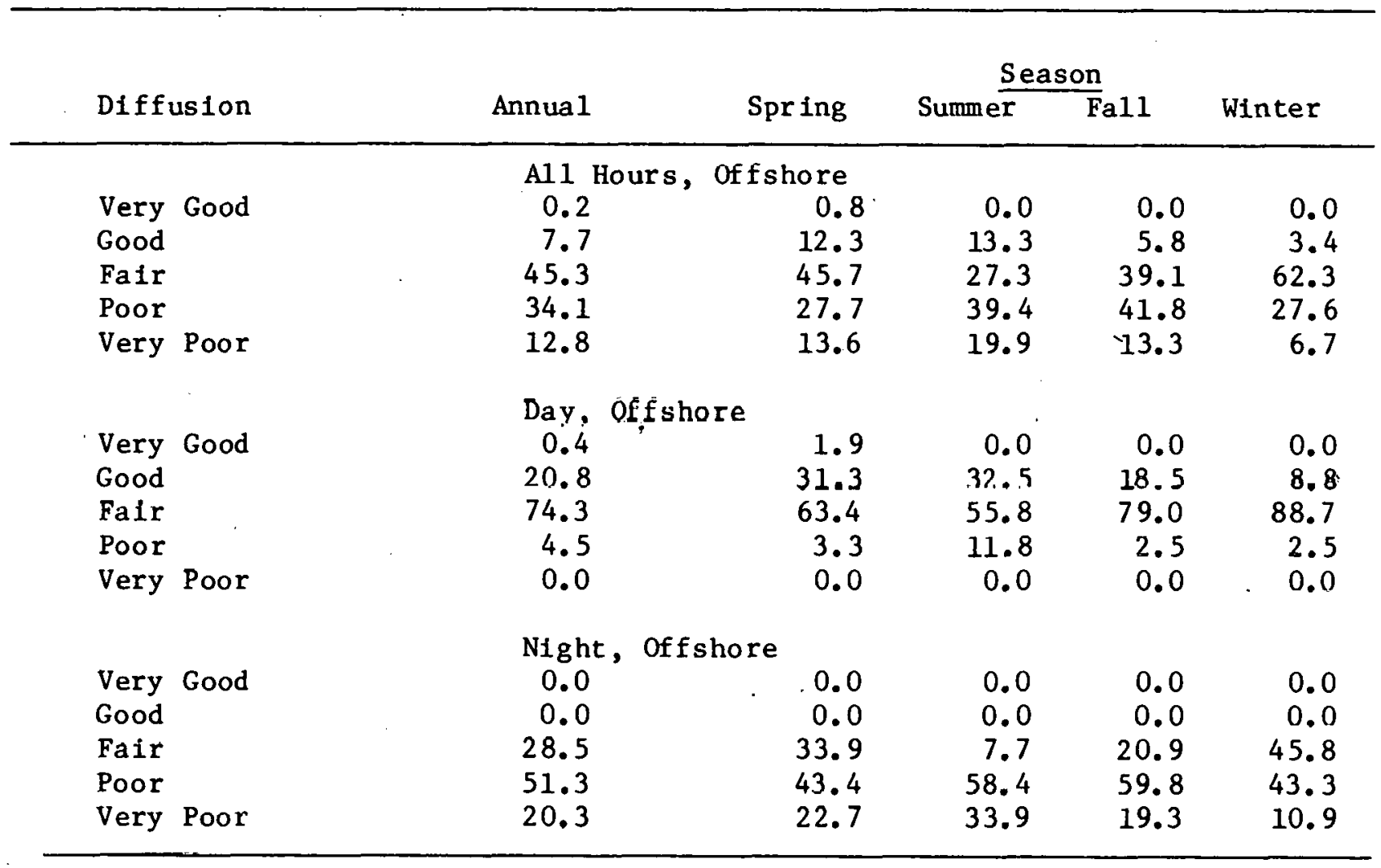




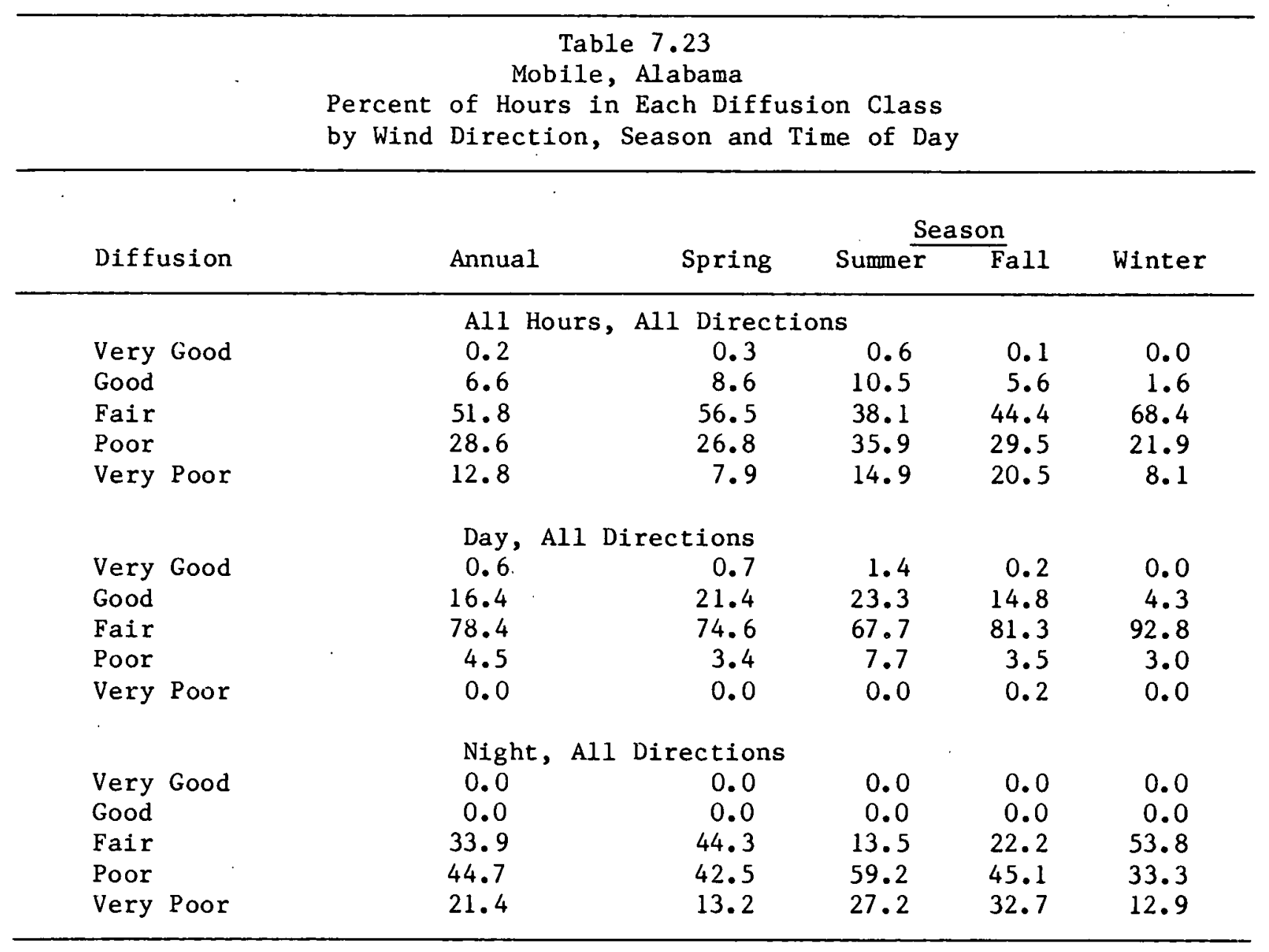


Table 7.23 (continued)

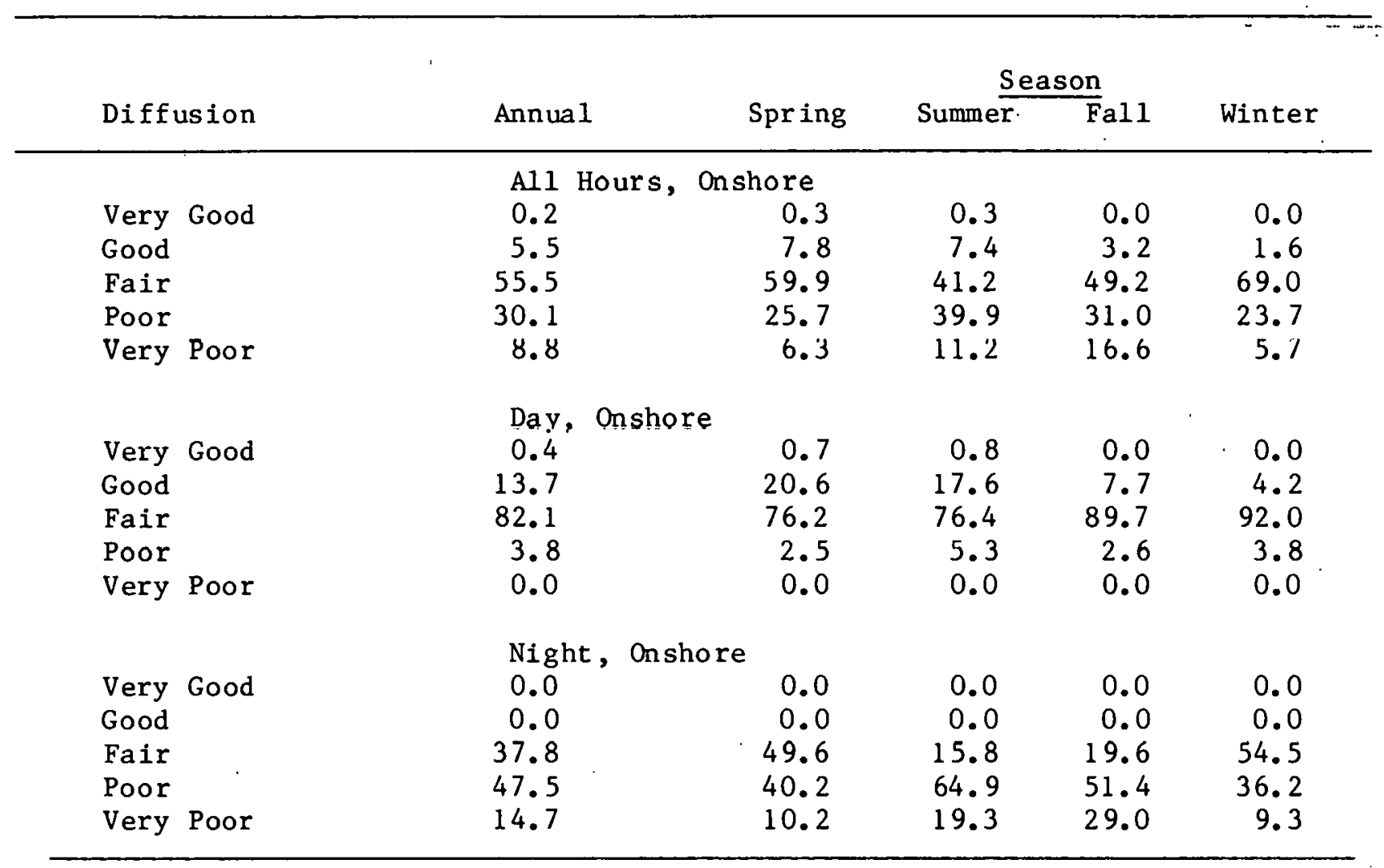


Table 7.23 (continued)

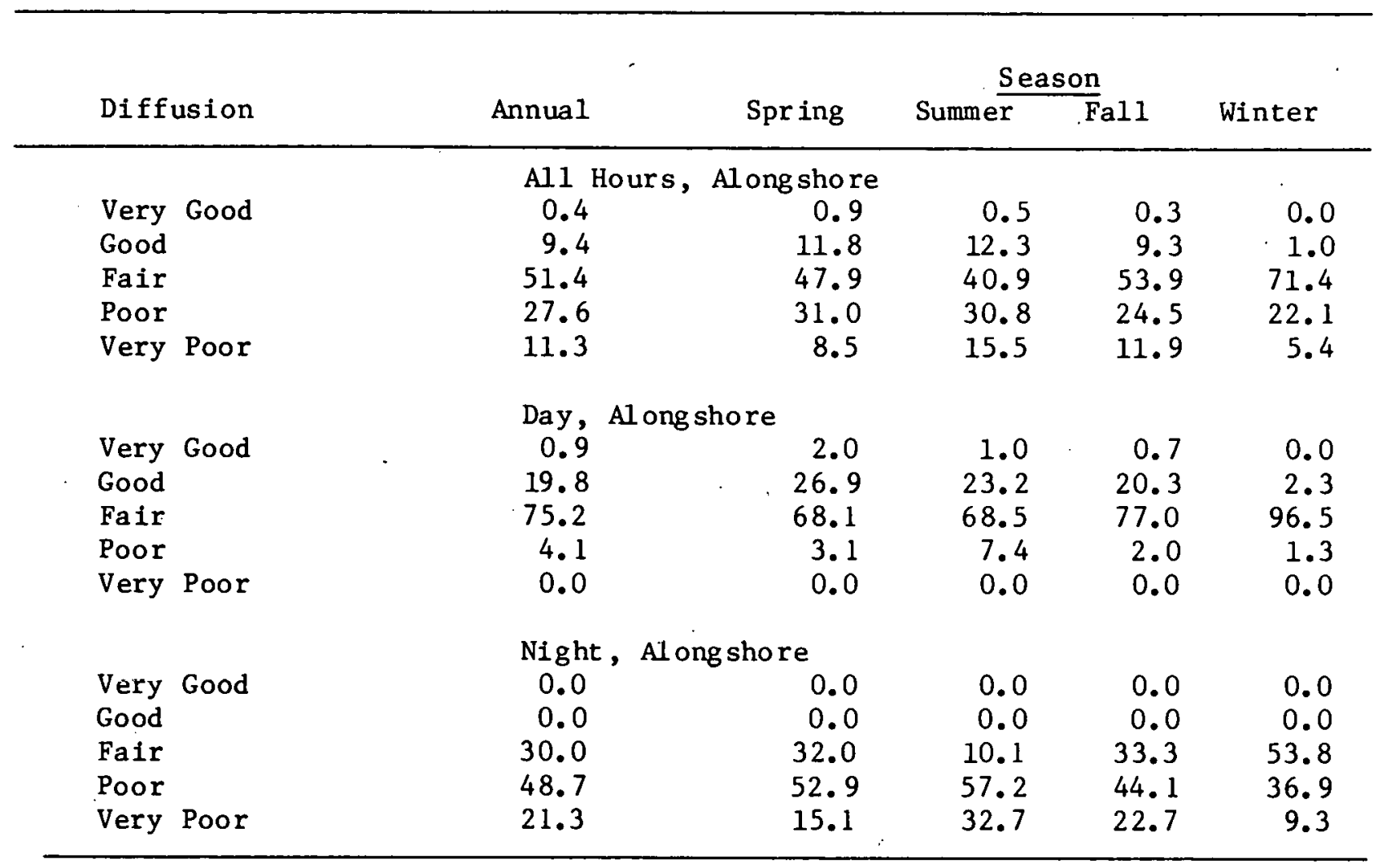


Table 7.23 (continued)

\begin{tabular}{|c|c|c|c|c|c|}
\hline \multirow[b]{2}{*}{ Diffusion } & \multirow[b]{2}{*}{ Annual } & \multirow[b]{2}{*}{ Spring } & \multicolumn{2}{|c|}{ Season } & \multirow[b]{2}{*}{ Winter } \\
\hline & & & Summer & $\bar{F}$ all & \\
\hline $\begin{array}{l}\text { Very Good } \\
\text { Good } \\
\text { Fair } \\
\text { Poor } \\
\text { Very Poor }\end{array}$ & $\begin{array}{c}\mathrm{A} 11 \mathrm{H} \\
0.2 \\
7.0 \\
53.9 \\
29.6 \\
9.3\end{array}$ & $\begin{array}{r}\text { Hours, Offshore } \\
0.0 \\
8.4 \\
58.6 \\
27.0 \\
5.9\end{array}$ & $\begin{array}{l}1.3 \\
15.0 \\
35.4 \\
35.7 \\
12.6\end{array}$ & $\begin{array}{r}0.0 \\
5.8 \\
45.9 \\
34.5 \\
13.8\end{array}$ & $\begin{array}{r}0.0 \\
2.0 \\
71.3 \\
21.5 \\
5.2\end{array}$ \\
\hline $\begin{array}{l}\text { Very Good } \\
\text { Good } \\
\text { Fair } \\
\text { Poor } \\
\text { Very Poor }\end{array}$ & $\begin{array}{r}\text { Day, } \\
0.6 \\
17.6 \\
18.5 \\
3.4 \\
0.0\end{array}$ & $\begin{array}{r}0.0 \\
19.9 \\
70.5 \\
3.6 \\
0.0\end{array}$ & $\begin{array}{r}2.6 \\
31.8 \\
57.5 \\
8.0 \\
0.0\end{array}$ & $\begin{array}{r}0.0 \\
16.4 \\
81.7 \\
2.0 \\
0.0\end{array}$ & $\begin{array}{r}0.0 \\
5.4 \\
9.3 .8 \\
0.9 \\
0.0\end{array}$ \\
\hline $\begin{array}{l}\text { Very Good } \\
\text { Good } \\
\text { Fair } \\
\text { Poor } \\
\text { Very Poor }\end{array}$ & $\begin{array}{l}\text { Night } \\
0.0 \\
0.0 \\
37.7 \\
46.8 \\
15.5\end{array}$ & $\begin{array}{r}\text {, Off shore } \\
0.0 \\
0.0 \\
45.5 \\
44.3 \\
10.2\end{array}$ & $\begin{array}{r}0.0 \\
0.0 \\
16.3 \\
59.5 \\
24.2\end{array}$ & $\begin{array}{r}0.0 \\
0.0 \\
25.6 \\
52.7 \\
21.7\end{array}$ & $\begin{array}{r}0.0 \\
0.0 \\
58.0 \\
33.7 \\
8.3\end{array}$ \\
\hline
\end{tabular}




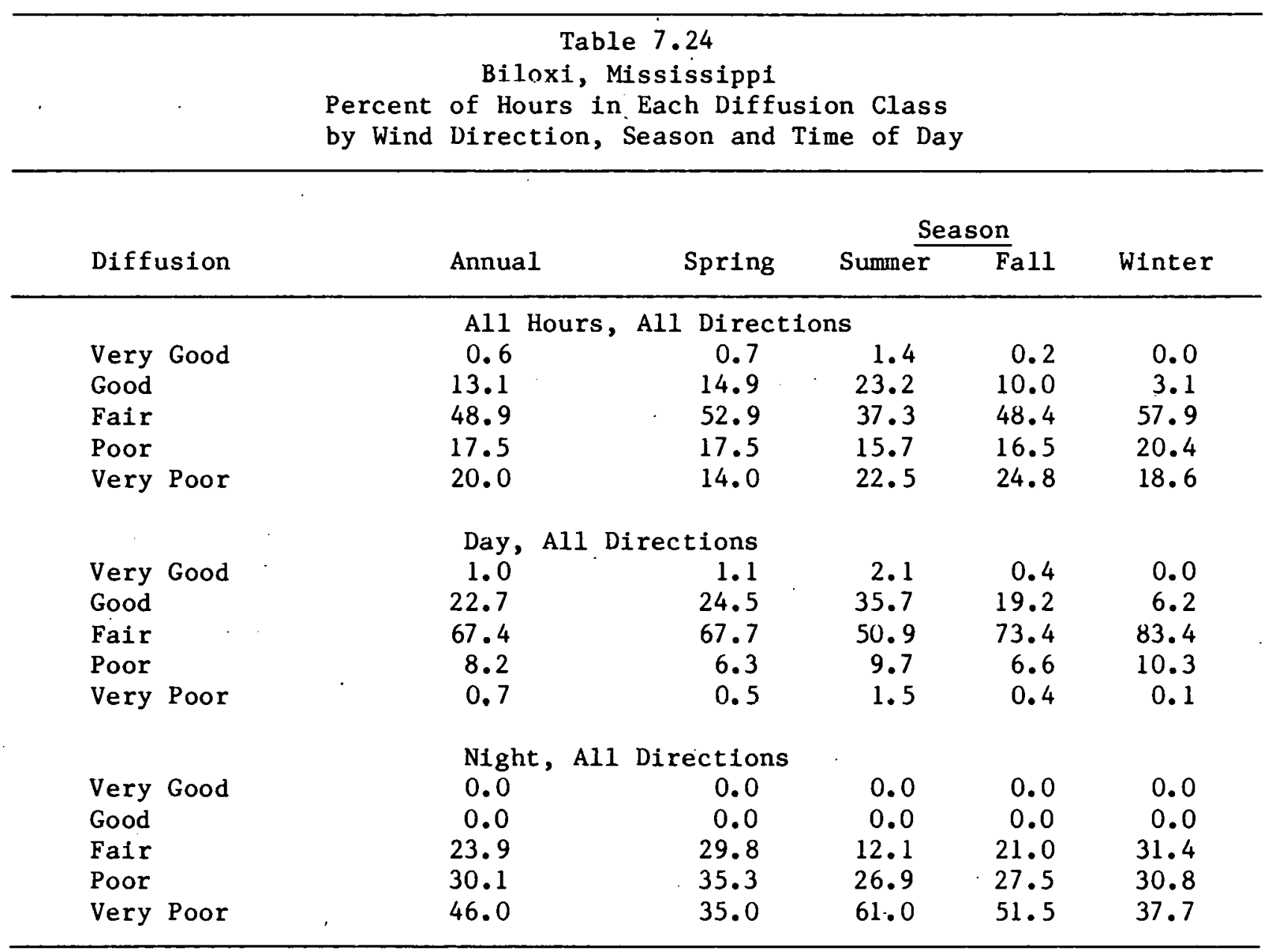


Table 7.24 (continued)

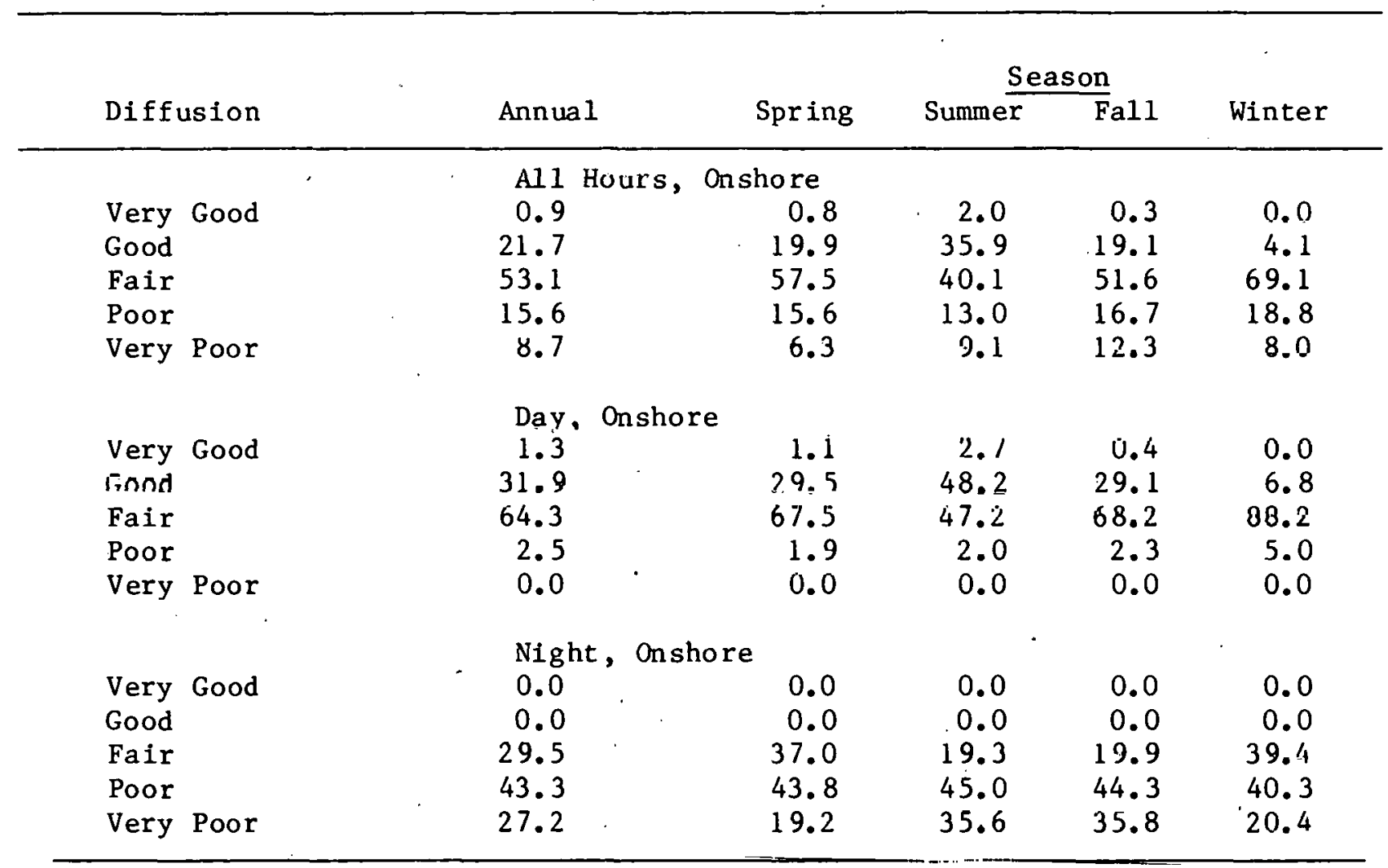


Table 7.24 (continued)

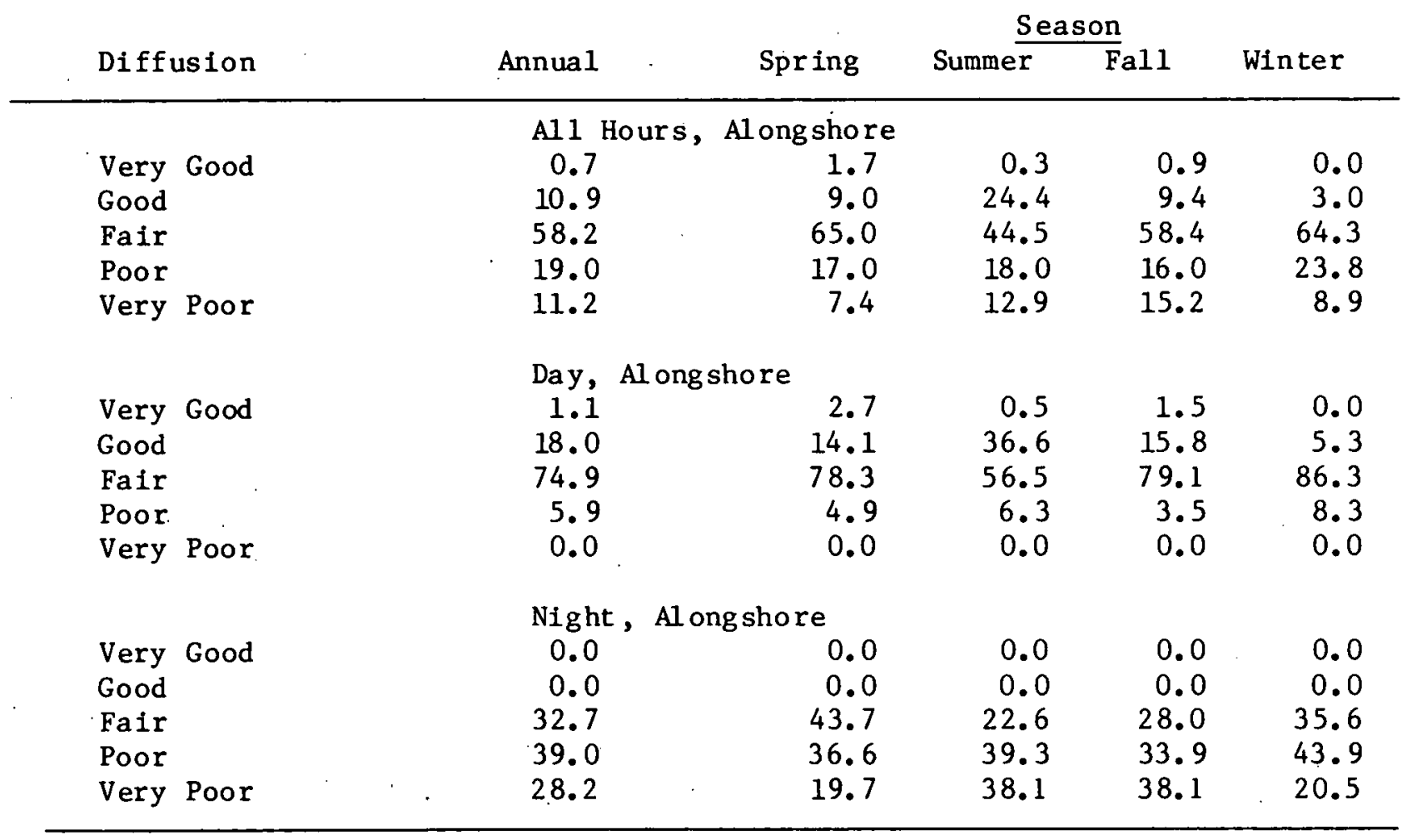


Table 7.24 (continued)

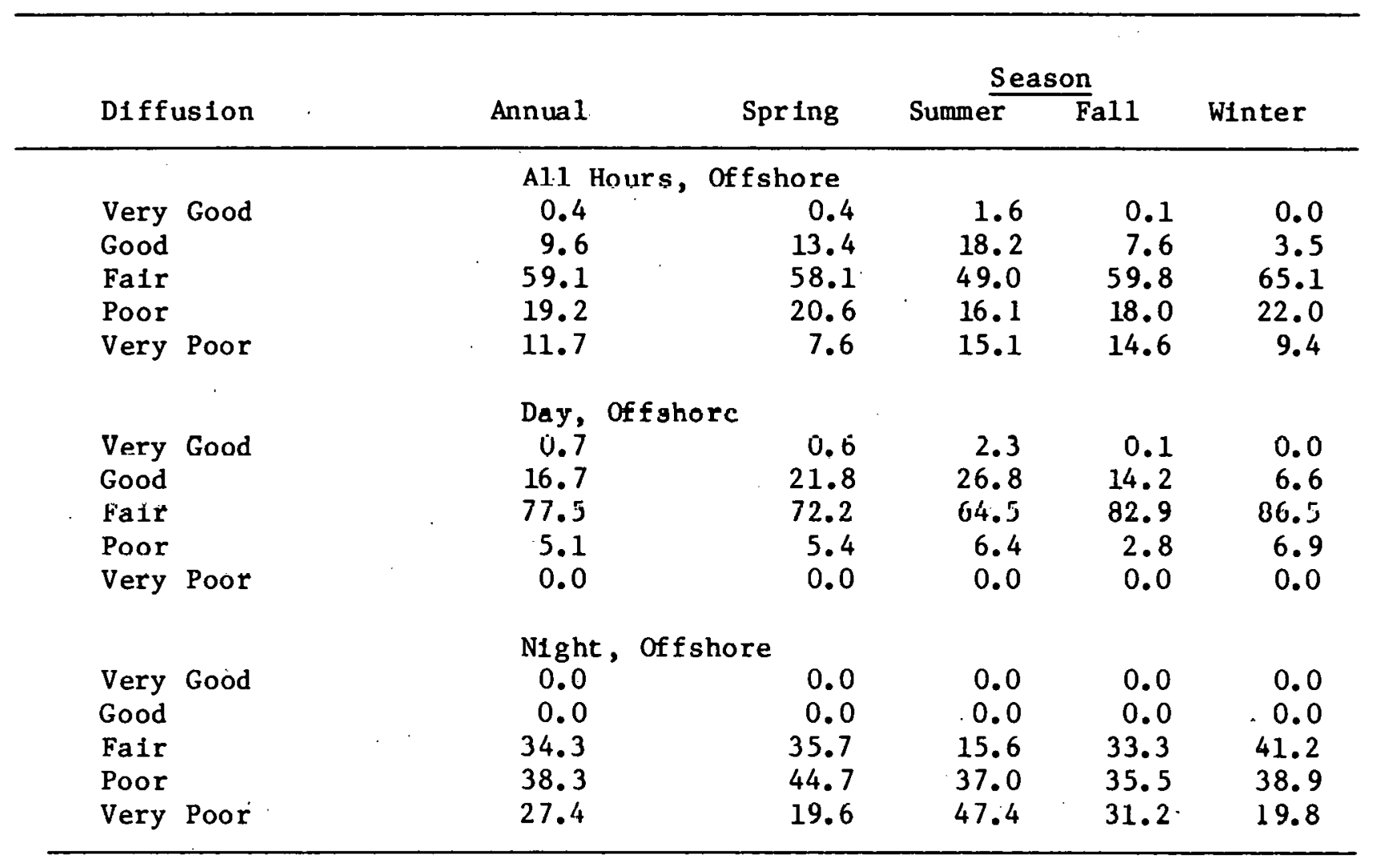


Table 7.25

New Orleans, Louisiana

Percent of Hours in Each Diffusion Class

by Wind Direction, Season and Time of Day

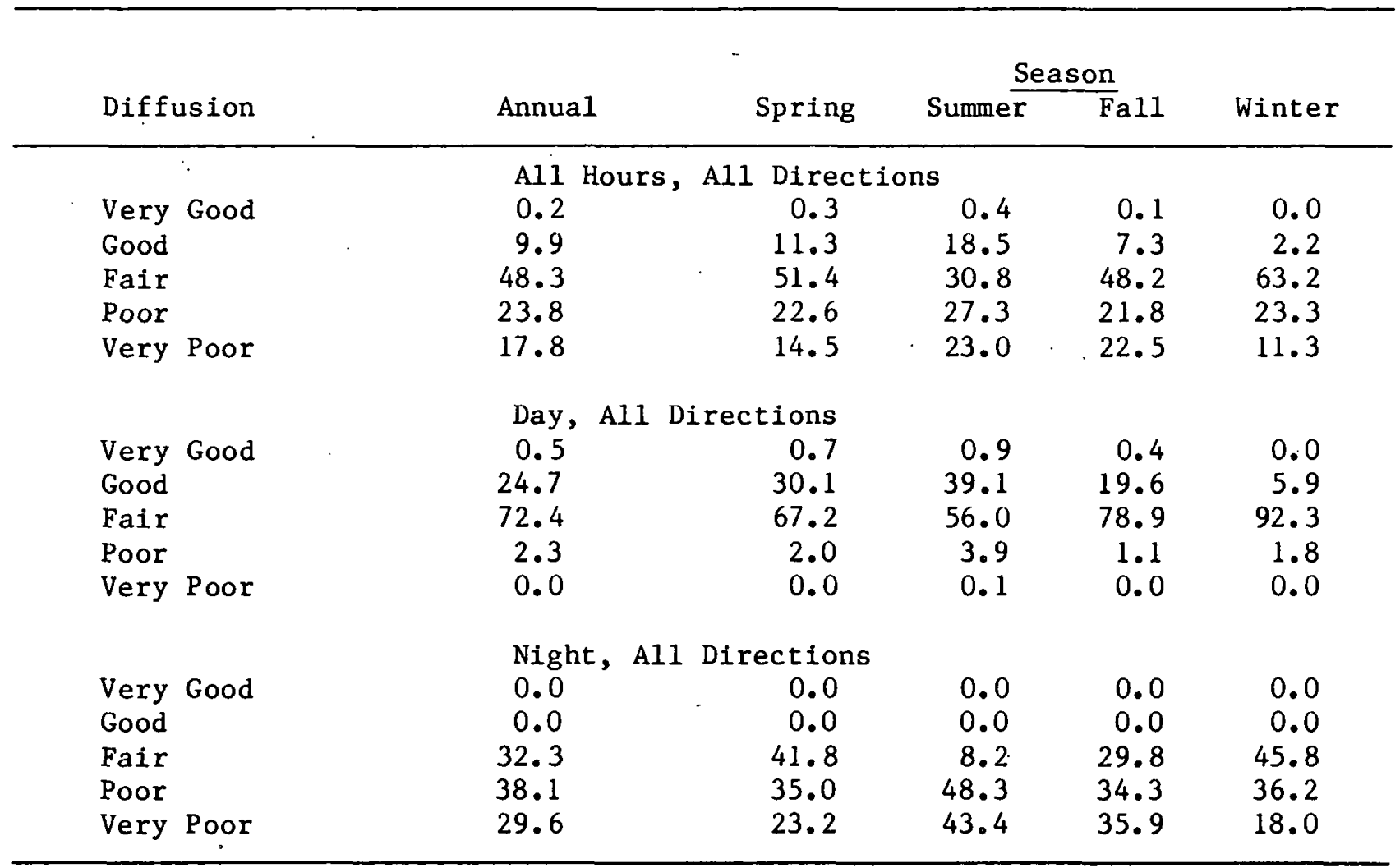


Table 7.25 (continued)

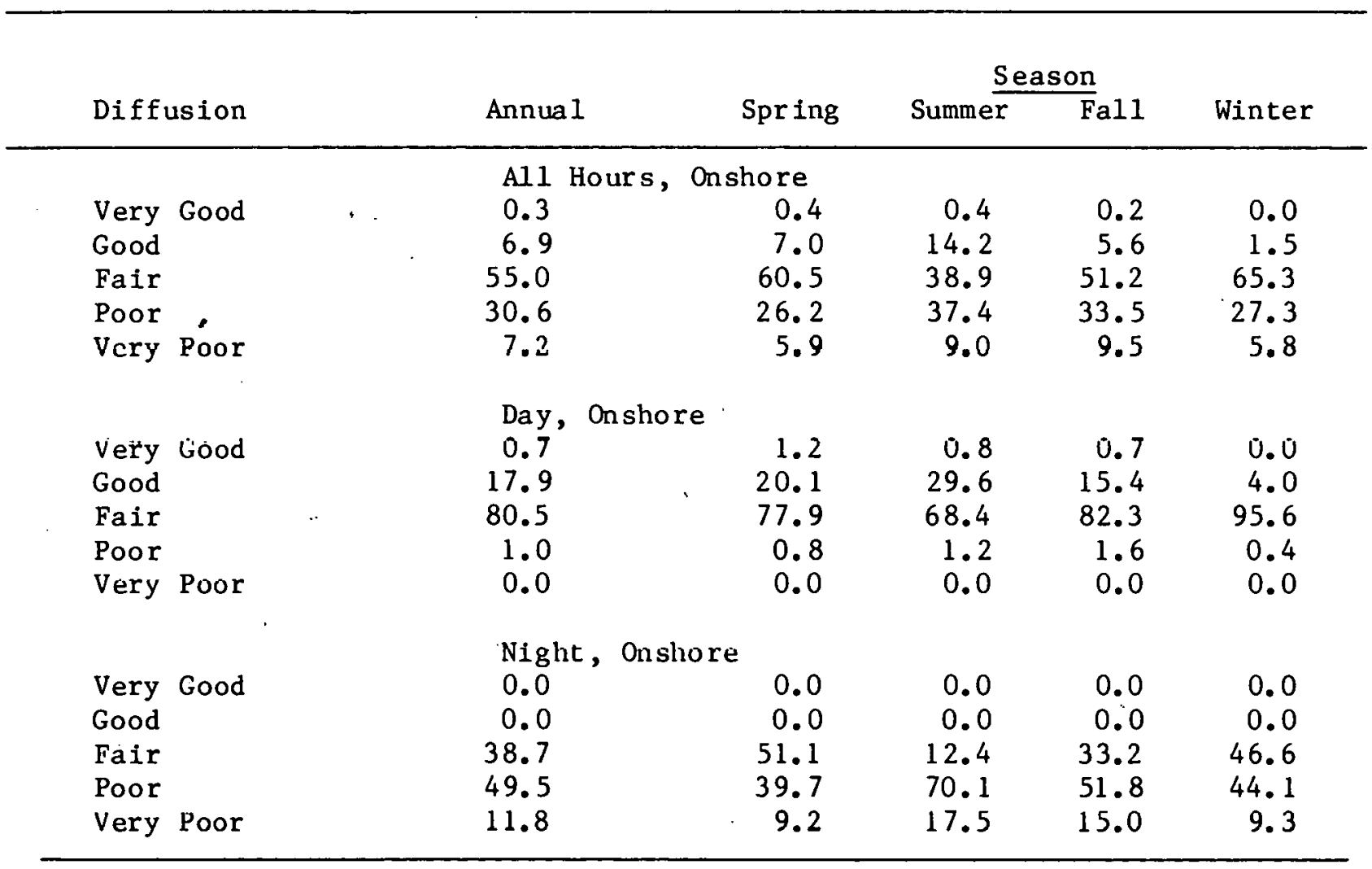


Table 7.25 (continued).

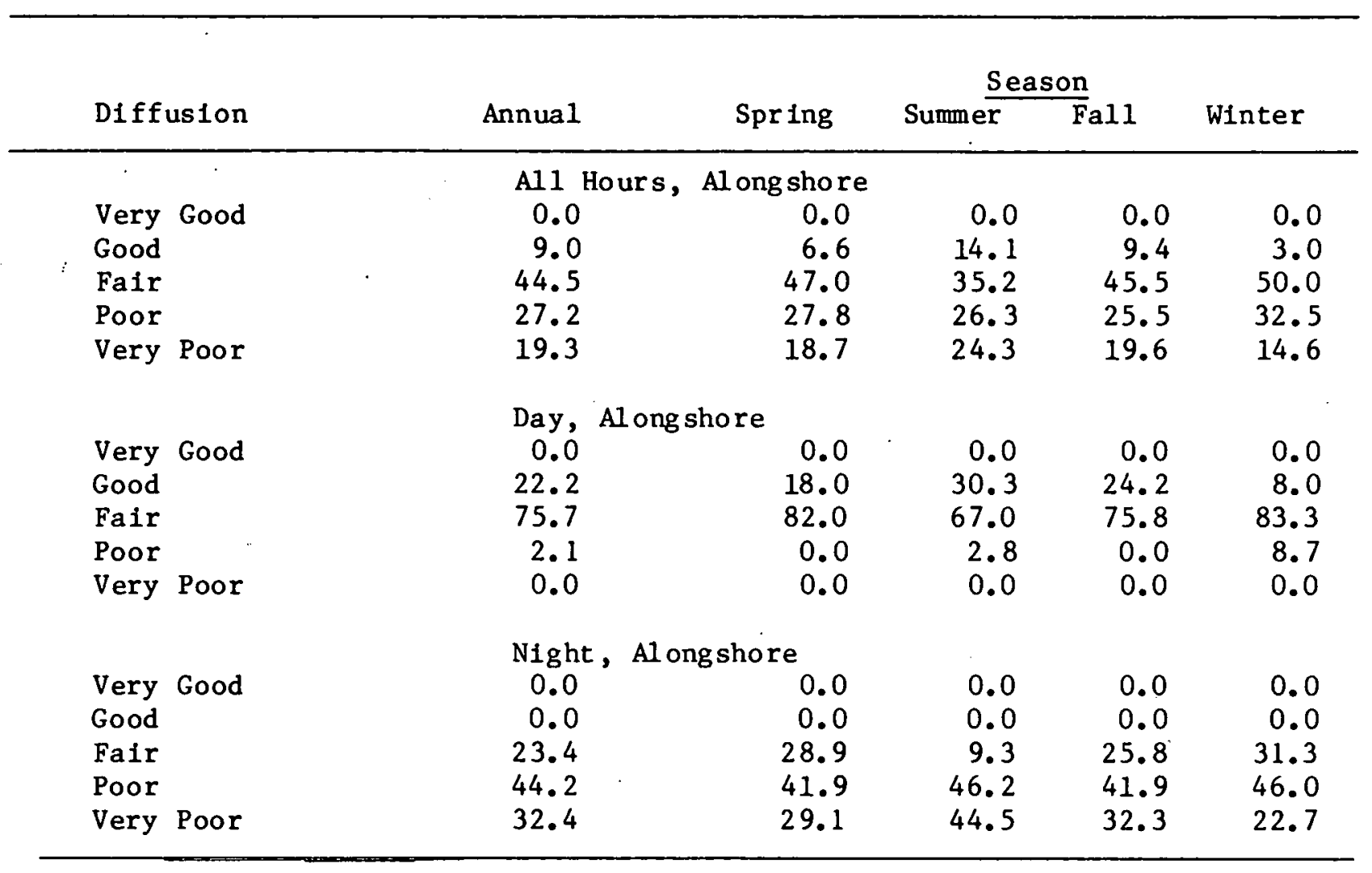


Table 7.25 (continued)

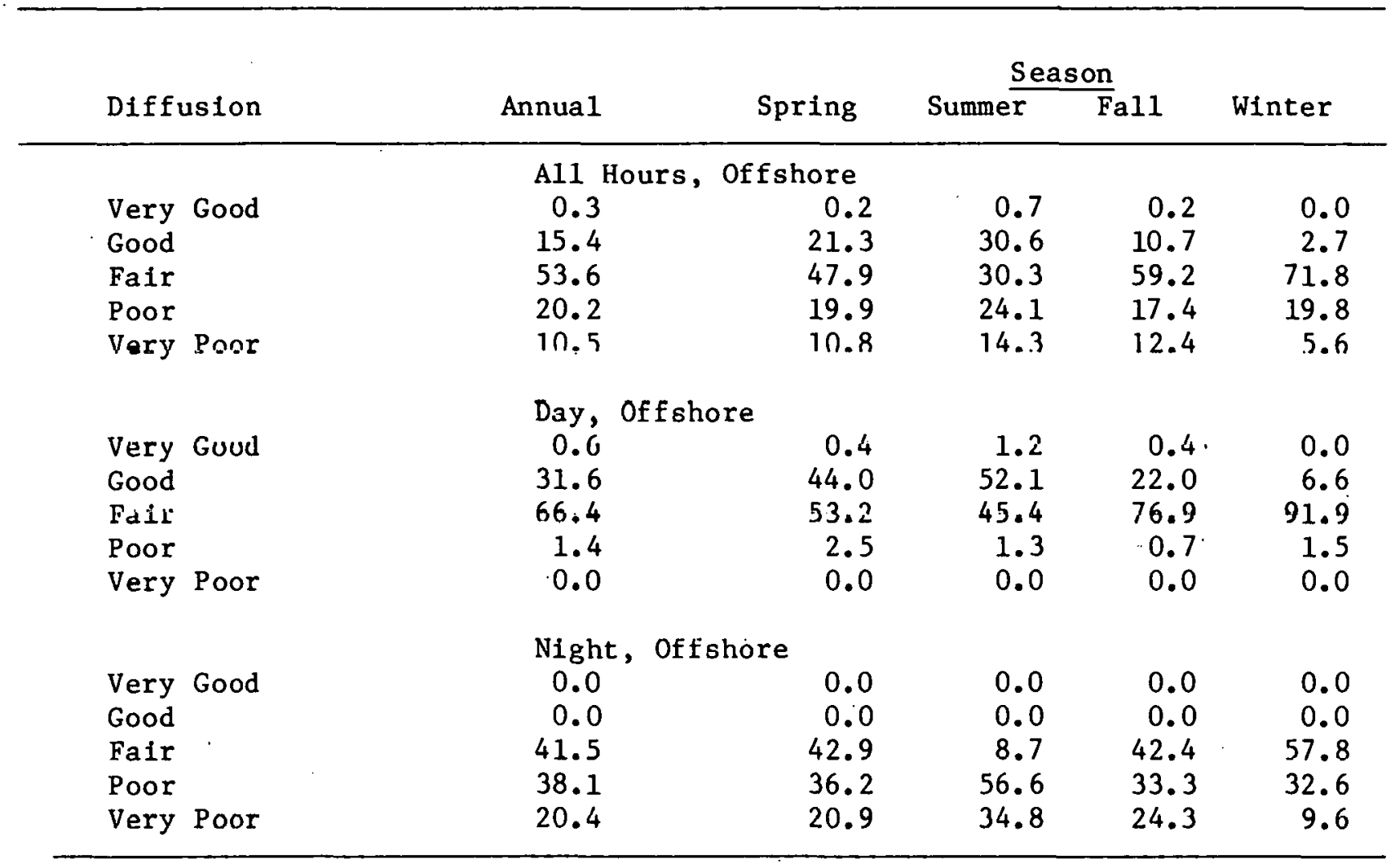


Table 7.26

Boothville, Louisiana

Percent of Hours in Each Diffusion Class

by Wind Direction, Season and Time of Day

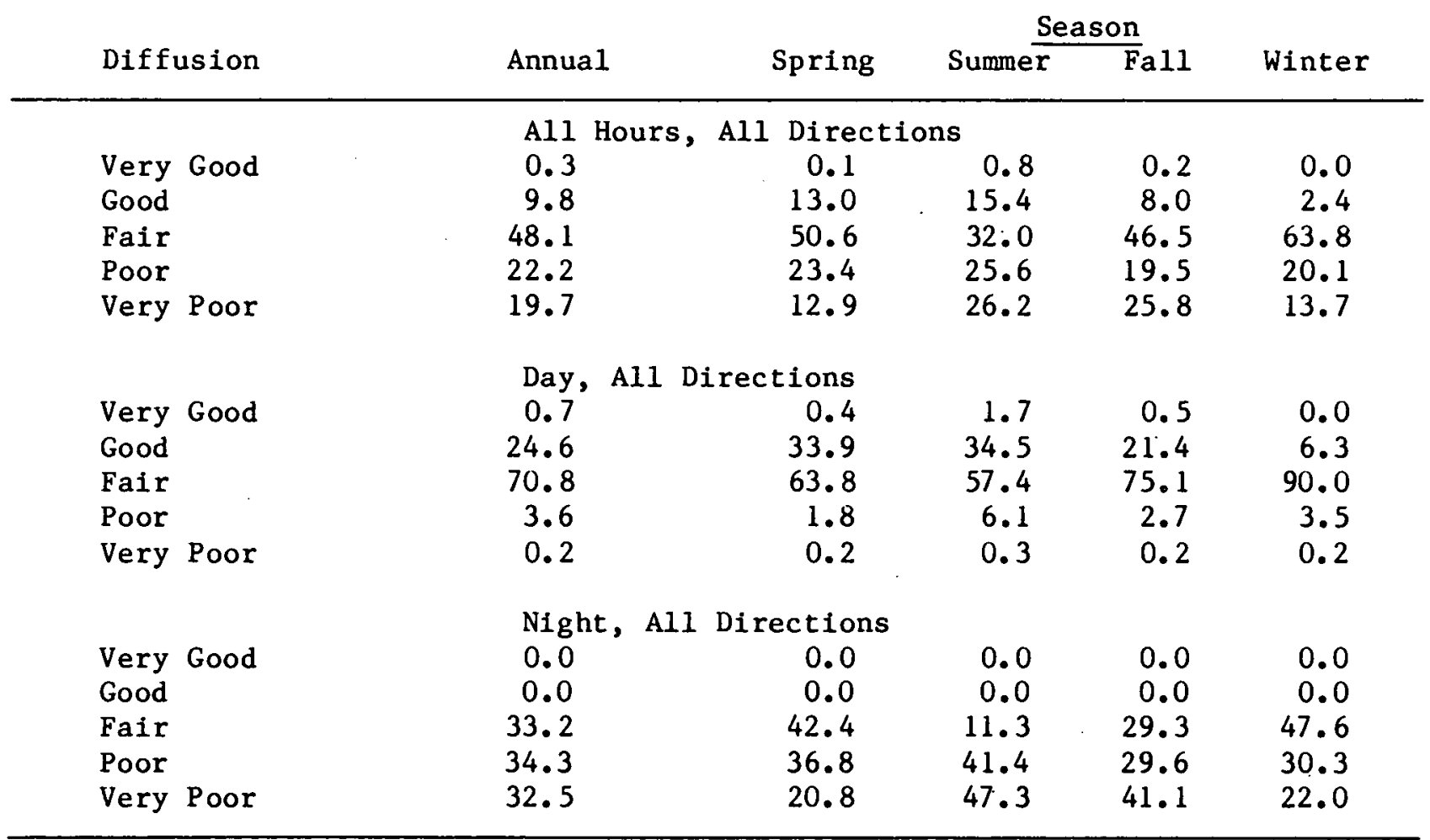


Table 7.26 (continued)

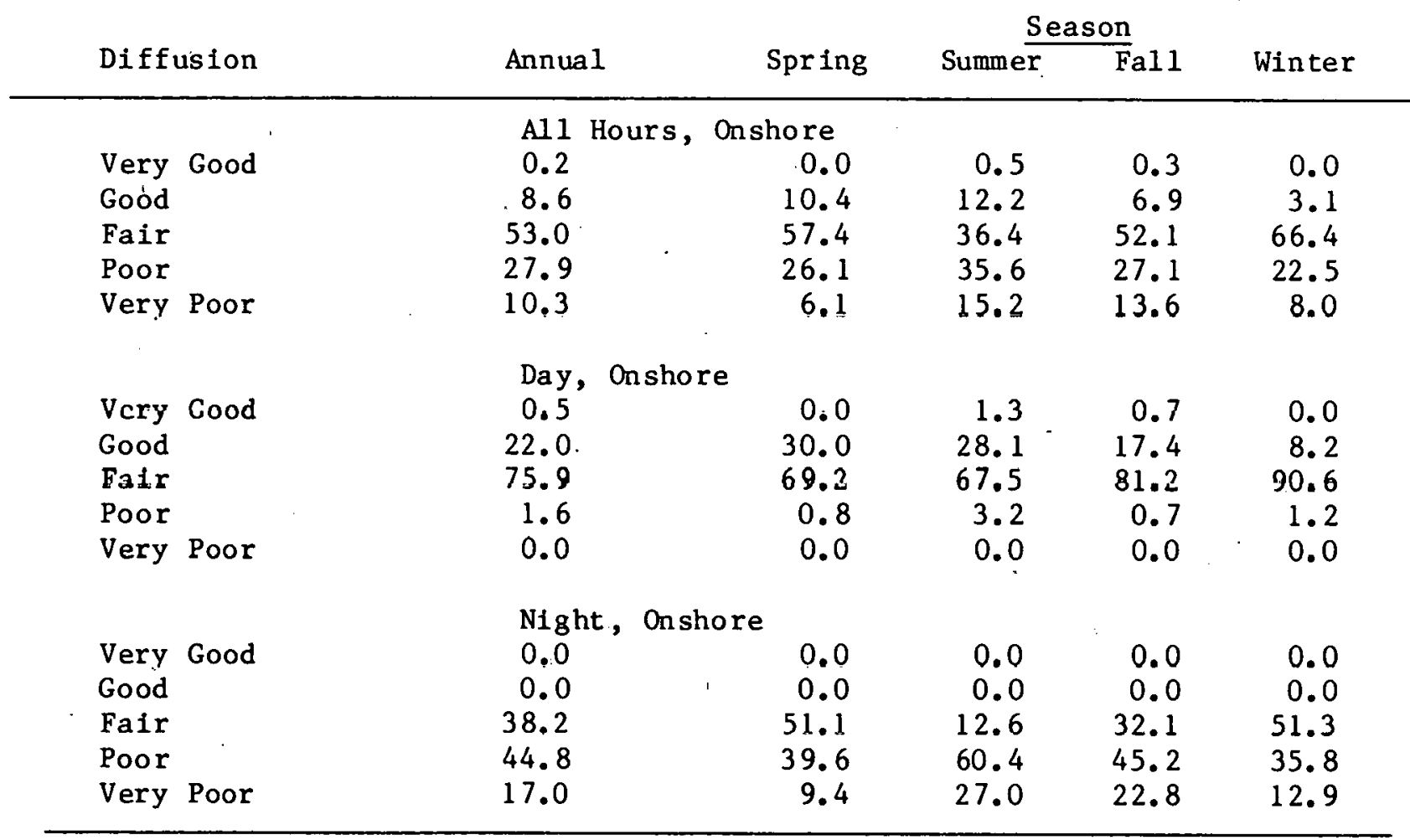


Table 7.26 (continued)

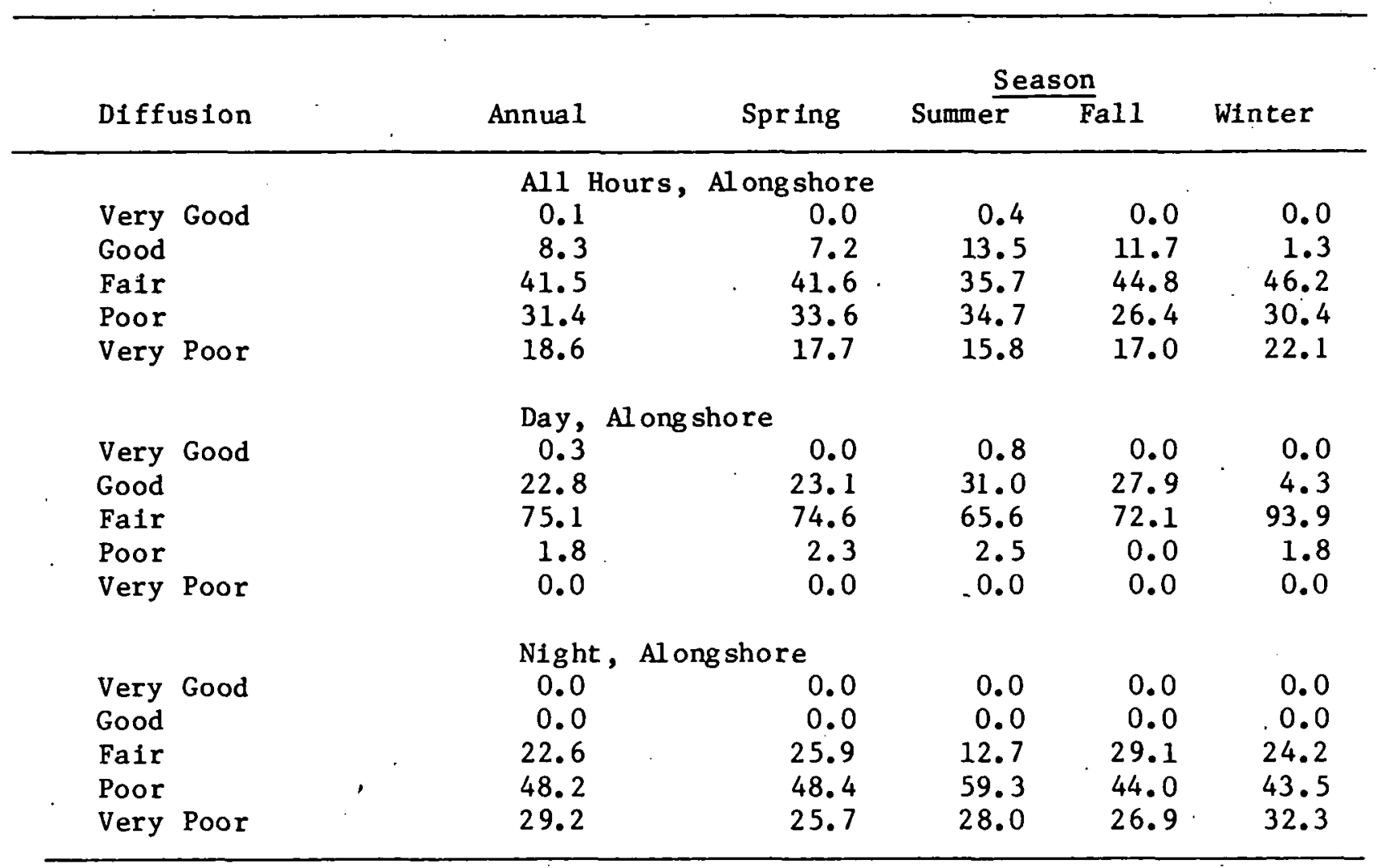


Table 7.26 (continued)

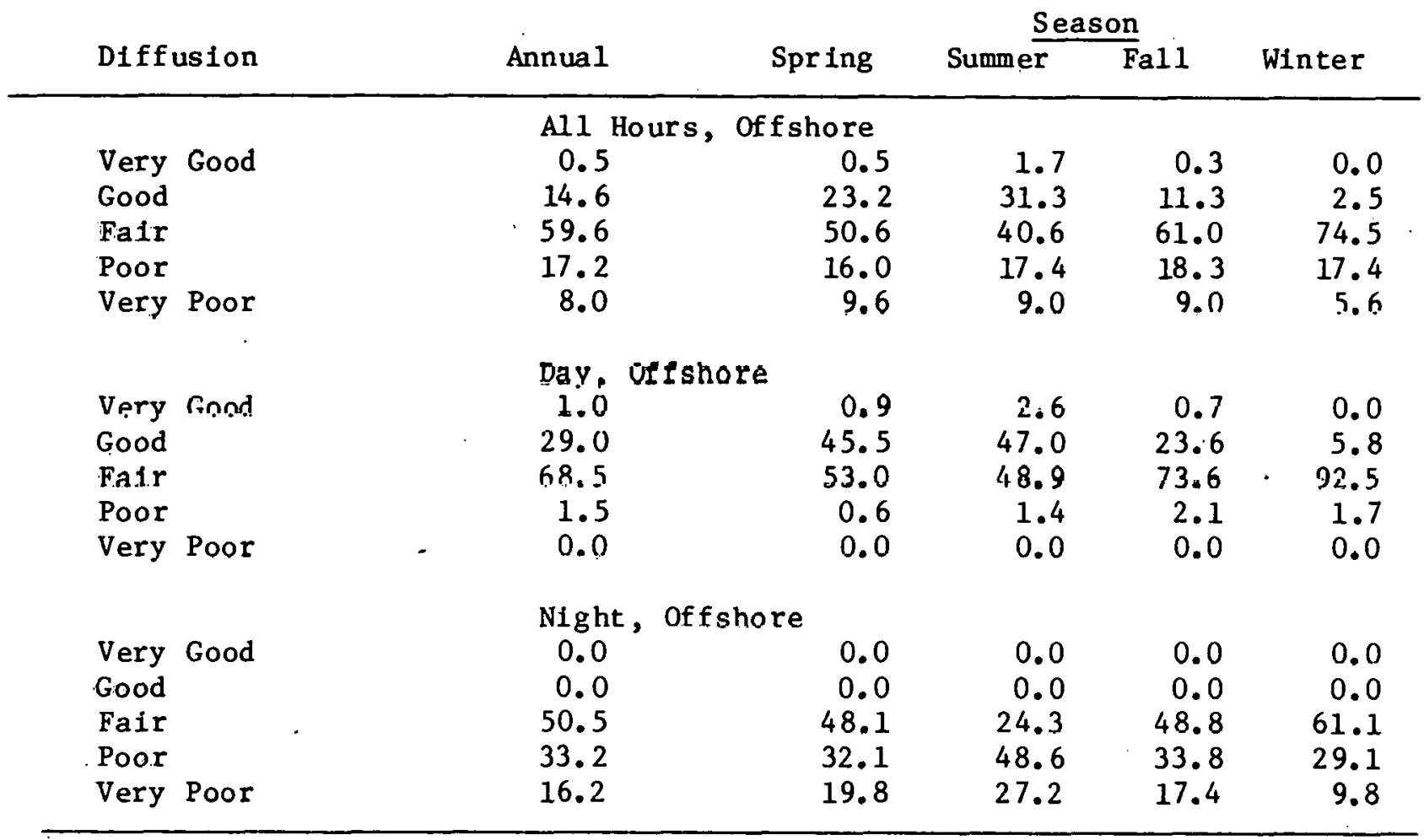




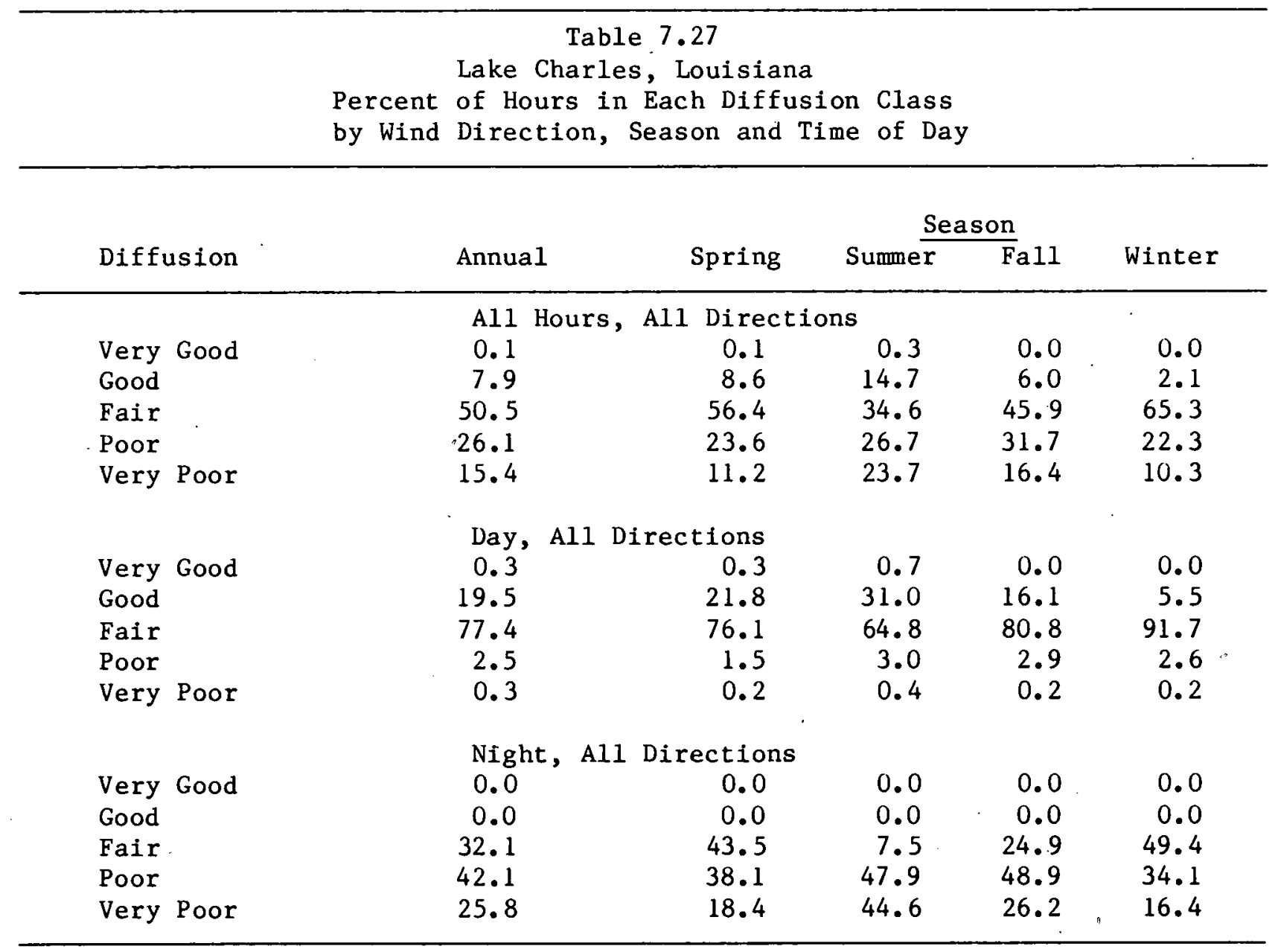


Table 7.27 (continued)

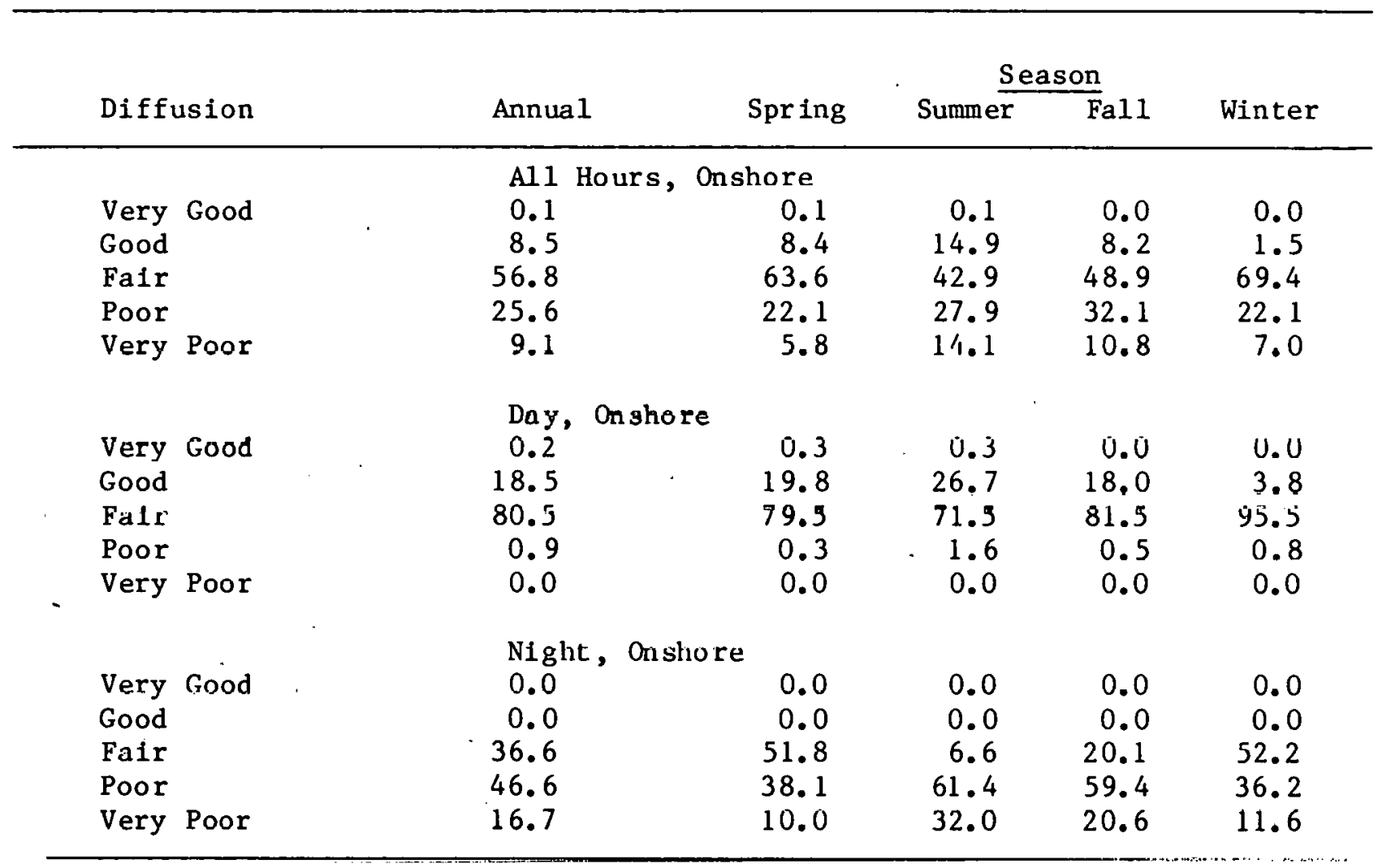


Table 7.27 (continued)

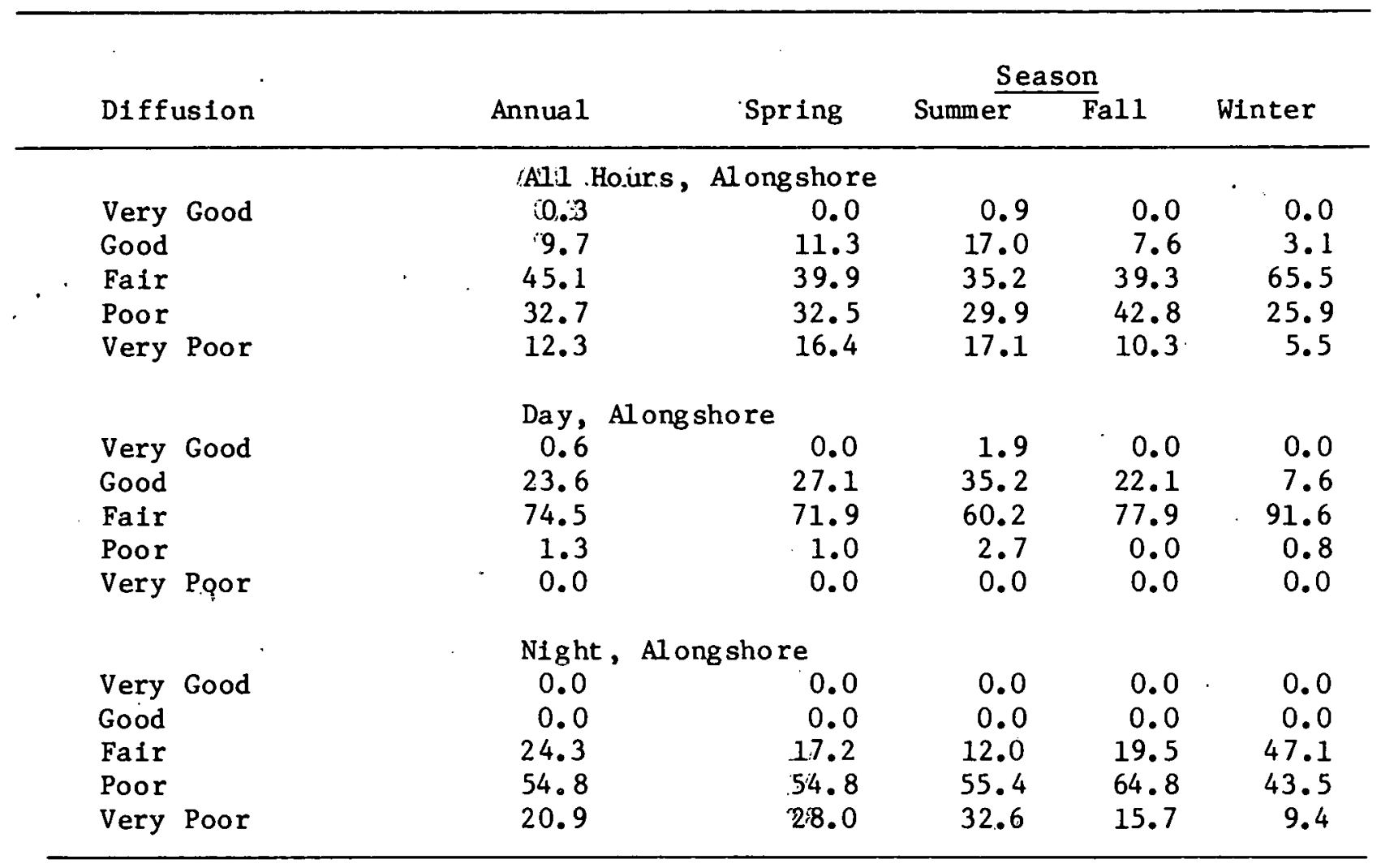


Table 7.27 (continued)

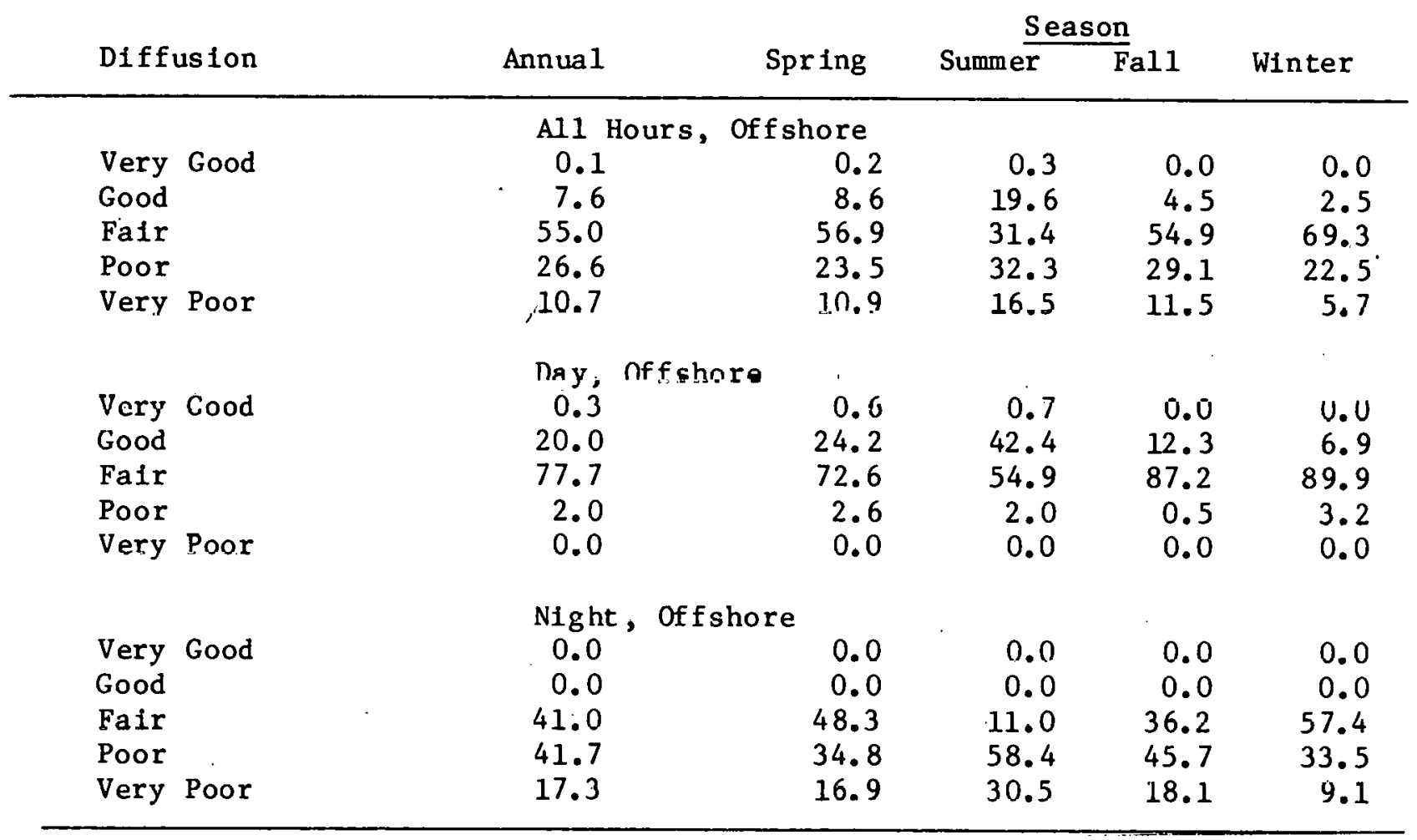




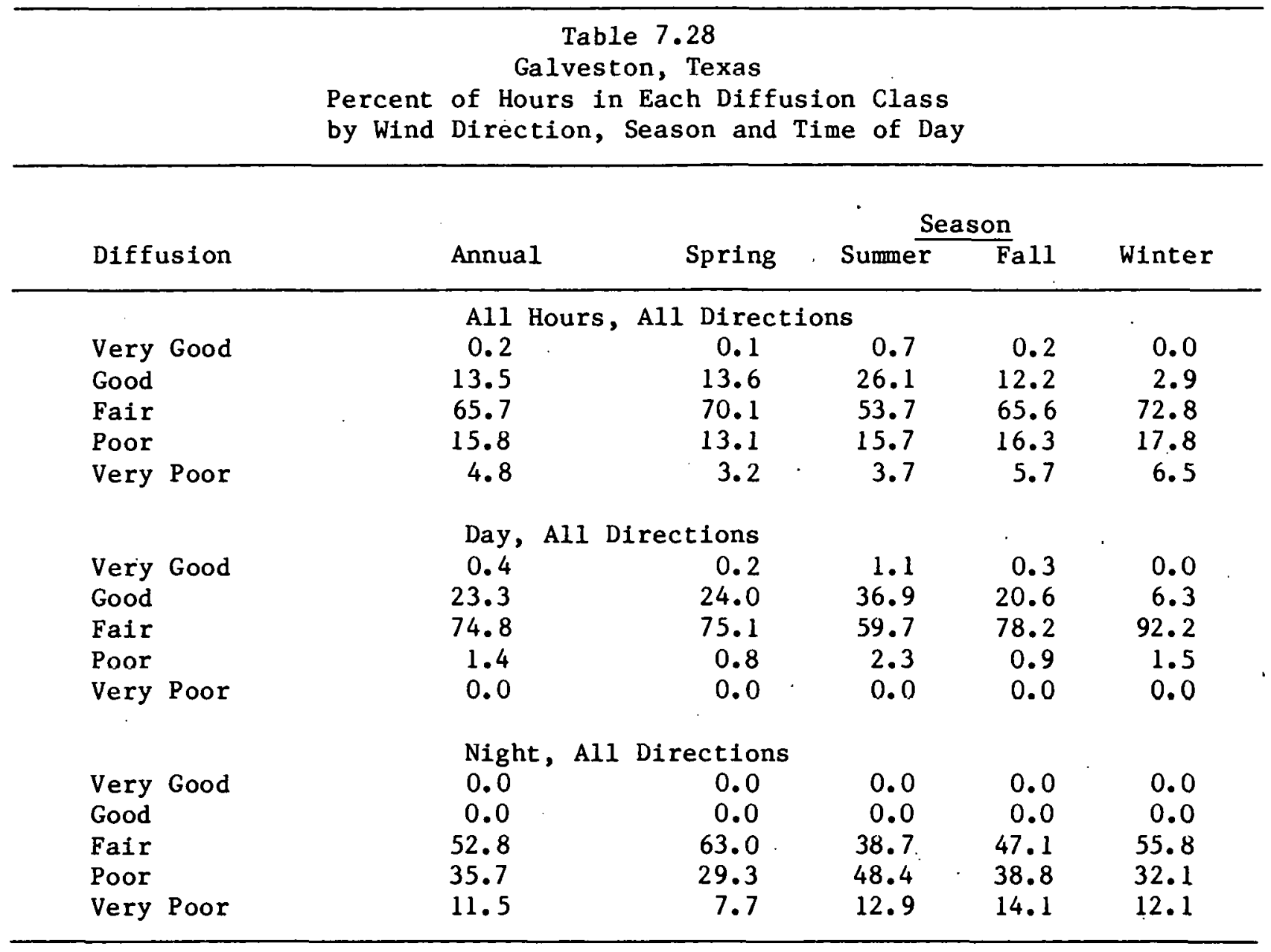


Table 7.28 (continued)

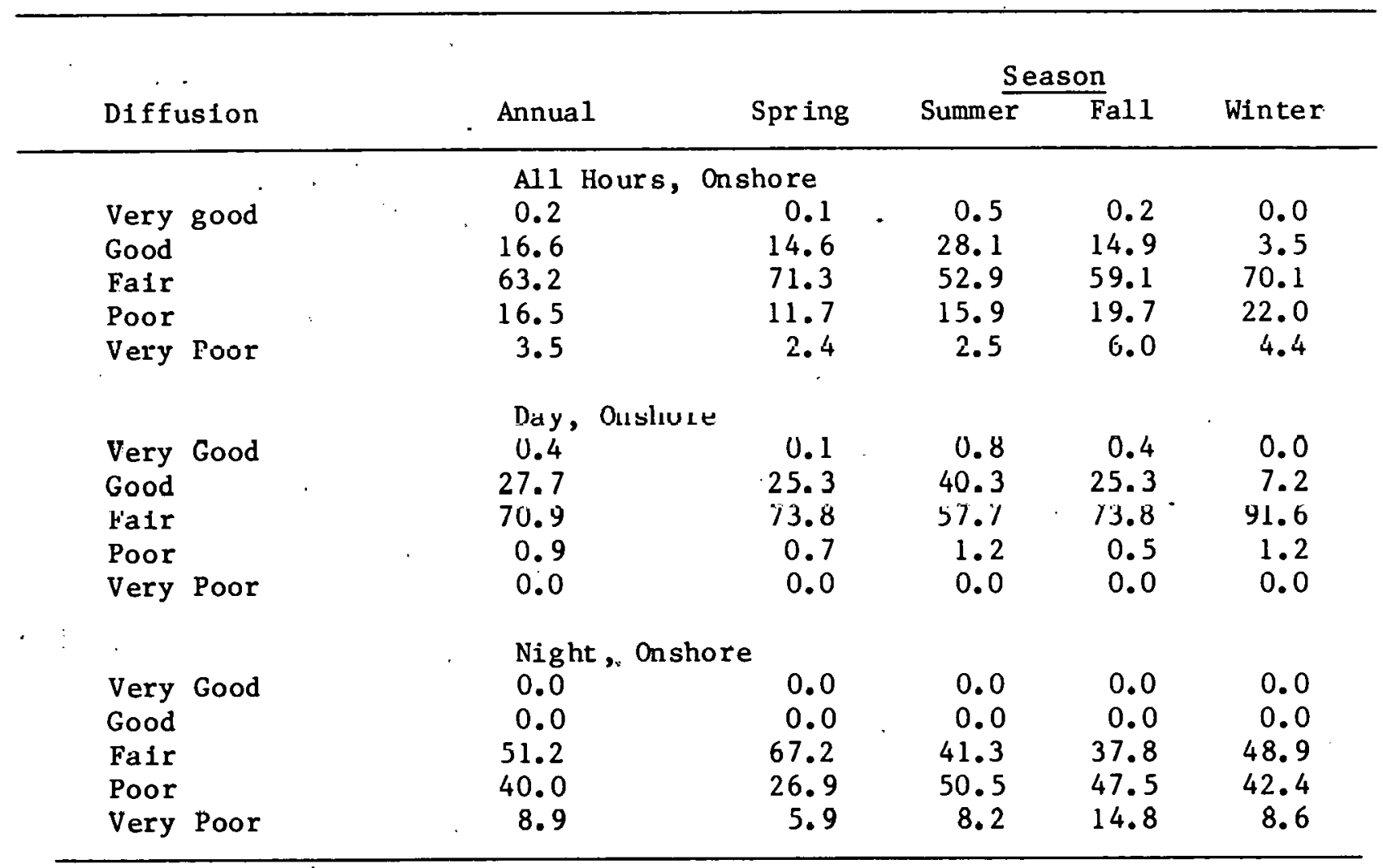


Table 7.28 (continued)

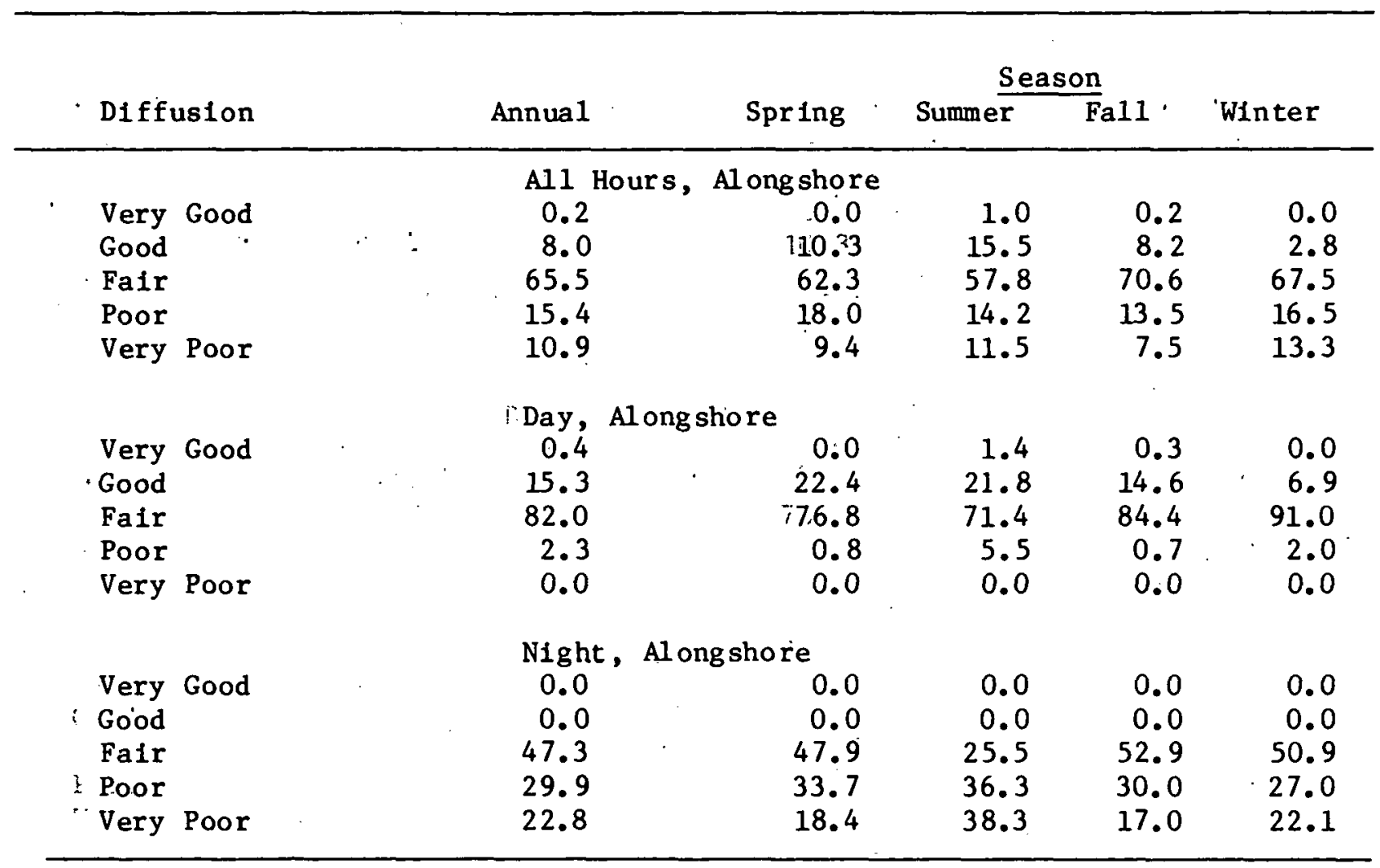


Table 7.28 (continued)

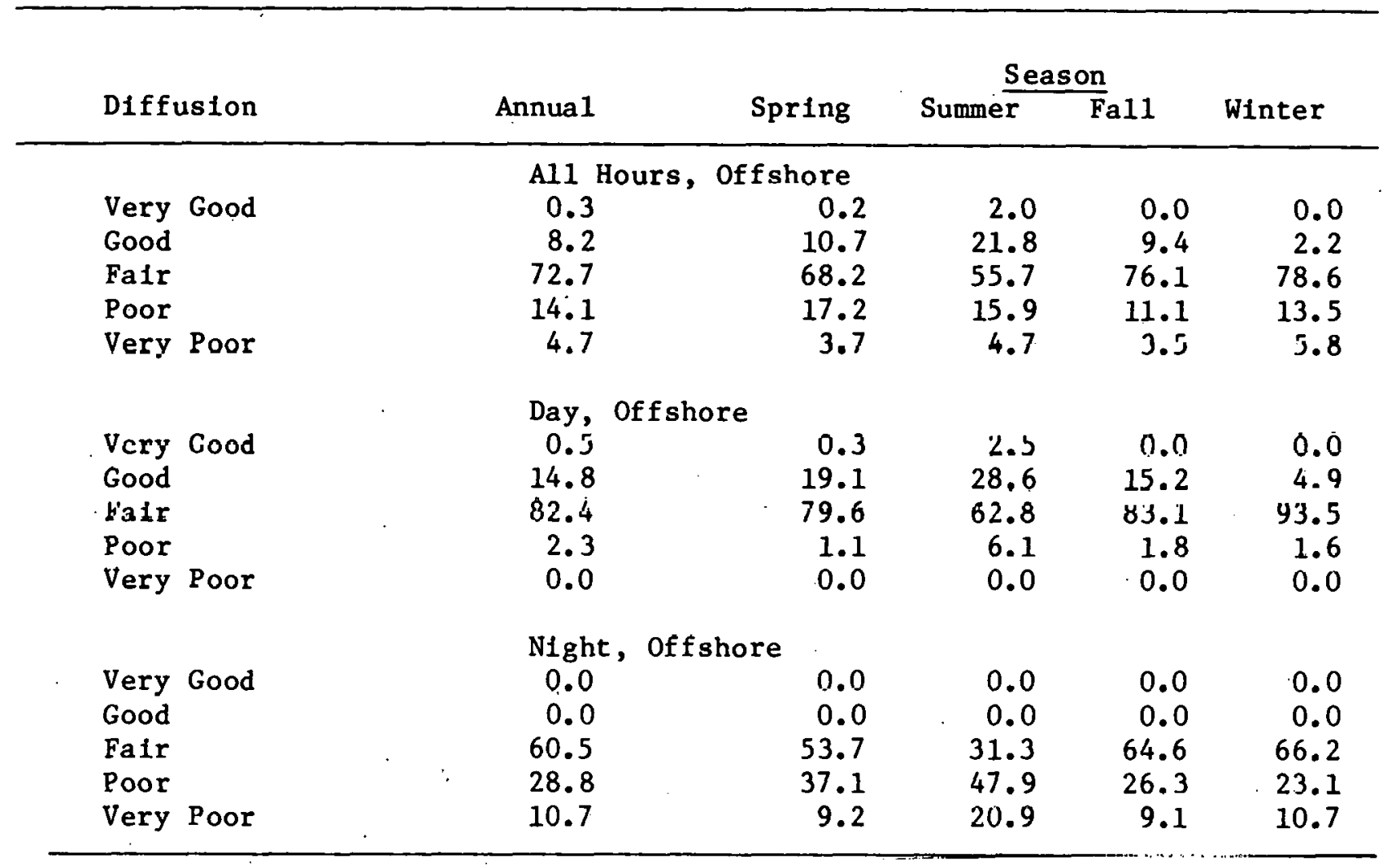




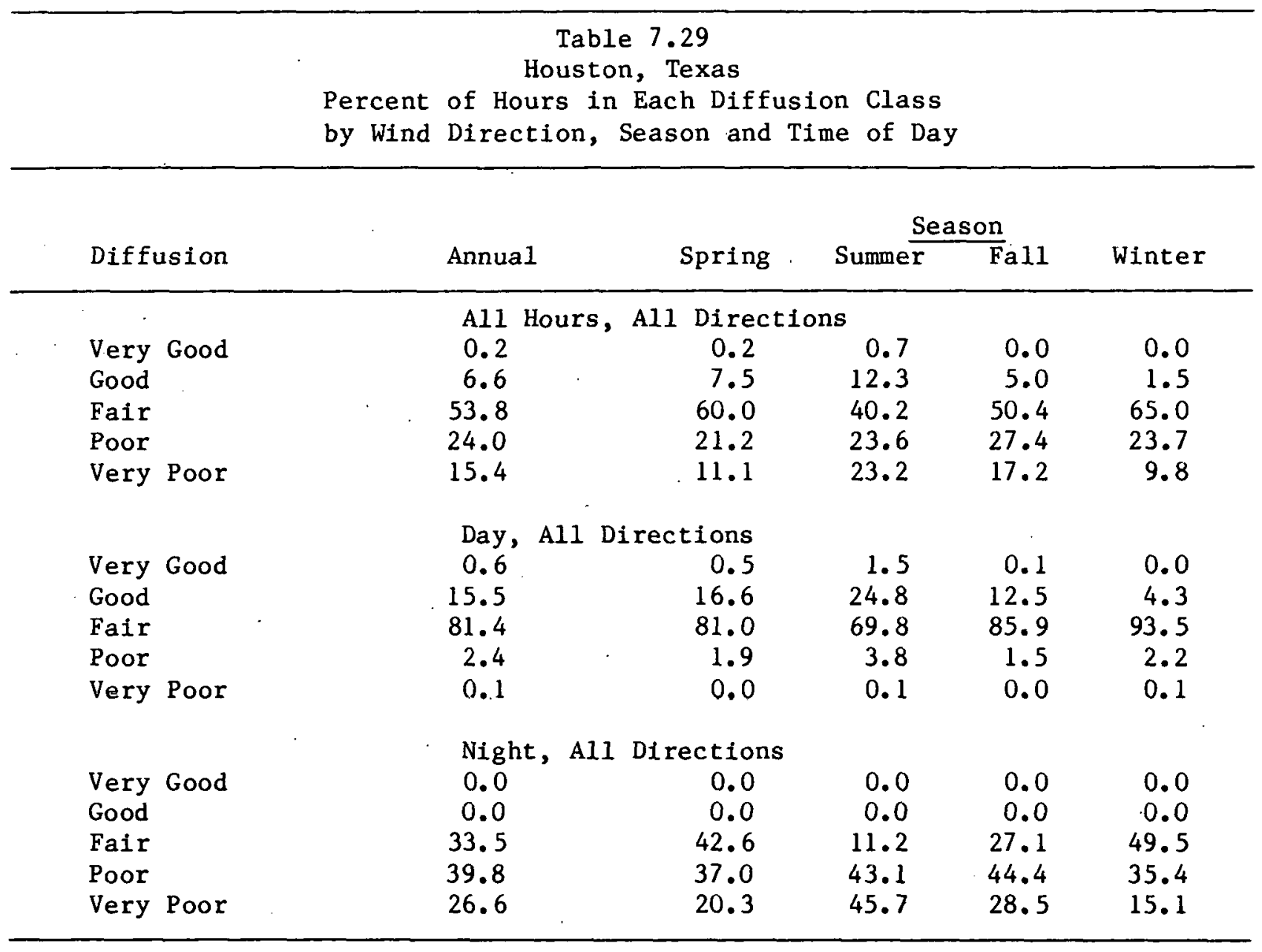


Tablie: 7:.29: (continued)

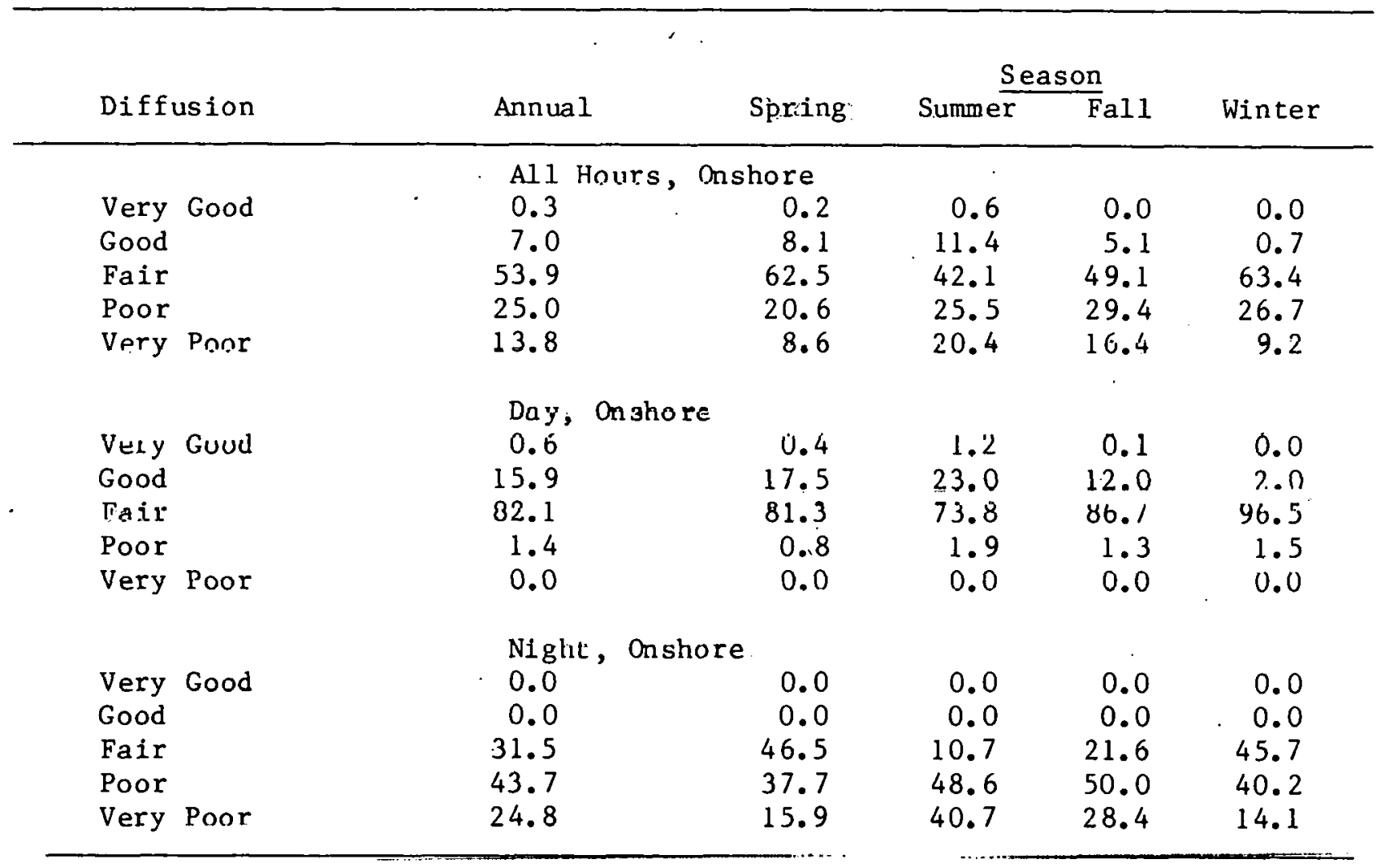


Table 7.29 (continued)

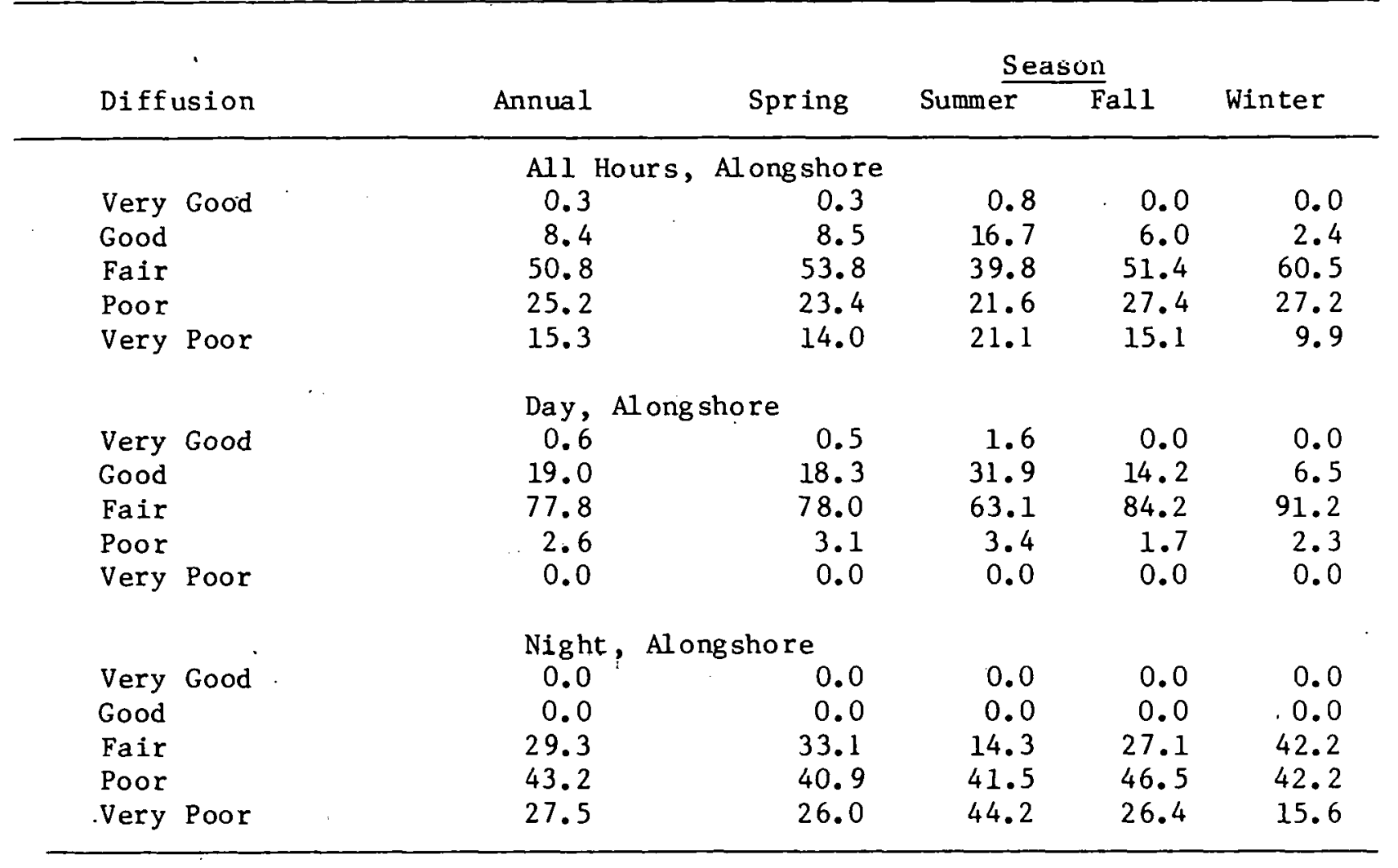


Table 7.29 (continued)

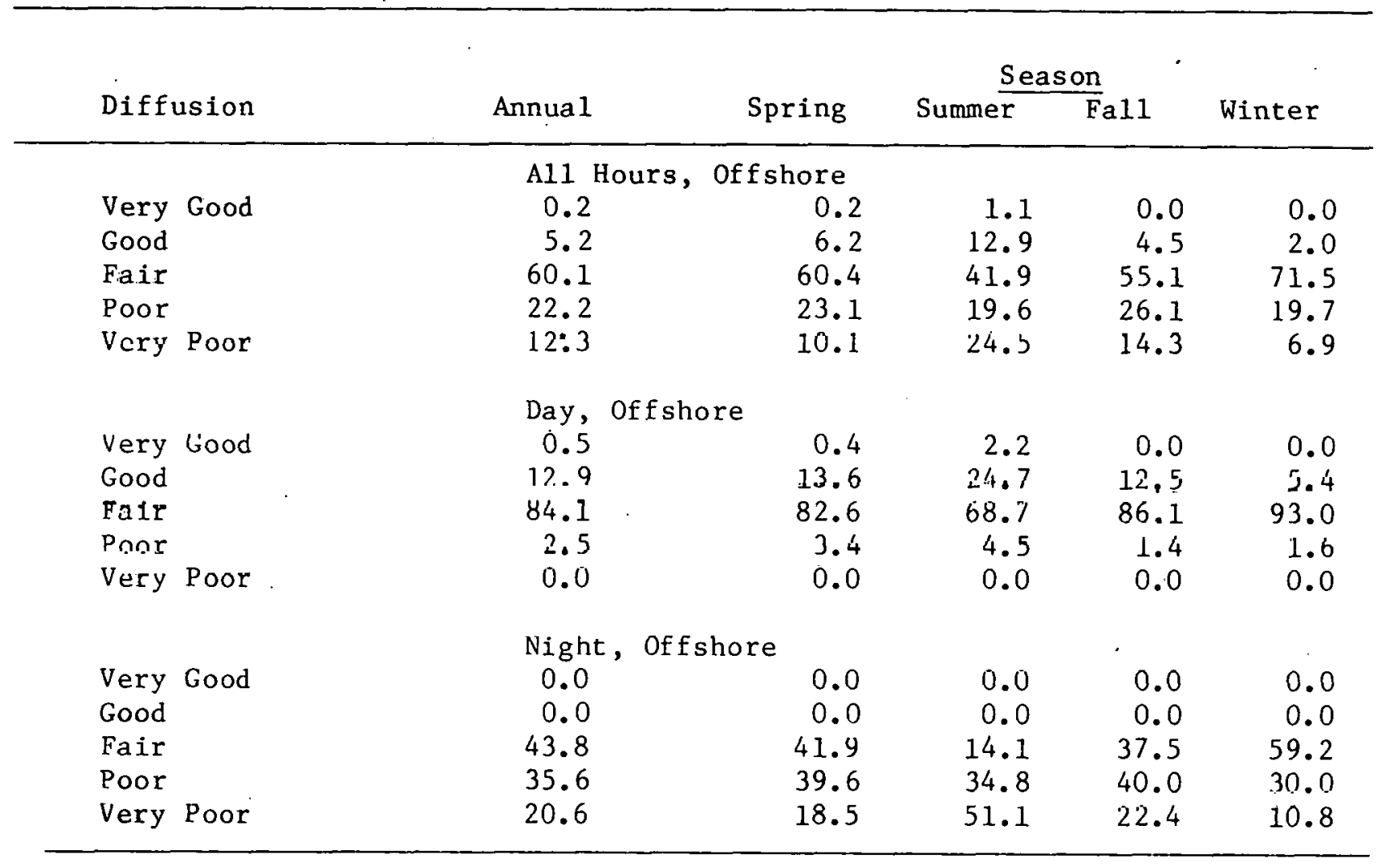


Table 7.30

Brownsville, Texas

Percent of Hours in Each Diffusion Class

by Wind Direction, Season and Time of Day

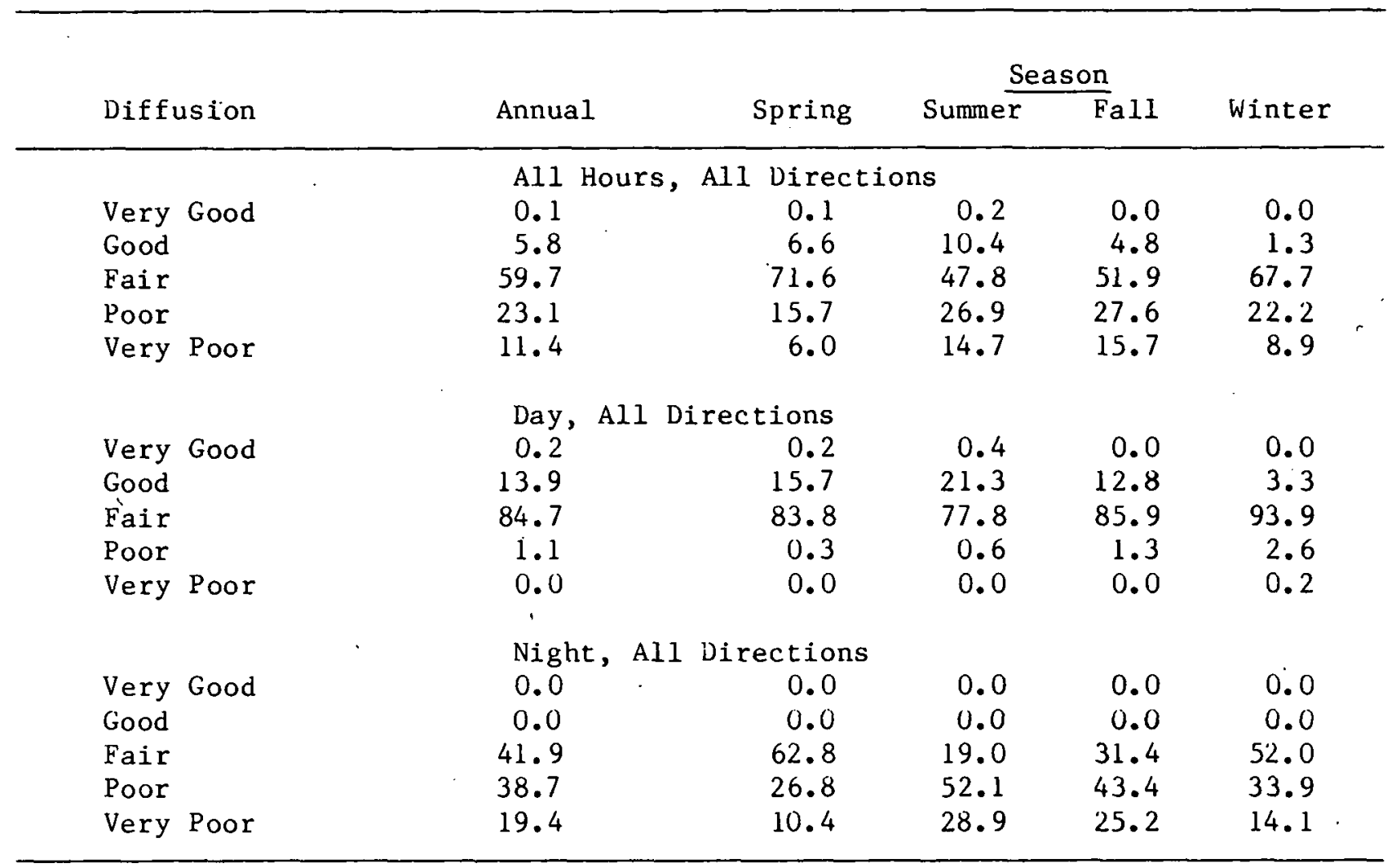


Table 7.30 (continued)

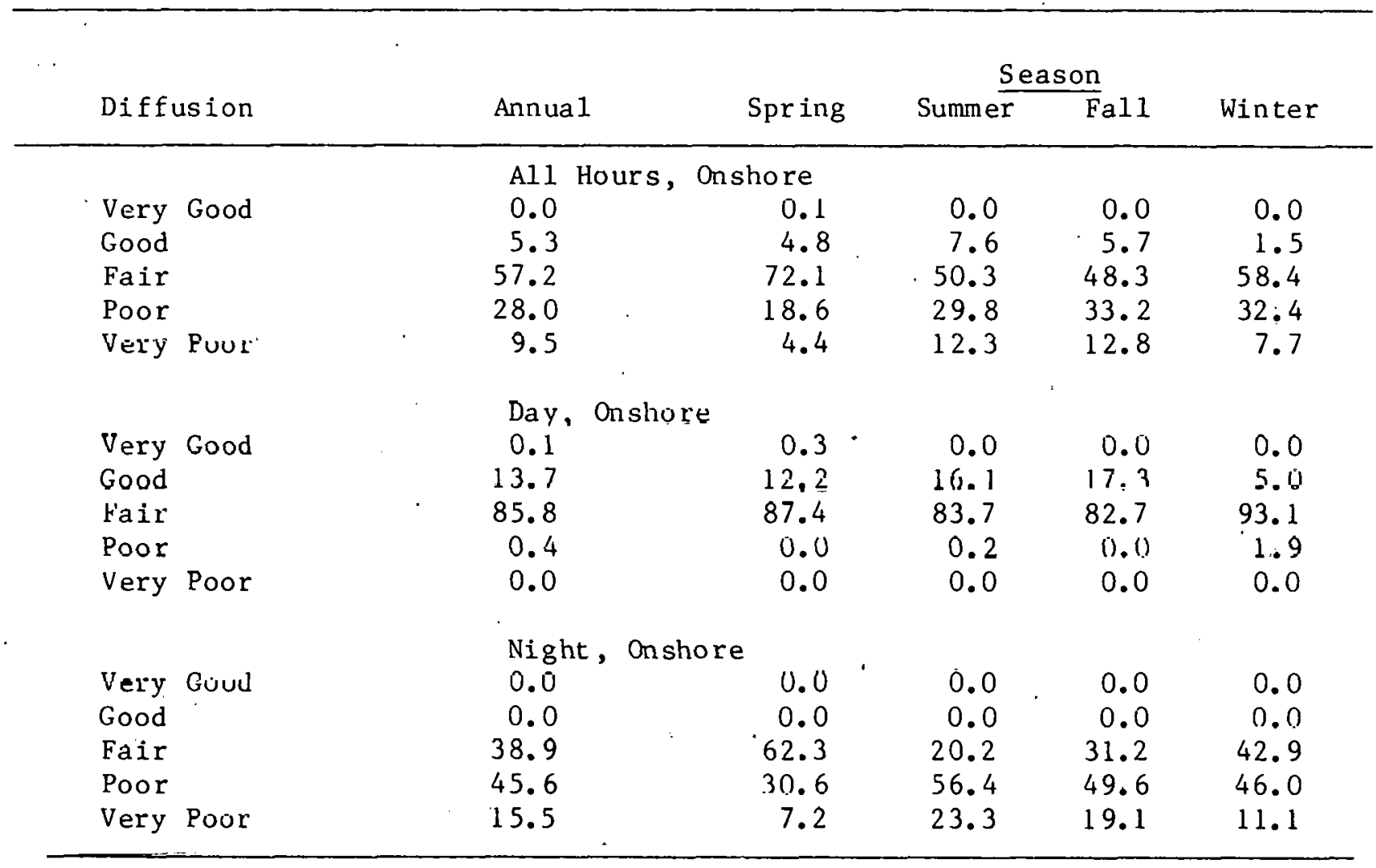


Table 7.30 (continued)

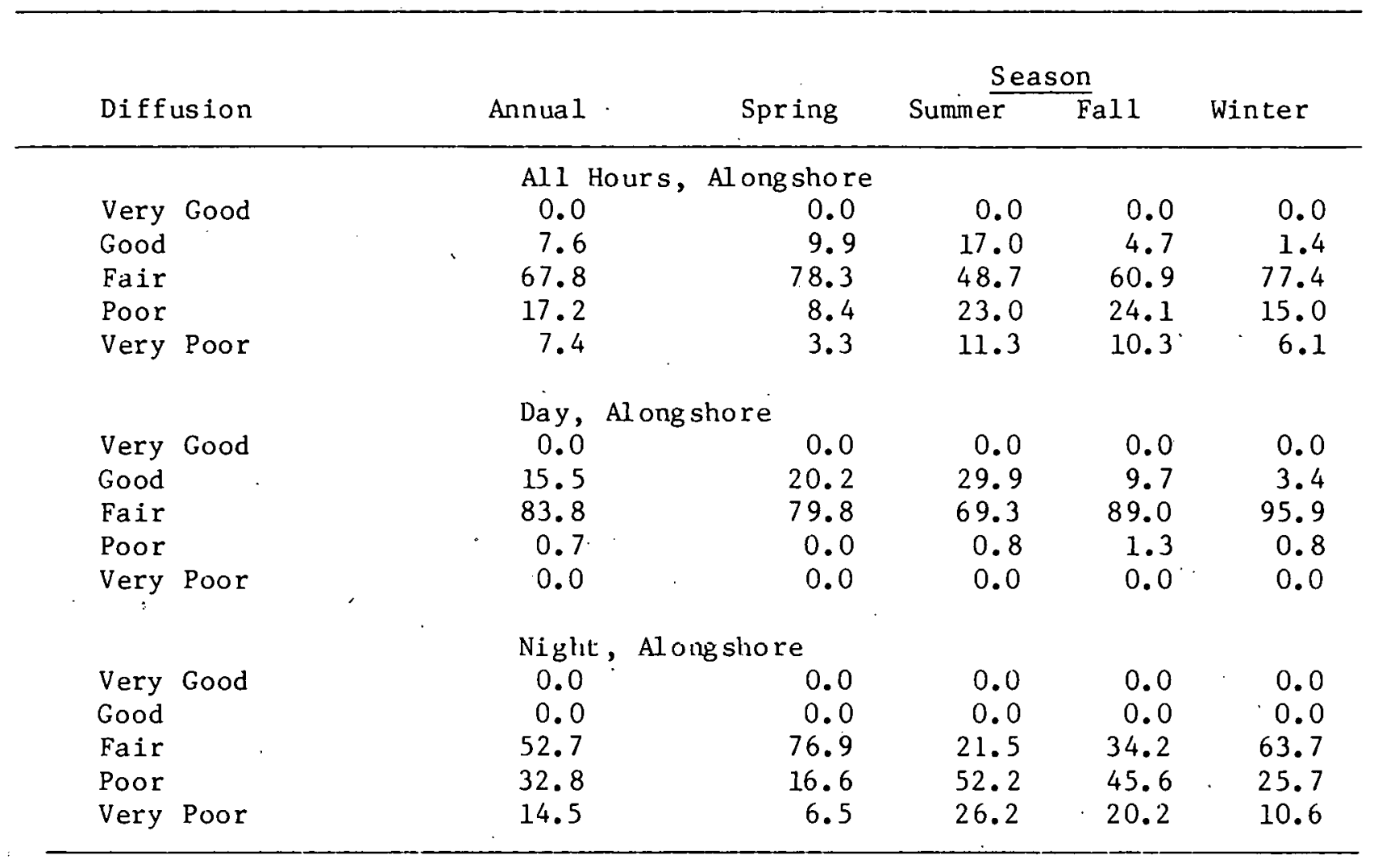


Table 7.30 (continued)

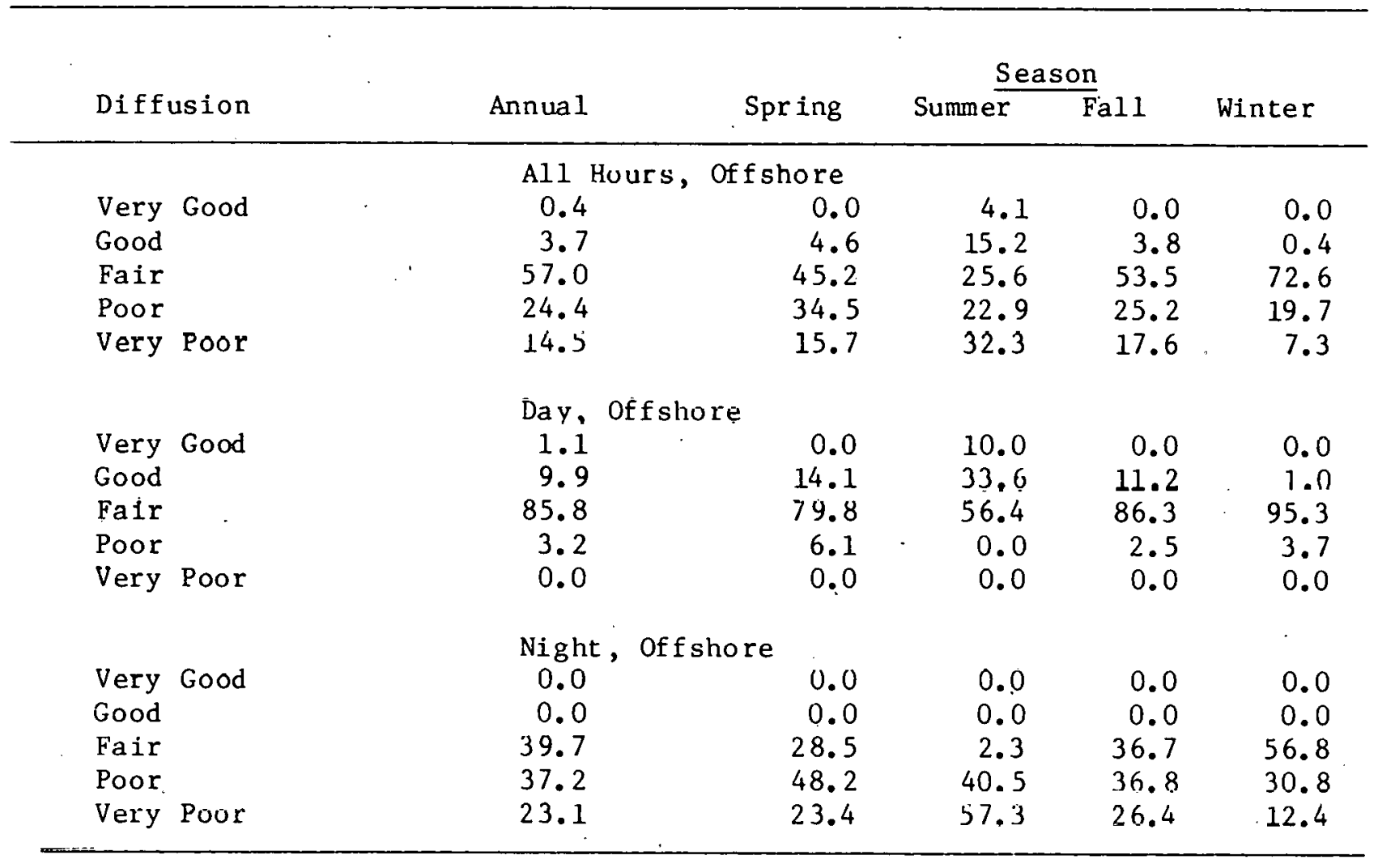




\begin{tabular}{|c|c|c|c|c|c|c|c|c|}
\hline \multicolumn{9}{|c|}{$\begin{array}{l}\text { Percent of Hours in Each Combination of Wind Direction } \\
\text { and Diffusion Rating by Season and Time of Day }\end{array}$} \\
\hline $\begin{array}{l}\text { Diffusion } \\
\text { Rating }\end{array}$ & $\begin{array}{l}\text { Off- } \\
\text { shore }\end{array}$ & $\begin{array}{l}\text { Along- } \\
\text { shore }\end{array}$ & $\begin{array}{l}\text { On- } \\
\text { shore }\end{array}$ & Calm & $\begin{array}{l}\text { Off- } \\
\text { shore }\end{array}$ & $\begin{array}{l}\text { Along- } \\
\text { shore }\end{array}$ & $\begin{array}{l}\text { On- } \\
\text { shore }\end{array}$ & Calm \\
\hline $\begin{array}{l}\text { Very Good } \\
\text { Good } \\
\text { Fair } \\
\text { Poor } \\
\text { Very Poor }\end{array}$ & $\begin{array}{r}0.0 \\
1.6 \\
26.6 \\
15.2 \\
7.5\end{array}$ & $\begin{array}{c}\text { Al1 Ho } \\
0.0 \\
0.6 \\
17.0 \\
6.1 \\
1.5\end{array}$ & $\begin{array}{r}1 \mathrm{ss} \\
0.0 \\
2.3 \\
14.7 \\
3.4 \\
1.2\end{array}$ & $\begin{array}{l}0.0 \\
0.0 \\
0.0 \\
0.3 \\
1.8\end{array}$ & & & & \\
\hline $\begin{array}{l}\text { Very Good } \\
\text { Good } \\
\text { Fair } \\
\text { Poor } \\
\text { Very Poor }\end{array}$ & $\begin{array}{r}0.0 \\
4.0 \\
38.7 \\
1.7 \\
0.0\end{array}$ & $\begin{array}{c}\text { Day } \\
0.0 \\
1.5 \\
20.6 \\
0.7 \\
0.0\end{array}$ & $\begin{array}{r}0.0 \\
5.5 \\
25.0 \\
1.3 \\
0.0\end{array}$ & $\begin{array}{l}0.0 \\
0.0 \\
0.0 \\
0.8 \\
0.1\end{array}$ & $\begin{array}{r}0.0 \\
0.0 \\
18.2 \\
24.7 \\
12.7\end{array}$ & $\begin{array}{l}\text { Nigh } \\
0.0 \\
0.0 \\
14.4 \\
9.9 \\
2.6\end{array}$ & $\begin{array}{l}0.0 \\
0.0 \\
7.6 \\
4.9 \\
2.1\end{array}$ & $\begin{array}{l}0.0 \\
0.0 \\
0.0 \\
0.0 \\
3.0\end{array}$ \\
\hline $\begin{array}{l}\text { Very Good } \\
\text { Good } \\
\text { Fair } \\
\text { Poor } \\
\text { Very Poor }\end{array}$ & $\begin{array}{r}0.0 \\
1.8 \\
29.2 \\
12.8 \\
5.6\end{array}$ & $\begin{array}{l}\text { Sprir } \\
0.0 \\
0.5 \\
15.5 \\
4.7 \\
1.6\end{array}$ & $\begin{array}{r}0.1 \\
2.5 \\
19.2 \\
3.0 \\
0.8\end{array}$ & $\begin{array}{l}0.0 \\
0.0 \\
0.0 \\
0.6 \\
2.2\end{array}$ & $\begin{array}{r}0.0 \\
4.3 \\
18.6 \\
11.2 \\
8.9\end{array}$ & $\begin{array}{c}\text { Summ } \\
0.0 \\
1.5 \\
17.3 \\
6.7 \\
2.0\end{array}$ & $\begin{array}{r}\text { r } \\
0.0 \\
5.6 \\
15.8 \\
4.8 \\
1.9\end{array}$ & $\begin{array}{l}0.0 \\
0.0 \\
0.0 \\
0.0 \\
1.4\end{array}$ \\
\hline $\begin{array}{l}\text { Very Good } \\
\text { Good } \\
\text { Fair } \\
\text { Pour } \\
\text { Very Poor }\end{array}$ & $\begin{array}{r}0.0 \\
0.5 \\
21.6 \\
15.8 \\
9.3\end{array}$ & $\begin{array}{l}\text { Fall } \\
0.0 \\
0.5 \\
19.1 \\
7.3 \\
1.2\end{array}$ & $\begin{array}{r}0.0 \\
0.8 \\
17.1 \\
3.6 \\
1.2\end{array}$ & $\begin{array}{l}0.0 \\
0.0 \\
0.0 \\
0.2 \\
1.8\end{array}$ & $\begin{array}{r}0.0 \\
0.0 \\
37.1 \\
21.1 \\
6.1\end{array}$ & $\begin{array}{c}\text { Wint } \\
0.0 \\
0.0 \\
15.9 \\
5.8 \\
1.3\end{array}$ & $\begin{array}{l}0 . \\
0.0 \\
0.1 \\
6.8 \\
2.1 \\
1.0\end{array}$ & $\begin{array}{l}0.0 \\
0.0 \\
0.0 \\
0.6 \\
1.8\end{array}$ \\
\hline
\end{tabular}




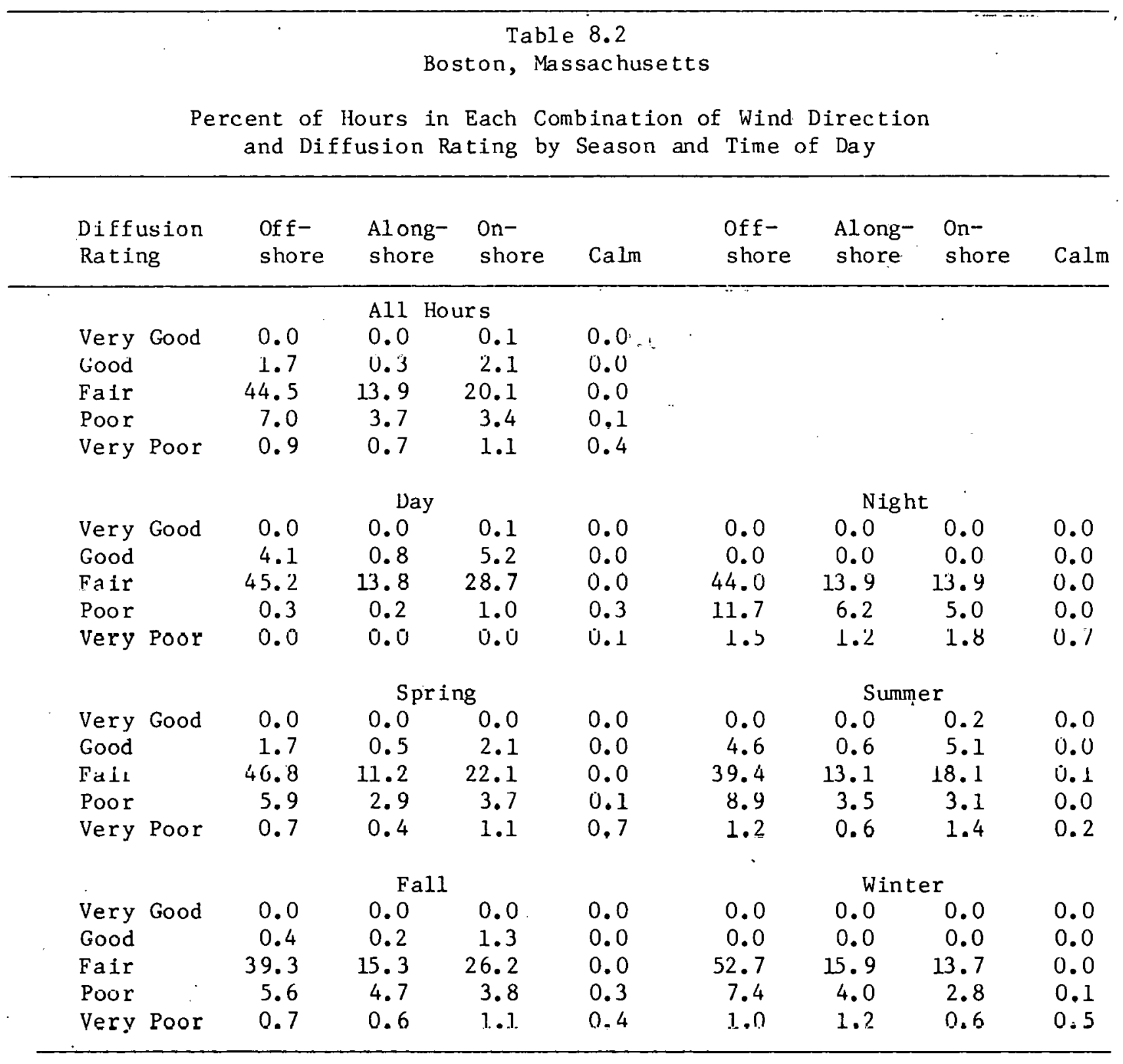




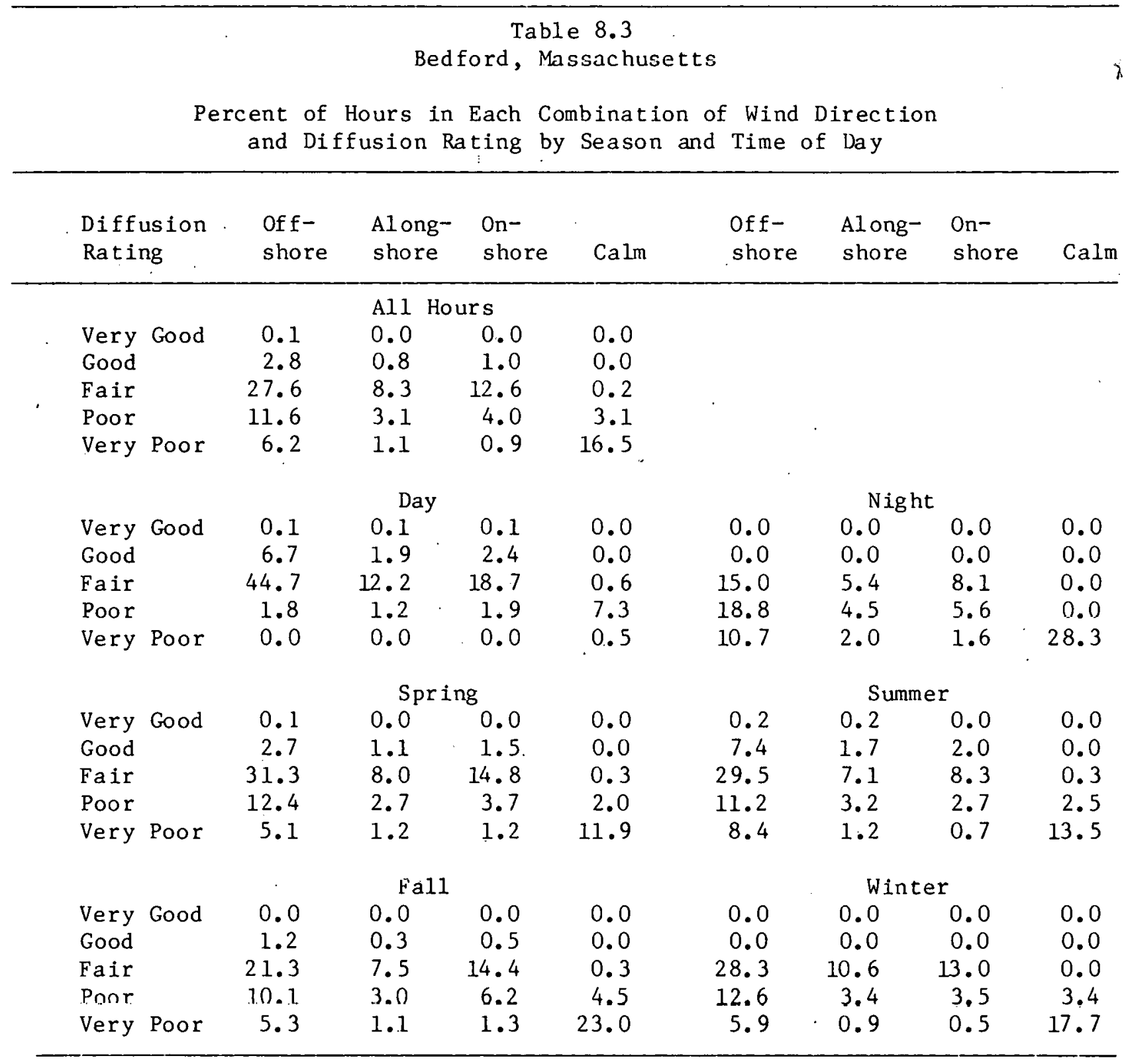


Table 8.4

Falmouth, Massachusetts

Percent of Hours in Each Combination of Wind Direction and Diffusion Rating by. Season and Time of Day

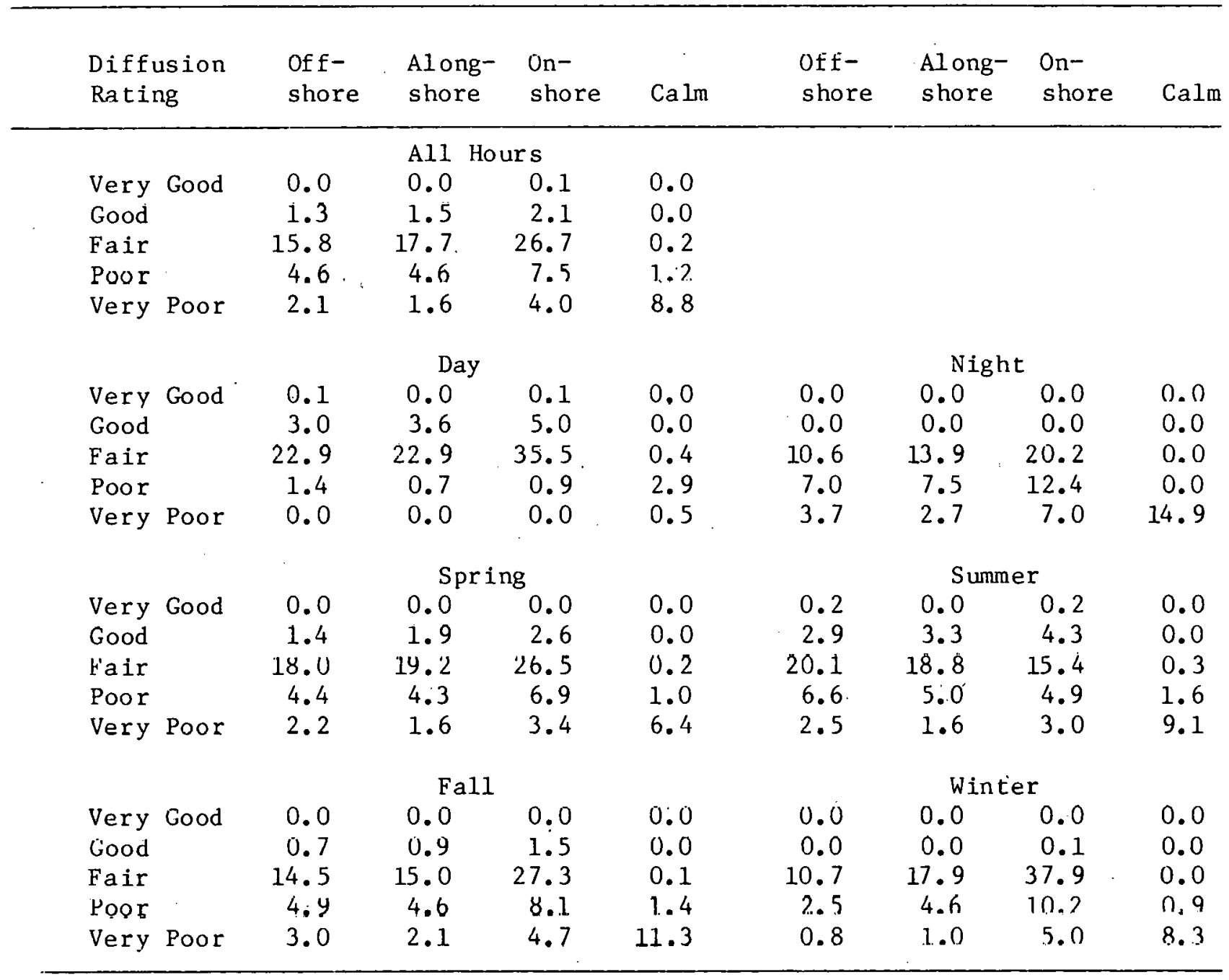


Table 8.5

Nantucket, Massachusetts

Percent of Hours in Each Combination of Wind Direction and Diffusion Rating by Season and Time of Day

\begin{tabular}{|c|c|c|c|c|c|c|c|c|}
\hline $\begin{array}{l}\text { Diffusion } \\
\text { Rating }\end{array}$ & $\begin{array}{l}\text { Off- } \\
\text { shore }\end{array}$ & $\begin{array}{l}\text { Along- } \\
\text { shore }\end{array}$ & $\begin{array}{l}\text { On- } \\
\text { shore }\end{array}$ & $\mathrm{Ca} 1 \mathrm{~m}$ & $\begin{array}{l}\text { Off- } \\
\text { shore }\end{array}$ & $\begin{array}{l}\text { Along- } \\
\text { shore }\end{array}$ & $\begin{array}{l}\text { On- } \\
\text { shore }\end{array}$ & Calm \\
\hline \multicolumn{9}{|c|}{ All Hours } \\
\hline Very Good & 0.0 & 0.1 & 0.0 & 0.0 & & & & \\
\hline Good & 1.9 & 0.7 & 1.2 & 0.0 & & & & \\
\hline Fair & 26.1 & 19.4 & 31.8 & 0.0 & & & & \\
\hline Poor & 5.3 & 4.0 & 6.3 & 0.0 & & & & \\
\hline Very Poor & 0.9 & 0.5 & 1.1 & 0.6 & & & & \\
\hline \multicolumn{5}{|c|}{ Day } & \multicolumn{4}{|c|}{ Night } \\
\hline Very Good & 0.1 & 0.1 & 0.0 & 0.0 & 0.0 & 0.0 & 0.0 & 0.0 \\
\hline Good & 4.4 & 1.7 & 2.8 & 0.0 & 0.0 & 0.0 & 0.0 & 0.0 \\
\hline Fair & 33.1 & 21.9 & $35: 2$ & 0.0 & 20.9 & 17.5 & 29.4 & 0.0 \\
\hline Poor & 0.2 & 0.0 & 0.2 & 0.1 & 9.1 & 7.0 & 10.8 & 0.0 \\
\hline \multirow[t]{2}{*}{ Very Poor } & 0.0 & 0.0 & 0.0 & 0.0 & 1.6 & 0.8 & 1.9 & 1.0 \\
\hline & \multicolumn{4}{|c|}{ Spring } & \multicolumn{4}{|c|}{ Summer } \\
\hline Very Good & 0.0 & 0.1 & 0.0 & 0.0 & 0.1 & 0.1 & 0.0 & 0.0 \\
\hline Good & 1.6 & 0.8 & 1.0 & 0.0 & 5.2 & 1.3 & 2.7 & 0.0 \\
\hline Fair & 30.2 & 17.9 & 30.1 & 0.0 & 37.2 & 14.2 & 17.4 & 0.0 \\
\hline Poor & 6.0 & 4.3 & 5.6 & $0.0^{\circ}$ & 8.6 & 4.2 & 4.8 & 0.1 \\
\hline \multirow[t]{2}{*}{ Very Poor } & 0.7 & 0.5 & 1.0 & 0.3 & 1.6 & 0.6 & 0.9 & 1.0 \\
\hline & \multicolumn{4}{|c|}{ Fall } & \multicolumn{4}{|c|}{ Winter } \\
\hline Very Good & 0.0 & 0.0 & 0.0 & 0.0 & 0.0 & 0.0 & 0.0 & 0.0 \\
\hline Good & 0.5 & 0.8 & 1.0 & 0.0 & 0.0 & 0.0 & 0.1 & 0.0 \\
\hline Fair & 21.4 & 22.9 & 33.7 & 0.0 & 15.0 & 23.0 & 46.4 & 0.0 \\
\hline Poor & 4.6 & 5.3 & 6.8 & 0.1 & 2.0 & 2.4 & 8.2 & 0.0 \\
\hline Very Poor & 0.8 & 0.6 & 0.9 & 0.5 & 0.6 & 0.3 & 1.7 & 0.5 \\
\hline
\end{tabular}




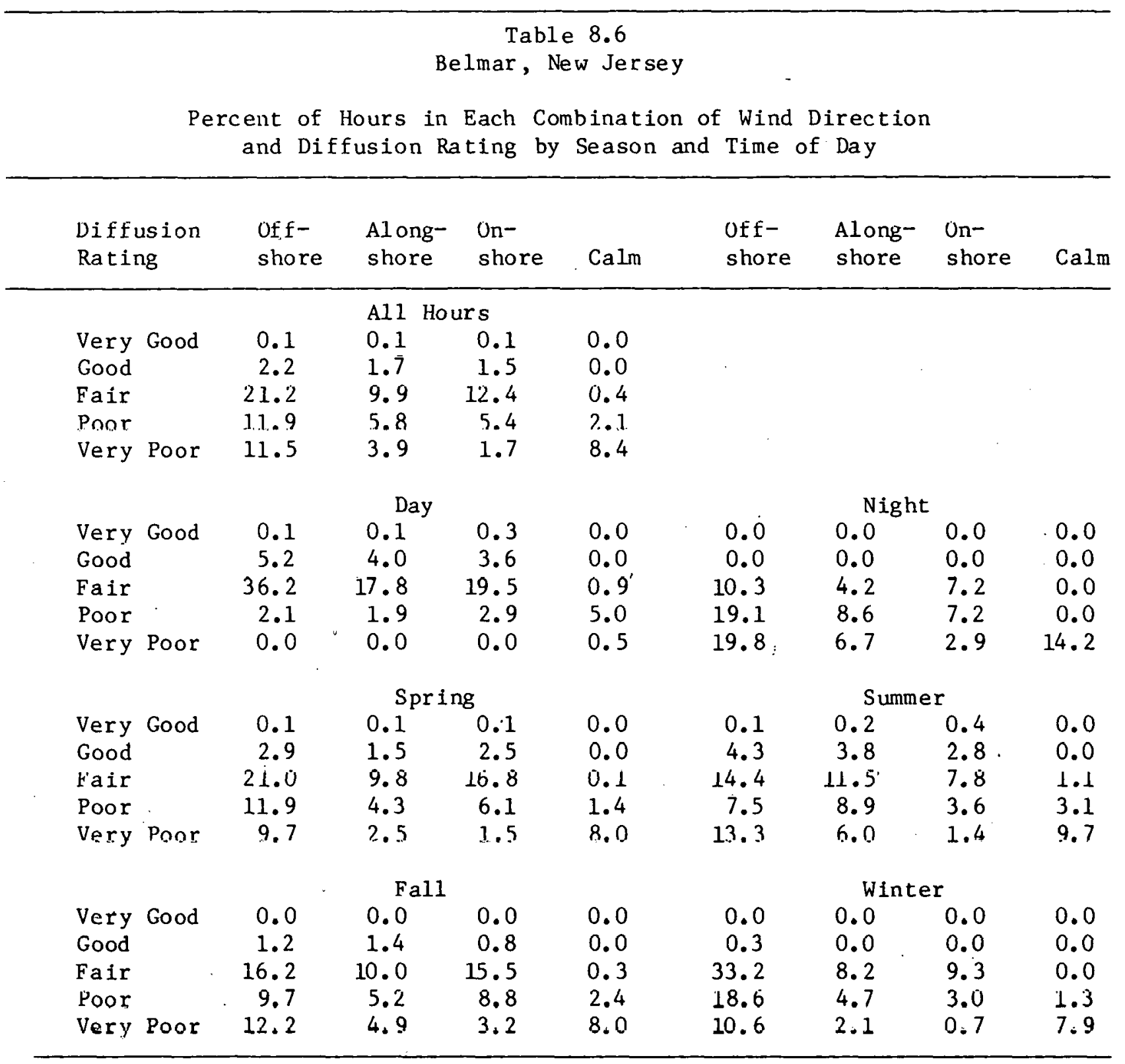


Table 8.7

Lakehurst, New Jersey

Percent of Hours in Each Combination of Wind Direction and Diffusion Rating, by Season and Time of Day

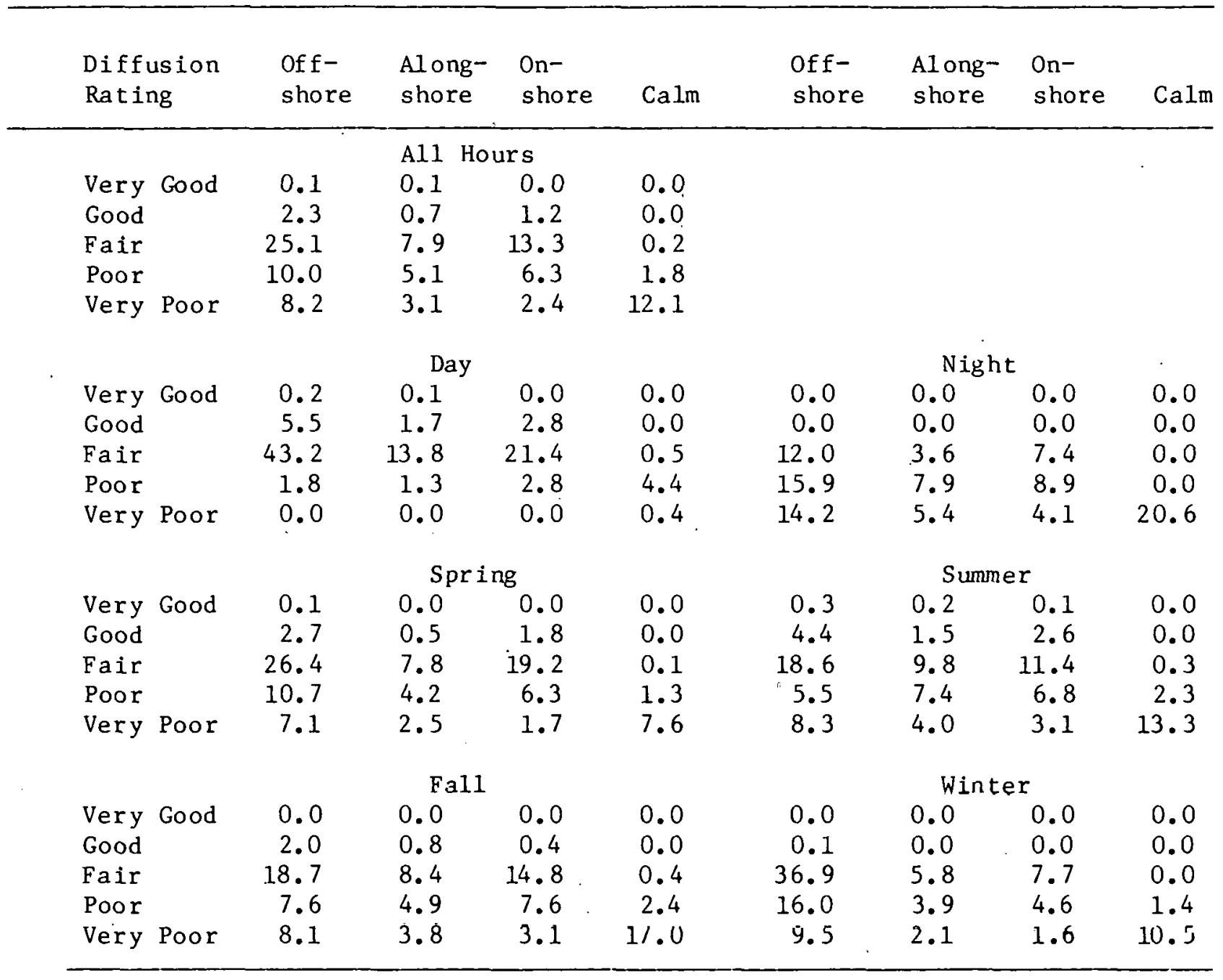




\begin{tabular}{|c|c|c|c|c|c|c|c|c|}
\hline \multicolumn{9}{|c|}{ Table 8.8} \\
\hline \multicolumn{9}{|c|}{$\begin{array}{l}\text { Percent of Hours in Each Combination of Wind Direction } \\
\text { and Diffusion Rating by Season and Time of Day }\end{array}$} \\
\hline $\begin{array}{l}\text { Diffusion } \\
\text { Rating }\end{array}$ & $\begin{array}{l}\text { Off- } \\
\text { shore }\end{array}$ & $\begin{array}{l}\text { Al ong- } \\
\text { shore }\end{array}$ & $\begin{array}{l}\text { On- } \\
\text { shore }\end{array}$ & Calm & $\begin{array}{l}\text { Off- } \\
\text { shore }\end{array}$ & $\begin{array}{l}\text { Along- } \\
\text { shore }\end{array}$ & $\begin{array}{l}\text { On- } \\
\text { shore }\end{array}$ & Calm \\
\hline \multicolumn{9}{|c|}{ Al1 Hours } \\
\hline Very Good & 0.1 & 0.0 & 0.0 & 0.0 & & & & \\
\hline Good & 2.1 & 0.9 & 2.5 & 0.0 & & & & \\
\hline Fair & 27.7 & 14.8 & 18,8 & 0.1 & & & . & \\
\hline Poor & 11.3 & 6.1 & 5.0 & 0.9 & & & & \\
\hline \multirow[t]{2}{*}{ Very Poor } & 2.4 & 1.0 & 0.9 & 5.4 & & & & \\
\hline & \multicolumn{4}{|c|}{ Day } & \multicolumn{4}{|c|}{ Night } \\
\hline Very Good & 0.1 & 0.0 & 0.0 & 0.0 & 0.0 & 0.0 & 0.0 & 0.0 \\
\hline Good & 5.0 & 2.1 & 5.9 & 0.0 & 0.0 & 0.0 & 0.0 & 0.0 \\
\hline Fair & 37.6 & 17.8 & 26.9 & 0.2 & 20.6 & 12.6 & 13.0 & 0.0 \\
\hline Poor & 0.7 & 0.6 & 0.7 & 2.2 & 19.0 & 10.1 & 8.1 & 0.0 \\
\hline Very Poor & 0.0 & 0.0 & 0.0 & 0.0 & 4.1 & 1.8 & 1.5 & 9.2 \\
\hline & \multicolumn{4}{|c|}{ Spring } & \multicolumn{4}{|c|}{ Summer } \\
\hline Very Good & 0.0 & 0.0 & 0.0 & 0.0 & 0.2 & 0.0 & 0.1 & 0.0 \\
\hline Good & 2.2 & 0.3 & 2.2 & 0.0 & 4.9 & 2.8 & 6.2 & 0.0 \\
\hline Fair & 32.7 & 15.8 & 17.7 & 0.3 & 16.0 & 14.0 & 20.5 & 0.0 \\
\hline Poor & 11.6 & 4.8 & 3.7 & 1.0 & 9.2 & 9.3 & 6.7 & 1.2 \\
\hline \multirow[t]{2}{*}{ Very Poor } & 1.7 & 0.7 & 0.7 & 4.5 & 2.2 & 1.4 & 1.2 & 4.1 \\
\hline & \multicolumn{4}{|c|}{ Fall } & \multicolumn{4}{|c|}{ Winter } \\
\hline Very Good & 0.0 & 0.0 & 0.0 & 0.0 & 0.0 & 0.0 & 0.0 & 0.0 \\
\hline Good & 1.0 & 0.5 & 1.3 & 0.0 & 0.1 & 0.0 & 0.1 & 0.0 \\
\hline Fair & 21.0 & 15.0 & 24.2 & 0.1 & 41.3 & 14.3 & 12.8 & 0.0 \\
\hline Poor & 10.0 & 5.8 & 6.9 & 1.1 & 14.5 & 4.6 & 2.7 & 0.3 \\
\hline Very Poor & 3.4 & 1.2 & 1.0 & 7.2 & 2.2 & 0.8 & 0.6 & 5.7 \\
\hline
\end{tabular}


Table 8.9

Wilming ton, Delaware

Percent of Hours in Each Combination of Wind Direction and Diffusion Rating by Season and Time of Day

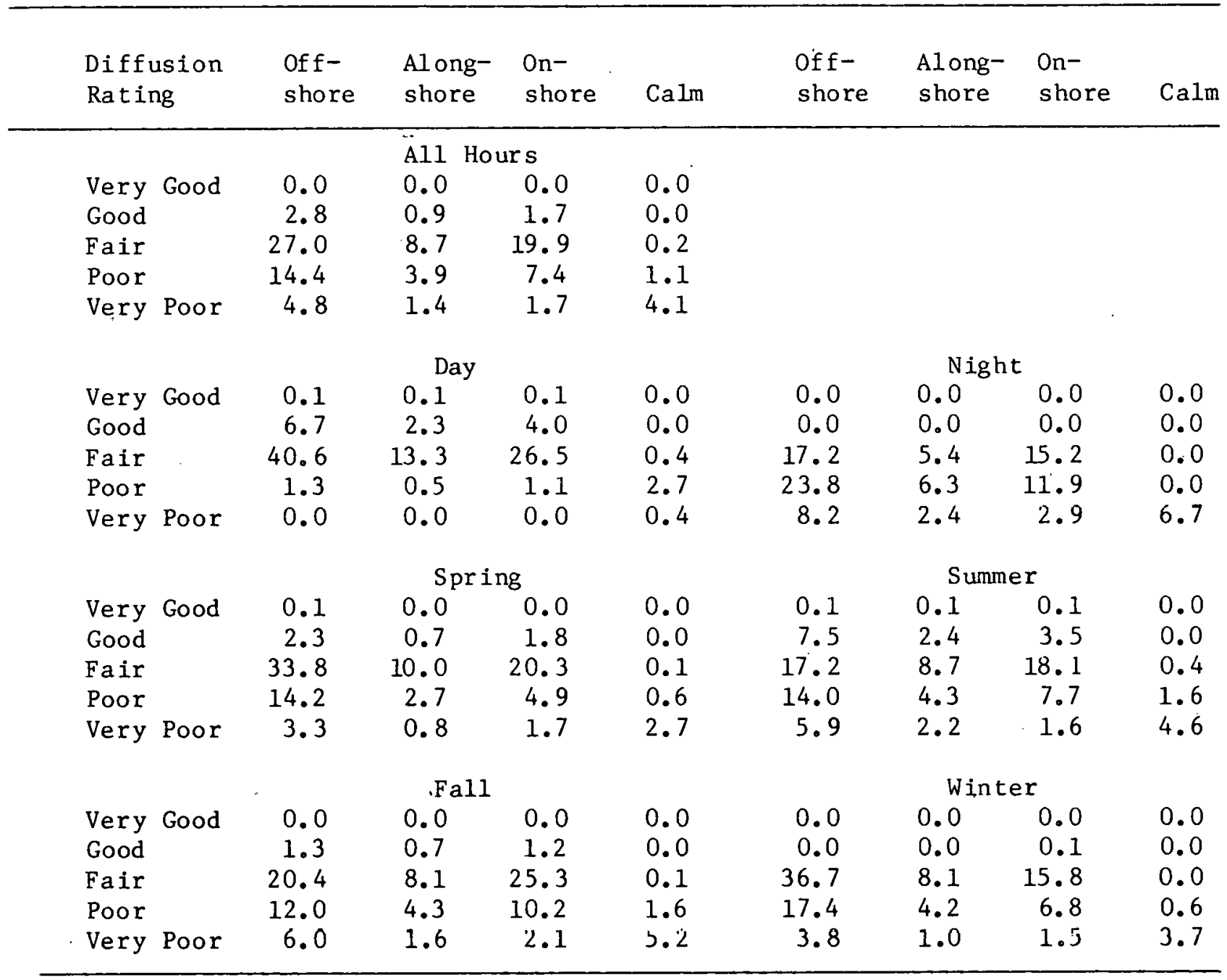




\begin{tabular}{|c|c|c|c|c|c|c|c|c|}
\hline \multicolumn{9}{|c|}{$\begin{array}{l}\text { Table } 8.10 \\
\text { Wallons }\end{array}$} \\
\hline \multicolumn{9}{|c|}{$\begin{array}{l}\text { Percent of Hours in Each Combination of Wind Direction } \\
\text { and Diffusion Rating by Season and Time of Day }\end{array}$} \\
\hline $\begin{array}{l}\text { Diffusion } \\
\text { Rating }\end{array}$ & $\begin{array}{l}\text { Off- } \\
\text { shore }\end{array}$ & $\begin{array}{l}\text { Al ong- } \\
\text { shore }\end{array}$ & $\begin{array}{l}\text { On- } \\
\text { shore }\end{array}$ & $\mathrm{Calm}$ & $\begin{array}{l}\text { Off- } \\
\text { shore }\end{array}$ & $\begin{array}{l}\text { Along- } \\
\text { shore }\end{array}$ & $\begin{array}{l}\text { On- } \\
\text { shore }\end{array}$ & $\mathrm{Calm}$ \\
\hline \multicolumn{9}{|c|}{ Al1 Hours } \\
\hline Very Good & 0.1 & 0.0 & 0.2 & 0.0 & & & & \\
\hline Good & 2.5 & 0.8 & 6.9 & 0.0 & & & & \\
\hline Fair & 24.6 & 18.0 & 23.9 & 0.1 & & & & \\
\hline Poor & 5.9 & 4.9 & 5.6 & 0.8 & & & & \\
\hline Verty & 2.0 & 1.4 & 1.2 & 1.5 & & & & \\
\hline & \multicolumn{3}{|c|}{ Day } & & \multicolumn{4}{|c|}{ Night } \\
\hline Very Good & 0.1 & 0.0 & 0.3 & 0.0 & 0.0 & 0.0 & 0.0 & 0.0 \\
\hline Good & 4.2 & 1.3 & 11.3 & 0.0 & 0.0 & 0.0 & 0.0 & 0.0 \\
\hline Fair & 29.2 & 18.2 & 31.0 & 0.1 & 17.6 & 17.8 & 12.9 & 0.0 \\
\hline Poor & 0.8 & 0.7 & 1.7 & 1.3 & 13.8 & 11.4 & 11.7 & 0.0 \\
\hline \multirow[t]{2}{*}{ Very } & 0.0 & 0.0 & 0.0 & 0.2 & 5.1 & 3.5 & 2.9 & 3.7 \\
\hline & \multicolumn{4}{|c|}{ Spring } & \multicolumn{4}{|c|}{ Summer } \\
\hline Very Good & 0.0 & $0.0^{\mathrm{T}}$ & 0.0 & 0.0 & 0.2 & 0.0 & 1.3 & 0.0 \\
\hline Good & 3.7 & 0.4 & 5.8 & 0.0 & 4.6 & 2.5 & 16.8 & 0.0 \\
\hline Fair & 30.2 & 1.6 .4 & 22.5 & 0.0 & 13.0 & 15.1 & 29.0 & 0.1 \\
\hline Poor & 5.3 & 4.8 & 6.2 & 0.7 & 1.2 & 3.2 & 6.4 & 1.6 \\
\hline \multirow[t]{2}{*}{ Very Poor } & 1.6 & 1.1 & 0.6 & 1.1 & 1.0 & 0.7 & 2.3 & 0.9 \\
\hline & \multicolumn{3}{|c|}{ Fall } & & \multicolumn{4}{|c|}{ Winter } \\
\hline Very Good & 0.0 & $0 . \dot{0}$ & 0.0 & 0.0 & 0.0 & 0.0 & 0.0 & 0.0 \\
\hline Good & 1.5 & 0.2 & 5.6 & 0.0 & 0.4 & 0.0 & 0.2 & 0.0 \\
\hline Fair & 19.0 & 19.4 & 30.3 & 0.1 & 35.1 & 21.0 & 14.2 & 0.0 \\
\hline Poor & 5.8 & 4.6 & 5.8 & 0.3 & 10.9 & 6.6 & $4.0^{\circ}$ & 0.7 \\
\hline Very Poor & 2.7 & 1.8 & 1.4 & 1.6 & 2.6 & 1.7 & 0.4 & 2.5 \\
\hline
\end{tabular}




\begin{tabular}{|c|c|c|c|c|c|c|c|c|}
\hline \multicolumn{9}{|c|}{$\begin{array}{c}\text { Percent of Hours in Each Combination of Wind Direction } \\
\text { and Diffusion Rating by Season and Time of Day }\end{array}$} \\
\hline $\begin{array}{l}\text { Diffusion } \\
\text { Rating }\end{array}$ & $\begin{array}{l}\text { Off- } \\
\text { shore }\end{array}$ & $\begin{array}{l}\text { Along- } \\
\text { shore }\end{array}$ & $\begin{array}{l}\text { On- } \\
\text { shore }\end{array}$ & $\mathrm{Ca} 1 \mathrm{~m}$ & $\begin{array}{l}\text { Off- } \\
\text { shore }\end{array}$ & $\begin{array}{l}\text { Along- } \\
\text { shore }\end{array}$ & $\begin{array}{l}\text { On- } \\
\text { shore }\end{array}$ & Calm \\
\hline $\begin{array}{l}\text { Very Good } \\
\text { Good } \\
\text { Fair } \\
\text { Poor } \\
\text { Very Poor }\end{array}$ & $\begin{array}{r}0.0 \\
1.8 \\
19.1 \\
5.4 \\
1.2\end{array}$ & $\begin{array}{r}\text { Al1 Ho } \\
0.0 \\
2.8 \\
24.0 \\
9.3 \\
2.2\end{array}$ & $\begin{array}{l}\text { urs } \\
0.1 \\
2.4 \\
17.6 \\
9.3 \\
3.3\end{array}$ & $\begin{array}{l}0.0 \\
0.0 \\
0.0 \\
0.3 \\
1.2\end{array}$ & & . & & \\
\hline $\begin{array}{l}\text { Very Good } \\
\text { Good } \\
\text { Fair } \\
\text { Poor } \\
\text { Very Poor }\end{array}$ & $\begin{array}{r}0.0 \\
4.2 \\
25.2 \\
0.2 \\
0.0\end{array}$ & $\begin{array}{c}\text { Day } \\
0.1 \\
6.6 \\
30.4 \\
0.6 \\
0.0\end{array}$ & $\begin{array}{r}0.1 \\
5.6 \\
25.3 \\
0.9 \\
0.0\end{array}$ & $\begin{array}{l}0.0 \\
0.0 \\
0.0 \\
0.6 \\
0.0\end{array}$ & $\begin{array}{r}0.0 \\
0.0 \\
14.6 \\
9.2 \\
2.2\end{array}$ & $\begin{array}{l}\text { Nigh } \\
0.0 \\
0.0 \\
19.4 \\
15.7 \\
3.9\end{array}$ & $\begin{array}{r}0.0 \\
0.0 \\
12.0 \\
15.5 \\
5.7\end{array}$ & $\begin{array}{l}0.0 \\
0.0 \\
0.0 \\
0.0 \\
2.0\end{array}$ \\
\hline $\begin{array}{l}\text { Very Good } \\
\text { Good } \\
\text { Fair } \\
\text { Poor } \\
\text { Very Poor }\end{array}$ & $\begin{array}{r}0.0 \\
3.0 \\
22.3 \\
6.1 \\
0.6\end{array}$ & $\begin{array}{l}\quad \text { Sprir } \\
0.0 \\
2.8 \\
23.6 \\
7.7 \\
1.1\end{array}$ & $\begin{array}{l}\text { ng } \\
0.1 \\
2.4 \\
18.2 \\
9.1 \\
2.2\end{array}$ & $\begin{array}{l}0.0 \\
0.0 \\
0.0 \\
0.1 \\
0.7\end{array}$ & $\begin{array}{r}0.0 \\
2.9 \\
12.0 \\
4.0 \\
1.5\end{array}$ & $\begin{array}{c}\text { Summe } \\
0.1 \\
5.9 \\
19.4 \\
11.8 \\
2.8\end{array}$ & $\begin{array}{r}\text { er } \\
0.1 \\
5.3 \\
16.8 \\
10.5 \\
4.2\end{array}$ & $\begin{array}{l}0.0 \\
0.0 \\
0.0 \\
0.7 \\
1.8\end{array}$ \\
\hline $\begin{array}{l}\text { Very Good } \\
\text { Good } \\
\text { Fair } \\
\text { Poor } \\
\text { Very Poor }\end{array}$ & $\begin{array}{r}0.0 \\
1.0 \\
14.1 \\
3.8 \\
2.0\end{array}$ & $\begin{array}{l}\quad \text { Fall } \\
0.0 \\
2.1 \\
20.8 \\
9.0 \\
3.1\end{array}$ & $\begin{array}{r}0.0 \\
1.6 \\
24.0 \\
12.0 \\
4.7\end{array}$ & $\begin{array}{l}0.0 \\
0.0 \\
0.0 \\
0.1 \\
1.8\end{array}$ & $\begin{array}{r}0.0 \\
0.2 \\
28.1 \\
7.8 \\
U . \dot{9}\end{array}$ & $\begin{array}{l}\text { Winte } \\
0.0 \\
0.2 \\
32.5 \\
8.9 \\
1.9\end{array}$ & $\begin{array}{r}\text { er } \\
0.0 \\
0.1 \\
11.2 \\
5.6 \\
2.0\end{array}$ & $\begin{array}{l}0.0 \\
0.0 \\
0.0 \\
0.1 \\
0.4\end{array}$ \\
\hline
\end{tabular}


Table 8.12

Cape Hatteras, North Carolina

Percent of Hours in Each Combination of Wind Direction and Diffusion Rating by Season and Time of Day

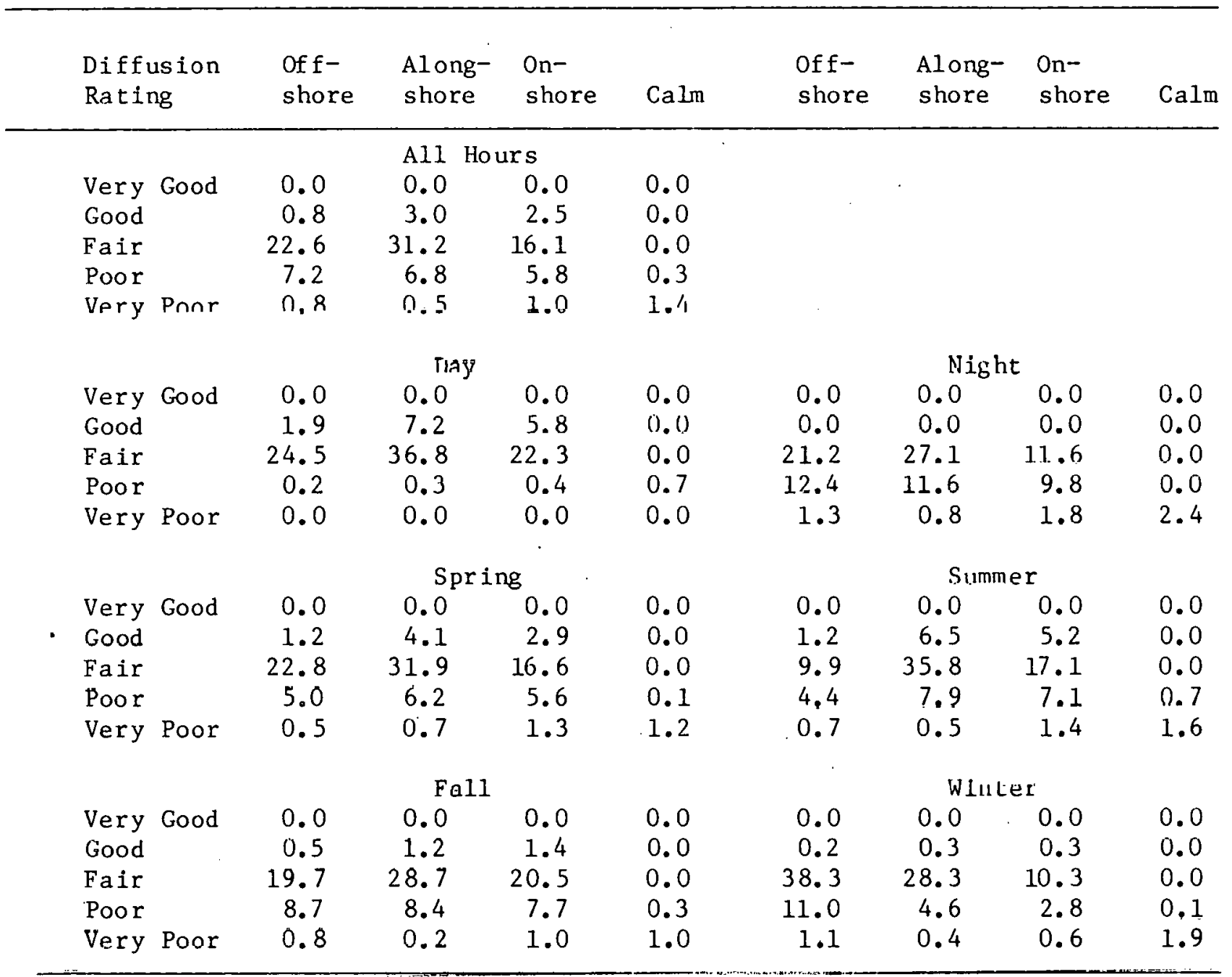




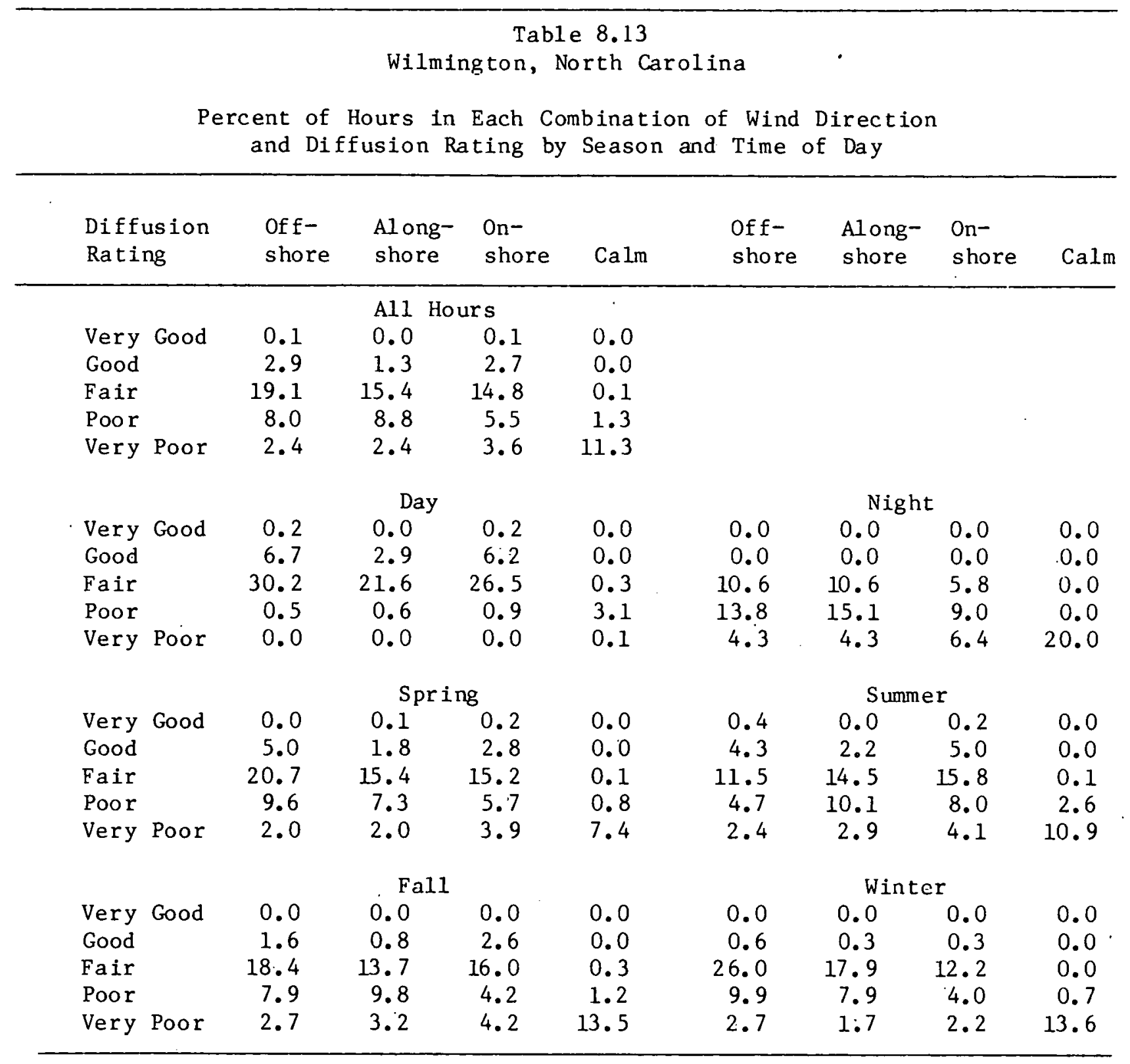




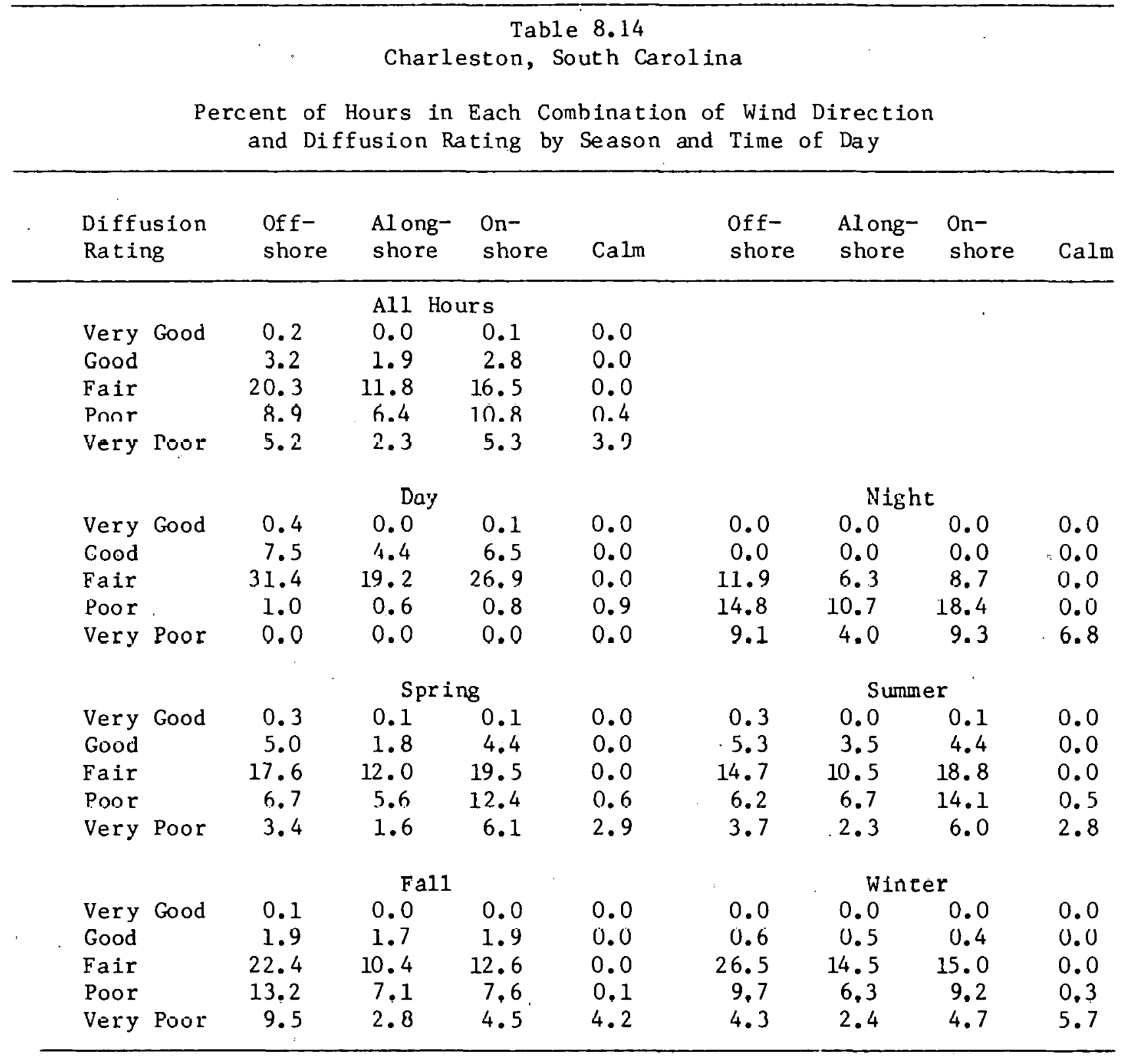




\begin{tabular}{|c|c|c|c|c|c|c|c|c|}
\hline \multicolumn{9}{|c|}{$\begin{array}{c}\text { Percent of Hours in Each Combination of Wind Direction } \\
\text { and Diffusion Rating by Season and Time of Day }\end{array}$} \\
\hline $\begin{array}{l}\text { Diffusion } \\
\text { Rating }\end{array}$ & $\begin{array}{l}\text { Off- } \\
\text { shore }\end{array}$ & $\begin{array}{l}\text { Along- } \\
\text { shore }\end{array}$ & $\begin{array}{l}\text { On- } \\
\text { shore }\end{array}$ & $\mathrm{Calm}$ & $\begin{array}{l}\text { Off- } \\
\text { shore }\end{array}$ & $\begin{array}{l}\text { Along- } \\
\text { shore }\end{array}$ & $\begin{array}{l}\text { On- } \\
\text { shore }\end{array}$ & Calm \\
\hline $\begin{array}{l}\text { Very Good } \\
\text { Good } \\
\text { Fair } \\
\text { Poor } \\
\text { Very, Poor }\end{array}$ & $\begin{array}{r}0.2 \\
2.8 \\
14.7 \\
7.3 \\
7.8\end{array}$ & $\begin{array}{l}\text { A11 Hou } \\
0.1 \\
1.4 \\
6.9 \\
4.4 \\
4.1\end{array}$ & $\begin{array}{l}\text { urs } \\
0.2 \\
3.4 \\
12.9 \\
5.2 \\
5.2\end{array}$ & $\begin{array}{r}0.0 \\
0.0 \\
0.5 \\
2.4 \\
20.5\end{array}$ & & & & \\
\hline $\begin{array}{l}\text { Very Good } \\
\text { Good } \\
\text { Fair } \\
\text { Poor } \\
\text { Very Poor }\end{array}$ & $\begin{array}{r}0.4 \\
6.6 \\
29.6 \\
2.8 \\
0.0\end{array}$ & $\begin{array}{c}\text { Day } \\
0.2 \\
3.2 \\
11.7 \\
1.1 \\
0.0\end{array}$ & $\begin{array}{r}0.5 \\
8.0 \\
26.7 \\
1.8 \\
0.0\end{array}$ & $\begin{array}{l}0.0 \\
0.0 \\
1.1 \\
5.7 \\
0.6\end{array}$ & $\begin{array}{r}0.0 \\
0.0 \\
3.8 \\
10.6 \\
13.5\end{array}$ & $\begin{array}{l}\quad \text { Nigh } \\
0.0 \\
0.0 \\
3.4 \\
6.9 \\
7.1\end{array}$ & $\begin{array}{l} \\
0.0 \\
0.0 \\
2.9 \\
7.8 \\
9.0\end{array}$ & $\begin{array}{r}0.0 \\
0.0 \\
0.0 \\
0.0 \\
35.1\end{array}$ \\
\hline $\begin{array}{l}\text { Very Good } \\
\text { Good } \\
\text { Fair } \\
\text { Poor } \\
\text { Very Poor }\end{array}$ & $\begin{array}{r}0.2 \\
4.3 \\
15.8 \\
8.1 \\
8.6\end{array}$ & $\begin{array}{l}\quad \text { Sprin } \\
0.2 \\
1.6 \\
5.8 \\
3.8 \\
2.2\end{array}$ & $\begin{array}{r}\text { ng } \\
0.4 \\
5.9 \\
11.5 \\
5.8 \\
6.9\end{array}$ & $\begin{array}{r}0.0 \\
0.0 \\
0.5 \\
2.4 \\
15.9\end{array}$ & $\begin{array}{r}0.5 \\
4.3 \\
13.9 \\
5.4 \\
6.0\end{array}$ & $\begin{array}{l}\quad \text { Summ } \\
0.2 \\
1.7 \\
5.2 \\
4.5 \\
5.8\end{array}$ & $\begin{array}{r}\text { er } \\
0.4 \\
5.0 \\
11.7 \\
5.8 \\
4.5\end{array}$ & $\begin{array}{r}0.0 \\
0.0 \\
0.8 \\
5.4 \\
18.8\end{array}$ \\
\hline $\begin{array}{l}\text { Very Good } \\
\text { Good } \\
\text { Fair } \\
\text { Poor } \\
\text { Very Poor }\end{array}$ & $\begin{array}{r}0.0 \\
1.7 \\
10.2 \\
5.4 \\
8.4\end{array}$ & $\begin{array}{l}\quad \text { Fall } \\
0.0 \\
1.6 \\
7.9 \\
4.9 \\
4.1\end{array}$ & $\begin{array}{r}0.0 \\
2.1 \\
15.9 \\
5.1 \\
5.9\end{array}$ & $\begin{array}{r}0.0 \\
0.0 \\
0.3 \\
0.5 \\
26.0\end{array}$ & $\begin{array}{r}0.0 \\
0.8 \\
19.0 \\
10.4 \\
8.1\end{array}$ & $\begin{array}{l}\quad \text { Wint } \\
0.0 \\
0.5 \\
8.8 \\
4.4 \\
4.3\end{array}$ & $\begin{array}{r}\text { er } \\
0.0 \\
0.5 \\
12.6 \\
4.2 \\
3.3\end{array}$ & $\begin{array}{r}0.0 \\
0.0 \\
0.2 \\
1.3 \\
21.4\end{array}$ \\
\hline
\end{tabular}


Table 8.16

Cape Kennedy, Florida

Percent of Hours in Each Combination of Wind Direction and Diffusion Rating by Season and Time of Day

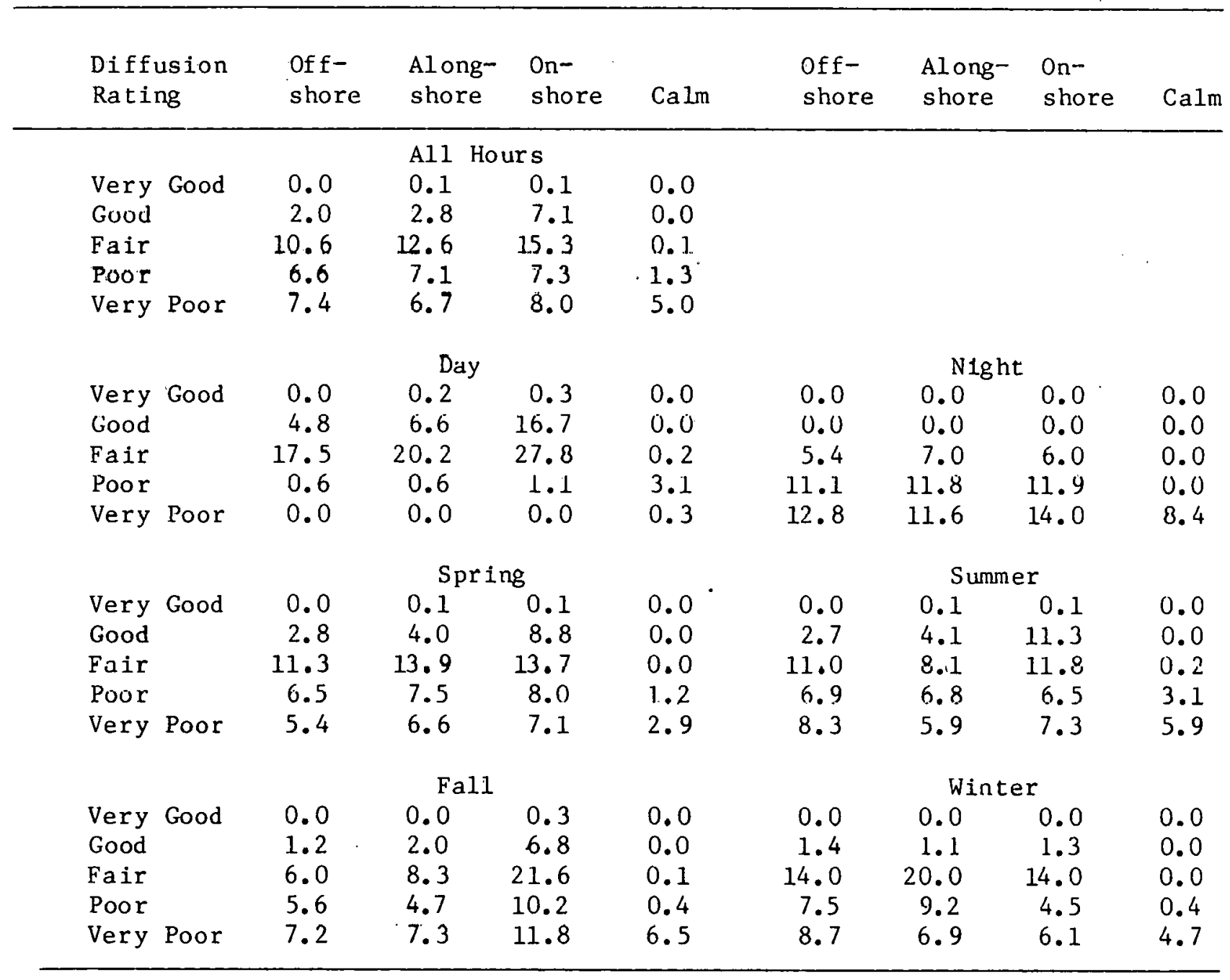




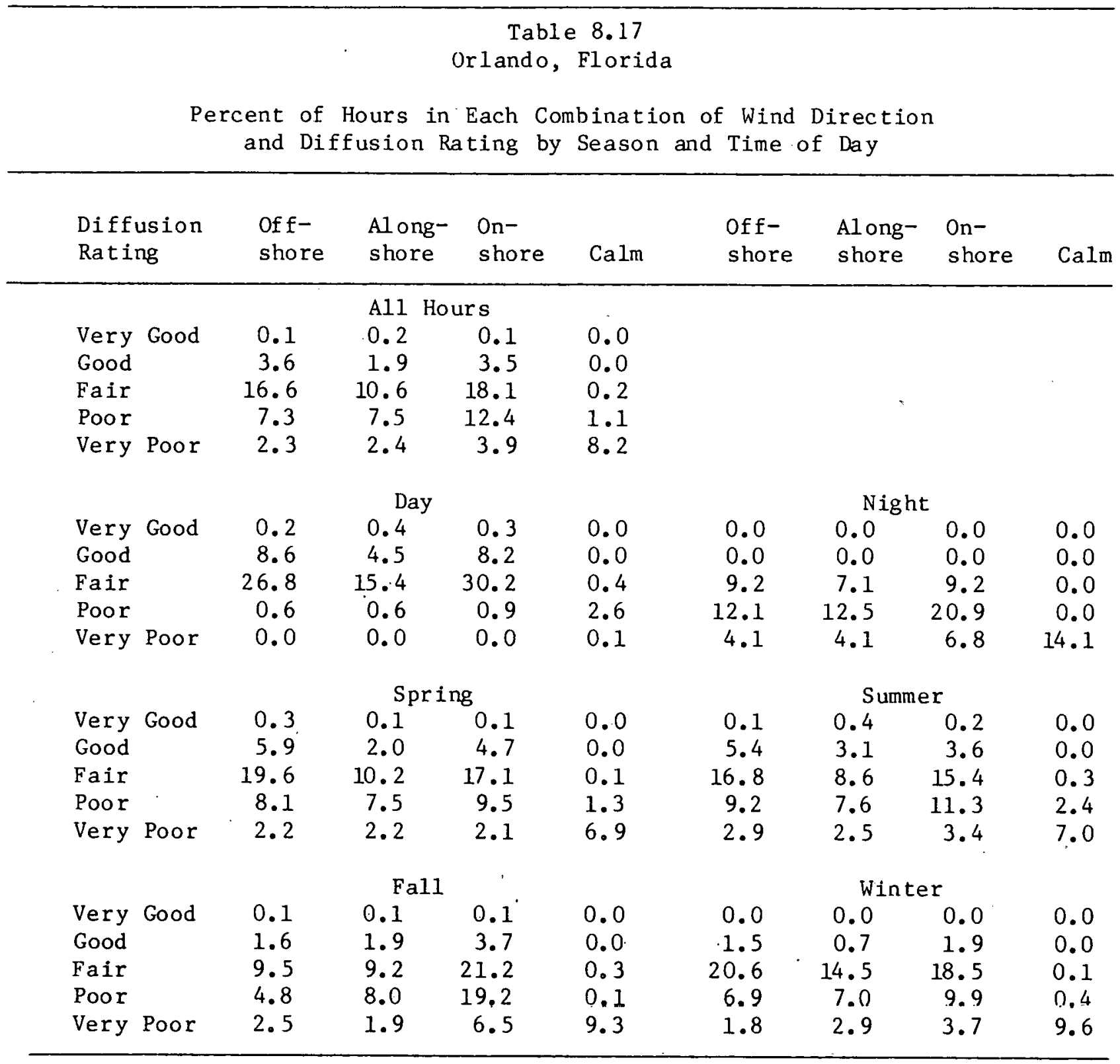


Table 8.18

Miami, Florida

Percent of Hours in Each Combination of Wind Direction and Diffusion Ratiing: by, Sèason and Time. of Day

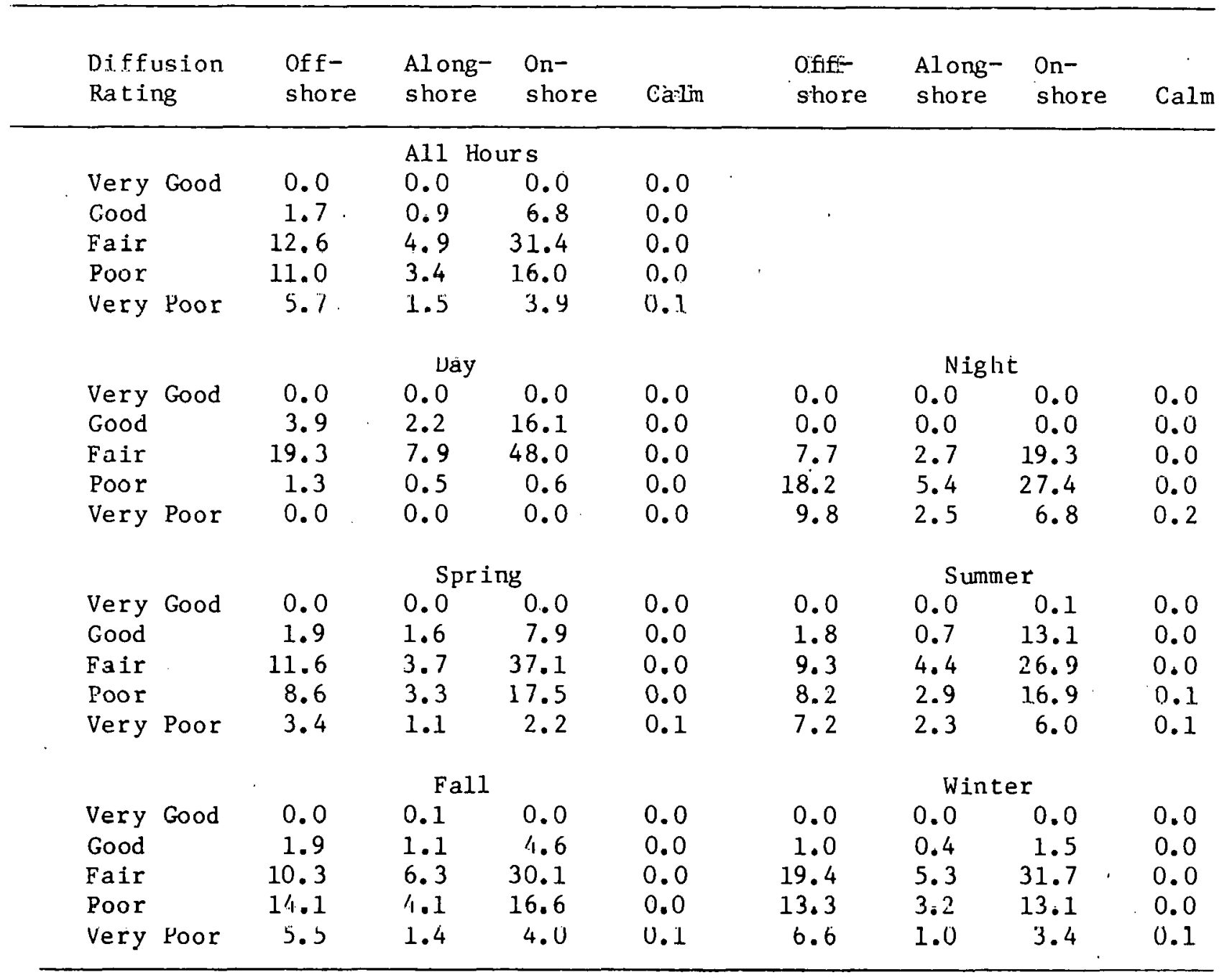




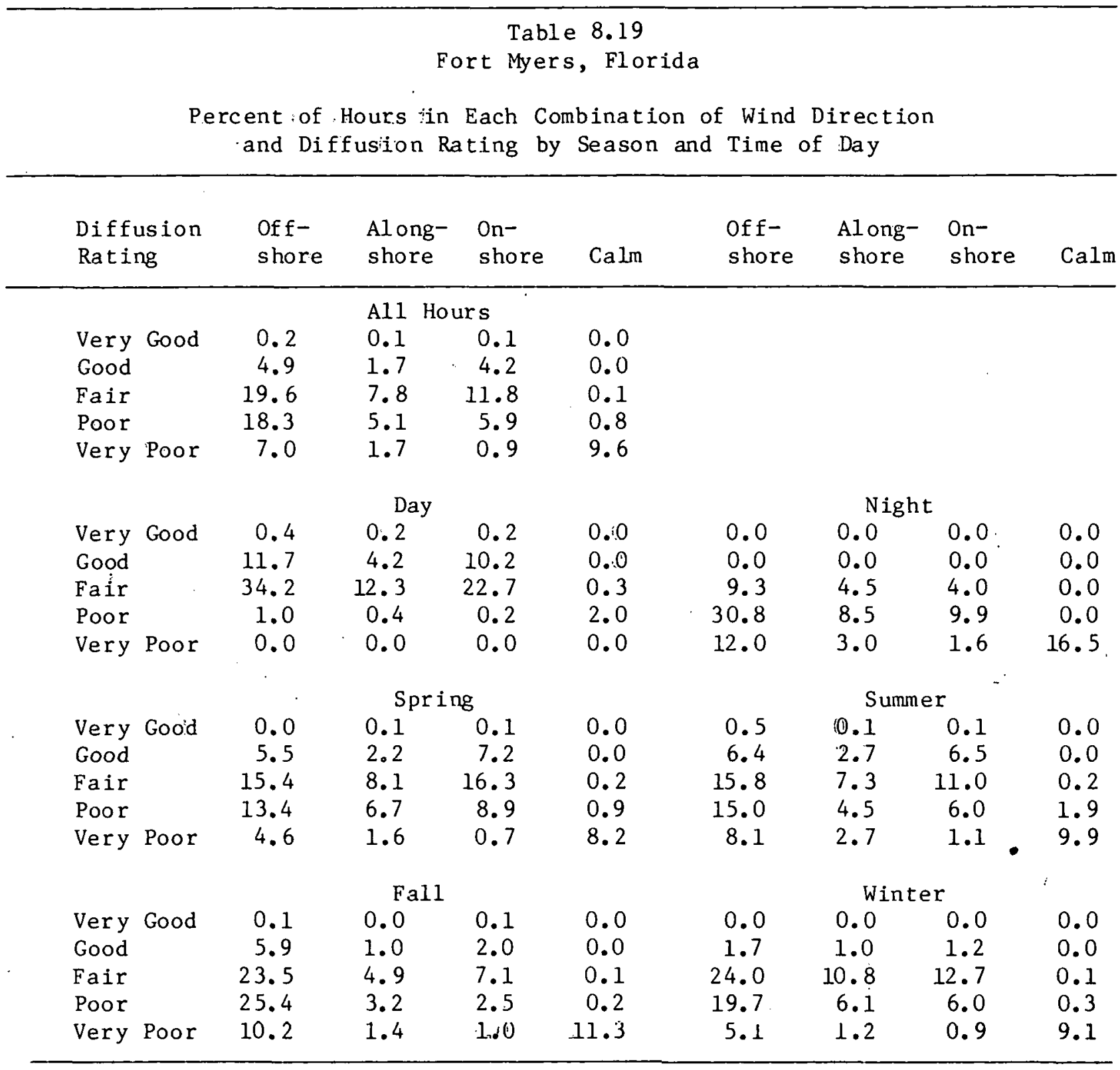




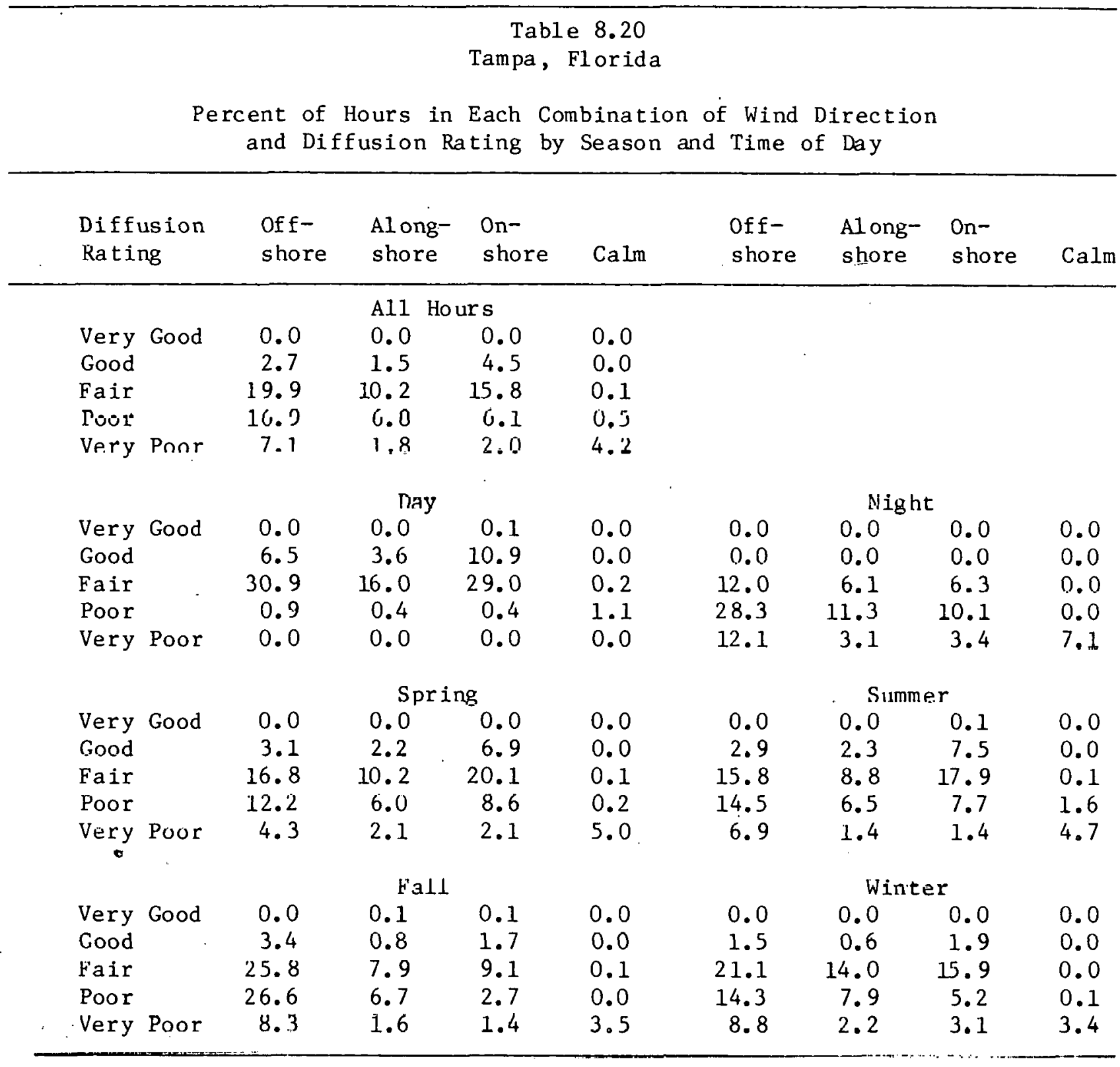


Table 8.21

Tallahassee, Florida

Percent of Hours in Each Combination of Wind Direction and Diffusion Rating by Season and Time of Day

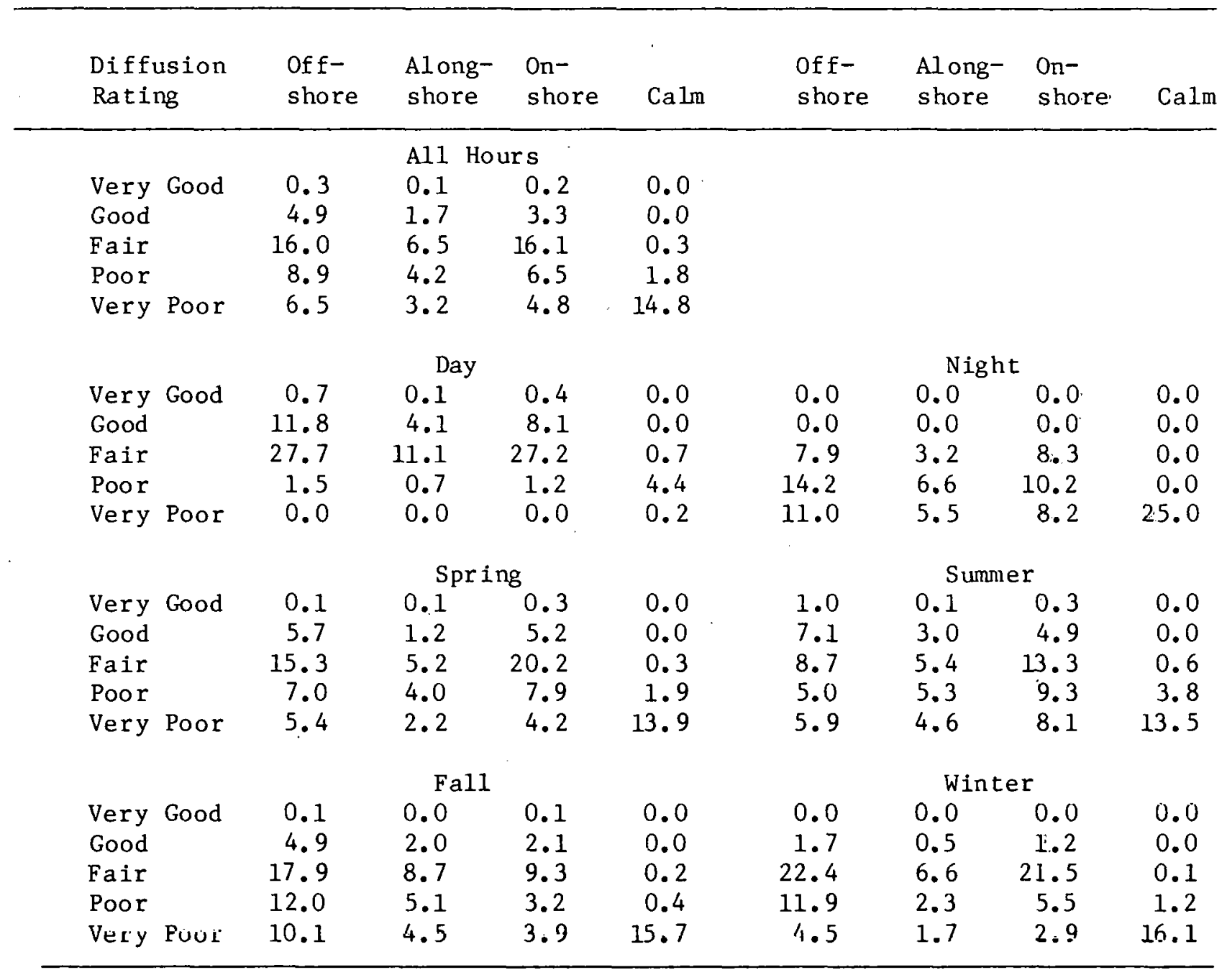




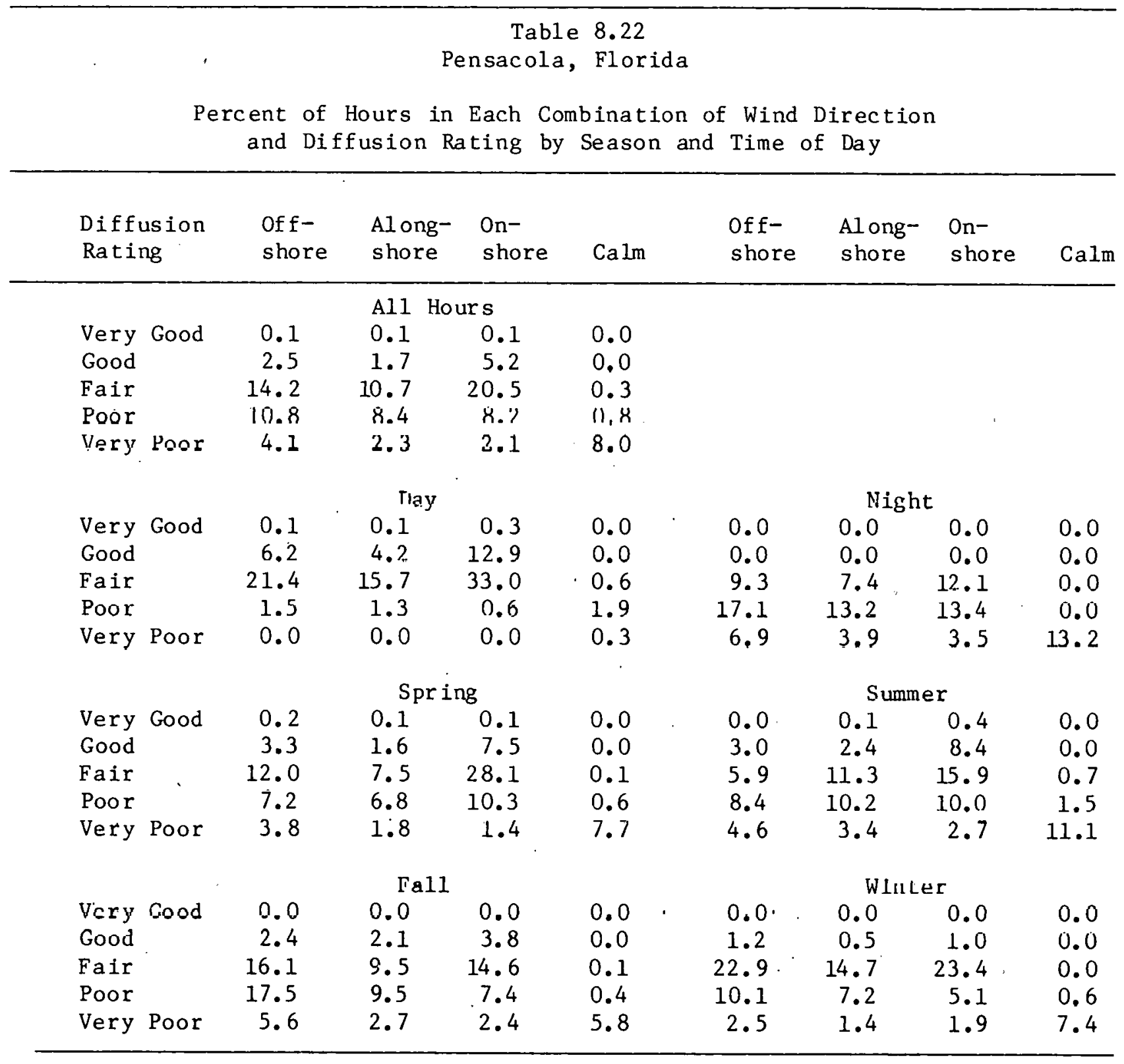




\begin{tabular}{|c|c|c|c|c|c|c|c|c|}
\hline \multicolumn{9}{|c|}{$\begin{array}{c}\text { Table } 8.23 \\
\text { Mobile, Alabama }\end{array}$} \\
\hline \multicolumn{9}{|c|}{$\begin{array}{c}\text { Percent of Hours in Each Combination of Wind Direction } \\
\text { and Diffusion Rating by Season and Time of Day }\end{array}$} \\
\hline $\begin{array}{l}\text { Diffusion } \\
\text { Rating }\end{array}$ & $\begin{array}{l}\text { Off- } \\
\text { shore }\end{array}$ & $\begin{array}{l}\text { Along- } \\
\text { shore }\end{array}$ & $\begin{array}{l}\text { On- } \\
\text { shore }\end{array}$ & Calm & $\begin{array}{l}\text { Off- } \\
\text { shore }\end{array}$ & $\begin{array}{l}\text { Alon } \\
\text { shor }\end{array}$ & $\begin{array}{l}\text { On- } \\
\text { shore }\end{array}$ & $\mathrm{Calm}$ \\
\hline \multicolumn{9}{|c|}{ All Hours } \\
\hline Very Good & 0.1 & 0.1 & 0.1 & 0.0 & & & & \multirow{5}{*}{ - } \\
\hline Good & 2.6 & 1.8 & 2.1 & 0.0 & & & & \\
\hline Fair & 20.1 & 10.0 & 21.5 & 0.1 & & & & \\
\hline Poor & 11.1 & 5.4 & 11.7 & 0.4 & & & & \\
\hline \multirow[t]{2}{*}{ Very Poor } & 3.5 & 2.2 & 3.4 & 3.7 & & & & \\
\hline & & Day & & & \multicolumn{3}{|c|}{ Night } & \\
\hline Very Good & 0.2 & 0.2 & 0.2 & 0.0 & 0.0 & 0.0 & 0.0 & 0.0 \\
\hline Good & 6.5 & 4.6 & 5.3 & 0.0 & 0.0 & 0.0 & 0.0 & 0.0 \\
\hline Fair & 29.1 & 17.4 & 31.6 & 0.3 & 14.1 & 5.1 & 14.7 & 0.0 \\
\hline Poor & 1.2 & 0.9 & 1.5 & 0.9 & 17.7 & 8.4 & 18.6 & 0.0 \\
\hline \multirow[t]{2}{*}{ Very Poor } & 0.0 & 0.0 & 0.0 & 0.0 & 5.8 & 3.7 & 5.7 & 6.1 \\
\hline & \multicolumn{4}{|c|}{ Spring } & \multicolumn{4}{|c|}{ Summer } \\
\hline Very Good & 0.0 & 0.1 & 0.1 . & 0.0 & 0.3 & 0.1 & 0.1 & 0.0 \\
\hline Good & 2.8 & 1.9 & 3.9 & 0.0 & 4.2 & 3.2 & 3.1 & 0.0 \\
\hline Fair & 19.2 & 7.5 & 29.6 & 0.1 & 10.2 & 10.6 & 17.3 & 0.0 \\
\hline Poor & 9.0 & 4.9 & 12.8 & 0.2 & 10.5 & 8.1 & 16.8 & 0.5 \\
\hline \multirow[t]{2}{*}{ Very Poor } & 1.9 & 1.4 & 3.1 & 1.4 & 3.5 & 4.1 & 4.8 & 2.6 \\
\hline & \multicolumn{3}{|c|}{ Fal.1. } & & \multicolumn{4}{|c|}{ Winter } \\
\hline Very Good & 0.0 & 0.1 & 0.0 & 0.0 & 0.0 & 0.0 & 0.0 & 0.0 \\
\hline Good & 2.6 & 2.1 & 0.8 & 0.0 & 0.8 & 0.1 & 0.6 & 0.0 \\
\hline Fair & 20.6 & 11.9 & 11.6 & 0.3 & 30.8 & 10.1 & 27.4 & 0.1 \\
\hline Poor & 15.5 & 5.6 & 8.0 & 0.5 & 9.4 & 3.1 & 9.2 & 0.3 \\
\hline Very foor & 6.3 & 2.7 & 3.6 & $\% . \dot{y}$ & 2.3 & 0.8 & 2.2 & 2.8 \\
\hline
\end{tabular}


Table 8.24

Biloxi, Mississippi

Percent of Hours in Each Combination of Wind Direction and Diffusion Rating by Season and Time of Day

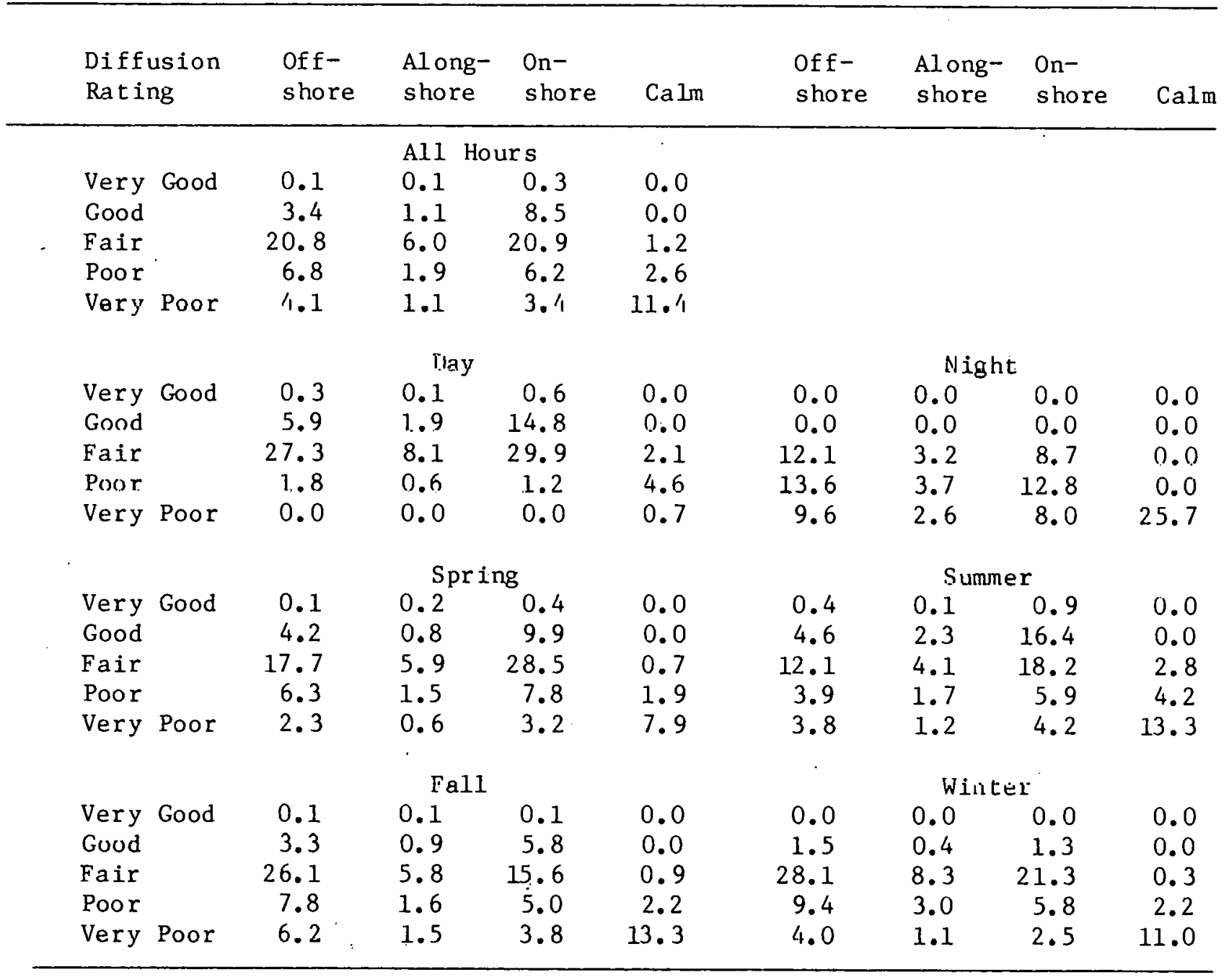


Table 8.25

New Orleans, Louisiana

Percent of Hours in Each Combination of Wind Direction and Diffusion Rating by Season and Time of Day

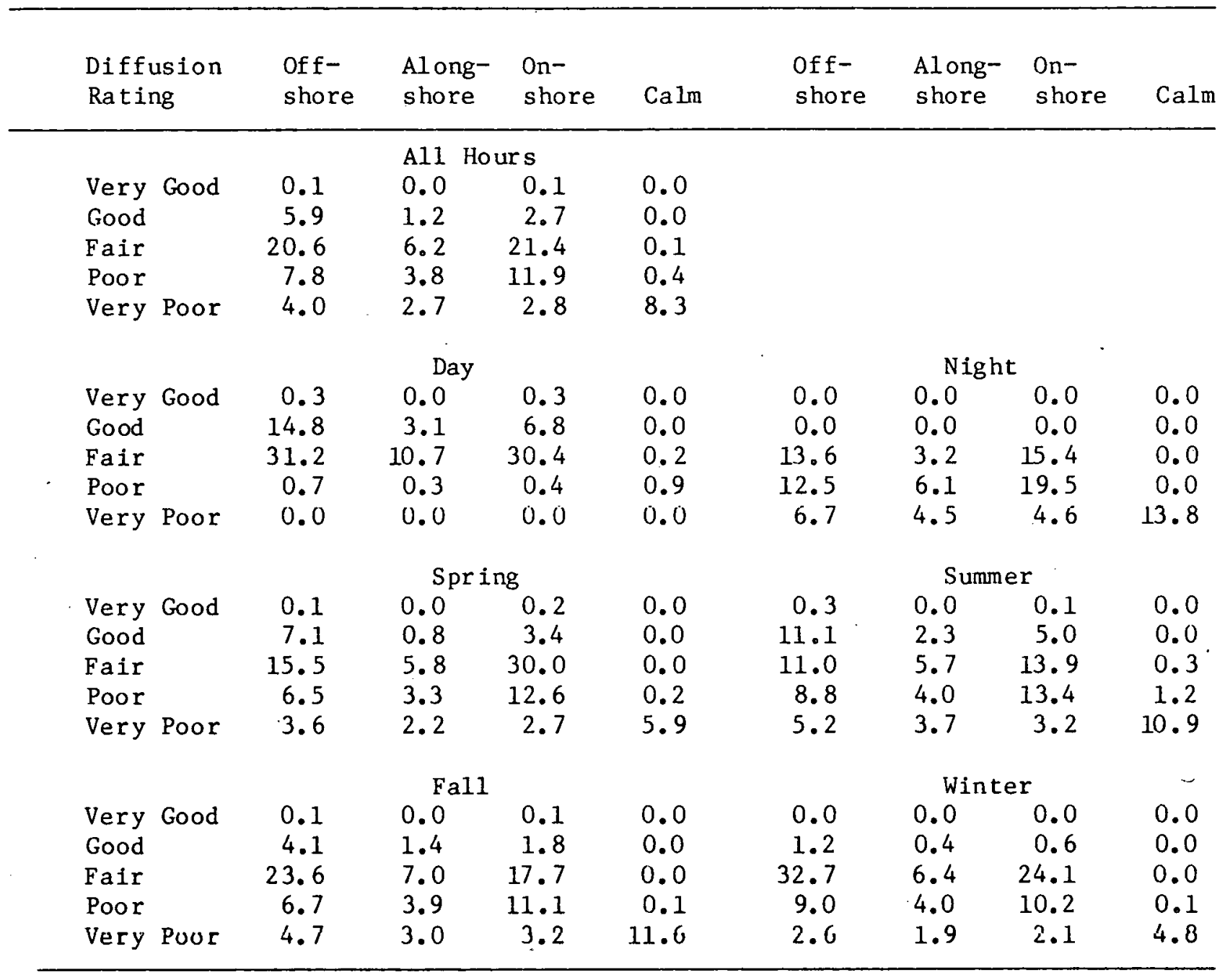




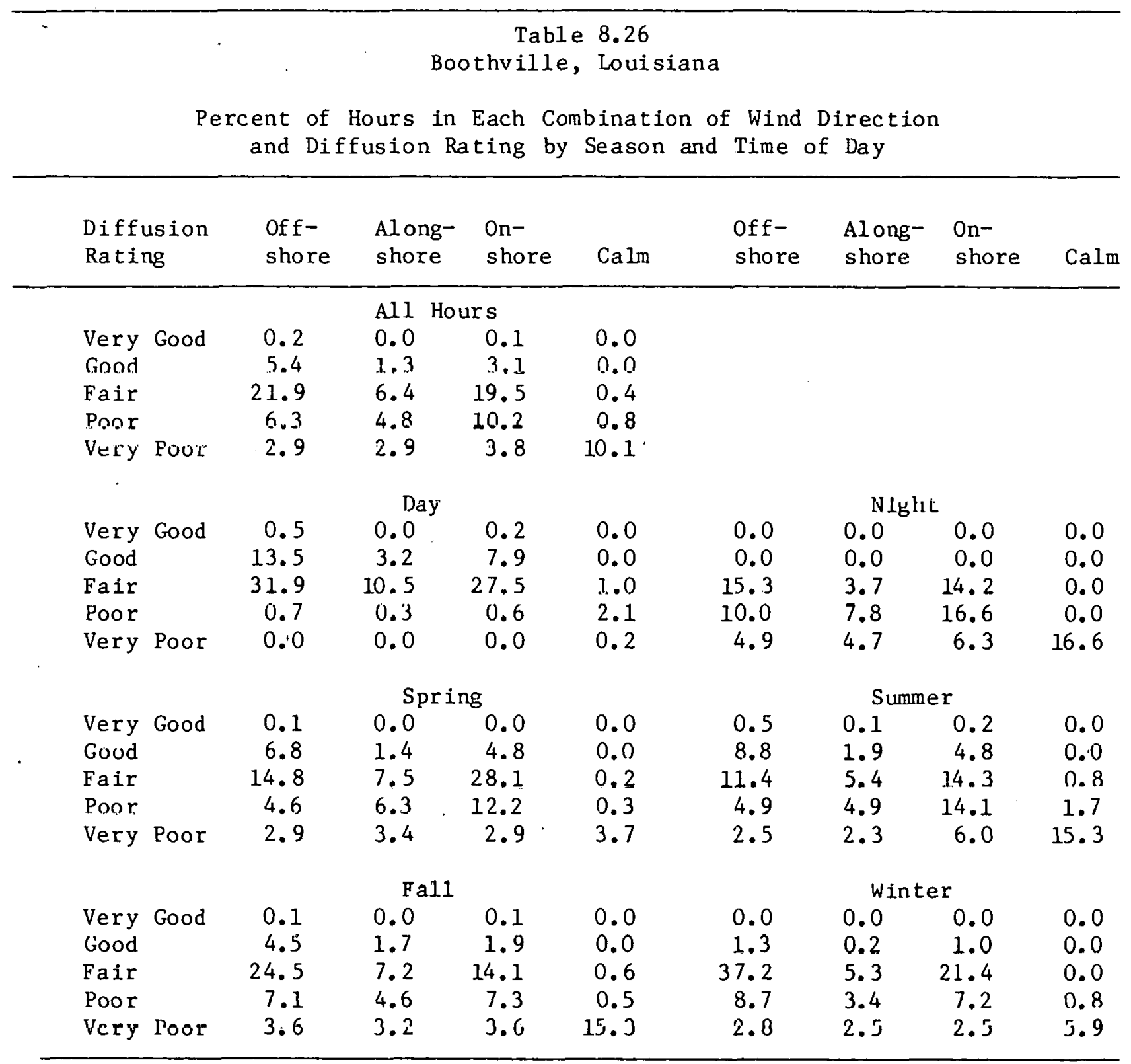




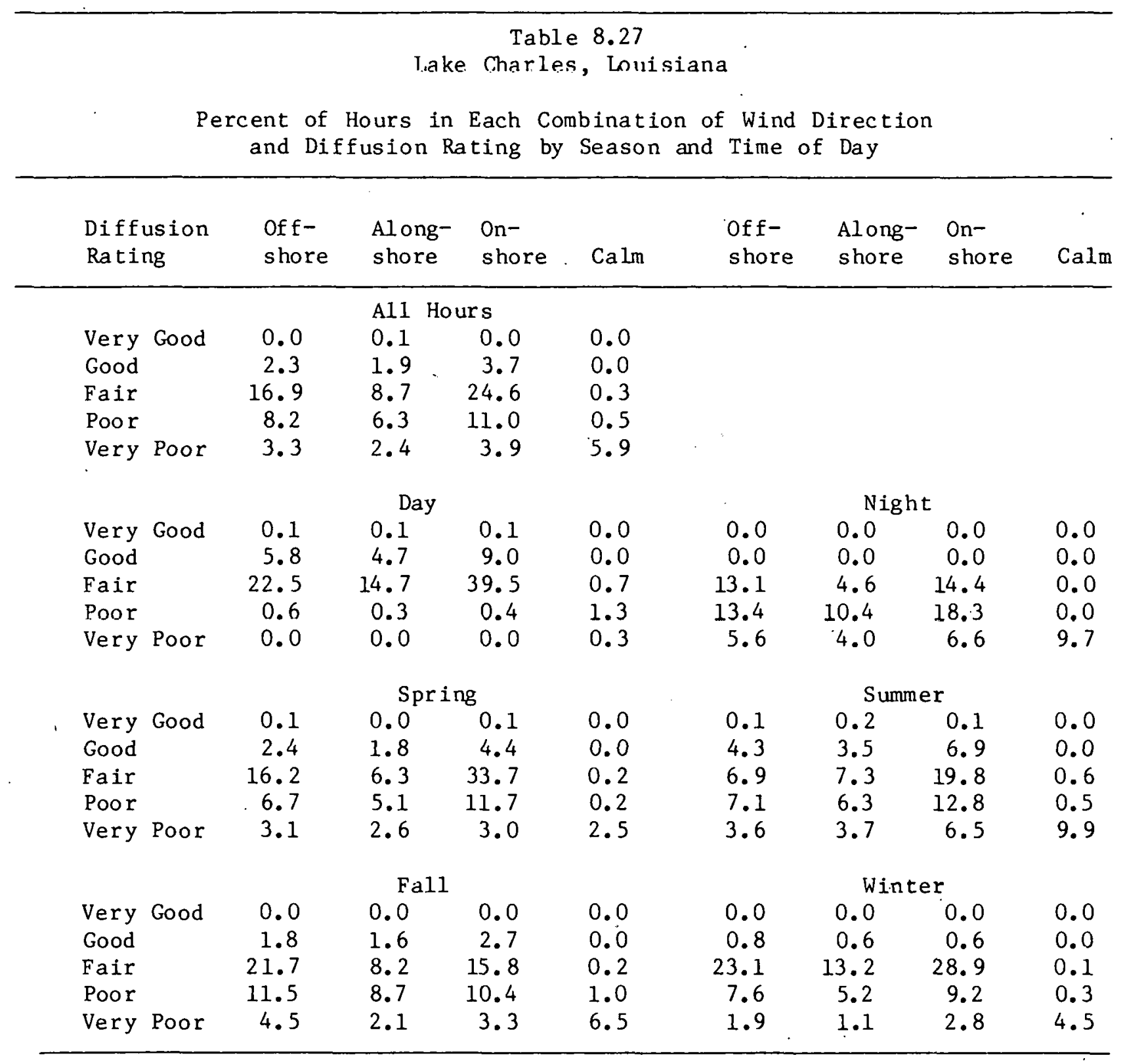




\begin{tabular}{|c|c|c|c|c|c|c|c|c|}
\hline \multicolumn{9}{|c|}{ Galvesto } \\
\hline \multicolumn{9}{|c|}{$\begin{array}{c}\text { Percent of Hours in Each Combination of Wind Direction } \\
\text { and Diffusion Rating by Season and Time of Day }\end{array}$} \\
\hline $\begin{array}{l}\text { Diffusion } \\
\text { Rating }\end{array}$ & $\begin{array}{l}\text { Off- } \\
\text { shore }\end{array}$ & $\begin{array}{l}\text { Along- } \\
\text { shore }\end{array}$ & $\begin{array}{l}\text { On- } \\
\text { shore }\end{array}$ & Calm & $\begin{array}{l}\text { Off- } \\
\text { shore }\end{array}$ & $\begin{array}{l}\text { Along- } \\
\text { shore }\end{array}$ & $\begin{array}{l}\text { On- } \\
\text { shore }\end{array}$ & Calm \\
\hline \multicolumn{9}{|c|}{ A11 Hours } \\
\hline Very Good & 0.1 & 0.0 & 0.2 & 0.0 & & & & \\
\hline Good & 1.9 & 1.1 & 10.5 & 0.0 & & & & \\
\hline Fair & 16.9 & 8.7 & 40.1 & 0.0 & & & & \\
\hline Poor & 3.3 & 2.0 & 10.4 & 0.0 & & & & \\
\hline Very & 1.1 & 1.4 & 2.2 & 0.0 & & & & \\
\hline & \multicolumn{4}{|c|}{ Day } & \multicolumn{4}{|c|}{ Night } \\
\hline Very Good & 0.1 & 0.1 & 0.3 & 0.0 & 0.0 & 0.0 & 0.0 & 0.0 \\
\hline Good & 3.3 & 1.8 & 18.2 & 0.0 & 0.0 & 0.0 & 0.0 & 0.0 \\
\hline Fair & 18.5 & 9.8 & 46.5 & 0.0 & 14.8 & 7.1 & 30.9 & 0.0 \\
\hline Poor & 0.5 & 0.3 & 0.6 & 0.0 & 7.0 & 4.5 & 24.1 & 0.0 \\
\hline Very & 0.0 & 0.0 & 0.0 & 0.0 & 2.6 & 3.5 & 5.3 & 0.1 \\
\hline & \multicolumn{4}{|c|}{ Spring } & \multicolumn{4}{|c|}{ Summer } \\
\hline Very Good & 0.0 & 0.0 & 0.1 & 0.0 & 0.2 & 0.1 & 0.4 & 0.0 \\
\hline Good & 2.0 & 0.7 & 10.8 & 0.0 & 2.5 & 1.6 & 22.0 & 0.0 \\
\hline Fair & 12.6 & 4.4 & 53.0 & 0.0 & 6.5 & 5.8 & 41.4 & 0.0 \\
\hline Poor & 3.2 & 1.3 & 8.7 & 0.0 & 1.9 & 1.4 & 12.5 & 0.0 \\
\hline \multirow[t]{2}{*}{ Very Poor } & 0.7 & 0.7 & 1.8 & 0.0 & 0.6 & $1 . \%$ & 2.0 & 0.0 \\
\hline & \multicolumn{3}{|c|}{ Fal1 } & & \multicolumn{4}{|c|}{ Winter } \\
\hline Very Good & 0.0 & 0.1 & 0.1 & 0.0 & 0.0 & 0.0 & 0.0 & 0.0 \\
\hline Good & 2.4 & 1.5 & 8.3 & 0.0 & 0.8 & 0.5 & $1.5^{\circ}$ & 0.0 \\
\hline Fair & 19.7 & 13.0 & 32.9 & 0.0 & 29.0 & 12.2 & 31.5 & 0.0 \\
\hline Poor & 2.8 & 2.5 & 11.0 & 0.0 & 5.0 & 3.0 & 9.8 & 0.0 \\
\hline Very Poor & 0.9 & 1.4 & 3.4 & 0.1 & 2.2 & 2.4 & 2.0 & 0.0 \\
\hline
\end{tabular}


Table 8.29

Houston, Texas

Percent of Hours in Each Combination of Wind Direction and Diffusion Rating by Season and Time of Day

\begin{tabular}{|c|c|c|c|c|c|c|c|c|}
\hline $\begin{array}{l}\text { Diffusion } \\
\text { Rating }\end{array}$ & $\begin{array}{l}\text { Off- } \\
\text { shore }\end{array}$ & $\begin{array}{l}\text { Along- } \\
\text { shore }\end{array}$ & $\begin{array}{l}\text { On- } \\
\text { shore }\end{array}$ & $\mathrm{Calm}$ & $\begin{array}{l}\text { Off- } \\
\text { shore }\end{array}$ & $\begin{array}{l}\text { Along- } \\
\text { shore }\end{array}$ & $\begin{array}{l}\text { On- } \\
\text { shore }\end{array}$ & $\mathrm{Calm}$ \\
\hline \multicolumn{9}{|c|}{ Al1 Hours } \\
\hline Very Good & 0.1 & 0.0 & 0.1 & 0.0 & & & & \\
\hline Good & 1.4 & 1.4 & 3.8 & 0.0 & & & & \\
\hline Fair & 16.3 & 8.5 & 29.1 & 0.0 & & & & \\
\hline Poor & 6.0 & 4.2 & 13.5 & 0.2 & & & & \\
\hline Very Poor & 3.3 & 2.6 & 7.4 & 2.0 & & & & \\
\hline \multicolumn{5}{|c|}{ Day } & \multicolumn{4}{|c|}{ Night } \\
\hline Very Good & 0.1 & 0.1 & 0.3 & 0.0 & 0.0 & 0.0 & 0.0 & 0.0 \\
\hline Good & 3.3 & 3.3 & 3.9 & 0.0 & 0.0 & 0.0 & 0.0 & 0.0 \\
\hline Fair & 21.7 & 13.5 & 46.1 & 0.1 & 12.3 & 4.7 & 16.5 & 0.0 \\
\hline Poor & 0.6 & 0.4 & 0.8 & 0.6 & 10.0 & 7.0 & 22.9 & 0.0 \\
\hline \multirow[t]{2}{*}{ Very Poor } & 0.0 & 0.0 & 0.0 & 0.1 & 5.8 & 4.4 & 12.9 & 3.5 \\
\hline & \multicolumn{4}{|c|}{ Spring } & \multicolumn{4}{|c|}{ Summer } \\
\hline Very Good & 0.0 & $0.0^{\circ}$ & 0.1 & 0.0 & 0.2 & 0.2 & 0.4 & 0.0 \\
\hline Good & 1.4 & 0.8 & 5.3 & 0.0 & 2.0 & 3.2 & 7.0 & 0.0 \\
\hline Fair & 13.5 & 5.1 & 41.4 & 0.0 & 6.7 & 7.5 & 25.9 & 0.1 \\
\hline Poor & 5.1 & 2.2 & 13.7 & 0.1 & 3.2 & 4.2 & 15.7 & 0.6 \\
\hline \multirow[t]{2}{*}{ Very Poor } & 2.2 & 1.4 & 5.7 & 1.9 & 3.9 & 4.0 & 12.6 & 2.6 \\
\hline & \multicolumn{4}{|c|}{ Fall } & \multicolumn{4}{|c|}{ Winter } \\
\hline Very Good & 0.0 & 0.0 & 0.0 & 0.0 & 0.0 & 0.0 & 0.0 & 0.0 \\
\hline Good & 1.4 & 1.1 & 2.4 & 0.0 & 0.8 & 0.5 & 0.3 & 0.0 \\
\hline Fair & 17.3 & 9.8 & 23.3 & 0.0 & 27.9 & 11.5 & 25.6 & 0.0 \\
\hline Poor & 8.2 & 5.2 & 14.0 & 0.0 & 7.7 & 5.2 & 10.6 & 0.2 \\
\hline Very Poor & 4.5 & 2.9 & 7.8 & 2.1 & 2.7 & 1.9 & 3.7 & 1.5 \\
\hline
\end{tabular}




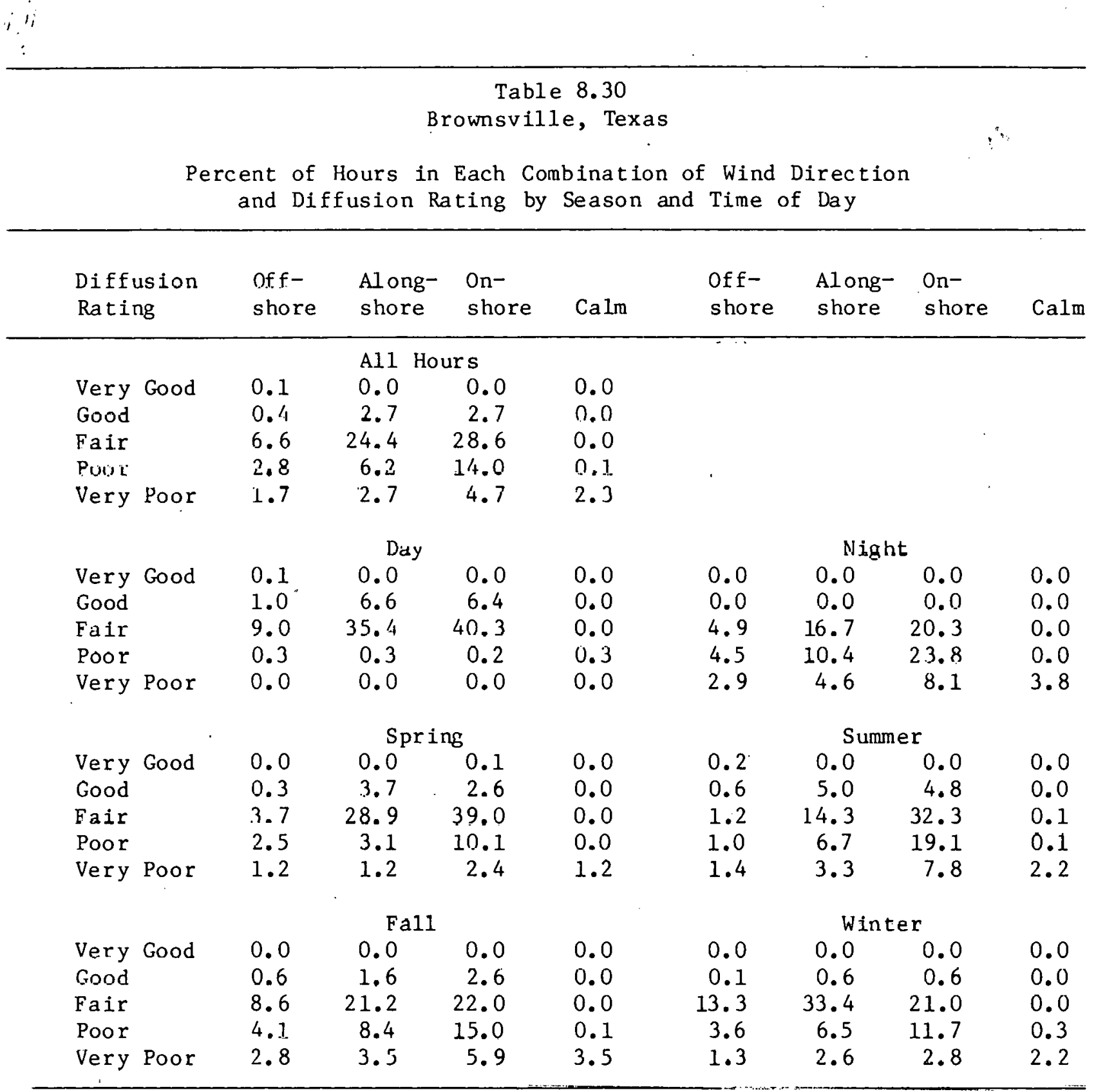




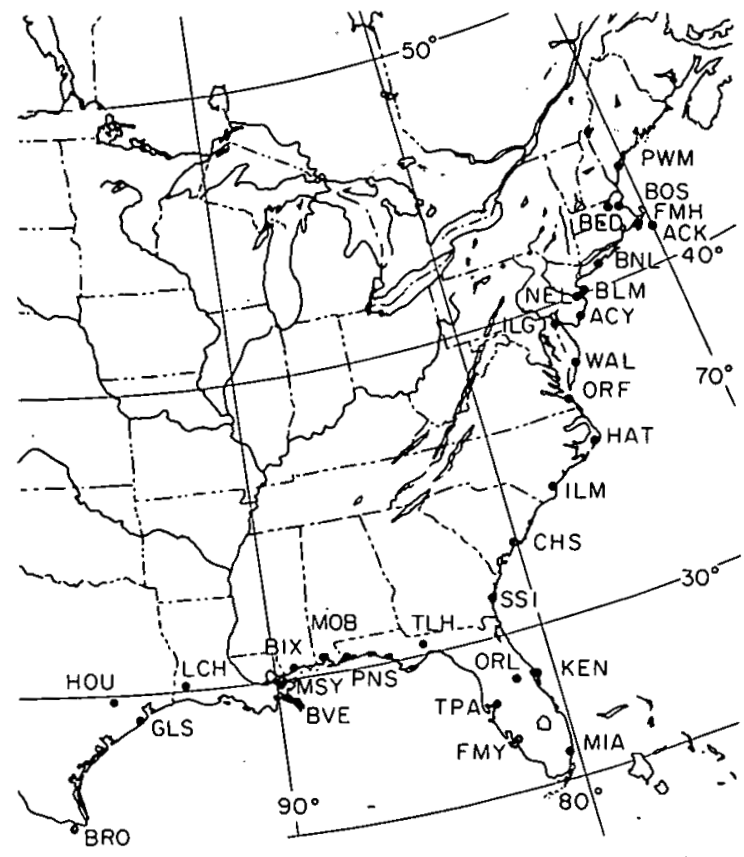

Figure 1. Map of the study area showing Brookhaven National Laboratory (BNL) and the stations from which data were used. Station names are given in Table 1

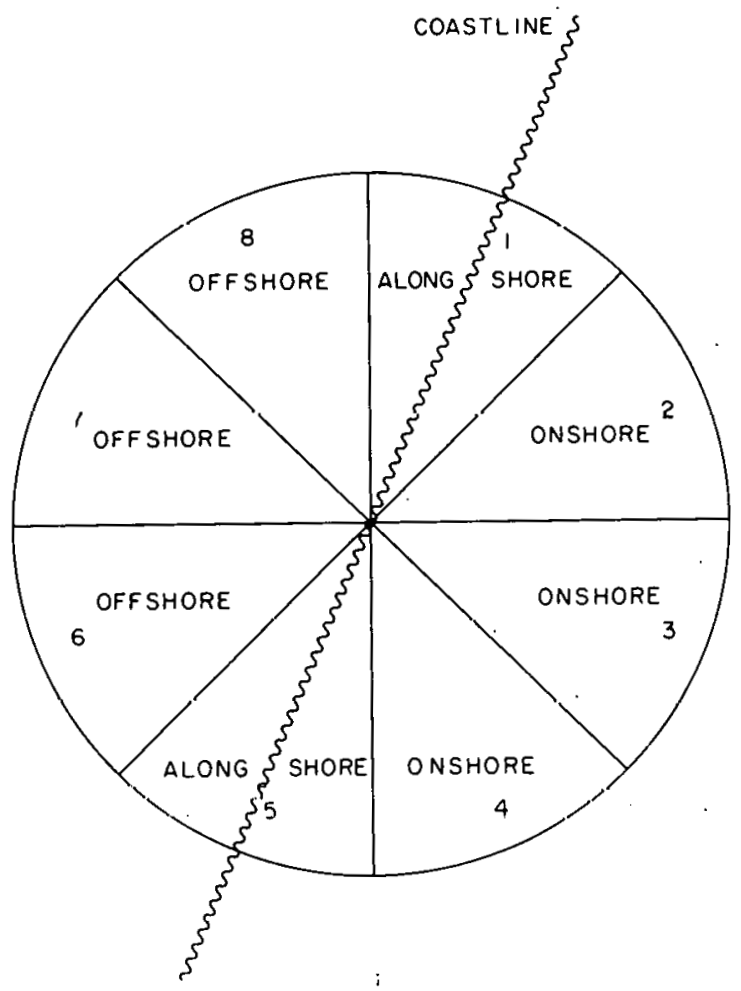

Figure 2. Numbcr and decignation of wind direction sectors relative to the coastline 